$Y-12$

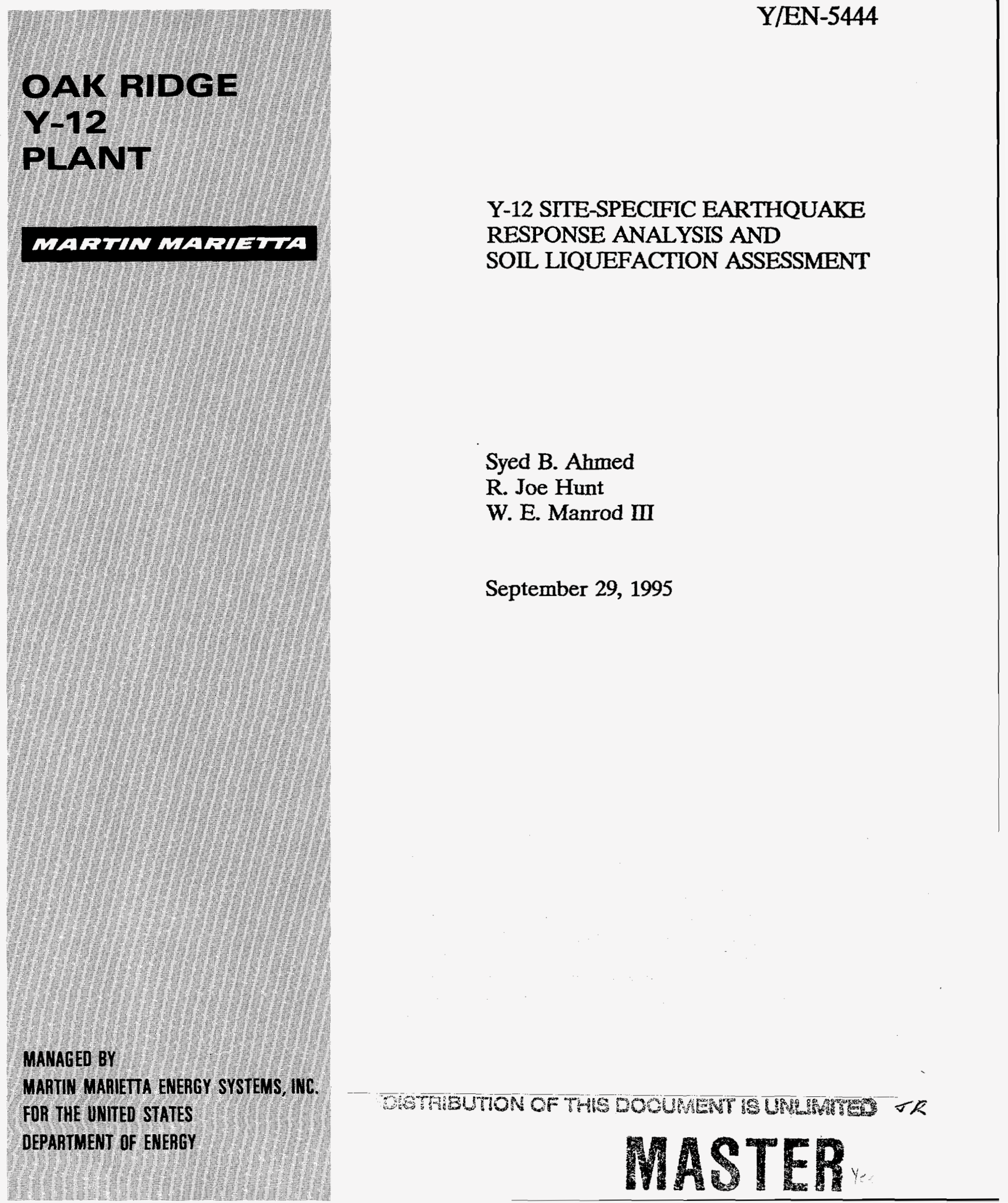




\section{DISCLAIMER}

This report was prepared as an account of work sponsored by an agency of the United States Government. Neither the United States Government nor any agency thereof, nor any of their employees, makes any warranty, express or implied, or assumes any legal liability or responsibility for the accuracy, completeness, or usefulness of any information, apparatus, product, or process disclosed, or represents that its use would not infringe privately owned rights. Reference herein to any specific commercial product, process, or service by trade name, trademark, manufacturer, or otherwise, does not necessarily constitute or imply its endorsement, recommendation, or favoring by the United States Government or any agency thereof. The views and opinions of authors expressed herein do not necessarily state or reflect those of the United States Government or any agency thereof. 


\section{DISCLAMMER}

Portions of this document may be illegible in electronic image products. Images are produced from the best available original document. 
Central Engineering Services Division

\title{
Y-12 SITE-SPECIFIC EARTHQUAKE RESPONSE ANALYSIS AND SOIL LIQUEFACTION ASSESSMENT
}

Date Issued--September 1995

\author{
Prepared by \\ Central Engineering Services \\ Oak Ridge K-25 Site \\ Oak Ridge, Tennessee \\ operated by
}

LOCKHEED MARTIN ENERGY SYSTEMS, INC. for the

U.S. DEPARTMENT OF ENERGY under contract DE-AC05-84OR21400 
FIGURES $\ldots \ldots \ldots \ldots \ldots \ldots \ldots \ldots \ldots \ldots \ldots \ldots \ldots \ldots \ldots \ldots \ldots \ldots$ iv

TABLES $\ldots \ldots \ldots \ldots \ldots \ldots \ldots \ldots \ldots \ldots \ldots \ldots \ldots \ldots \ldots \ldots \ldots \ldots \ldots \ldots \ldots$

SUMMARY AND CONCLUSIONS $\ldots \ldots \ldots \ldots \ldots \ldots \ldots \ldots \ldots \ldots \ldots \ldots \ldots \ldots \ldots$

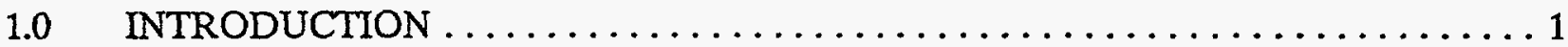

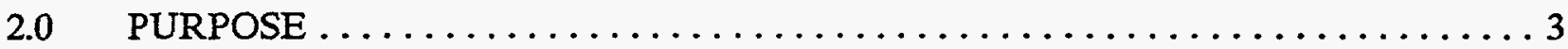

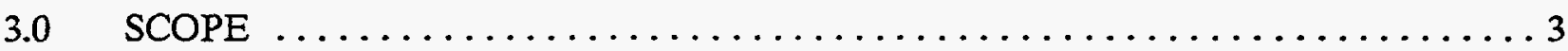

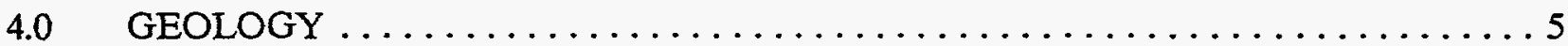

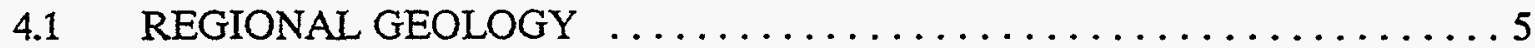

4.2 AREA GEOLOGY $\ldots \ldots \ldots \ldots \ldots \ldots \ldots \ldots \ldots \ldots \ldots \ldots \ldots$

$5.0 \quad$ SEISMOLOGY $\ldots \ldots \ldots \ldots \ldots \ldots \ldots \ldots \ldots \ldots \ldots \ldots \ldots \ldots \ldots \ldots$

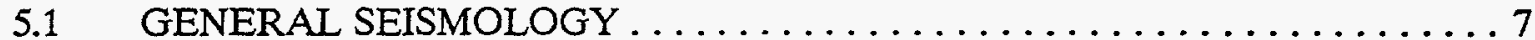

5.2 EARTHQUAKES AFFECTING THE OAK RIDGE RESERVATION $\ldots \ldots 10$

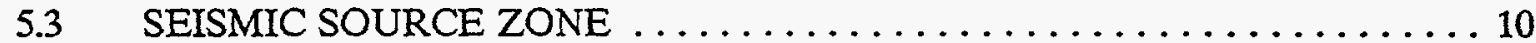

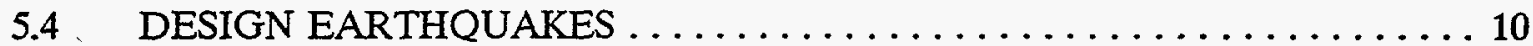

6.0 GEOTECHNICAL INVESTIGATIONS $\ldots \ldots \ldots \ldots \ldots \ldots \ldots \ldots \ldots \ldots \ldots$

6.1 PREPARATION OF DRAWINGS $\ldots \ldots \ldots \ldots \ldots \ldots \ldots \ldots \ldots \ldots$

6.2 SUBSURFACE PROFILES $\ldots \ldots \ldots \ldots \ldots \ldots \ldots \ldots \ldots \ldots \ldots \ldots \ldots$

6.3 MATERIAL PROPERTIES ........................ 19

7.0 SITE-SPECIFIC EARTHQUAKE RESPONSE ANALYSIS $\ldots \ldots \ldots \ldots \ldots \ldots \ldots 24$

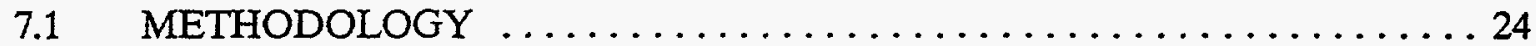

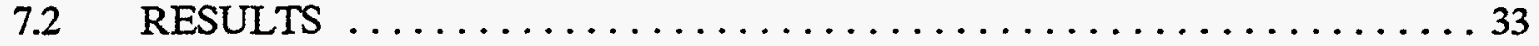

8.0 DESIGN RESPONSE SPECTRA CURVES $\ldots \ldots \ldots \ldots \ldots \ldots \ldots \ldots \ldots \ldots \ldots \ldots$

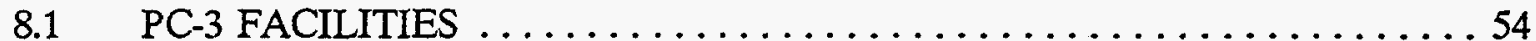

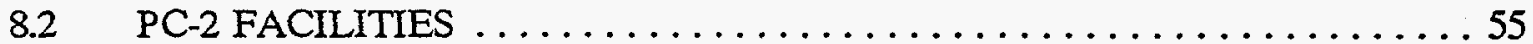

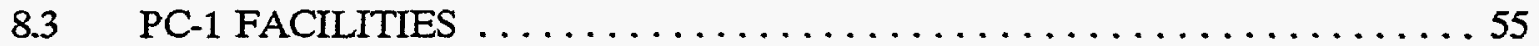

9.0 SOIL LIQUEFACTION ASSESSMENT $\ldots \ldots \ldots \ldots \ldots \ldots \ldots \ldots \ldots \ldots \ldots \ldots \ldots$

9.1 LIQUEFACTION ANAIYYSIS OF SILTS AND CLAYS $\ldots \ldots \ldots \ldots \ldots \ldots 72$

9.2 LIQUEFACTION ANALYSIS OF SANDY SOILS $\ldots \ldots \ldots \ldots \ldots \ldots \ldots \ldots$

9.3 CONCLUSION OF LIQUEFACTION ANALYSIS $\ldots \ldots \ldots \ldots \ldots \ldots \ldots \ldots \ldots$

$10.0 \quad$ REFERENCES $\ldots \ldots \ldots \ldots \ldots \ldots \ldots \ldots \ldots \ldots \ldots \ldots \ldots \ldots \ldots \ldots$ 
CONTENTS (cont'd.)

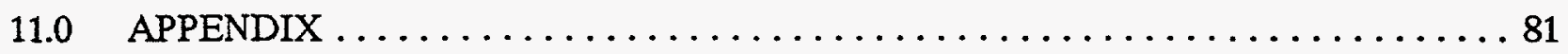

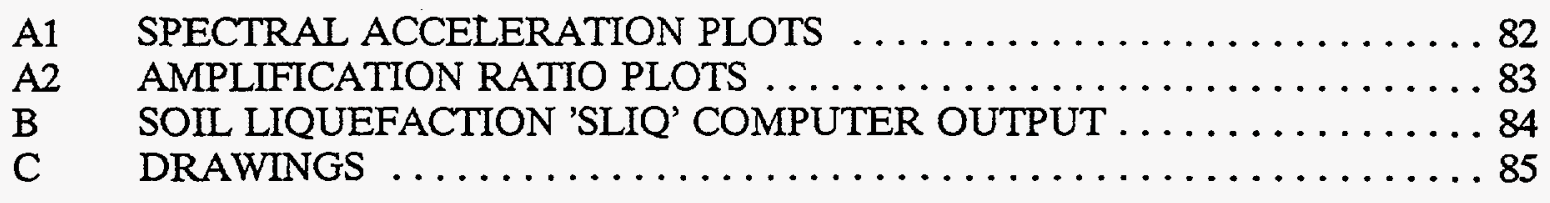




\section{FIGURES}

\section{$\underline{\text { PAGE }}$}

1. Location of $\mathrm{Y}-12$ Plant on Oak Ridge Reservation $\ldots \ldots \ldots \ldots \ldots \ldots \ldots \ldots \ldots \ldots \ldots$

2. Location of Performance Category PC-3 Structures (Considered in the Study) . . . . . 4

3. Location of Ridges, Valleys and Faults on the Oak Ridge Reservation $\ldots \ldots \ldots \ldots \ldots 6$

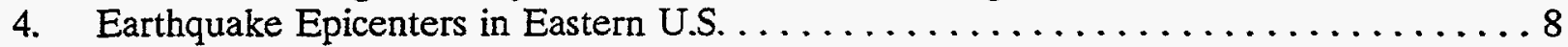

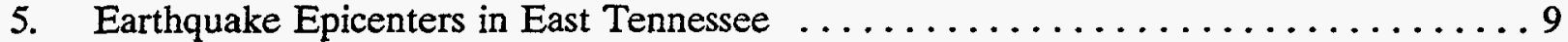

6. Site Specific Earthquake Hazard Curve for Oak Ridge Reservation $\ldots \ldots \ldots \ldots \ldots 12$

7. Oak Ridge - Site Specific Response Spectra for Horizontal Rock Motion ......... 13

8. Oak Ridge Reservation, Y-12 Plant Site, Time History of Acceleration

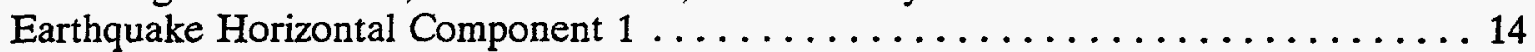

9. Oak Ridge Reservation, Y-12 Plant Site, Time History of Acceleration

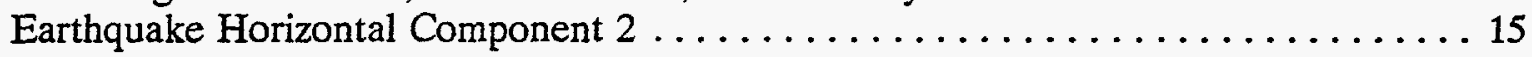

10. Oak Ridge Reservation - Y-12 Plant Site - Horizontal 1 2000-Year Spectrum, 5\% Damping ............................. 16

11. Oak Ridge Reservation - Y-12 Plant Site - Horizontal 2 2000-Year Spectrum, 5\% Damping ............................ 17

12. Representative Soil Columns for SHAKE Analysis .................... 21

13. Shear Wave Velocity Vs. Depth - Weathered Rock (Shale) and Fresh Rock ...... 22

14. Three Primary Control Points for A Site Response Analysis .............. 32

15. Schematic Representation of Soil Response Analysis ................... 34

16. Design Response Spectra for PC-3 Facilities Supported on Fresh Rock . . . . . . . . 60

17. Design Response Spectra for PC-3 Facilities Supported on

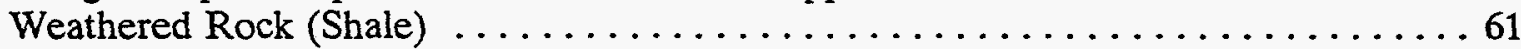

18. Design Response Spectra for PC-3 Facilities Supported on Soil Overburden

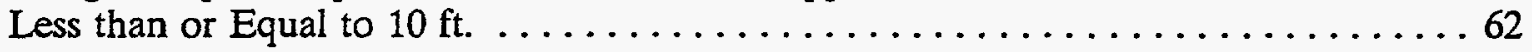

19. Design Response Spectra for PC-3 Facilities Supported on Soil Overburden

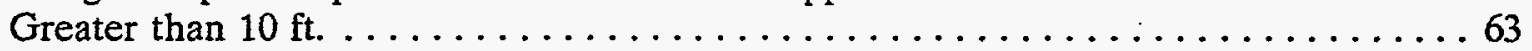

20. Design Response Spectra for PC-2 Facilities Supported on Fresh Rock . . . . . . . . 64

21. Design Response Spectra for PC-2 Facilities Supported on

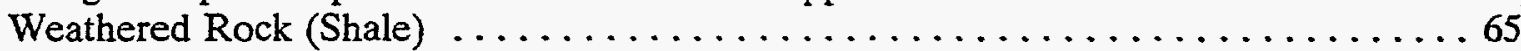

22. Design Response Spectra for PC-2 Facilities Supported on Soil Overburden

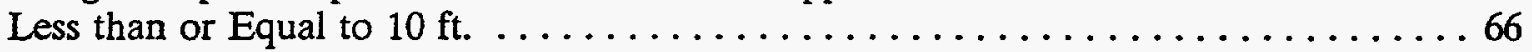

23. Design Response Spectra for PC-2 Facilities Supported on Soil Overburden

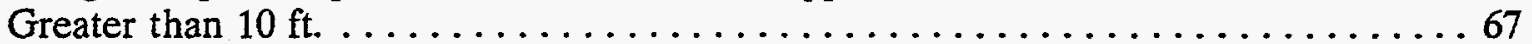

24. Design Response Spectra for PC-1 Facilities Supported on Fresh Rock . . . . . . . 68

25. Design Response Spectra for PC-1 Facilities Supported on Weathered Rock (Shale) $\ldots \ldots \ldots \ldots \ldots \ldots \ldots \ldots \ldots \ldots \ldots \ldots . \ldots \ldots$

26. Design Response Spectra for PC-1 Facilities Supported on Soil Overburden

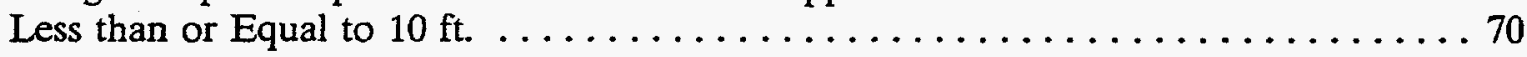

27. Design Response Spectra for PC-1 Facilities Supported on Soil Overburden

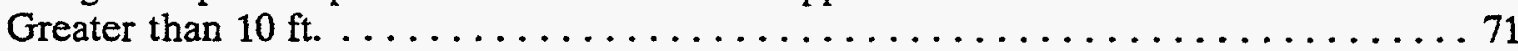

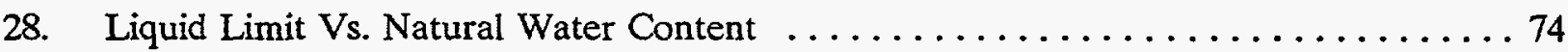

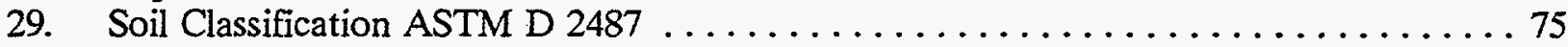


1. Representative Soil Columns used in SHAKE Analysis ............... 20

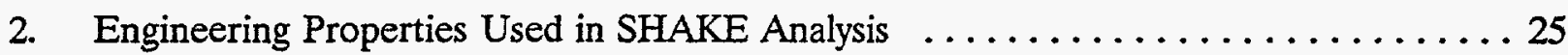

3. Normalized Shear Modulus and Damping Vs. Shear Strain for Soil ........... 26

4. Normalized Shear Modulus and Damping Vs. Shear Strain for Weathered Rock (Shale) and Fresh Rock ..................... 27

5. Index Properties and Soil Classification $\ldots \ldots \ldots \ldots \ldots \ldots \ldots \ldots \ldots \ldots \ldots \ldots \ldots \ldots$

6. Summary of Amplification Ratios for $2 \%$ Damping $\ldots \ldots \ldots \ldots \ldots \ldots \ldots \ldots \ldots$

7. Summary of Amplification Ratios for $5 \%$ Damping ................... 39

8. Summary of Amplification Ratios for $7 \%$ Damping $\ldots \ldots \ldots \ldots \ldots \ldots \ldots \ldots \ldots \ldots$

9. Summary of Amplification Ratios for $10 \%$ Damping $\ldots \ldots \ldots \ldots \ldots \ldots \ldots$

10. Summary of Amplification Ratios for $12 \%$ Damping $\ldots \ldots \ldots \ldots \ldots \ldots \ldots$

11. Summary of Amplification Ratios for $15 \%$ Damping $\ldots \ldots \ldots \ldots \ldots \ldots \ldots \ldots \ldots$

12. Design Response Spectra for PC-3 Facilities Supported on Fresh Rock $\ldots \ldots \ldots \ldots 6$

13. Design Response Spectra for PC-3 Facilities Supported on Weathered (Rock) Shale . 57

14. Design Response Spectra for PC-3 Facilities Supported on Thin Overburden

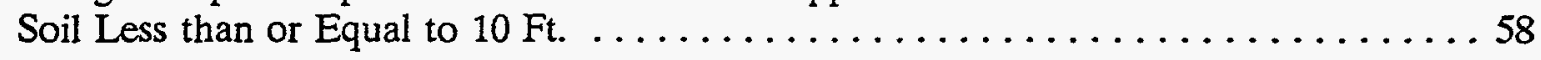

15. Design Response Spectra for PC-3 Facilities Supported on Overburden Soil Greater than $10 \mathrm{Ft}$. 


\section{SUMMARY AND CONCLUSIONS}

A site-specific earthquake response analysis and soil liquefaction assessment were performed for the Oak Ridge Y-12 Plant. The main purpose of these studies was to use the results of the analyses for evaluating the safety of the performance category $-1,-2$, and -3 facilities against the natural phenomena seismic hazards.

Earthquake response was determined for seven (7), one dimensional soil columns (Fig. 12) using two horizontal components of the PC-3 design basis 2000-year seismic event.

The computer program SHAKE 91 (Ref. 7) was used to calculate the absolute response accelerations on top of ground (soil/weathered shale) and rock outcrop. The SHAKE program has been validated for horizontal response calculations at periods less than 2.0 second at several sites and consequently is widely accepted in the geotechnical earthquake engineering area for site response analysis.

Geotechnical data available from the past investigations at the Y-12 site (Refs. 15 through 28) was used to develop forty two (42) soil columns. A relatively wide range of site conditions in terms of height of soil layer and weathered (rock) shale were encountered at the site. From the forty-two (42) soil columns, seven (7) representative one dimensional soil columns were selected to represent the Y-12 Plant site. Although the locations of the borings and the monitoring wells are scattered, the range of subsurface conditions like stratigraphy, depth to groundwater and rock; and type of overburden materials are judged to be adequately represented by the soil columns and the engineering properties of the materials used in the response analysis.

The range of soil thickness used in the analysis was 10 to $30 \mathrm{ft}$. Weathered shale underlying the soil varied from 10 to $20 \mathrm{ft}$. The highest column used in the analysis was $50 \mathrm{ft}(30 \mathrm{ft}$ soil +20 $\mathrm{ft}$ weathered shale) and the shortest being $10 \mathrm{ft}$. weathered shale overlying fresh rock. A fairly wide spectrum of shear wave velocities for soil $(500,700$ and $1000 \mathrm{fps})$ and weathered shale (1300, 1800 and 2,500 fps) were used to bracket soft, stiff and very dense materials. Therefore, the ranges of subsurface parameters modeled in the SHAKE 91 program are considered to be adequate.

For each soil column, free-field response spectra was determined using six (6) damping values; $2,5,7,10,12$ and 15 percent. Amplification ratio, defined as the ratio between the absolute response accelerations at top of ground (soil/weathered shale) and rock outcrop, was calculated for all seven profiles using the lower bound, mean and upper bound shear wave velocities (Tables 6 through 11).

Considering the mean range soil properties and 5 percent damping, the peak soil amplification ratio varied from 3.8 to 4.5 at periods ranging between .06 and .2 second. For weathered shale, the peak amplification ratio varied from 1.2 to 1.4 at .04 second.

Based on the amplification ratios, site-specific earthquake design response spectra for horizontal soil motion and weathered rock motion were developed for use in the seismic evaluation of the PC $-1,-2$, and -3 facilities at the Y-12 Plant (Figures 16 through 27). 
Results of the liquefaction study indicate that the soils encountered at the Y-12 site are not susceptible to liquefaction (Figures 28 and 29). Consequently, it is concluded that the PC $-1,-2$, and -3 soil-supported facilities at the Y-12 Plant should remain stable against liquefaction during and after the design seismic events. 
The Y-12 Plant, owned by the U.S. Department of Energy (DOE) and operated by Lockheed Martin Energy Systems (LMES), is located about three (3) miles from the center of Oak Ridge, Tennessee. The Y-12 Plant, situated in eastern Bear Creek Valley, is bounded on the south by Chestnut Ridge and on the north by Pine Ridge (Fig. 1). The plant was originally constructed in the early 1940's as part of the Manhattan Project. The primary mission has been production of nuclear weapon components and now the mission is to maintain the production capacity and dismantle nuclear weapon components.

The facilities at Y-12 are currently being evaluated for safety in response to natural seismic hazards. Design and evaluation guidelines to evaluate the effects of earthquakes and other natural hazards on DOE facilities are defined in DOE Order 5480.28, Natural Phenomena Hazards Mitigation (Ref. 1) and the following five DOE Standards:

DOE-STD-1020-94, Natural Phenomena Hazards Design and Evaluation Criteria for Department of Energy Facilities (Ref. 2)

DOE-STD-1021-93, Natural Phenomena Hazards Performance Categorization Guidelines for Structures, Systems, and Components (Ref. 3)

DOE-STD-1022-94, Natural Phenomena Hazards Site Characterization Criteria (Ref. 4)

DOE-STD-1023 (Draft, November 1994), Natural Phenomena Hazards Assessment Criteria (Ref. 5)

DOE-STD-1024-92, Guidelines for Use of Probabilistic Seismic Hazard Curves at Department of Energy Sites (Ref. 6)

These DOE guidelines establish natural phenomena performance categories for structures, systems, and components (SSCs) in facilities as PC-0, PC-1, PC-2, PC-3, and PC-4. PC-0 SSCs do not require any natural phenomena considerations. The guidelines also establish the annual probability of exceedance of the seismic hazard input for each PC category. These annual probabilities of exceedance for each PC category are as follows:

PC CATEGORY

PC-1

PC-2

PC-3

PC-4

\section{ANNUAL PROBABILITY RETURN PERIOD}

$$
2 \times 10^{-3}
$$

500 yrs.

$1 \times 10^{-3}$

1000 yrs.

2000 yrs.

10000 yrs.

There are PC-0, PC-1, PC-2, and PC-3 SSCs at Y-12, but no facilities have been classified as PC-4 to date. 


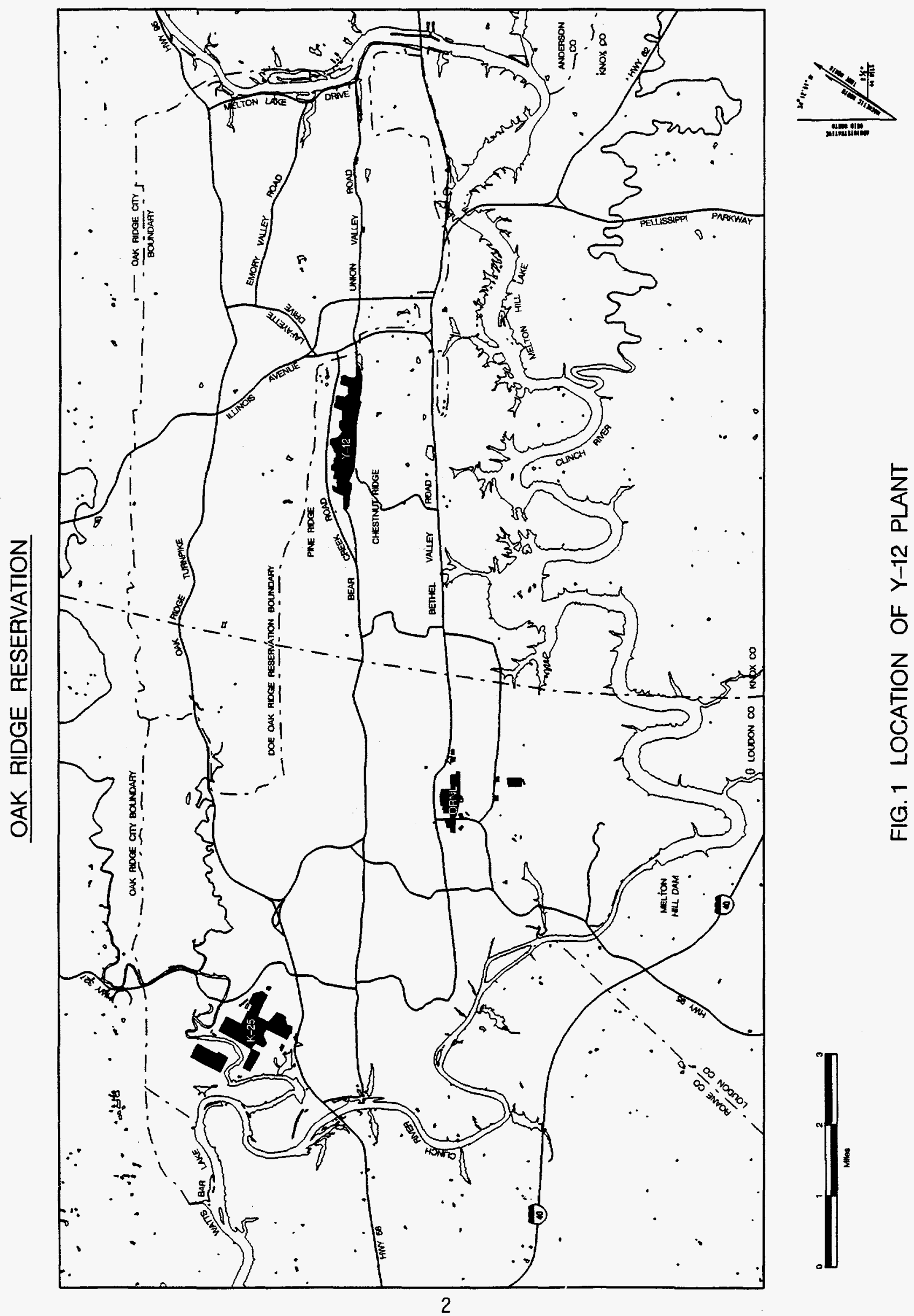


The PC structures are supported by foundations bearing on sound (fresh) rock or weathered rock (shale) or soil. The seismic evaluation requires the ground motion characteristics of the foundation bearing stratum. The top of ground motion, i.e. soil and weathered shale spectra are not available for the evaluation of the structures supported on soil or weathered shale deposit. Additionally, there is also a need to determine if the soil-supported structures are safe against soil liquefaction during and after the seismic event.

2.0

\section{PURPOSE}

There are two main purposes of this study:

(1) To calculate a reasonable range of expected site-specific free-field soil earthquake responses at Y-12 for the 2000-year return period earthquake, using geotechnical and geophysical information collected at the site during previous investigations for facilities at the site. The response will be calculated independently for two components of horizontal motion at the 2000-year hazard level. The emphasis on evaluating the 2000-year event is because there are no PC-4 SSCs at Y-12 and because the 1000 and 500-year hazard levels can be scaled from the 2000-year event. The calculated responses will be used to define free-field ground response spectra for the different soil and weathered shale conditions at $Y-12$. The free-field ground response spectra will be used for the seismic designs/evaluations of SSCs at Y-12.

(2) To assess the liquefaction potential of the foundation soils at $Y-12$ site in order to assure the stability of those SSCs supported on soil deposit against liquefaction during and after the seismic event.

A site-specific response analysis will be conducted using SHAKE 91 computer program (Ref. 7). Soil profiles will be developed at several locations at or in the vicinity of PC-3 SSCs 9204-2, 92042E, 9212, 9215, 9720-5, 9995, 9996 and 9998 (Fig. 2). A wide range of engineering properties covering unit weights, shear wave velocities, normalized shear moduli and damping ratios will be evaluated for soil, weathered shale, and fresh rock. Free-field earthquake response will be calculated for a 2000-year event which represents the Design Basis Earthquake (DBE) for the PC-3 facilities. The response will be determined for two components of horizontal motion. It was decided that the earthquake time history for fresh rock originally determined by Risk Engineering for 1000-year return period (Ref. 8) could be used for 2000-year period for this study. The two horizontal components of the time history of acceleration (Figs. 8 and 9) will be used as input ground motion in the SHAKE analysis.

SHAKE program will generate the absolute response spectral accelerations at the top of soil, weathered shale, and fresh rock. Amplification ratios will be calculated by dividing the response spectral acceleration at the top of soil/weathered shale by the response spectral acceleration at the top of fresh rock.

Design spectra curves for horizontal ground motion will be developed for PC-3, PC-2, and PC-1 facilities supported on weathered (rock) shale, or soil by applying the amplification ratios to the top of fresh rock response spectra. 


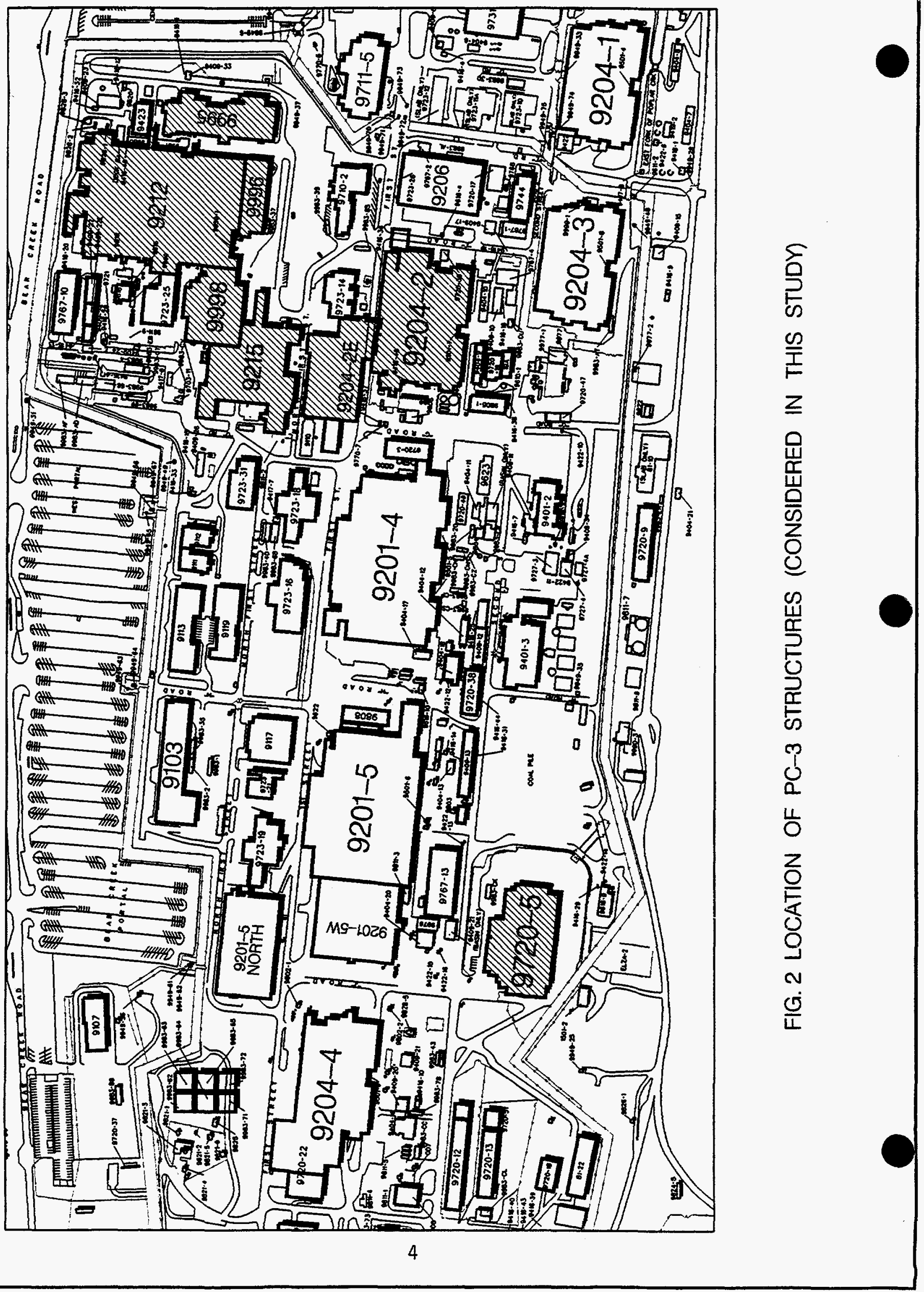


Vertical ground motion calculations will not be performed. The vertical ground motion will be assumed to be two-thirds of the horizontal ground motion (same ratio as rock).

Additionally, the soils present at the $\mathrm{Y}-12$ site will be assessed for liquefaction potential using simplified procedures recommended by Seed, et. al. (Ref. 9). These procedures are based upon soil index properties, grain size distribution, standard penetration test (SPT) blow counts (N) and cyclic stress ratio (CSR) for a given magnitude of earthquake and peak ground acceleration. If sandy soils are encountered, computer program SLIQ (Ref. 10) will be used. SLIQ is based upon Seed's procedures (Ref. 11 and 12) employing the relationship between CSR and $N$ for evaluating liquefaction potential for sands and silty sands.

\subsection{GEOLOGY}

\subsection{REGIONAL GEOLOGY}

The Oak Ridge Reservation (ORR) is located in the western part of the Valley and Ridge Province at the narrowest part of the Appalachian foreland fold-thrust belt. Here, the Valley and Ridge Province is dominated by several west-directed thrust faults that formed when the huge Blue Ridge sheet to the east pushed the Valley and Ridge sedimentary succession in front of it. The ORR contains a variety of geologic structures. The map-scale structure of the ORR is dominated by a uniform southeast dip of sedimentary layering interrupted only by the two large thrust faults, the Copper Creek and the White Oak Mountain faults, and the East Fork Ridge (and Pilot Knob) syncline in the footwall of the White Oak Mountain thrust. A simplified map of the ORR showing the valleys, ridges and the faults is presented in Fig. 3.

The bedrock geology exposed in the ORR is composed entirely of sedimentary rocks that range in age from Early Cambrian to Early Mississippian. This stratigraphy formed as part of the early Paleozoic drift (ocean opening) succession. The carbonate bank was developed. Uplift and erosion of the carbonate bank occurred in early Middle Ordovician time forming the regional post-Knox unconfirmity, and the carbonate bank was reestablished during the middle Ordovician only to be destroyed later in the Ordovician by development of a clastic wedge in the Late Ordovician and Silurian. This was followed by erosion and formation of another unconfirmity, then by the deposition of the Upper Devonian-Early Carboniferous clastic wedge.

Nine major stratigraphic units have been recognized previously in the ORR: Rome Formation, Conasauga Group, Knox Group, Chickamauga Group, Reedsville Shale, Sequatchie Formation, Rockwood Formation, Chattanooga Shale, and Fort Payne Formation. Detailed studies of surface geology and core over the past decade have permitted subdivision of the Conasauga, Knox, and Chickamauga Groups. The Conasauga, and to a much lesser extent, the Knox and Chickamauga Groups, have served as the principal units for disposal of radionuclides and other waste materials in the ORR.

Outcrop-scale structures consist of inclined, faulted, and folded bedding, and joints. Joints (systematic fractures) are the most common structures present here, and several sets of joints with different orientations have been recognized. The dominant joint sets are oriented northeast and northwest, with lesser north-south and east-west sets. These structures are probably the most 


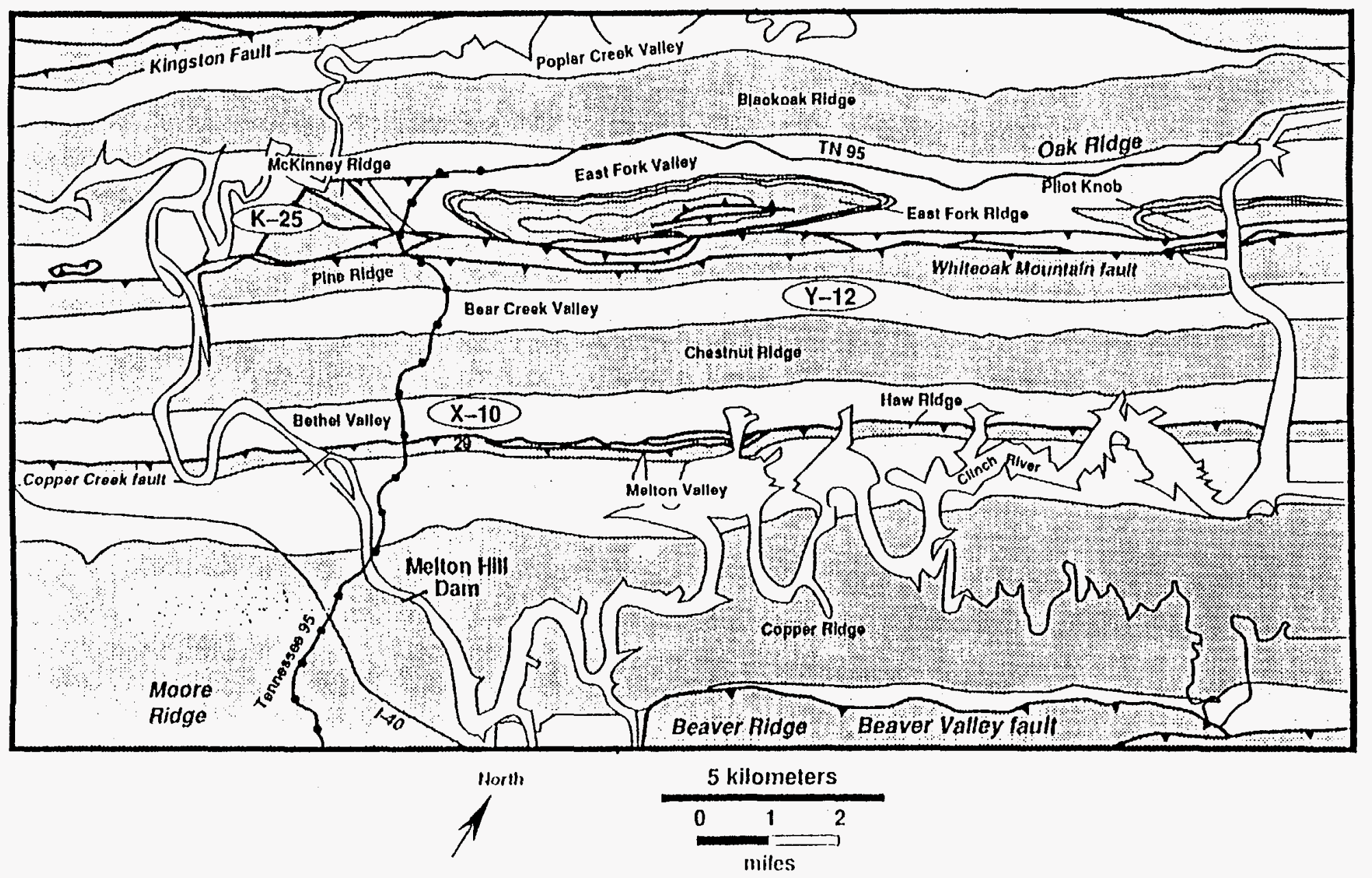

Fig. 3 Locations of Ridges, Valleys and Faults on the Oak Ridge Reservation 
important in the ORR because they, along with bedding and local karst, form the conduit system that controls groundwater movement.

The mantle of unconsolidated residual materials, or regolith, derived by in situ weathering of bedrock, is composed mostly of silty clays and clayey silts. The water table typically is near the interface between regolith and bedrock. In a normal year, the water table is lowest during September-October and highest during February-March.

A detailed geology of the ORR is described in Ref. 13.

\subsection{AREA GEOLOGY}

The ORR consists of approximately 36,000 acres bounded by the Clinch River on the east and south and to the west and north, at approximately 5 to $10 \mathrm{~km}$, by the Cumberland Mountains. The reservation is situated in the Valley and Ridge Province.

Faulting in the Valley and Ridge is quite extensive, with a southeast to northwest trend. The White Oak mountain fault, an inactive fault, crosses the ORR at the Oak Ridge Gaseous Diffusion Plant (ORGDP). The ORGDP, Oak Ridge National Laboratory (ORNL), and Y-12 facilities are all located in long, narrow, northeasterly trending valleys bordered by narrow ridges. Ridge levels average 200 to 300 feet above the elevation of the valleys and have steep northwest slopes and flattened southeast slopes which reflect the southeast dip of the underlying rock.

The Y-12 facility is in Bear Creek Valley. It is flanked on the northwest by Pine Ridge and on the southeast by Chestnut Ridge. Bedrock is southeast dipping limestone, dolomite, and argillaceous shale of the Conasauga Group of Cambrian age which, in turn, overlies sandy shales and sandstones of the Cambrian Rome Formation. At the base of the unit, the Conasauga rocks are predominantly shales, with an increase in the percentage of limestone layers toward the top of the unit. The bed strike is approximately N $55 \mathrm{E}$, and the dip is about 45 to $60 \mathrm{SE}$. Along the westside of Pine Ridge is a prominent southeast dipping thrust fault which indicates that the Y-12 Plant is located on the southeast dipping limb of a syncline that contains a sequence of Cambrian and Ordovician rocks. The Rome Formation is the bottom portion of a thrust fault sheet, and the entire sequence of rocks has been thrust westward over Silurian and Ordovician rocks. A number of similar folded and faulted geologic structures occur parallel to Pine Ridge and constitute the structural pattern of the area.

\subsection{SEISMOLOGY}

\subsection{GENERAL SEISMOLOGY}

Seismic activity in the vicinity of the ORR has primarily occurred in the Valley and Ridge Province, although some historical activity has occurred in the Cumberland Plateau. To the east of the Valley and Ridge province, a similar amount of seismic activity has occurred in the Blue Ridge province, and some scattered activity has occurred in the Piedmont province. Major historical activity has occurred in a northwesterly band of the Atlantic Coastal Plain province centered in South Carolina. The seismic activity described here is shown in Fig. 4. Additionally, the earthquake epicenters in east Tennessee, as recorded from 1977 to 1991 are shown on Fig. 5. 


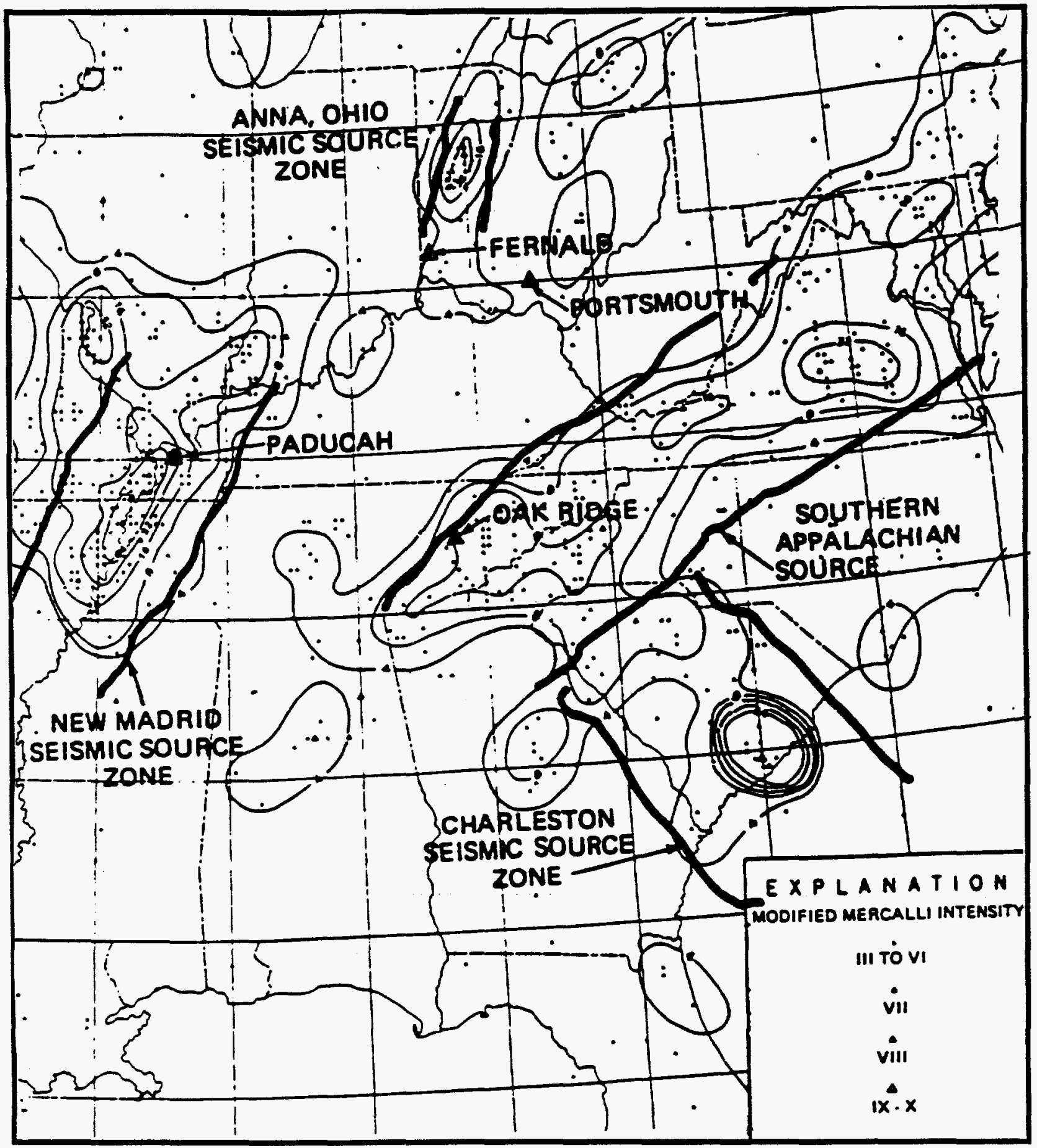

FIG. 4 EARTHQUAKE EPICENTERS IN EASTERN U.S. 


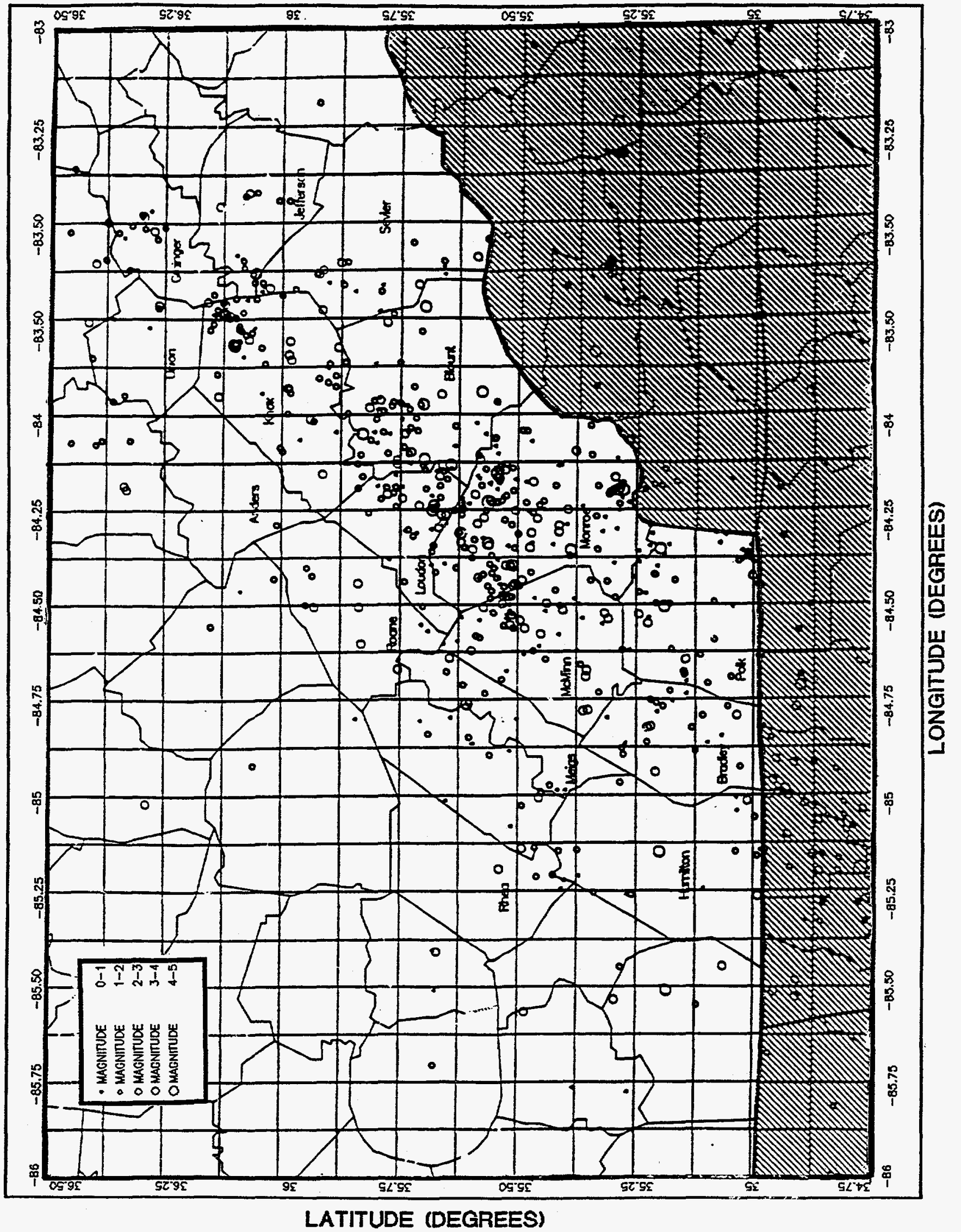

FIG.5 EARTHQUAKE EPICENTERS IN EAST TENNESSEE,

AS RECORDED ON SEISMIC NETWORK, 1977 - 1991 


\subsection{EARTHQUAKES AFFECTING THE OAK RIDGE RESERVATION}

Of the earthquakes that are known to have occurred in the above regions, only three may have had any significant impact on the ORR. In 1886, the Charleston, South Carolina, earthquake occurred $520 \mathrm{~km}$ ( 325 miles) from the Reservation. This earthquake has been estimated to have had a Modified Mercalli Intensity (MMI) of X at the epicenter, and the effects at the ORR area have been estimated to have been an MMI of VI.

Following the 1886 Charleston, South Carolina, earthquake, an earthquake occurred in Giles County, Virginia, on May 31, 1897, $360 \mathrm{~km}$ (228 miles) from the ORR. This earthquake has been estimated to have an epicentral MMI from VII to VIII and at the ORR an MMI somewhere between V and VII.

The third earthquake was nearest the ORR, occurring just northwest of Knoxville on March 28, 1913, approximately $60 \mathrm{~km}$ ( 37 miles) from the Reservation. It has been estimated to have had an epicentral MMI of VII and an MMI of V to VI in the ORR.

The most recent significant earthquake to occur in the Appalachian area occurred on November 30, 1973, at Maryville, $34 \mathrm{~km}$ (21 miles) southeast of the ORR. This earthquake was estimated as having an epicentral MMI of VI and an MMI of IV in the ORR. Instrumental seismograph readings of this earthquake placed the magnitude at a value of $4.6 \mathrm{~m}_{\mathrm{b}}$. The recording instrument at the Y-12 Plant recorded a peak acceleration of $0.024 \mathrm{~g}$. No damage occurred at the Y-12 Plant facilities from this earthquake. Two other earthquakes are worthy of discussion that did not occur in the above provinces. The first is the series of earthquakes that occurred near New Madrid, Missouri, $450 \mathrm{~km}$ ( 280 miles) west of the area of ORR during the months of December 1811 , January 1812 , and February 1812. Three of these earthquakes have been estimated as having an epicentral MMI of X to XII and an MMI of V to VI at the ORR.

The second earthquake to discuss is significant because of it's recent occurrence, June 27, 1980 . This earthquake occurred in Northern Kentucky approximately $290 \mathrm{~km}$ (180 miles) north of the ORR. The earthquake was estimated to have had an epicentral MMI of VII and a magnitude from instrumental records of $5.2 \mathrm{~m}_{\mathrm{b}}$. Some areas of the ORR experienced an MMI of I to II.

\section{SEISMIC SOURCE ZONE}

A recent seismic hazard study has indicated that the major potential for earthquake damage to the ORR is the seismic source zone of the Valley and Ridge province. Although there is considerable micro-earthquake activity occurring in the area, none of the activity can be attributed to known faulting.

\subsection{DESIGN EARTHQUAKES}

The determination of the earthquake hazard and the selection or derivation of appropriate design time history records for the rock outcrop at Y-12 represent the first step of a site response analysis. Based on the DOE Order 5480.28, DOE-STD-1020, 1022, 1023, and 1024, probabilistic 
methods of hazard analysis were used to derive parameters defining the design events and to develop corresponding synthetic records.

The probabilistic assessment of seismic hazard was conducted by Risk Engineering, Inc. (Ref. 8). The results from Risk Engineering were used to define the rock outcrop seismic hazard and rock outcrop design response spectra. The results are summarized in report ES/CNPE-95/2, (Ref. 14). The seismic hazard results for peak rock acceleration are as follows:

\begin{tabular}{ccc} 
PC CATEGORY & RETURN PERIOD & PEAK ROCK ACCELERATION \\
\cline { 1 - 2 } PC-1 & 500 & $0.06 \mathrm{G}$ \\
PC-2 & 1000 & $0.08 \mathrm{G}$ \\
PC-3 & 2000 & $0.12 \mathrm{G}$ \\
PC-4 & 10000 & $0.26 \mathrm{G}$
\end{tabular}

The seismic hazard curve for the peak rock acceleration versus annual probability of exceedance is shown in Figure 6. The design response spectra for 5 percent damping are shown in Figure 7.

Synthetic earthquake records were developed by Risk Engineering, Inc. to represent rock outcrop motions. Two components of horizontal motion were provided. The time histories of acceleration for these horizontal components for the 2000-year earthquake are shown in Figures 8 and 9. The acceleration response spectra of these records are shown in Figs. 10 and 11 compared to the rock outcrop spectra. The acceleration response spectra from these time histories envelop the rock outcrop spectra in the frequency range of the Y-12 soil deposits and therefore are considered adequate to determine amplification ratios which can be used to scale the rock spectra to represent the free-field soil spectra.

\subsection{GEOTECHNICAL INVESTIGATIONS}

Geotechnical data available from the past site investigations were used in this study. New geotechnical explorations or laboratory testing for determining the soil and weathered shale response spectra and soil liquefaction potential were not performed because it was judged that the existing data was sufficient to define the ground motions and evaluate liquefaction potential.

The main sources of the past geotechnical data are the (1) Y-12 Updated Subsurface Data Base for Bear Creek Valley, Chestnut Ridge, and Parts of Bethel Valley, October, 1993 (Ref. 15), (2) Y-12 Plant, Groundwater Quality Report for the Upper East Fork Poplar Creek Hydrologic Regime Feb. 1994 (Ref. 16), and (3) The subsurface Investigation reports listed in Refs. 17 through 28.

Since the past geotechnical investigation programs were not designed for the purpose of this study, the above referenced reports did not contain all the information needed to define the 


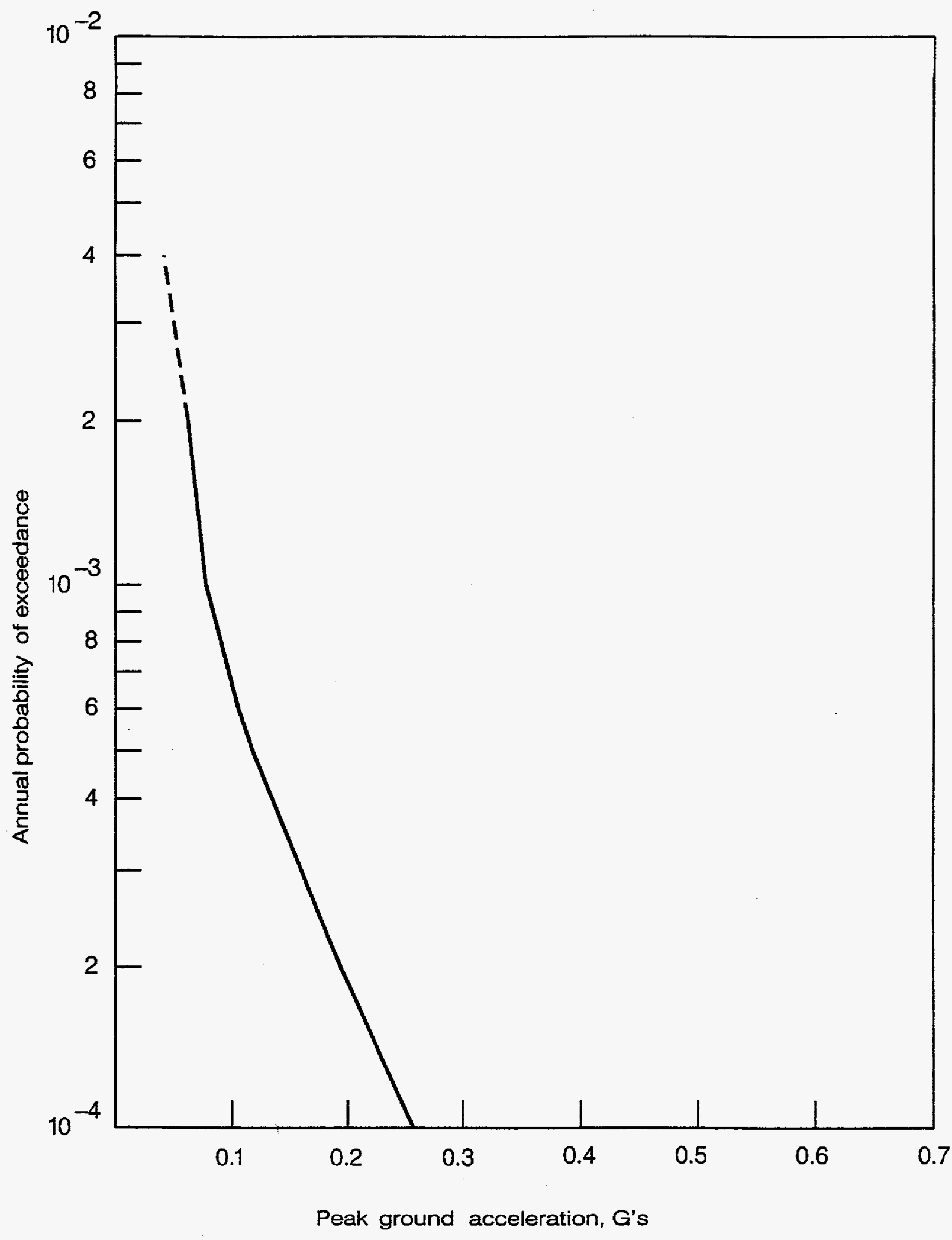

FIG. 6 SITE SPECIFIC EARTHQUAKE HAZARD CURVE FOR OAK RIDGE RESERVATION 


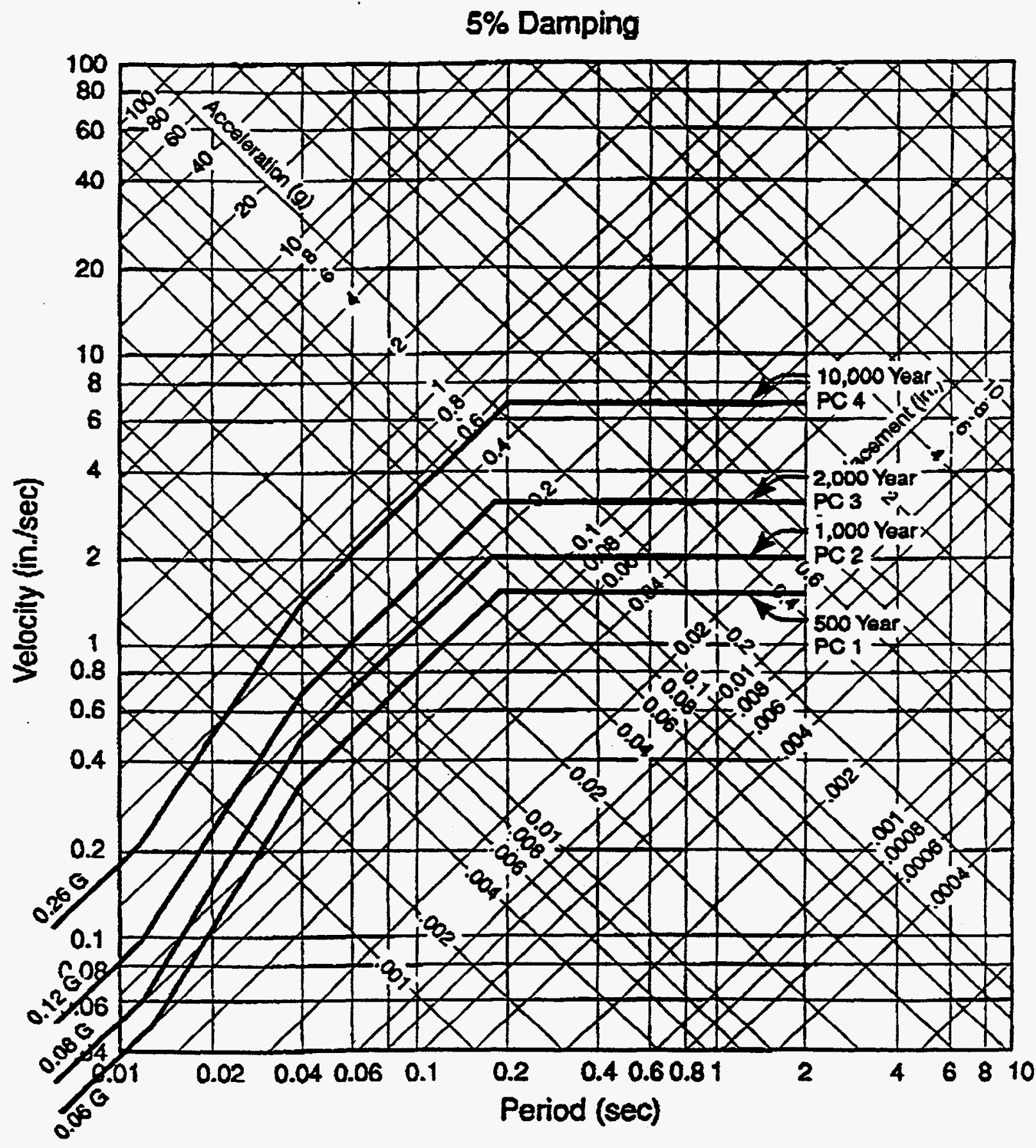

FIG. 7 OAK RIDGE-SITE SPECIFIC RESPONSE SPECTRA FOR HORIZONTAL

ROCK MOTION 


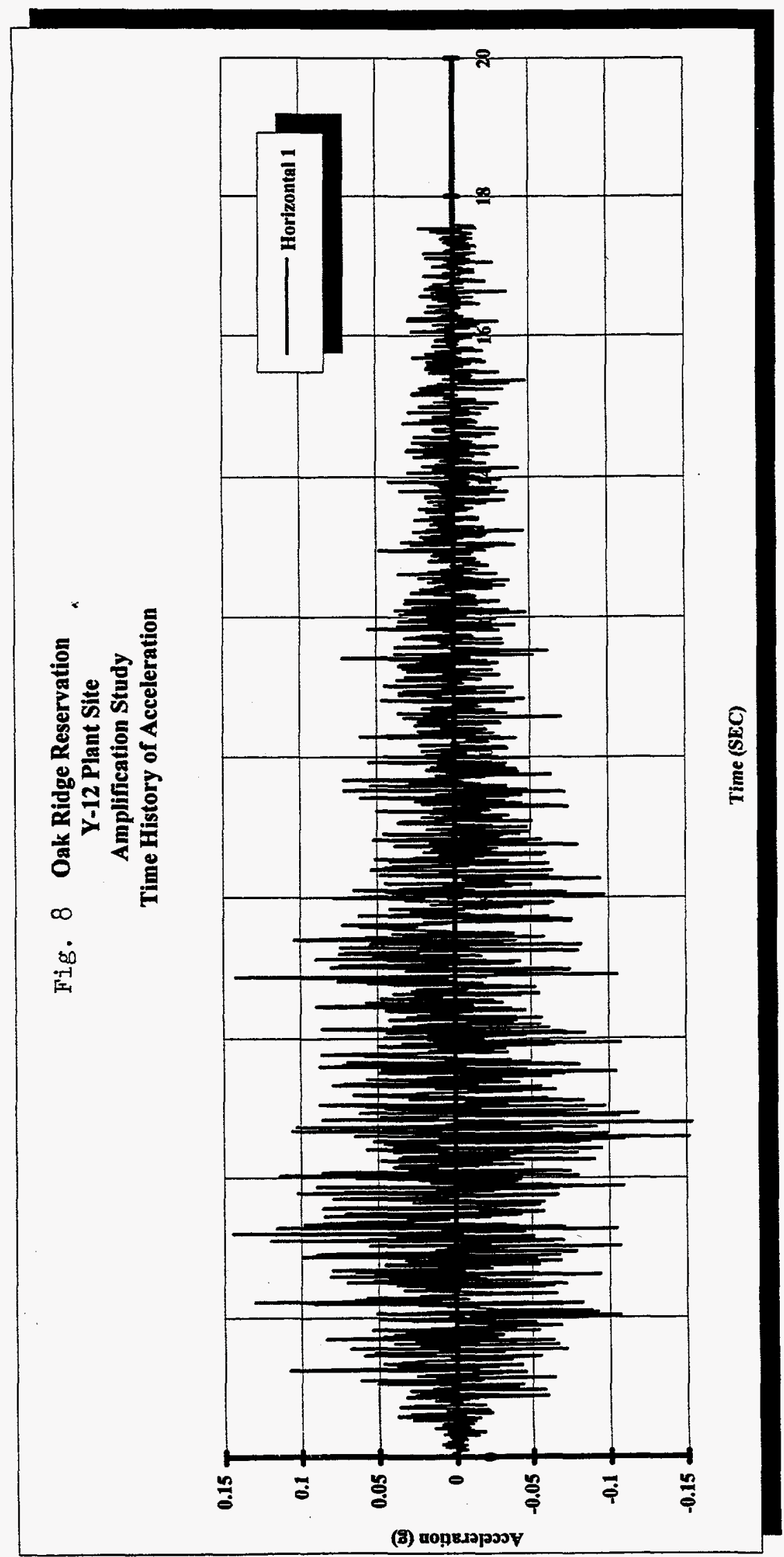




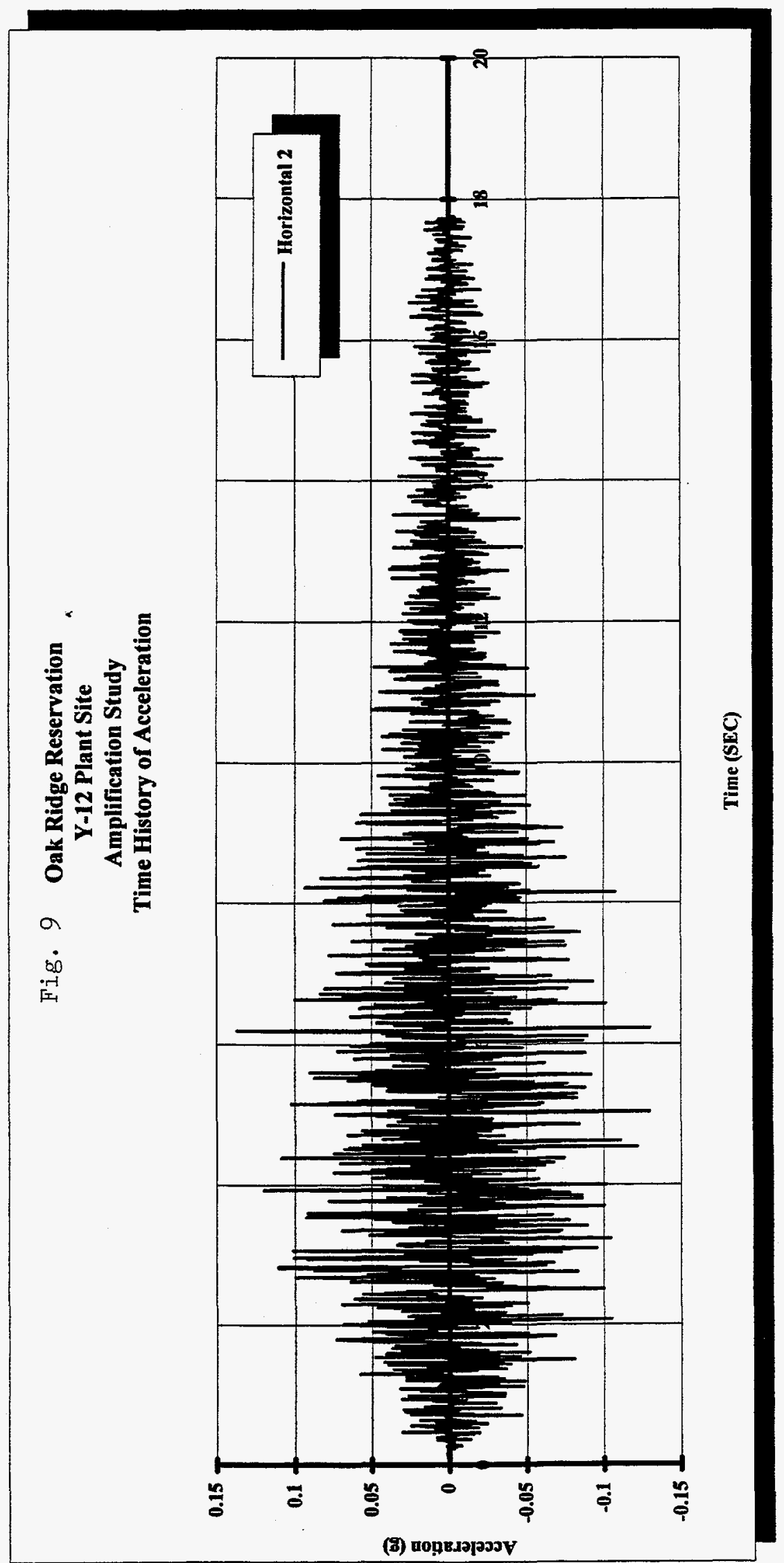


Oak Ridge Reservation

Fig. 10 Y-12 Plant Site - Horizontal 1 - 2000 YR Spectrum - 5\% Damping

Comparison of Original Oak Ridge with Current 2000 YR Smooth Spectrum

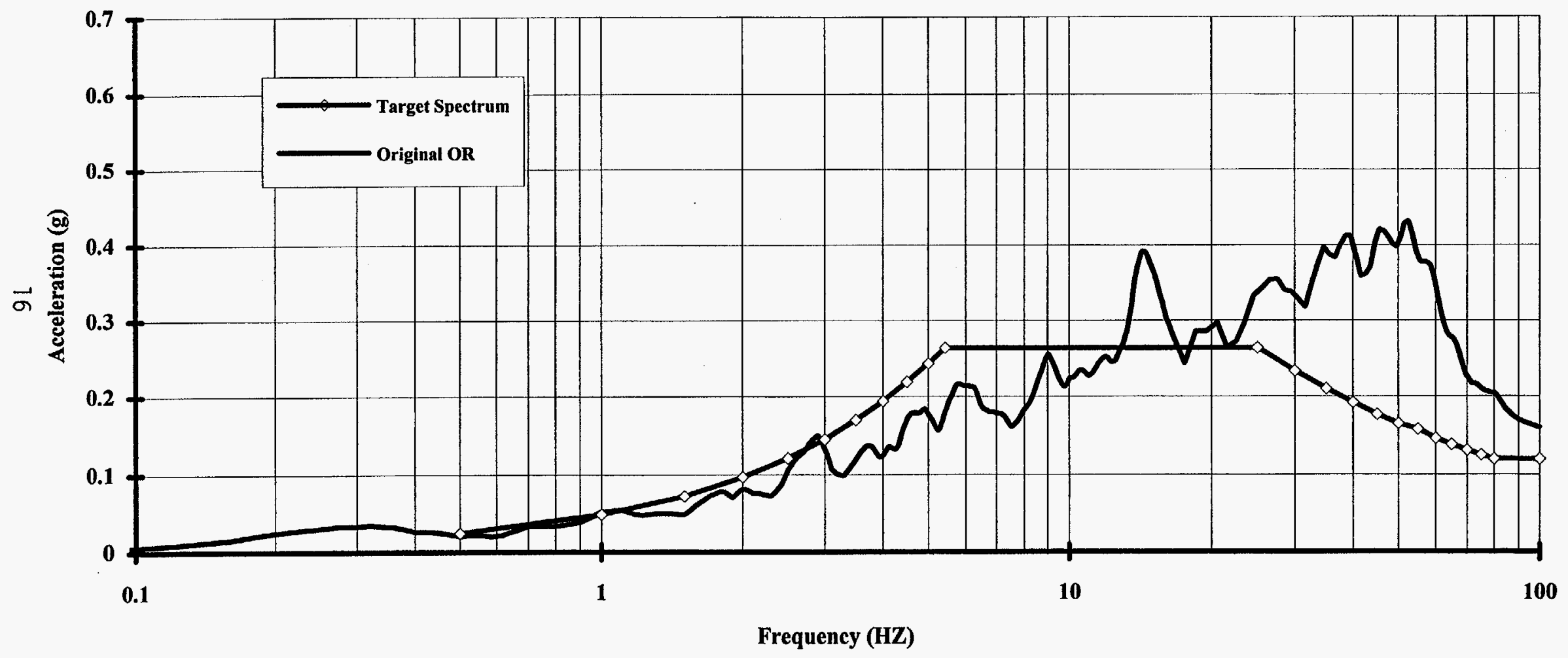



required response spectra and assess the soil liquefaction potential. The gaps in the data and the soil properties were filled in using engineering judgement based upon information available in published literature on similar materials.

Pertinent data from the past geotechnical reports were compiled. Information relating to depths of fresh rock and weathered rock (shale) were generally obtained from References 15 and 16. Engineering properties and profiles were basically derived from subsurface investigation reports (Refs. 17 through 28). Dynamic properties like normalized shear moduli and damping ratios, and shear strain were obtained from published literature.

\subsection{PREPARATION OF DRAWINGS}

The following drawings were prepared to show the locations of soil borings, soundings, and monitoring wells, and incorporate the geotechnical information, e.g., depths to fresh rock and weathered rock (shale), soil stratification, groundwater elevation, and original ground contours.

(1) Drawing Y-12 SARUP SUPPORT
- Boring Location Plan, C2E 900000 A952

- 1943 Contours, C2E 900000A953

- Top of fresh rock Contours, C2E 900000A954

- Groundwater Contours, C2E 900000A955

- Top of Weathered Rock (Shale) Contours, C2E 900000A956

- Soil Profiles (Columns), Bldg. 9212, C2E 900000A957

- Soil Profiles (Columns), Bldgs. 9998, 9996, 9215, 9204-2 \& -2E, 9720-5, C2E 900000A958

- Soil Profiles (Columns), Bldg. 9995, C2E 900000A959

Drawing 1 presents the locations of soil borings, soundings, and groundwater monitoring wells. Original ground contours depicting the site topography in 1943, prior to the construction of Y-12 Plant are shown on Drawing 2. References 15 and 16 provided a significant amount of data relating to the depths of fresh rock and weathered rock (shale). This data was used to generate top of fresh rock and weathered rock (shale) contours shown on Drawings (3) and (5). Groundwater information was taken directly from Ref. 16 . In areas where the information was not available, groundwater contours were extrapolated based on the trend of the watertable. Drawing 4 shows the groundwater contours generated at the site. All (8) drawings are included in Appendix $\mathrm{C}$ of this report.

\subsection{SUBSURFACE PROFILES}

Subsurface profiles were developed using field exploratory data from several geotechnical investigations listed in Refs. 17 through 28. Elevations of fresh rock, weathered rock (shale) and groundwater were derived from the contours discussed in Section 6.1. Subsurface profiles consisting of soil and weathered shale strata overlying fresh rock were designated as soil columns for use in the seismic response analysis and soil liquefaction assessment. 
For the purpose of this study, a given height of soil deposit was treated as a single layer of homogeneous soil mass for the following reasons:

(1) The geotechnical investigations revealed that the overburden soils are predominantly silty clays or clayey silts. Coarse-grained soils are generally not present at the site. Consequently, we are dealing with only one major type of soil i.e fine-grained soil.

(2) Variation in shear strength of the overburden soil (soft, medium, stiff, very stiff and hard) can be estimated to a reasonable degree by assigning a wide spectrum of shear wave velocities, i.e., lower bound, mean, and upper bound in the response analyses using the criteria recommended by the Nuclear Regulatory Commission (Ref. 29).

(3) The soil overburden is shallow, generally, not exceeding $30 \mathrm{ft}$.

(4) The single layer assumption will simplify the analyses specially when detailed (layer by layer) soil properties are not available since the referenced geotechnical investigations were not designed for the purpose of this study.

Weathered (rock) shale was also considered as a single layer due to similar reasons discussed above. The subsurface profiles were simplified to constitute columns of soil overlying weathered shale. A total of forty-two (42) simplified subsurface profiles were developed at several locations in the vicinity of the PC-3 structures. For the purpose of this report the subsurface profiles will be referred to as the soil columns. The 42 soil columns are presented on Drawings 6,7 , and 8 . Grade elevation, bottom of foundations of the structures, groundwater table, top of weathered shale and fresh rock are marked on individual soil columns.

In order to simplify the analysis, seven representative soil columns were modelled for the SHAKE 91 computer input. Thicknesses of soil and weathered shale for each soil column (model) are listed in Table 1. Each model is pictorially presented on Fig. 12. It is believed that the seven models should be sufficient to cover the ranges of soil and weathered rock (shale) thicknesses encountered at the Y-12 SSCs.

\subsection{MATERIAL PROPERTIES}

Material properties required to calculate the amplification ratios by SHAKE analysis and for determining liquefaction potential of soils are discussed below.

\subsubsection{SHAKE 91 PROGRAM}

The SHAKE 91 analysis requires unit weight, shear wave velocity or shear modulus, normalized shear modulus vs. shear strain, and damping ratio vs. shear strain.

Table 2, presents unit weights and shear wave velocities for soil, weathered shale and fresh rock. Unit weight for soil is calculated from the laboratory test results reported in refs. 18, 19, 20, 24, 25 , and 26. Shear wave velocities for the soils at the Y-12 site are not available. The most extensive testing performed in East Tennessee relating to shear wave velocity and shear modulus on soils similar to those at Y-12 has been at TVA Watts Bar Nuclear Plant (Ref. 30). Therefore, 
Table 1

Representative Soil Columns For

SHAKE Analysis

\begin{tabular}{|c|c|c|c|c|c|c|c|}
\hline Soil Column No. & 1 & 2 & 3 & 4 & 5 & 6 & 7 \\
\hline Soil Thickness (Ft.) & 10 & 10 & 20 & 20 & 30 & 30 & 0 \\
\hline $\begin{array}{l}\text { Weathered Rock (Shale) } \\
\text { Thickness (Ft.) }\end{array}$ & 10 & 20 & 10 & 20 & 10 & 20 & 10 \\
\hline Fresh Rock & \multicolumn{7}{|c|}{ Half-Space Elastic } \\
\hline
\end{tabular}



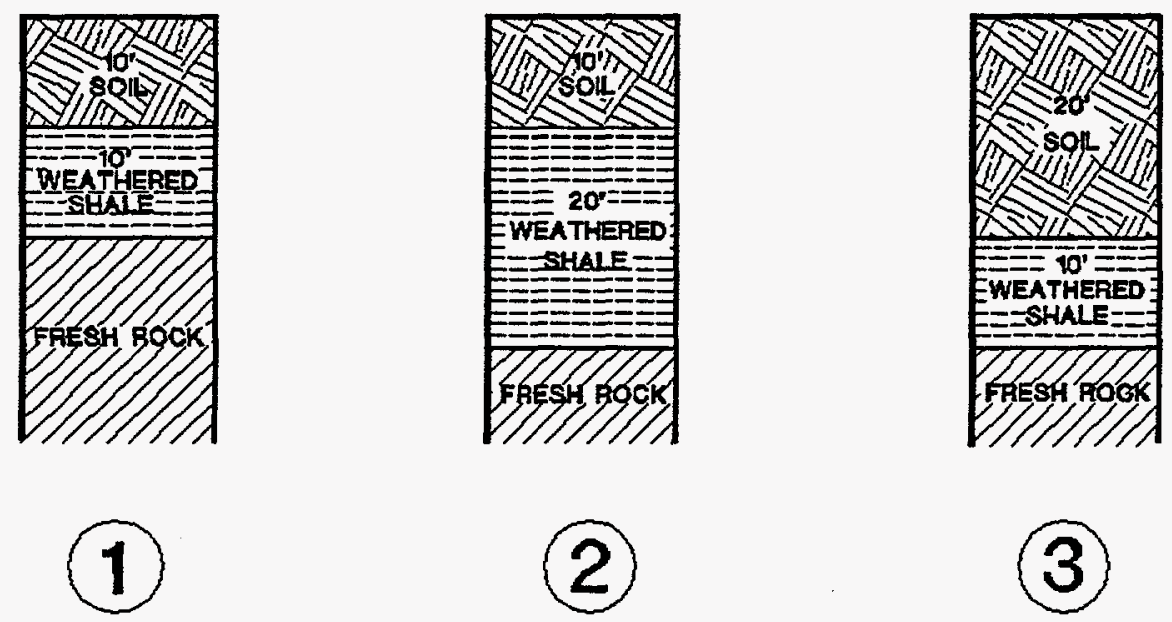

(2)
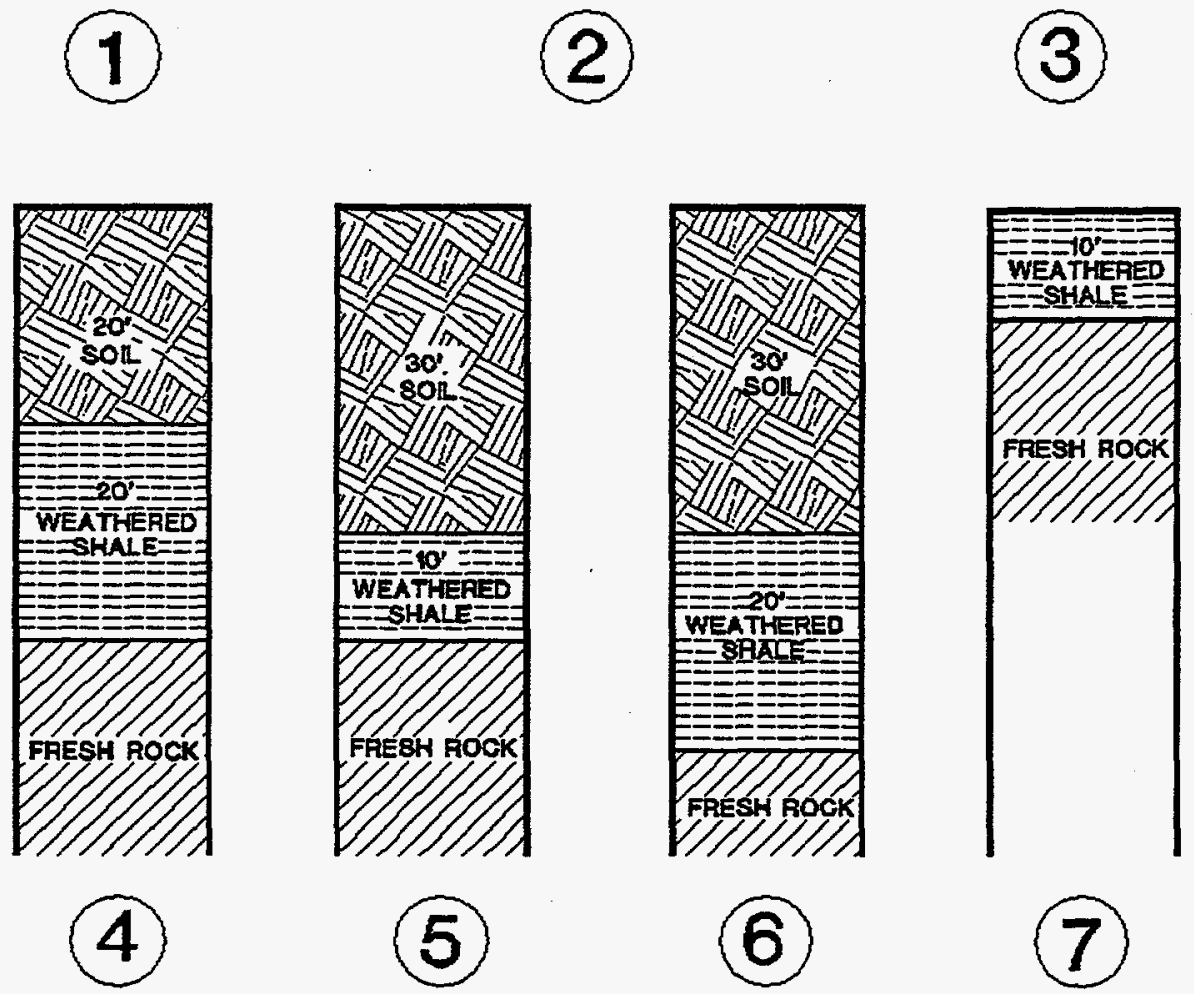

(5)
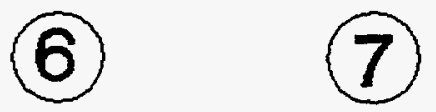

FIG. 12 REPRESENTATIVE SOLL COLUMNS FOR SHAKE ANALYSIS 


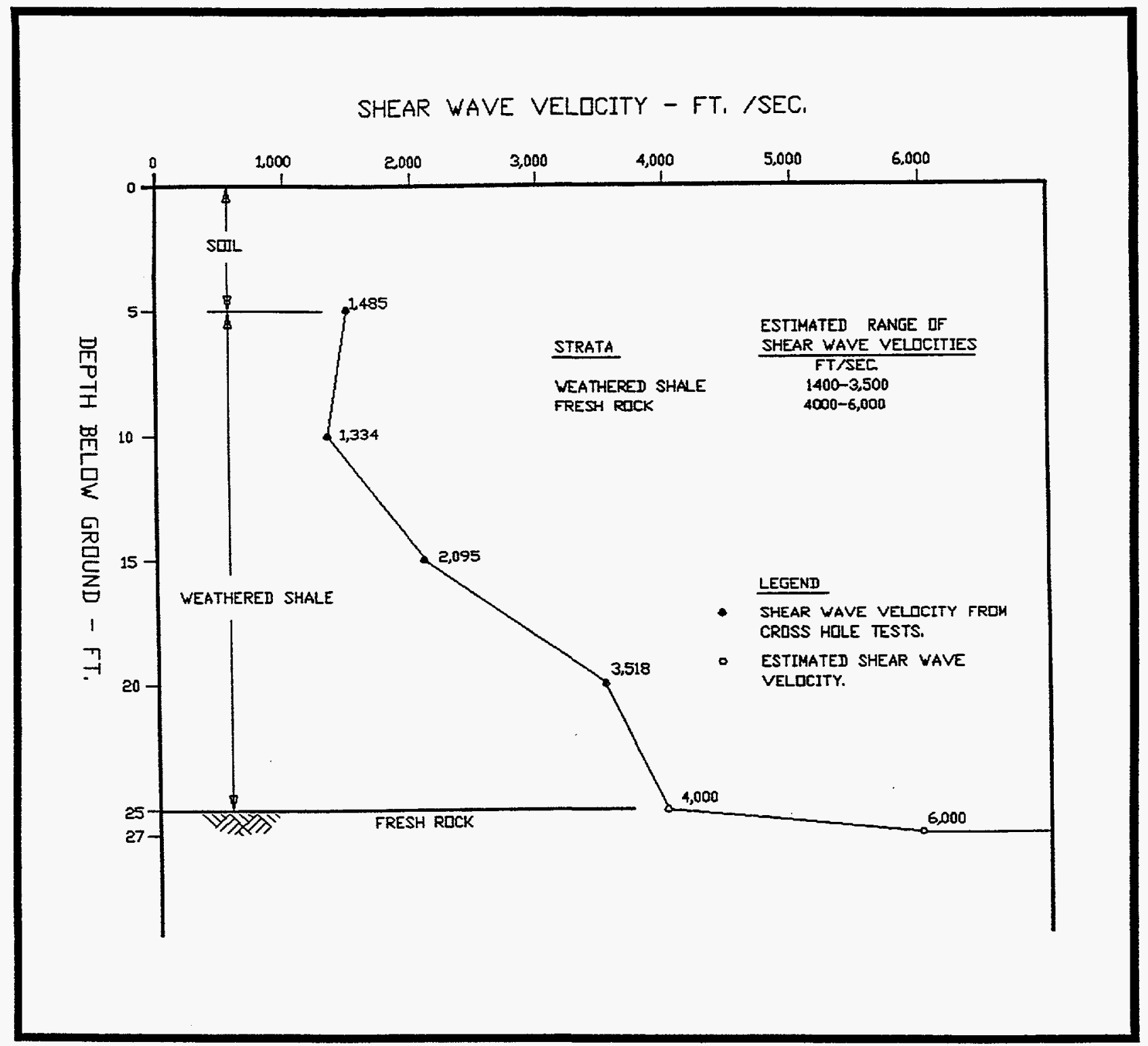

FIG. 13 SEAR WAVE VELOCITY VS DEPTH WHEATHERED ROCK (SHALE) AND FRESH ROCK 
the mean shear wave velocity of soil $=700 \mathrm{fps}$ was calculated from the data reported in Ref. (30).

For weathered (rock) shale, shear wave velocity is determined from the geophysical Cross Hole Tests conducted in the vicinity area of Building 9206 at Y-12. Fig. 13 presents a plot of shear wave velocity vs. depth for weathered (rock) shale. The unit weight for weathered (rock) shale was obtained from Ref. 30.

There may be areas where the weathered rock could be shaly limestone. The SHAKE analysis, however, requires only the shear wave velocity and does not distinguish between the rock types. The range of shear wave velocities used in the analysis covers the types of weathered rock in the area.

Unit weight and shear wave velocity for fresh rock listed in Table 2 were estimated from Ref. 30 and 28 , respectively.

The lower and upper bound values of shear wave velocities are computed in accordance with the recommendations made by Nuclear Regulatory Commission (Ref. 29). The lower bound, mean and upper bound properties shown in Table 2 should cover a wide range of overburden soil/weathered (rock) shale deposits varying from soft to very dense consistencies.

The normalized moduli and damping ratio vs. shear strain data for soil, weathered shale and fresh rock are presented in Tables 3 and 4 . For soil, this data has been adequately determined by using the curve for a Plasticity Index of 10-15 given in Ref. 32. At the Y-12 site, the computed mean value of Plasticity Index of soil is on the order of 14 .

In the absence of tests on weathered shale and fresh rock at the Y-12 site, the dynamic moduli and damping ratios are assumed from the recommendations made in Ref. 30. The assumptions are justified in view of the similarity between the materials encountered at the Y-12 site and the Watts Bar Nuclear Plant.

\subsubsection{SOIL LIQUEFACTION POTENTIAL}

Liquefaction analysis will be performed by using the simplified methods presented by Seed et. al., (Ref. 9) for cohesive soils and sandy soils with varying percentages of fines. These procedures require the following engineering properties: Natural water content, Atterberg limits, Grain size distribution, Unit weight, $\mathrm{N}$ values and CSR.

Table 5 presents the soil properties collected from various geotechnical investigation reports (Refs. 18, 20, 24, 27, and 28). These properties include natural water content, Atterberg limits, plasticity index, grain size distribution (percent finer than $.005 \mathrm{~mm}$ ) and soil classification. Although the information on grain size distribution is very limited, Atterberg limits and natural water content data should be sufficient to assess the liquefaction potential of the cohesive soils.

Cyclic triaxial, resonant column, or simple shear tests were not conducted on the soils at the Y-12 site since the past geotechnical investigation programs were not designed for soil liquefaction analysis. Empirical formulation for calculating the CSR have been used in the SLIQ computer program. $\mathrm{N}$ value is generally used for liquefaction analysis of sandy soils by field performance 
procedures. Sands and sandy soils are generally not present at Y-12 site. Out of several soil investigations' results considered in this study, only one boring (Ref. 27) indicated a small pocket of silty sand (approximately 18 in. thick) with a $N$ value of 8 . This information will be used as input to the SLIQ Program.

\subsection{SITE-SPECIFIC EARTHQUAKE RESPONSE ANALYSIS}

\subsection{METHODOLOGY}

A site response analysis, sometimes referred to as soil amplification analysis, involves the determination of components of ground motion for seismic design or evaluation. Typically, as in this study, that determination is made for a "free-field",i.e., the response at ground surface of an ideal soil deposit (horizontal layers extending to infinity) to a spatially-uniform, horizontal motion applied at the base. The conceptual relationship between free-field response with respect to two other primary control points, rock outcrop and base rock in a site response analysis is shown in Fig. 14. The motions at these points, as well as any other point in the vertical profile are unique.

Design earthquake motions are most often specified as corresponding to rock outcrop. Mathematical expressions (transfer functions) are then used to find the equivalent motion for the base rock and then seismic waves are propagated through the soil column to determine the freefield motion at the surface.

The determination of site-specific earthquake response of soil deposits generally involves three basic steps:

a. Determination of earthquake hazard and the selection or derivation of design motions.

b. Idealization of stratigraphy and selection of material properties.

c. Calculation and evaluation of site response.

For this study, step (a) was conducted by Risk Engineering, Inc. (Ref. 8) and is summarized in ES/CNPE-95/2 (Ref. 14). This study addresses steps (b) and (c).

The computer program, SHAKE 91 (Ref. 7), is used for this task to calculate the site responses. SHAKE 91 is a one dimensional, total stress code that solves the wave equation in the frequency domain (complex response technique). The soil profile is represented with an idealized soil column of homogeneous, visco elastic layers of infinite extent. SHAKE 91 can be used to determine the site responses from horizontal motions, but is not easily adapted for calculating vertical motions. Therefore, the vertical motions are assumed to be two-thirds of the horizontal ground motions, just like the vertical rock outcrop motions are assumed to be two-thirds of the horizontal rock outcrop motions.

Schematic representation of soil response analysis is depicted in Fig. 15. 
$\underline{\text { Table } 2}$

Engineering Properties Used in SHAKE Analysis

\begin{tabular}{||l|l|c|c|c||}
\hline \hline Stratum & $\begin{array}{l}\text { Unit } \\
\text { Weight } \\
\text { (PCF) }\end{array}$ & \multicolumn{3}{|c|}{$\begin{array}{c}\text { Shear Wave Velocity } \\
\text { (FPS) }\end{array}$} \\
\cline { 2 - 5 } & & $\begin{array}{l}\text { Lower } \\
\text { Bound }\end{array}$ & Mean & $\begin{array}{l}\text { Upper } \\
\text { Bound }\end{array}$ \\
\hline Soil & 120 & 500 & 700 & 1,000 \\
\hline Weathered Rock (Shale) & 127 & 1,300 & 1,800 & 2,500 \\
\hline Fresh Rock & 150 & \multicolumn{3}{|c|}{4,000} \\
\hline
\end{tabular}


Table 3

Normalized Shear Modulus \&

Damping Vs. Shear Strain for Soil

Shear Strain

$\longrightarrow$

.0001

.001

.00316

.010

.0316

.1

\begin{tabular}{cc}
$\stackrel{\mathrm{G}}{\mathrm{Gmax} .}$ & \multicolumn{2}{c}{$\begin{array}{c}\text { Damping } \\
\text { \% }\end{array}$} \\
1.00 & 2.00 \\
0.997 & 2.50 \\
0.974 & 3.50 \\
0.881 & 4.75 \\
0.674 & 6.50 \\
0.425 & 9.25
\end{tabular}




\section{$\underline{\text { Table } 4}$}

Normalized Shear Modulus \& Damping Vs.

Shear Strain for Weathered Shale and Fresh Rock

\begin{tabular}{l} 
Shear Strain \\
\hline .0001 \\
.0003 \\
.001 \\
.003 \\
.01 \\
.03 \\
.10 \\
1.00
\end{tabular}

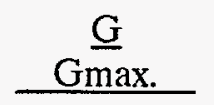

1.00

1.00

0.9875

0.9525

0.900

0.810

0.725

0.550
1.50

Damping

$\%$

0.40

0.80

3.00

4.60 


\begin{tabular}{|c|c|c|c|c|c|c|c|c|}
\hline & Natural & Liquid & & Plastic & Plasticity & & Grain & \\
\hline & Water content & Limit & & Limit & Index & Soil & Size & \\
\hline$\#$ & $\frac{9}{8}$ & 8 & $0.9 * \mathrm{LL}$ & 8 & LL-PL & Classification & $.005 \mathrm{~mm}$ & Reference \\
\hline & $(W C)$ & (LL) & & (PL) & (PI) & & (\% Finer) & \\
\hline 1 & 24.70 & 51.50 & 46.35 & 24.30 & 27.20 & $\mathrm{CH}$ & & Ref.18-Boring 11A \\
\hline 2 & 20.40 & 29.00 & 26.10 & 19.60 & 9.40 & CL & & $t$ \\
\hline 3 & 22.90 & 38.90 & 35.01 & 21.60 & 17.30 & $\overline{C L}$ & & $\operatorname{Ref.20}$-Boring 1 \\
\hline 4 & 21.60 & 40.40 & 36.36 & 23.20 & 17.20 & CL & & \\
\hline 5 & 16.40 & 42.20 & 37.98 & 26.60 & 15.60 & ML & & \\
\hline 6 & 15.80 & 36.10 & 32.49 & 24.20 & 11.90 & ML & & \\
\hline 7 & 25.40 & 36.90 & 33.21 & 24.50 & 12.40 & ML & & Ref.20-Boring 2 \\
\hline 8 & 26.90 & 43.60 & 39.24 & 29.00 & 14.60 & ML & & \\
\hline 9 & 13.70 & 28.90 & 26.01 & 20.10 & 8.80 & $\mathrm{CL}$ & & $\downarrow$ \\
\hline 10 & 23.60 & 40.10 & 36.09 & 22.80 & 17.30 & CL & & Ref.20-Boring $2 \mathrm{~A}$ \\
\hline 11 & 29.80 & 41.80 & 37.62 & 25.00 & 16.80 & ML & & \\
\hline 12 & 29.90 & 38.60 & 34.74 & 21.80 & 16.80 & CL & & \\
\hline 13 & 18.60 & 37.50 & 33.75 & 21.20 & 16.30 & $\mathrm{CL}$ & & Ref.20-Boring 3 \\
\hline 14 & 21.50 & 38.10 & 34.29 & 22.00 & 16.10 & CL & & \\
\hline 15 & 22.40 & 32.80 & 29.52 & 23.10 & 9.70 & ML & & \\
\hline 16 & 22.60 & 39.20 & 35.28 & 25.00 & 14.20 & ML & & \\
\hline 17 & 34.90 & 43.10 & 38.79 & 30.90 & 12.20 & ML & & Ref. 20 -Boring 4 \\
\hline 18 & 21.70 & 38.60 & 34.74 & 21.90 & 16.70 & CL & & \\
\hline 19 & 22.10 & 36.90 & 33.21 & 22.40 & 14.50 & $\mathrm{CL}$ & & \\
\hline 20 & 23.20 & 39.80 & 35.82 & 21.10 & 18.70 & $\mathrm{CL}$ & & $\downarrow$ \\
\hline 21 & 15.00 & 40.20 & 36.18 & 26.60 & 13.60 & ML & & Ref.20-Boring 5 \\
\hline 22 & 17.50 & 42.10 & 37.89 & 26.50 & 15.60 & ML & & \\
\hline 23 & 19.20 & 36.30 & 32.67 & 22.40 & 13.90 & CL. & & \\
\hline 24 & 11.90 & 32.20 & 28.98 & 25.00 & 7.10 & ML & & W \\
\hline 25 & 24.70 & 39.80 & 35.82 & 23.00 & 16.80 & $\mathrm{CL}$ & & Ref.20-Boring 6 \\
\hline 26 & 23.50 & 37.40 & 33.66 & 25.20 & 12.20 & ML & & \\
\hline 27 & 33.90 & 36.00 & 32.40 & 20.60 & 15.40 & $\mathrm{CL}$ & & \\
\hline 28 & 11.90 & 39.90 & 35.91 & 24.00 & 15.90 & CL & & $\Psi$ \\
\hline 29 & 17.60 & 41.60 & 37.44 & 26.40 & 15.20 & ML & & Ref. 20 -Boring 7 \\
\hline 30 & 28.10 & 36.40 & 32.76 & 21.10 & 15.30 & CL & & \\
\hline
\end{tabular}


'TABLE 5 Index Properties and Soil Classification (cont'd.)

\begin{tabular}{|c|c|c|c|c|c|c|c|c|}
\hline & Natural & Liquid & & Plastic & Plasticity & & Grain & \\
\hline & Water content & Iimit & & Limit & Index & Soil & Size & \\
\hline$\#$ & $\frac{8}{8}$ & 8 & $0.9 * \mathrm{LL}$ & $\frac{9}{6}$ & LL-PL & Classification & $.005 \mathrm{~mm}$ & Reference \\
\hline & (WC) & (LL) & & (PL) & (PI) & & (\% Finer) & \\
\hline 31 & 24.30 & 40.70 & 36.63 & 21.90 & 18.80 & CL & & \\
\hline 32 & 25.30 & 28.20 & 25.38 & 25.40 & 2.90 & ML & & \\
\hline 33 & 6.80 & 28.80 & 25.92 & 22.60 & 6.20 & ML & & $\downarrow$ \\
\hline 34 & 18.80 & 35.60 & 32.04 & 23.00 & 12.60 & CL & & Ref.20 -Boring 8 \\
\hline 35 & 19.20 & 36.10 & 32.49 & 24.50 & 11.60 & ML & & \\
\hline 36 & 20.50 & 34.10 & 30.69 & 22.80 & 11.30 & ML & & \\
\hline 37 & 15.30 & 36.90 & 33.21 & 20.00 & 16.90 & CL & & \\
\hline 38 & 5.90 & 27.30 & 24.57 & 20.90 & 6.40 & ML & & $y$ \\
\hline 39 & 24.90 & 38.80 & 34.92 & 23.80 & 15.00 & ML & & Ref.20-Boring 9 \\
\hline 40 & 22.70 & 41.10 & 36.99 & 25.20 & 15.90 & ML & & \\
\hline 41 & 22.70 & 40.40 & 36.36 & 26.00 & 14.40 & ML & & \\
\hline 42 & 8.20 & 28.00 & 25.20 & 21.10 & 6.90 & ML & & $t$ \\
\hline 43 & 25.10 & 38.90 & 35.01 & 28.00 & 10.90 & ML & & Ref.20-Boring 10 \\
\hline 44 & 24.90 & 41.20 & 37.08 & 28.80 & 12.40 & ML & & \\
\hline 45 & 25.50 & 43.10 & 38.79 & 26.40 & 16.70 & ML & & \\
\hline 46 & 11.30 & 34.20 & 30.78 & 26.10 & 8.10 & ML & & \\
\hline 47 & 8.70 & 27.80 & 25.02 & 22.10 & 5.70 & $\mathrm{CL}$ & & $\psi$ \\
\hline 48 & 22.10 & 41.20 & 37.08 & 23.10 & 18.10 & CL & & Ref. 24, Boring 1 \\
\hline 49 & 17.40 & 34.20 & 30.78 & 19.40 & 14.80 & $C L$ & & \\
\hline 50 & 16.10 & 34.80 & 31.32 & 21.20 & 13.60 & $\mathrm{CL}$ & & 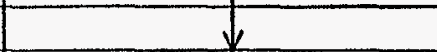 \\
\hline 51 & 28.70 & 41.60 & 37.44 & 24.30 & 17.30 & CL & & Ref. 24, Boring 2 \\
\hline 52 & 21.80 & 43.60 & 39.24 & 25.00 & 18.60 & CL & & \\
\hline 53 & 19.10 & 36.10 & 32.49 & 16.70 & 19.40 & CL & & $\psi$ \\
\hline 54 & 22.70 & 37.20 & 33.48 & 19.10 & 18.10 & CL & & Ref. 24 , Boring 3 \\
\hline 55 & 20.60 & 39.80 & 35.82 & 22.90 & 16.90 & CL & & \\
\hline 56 & 18.40 & 36.80 & 33.12 & 19.80 & 17.00 & CL & & 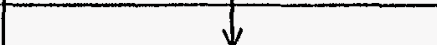 \\
\hline 57 & 11.60 & 36.20 & 32.58 & 19.00 & 17.20 & $C L$ & & Ref. 24 , Boring 4 \\
\hline 58 & 21.00 & 35.20 & 31.68 & 17.80 & 17.40 & $\mathrm{CL}$ & & \\
\hline 59 & 6.50 & 31.60 & 28.44 & 17.40 & 14.20 & CL & & \pm \\
\hline 60 & 18.20 & 32.10 & 28.89 & 20.00 & 12.10 & $\mathrm{CL}$ & & Ref. 27 , Boring 1 \\
\hline
\end{tabular}


TABLE 5 Index Properties and Soil Classification (cont'd.)

\begin{tabular}{|c|c|c|c|c|c|c|c|c|}
\hline & Natural & Liquid & & Plastic & Plasticity & & Grain & \\
\hline & Water Content & Limit & & Limit & Index & Soil & Size & \\
\hline$\#$ & 8 & $\frac{9}{8}$ & $0.9 * L L$ & 8 & $\mathbf{L}-\mathbf{P L}$ & Classification & $.005 \mathrm{~mm}$ & Reference \\
\hline & (WC) & (LL) & & (PL) & (PI) & & $(\&$ Finer $)$ & \\
\hline 61 & 12.20 & 33.20 & 29.88 & 19.10 & 14.10 & CL & & \\
\hline 62 & 24.30 & 39.40 & 35.46 & 23.10 & 16.30 & CL & & \\
\hline 63 & 24.50 & 38.10 & 34.29 & 23.30 & 14.80 & $\overrightarrow{C L}$ & & \\
\hline 64 & 20.70 & 39.20 & 35.28 & 23.60 & 15.60 & CL & & \\
\hline 65 & 22.40 & 36.20 & 32.58 & 20.40 & 15.80 & CL & & \\
\hline 66 & 17.40 & 35.40 & 31.86 & 19.00 & 16.40 & $\mathrm{CL}$ & & $\downarrow$ \\
\hline 67 & 20.00 & 36.20 & 32.58 & 21.10 & 15.10 & $\mathrm{CL}$ & & Ref. 27 , Boring 2 \\
\hline 68 & 18.80 & 34.20 & 30.78 & 21.60 & 12.60 & CL & & \\
\hline 69 & 26.70 & 37.50 & 33.75 & 27.30 & 10.20 & ML & & \\
\hline 70 & 15.60 & 34.60 & 31.14 & 21.50 & 13.10 & $\mathrm{CL}$ & & \\
\hline 71 & 25.00 & 37.20 & 33.48 & 20.10 & 17.10 & CL & & \\
\hline 72 & 17.90 & 34.40 & 30.96 & 19.40 & 15.00 & CL & & $\downarrow$ \\
\hline 73 & 16.30 & 32.10 & 28.89 & 17.70 & 14.40 & CL & & Ref. 27 , Boring 3 \\
\hline 74 & 15.50 & 34.20 & 30.78 & 22.90 & 11.30 & ML & & \\
\hline 75 & 22.60 & 35.60 & 32.04 & 19.10 & 16.50 & $\mathbf{C L}$ & & $\therefore$ \\
\hline 76 & 19.70 & 36.90 & 33.21 & 19.50 & 17.40 & CL & & \\
\hline 77 & 15.00 & 35.10 & 31.59 & 17.60 & 17.50 & $\mathrm{CL}$ & & \\
\hline 78 & 17.80 & 34.70 & 31.23 & 19.00 & 15.70 & CL & & $\sqrt{2}$ \\
\hline 79 & 24.10 & 34.70 & 31.23 & 20.40 & 14.30 & CL & & Ref. 27 , Boring 4 \\
\hline 80 & 23.00 & 35.10 & 31.59 & 22.90 & 12.20 & CL & & \\
\hline 81 & 22.90 & 28.80 & 25.92 & 17.40 & 11.40 & $\mathrm{CL}$ & & \\
\hline 82 & 17.50 & 27.80 & 25.02 & 15.90 & 11.90 & CL & & \\
\hline 83 & 24.10 & 32.60 & 29.34 & 16.50 & 16.10 & $\mathrm{CL}$ & & \\
\hline 84 & 21.50 & 33.30 & 29.97 & 18.30 & 15.00 & CL & & \\
\hline 85 & 22.10 & 32.60 & 29.34 & 18.40 & 14.20 & CL & & $\sqrt{k}$ \\
\hline 86 & 16.50 & 44.00 & 39.60 & 29.00 & 15.00 & ML & 34 & Ref. 28 , Boring $v-1$ \\
\hline 87 & 18.80 & 33.00 & 29.70 & 32.00 & 1.00 & $\mathrm{ML}$ & & \\
\hline 88 & 12.80 & 29.00 & 26.10 & 24.00 & 5.00 & ML & 12 & $\sqrt{2}$ \\
\hline 89 & 27.70 & 42.00 & 37.80 & 21.00 & 21.00 & CL & & Ref. 28, Boring $V-2$ \\
\hline 90 & 20.30 & 50.00 & 45.00 & 23.00 & 27.00 & $\mathrm{CH}$ & & Ref. 28 , Boring $\mathrm{V}-3$ \\
\hline
\end{tabular}


TABLE 5 Index Properties and Soil Classification (cont'd.)

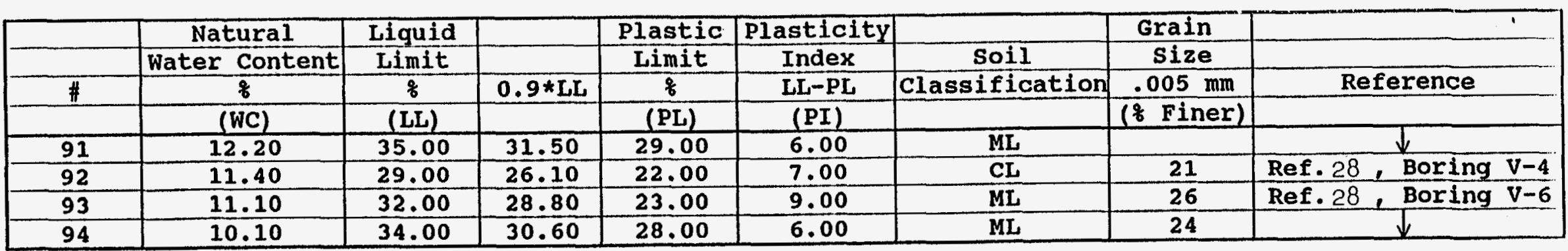




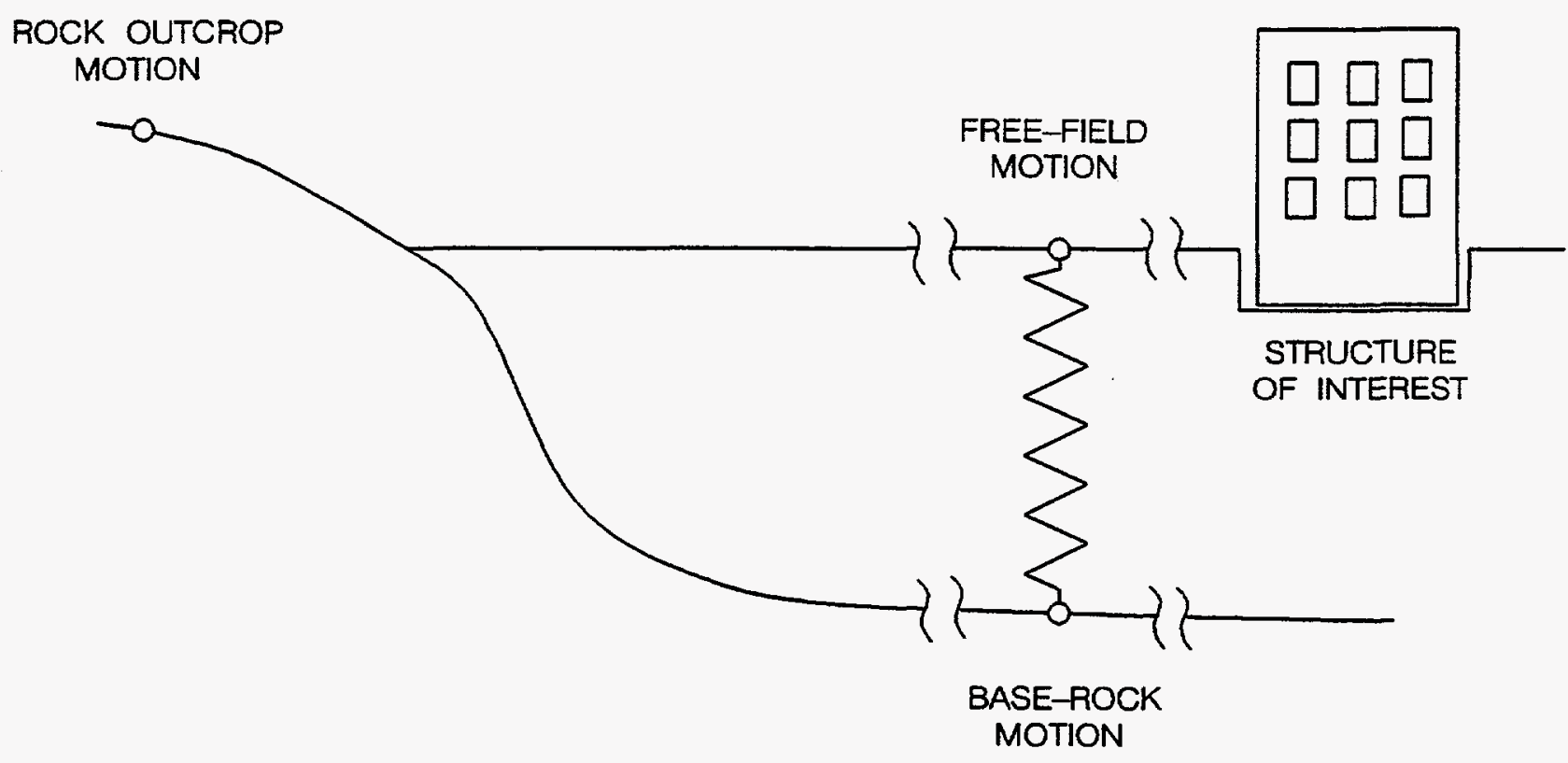

FIG. 14 THREE PRIMARY CONTROL POINTS FOR A SITE RESPONSE ANALYSIS 
The primary focus of this study was to calculate the free field acceleration response spectra for the soil/weathered shale and determine the amplification ratios between the free field and rock outcrop motions. The scope of this study requires response analysis for damping ratios of 2, 5, $7,10,12$, and 15 percent. For the purpose of this analysis, data is presented for spectral periods up to 10 seconds to define the peak response values.

The SHAKE 91 output consists of two files. The first file contains input information, the results of iteration of modulus reduction and damping ratios to arrive at the strain compatible materials properties, calculated maximum shear stresses and strains, peak accelerations, response spectrum and fourier spectrum, etc. The second output file contains all the time histories desired. Since the main objective of this study is to determine soil amplification ratios, this report will focus on the spectral accelerations and the amplification ratios considering the two components of the 2000-year seismic event, seven representative soil columns and six levels of damping values. Therefore, the second output file is not required and consequently it is not included in this report. The filing system for identifying the individual SHAKE runs are explained on page 35 . Tables 6 through 11 present the summary of the amplification ratios for seven soil columns, two horizontal components of the earthquake and six damping ratios.

Plots of absolute response accelerations at top of ground (soil/weathered shale) and fresh rock are shown in Appendix A1. Plots of soil amplification ratios vs. spectral period are presented in Appendix A2.

Considering the mean values of material properties with 5 percent damping: (a) The peak soil amplification ratio varied between 3.8 and 4.5 at spectral periods ranging from .06 to .2 second, and (b) The peak weathered shale amplification ratio varied from 1.2 to 1.4 at .04 second.

For the same conditions as above with the exception of damping changed to 10 percent, the peak soil amplification ratio varied from 3.3 to 4.4 at periods ranging between $: 06$ and .2 seconds, and the peak amplification ratio for weathered shale was on the order of 1.3 at an spectral period of .04 second.

Considering all seven soil columns, lower bound, mean and upper bound material properties, a general range of peak amplification ratios occurred at spectral periods between .04 and .3 second. The highest peak amplification ratio was 9.7 at spectral period of .1 second for soil column 1 (10 $\mathrm{ft}$. soil over $10 \mathrm{ft}$. weathered shale), with a damping value of 2 percent for lower bound shear wave velocities and earthquake component 2 . 
ROCK OUTCROP
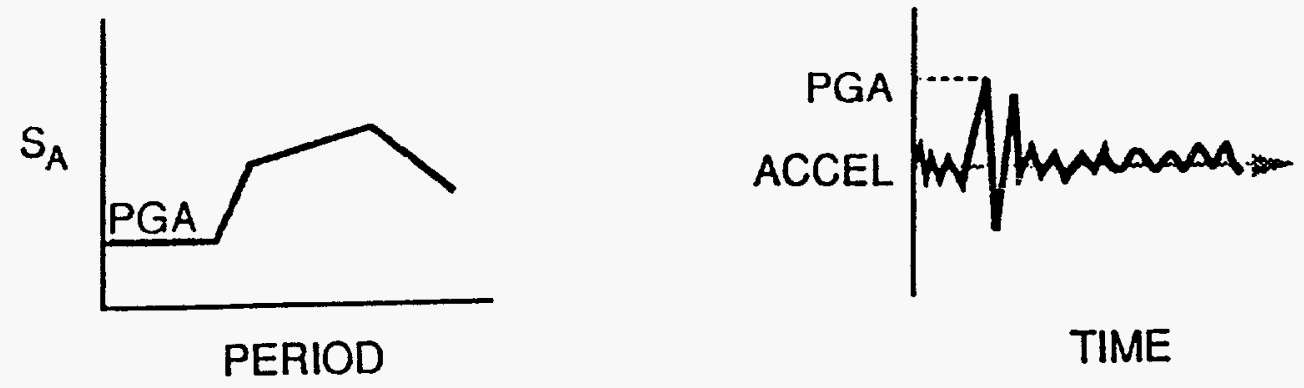

TIME

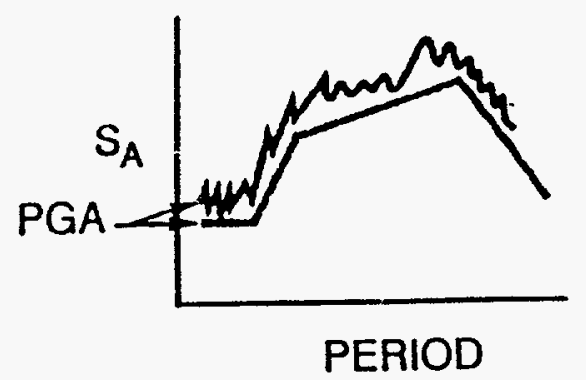

SURFACE

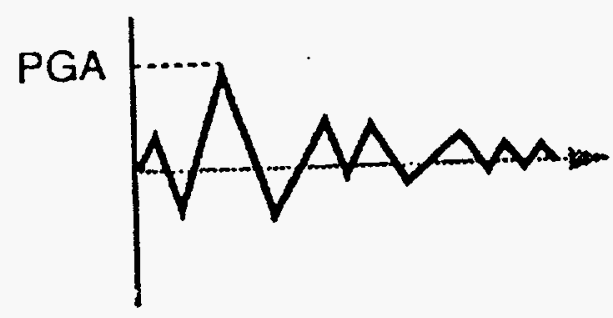

TIME

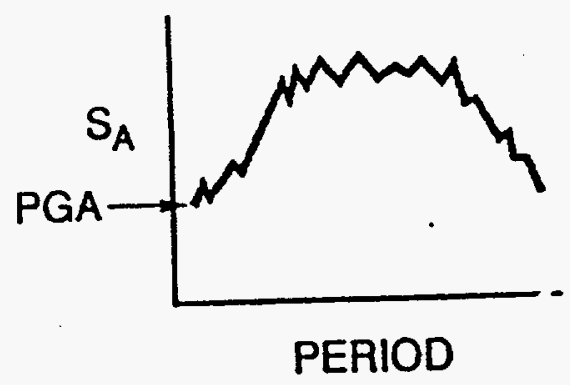

\section{SURFACE}
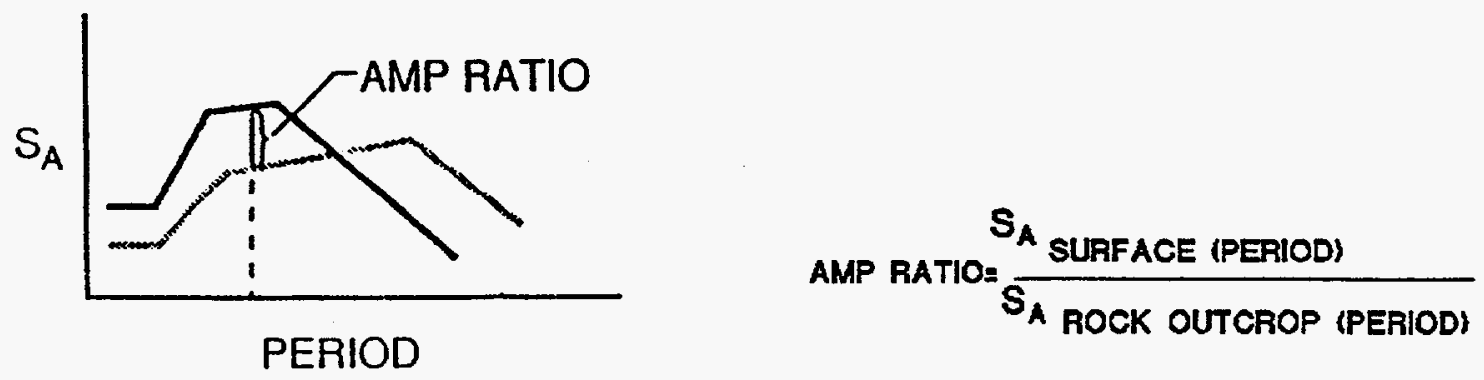

FIG.15 SCHEMATIC REPRESENTATION OF SOIL RESPONSE ANALYSIS 


\section{Shake 91 Filing System}

File Names

$\begin{array}{lll}\text { Y1M1 } & \text { Y1L1 } & \text { Y1U1 } \\ \text { Y1M2 } & \text { Y1L2 } & \text { Y1U2 } \\ \text { Y1M3 } & \text { Y1L3 } & \text { Y1U3 } \\ \text { Y1M4 } & \text { Y1L4 } & \text { Y1U4 } \\ \text { Y1M5 } & \text { Y1L5 } & \text { Y1U5 } \\ \text { Y1M6 } & \text { Y1L6 } & \text { Y1U6 } \\ \text { Y1M7 } & \text { Y1L7 } & \text { Y1U7 } \\ & & \\ \text { Y2M1 } & \text { Y2L1 } & \text { Y2U1 } \\ \text { Y2M2 } & \text { Y2L2 } & \text { Y2U2 } \\ \text { Y2M3 } & \text { Y2L3 } & \text { Y2U3 } \\ \text { Y2M4 } & \text { Y2L4 } & \text { Y2U4 } \\ \text { Y2M5 } & \text { Y2L5 } & \text { Y2U5 } \\ \text { Y2M6 } & \text { Y2L6 } & \text { Y2U6 } \\ \text { Y2M7 } & \text { Y2L7 } & \text { Y2U7 }\end{array}$

File Name System

$\begin{array}{lll}\mathrm{Y}= & \mathrm{Y}-12 & \text { SARUP } \\ 1,2 & = & \text { Earthquake History } 1000 \text { yrs. Horiz. Component } 1 \text { or 2. } \\ \mathrm{L}, \mathrm{M}, \mathrm{U} & = & \text { Lower Bound, Mean, Upper Bound Shear Wave Velocity } \\ 1,2,3,4,5,6,7 & = & \text { Soil Column \#1,2,3, 4, 5, 6,7 } \\ \text { Y2L3 } & = & \begin{array}{l}\text { Y12 SARUP, EQ History Horiz. Comp 2, Lower Bound Shear wave Velocity, } \\ \text { Soil Column \#3 [For damping ratios .02, .05, .07, .10,.12 \&.15] }\end{array} \\ \text { Y2L3.DAT } & = & \text { Data File } \\ \text { Y2L3.01 } & = & \text { Output File 1 } \\ \text { Y2L3.02 } & = & \text { Output File 2 }\end{array}$


TABLE 6 Summary of Amplification Ratios

$$
\text { Damping }=2 \%
$$

$\mathrm{L}=$ Lower Bound Properties, $M=$ Median Properties, $U=$ Upper Bound Properties

\begin{tabular}{|c|c|c|c|c|c|c|c|c|c|c|c|c|c|c|c|c|c|c|c|c|c|c|c|c|}
\hline & & & & & & & & & & & & & & & & & & & & & & & & \\
\hline \multirow{4}{*}{\begin{tabular}{|l|} 
Profile \\
Property \\
History \\
Period, (sec) \\
\end{tabular}} & \multicolumn{6}{|c|}{1} & \multicolumn{6}{|c|}{$\frac{1}{2}$} & \multicolumn{6}{|c|}{3} & \multicolumn{6}{|c|}{4} \\
\hline & \multicolumn{2}{|c|}{$\mathrm{L}$} & \multicolumn{2}{|c|}{ M } & \multicolumn{2}{|c|}{$\mathbf{U}$} & \multicolumn{2}{|c|}{$\mathbf{L}$} & \multicolumn{2}{|c|}{$\bar{M}$} & \multicolumn{2}{|c|}{$\bar{U}$} & \multicolumn{2}{|c|}{ L } & \multicolumn{2}{|c|}{$\bar{M}$} & \multicolumn{2}{|c|}{$\bar{U}$} & \multicolumn{2}{|c|}{$\mathrm{L}$} & \multicolumn{2}{|c|}{$M$} & \multicolumn{2}{|c|}{$\bar{U}$} \\
\hline & 1 & 2 & 1 & 2 & 1 & 2 & 1 & 2 & 1 & 2 & 1 & 2 & 1 & 2 & 1 & 2 & 1 & 2 & 1 & 2 & 1 & 2 & 1 & 2 \\
\hline & \multicolumn{24}{|c|}{ Amplification Ratio at Period, $(\mathrm{sec})$} \\
\hline 0.01 & 3.10 & 2.85 & 3.11 & 2.42 & 2.87 & 2.49 & 3.25 & 2.84 & 3.44 & 2.68 & 2.38 & 2.04 & 2.68 & 2.11 & 2.50 & 2.04 & 2.35 & 1.98 & 2.87 & 2.52 & 2.38 & 2.12 & 2.31 & $\overline{2.24}$ \\
\hline 0.03 & 2.98 & 3.07 & 2.96 & 2.36 & 3.52 & 3.08 & 3.02 & 2.68 & 3.31 & 2.55 & 2.58 & 2.08 & 2.31 & 2.07 & 2.43 & 1.91 & 2.14 & 1.83 & $-\frac{1}{2.58}$ & 2.36 & 2.22 & $\frac{2.10}{2.10}$ & 2.19 & 2,13 \\
\hline 0.04 & 2.79 & 3.04 & 1.93 & 2.04 & 4.56 & 5.04 & 1.86 & 2.04 & 2.66 & 2.85 & 2.57 & 2.76 & $\frac{2.0}{2.08}$ & 2.11 & 2.78 & 2.54 & $\frac{2.14}{1.26}$ & $\frac{1.00}{1.60}$ & $\begin{array}{l}2.00 \\
1.62\end{array}$ & $\frac{4.00}{1.69}$ & \begin{tabular}{c|}
$\frac{2.24}{2.05}$ \\
\end{tabular} & $\frac{2.10}{2.08}$ & $\frac{2.10}{1.72}$ & $\frac{4.10}{1.96}$ \\
\hline 0.05 & 1.91 & 1.85 & 2.65 & 2.22 & 7.39 & -7.72 & 2.62 & 2.52 & 2.51 & 2.53 & 3.23 & 3.07 & 1.69 & 1.63 & 1.64 & 1.79 & 1.34 & 1.41 & 2.49 & 2.47 & 2.37 & 2.25 & 1.58 & 1.47 \\
\hline 0.06 & 1.98 & 2.02 & 4.39 & 3.31 & 3.06 & 3.33 & 3.18 & 2.81 & 3.06 & 2.61 & 3.17 & 2.73 & 3.16 & 2.58 & 1.44 & 1.36 & 1.79 & 1.59 & 2.59 & 2.58 & 2.02 & $\begin{array}{l}2.24 \\
1.79\end{array}$ & 1.81 & 1.62 \\
\hline 0.07 & 2.87 & 2.84 & 4.02 & 4.33 & 1.92 & 2.08 & 2.96 & 2.72 & 3.99 & 4.03 & 2.22 & 2.10 & 2.02 & 2.14 & 1.53 & 1.37 & 2.77 & 2.68 & 2.94 & 2.93 & 1.60 & 1.71 & 2.47 & 2.53 \\
\hline 0.08 & 4.10 & 3.78 & 3.52 & 3.03 & 1.86 & 1.64 & 3.02 & 2.67 & 4.03 & 4.11 & 1.95 & 1.78 & 1.51 & 1.42 & 1.66 & 1.40 & 3.16 & 3.24 & 2.54 & 2.56 & 1.67 & 1.58 & 2.92 & 3.01 \\
\hline 0.09 & 8.30 & 8.48 & 2.70 & 2.24 & 1.57 & 1.47 & 3.73 & 3.83 & 3.50 & 3.14 & 1.68 & 1.67 & 1.57 & 1.39 & 2.18 & 2.03 & 3.68 & 3.34 & 2.11 & 1.84 & 2.08 & 2.15 & 3.65 & 3.45 \\
\hline 0.1 & 7.80 & 9.73 & 2.09 & 1.99 & 1.42 & 1.37 & 4.49 & 4.28 & 2.62 & 2.74 & 1.46 & 1.45 & 1.44 & 1.55 & 2.64 & 2.52 & 2.65 & 3.07 & 1.77 & 1.91 & 2.38 & $\frac{.10}{2.20}$ & $\frac{0.04}{3.04}$ & $\frac{0.70}{3.41}$ \\
\hline 0.11 & 4.65 & 3.92 & 1.89 & 1.64 & 1.39 & 1.29 & 4.90 & 4.98 & 2.41 & 2.05 & 1.45 & 1.35 & 1.60 & 1.65 & 3.41 & 3.53 & 2.52 & 2.22 & 1.66 & 1.84 & $\frac{2.00}{2.99}$ & $\frac{2.24}{2.96}$ & $\frac{0.04}{2.74}$ & $\frac{0.41}{2.40}$ \\
\hline 0.12 & 3.96 & 3.93 & 1.77 & 1.62 & 1.29 & 1.31 & 4.67 & 5.07 & 2.31 & 2.07 & 1.36 & 1.33 & 1.86 & 1.83 & 3.91 & 3.91 & 2.38 & 2.22 & 1.94 & 1.78 & 3.47 & 3.45 & 2.59 & 2.42 \\
\hline 0.13 & 2.91 & 2.96 & 1.66 & 1.47 & 1.22 & 1.19 & 4.15 & 4.06 & 1.97 & 1.81 & 1.27 & 1.23 & 1.91 & 2.29 & 4.25 & 4.36 & 2.01 & 1.95 & 2.03 & 2.17 & 4.05 & 4.29 & 2.22 & 2.14 \\
\hline 0.14 & 2.22 & 2.63 & 1.38 & 1.46 & 1.17 & 1.21 & 3.27 & 3.50 & 1.64 & 1.77 & 1.21 & 1.25 & 2.46 & 2.25 & 3.98 & 4.23 & 1.68 & 1.82 & 2.45 & 2.15 & 4.36 & 4.60 & 1.82 & 1.92 \\
\hline 0.15 & 2.00 & 2.14 & 1.30 & 1.34 & 1.15 & 1.14 & 2.61 & 2.82 & 1.54 & 1.55 & 1.19 & 1.17 & 2.79 & 3.37 & 3.09 & 3.09 & 1.57 & 1.62 & 2.60 & 2.99 & 3.68 & $\frac{7.00}{3.54}$ & 1.63 & 1.75 \\
\hline 0.16 & 1.77 & 1.67 & 1.24 & 1.18 & 1.13 & 1.14 & 2.17 & 2.17 & 1.39 & 1.35 & 1.14 & 1.16 & 3.44 & 3.61 & 2.51 & 2.56 & 1.47 & 1.37 & 3.04 & 3.19 & 3.05 & \begin{tabular}{|c|}
.11 \\
.11
\end{tabular} & 1.57 & 1.46 \\
\hline 0.17 & 1.65 & 1.75 & 1.21 & $1.2 \overline{3}$ & 1.11 & 1.12 & 2.22 & 2.30 & 1.36 & 1.42 & 1.12 & $1.1 \overline{6}$ & 4.39 & 4.58 & 2.55 & 2.38 & 1.40 & 1.45 & 3.70 & 3.71 & 2.98 & 2.77 & 1.50 & 1.52 \\
\hline 0.18 & 1.52 & $1.5 \bar{B}$ & 1.17 & 1.25 & 1.10 & 1.09 & 1.83 & 2.04 & 1.27 & 1.36 & 1.11 & 1.13 & 5.42 & 5.10 & 2.04 & 2.15 & 1.31 & 1.36 & 4.83 & 4.53 & 2.28 & 2.41 & 1.39 & 1.44 \\
\hline 0.19 & 1.50 & 1.44 & 1.17 & 1.14 & 1.10 & 1.07 & 1.85 & 1.83 & 1.27 & 1.24 & 1.11 & 1.08 & 5.34 & 5.25 & 2.01 & 1.95 & 1.28 & 1.27 & 5.18 & 5.46 & 2.25 & 2.20 & 1.35 & 1.32 \\
\hline 0.2 & 1.52 & 1.35 & 1.22 & 1.15 & 1.09 & 1.09 & 1.82 & 1.61 & 1.33 & $1 . \overline{22}$ & 1.14 & 1.11 & 5.08 & 4.46 & 1.94 & 1.76 & 1.35 & 1.22 & 5.44 & 4.98 & 2.12 & 1.98 & 1.41 & 1.28 \\
\hline 0.3 & 1.40 & 1.53 & 1.15 & 1.16 & 1.08 & 1.09 & 1.61 & 1.92 & 1.27 & 1.26 & 1.10 & 1.09 & 3.82 & 4.22 & 1.76 & 1.88 & 1.30 & 1.31 & 4.38 & $4 . \overline{87}$ & 1.99 & 2.06 & 1.33 & 1.39 \\
\hline 0.4 & 1.17 & 1.16 & 1.07 & 1.10 & 1.04 & 1.05 & 1.21 & 1.30 & 1.11 & 1.12 & 1.04 & 1.05 & 1.84 & 1.94 & 1.25 & 1.36 & 1.12 & 1.12 & 2.10 & 2.28 & 1.30 & 1.42 & 1.13 & 1.14 \\
\hline 0.5 & 1.15 & 1.12 & 1.04 & 1.04 & 1.02 & 1.03 & 1.18 & 1.22 & 1.08 & 1.06 & 1.03 & 1.03 & 1.45 & 1.43 & 1.20 & 1.21 & 1.09 & 1.07 & 1.64 & 1.46 & 1.24 & 1.23 & 1.11 & 1.09 \\
\hline 0.6 & 1.07 & 1.06 & 1.05 & 1.03 & 1.02 & 1.02 & 1.14 & 1.08 & 1.06 & 1.04 & 1.03 & 1.02 & 1.33 & 1.35 & 1.16 & 1.08 & 1.05 & 1.04 & 1.38 & 1.37 & 1.18 & 1.11 & 1.06 & 1.05 \\
\hline 0.8 & 1.06 & 1.04 & 1.04 & 1.02 & 1.01 & 1.01 & 1.08 & 1.05 & 1.05 & 1.03 & 1.02 & 1.01 & 1.24 & 1.16 & 1.07 & 1.06 & 1.04 & 1.03 & 1.25 & 1.25 & 1.08 & 1.07 & 1.04 & 1.03 \\
\hline 1 & 1.04 & 1.05 & 1.02 & 1.02 & 1.01 & 1.01 & 1.05 & 1.05 & 1.03 & 1.03 & 1.01 & 1.01 & 1.16 & 1.09 & 1.06 & 1.05 & 1.03 & 1.03 & 1.17 & 1.12 & 1.07 & 1.06 & 1.03 & 1.04 \\
\hline 2 & 1.04 & 1.03 & 1.02 & 1.02 & 1.01 & 1.01 & 1.04 & 1.05 & 1.02 & 1.03 & 1.01 & 1.01 & 1.08 & 1.11 & 1.03 & 1.05 & 1.02 & 1.02 & 1.10 & 1.15 & 1.03 & 1.06 & 1.02 & 1.03 \\
\hline
\end{tabular}


TABLE 6 Summary Amplification Ratios (Cont'd.)

Damping $=2 \%$

$L=$ Lower Bound Properties, $M=$ Median Properties, $U=$ Upper Bound Properties

\begin{tabular}{|c|c|c|c|c|c|c|c|c|c|c|c|c|c|c|c|c|c|c|}
\hline \multirow{4}{*}{\begin{tabular}{|l|} 
Profile \\
Property \\
History \\
\end{tabular}} & \multirow{2}{*}{\multicolumn{6}{|c|}{5}} & & \\
\hline & & & & & & & & & & & & & & & & & & \\
\hline & \multicolumn{2}{|c|}{ L } & \multicolumn{2}{|c|}{$M$} & \multicolumn{2}{|c|}{$U$} & \multicolumn{2}{|l|}{$L$} & \multicolumn{2}{|c|}{$M$} & \multicolumn{2}{|c|}{$\bar{U}$} & \multicolumn{2}{|c|}{$L$} & \multicolumn{2}{|c|}{$M$} & \multicolumn{2}{|c|}{$U$} \\
\hline & 1 & 2 & 1 & 2 & 1 & 2 & 1 & 2 & 1 & 2 & 1 & 2 & 1 & 2 & 1 & 2 & 1 & 2 \\
\hline Period, (sec) & & & & & & & Amp & lificati & n Ratic & at $P_{E}$ & od, (se & & & & & & & \\
\hline 0.01 & 2.35 & 2.14 & 2.32 & 1.79 & 2.10 & 1.81 & 2.60 & 2.01 & 2.53 & 2.11 & 2.00 & 1.73 & 1.35 & 1.34 & 1.12 & 1.12 & 1.04 & 1.03 \\
\hline 0.03 & 2.11 & 1.98 & 2.06 & 1.74 & 2.06 & 1.69 & 2.27 & 1.78 & 2.30 & 1.94 & 1.90 & 1.74 & 1.52 & 1.37 & 1.14 & 1.13 & 1.04 & 1.03 \\
\hline 0.04 & 2.02 & 1.89 & 1.62 & 1.77 & 2.48 & 2.23 & 1.35 & 1.55 & 1.81 & 1.97 & 1.98 & 2.20 & 1.93 & 2.01 & 1.33 & 1.30 & 1.14 & 1.09 \\
\hline 0.05 & 1.84 & 1.43 & 1.45 & 1.38 & 1.48 & 1.58 & 2.09 & 1.89 & 1.97 & 1.95 & 1.88 & 2.07 & $1.5 \overline{8}$ & 1.65 & 1.23 & 1.25 & 1.10 & 1.10 \\
\hline 0.06 & 1.76 & 1.79 & 2.78 & 2.24 & 1.30 & 1.23 & 2.34 & 1.75 & 2.72 & 2.18 & 1.51 & 1.25 & 1.51 & 1.30 & 1.26 & 1.11 & 1.15 & 1.03 \\
\hline 0.07 & 1.35 & $1.4 \overline{6}$ & 1.78 & 1.98 & 1.42 & 1.28 & 2.26 & 2.23 & 2.31 & 2.51 & 1.34 & 1.38 & 1.32 & 1.22 & 1.16 & 1.08 & 1.09 & 1.02 \\
\hline 0.08 & 1.81 & 1.57 & 1.39 & 1.31 & 1.51 & 1.29 & 2.26 & 1.94 & 1.85 & 1.72 & 1.43 & 1.33 & 1.19 & 1.17 & 1.09 & 1.08 & 1.04 & 1.03 \\
\hline 0.09 & 2.98 & 2.52 & 1.42 & 1.27 & 1.99 & 1.85 & 2.83 & 2.66 & 1.60 & 1.45 & 1.88 & 1.90 & 1.18 & 1.13 & 1.09 & 1.05 & 1.04 & 1.01 \\
\hline 0.1 & 2.07 & 2.40 & 1.30 & 1.43 & 2.35 & 2.23 & 2.84 & 2.86 & 1.43 & 1.57 & 2.15 & 2.05 & 1.09 & 1.13 & 1.03 & 1.07 & 1.00 & 1.04 \\
\hline 0.11 & 1.54 & 1.50 & 1.40 & 1.51 & 2.90 & 2.96 & 2.06 & 2.00 & 1.36 & 1.56 & 2.68 & 2.73 & 1.09 & 1.07 & 1.03 & 1.03 & 1.00 & 1.00 \\
\hline 0.12 & 1.27 & 1.35 & 1.65 & 1.63 & 3.28 & 3.17 & 1.81 & 1.89 & 1.66 & 1.55 & 3.07 & 2.99 & 1.10 & 1.08 & 1.05 & 1.03 & 1.02 & 1.00 \\
\hline 0.13 & 1.15 & 1.26 & 1.75 & 1.99 & 3.38 & 3.51 & 1.58 & 1.51 & 1.77 & 1.95 & 3.32 & 3.48 & 1.08 & 1.08 & 1.05 & 1.04 & 1.03 & 1.02 \\
\hline 0.14 & 1.58 & 1.34 & 2.21 & 2.01 & 3.34 & 3.55 & 1.73 & 1.43 & 2.22 & 1.94 & 3.40 & 3.66 & 1.07 & 1.08 & 1.04 & 1.04 & 1.02 & 1.02 \\
\hline 0.15 & 1.23 & 1.64 & 2.44 & 2.87 & 2.81 & 2.76 & 1.33 & 1.66 & 2.32 & 2.67 & 3.00 & 2.92 & 1.05 & 1.04 & 1.02 & 1.02 & 1.01 & 1.01 \\
\hline 0.16 & 1.52 & 1.67 & 3.06 & 3.04 & 2.38 & 2.41 & 1.51 & 1.77 & 2.83 & 2.84 & 2.60 & 2.64 & 1.04 & 1.04 & 1.01 & 1.02 & 1.00 & 1.01 \\
\hline 0.17 & 1.37 & 1.26 & 3.77 & 3.78 & 2.39 & 2.24 & 1.42 & 1.28 & 3.40 & 3.37 & 2.55 & 2.44 & 1.02 & 1.04 & 1.01 & 1.02 & 1.00 & 1.01 \\
\hline 0.18 & 1.70 & 1.58 & 4.53 & 4.33 & 1.91 & 2.01 & 1.61 & 1.59 & 4.28 & 3.99 & 2.03 & 2.14 & 1.04 & 1.04 & 1.02 & 1.02 & 1.01 & 1.01 \\
\hline 0.19 & 1.69 & 2.06 & 4.51 & 4.54 & 1.93 & 1.87 & 1.66 & 2.05 & 4.41 & 4.60 & 2.03 & 1.99 & 1.02 & 1.02 & 1.01 & 1.01 & 1.00 & 1.00 \\
\hline 0.2 & 1.89 & 1.68 & 4.47 & 3.96 & 1.87 & 1.72 & 1.74 & 1.66 & 4.58 & 4.13 & 1.95 & 1.80 & 1.04 & 1.04 & 1.02 & 1.02 & 1.01 & 1.01 \\
\hline 0.3 & 4.88 & 5.22 & 3.42 & 3.83 & 1.72 & 1.83 & 4.61 & 5.44 & 3.67 & 4.11 & 1.82 & 1.91 & 1.03 & 1.03 & 1.02 & 1.01 & 1.01 & 1.01 \\
\hline 0.4 & 3.49 & 4.85 & 1.78 & 1.94 & 1.24 & 1.33 & 4.13 & 5.28 & 1.92 & 2.10 & 1.26 & 1.36 & 1.02 & 1.01 & 1.01 & 1.01 & 1.01 & 1.01 \\
\hline 0.5 & 2.21 & 2.45 & 1.45 & 1.40 & 1.17 & 1.19 & 2.41 & 2.55 & 1.55 & 1.40 & 1.20 & 1.21 & 1.01 & 1.01 & 1.00 & 1.00 & 1.00 & 1.00 \\
\hline 0.6 & 1.60 & 1.70 & 1.31 & 1.32 & 1.14 & 1.08 & 1.72 & 1.82 & 1.33 & 1.33 & 1.16 & 1.09 & 1.01 & 1.01 & 1.00 & 1.00 & 1.00 & 1.00 \\
\hline 0.8 & 1.43 & 1.46 & 1.21 & 1.17 & 1.06 & 1.06 & 1.52 & 1.50 & 1.22 & 1.21 & 1.07 & 1.07 & 1.01 & 1.01 & 1.00 & 1.00 & 1.00 & 1.00 \\
\hline 1 & 1.21 & 1.29 & 1.14 & 1.09 & 1.05 & 1.05 & 1.23 & 1.32 & 1.15 & 1.10 & 1.06 & 1.05 & 1.00 & 1.00 & 1.00 & 1.00 & 1.00 & 1.00 \\
\hline 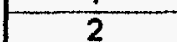 & 1.12 & 1.31 & 1.08 & 1.12 & 1.03 & 1.05 & 1.13 & 1.34 & 1.09 & 1.14 & 1.03 & 1.05 & 1.00 & 1.00 & 1.00 & 1.00 & 1.00 & 1.00 \\
\hline
\end{tabular}


TABLE 6 Summary Amplification Ratios (Cont'd.) Damping $=2 \%$

$L=$ Lower Bound Properties, $M=$ Median Properties, $U=$ Upper Bound Properties

\begin{tabular}{|c|c|c|c|c|c|c|c|c|c|c|c|c|c|c|c|c|}
\hline & Total & Average & $\operatorname{Max}$ & $85 \%$ & Total & Average & Max & $85 \%$ & Total & Average & Max & $85 \%$ & Total & Average & Max & $85 \%$ \\
\hline \multicolumn{17}{|l|}{ Profile } \\
\hline Property & $L$ & $L$ & $\mathbf{L}$ & $L$ & $M$ & $M$ & $M$ & $\bar{M}$ & $\mathbf{U}$ & $\mathrm{U}$ & $\mathrm{U}$ & $u$ & All & All & All & All \\
\hline \multicolumn{17}{|l|}{ History } \\
\hline \multicolumn{17}{|c|}{ Period, (sec) } \\
\hline 0.01 & 34.00 & 2.43 & 3.25 & 3.10 & 31.67 & 2.26 & 3.44 & 3.11 & 28.38 & 2.03 & 2.87 & 2.49 & 94.05 & 2.24 & 3.44 & 2.87 \\
\hline 0.03 & 32.12 & 2.29 & 3.07 & 3.02 & 30.15 & 2.15 & 3.31 & 2.96 & 29.02 & 2.07 & 3.52 & 3.08 & 91.29 & 2.17 & 3.52 & 2.98 \\
\hline 0.04 & 27.99 & 2.00 & 3.04 & 2.79 & 28.77 & 2.06 & 2.85 & 2.83 & 32.59 & 2.33 & 5.04 & 4.56 & 89.35 & 2.13 & 5.04 & 2.79 \\
\hline 0.05 & 27.67 & 1.98 & 2.62 & 2.52 & 27.19 & 1.94 & 2.65 & 2.53 & 36.41 & 2.60 & 7.72 & 7.39 & 91.27 & 2.17 & 7.72 & 2.62 \\
\hline 0.06 & 31.35 & 2.24 & 3.18 & 3.16 & 32.26 & 2.30 & 4.39 & 3.31 & 26.59 & 1.90 & 3.33 & 3.17 & 90.20 & 2.15 & 4.39 & $\overline{3.16}$ \\
\hline 0.07 & 31.25 & 2.23 & 2.96 & 2.94 & 33.41 & 2.39 & 4.33 & 4.03 & 26.30 & 1.88 & 2.77 & 2.68 & 90.96 & 2.17 & 4.33 & 2.94 \\
\hline 0.08 & 31.56 & 2.25 & 4.10 & 3.78 & 29.42 & 2.10 & 4.11 & 4.03 & 27.19 & 1.94 & 3.24 & 3.16 & 88.17 & 2.10 & 4.11 & 3.24 \\
\hline 0.09 & 44.57 & 3.18 & 8.48 & 8.30 & 27.89 & 1.99 & 3.50 & 3.14 & 30.19 & 2.16 & 3.68 & 3.65 & 102.65 & 2.44 & 8.48 & 3.65 \\
\hline 0.1 & 45.33 & 3.24 & 9.73 & 7.80 & 27.00 & 1.93 & 2.74 & 2.64 & 28.68 & 2.05 & 3.41 & 3.07 & 101.01 & 2.41 & 9.73 & 3.07 \\
\hline 0.11 & 34.49 & 2.46 & 4.98 & 4.90 & 28.78 & 2.06 & 3.53 & 3.41 & 28.63 & 2.05 & 2.96 & 2.90 & 91.90 & 2.19 & 4.98 & 3.41 \\
\hline 0.12 & 33.52 & 2.39 & 5.07 & 4.67 & 31.10 & 2.22 & 3.91 & 3.91 & 29.43 & 2.10 & 3.28 & 3.17 & 94.05 & 2.24 & 5.07 & 3.91 \\
\hline 0.13 & 30.14 & 2.15 & 4.15 & 4.06 & 33.40 & 2.39 & 4.36 & 4.29 & 28.97 & 2.07 & 3.51 & 3.48 & 92.51 & 2.20 & 4.36 & 4.05 \\
\hline 0.14 & 29.16 & 2.08 & 3.50 & 3.27 & 33.89 & 2.42 & 4.60 & 4.36 & 28.08 & 2.01 & 3.66 & 3.55 & 91.13 & 2.17 & 4.60 & 3.55 \\
\hline 0.15 & 29.28 & 2.09 & 3.37 & 2.99 & 31.48 & 2.25 & 3.68 & 3.54 & 24.72 & 1.77 & 3.00 & 2.92 & 85.48 & 2.04 & 3.68 & 3.00 \\
\hline 0.16 & 29.59 & 2.11 & 3.61 & 3.44 & 30.20 & 2.16 & 3.11 & 3.06 & 22.47 & 1.61 & 2.64 & 2.60 & 82.27 & 1.96 & 3.61 & 3.05 \\
\hline 0.17 & 31.70 & 2.26 & 4.58 & 4.39 & 32.25 & 2.30 & 3.78 & 3.77 & 22.00 & 1.57 & 2.55 & 2.44 & 85.95 & 2.05 & 4.58 & 3.70 \\
\hline 0.18 & 35.41 & 2.53 & 5.42 & 5.10 & 33.10 & 2.36 & 4.53 & 4.33 & 20.03 & 1.43 & 2.14 & 2.03 & 88.54 & 2.11 & 5.42 & 4.33 \\
\hline 0.19 & 37.36 & 2.67 & 5.46 & $\overline{5.34}$ & 33.30 & 2.38 & 4.60 & 4.54 & 19.40 & 1.39 & 2.03 & 1.99 & 90.05 & 2.14 & 5.46 & 4.54 \\
\hline 0.2 & 35.31 & 2.52 & 5.44 & 5.08 & 31.89 & 2.28 & 4.58 & 4.47 & 19.04 & 1.36 & \begin{tabular}{|c|}
1.95 \\
\end{tabular} & 1.87 & 86.24 & 2.05 & 5.44 & 4.46 \\
\hline 0.3 & 45.95 & 3.28 & 5.44 & 5.22 & 29.60 & 2.11 & 4.11 & 3.83 & 18.98 & 1.36 & 1.91 & 1.83 & 94.53 & 2.25 & 5.44 & 4.38 \\
\hline 0.4 & 32.77 & 2.34 & 5.28 & 4.85 & 19.50 & 1.39 & 2.10 & 1.94 & 15.90 & 1.14 & 1.36 & 1.33 & 68.18 & 1.62 & 5.28 & 2.10 \\
\hline 0.5 & 22.28 & 1.59 & 2.55 & 2.45 & 16.91 & 1.21 & 1.55 & 1.45 & 15.24 & 1.09 & 1.21 & 1.20 & 54.43 & 1.30 & 2.55 & 1.55 \\
\hline 0.6 & 18.63 & 1.33 & 1.82 & 1.72 & 16.02 & 1.14 & 1.33 & 1.33 & 14.76 & 1.05 & 1.16 & 1.14 & 49.41 & 1.18 & 1.82 & 1.37 \\
\hline 0.8 & 17.04 & 1.22 & 1.52 & 1.50 & 15.22 & 1.09 & 1.22 & 1.21 & 14.44 & 1.03 & 1.07 & 1.07 & 46.71 & 1.11 & 1.52 & 1.25 \\
\hline 1 & 15.79 & 1.13 & 1.32 & 1.29 & 14.82 & 1.06 & 1.15 & 1.14 & 14.39 & 1.03 & 1.06 & 1.05 & 45.00 & 1.07 & 1.32 & 1.16 \\
\hline 2 & 15.52 & 1.11 & 1.34 & 1.31 & 14.69 & 1.05 & 1.14 & 1.12 & 14.30 & 1.02 & 1.05 & 1.05 & 44.51 & 1.06 & 1.34 & 1.12 \\
\hline
\end{tabular}


TABLE 7 Summary of Amplification Ratios Damping $=5 \%$

$L=$ Lower Bound Properties, $M=$ Median Properties, $U=$ Upper Bound Properties

$\omega$

\begin{tabular}{|c|c|c|c|c|c|c|c|c|c|c|c|c|c|c|c|c|c|c|c|c|c|c|c|c|}
\hline \multirow{4}{*}{$\begin{array}{l}\text { Profile } \\
\text { Property } \\
\text { History } \\
\text { Period, (sec) } \\
\end{array}$} & \multicolumn{6}{|c|}{1} & \multicolumn{6}{|c|}{2} & \multicolumn{6}{|c|}{3} & \\
\hline & \multicolumn{2}{|c|}{$\mathrm{L}$} & \multicolumn{2}{|c|}{$M$} & \multicolumn{2}{|c|}{ U } & \multicolumn{2}{|c|}{$\mathbf{L}$} & \multicolumn{2}{|c|}{$M$} & \multicolumn{2}{|c|}{$\mathbf{U}$} & \multicolumn{2}{|c|}{$L$} & \multicolumn{2}{|c|}{$M$} & \multicolumn{2}{|c|}{$U$} & \multicolumn{2}{|c|}{$\mathrm{L}$} & \multicolumn{2}{|c|}{$M$} & \multicolumn{2}{|c|}{$\bar{U}$} \\
\hline & 1 & 2 & 1 & 2 & 1 & 2 & 1 & 2 & 1 & 2 & 1 & 2 & 1 & 2 & 1 & 2 & 1 & 2 & 1 & 2 & 1 & 2 & 1 & 2 \\
\hline & \multicolumn{24}{|c|}{ Amplification Ratio at Period, (sec) } \\
\hline 0.01 & 3.10 & 2.85 & 3.11 & 2.42 & 2.87 & 2.49 & 3.26 & 2.83 & 3.44 & 2.68 & 2.38 & 2.04 & 2.68 & 2.11 & 2.50 & 2.04 & 2.35 & 1.98 & 2.87 & 2.52 & 2.38 & 2.12 & 2.31 & 2.24 \\
\hline 0.03 & 3.00 & 3.10 & 2.96 & $2 . \overline{36}$ & 3.47 & 3.10 & 3.02 & 2.69 & 3.33 & 2.57 & 2.60 & 2.08 & 2.32 & 2.08 & 2.42 & 1.90 & 2.16 & 1.85 & 2.59 & 2.36 & 2.22 & 2.10 & 2.20 & 2.14 \\
\hline 0.04 & 2.64 & 3.00 & 2.12 & 2.51 & 4.79 & 4.88 & 2.27 & 2.37 & 3.06 & 3.01 & 2.93 & 2.72 & 2.11 & 2.12 & 2.56 & 2.52 & 1.73 & 1.96 & 1.98 & 2.12 & 2.16 & 2.31 & 1.87 & 2.16 \\
\hline 0.05 & 2.19 & 2.16 & 2.77 & 2.36 & 5.94 & 5.17 & 2.60 & 2.90 & 2.50 & 2.43 & 2.93 & 2.97 & 1.79 & 1.98 & 1.63 & 1.75 & 1.41 & 1.47 & 2.48 & 2.37 & 2.08 & 2.28 & 1.69 & 1.58 \\
\hline 0.06 & 2.46 & 2.01 & 4.15 & 3.25 & 2.93 & 3.31 & 2.98 & 2.73 & 3.03 & 2.50 & 2.85 & 2.65 & 2.77 & 2.67 & 1.51 & 1.46 & 1.98 & 1.56 & 2.54 & 2.67 & 1.91 & 1.74 & 2.05 & 1.61 \\
\hline 0.07 & 2.83 & 3.23 & 3.95 & 3.84 & 1.82 & 1.8 & 2.90 & 2.78 & 3.86 & 3.74 & 2.03 & 2.11 & 1.88 & 2.00 & 1.52 & 1.59 & 2.59 & 2.77 & 2.75 & 2.62 & 1.63 & 1.74 & 2.40 & 2.59 \\
\hline 0.08 & 3.65 & 3.95 & 3.48 & 2.79 & 1.89 & 1.78 & 3.30 & 2.92 & 4.08 & 3.78 & 1.94 & 1.73 & 1.51 & 1.56 & 1.80 & 1.68 & 3.03 & 3.02 & 2.67 & 2.45 & 1.92 & 1.82 & 2.87 & 2.85 \\
\hline 0.09 & 7.05 & 7.85 & 2.66 & 2.17 & 1.56 & 1.53 & 3.86 & 3.57 & 3.57 & 3.05 & 1.72 & 1.59 & 1.73 & 1.55 & 2.43 & 2.10 & 3.58 & 3.16 & 2.42 & 1.94 & 2.23 & 2.16 & 3.39 & 3.34 \\
\hline 0.1 & 6.23 & 6.71 & 2.21 & 2.08 & 1.42 & 1.43 & 4.56 & 4.09 & 2.74 & 2.75 & 1.53 & 1.58 & 1.74 & 1.77 & 2.79 & 2.63 & 2.77 & 3.07 & 2.16 & 2.19 & 2.53 & 2.43 & 2.85 & 3.11 \\
\hline 0.11 & 4.81 & 3.82 & 2.03 & 1.74 & 1.36 & 1.28 & 4.69 & 4.89 & 2.47 & 2.12 & 1.43 & 1.40 & 1.81 & 1.87 & 3.45 & 3.43 & 2.57 & 2.23 & 1.82 & 2.00 & 2.98 & 3.06 & 2.73 & 2.50 \\
\hline 0.1 & 4.07 & 3.44 & 1.89 & 1.63 & 1.30 & 1.23 & 4.83 & 5.30 & 2.33 & 2.16 & 1.44 & 1.29 & 2.04 & 2.30 & 3.90 & 3.88 & 2.47 & 2.16 & 2.12 & 2.11 & 3.42 & 3.64 & 2.64 & 2.49 \\
\hline 0.13 & 2.95 & 2.56 & 1.68 & 1.44 & 1.23 & 1.17 & 4.23 & 3.89 & 2.04 & 1.65 & 1.32 & 1.19 & 2.41 & 2.47 & 3.84 & 3.85 & 1.96 & 1.74 & 2.33 & 2.42 & 3.89 & 3.64 & 2.23 & 1.93 \\
\hline 0.14 & 2.18 & 2.40 & 1.36 & 1.41 & 1.15 & 1.24 & 3.40 & 3.48 & 1.55 & 1.71 & 1.17 & 1.23 & 2.70 & 3.11 & 3.82 & 3.89 & 1.63 & 1.78 & 2.61 & 2.96 & 4.05 & 4.19 & 1.76 & 1.91 \\
\hline 0.1 & 1.95 & 1.90 & 1.32 & 1.34 & 1.17 & 1.18 & 2.64 & 2.91 & 1.46 & 1.61 & 1.21 & 1.20 & 3.08 & 3.58 & 2.74 & 3.04 & 1.52 & 1.66 & 2.81 & 3.36 & 3.20 & 3.16 & 1.62 & 1.76 \\
\hline 0.16 & 1.82 & 1.77 & 1.31 & 1.28 & 1.14 & 1.17 & 2.48 & 2.54 & 1.46 & 1.41 & 1.18 & 1.19 & 3.74 & 3.89 & 2.68 & 2.81 & 1.50 & 1.45 & 3.02 & 3.37 & 2.97 & 2.94 & 1.62 & 1.59 \\
\hline 0.17 & 1.74 & 1.72 & 1.26 & 1.27 & 1.10 & 1.13 & 2.38 & 2.31 & 1.42 & 1.39 & 1.15 & 1.16 & 4.51 & 4.47 & 2.74 & 2.49 & 1.47 & 1.40 & 3.68 & 3.81 & 3.16 & 2.77 & 1.56 & 1.49 \\
\hline 0.18 & 1.49 & 1.66 & 1.19 & 1.28 & 1.11 & 1.11 & 1.92 & 2.11 & 1.27 & 1.38 & 1.13 & 1.13 & 5.00 & 4.74 & 2.11 & 2.31 & 1.27 & 1.37 & 4.81 & 4.12 & 2.42 & 2.47 & 1.34 & 1.47 \\
\hline 0.19 & 1.46 & 1.61 & 1.17 & 1.22 & 1.12 & 1.09 & 1.78 & 2.07 & 1.27 & 1.30 & 1.11 & 1.15 & 5.00 & 4.95 & 1.92 & 2.35 & 1.29 & 1.34 & 5.09 & 4.97 & 2.14 & 2.62 & 1.33 & 1.39 \\
\hline 0.2 & 1.58 & 1.35 & 1.22 & 1.12 & 1.12 & 1.09 & 1.85 & 1.60 & 1.35 & 1.17 & 1.14 & 1.10 & 5.08 & 3.90 & 1.92 & 1.70 & 1.37 & 1.17 & 5.23 & 4.40 & 2.12 & 1.90 & 1.45 & 1.23 \\
\hline 0.3 & 1.50 & 1.53 & 1.18 & 1.18 & 1.09 & 1.09 & 1.74 & 1.87 & 1.31 & 1.26 & 1.10 & 1.11 & 4.41 & 3.87 & 1.81 & 1.87 & 1.33 & 1.31 & 4.45 & 4.48 & 2.03 & 2.11 & 1.37 & 1.37 \\
\hline 0 & 1.22 & 1.16 & 1.09 & 1.08 & 1.05 & 1.06 & 1.26 & 1.30 & 1.14 & 1.11 & 1.06 & 1.06 & 2.00 & 2.09 & 1.31 & 1.39 & 1.14 & 1.11 & 2.20 & 2.46 & 1.38 & 1.45 & 1.16 & 1.13 \\
\hline 0 & 1.09 & 1.19 & 1.07 & 1.06 & 1.03 & 1.03 & 1.20 & 1.28 & 1.09 & 1.11 & 1.03 & 1.04 & 1.53 & 1.52 & 1.26 & 1.26 & 1.08 & 1.12 & 1.74 & 1.56 & 1.30 & 1.28 & 1.08 & 1.13 \\
\hline 0.0 & 1.08 & 1.12 & 1.08 & 1.04 & 1.03 & 1.04 & 1.17 & 1.19 & 1.08 & 1.08 & 1.04 & 1.04 & 1.43 & 1.53 & 1.20 & 1.23 & 1.06 & 1.09 & 1.47 & 1.55 & 1.22 & 1.25 & 1.07 & 1.09 \\
\hline 0.8 & 1.08 & 1.05 & 1.05 & 1.02 & 1.02 & 1.01 & 1.10 & 1.06 & 1.05 & 1.04 & 1.03 & 1.02 & 1.25 & 1.19 & 1.09 & 1.07 & 1.04 & 1.03 & 1.27 & 1.27 & 1.10 & 1.09 & 1.05 & 1.03 \\
\hline 1 & 1.07 & 1.06 & 1.04 & 1.03 & 1.01 & 1.01 & 1.08 & 1.07 & 1.05 & 1.04 & 1.02 & 1.02 & 1.23 & 1.14 & 1.08 & 1.05 & 1.04 & 1.04 & 1.25 & 1.18 & 1.09 & 1.05 & 1.05 & 1.04 \\
\hline 2 & 1.06 & 1.06 & 1.03 & 1.03 & 1.01 & 1.02 & 1.05 & 1.07 & 1.04 & 1.04 & 1.01 & 1.02 & 1.12 & 1.13 & 1.05 & 1.05 & 1.04 & 1.04 & 1.14 & 1.17 & 1.06 & 1.06 & 1.04 & 1.04 \\
\hline
\end{tabular}


TABLE 7 Summary of Amplification Ratios (Cont'd.)

Damping $=5 \%$

$\mathrm{L}=$ Lower Bound Properties, $\mathrm{M}=$ Median Properties, $\mathrm{U}=$ Upper Bound Properties

\begin{tabular}{|c|c|c|c|c|c|c|c|c|c|c|c|c|c|c|c|c|c|c|}
\hline \multirow{4}{*}{\begin{tabular}{|l|} 
Profile \\
Property \\
History
\end{tabular}} & & & & & & & & & & & & & & & & & & \\
\hline & \multicolumn{6}{|c|}{5} & \multicolumn{6}{|c|}{6} & \multicolumn{6}{|c|}{7} \\
\hline & \multicolumn{2}{|c|}{ L } & \multicolumn{2}{|c|}{$\bar{M}$} & \multicolumn{2}{|c|}{$\bar{U}$} & \multicolumn{2}{|c|}{$\mathbf{L}$} & \multicolumn{2}{|c|}{$M$} & \multicolumn{2}{|c|}{ U } & \multicolumn{2}{|c|}{$\mathrm{L}$} & \multicolumn{2}{|c|}{$\bar{M}$} & \multicolumn{2}{|c|}{$\mathrm{U}$} \\
\hline & 1 & 2 & 1 & 2 & 1 & 2 & 1 & 2 & 1 & 2 & 1 & 2 & 1 & 2 & 1 & 2 & 1 & 2 \\
\hline Period, (se & \multicolumn{18}{|c|}{ Amplification Ratio at Period, (sec) } \\
\hline 0.01 & 2.35 & 2.14 & 2.32 & 1.79 & 2.10 & 1.81 & 2.59 & 2.01 & 2.53 & 2.11 & 2.00 & 1.73 & 1.35 & 1.34 & 1.12 & 1.12 & 1.04 & 1.03 \\
\hline 0.03 & 2.11 & 1.98 & 2.06 & 1.74 & 2.06 & 1.68 & 2.27 & 1.78 & 2.30 & 1.95 & 1.89 & 1.74 & 1.51 & 1.35 & 1.13 & 1.12 & 1.04 & 1.03 \\
\hline 0.04 & 1.96 & 1.93 & 1.76 & 1.86 & 2.28 & 2.24 & 1.60 & 1.71 & 2.27 & 2.10 & 2.14 & 2.22 & 1.77 & 2.05 & 1.23 & 1.42 & 1.07 & 1.22 \\
\hline 0.05 & 1.90 & 1.67 & 1.54 & 1.61 & 1.40 & 1.56 & 2.05 & 1.81 & 1.97 & 1.78 & 1.58 & 1.92 & 1.58 & 1.44 & 1.20 & 1.16 & 1.06 & 1.05 \\
\hline 0.06 & 1.72 & 1.79 & 2.42 & 2.32 & 1.36 & 1.32 & 2.25 & 1.77 & 2.78 & 2.15 & 1.41 & 1.47 & 1.35 & 1.35 & 1.11 & 1.14 & 1.02 & 1.05 \\
\hline 0.07 & 1.27 & 1.73 & 1.72 & 1.83 & 1.40 & 1.48 & 2.11 & 2.25 & 2.18 & 2.18 & 1.37 & 1.46 & 1.32 & 1.20 & 1.16 & 1.06 & 1.10 & 1.01 \\
\hline 0.08 & 1.83 & 1.58 & 1.39 & 1.39 & 1.63 & 1.50 & 2.14 & 1.91 & 1.94 & 1.74 & 1.63 & 1.56 & 1.22 & 1.22 & 1.10 & 1.10 & 1.05 & 1.05 \\
\hline 0.09 & 2.78 & 2.21 & 1.54 & 1.41 & 2.19 & 1.87 & 2.64 & 2.42 & 1.75 & 1.51 & 2.08 & 1.90 & 1.18 & 1.14 & 1.08 & 1.06 & 1.03 & 1.02 \\
\hline 0.1 & 2.16 & 2.31 & 1.59 & 1.63 & 2.43 & 2.23 & 2.78 & 2.58 & 1.76 & 1.72 & 2.22 & 2.17 & 1.11 & 1.09 & 1.04 & 1.03 & 1.01 & 1.00 \\
\hline 0.1 & 1.50 & 1.44 & 1.56 & 1.69 & 2.89 & 2.91 & 2.09 & 2.05 & 1.50 & 1.69 & 2.60 & 2.70 & 1.08 & 1.11 & 1.03 & 1.06 & 1.01 & 1.04 \\
\hline 0.12 & 1.22 & 1.54 & 1.80 & 2.04 & 3.18 & 3.20 & 1.82 & 2.00 & 1.80 & 1.96 & 3.03 & 3.17 & 1.11 & 1.12 & 1.05 & 1.06 & 1.01 & 1.03 \\
\hline 0.13 & 1.36 & 1.28 & 2.06 & 2.19 & 3.14 & 3.24 & 1.67 & 1.60 & 1.94 & 2.20 & 3.19 & 3.20 & 1.09 & 1.06 & 1.05 & 1.03 & 1.03 & 1.01 \\
\hline 0.14 & 1.80 & 1.61 & 2.38 & 2.67 & 3.18 & 3.19 & 1.92 & 1.68 & 2.33 & 2.57 & 3.21 & 3.25 & 1.04 & 1.06 & 1.02 & 1.02 & 1.01 & 1.01 \\
\hline 0.15 & 1.48 & 1.83 & 2.68 & 3.11 & 2.51 & 2.70 & 1.55 & 1.87 & 2.42 & 2.93 & 2.69 & 2.82 & 1.05 & 1.08 & 1.02 & 1.04 & 1.01 & 1.03 \\
\hline 0.16 & 1.56 & 1.71 & 3.19 & 3.26 & 2.48 & 2.53 & 1.57 & 1.77 & 2.79 & 3.01 & 2.58 & 2.58 & 1.05 & 1.05 & 1.03 & 1.02 & 1.01 & 1.01 \\
\hline 0.17 & 1.55 & 1.31 & 3.83 & 3.76 & 2.49 & 2.25 & 1.58 & 1.43 & 3.38 & 3.41 & 2.71 & 2.36 & 1.05 & 1.03 & 1.03 & 1.01 & 1.02 & 1.00 \\
\hline 0.1 & $1 . \overline{83}$ & 1.49 & 4.26 & 3.93 & 1.98 & 2.13 & 1.70 & 1.52 & 4.12 & 3.61 & 2.10 & 2.19 & 1.03 & 1.04 & 1.01 & 1.02 & 1.00 & 1.01 \\
\hline 0.19 & 1.73 & 2.33 & 4.30 & 4.21 & 1.84 & 2.19 & 1.60 & 2.32 & 4.29 & $\overline{4.14}$ & 1.95 & 2.29 & 1.02 & 1.02 & 1.01 & 1.00 & 1.00 & 1.00 \\
\hline 0.2 & 1.93 & 2.04 & 4.39 & 3.51 & 1.87 & 1.64 & 1.82 & 2.08 & 4.47 & 3.63 & 1.97 & 1.74 & 1.04 & 1.03 & 1.02 & 1.01 & 1.01 & 1.00 \\
\hline 0.3 & 4.29 & 4.46 & 3.79 & 3.54 & 1.74 & 1.79 & 4.26 & 4.69 & 3.78 & 3.74 & 1.86 & 1.89 & 1.03 & 1.03 & 1.02 & 1.01 & 1.01 & 1.01 \\
\hline 0.4 & 3.67 & 4.08 & 1.91 & 2.07 & 1.30 & 1.34 & 3.94 & 4.37 & 1.99 & 2.26 & 1.33 & 1.38 & 1.02 & 1.02 & 1.01 & 1.01 & 1.00 & 1.00 \\
\hline 0.5 & 2.19 & 2.58 & 1.53 & 1.48 & 1.23 & 1.23 & 2.36 & 2.65 & 1.63 & 1.48 & 1.26 & 1.25 & 1.01 & 1.01 & 1.01 & 1.01 & 1.00 & 1.00 \\
\hline 0.6 & 1.67 & 1.91 & 1.39 & 1.48 & 1.18 & 1.20 & 1.74 & 2.10 & 1.41 & 1.50 & 1.19 & 1.21 & 1.01 & 1.01 & 1.00 & 1.01 & 1.00 & 1.00 \\
\hline 0.8 & 1.44 & 1.47 & 1.22 & 1.19 & 1.08 & 1.07 & 1.55 & 1.51 & 1.24 & 1.23 & 1.09 & 1.07 & 1.01 & 1.01 & 1.00 & 1.00 & 1.00 & 1.00 \\
\hline 1 & 1.26 & 1.35 & 1.20 & 1.13 & 1.07 & 1.05 & 1.30 & 1.41 & 1.21 & 1.16 & 1.08 & 1.05 & 1.00 & 1.00 & 1.00 & 1.00 & 1.00 & 1.00 \\
\hline 2 & 1.17 & 1.30 & 1.11 & 1.12 & 1.04 & 1.05 & 1.18 & 1.32 & 1.12 & 1.14 & 1.05 & 1.05 & 1.00 & 1.00 & 1.00 & 1.00 & 1.00 & 1.00 \\
\hline
\end{tabular}


TABLE 7 Summary of Amplification Ratios (Cont'd.) Damping $=5 \%$

$L=$ Lower Bound Properties, $M=$ Median Properties, $U=$ Upper Bound Properties

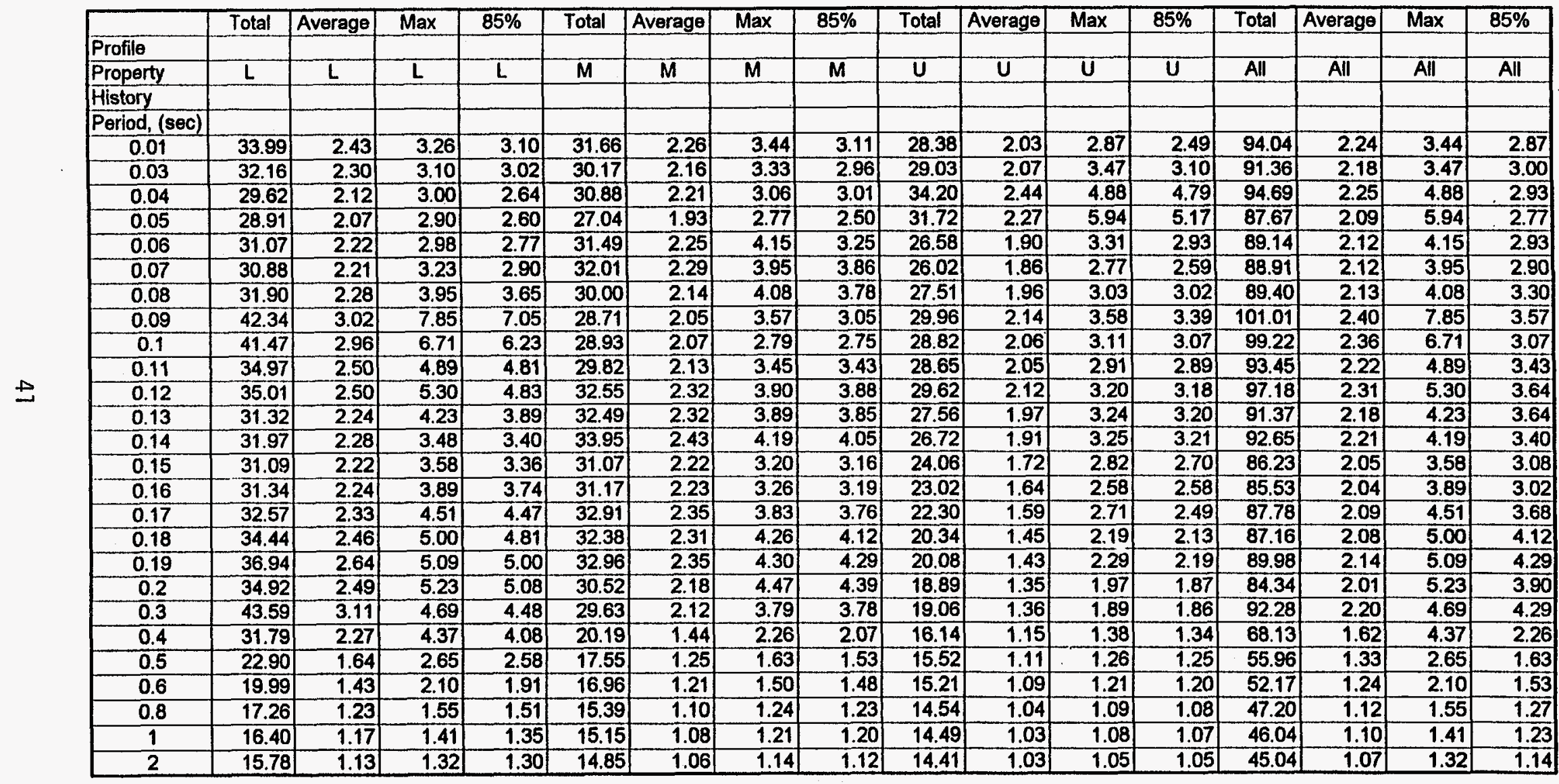


TABLE 8 Summary of Amplification Ratios

Damping $=7 \%$

$L=$ Lower Bound Properties, $M=$ Median Properties, $U=$ Upper Bound Properties

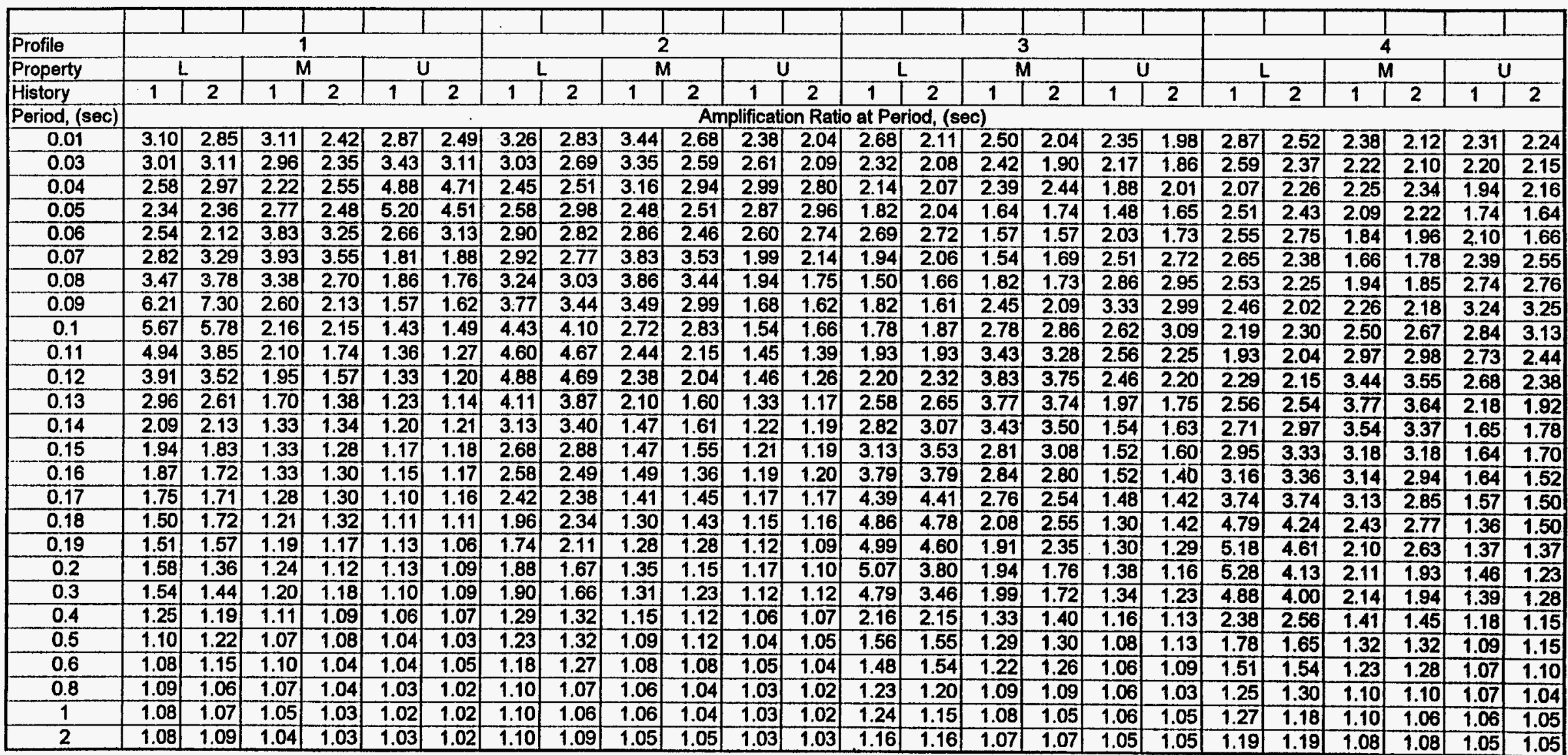


TABLE 8 Summary of Amplification Ratios (Cont'd.) Damping $=7 \%$

$L=$ Lower Bound Properties, $M=$ Median Properties, $U=$ Upper Bound Properties

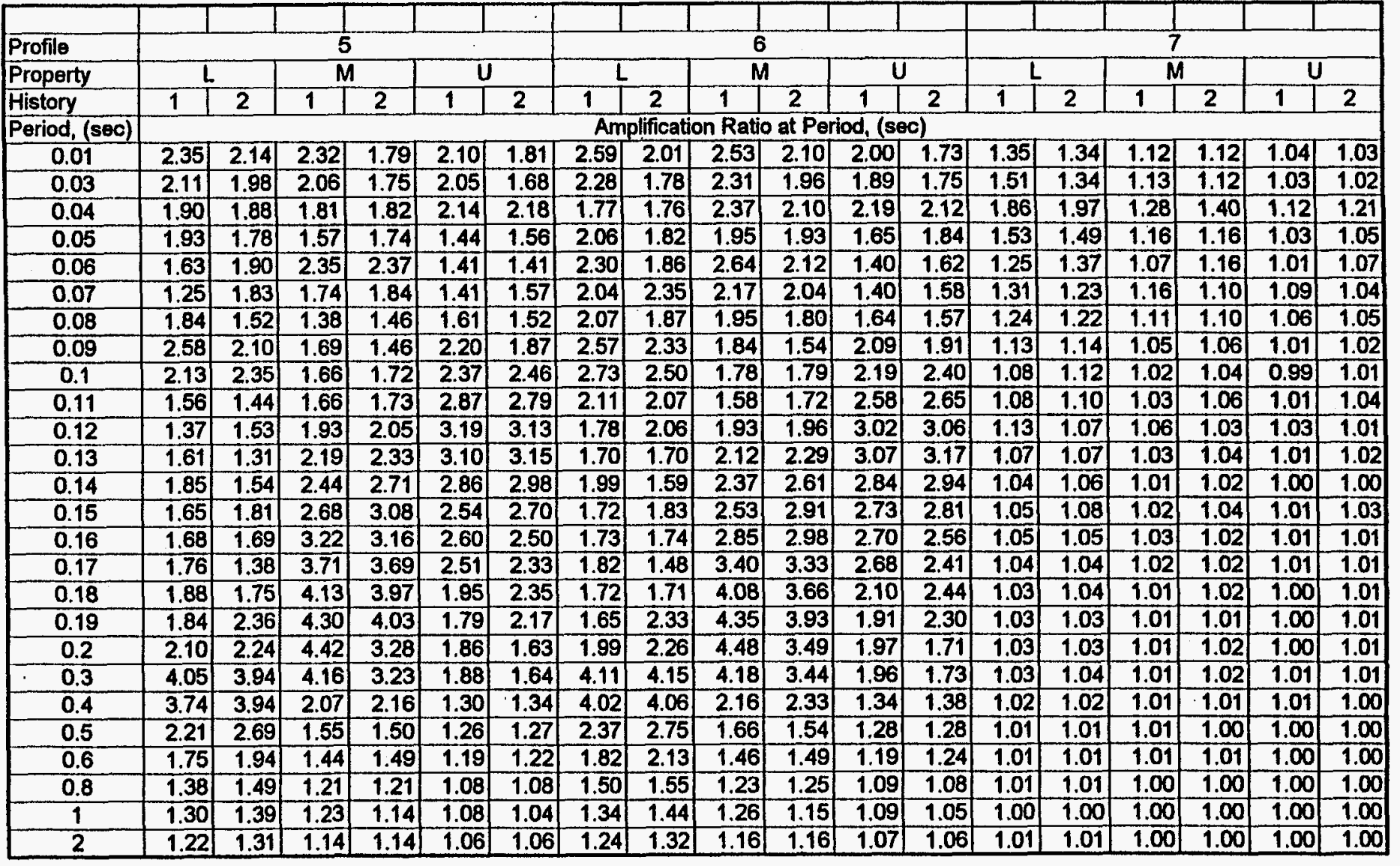


TABLE 8 Summary of Amplification Ratios (Cont'd.) Damping $=7 \%$

L = Lower Bound Properties, $M=$ Median Properties, $U=$ Upper Bound Properties

\begin{tabular}{|c|c|c|c|c|c|c|c|c|c|c|c|c|c|c|c|c|}
\hline & Total & Average & Max & $85 \%$ & Total & Average & Max & $85 \%$ & Total & Average & Max & $85 \%$ & Total & Average & Max & $85 \%$ \\
\hline \multicolumn{17}{|l|}{ Profile } \\
\hline Propenty & $L$ & $L$ & $L$ & $L$ & $M$ & $M$ & $M$ & $M$ & $\mathbf{U}$ & $U$ & U & $\mathrm{U}$ & All & All & All & All \\
\hline \multicolumn{17}{|l|}{ History } \\
\hline \multicolumn{17}{|c|}{ Period, (sec) } \\
\hline 0.01 & 33.99 & 2.43 & 3.26 & 3.10 & 31.66 & 2.26 & 3.44 & 3.11 & 28.39 & 2.03 & 2.87 & 2.49 & 94.04 & 2.24 & 3.44 & 2.87 \\
\hline 0.03 & 32.21 & 2.30 & 3.11 & 3.03 & 30.21 & 2.16 & 3.35 & 2.96 & 29.04 & 2.07 & 3.43 & 3.11 & 91.45 & 2.18 & 3.43 & 3.01 \\
\hline 0.04 & 30.19 & 2.16 & 2.97 & 2.58 & 31.06 & 2.22 & 3.16 & 2.94 & 34.32 & 2.45 & 4.88 & 4.71 & 95.57 & 2.28 & 4.88 & 2.94 \\
\hline 0.05 & 29.67 & 2.12 & 2.98 & 2.58 & 27.44 & 1.96 & 2.77 & 2.51 & 30.62 & 2.19 & 5.20 & 4.51 & 87.73 & 2.09 & 5.20 & 2.77 \\
\hline 0.06 & 31.42 & 2.24 & 2.90 & 2.82 & 31.05 & 2.22 & 3.83 & 3.25 & 26.58 & 1.90 & 3.13 & 2.74 & 89.04 & 2.12 & 3.83 & 2.82 \\
\hline 0.07 & 30.83 & 2.20 & 3.29 & 2.92 & 31.54 & 2.25 & 3.93 & 3.83 & 26.10 & 1.86 & 2.72 & 2.55 & 88.47 & 2.11 & 3.93 & 2.92 \\
\hline 0.08 & 31.23 & 2.23 & 3.78 & 3.47 & 29.51 & 2.11 & 3.86 & 3.44 & 27.08 & 1.93 & 2.95 & 2.86 & 87.82 & 2.09 & 3.86 & 3.24 \\
\hline 0.09 & 40.46 & 2.89 & 7.30 & 6.21 & 28.81 & 2.06 & 3.49 & 2.99 & 29.38 & 2.10 & 3.33 & 3.25 & 98.66 & 2.35 & 7.30 & 3.33 \\
\hline 0.1 & 40.01 & 2.86 & 5.78 & 5.67 & 29.69 & 2.12 & 2.86 & 2.83 & 29.22 & 2.09 & 3.13 & 3.09 & 98.92 & 2.36 & 5.78 & 3.09 \\
\hline 0.11 & 35.26 & 2.52 & 4.94 & 4.67 & 29.88 & 2.13 & 3.43 & 3.28 & 28.39 & 2.03 & 2.87 & 2.79 & 93.52 & 2.23 & 4.94 & 3.28 \\
\hline 0.12 & 34.92 & 2.49 & 4.88 & 4.69 & 32.49 & 2.32 & 3.83 & 3.75 & 29.39 & 2.10 & 3.19 & 3.13 & 96.80 & 2.30 & 4.88 & 3.55 \\
\hline 0.13 & 32.32 & 2.31 & 4.11 & 3.87 & 32.70 & 2.34 & 3.77 & 3.77 & 27.22 & 1.94 & 3.17 & 3.15 & 92.23 & 2.20 & 4.11 & 3.64 \\
\hline 0.14 & 31.39 & 2.24 & 3.40 & 3.13 & 31.75 & 2.27 & 3.54 & 3.50 & 25.04 & 1.79 & 2.98 & 2.94 & 88.18 & 2.10 & 3.54 & 3.13 \\
\hline 0.15 & 31.40 & 2.24 & 3.53 & 3.33 & 31.13 & 2.22 & 3.18 & 3.18 & 24.03 & 1.72 & 2.81 & 2.73 & 86.56 & 2.06 & 3.53 & 3.08 \\
\hline 0.16 & 31.71 & 2.26 & 3.79 & 3.79 & 31.45 & 2.25 & 3.22 & 3.16 & 23.17 & 1.65 & 2.70 & 2.60 & 86.32 & 2.06 & 3.79 & 3.16 \\
\hline 0.17 & 33.08 & 2.36 & 4.41 & 4.39 & 32.91 & 2.35 & 3.71 & 3.69 & 22.52 & 1.61 & 2.68 & 2.51 & 88.50 & 2.11 & 4.41 & 3.69 \\
\hline 0.18 & 35.31 & 2.52 & 4.86 & 4.79 & 32.97 & 2.36 & 4.13 & 4.08 & 20.97 & 1.50 & 2.44 & 2.35 & 89.26 & 2.13 & 4.86 & 4.08 \\
\hline 0.19 & 36.53 & 2.61 & 5.18 & 4.99 & 32.55 & 2.33 & 4.35 & 4.30 & 19.91 & 1.42 & 2.30 & 2.17 & 88.99 & 2.12 & 5.18 & 4.30 \\
\hline 0.2 & 35.43 & 2.53 & 5.28 & 5.07 & 30.29 & 2.16 & 4.48 & 4.42 & 18.90 & 1.35 & 1.97 & 1.86 & 84.62 & 2.01 & 5.28 & 3.80 \\
\hline 0.3 & 41.99 & 3.00 & 4.88 & $\frac{.79}{4.79}$ & 29.76 & 2.13 & 4.18 & 4.16 & 18.91 & 1.35 & 1.96 & 1.88 & 90.65 & 2.16 & 4.88 & 4.11 \\
\hline 0.4 & 32,10 & 2.29 & 4.06 & 4.02 & 20.79 & 1.49 & 2.33 & 2.16 & 16.24 & 1.16 & 1.38 & 1.34 & 69.13 & 1.65 & 4.06 & 2.38 \\
\hline 0.5 & 23.45 & 1.68 & 2.75 & 2.69 & 17.85 & 1.27 & 1.66 & 1.55 & 15.70 & 1.12 & 1.28 & 1.28 & 57.00 & 1.36 & 2.75 & 1.66 \\
\hline 0.6 & 20.43 & 1.46 & 2.13 & 1.94 & 17.19 & 1.23 & 1.49 & 1.49 & 15.34 & 1.10 & 1.24 & 1.22 & 52.96 & 1.26 & 2.13 & 1.54 \\
\hline 0.8 & 17.24 & 1.23 & 1.55 & 1.50 & 15.50 & 1.11 & 1.25 & 1.23 & 14.63 & 1.05 & 1.09 & 1.08 & 47.37 & 1.13 & 1.55 & 1.25 \\
\hline 1 & 16.63 & 1.19 & 1.44 & 1.39 & 15.25 & 1.09 & 1.26 & 1.23 & 14.56 & 1.04 & 1.09 & 1.08 & 46.44 & 1.11 & 1.44 & 1.26 \\
\hline 2 & 16.12 & 1.15 & 1.32 & 1.31 & 15.08 & 1.08 & 1.16 & 1.16 & 14.57 & 1.04 & 1.07 & 1.06 & 45.77 & 1.09 & 1.32 & 1.19 \\
\hline
\end{tabular}


TABLE 9 Summary of Amplification Ratios Damping $=10 \%$

L E Lower Bound Properties, $M=$ Median Properties, $U=$ Upper Bound Properties

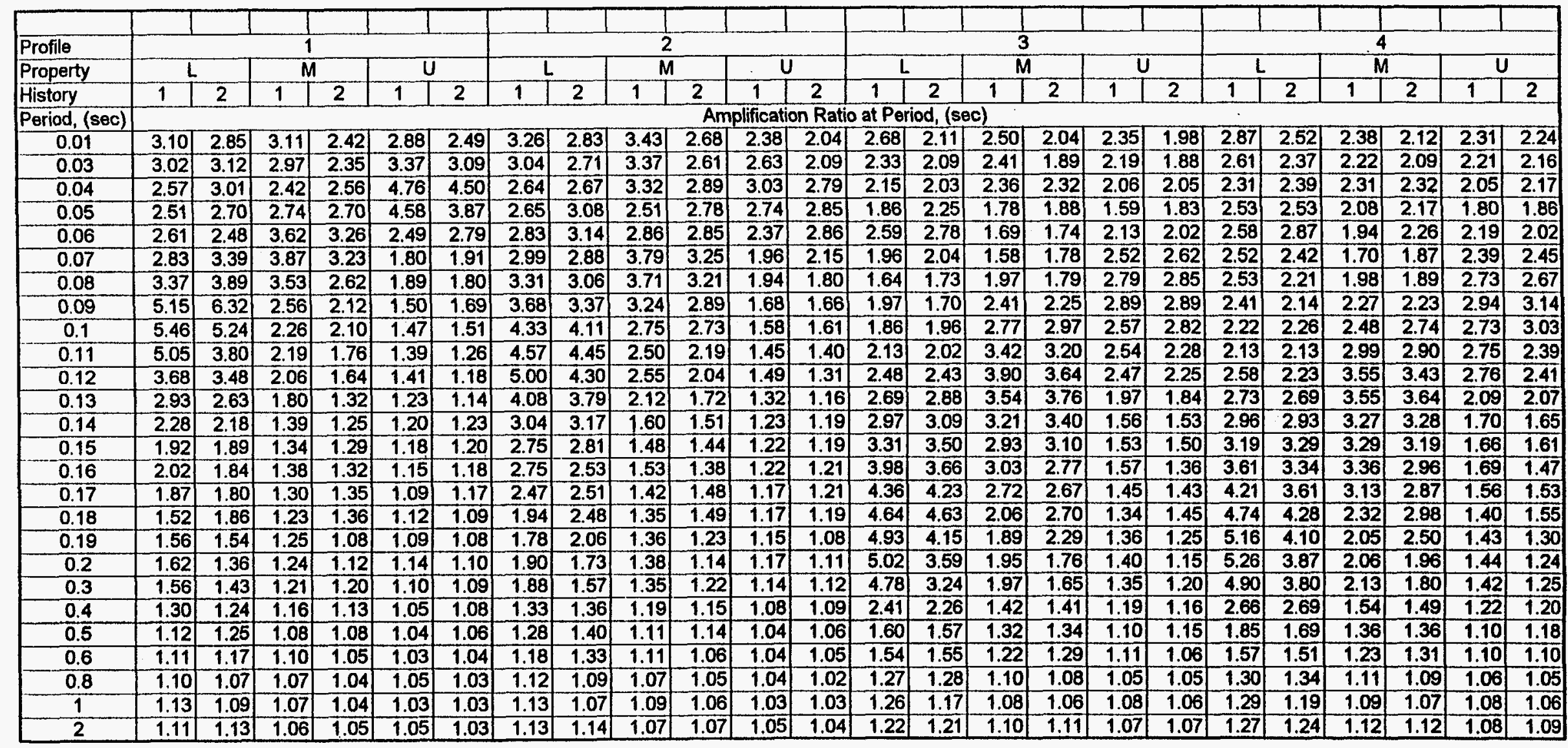


TABLE 9 Summary of Amplification Ratios (Cont'd.)

Damping $=10 \%$

$L=$ Lower Bound Properties, $M=$ Median Properties, $U=$ Upper Bound Properties

\begin{tabular}{|c|c|c|c|c|c|c|c|c|c|c|c|c|c|c|c|c|c|c|}
\hline \multirow{4}{*}{$\begin{array}{l}\text { Profile } \\
\text { Propenty } \\
\text { History }\end{array}$} & & & & & & & & & & & & & & & & & & \\
\hline & \multicolumn{6}{|c|}{ 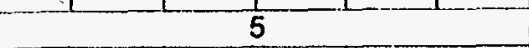 } & \multicolumn{6}{|c|}{6} & \multicolumn{6}{|c|}{7} \\
\hline & \multicolumn{2}{|c|}{$L$} & \multicolumn{2}{|c|}{$M$} & \multicolumn{2}{|c|}{$\bar{U}$} & \multicolumn{2}{|l|}{ L } & \multicolumn{2}{|c|}{$M$} & \multicolumn{2}{|c|}{$\mathrm{U}$} & \multicolumn{2}{|c|}{$\mathrm{L}$} & \multicolumn{2}{|c|}{$\bar{M}$} & \multicolumn{2}{|c|}{$\mathrm{U}$} \\
\hline & 1 & 2 & 1 & 2 & 1 & 2 & 1 & 2 & 1 & 2 & 1 & 2 & 1 & 2 & 1 & 2 & 1 & 2 \\
\hline Period, (sec) & \multicolumn{18}{|c|}{ Amplification Ratio at Period, (sec) } \\
\hline 0.01 & 2.35 & 2.14 & 2.31 & 1.79 & 2.10 & 1.81 & 2.59 & 2.01 & 2.53 & 2.10 & 2.00 & 1.73 & 1.00 & 1.34 & 1.14 & 1.12 & 1.04 & 1.03 \\
\hline 0.03 & 2.12 & 1.98 & 2.06 & 1.76 & 2.05 & 1.67 & 2.29 & 1.79 & 2.32 & 1.97 & 1.88 & 1.75 & 1.50 & 1.33 & 1.13 & 1.11 & 1.02 & 1.02 \\
\hline 0.04 & 1.96 & 1.80 & 1.85 & 1.79 & 2.06 & 2.09 & 1.95 & 1.78 & 2.46 & 2.13 & 2.20 & 1.94 & 1.91 & 1.85 & 1.34 & 1.34 & 1.16 & 1.17 \\
\hline 0.05 & 1.96 & 1.87 & 1.60 & 1.92 & 1.60 & 1.58 & 2.06 & 1.91 & 1.87 & 2.13 & 1.77 & 1.86 & 1.47 & 1.55 & 1.12 & 1.21 & 1.02 & 1.10 \\
\hline 0.06 & 1.66 & 2.07 & 2.26 & 2.44 & 1.51 & 1.56 & 2.37 & 2.00 & 2.49 & 2.22 & 1.52 & 1.84 & 1.23 & 1.40 & 1.07 & 1.18 & 1.01 & 1.09 \\
\hline 0.07 & 1.31 & 1.88 & 1.76 & 1.83 & 1.45 & $1 . \overline{66}$ & 1.95 & 2.40 & 2.12 & 2.01 & 1.44 & 1.70 & 1.31 & 1.28 & 1.16 & 1.10 & 1.09 & 1.05 \\
\hline 0.08 & 1.90 & 1.64 & 1.48 & 1.52 & 1.74 & 1.59 & 2.01 & 2.03 & 1.93 & 1.89 & 1.67 & 1.60 & 1.26 & 1.24 & 1.14 & 1.11 & 1.08 & 1.05 \\
\hline 0 . & 2.36 & 1.99 & 1.82 & 1.53 & 2.17 & 1.98 & 2.53 & 2.20 & 1.97 & 1.66 & 2.04 & 1.93 & 1.13 & 1.15 & 1.05 & 1.06 & 1.02 & 1.02 \\
\hline & 2.08 & 2.20 & 1.74 & 1.74 & 2.36 & 2.57 & 2.66 & 2.27 & 1.89 & 1.84 & 2.21 & 2.47 & 1.08 & 1.11 & 1.03 & 1.05 & 1.00 & 1.02 \\
\hline & 1.72 & 1.46 & 1.82 & 1.81 & 2.86 & 2.68 & 2.16 & 2.11 & 1.72 & 1.77 & 2.64 & 2.58 & 1.08 & 1.10 & 1.03 & 1.06 & .01 & 1.03 \\
\hline & 1.70 & 1.55 & 2.16 & 2.13 & 3.22 & 3.04 & 1.89 & 2.11 & 2.16 & 2.01 & 3.08 & 2.92 & 1.16 & 1.09 & 1.08 & 1.05 & 1.04 & 1.03 \\
\hline & 1.83 & 1.44 & 2.27 & 2.52 & 2.94 & 3.14 & 1.96 & 1.79 & 2.25 & 2.43 & 2.97 & 3.14 & 1.05 & 1.08 & 1.02 & 1.04 & 1.00 & 1.03 \\
\hline & 1.95 & 1.55 & 2.55 & 2.71 & 2.68 & 2.91 & 2.11 & 1.56 & 2.54 & 2.62 & 2.77 & 2.82 & 1.04 & 1.04 & 1.01 & 1.01 & 1.00 & 1.00 \\
\hline & 1.88 & 1.81 & 2.81 & 3.04 & 2.65 & 2.69 & 1.96 & 1.80 & 2.74 & 2.86 & 2.82 & 2.76 & 1.06 & 1.07 & 1.03 & 1.04 & 1.01 & 1.02 \\
\hline 0 & 1.99 & 1.76 & 3.38 & 3.12 & 2.76 & 2.46 & 2.07 & 1.81 & 3.13 & 2.96 & 2.86 & 2.55 & 1.06 & 1.06 & 1.03 & 1.02 & 1.02 & 1.01 \\
\hline & 2.02 & 1.74 & 3.70 & 3.51 & 2.49 & 2.44 & 2.10 & 1.75 & 3.60 & 3.17 & 2.63 & 2.48 & 1.04 & 1.05 & 1.02 & 1.03 & 1.00 & 1.02 \\
\hline & 2.00 & 2.11 & 3.96 & 3.84 & 1.89 & 2.47 & 1.89 & 2.10 & 4.01 & 3.67 & 2.02 & 2.61 & 1.04 & 1.04 & 1.02 & 1.02 & 1.01 & 1.01 \\
\hline 19 & 2.02 & 2.36 & 4.23 & 3.60 & 1.78 & 2.07 & 1.87 & 2.35 & 4.33 & 3.47 & 1.88 & 2.17 & 1.03 & 1.03 & 1.02 & 1.01 & 1.01 & 1.01 \\
\hline & 2.29 & 2.43 & 4.35 & 3.12 & 1.88 & 1.63 & 2.18 & 2.45 & 4.40 & 3.26 & 1.93 & 1.69 & 1.05 & 1.04 & 1.02 & 1.02 & 1.01 & 1.01 \\
\hline & 3.79 & 3.64 & 4.10 & 2.99 & 1.88 & 1.61 & 3.99 & 3.71 & 4.18 & 3.24 & 1.96 & 1.68 & 1.04 & 1.03 & 1.02 & 1.02 & 1.01 & 1.01 \\
\hline 0. & 3.65 & 3.81 & 2.30 & 2.24 & 1.39 & 1.38 & 3.94 & 3.86 & 2.44 & 2.45 & 1.45 & 1.43 & 1.02 & 1.02 & 1.01 & 1.01 & 1.00 & 1.00 \\
\hline 0.5 & 2.23 & 2.68 & 1.58 & 1.51 & 1.29 & 1.30 & 2.39 & 2.73 & 1.70 & 1.55 & 1.31 & 1.31 & 1.01 & 1.02 & 1.01 & 1.01 & 1.00 & 1.01 \\
\hline 0.6 & 1.88 & 2.09 & 1.48 & 1.49 & 1.19 & 1.25 & 1.96 & 2.16 & 1.51 & 1.46 & 1.20 & 1.26 & 1.01 & 1.01 & 1.01 & 1.01 & 1.00 & 1.00 \\
\hline 0.8 & 1.57 & 1.53 & 1.26 & 1.25 & 1.09 & 1.08 & 1.63 & 1.59 & 1.28 & 1.29 & 1.09 & 1.08 & 1.02 & 1.01 & 1.01 & 1.00 & 1.01 & 1.00 \\
\hline 1 & 1.33 & 1.51 & 1.24 & 1.16 & 1.07 & 1.06 & 1.36 & 1.56 & 1.27 & 1.16 & 1.07 & 1.06 & 1.01 & 1.01 & -1.00 & 1.00 & 1.00 & 1.00 \\
\hline 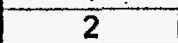 & 1.27 & 1.32 & 1.21 & 1.19 & 1.09 & 1.09 & 1.31 & 1.32 & 1.23 & 1.20 & 1.10 & 1.09 & 1.01 & 1.01 & 1.00 & 1.01 & 1.00 & 1.00 \\
\hline
\end{tabular}


TABLE 9 Summary of Amplification Ratios (Cont'd.)

$$
\text { Damping }=10 \%
$$

$\mathrm{L}=$ Lower Bound Properties, $M=$ Median Properties, $U=$ Upper Bound Properties

\begin{tabular}{|c|c|c|c|c|c|c|c|c|c|c|c|c|c|c|c|c|}
\hline & Total & Average & Max & $85 \%$ & Total & Average & Max & $85 \%$ & Total & Average & Max & $85 \%$ & Total & Average & Max & $85 \%$ \\
\hline \multicolumn{17}{|l|}{ Profile } \\
\hline Property & $L$ & L & $\mathbf{L}$ & L & $M$ & $M$ & $M$ & $M$ & $\mathrm{U}$ & U & $\mathrm{U}$ & $\mathrm{U}$ & All & All & All & All \\
\hline \multicolumn{17}{|l|}{ History } \\
\hline \multicolumn{17}{|l|}{ Period, (sec) } \\
\hline 0.01 & 33.99 & 2.43 & 3.26 & 3.10 & 31.66 & 2.26 & 3.43 & 3.11 & 28.39 & 2.03 & 2.88 & 2.49 & 94.04 & 2.24 & 3.43 & 2.87 \\
\hline 0.03 & 32.28 & 2.31 & 3.12 & 3.04 & 30.27 & 2.16 & 3.37 & 2.97 & 29.02 & 2.07 & 3.37 & 3.09 & 91.58 & 2.18 & 3.37 & 3.02 \\
\hline 0.04 & 31.03 & 2.22 & 3.01 & 2.67 & 31.40 & 2.24 & 3.32 & 2.89 & 34.02 & 2.43 & 4.76 & 4.50 & 96.45 & 2.30 & 4.76 & 2.89 \\
\hline 0.05 & 30.92 & 2.21 & 3.08 & 2.70 & 28.51 & 2.04 & 2.78 & 2.74 & 30.04 & 2.15 & 4.58 & 3.87 & 89.48 & 2.13 & 4.58 & 2.74 \\
\hline 0.06 & 32.63 & 2.33 & 3.14 & 2.87 & 31.90 & 2.28 & 3.62 & 3.26 & 27.41 & 1.96 & 2.86 & 2.79 & 91.94 & 2.19 & 3.62 & 2.86 \\
\hline 0.07 & 31.18 & 2.23 & 3.39 & 2.99 & 31.07 & 2.22 & 3.87 & 3.79 & 26.19 & 1.87 & 2.62 & 2.52 & 88.44 & 2.11 & 3.87 & 2.99 \\
\hline 0.08 & 31.83 & 2.27 & 3.89 & 3.37 & 29.77 & 2.13 & 3.71 & 3.53 & 27.21 & 1.94 & 2.85 & 2.79 & 88.82 & 2.11 & 3.89 & 3.21 \\
\hline 0.09 & 38.10 & 2.72 & 6.32 & 5.15 & 29.07 & 2.08 & 3.24 & 2.89 & 28.55 & 2.04 & 3.14 & 2.94 & 95.72 & 2.28 & 6.32 & 3.14 \\
\hline 0.1 & 38.84 & 2.77 & 5.46 & 5.24 & 30.08 & 2.15 & 2.97 & 2.77 & 28.95 & 2.07 & 3.03 & 2.82 & 97.87 & 2.33 & 5.46 & 2.97 \\
\hline 0.11 & 35.92 & 2.57 & 5.05 & 4.57 & 30.35 & 2.17 & 3.42 & 3.20 & 28.27 & 2.02 & 2.86 & 2.75 & 94.53 & 2.25 & 5.05 & 3.20 \\
\hline 0.12 & 35.69 & 2.55 & 5.00 & 4.30 & 33.41 & 2.39 & 3.90 & 3.64 & 29.61 & 2.12 & 3.22 & 3.08 & 98.72 & 2.35 & 5.00 & 3.55 \\
\hline 0.13 & 33.55 & 2.40 & 4.08 & 3.79 & 32.97 & 2.35 & 3.76 & 3.64 & 27.03 & 1.93 & 3.14 & 3.14 & 93.55 & 2.23 & 4.08 & 3.54 \\
\hline 0.14 & 31.87 & 2.28 & 3.17 & 3.09 & 31.36 & 2.24 & 3.40 & 3.28 & 24.47 & 1.75 & 2.91 & 2.82 & 87.70 & 2.09 & 3.40 & 3.09 \\
\hline 0.15 & 32.24 & 2.30 & 3.50 & 3.31 & 31.59 & 2.26 & 3.29 & 3.19 & 24.04 & 1.72 & 2.82 & 2.76 & 87.88 & 2.09 & 3.50 & 3.19 \\
\hline 0.16 & 33.47 & 2.39 & 3.98 & 3.66 & 32.37 & 2.31 & 3.38 & 3.36 & 23.51 & 1.68 & 2.86 & 2.76 & 89.35 & 2.13 & 3.98 & 3.34 \\
\hline 0.17 & 34.76 & $2.4 \overline{8}$ & 4.36 & 4.23 & 32.96 & 2.35 & 3.70 & 3.60 & 22.66 & 1.62 & 2.63 & 2.49 & 90.38 & 2.15 & 4.36 & 3.60 \\
\hline 0.18 & 36.26 & 2.59 & 4.74 & 4.64 & 33.01 & 2.36 & 4.01 & 3.96 & 21.32 & 1.52 & $2.6^{1}$ & 2.47 & 90.59 & 2.16 & 4.74 & 3.96 \\
\hline 0.19 & 35.93 & 2.57 & 5.16 & 4.93 & 31.32 & 2.24 & 4.33 & 4.23 & 19.65 & 1.40 & 2.17 & 2.07 & 86.90 & 2.07 & 5.16 & 4.10 \\
\hline 0.2 & 35.78 & 2.56 & 5.26 & 5.02 & 29.78 & 2.13 & 4.40 & 4.35 & 18.90 & 1.35 & 1.93 & 1.88 & 84.46 & 2.01 & 5.26 & 3.59 \\
\hline 0.3 & 40.36 & 2.88 & 4.90 & 4.78 & 29.09 & 2.08 & 4.18 & 4.10 & 18.83 & 1.35 & 1.96 & 1.88 & 88.28 & 2.10 & 4.90 & 3.80 \\
\hline 0.4 & 32.56 & 2.33 & 3.94 & 3.86 & 21.93 & 1.57 & 2.45 & 2.44 & 16.73 & 1.19 & 1.45 & 1.43 & 71.22 & 1.70 & 3.94 & 2.66 \\
\hline 0.5 & 23.81 & 1.70 & 2.73 & 2.68 & 18.16 & 1.30 & 1.70 & 1.58 & 15.97 & 1.14 & 1.31 & 1.31 & 57.94 & 1.38 & 2.73 & 1.70 \\
\hline 0.6 & 21.08 & 1.51 & 2.16 & 2.09 & 17.32 & 1.24 & 1.51 & 1.49 & 15.44 & 1.10 & 1.26 & 1.25 & 53.84 & 1.28 & 2.16 & 1.55 \\
\hline 0.8 & 17.91 & 1.28 & 1.63 & 1.59 & 15.69 & 1.12 & 1.29 & 1.28 & 14.71 & 1.05 & 1.09 & 1.09 & 48.31 & 1.15 & 1.63 & 1.30 \\
\hline 1 & 17.12 & 1.22 & 1.56 & 1.51 & 15.41 & 1.10 & 1.27 & 1.24 & 14.67 & 1.05 & 1.08 & 1.08 & 47.20 & 1.12 & 1.56 & 1.27 \\
\hline 2 & 16.70 & 1.19 & 1.32 & 1.32 & 15.53 & 1.11 & 1.23 & 1.21 & 14.87 & 1.06 & 1.10 & 1.09 & 47.10 & 1.12 & 1.32 & 1.24 \\
\hline
\end{tabular}


TABLE 10 Summary of Amplification Ratios

Damping $=12 \%$

$\mathrm{L}=$ Lower Bound Properties, $M=$ Median Properties, $U=$ Upper Bound Properties

\begin{tabular}{|c|c|c|c|c|c|c|c|c|c|c|c|c|c|c|c|c|c|c|c|c|c|c|c|c|}
\hline & & & & & & & & & & & & & & & & & & & & & & & & \\
\hline Profile & \multicolumn{6}{|c|}{1} & \multicolumn{6}{|c|}{2} & \multicolumn{6}{|c|}{3} & \multicolumn{6}{|c|}{4} \\
\hline Property & \multicolumn{2}{|c|}{$\mathrm{L}$} & \multicolumn{2}{|c|}{$\bar{M}$} & \multicolumn{2}{|c|}{$\mathbf{U}$} & \multicolumn{2}{|c|}{$L$} & \multicolumn{2}{|c|}{$M$} & \multicolumn{2}{|c|}{ U } & \multicolumn{2}{|c|}{$\mathbf{L}$} & \multicolumn{2}{|c|}{$\bar{M}$} & \multicolumn{2}{|c|}{$\mathbf{U}$} & \multicolumn{2}{|c|}{$\mathbf{L}$} & \multicolumn{2}{|c|}{$M$} & \multicolumn{2}{|c|}{$\mathrm{U}$} \\
\hline History & 1 & 2 & 1 & 2 & 1 & 2 & 1 & 2 & 1 & 2 & 1 & 2 & 1 & 2 & 1 & 2 & 1 & 2 & 1 & 2 & 1 & 2 & 1 & 2 \\
\hline Period, (sec) & \multicolumn{24}{|c|}{ Amplification Ratio at Period, (sec) } \\
\hline 0.01 & 3.10 & 2.84 & 3.10 & 2.42 & 2.88 & 2.50 & 3.26 & 2.83 & 3.43 & 2.68 & 2.38 & 2.04 & 2.68 & 2.11 & 2.50 & 2.04 & 2.35 & 1.98 & 2.87 & 2.52 & 2.38 & 2.12 & 2.31 & 2.24 \\
\hline 0.03 & 3.01 & 3.13 & 2.98 & 2.36 & 3.34 & 3.09 & 3.04 & 2.71 & 3.39 & 2.62 & 2.64 & 2.10 & 2.34 & 2.09 & 2.41 & 1.89 & 2.20 & 1.89 & 2.62 & 2.38 & 2.23 & 2.09 & 2.22 & 2.17 \\
\hline 0.04 & 2.58 & 3.06 & 2.49 & 2.58 & 4.59 & 4.37 & 2.67 & 2.77 & 3.36 & 2.91 & 2.99 & 2.77 & 2.11 & 2.04 & 2.33 & 2.27 & 2.12 & 2.09 & 2.38 & 2.47 & 2.28 & 2.31 & 2.07 & 2.20 \\
\hline 0.05 & 2.60 & 2.80 & 2.72 & 2.77 & 4.22 & 3.77 & 2.71 & 3.14 & 2.52 & 2.95 & 2.68 & 2.76 & 1.88 & 2.34 & 1.85 & 1.99 & 1.67 & 1.96 & 2.55 & 2.67 & 2.14 & 2.22 & 1.87 & 1.99 \\
\hline 0.06 & 2.66 & 2.71 & 3.64 & 3.16 & 2.51 & 2.78 & 2.90 & 3.26 & 3.04 & 2.96 & 2.37 & 2.82 & 2.54 & 2.72 & 1.75 & 1.82 & 2.29 & 2.10 & 2.62 & 2.84 & 1.98 & 2.36 & 2.29 & 2.10 \\
\hline 0.07 & 2.85 & 3.51 & 3.82 & 3.04 & 1.80 & 1.96 & 3.05 & 2.99 & 3.78 & 3.30 & 1.96 & 2.14 & 1.96 & 2.04 & 1.63 & 1.83 & 2.53 & 2.57 & 2.50 & 2.43 & 1.74 & 1.96 & 2.39 & 2.42 \\
\hline 0.08 & 3.39 & 3.98 & 3.41 & 2.57 & 1.91 & 1.80 & 3.34 & 3.04 & 3.43 & 3.10 & 1.87 & 1.83 & 1.70 & 1.76 & 1.96 & 1.90 & 2.73 & 2.76 & 2.42 & 2.26 & 1.91 & 1.90 & 2.60 & 2.63 \\
\hline 0.09 & $4 . \overline{77}$ & 5.90 & 2.78 & $\overline{2.16}$ & 1.59 & 1.71 & $3 . \overline{83}$ & 3.36 & $\overline{3.10}$ & 2.84 & 1.73 & 1.69 & 2.04 & 1.76 & 2.41 & 2.36 & 2.80 & 2.86 & 2.48 & 2.29 & 2.29 & 2.28 & 2.79 & 3.08 \\
\hline 0.1 & 5.37 & 5.00 & 2.39 & 2.03 & 1.46 & 1.50 & 4.29 & 4.14 & 2.78 & 2.59 & 1.63 & 1.54 & 1.97 & 1.97 & $2.7 \overline{7}$ & 2.96 & 2.60 & 2.70 & 2.31 & 2.27 & 2.54 & 2.71 & 2.70 & 2.89 \\
\hline 0.11 & 5.00 & 3.76 & 2.24 & 1.78 & 1.39 & 1.25 & 4.65 & 4.36 & 2.56 & 2.21 & 1.48 & 1.41 & 2.27 & 2.09 & 3.42 & 3.18 & 2.53 & 2.32 & 2.26 & 2.17 & 3.06 & 2.86 & 2.76 & 2.42 \\
\hline 0.12 & 3.53 & 3.51 & 2.07 & 1.66 & 1.42 & 1.17 & 4.90 & 4.30 & 2.58 & 2.07 & 1.47 & 1.33 & 2.61 & 2.53 & 3.80 & 3.57 & 2.46 & 2.26 & 2.69 & 2.33 & 3.51 & 3.36 & 2.72 & 2.46 \\
\hline 0.13 & 3.02 & 2.62 & 1.84 & 1.38 & 1.26 & 1.14 & 4.08 & 3.84 & 2.12 & 1.81 & 1.32 & 1.16 & 2.75 & 3.01 & 3.47 & 3.76 & 1.97 & 1.96 & 2.80 & 2.78 & 3.47 & 3.63 & 2.11 & 2.14 \\
\hline 0.14 & 2.36 & 2.27 & 1.45 & 1.25 & 1.20 & 1.22 & 3.07 & 3.14 & 1.71 & 1.45 & 1.23 & 1.19 & 3.09 & 3.11 & 3.18 & 3.34 & 1.63 & 1.54 & 3.14 & 2.93 & 3.26 & 3.22 & 1.80 & $1 . \overline{73}$ \\
\hline 0.85 & 2.05 & 1.98 & 1.34 & 1.29 & 1.19 & 1.21 & 2.77 & 2.82 & 1.52 & 1.38 & 1.21 & 1.20 & 3.38 & 3.48 & $\overline{2.99}$ & $\overline{3.10}$ & 1.53 & 1.45 & $\overline{3.36}$ & 3.26 & 3.34 & 3.17 & 1.66 & 1.59 \\
\hline 0.16 & 2.11 & 1.92 & 1.38 & 1.34 & 1.17 & 1.19 & 2.76 & 2.57 & 1.53 & 1.39 & 1.20 & 1.22 & 4.01 & 3.59 & 3.04 & 2.74 & 1.58 & 1.41 & 3.82 & 3.33 & 3.40 & 2.98 & 1.69 & 1.52 \\
\hline 0.17 & 1.98 & 1.89 & 1.27 & 1.39 & 1.15 & 1.17 & 2.47 & 2.60 & 1.44 & 1.51 & 1.17 & 1.24 & 4.36 & 4.13 & 2.63 & 2.76 & 1.47 & 1.45 & 4.32 & 3.65 & 3.06 & 2.88 & 1.51 & 1.55 \\
\hline 0.18 & 1.60 & 1.86 & 1.25 & 1.36 & 1.12 & 1.11 & 1.91 & 2.48 & 1.37 & 1.51 & 1.18 & 1.18 & 4.49 & 4.43 & 2.01 & 2.78 & 1.37 & 1.45 & 4.71 & 4.18 & 2.26 & 3.02 & 1.42 & 1.59 \\
\hline 0.19 & 1.59 & 1.54 & 1.25 & 1.08 & 1.08 & 1.09 & 1.79 & 2.09 & 1.38 & 1.24 & 1.14 & 1.08 & 4.77 & 3.97 & 1.84 & 2.26 & 1.37 & 1.26 & 5.01 & 3.85 & 2.04 & 2.45 & 1.43 & 1.28 \\
\hline 0.2 & 1.60 & 1.39 & 1.22 & 1.12 & 1.13 & 1.11 & 1.89 & 1.73 & 1.37 & 1.14 & 1.15 & 1.10 & 4.98 & 3.49 & 1.94 & $1 . \overline{80}$ & 1.40 & 1.18 & 5.15 & 3.71 & 2.11 & 2.00 & 1.45 & 1.24 \\
\hline 0.3 & 1.60 & 1.44 & 1.21 & 1.22 & 1.11 & 1.10 & $1 . \overline{9} 2$ & 1.58 & 1.36 & 1.22 & 1.16 & 1.13 & 4.82 & 3.22 & 1.96 & 1.63 & 1.35 & 1.21 & 4.99 & 3.69 & 2.15 & 1.79 & 1.43 & 1.25 \\
\hline 0.4 & 1.34 & 1.27 & 1.17 & 1.14 & 1.08 & 1.09 & 1.34 & 1.42 & 1.22 & 1.17 & 1.09 & 1.11 & 2.51 & 2.30 & 1.44 & 1.42 & 1.20 & 1.17 & 2.77 & 2.75 & 1.57 & 1.50 & 1.24 & 1.20 \\
\hline 0.5 & 1.15 & 1.28 & 1.09 & 1.09 & 1.05 & 1.07 & 1.31 & 1.46 & 1.12 & 1.16 & 1.05 & 1.07 & 1.63 & 1.58 & 1.34 & 1.37 & 1.10 & 1.17 & 1.90 & 1.70 & 1.37 & 1.39 & 1.10 & 1.21 \\
\hline 0.6 & 1.12 & 1.17 & 1.08 & 1.05 & 1.04 & 1.05 & 1.17 & 1.33 & 1.12 & 1.06 & 1.04 & 1.06 & 1.55 & 1.46 & 1.22 & 1.25 & 1.12 & 1.07 & 1.60 & 1.44 & 1.23 & 1.27 & 1.12 & 1.08 \\
\hline 0.8 & 1.10 & 1.06 & 1.07 & 1.04 & 1.05 & 1.05 & 1.12 & 1.12 & 1.07 & 1.05 & 1.05 & 1.05 & 1.30 & 1.31 & 1.10 & 1.08 & 1.06 & 1.03 & 1.32 & 1.35 & 1.11 & 1.11 & 1.07 & 1.03 \\
\hline 1 & 1.12 & 1.11 & 1.08 & 1.05 & 1.04 & 1.04 & 1.11 & 1.10 & 1.09 & 1.08 & 1.04 & 1.03 & 1.24 & 1.20 & 1.10 & 1.07 & 1.07 & 1.07 & 1.27 & 1.18 & 1.10 & 1.08 & 1.08 & 1.08 \\
\hline 2 & 1.14 & 1.17 & 1.07 & 1.05 & 1.07 & 1.05 & 1.14 & 1.16 & 1.09 & 1.08 & 1.05 & 1.05 & 1.24 & 1.24 & 1.11 & 1.12 & 1.09 & 1.08 & $1 . \overline{31}$ & 1.26 & 1.13 & 1.13 & 1.09 & 1.10 \\
\hline
\end{tabular}


TABLE 10 Summary of Amplification Ratios (Cont'd.) Damping $=12 \%$

$\mathrm{L}=$ Lower Bound Properties, $M=$ Median Properties, $U=$ Upper Bound Properties

\begin{tabular}{|c|c|c|c|c|c|c|c|c|c|c|c|c|c|c|c|c|c|c|}
\hline & \multirow{2}{*}{\multicolumn{6}{|c|}{5}} & \multirow{2}{*}{\multicolumn{6}{|c|}{6}} & \multirow{2}{*}{\multicolumn{6}{|c|}{7}} \\
\hline \multirow{3}{*}{$\begin{array}{l}\text { Profile } \\
\text { Property } \\
\text { History } \\
\end{array}$} & & & & & & & & & & & & & & & & & & \\
\hline & \multicolumn{2}{|c|}{ L } & \multicolumn{2}{|c|}{$M$} & \multicolumn{2}{|c|}{$\mathbf{U}$} & \multirow{2}{*}{\multicolumn{2}{|c|}{ L }} & \multicolumn{2}{|c|}{$\mathbf{M}$} & \multicolumn{2}{|c|}{$u$} & \multicolumn{2}{|c|}{$\mathrm{L}$} & \multicolumn{2}{|c|}{$M$} & \multicolumn{2}{|c|}{$U$} \\
\hline & 1 & 2 & 1 & 2 & 1 & 2 & & & 1 & 2 & 1 & 2 & 1 & 2 & 1 & 2 & 1 & 2 \\
\hline Period, (sec) & & & & & & & & 8 & & 60 & & & & & & & & \\
\hline 0.01 & 2.35 & 2.14 & 2.31 & 1.79 & 2.10 & 1.81 & 2.59 & 2.01 & 2.53 & 2.10 & 2.00 & 1.73 & 1.35 & 1.34 & 1.12 & 1.12 & 1.04 & 1.03 \\
\hline 0.03 & 2.13 & 1.98 & 2.07 & 1.76 & 2.05 & 1.67 & 2.30 & 1.79 & 2.32 & 1.98 & 1.88 & 1.75 & 1.50 & 1.32 & 1.13 & 1.11 & 1.02 & 1.02 \\
\hline 0.04 & 1.99 & 1.81 & 1.82 & 1.80 & 2.04 & 2.05 & 2.00 & 1.80 & 2.45 & 2.16 & 2.16 & 1.85 & 1.87 & 1.80 & 1.33 & 1.32 & 1.15 & 1.15 \\
\hline 0.05 & 1.98 & 1.91 & 1.61 & 2.01 & 1.65 & 1.70 & 2.06 & 2.02 & 1.89 & 2.22 & 1.80 & 1.85 & 1.42 & 1.57 & 1.12 & 1.23 & 1.02 & 1.11 \\
\hline 0.06 & 1.71 & 2.10 & 2.27 & 2.40 & 1.56 & 1.63 & 2.41 & 2.04 & 2.50 & 2.27 & 1.62 & 1.90 & 1.27 & 1.37 & 1.08 & 1.15 & 1.01 & 1.06 \\
\hline 0.07 & 1.38 & 1.90 & 1.76 & 1.81 & 1.47 & 1.71 & 1.90 & 2.42 & 2.09 & 2.04 & 1.49 & 1.76 & 1.32 & 1.31 & 1.16 & 1.12 & 1.09 & 1.05 \\
\hline 0.08 & 1.85 & 1.70 & 1.52 & 1.55 & 1.73 & 1.66 & 1.98 & 2.09 & 1.81 & 1.94 & 1.61 & 1.68 & 1.21 & 1.26 & 1.09 & 1.12 & 1.04 & 1.06 \\
\hline 0.09 & 2.31 & 2.02 & 1.89 & 1.58 & 2.16 & 2.08 & 2.57 & 2.14 & 2.02 & 1.74 & 2.02 & 1.96 & 1.14 & 1.16 & 1.06 & 1.06 & 1.02 & 1.02 \\
\hline 0.1 & 2.08 & 2.13 & 1.79 & 1.74 & 2.36 & 2.56 & 2.63 & 2.11 & 1.97 & 1.83 & 2.25 & 2.45 & 1.09 & 1.10 & 1.03 & 1.05 & 1.01 & 1.02 \\
\hline 0.11 & 1.82 & 1.59 & 1.93 & 1.86 & 2.86 & 2.66 & 2.20 & 2.14 & 1.83 & 1.81 & 2.70 & 2.55 & 1.08 & 1.10 & 1.04 & 1.06 & 1.01 & 1.03 \\
\hline 0.12 & 1.85 & 1.59 & 2.24 & 2.21 & 3.16 & 3.01 & 2.06 & 2.12 & 2.23 & 2.07 & 3.02 & 2.87 & 1.15 & 1.09 & 1.06 & 1.05 & 1.02 & 1.03 \\
\hline 0.1 & 1.95 & 1.57 & 2.36 & 2.62 & 2.88 & 3.14 & 2.10 & 1.88 & 2.31 & 2.51 & 2.88 & 3.11 & 1.06 & 1.09 & 1.02 & 1.05 & 1.01 & 1.03 \\
\hline 0.14 & 2.05 & 1.60 & 2.65 & 2.73 & 2.71 & 2.86 & 2.22 & 1.59 & 2.68 & 2.63 & 2.83 & 2.83 & 1.05 & 1.05 & 1.02 & 1.02 & 1.00 & 1.00 \\
\hline 0.15 & 2.01 & 1.83 & 2.93 & 3.02 & 2.70 & 2.68 & 2.12 & 1.82 & 2.88 & 2.83 & 2.85 & 2.72 & 1.05 & 1.07 & 1.02 & 1.03 & 1.01 & 1.01 \\
\hline 0.16 & 2.13 & 1.84 & 3.41 & 3.12 & 2.77 & 2.42 & 2.22 & 1.88 & 3.32 & 2.95 & 2.88 & 2.55 & 1.05 & 1.06 & 1.02 & 1.03 & 1.01 & 1.01 \\
\hline 0.17 & 2.11 & 1.95 & 3.77 & 3.42 & 2.39 & 2.51 & 2.21 & 1.97 & 3.70 & 3.20 & 2.57 & 2.57 & 1.04 & 1.05 & 1.02 & 1.03 & 1.01 & 1.02 \\
\hline 0.18 & 2.06 & 2.30 & 3.87 & 3.75 & 1.86 & 2.49 & 1.97 & 2.29 & 3.93 & 3.57 & 1.94 & 2.64 & 1.04 & 1.02 & 1.02 & 1.00 & 1.02 & 1.00 \\
\hline 0.19 & 2.10 & 2.41 & 4.09 & 3.39 & 1.78 & 2.05 & 1.95 & 2.41 & 4.21 & 3.26 & 1.86 & 2.12 & 1.02 & 1.03 & 1.01 & 1.01 & 1.00 & 1.01 \\
\hline 0.2 & 2.37 & 2.52 & 4.28 & 3.04 & 1.86 & 1.62 & 2.25 & 2.54 & 4.39 & 3.16 & 1.92 & 1.71 & 1.04 & 1.03 & 1.01 & 1.02 & 1.00 & 1.01 \\
\hline 0.3 & 3.61 & 3.59 & 4.18 & 2.98 & 1.87 & 1.59 & 3.79 & 3.66 & 4.24 & 3.16 & 1.98 & 1.68 & 1.04 & 1.03 & 1.02 & 1.01 & 1.01 & 1.01 \\
\hline 0.4 & 3.50 & 3.76 & 2.39 & 2.29 & 1.41 & 1.38 & 3.76 & 3.79 & 2.53 & 2.50 & 1.47 & 1.44 & 1.03 & 1.02 & 1.01 & 1.01 & 1.01 & 1.01 \\
\hline 0.5 & 2.24 & 2.64 & 1.60 & 1.51 & 1.30 & 1.33 & 2.40 & 2.67 & 1.73 & 1.56 & 1.32 & 1.34 & 1.01 & 1.02 & 1.01 & 1.01 & 1.00 & 1.01 \\
\hline 0.6 & 1.93 & 2.11 & 1.49 & 1.40 & 1.18 & 1.21 & 2.02 & 2.18 & 1.54 & 1.37 & 1.19 & 1.22 & 1.01 & 1.02 & 1.01 & 1.01 & 1.00 & 1.00 \\
\hline 0.8 & 1.68 & 1.55 & 1.28 & 1.28 & 1.09 & 1.07 & 1.76 & 1.63 & 1.30 & 1.28 & 1.10 & 1.08 & 1.02 & 1.01 & 1.01 & 1.00 & 1.01 & 1.00 \\
\hline 1 & 1.36 & 1.57 & 1.22 & 1.16 & 1.08 & 1.06 & 1.36 & 1.62 & 1.25 & 1.15 & 1.08 & 1.07 & 1.01 & 1.01 & 1.01 & 1.00 & 1.00 & 1.00 \\
\hline 2 & 1.29 & 1.31 & 1.23 & 1.21 & 1.10 & 1.10 & 1.33 & 1.31 & 1.26 & 1.22 & 1.11 & 1.10 & 1.01 & 1.02 & 1.00 & 1.01 & 1.00 & 1.00 \\
\hline
\end{tabular}


TABLE 10 Summary of Amplification Ratios (Cont'd.)

$$
\text { Damping }=12 \%
$$

$L=$ Lower Bound Properties, $M=$ Median Properties, $U=$ Upper Bound Properties

\begin{tabular}{|c|c|c|c|c|c|c|c|c|c|c|c|c|c|c|c|c|}
\hline & Total & Average & Max & $85 \%$ & Total & Average & Max & $85 \%$ & Total & Average & Max & $85 \%$ & Total & Average & Max & $85 \%$ \\
\hline \multicolumn{17}{|l|}{ Profile } \\
\hline Property & $L$ & $L$ & $L$ & $L$ & $M$ & $M$ & $M$ & $M$ & U & $\mathrm{U}$ & U & $\mathrm{U}$ & All & All & Ail & All \\
\hline \multicolumn{17}{|l|}{ History } \\
\hline \multicolumn{17}{|c|}{ Period, (sec) } \\
\hline 0.01 & 33.99 & 2.43 & 3.26 & 3.10 & 31.66 & 2.26 & 3.43 & 3.10 & 28.39 & 2.03 & 2.88 & 2.50 & 94.04 & 2.24 & 3.43 & 2.87 \\
\hline 0.03 & 32.33 & 2.31 & 3.13 & 3.04 & 30.33 & 2.17 & 3.39 & 2.98 & 29.03 & 2.07 & 3.34 & 3.09 & 91.70 & 2.18 & 3.39 & 3.01 \\
\hline 0.04 & 31.36 & 2.24 & 3.06 & 2.77 & 31.40 & 2.24 & 3.36 & 2.91 & 33.62 & 2.40 & 4.59 & 4.37 & 96.37 & 2.29 & 4.59 & 2.91 \\
\hline 0.05 & 31.65 & 2.26 & 3.14 & 2.80 & 29.23 & 2.09 & 2.95 & 2.77 & 30.06 & 2.15 & 4.22 & 3.77 & 90.94 & 2.17 & 4.22 & 2.77 \\
\hline 0.06 & 33.15 & 2.37 & 3.26 & 2.90 & 32.38 & 2.31 & 3.64 & 3.16 & 28.13 & 2.01 & 2.82 & 2.78 & 93.66 & 2.23 & 3.64 & 2.90 \\
\hline 0.07 & 31.57 & 2.25 & 3.51 & 3.05 & 31.06 & 2.22 & 3.82 & 3.78 & 26.33 & 1.88 & 2.57 & 2.53 & 88.96 & 2.12 & 3.82 & 3.04 \\
\hline 0.08 & 31.97 & 2.28 & 3.98 & 3.39 & 29.22 & 2.09 & 3.43 & 3.41 & 26.89 & 1.92 & 2.76 & 2.73 & 88.08 & 2.10 & 3.98 & 3.10 \\
\hline 0.09 & 37.76 & 2.70 & 5.90 & 4.77 & 29.56 & 2.11 & 3.10 & 2.84 & 28.51 & 2.04 & 3.08 & 2.86 & 95.84 & 2.28 & 5.90 & 3.08 \\
\hline 0.1 & 38.46 & 2.75 & 5.37 & 5.00 & 30.19 & 2.16 & 2.96 & 2.78 & 28.67 & 2.05 & 2.89 & 2.70 & 97.32 & 2.32 & 5.37 & 2.89 \\
\hline 0.11 & 36.48 & 2.61 & 5.00 & 4.65 & 30.83 & 2.20 & 3.42 & 3.18 & 28.38 & 2.03 & 2.86 & 2.76 & 95.68 & 2.28 & 5.00 & 3.18 \\
\hline 0.12 & 36.27 & 2.59 & 4.90 & 4.30 & 33.47 & 2.39 & 3.80 & 3.57 & 29.40 & 2.10 & 3.16 & 3.02 & 99.14 & 2.36 & 4.90 & 3.51 \\
\hline 0.13 & 34.55 & 2.47 & 4.08 & 3.84 & 33.36 & 2.38 & 3.76 & 3.63 & 27.11 & 1.94 & 3.14 & 3.11 & 95.02 & 2.26 & 4.08 & 3.47 \\
\hline 0.14 & 32.68 & $2.3 \overline{3}$ & 3.14 & 3.14 & 31.59 & 2.26 & 3.34 & 3.26 & 24.78 & 1.77 & 2.86 & 2.83 & 89.04 & 2.12 & 3.34 & 3.14 \\
\hline 0.15 & 33.00 & 2.36 & 3.48 & 3.38 & 31.84 & 2.27 & 3.34 & 3.17 & 23.99 & 1.71 & 2.85 & 2.72 & 88.83 & 2.12 & 3.48 & 3.17 \\
\hline 0.18 & 36.35 & 2.60 & 4.71 & 4.49 & 32.72 & 2.34 & 3.93 & 3.87 & 21.36 & 1.53 & 2.64 & 2.49 & 90.43 & 2.15 & 4.71 & 3.87 \\
\hline 0.19 & 35.53 & 2.54 & 5.01 & 4.77 & 30.52 & 2.18 & 4.21 & 4.09 & 19.55 & 1.40 & 2.12 & 2.05 & 85.61 & 2.04 & 5.01 & 3.85 \\
\hline 0.2 & 35.68 & 2.55 & 5.15 & 4.98 & 29.59 & 2.11 & 4.39 & 4.28 & 18.88 & 1.35 & 1.92 & 1.86 & 84.16 & 2.00 & 5.15 & 3.49 \\
\hline 0.3 & 39.99 & 2.86 & 4.99 & 4.82 & 29.13 & 2.08 & 4.24 & 4.18 & 18.88 & 1.35 & 1.98 & 1.87 & 88.00 & 2.10 & 4.99 & 3.69 \\
\hline 0.4 & 32.55 & 2.33 & 3.79 & 3.76 & 22.35 & 1.60 & 2.53 & 2.50 & 16.91 & 1.21 & 1.47 & 1.44 & 71.81 & 1.71 & 3.79 & 2.75 \\
\hline 0.5 & 24.00 & 1.71 & 2.67 & 2.64 & 18.35 & 1.31 & 1.73 & 1.60 & 16.11 & 1.15 & 1.34 & 1.33 & 58.47 & 1.39 & 2.67 & 1.73 \\
\hline 0.6 & 21.11 & 1.51 & 2.18 & 2.11 & 17.10 & 1.22 & 1.54 & 1.49 & 15.38 & 1.10 & 1.22 & 1.21 & 53.59 & 1.28 & 2.18 & 1.55 \\
\hline 0.8 & 18.34 & 1.31 & 1.76 & 1.68 & 15.79 & 1.13 & 1.30 & 1.28 & 14.74 & 1.05 & 1.10 & 1.09 & 48.88 & 1.16 & 1.76 & 1.32 \\
\hline 1 & 17.27 & 1.23 & 1.62 & 1.57 & 15.43 & 1.10 & 1.25 & 1.22 & 14.73 & 1.05 & 1.08 & 1.08 & 47.43 & 1.13 & 1.62 & 1.25 \\
\hline 2 & 16.93 & 1.21 & 1.33 & 1.31 & 15.72 & 1.12 & 1.26 & 1.23 & 15.01 & 1.07 & 1.11 & 1.10 & 47.65 & 1.13 & 1.33 & 1.26 \\
\hline
\end{tabular}


TABLE 11 Summary of Amplification Ratios

Damping $=15 \%$

$L=$ Lower Bound Properties, $M=$ Median Properties, $U=$ Upper Bound Properties

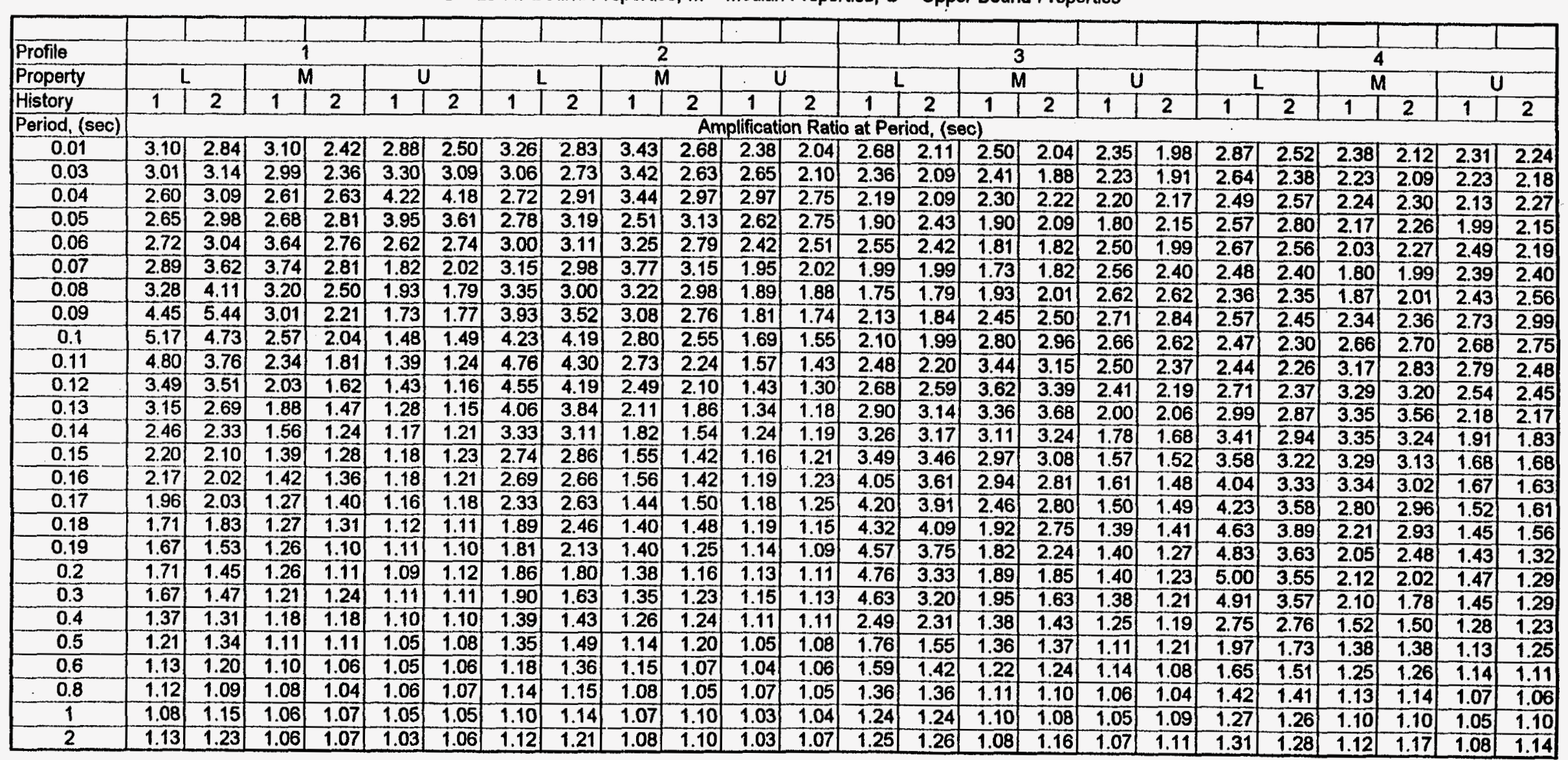


TABLE 11 Summary of Amplification Ratios (Cont'd.) Damping $=15 \%$

$\mathrm{L}=$ Lower Bound Properties, $M=$ Median Properties, $\mathrm{U}=$ Upper Bound Properties

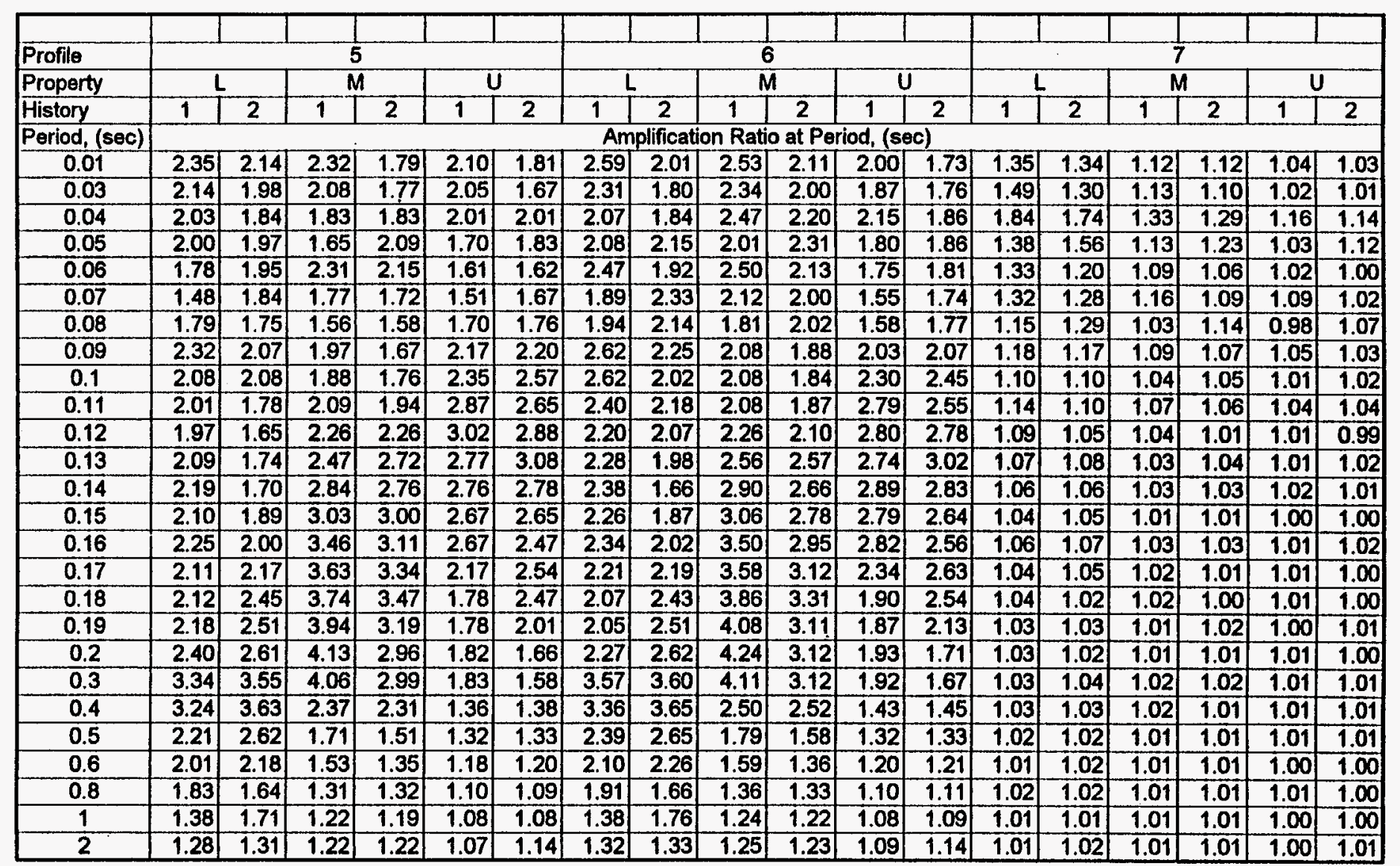


TABLE 11 Summary of Amplification Ratios (Cont'd.) Damping $=15 \%$

$\mathrm{L}=$ Lower Bound Properties, $M=$ Median Properties, $U=$ Upper Bound Properties

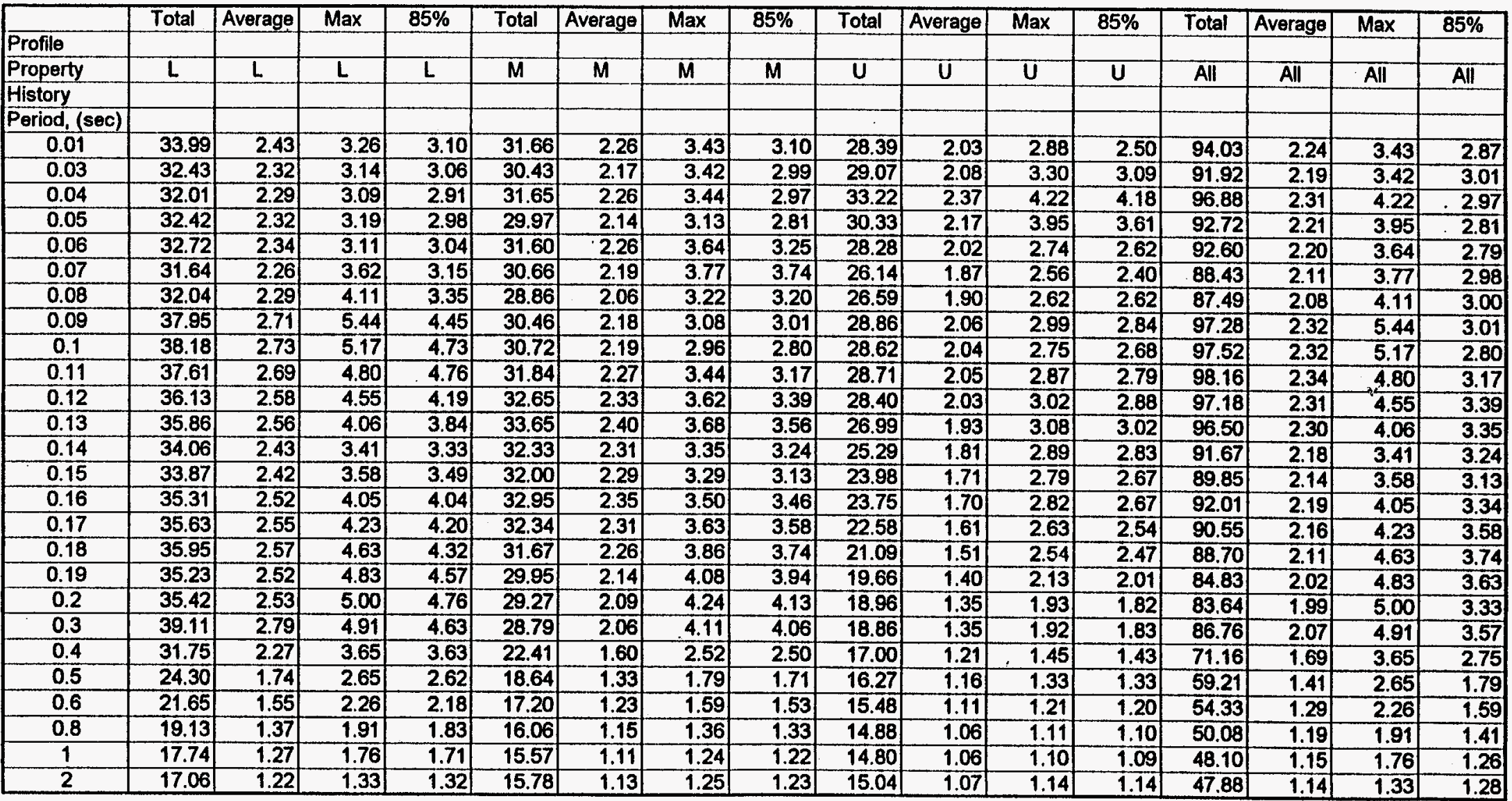




\subsection{DESIGN RESPONSE SPECTRA CURVES}

Calculations were performed to develop smooth top of ground (soil/weathered shale) design response spectra curves using the results of the foregoing SHAKE analysis. These design curves are to be used for the seismic evaluation of the structures founded on soil/weathered shale. The top of ground smooth response spectra was determined by multiplying the rock response spectra by the soil and weathered shale amplification ratios. This approach is represented by the schematic shown on Fig. 15.

A summary of amplification ratios for the seven soil columns considered is presented in Tables 6 through 11. The average, maximum and the 85 percent peak acceleration values were plotted to envelop the spectral acceleration values. This envelope was used to finalize the smooth top of ground design response spectra curve.

The Newmark and Hall data (Ref. 34) are used to define the differences in response spectra for different damping ratios. These differences are used to scale the 5 percent damped ground spectra to other damping ratios. These scaling factors are also validated by site studies for one soil profile.

It was decided to develop separate design response spectra curves for the following three groups of foundations.

(1) Soil columns 1 through 7 with lower bound, mean and upper bound shear wave velocities: This case is applicable to the seismic evaluation of structures founded on soil deposits thicker than $10 \mathrm{ft}$. (10 to $30 \mathrm{ft}$. of soil overlying 10 to $20 \mathrm{ft}$. weathered shale).

(2) Soil columns 1 and 7 with lower bound, mean and upper bound shear wave velocities: Response curve for this condition is applicable to structures founded on soil deposits equal to or less than $10 \mathrm{ft}$.

(3) Soil column 7 with lower bound, mean and upper bound shear wave velocities: This case is applicable to structures supported on weathered shale.

\subsection{PC-3 FACILITIES}

Smooth top of ground response curves were determined for the PC-3 SSCs 5 percent damping for the three cases discussed in Section 8.0. SHAKE analysis was performed at 2, 7, 10,12, and 15 percent damping, also. Formulations presented in Ref. (34) for the Newmark and Hall Spectra were used to develop the smooth response spectra curves for $2,7,10,12$ and 15 percent dampings from the 5\% damped spectra. Design spectra for 5\% damping developed from independent SHAKE run results was compared with the Newmark and Hall formulations which checked very closely with the independent SHAKE analysis results. Therefore, it should be justified to use Newmark and Hall method which resulted in time saving without any sacrifice in quality.

Smooth top of ground design response spectra curves for PC-3 facilities are presented in Tables 12 through 15 and Figures 16 through 19. 
For PC-2 facilities it was not considered necessary to perform the response analysis using SHAKE 91. Instead, the 1000-year seismic hazard level was linearly scaled of the PGA's from the 2000year event. Smooth top of ground design response curves for the PC-2 facilities are depicted in Figures 20 through 23.

\subsection{PC-1 FACILITIES}

Similar to PC-2 facilities, the 500-year seismic hazard level was scaled from the 2000 -year event. Figures 24 through 27 present the smooth top of ground design response curves for PC-1 facilities. 
Table 12 Design Response Spectra for PC-3 Facilities Supported on Fresh Rock

\begin{tabular}{|c|c|c|c|c|c|c|c|}
\hline \multirow{2}{*}{$\begin{array}{l}\text { Period } \\
\text { (Sec) }\end{array}$} & \multirow{2}{*}{$\begin{array}{l}\text { Frequency } \\
(\mathrm{Hz})\end{array}$} & \multicolumn{6}{|c|}{ Peak Ground Acceleration (g) at Damping } \\
\hline & & $2 \%$ & $5 \%$ & $7 \%$ & $10 \%$ & $12 \%$ & $15 \%$ \\
\hline .0125 & 80.0 & .12 & .12 & .12 & .12 & .12 & .12 \\
\hline .04 & 25.0 & .34 & .26 & .23 & .20 & .18 & .16 \\
\hline .18 & 5.55 & .34 & & & & & \\
\hline 19 & 5.26 & & .26 & & & & \\
\hline .20 & 5.0 & & & .23 & .20 & .18 & \\
\hline .22 & 4.55 & & & & & & .16 \\
\hline 2.0 & 0.5 & .030 & .024 & .023 & .02 & .019 & .018 \\
\hline
\end{tabular}

Note: For PC-1 and PC-2 Facilities, the Peak Ground Acceleration can be scaled from the Acceleration Values Given in this Table. 
Table 13 Design Response Spectra for PC-3 Facilities Supported on Weathered Rock (Shale)

\begin{tabular}{|c|c|c|c|c|c|c|c|}
\hline \multirow{2}{*}{$\begin{array}{l}\text { Period } \\
(\mathrm{Sec})\end{array}$} & \multirow{2}{*}{$\begin{array}{c}\text { Frequency } \\
(\mathrm{Hz})\end{array}$} & \multicolumn{6}{|c|}{ Peak Ground Acceleration (g) at Damping } \\
\hline & & $2 \%$ & $5 \%$ & $7 \%$ & $10 \%$ & $12 \%$ & $15 \%$ \\
\hline .0125 & 80.0 & .16 & .16 & .16 & .16 & .16 & .16 \\
\hline .04 & 25.0 & .45 & .35 & .31 & .27 & .25 & .23 \\
\hline .17 & 5.88 & 0.45 & & & & & \\
\hline .175 & 5.71 & & 0.35 & & & & \\
\hline .18 & 5.55 & & & 0.31 & & & \\
\hline .19 & 5.26 & & & & 0.27 & & \\
\hline .20 & 5.0 & & & & & .25 & .23 \\
\hline .40 & 2.5 & .20 & .15 & .14 & .13 & .12 & .11 \\
\hline 2.0 & 0.5 & .03 & .024 & .023 & .020 & .019 & .018 \\
\hline
\end{tabular}

Note: For PC-1 and PC-2 Facilities, the Peak Ground Acceleration can be scaled from the Acceleration Values Given in this Table. 
Table 14 Design Response Spectra for PC-3 Facilities

Supported on Thin Overburden Soil $\leq 10 \mathrm{Ft}$.

\begin{tabular}{|c|c|c|c|c|c|c|c|}
\hline \multirow{2}{*}{$\begin{array}{l}\text { Period } \\
\text { (Sec) }\end{array}$} & \multirow{2}{*}{$\begin{array}{c}\text { Frequency } \\
(\mathrm{Hz})\end{array}$} & \multicolumn{6}{|c|}{ Peak Ground Acceleration (g) at Damping } \\
\hline & & $2 \%$ & $5 \%$ & $7 \%$ & $10 \%$ & $12 \%$ & $15 \%$ \\
\hline .0125 & 80.0 & 0.28 & 0.28 & 0.28 & 0.28 & 0.28 & 0.28 \\
\hline .04 & 25.0 & 0.90 & 0.70 & 0.62 & 0.54 & 0.50 & 0.46 \\
\hline .09 & 11.11 & 0.90 & & & & & \\
\hline .10 & 10.0 & & 0.70 & 0.62 & 0.54 & 0.50 & \\
\hline .11 & 9.09 & & & & & & 0.46 \\
\hline .40 & 2.5 & 0.20 & 0.17 & 0.16 & 0.14 & 0.13 & 0.12 \\
\hline 2.0 & 0.5 & 0.03 & 0.024 & 0.023 & 0.020 & 0.019 & 0.018 \\
\hline
\end{tabular}

Note: For PC-1 and PC-2 Facilities, the Peak Ground Acceleration

Can Be Scaled from the Acceleration Values Given in this Table. 
Table 15 Design Response Spectra for PC-3 Facilities

Supported on Overburden Soil $>10 \mathrm{Ft}$.

\begin{tabular}{|c|c|c|c|c|c|c|c|}
\hline \multirow{2}{*}{$\begin{array}{l}\text { Period } \\
(\mathrm{Sec})\end{array}$} & \multirow{2}{*}{$\begin{array}{c}\text { Frequency } \\
(\mathrm{Hz})\end{array}$} & \multicolumn{6}{|c|}{ Peak Ground Acceleration (g) at Damping } \\
\hline & & $2 \%$ & $5 \%$ & $7 \%$ & $10 \%$ & $12 \%$ & $15 \%$ \\
\hline .0125 & 80.0 & 0.30 & 0.30 & 0.30 & 0.30 & 0.30 & 0.30 \\
\hline .04 & 25.0 & 1.03 & 0.80 & 0.71 & 0.62 & 0.58 & 0.52 \\
\hline .20 & 5.0 & 1.03 & 0.80 & & & & \\
\hline .21 & 4.76 & & & 0.71 & 0.62 & 0.58 & \\
\hline .23 & 4.35 & & & & & & 0.52 \\
\hline .40 & 2.5 & 0.52 & 0.40 & 0.38 & 0.34 & 0.30 & 0.28 \\
\hline 2.0 & 0.5 & 0.03 & 0.024 & 0.023 & 0.02 & 0.019 & 0.018 \\
\hline
\end{tabular}

Note: For PC-1 and PC-2 Facilities, the Peak Ground Acceleration can be scaled from the Acceleration Values Given in this Table. 


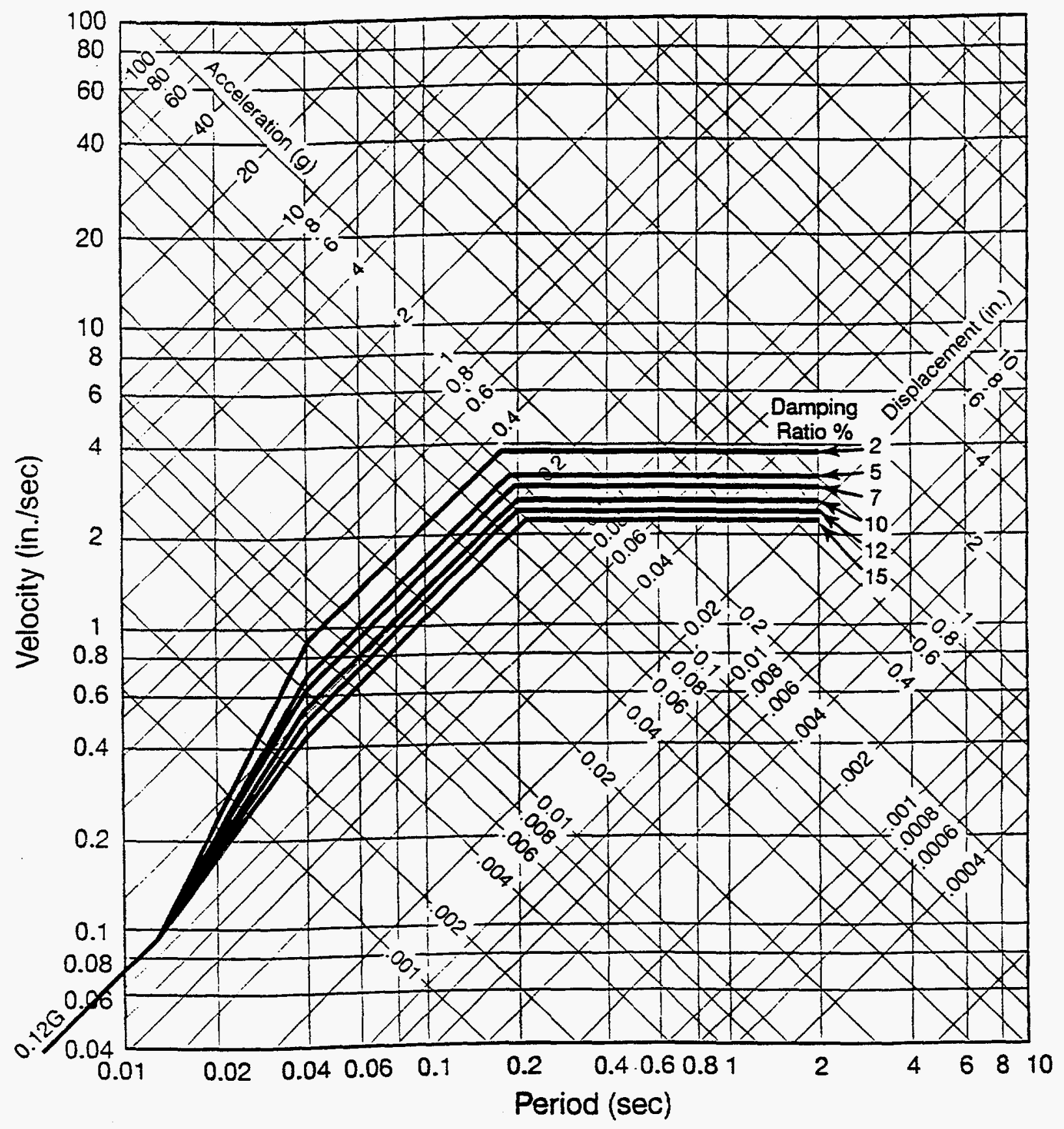

FIG. 16 Y-12 SITE SPECIFIC EARTHQUAKE DESIGN RESPONSE SPECTRA FOR HORIZONTAL ROCK MOTION 2000 - YEAR RETURN PERIOD PC-3 FACILITIES SUPPORTED ON FRESH ROCK 


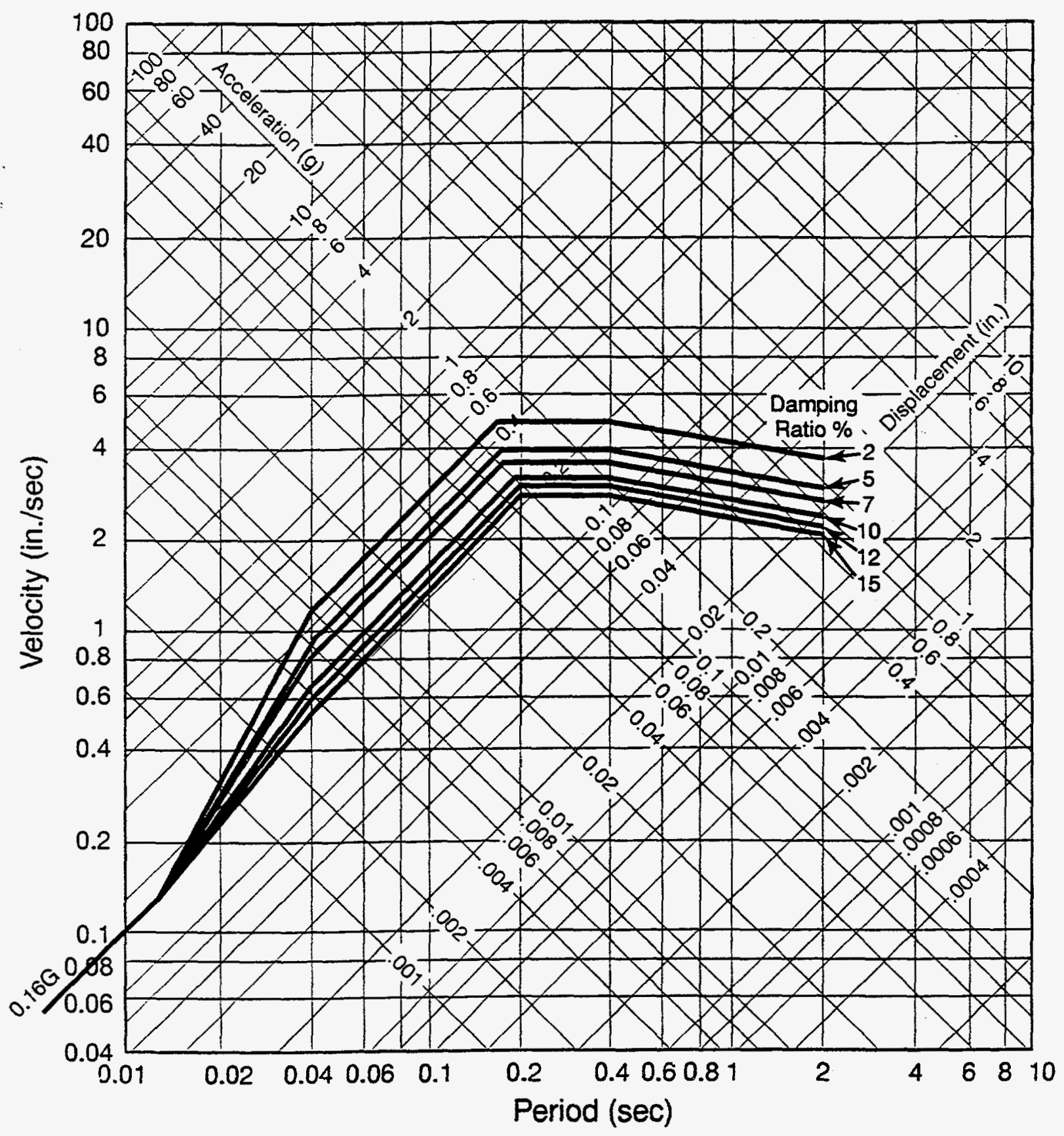

FIG. 17 Y-12 SITE SPECIFIC EARTHQUAKE DESIGN RESPONSE SPECTRA FOR HORIZONTAL WEATHERED ROCK (SHALE) MOTION 2000 - YEAR RETURN PERIOD

\author{
PC-3 FACILITIES SUPPORTED ON WEATHERED \\ ROCK (SHALE).
}




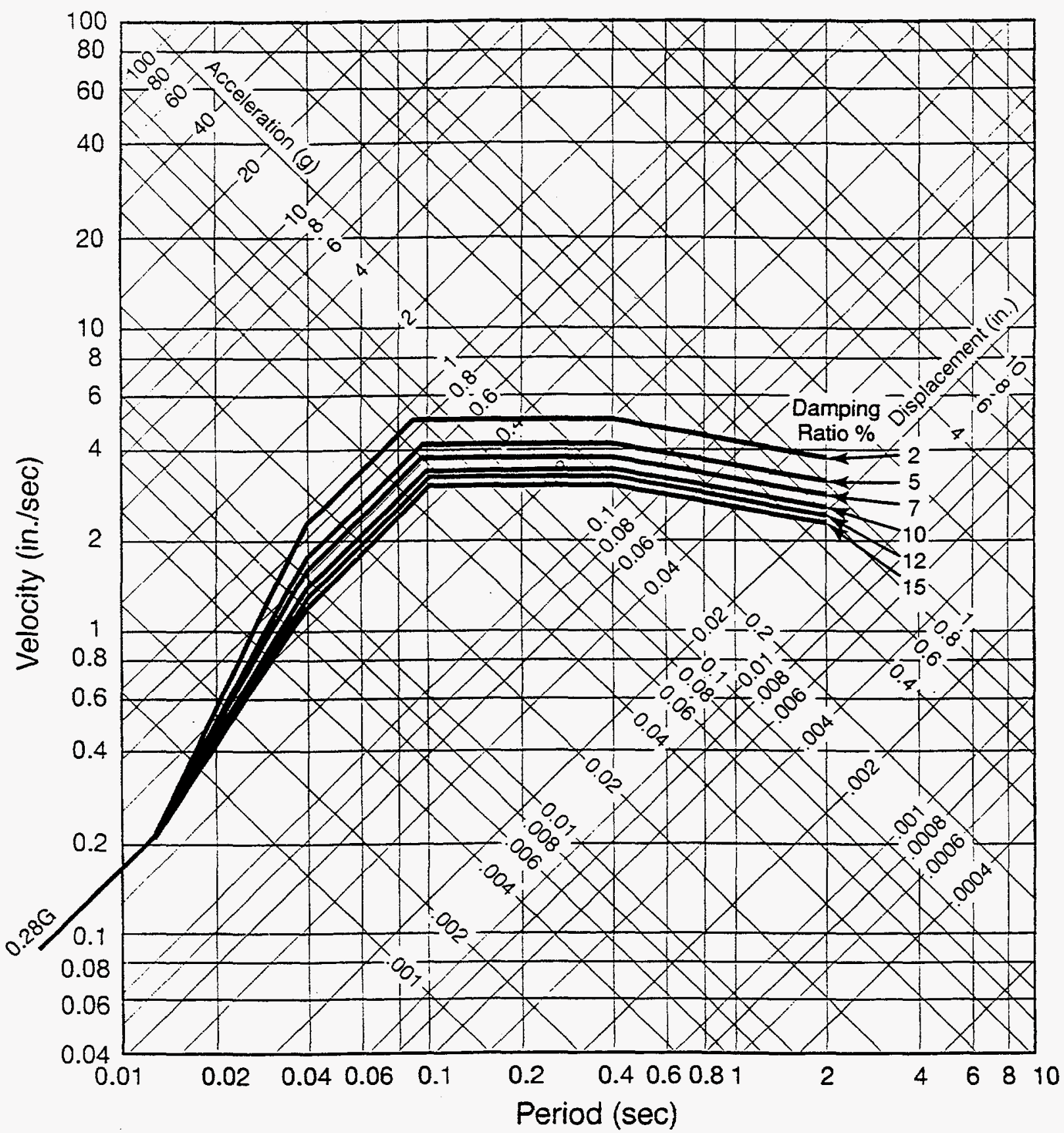

FIG. 18 Y-12 SITE SPECIFIC EARTHQUAKE DESIGN RESPONSE SPECTRA FOR HORIZONTAL SOIL MOTION SOIL OVERBURDEN $\leq 10 \mathrm{ft}$. 2000 - YEAR RETURN PERIOD

PC-3 FACILITIES SUPPORTED ON SOIL OVERBURDEN $\leq$ 1OFT 


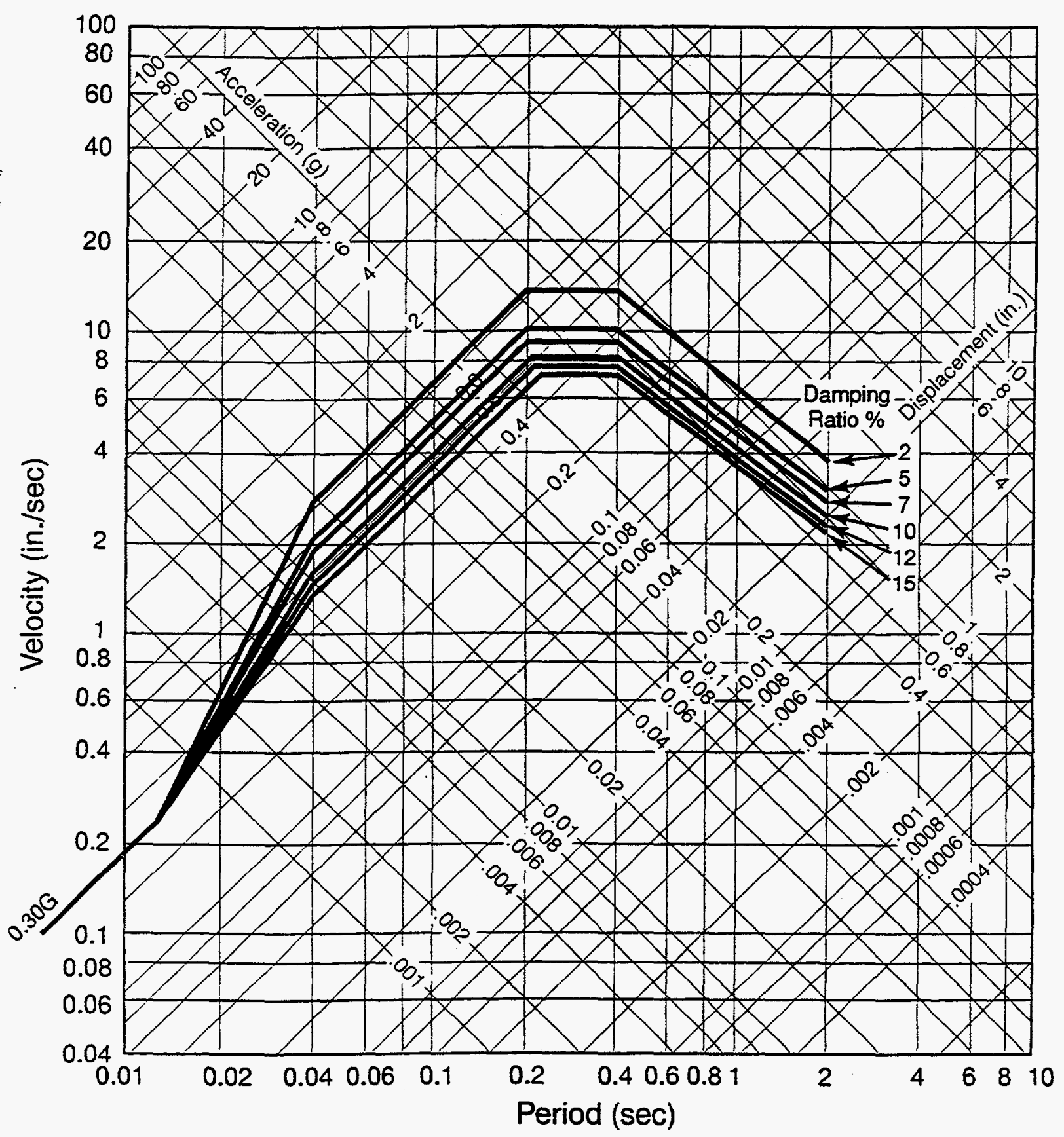

FIG. 19 Y-12 SITE SPECIFIC EARTHQUAKE DESIGN RESPONSE SPECTRA FOR HORIZONTAL SOIL MOTION SOIL OVERBURDEN > $10 \mathrm{ft}$. 2000 - YEAR RETURN PERIOD

\section{PC-3 FACILITIES SUPPORTED ON SOIL OVERBURDEN > 1OFT}




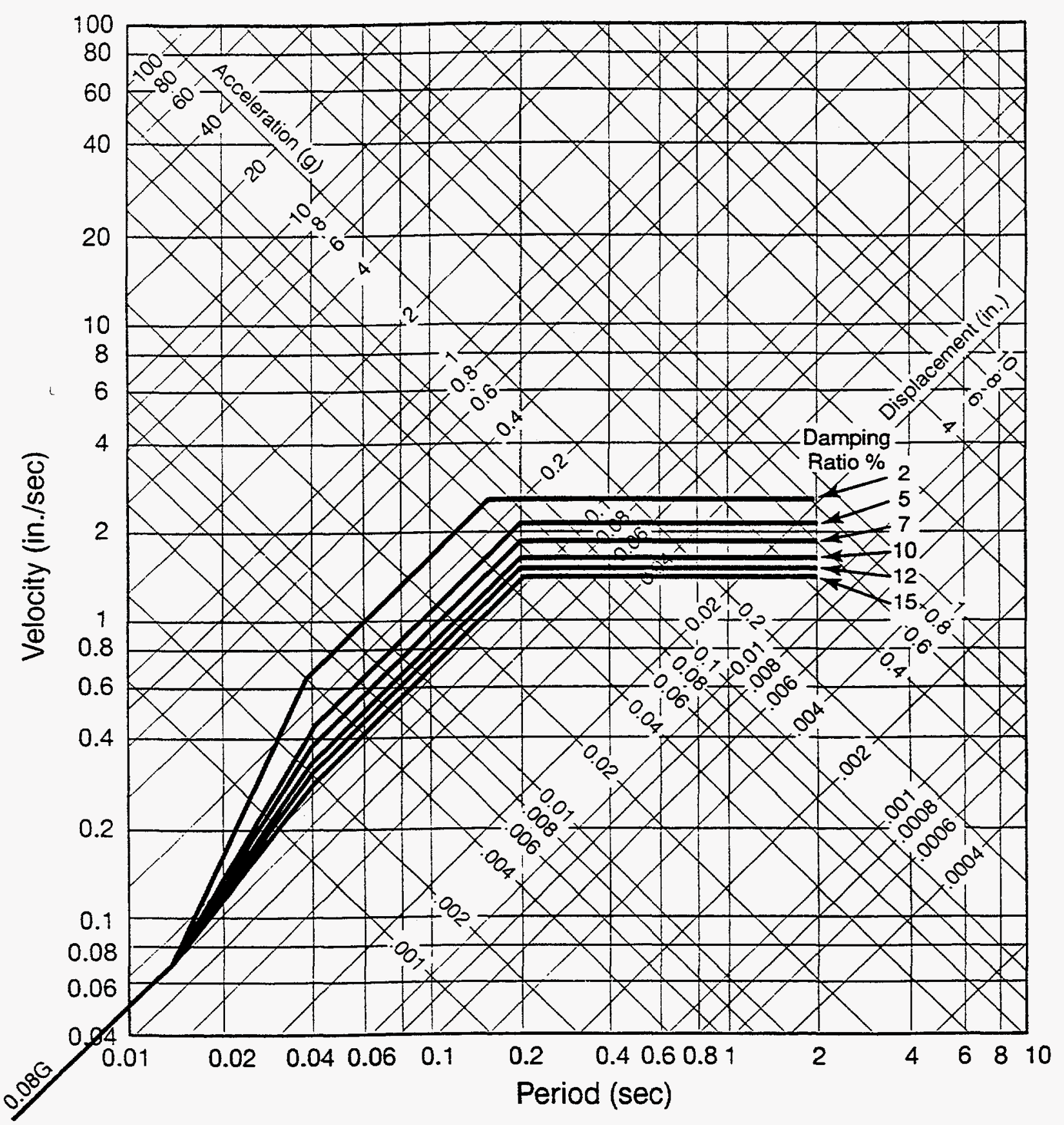

FIG. $20 \mathrm{Y}-12$ SITE SPECIFIC EARTHQUAKE DESIGN RESPONSE SPECTRA FOR HORIZONTAL ROCK MOTION 1000 - YEAR RETURN PERIOD 


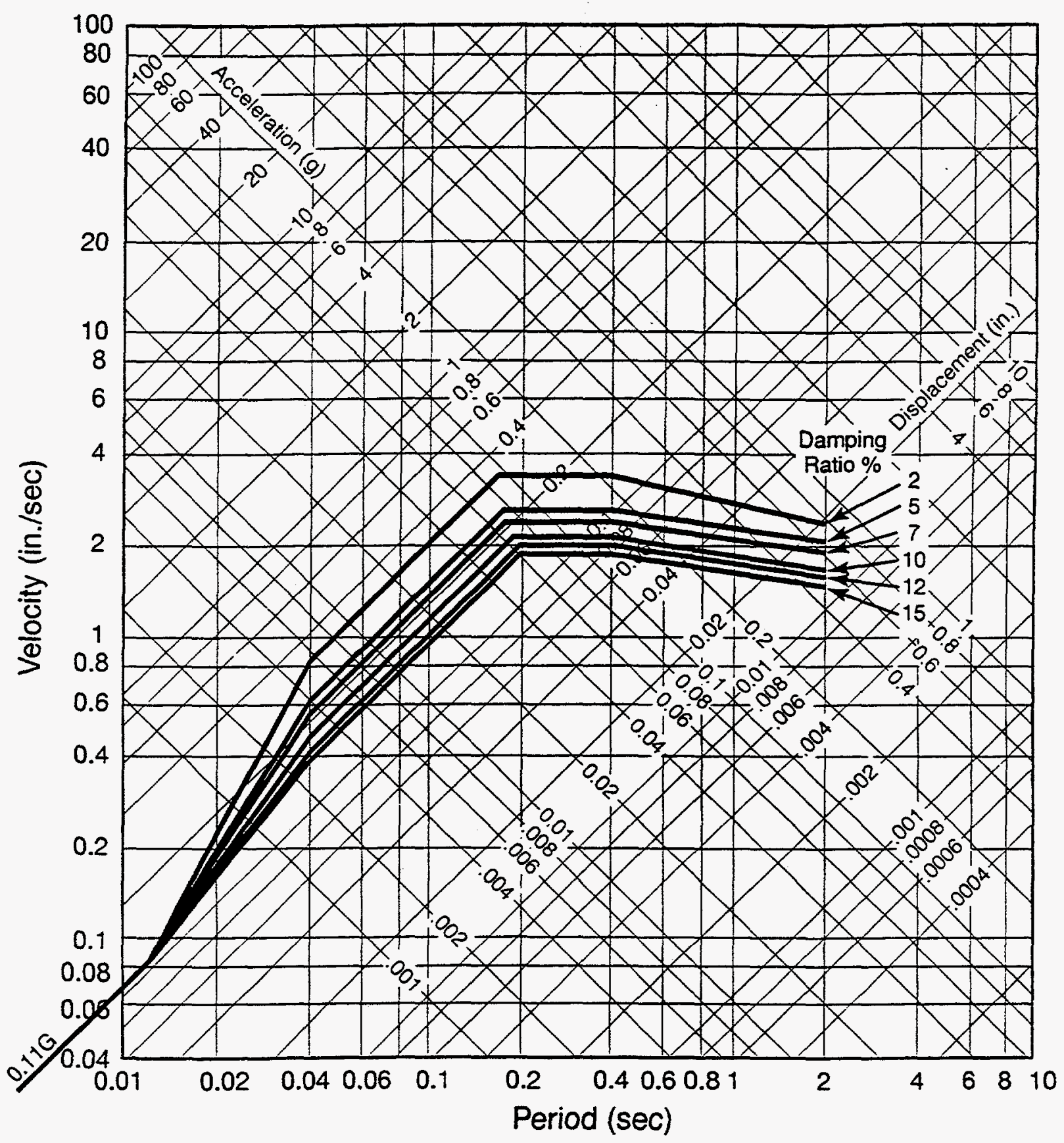

FIG. 2 I $Y-12$ SITE SPECIFIC EARTHQUAKE DESIGN RESPONSE SPECTRA FOR HORIZONTAL WEATHERED ROCK (SHALE) MOTION 1000 - YEAR RETURN PERIOD

PC-2 FACILITIES SUPPORTED ON WEATHERED ROCK (SHALE) 


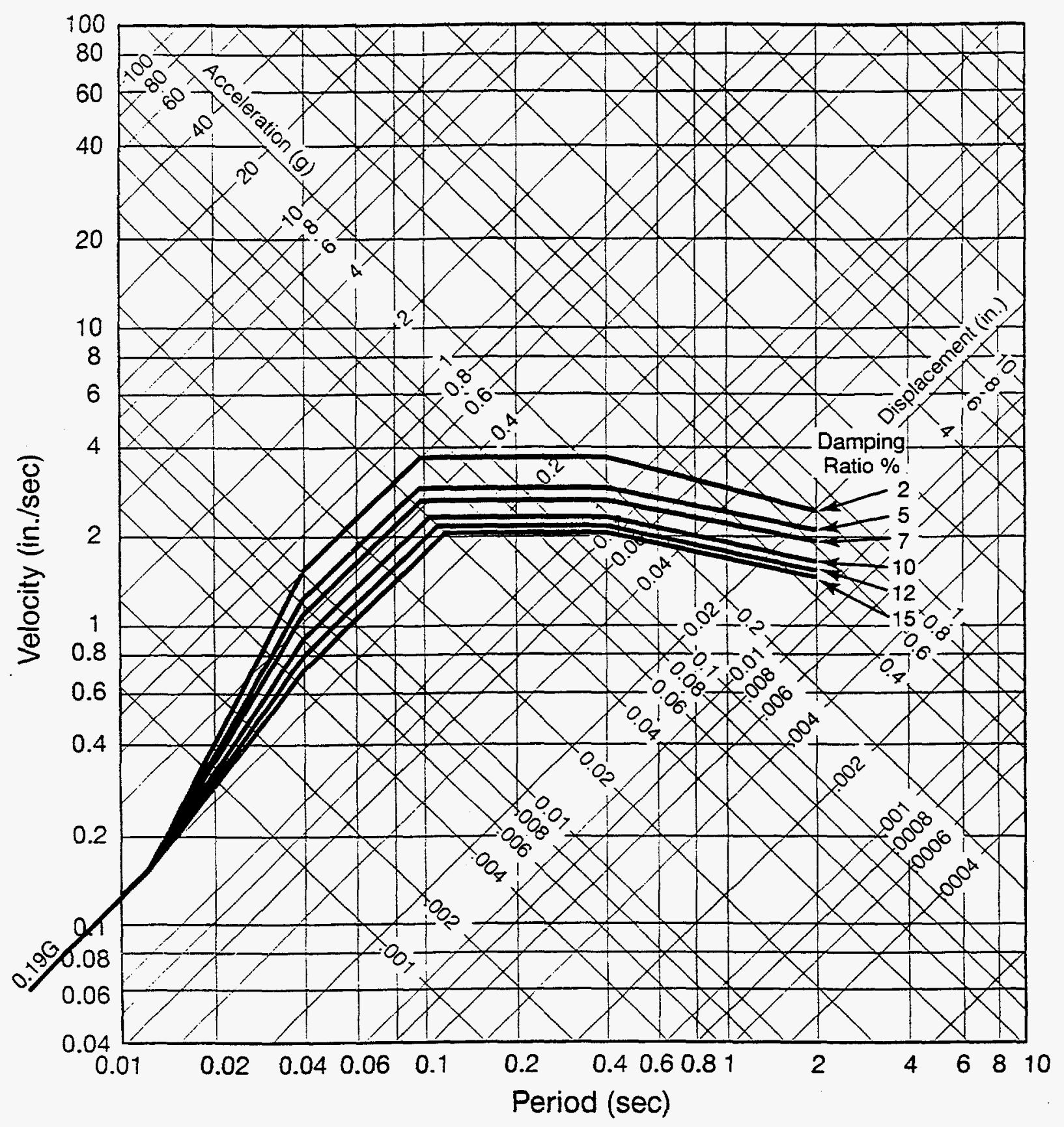

FIG. 22 Y-12 SITE SPECIFIC EARTHQUAKE DESIGN RESPONSE SPECTRA FOR HORIZONTAL SOIL MOTION SOIL OVERBURDEN $\leq 10 \mathrm{ft}$. 1000 - YEAR RETURN PERIOD

PC-2 FACILITIES SUPPORTED ON SOIL OVERBURDEN $\leq$ 1OFT. 


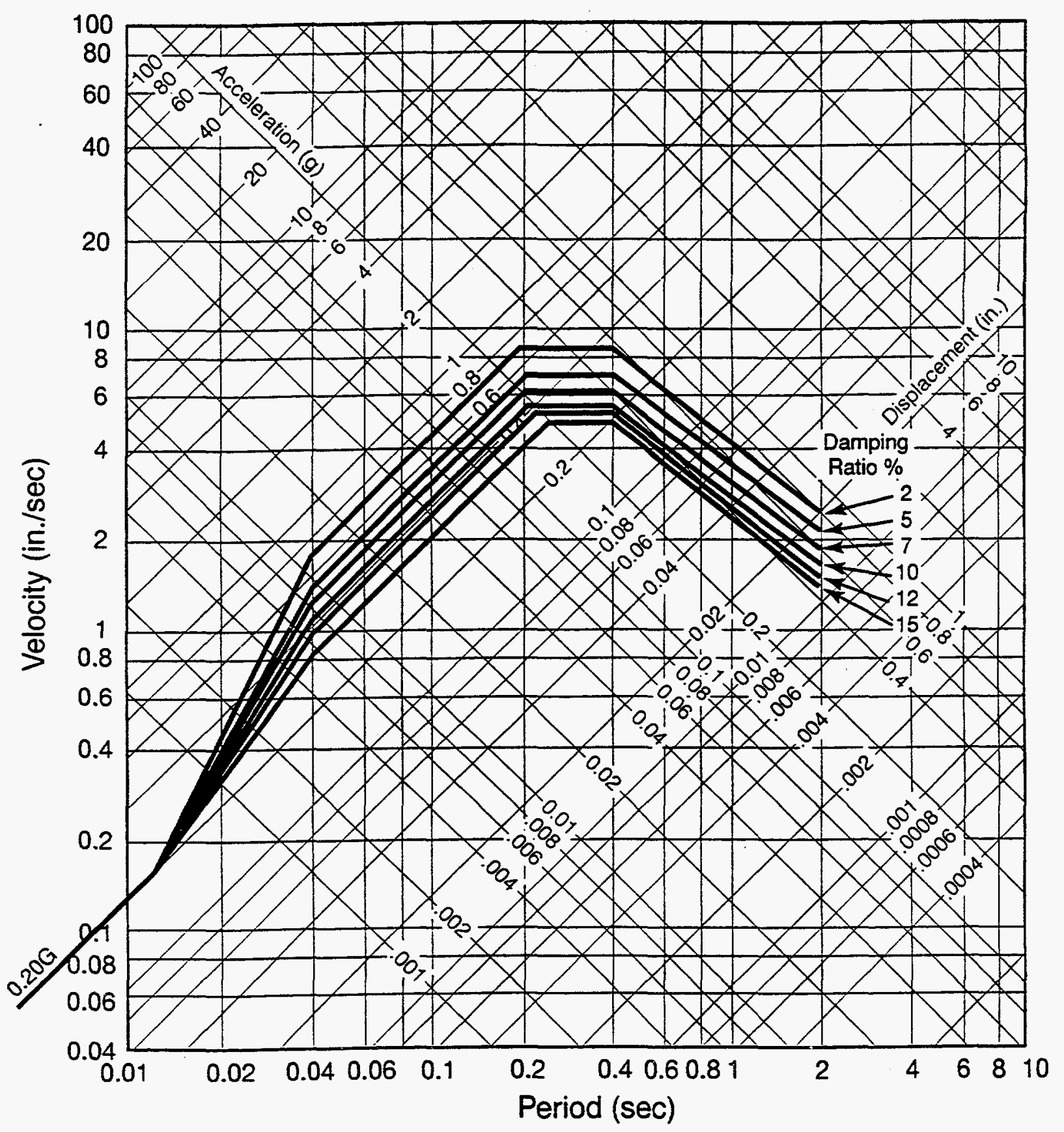

FIG. $23 \quad Y-12$ SITE SPECIFIC EARTHQUAKE DESIGN RESPONSE SPECTRA FOR HORIZONTAL SOIL MOTION SOIL OVERBURDEN $>10 \mathrm{ft}$. 1000 - YEAR RETURN PERIOD

PC-2 FACILITIES SUPPORTED ON SOIL OVERBURDEN > 1OFT. 


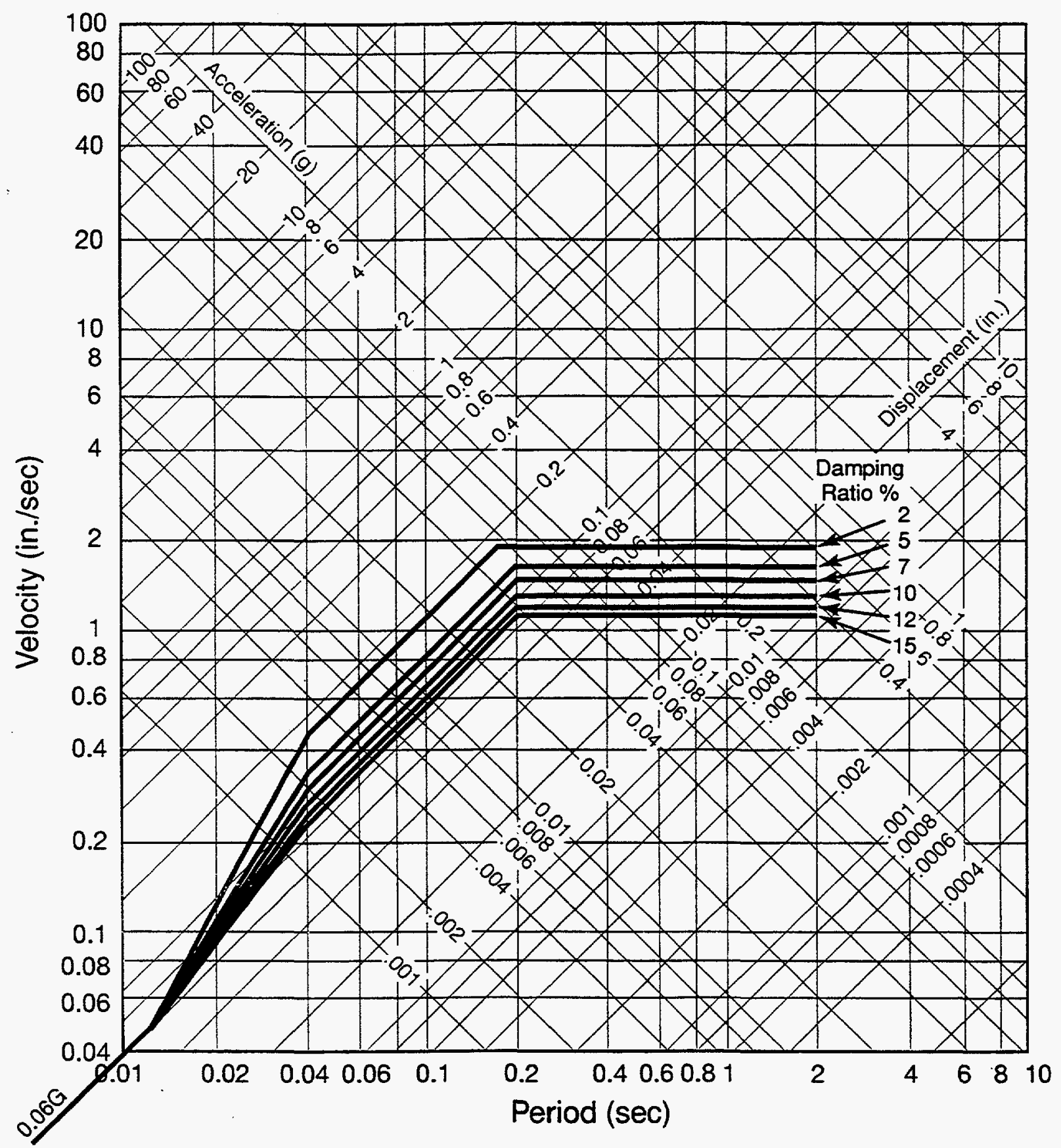

FIG. 24 Y-12 SITE SPECIFIC EARTHQUAKE DESIGN RESPONSE SPECTRA FOR HORIZONTAL ROCK MOTION 500 - YEAR RETURN PERIOD 


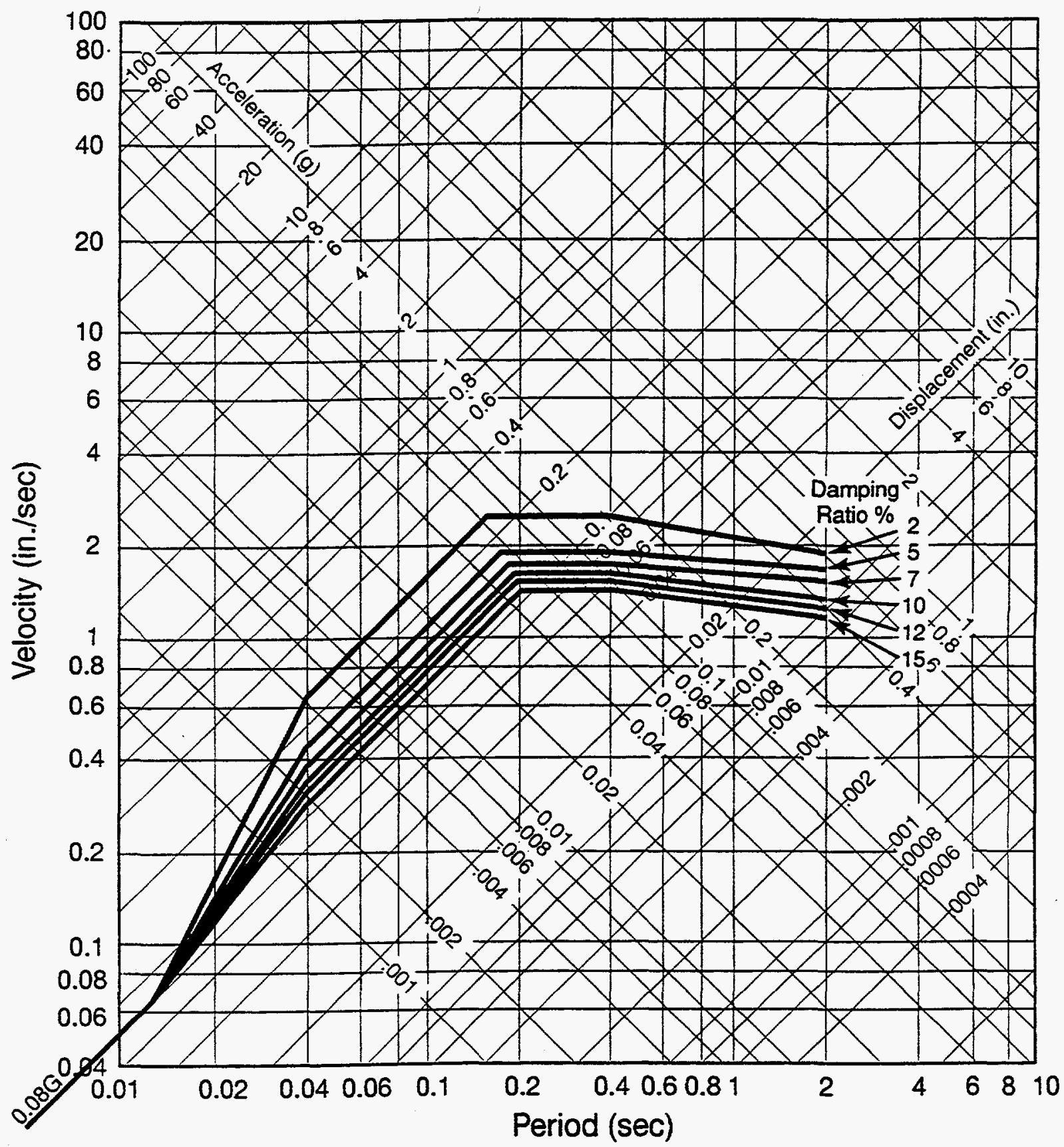

FIG. $25 \quad Y-12$ SITE SPECIFIC EARTHQUAKE DESIGN RESPONSE SPECTRA FOR HORIZONTAL WEATHERED ROCK (SHALE) MOTION 500 - YEAR RETURN PERIOD

\section{PC-1 FACILITIES SUPPORTED ON WEATHERED ROCK (SHALE)}




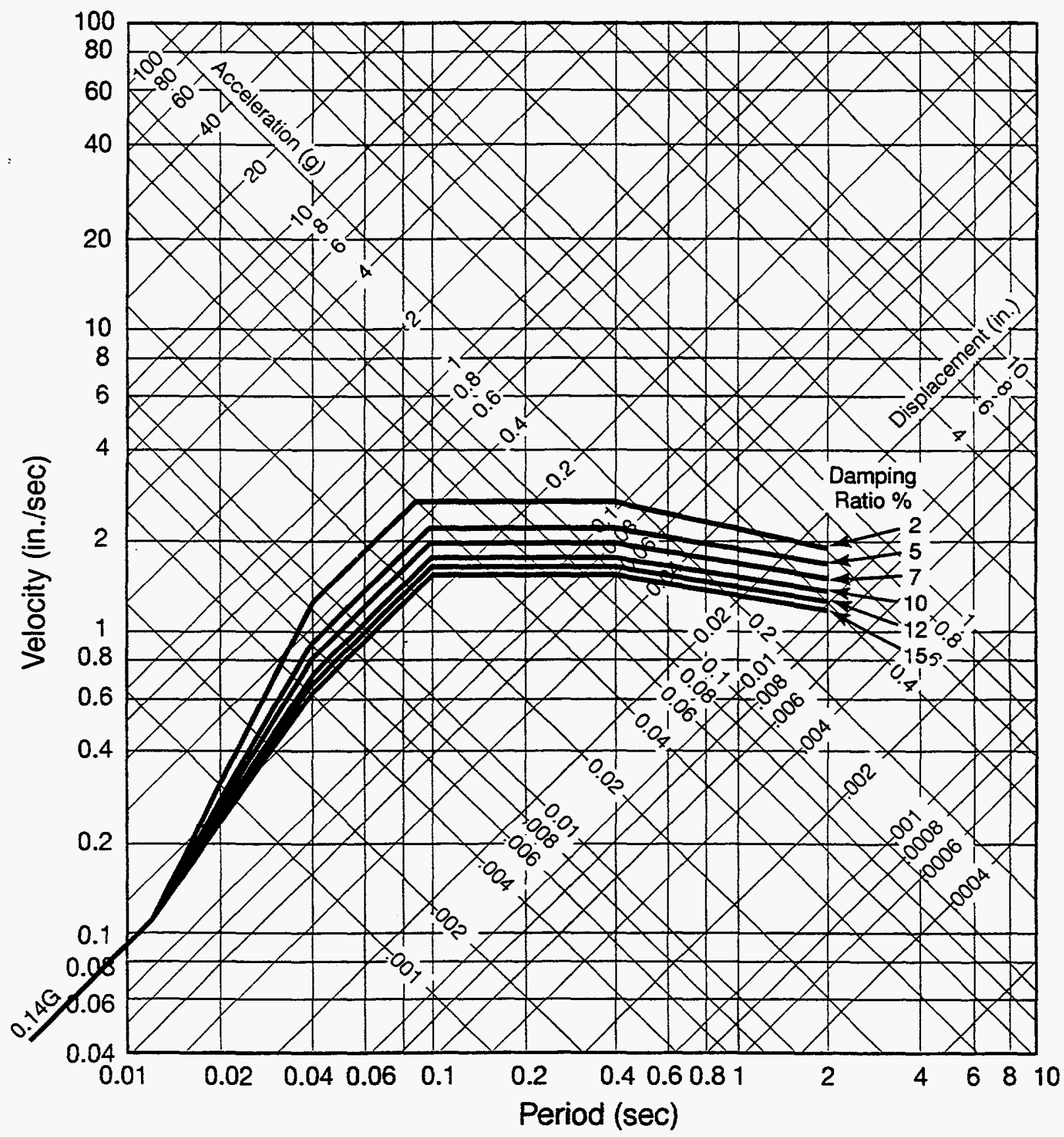

FIG. $26 \quad$ Y-12 SITE SPECIFIC EARTHQUAKE DESIGN RESPONSE SPECTRA FOR HORIZONTAL SOIL MOTION SOIL OVERBURDEN $\leq 10 \mathrm{ft}$. 500 - YEAR RETURN PERIOD

PC-1 FACILITIES SUPPORTED ON SOIL OVERBURDEN $\leq$ 1OFT. 


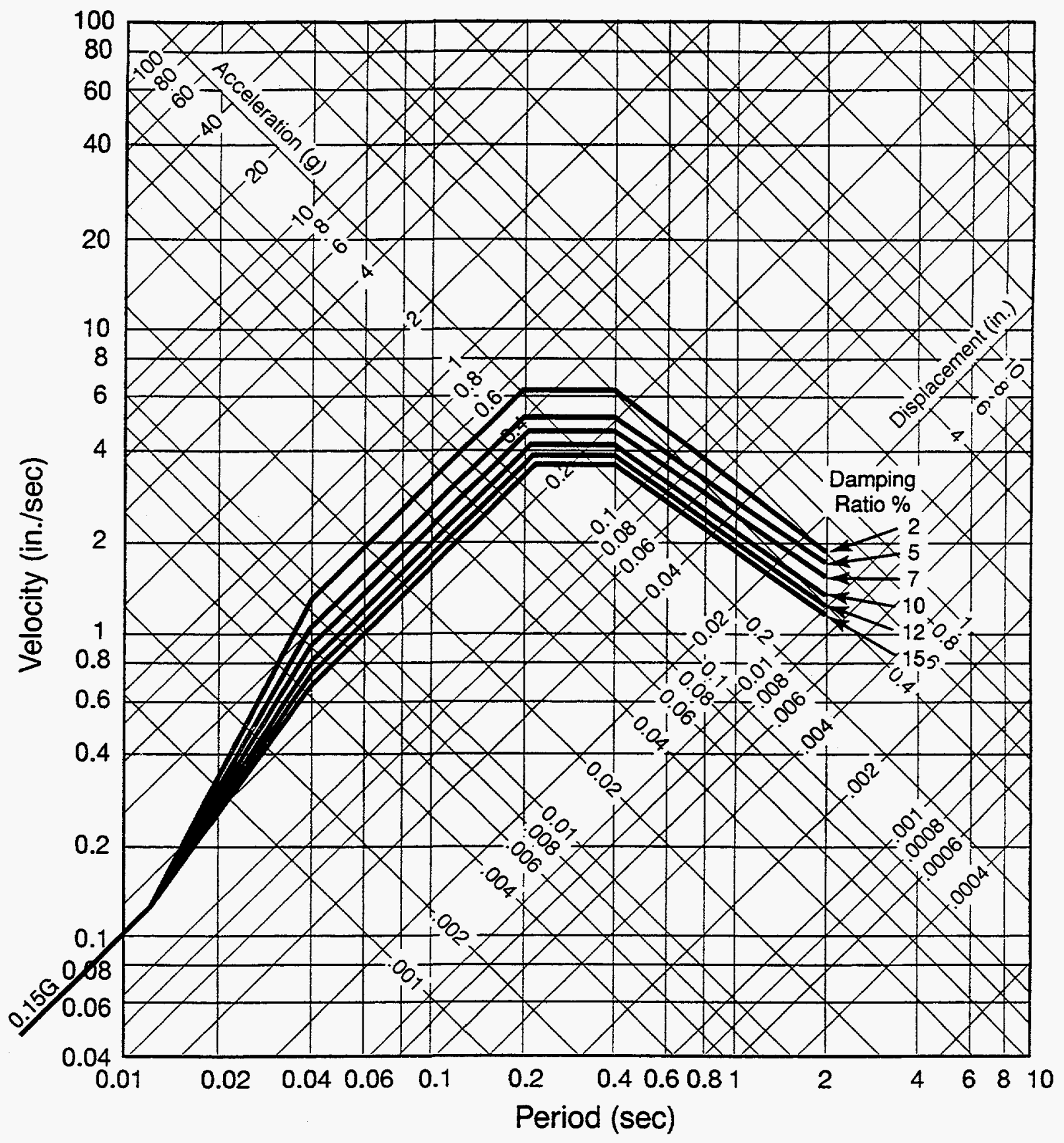

FIG. $27 \quad Y-12$ SITE SPECIFIC EARTHQUAKE DESIGN RESPONSE SPECTRA FOR HORIZONTAL SOIL MOTION SOIL OVERBURDEN > $10 \mathrm{ft}$. 500 - YEAR RETURN PERIOD

\section{PC-1 FACILITIES SUPPORTED ON SOIL OVERBURDEN > IOFT.}


Liquefaction, or the loss of shear strength due to an increase in pore water pressure, is a phenomenon associated with saturated cohesionless soils. The increase in pore pressure is a result of the densification of loose soil, which may occur due to ground shaking. The increase in the pore pressure results in a decrease in the intergranular or effective stress, with a corresponding decrease in strength and stiffness. This densification can be a problem with coarse-grained cohesionless soils such as sands.

The cohesion and plasticity exhibited by fine-grained soils (silts and clays) restrict the tendency for volume decrease and pore pressure increase during ground shaking. Although recent research suggests that fine-grained soils may liquefy, liquefaction generally occurs only if the fines are totally non-plastic. Non-plastic fines occur in materials that are produced by the crushing of rock, such as, rock flour or man-made materials such as mine tailings. Even low plasticity silts formed by the weathering of rock have sufficient plasticity to restrict the densification upon shaking. Thus, it is the presence of plastic fines that prohibit the rearrangement of particles leading to densification and liquefaction.

Geotechnical data from the investigation of the Y-12 site (Ref. 18 through 28 ) indicate that the subsurface materials are classified as silts and clays. This means that the overburden soil is primarily fine-grained. All samples for which Atterberg limits were determined were found to have plastic limits. Based on the reported plasticity, these materials should not be susceptible to liquefaction.

\subsection{LIQUEFACTION ANALYSIS OF SUTS AND CLAYS}

The visual examination of all SPT samples, and the results of the Atterberg limits and natural water content determinations (Ref. 18, 20,24, 27, and 28) suggest that the Y-12 foundation soils consist of silts and clays with substantial percentages of fine-grained materials.

To further demonstrate that the Y-12 SSCs foundation materials are not susceptible to liquefaction, a detailed evaluation of the potential for liquefaction has been performed. The evaluation is based upon methodologies reported by Seed, et al., for clayey soils and cohesionless soils with varying percentages of fines (Ref. 11 and 12). The liquefaction analysis is performed using the geotechnical information available in Ref. 18, 20, 24, 27, and 28.

1. According to Ref. 11, clayey soils may be vulnerable to liquefaction as a result of earth shaking if they have the following characteristics:

a) Percent finer than $.005 \mathrm{~mm}<15 \%$

b) Liquid Limit $<35$

c) Water Content $>.9 \mathrm{X}$ Liquid Limit

The data on liquid limit, plasticity index, natural water content and grain size distribution reported in Ref. 18, 20, 24, 27, and 28 are presented in Table 5. The liquid limit and natural water content values are plotted on Fig. 28. It is clear from this plot that out of a total 94 samples, 93 are outside the zone of potential liquefaction and only one sample is on the border line. 
Fig. 29 shows the plot of the liquid limit and plasticity index on the Plasticity Chart. The plot indicates that a majority of soils are silty clays (CL). Also a significant number of silty soils (ML) have liquid limit higher than 35 and therefore, they are not liquefiable. The remaining few samples have liquid limit below 35 but their natural water contents are less than 90 percent of the corresponding liquid limits (see Fig. 28). Consequently, the silty soils (ML) are not susceptible to liquefaction.

Grain size distribution data are available in Ref. 28 and this data is included in Table 5. Test results indicate that the percentages of particles finer than $.005 \mathrm{~mm}$ are 34, 26, 24, 21 and 12 . Thus, there is only one sample that contains fines below 15 percent but for all practical purposes 12 percent is fairly close to the required 15 percent fines. Furthermore, it can be argued that this ML sample has significantly lower water content (12.8\%) than the 0.9 times liquid limit $(26.1 \%)$ and therefore, this soil should be qualified as a nonliquefiable soil.

\subsection{LIQUEFACTION ANALYSIS OF SANDY SOIS}

Seed et. al., have developed the boundaries of liquefaction zones for sandy soils containing varying percentages of fines (Ref. 11 and 12). These boundaries are established on the basis of the $\mathrm{N}$ values and the CSRs of the soil deposit.

Review of the available geotechnical information (Ref. 17 through 28 ) indicates that sands or silty sands are generally not encountered at the Y-12 site. Out of several borings, only one boring V-1 (Ref. 27) drilled in the vicinity of Building 9206 revealed the presence of a small, thin and confined pocket of silty sand at 13.5 to $15 \mathrm{ft}$. depth just above the refusal. This pocket of silty sand will be evaluated for liquefaction potential.

Additionally, a cohesionless soil (sand or silty sand) must be fully saturated in order to liquefy. Geotechnical and hydrological investigations at the site indicate that groundwater is generally encountered in the weathered rock or fresh rock below the soil overburden. In general, the soils at the site are not completely saturated and consequently should not liquefy. Although there are indications of presence of groundwater (most probably perched water) within soil overburden at the north vault of building 9212 and in the vicinity areas of buildings $9215,9204-2,9204-2 \mathrm{E}$ and $9720-5$ but sandy soils are not present at these locations. Therefore, the overburden soils at the site are in general not susceptible to liquefaction.

\subsubsection{COMPUTER PROGRAM "SLIQ"}

Computer Program "SLIQ" (Ref. 10) evaluates liquefaction potential for sandy soils using the methodology presented by Seed, et. al., in Ref. 11 and 12. This methodology is based upon the relationship between the $\mathrm{N}$ values and the CSRs of the soil layer. The $\mathrm{N}$ values are corrected for overburden, hammer energy ratio, and very short drill rod lengths. Overburden correction is applied using the method recommended by Liao and Whitman (Ref. 33). For hammer energy ratio, a correction factor of 1.0 is generally used assuming a standard energy ratio of 60 percent of the free fall energy of the hammer (Ref. 12). 


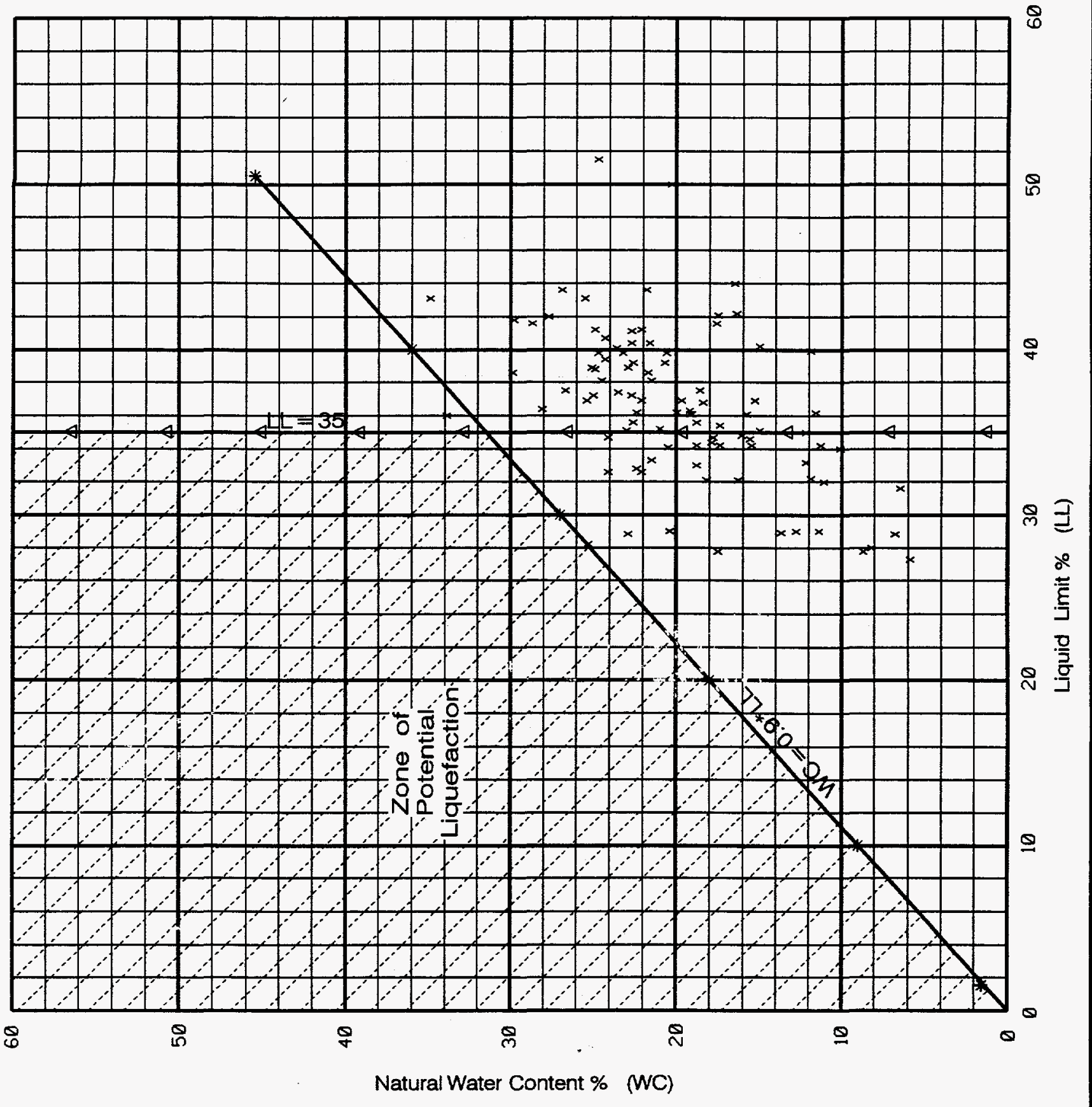

FIG. 28 LIQUID LIMIT VS NATURAL WATER CONTENT 


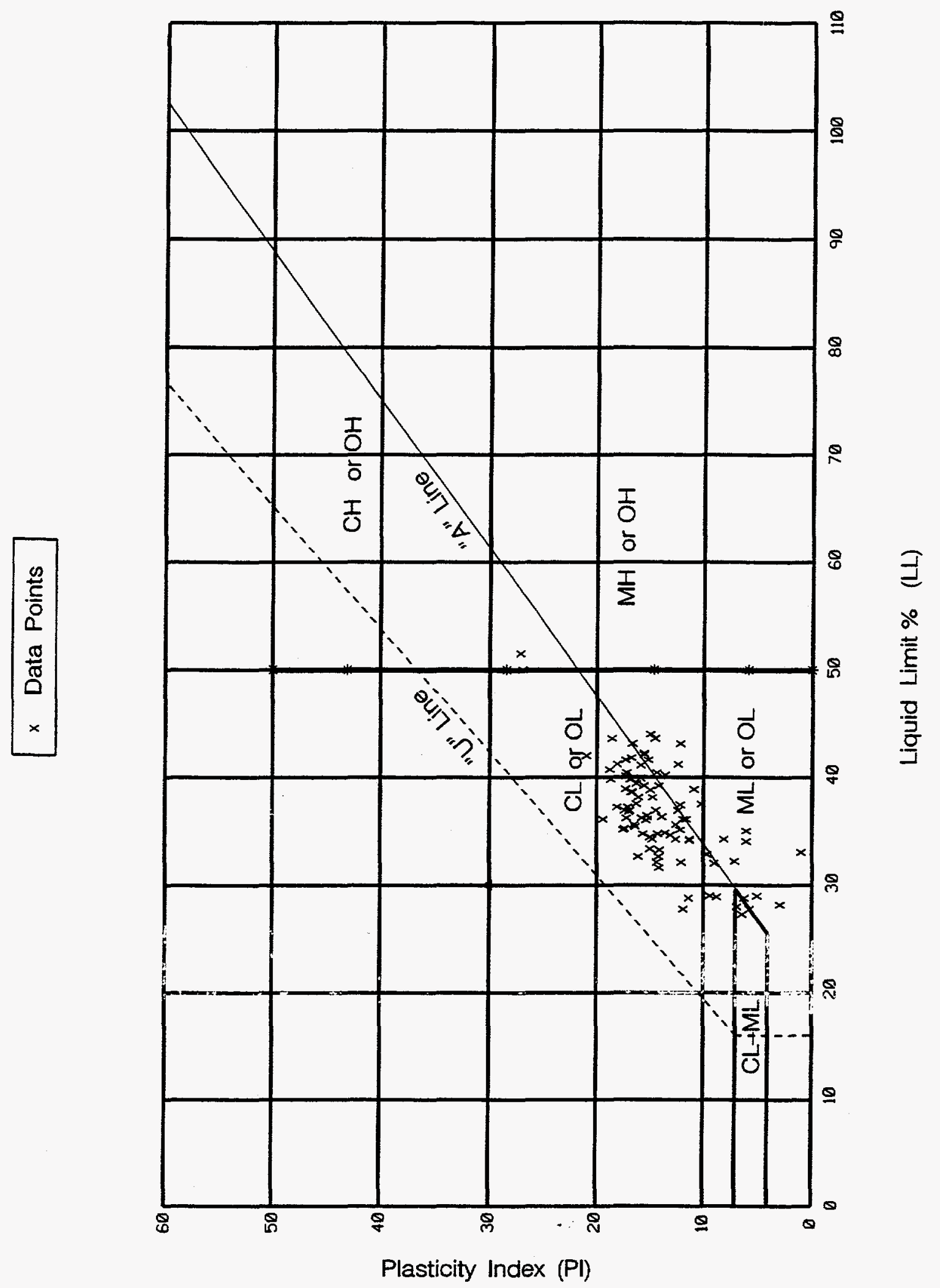

FIG. 29 SOIL CLASSIFICATION ASTM D 2487

(Plasticity Chart) 
The corrected $\mathrm{N}$ values are designated by the symbol $\left(\mathrm{N}_{1}\right)_{60}$. The CSR is calculated from the following equation:

$$
\frac{\tau_{\text {ave }}}{\sigma^{1}} \approx 0.65\left(\frac{a_{\max }}{g}\right)\left(\frac{\sigma}{\sigma^{1}}\right)\left(r_{d}\right)
$$

$\tau_{\text {ave }}=$ Average Cyclic Shear Stress

$\sigma \quad=$ Vertical total Stress

$\sigma^{1} \quad=$ Vertical Effective Stress

$a_{\max }=$ Maximum ground Acceleration

$\mathrm{g} \quad=$ Acceleration Due to Gravity

$r_{d} \quad=$ Stress reduction factor

Stress reduction factors are taken from Ref. (12).

Liquefaction analysis was performed using SLIQ program. Soil profiles from three borings V-1, V-3 and V-6 were used. The main objective of the SLIQ analysis was to determine if the thin pocket of silty sand is vulnerable to liquefaction. The input for this analysis is based upon the following assumptions which are very conservative.

(1) Groundwater was not encountered in borings V-1, V-3 and V-6. In this analysis water level was assumed to be at $5 \mathrm{ft}$. below the ground for borings V-1 and V-3, and at the ground surface and $2 \mathrm{ft}$. below the ground surface for boring V- 6 which makes the analysis extremely conservative.

(2) Grain size distribution curves indicate the percentage of -200 fines varying from 38 to 100 but the analysis was performed using 5, 10 and 35 percent fines for borings $V-1, V-3$ and V- 6 .

(3) A 2000-year peak ground acceleration equal to $.12 \mathrm{~g}$ for fresh rock and $.30 \mathrm{~g}$ for soil were used in the analysis.

\subsubsection{SLIQ ANALYSIS RESULTS}

The SLIQ output is presented in Appendix B of this report. The results of the analysis indicate that the materials encountered in the three borings should not liquefy. The factors of safety for liquefaction of the silty sand encountered in boring V-1 using PGA $=.12 \mathrm{~g}$ and water table at $5 \mathrm{ft}$. below ground, are 1.34, 1.61 and 2.25 for 5,10 and 35 percent fines contents, respectively. 
For the same water level but a $P G A=.30 \mathrm{~g}$, the safety factor for silty sand containing $35 \%$ of fines is .9 indicating initial liquefaction. Using $P G A=.30 \mathrm{~g}$ and waterlevel at $10 \mathrm{ft}$. depth, the safety factor is 1.04 indicating no liquefaction. However, assumptions for groundwater at $5 \mathrm{ft}$. below ground, and using $.30 \mathrm{~g}$ are extremely conservative and unrealistic. The application of .12 $\mathrm{g}$ is more realistic since the thin layer of silty sand is very close to the refusal or top of rock. The assumption of groundwater at $10 \mathrm{ft}$. is still conservative. Therefore, the silty sand pocket is not vulnerable to liquefaction.

\subsection{CONCLUSION OF LIQUEFACTION ANALYSIS}

The results of the foregoing liquefaction analysis of clays, silts and silty sand lead to the conclusion that the soils encountered at the Y-12 site are generally not vulnerable to liquefaction. This conclusion is applicable to $\mathrm{PC}-3,-2$, and -1 facilities located at $\mathrm{Y}-12$ plant. The evaluation was performed for the PC-3 earthquake level and indicated no liquefaction concerns. Since the PC-2 and -1 earthquake levels are lower, the results are applicable. Therefore, the design basis earthquakes for the 500,1000, and 2000 years return periods should not cause any adverse effect on the structural integrity of the soil supported facilities at the Y-12 site. 


\subsection{REFERENCES}

1. DOE Order 5480.28, Natural Phenomena Hazard Mitigation, U. S. Department of Energy, January 1993

2. DOE-STD-1020-94, Natural Phenomena Hazards Design and Evaluation Criteria for Department Of Energy Facilities, U.S. Department of Energy, April 1994

3. DOE-STD-1021-93, Natural Phenomena Hazards Performance Categorization Guidelines for Structures, Systems, and Components, U.S. Department of Energy, December 1992

4. DOE-STD-1022-94, Natural Phenomena Hazards Site Characterization Criteria, U. S. Department of Energy, March 1994

5. DOE-STD-1023 (Draft), Natural Phenomena Hazards Assessment Criteria, U.S. Department of Energy, November 1994

6. DOE-STD-1024-92, Guidelines for Use of Probabilistic Seismic Hazard Curves at Department of Energy Sites, U.S. Department of Energy, December 1992

7. 'SHAKE 91' Computer Program modified by I. M. Idriss \& Joseph I. Sun, (originally by P. B. Schnabel, J. Lysmer, \& H. B. Seed). Department of Civil \& Environmental Engineering, University of California, Davis, California

8. Y/EN-4683, Seismic Hazard Evaluation for Department of Energy Oak Ridge Reservations, Oak Ridge, Tennessee, Prepared by Risk Engineering, Inc. for Martin Marietta Energy Systems, Inc., September 1992

9. Seed, H. B., et al., "Simplified Procedures for Evaluating Soil Liquefaction Potential, ASCE Journal of the Soil Mechanics and Foundations Division, Vol. 97, No. SM 9

10. 'SLIQ' Computer Program for Analysis of Liquefaction Potential of Sands and Silty Sands, C. M. Shahbaz, 1992

11. Seed, H. B., et al., "Evaluation of Liquefaction Potential Using Field Performance Data", ASCE Journal of Geotechnical Engineering, Vol. 109, No. 3, 1983

12. Seed, H. B., et al., "Influence of SPT Procedures in Soil Liquefaction Resistance Evaluations", ASCE Journal of Geotechnical Engineering, Vol. 111, No. 12, 1985

13. "Status Report on the Geology of the Oak Ridge Reservation", by Hatcher, R.D., et. al., ORNL/TM-12074, October 1992. 
14. Seismic Hazard Criteria for the Oak Ridge, Tennessee; Paducah, Kentucky; and Portsmouth, Ohio; U.S. Department of Energy Reservations," Prepared by MS Technology for Lockheed Martin, October, 1995, ES/CNPE-95/2.

15. Y-12 Plant, Report Y/TS-881/R1 "Updated Subsurface Data Base for Bear Creek Valley, Chestnut Ridge, and Parts of Bethel Valley on the U.S. Department of Energy Oak Ridge Reservation" Revision 1, Oct. 1993

16. Y-12 Plant, Report Y/SUB/94-EAQ10C/2/P1 "Groundwater Quality Report for the Upper East Fork Poplar Creek Hydrogeologic Regime" Feb. 1994, Geotechnical Investigation Reports

17. USR-III Run 47810 Bldg. Addn./9206/Y12, Geotek 1986

18. 9215 Bldg. Foundation/9215/Y12; U.S.A.C.O.E., 1956

19. Foundation Investigation and Analysis Bldg. 9998, U.S.A.C.O.E., 1954

20. Subsurface Investigation for 9215 Building Addition, Geotek 1978

21. Pile Driving Data Bldg. 9212, North Extension, Austin Company, 1951

22. Building 9212 Foundation Information Analysis \& Boring, U.S.A.C.O.E. 1943-44

23. Proposed Addition to Building 9212 North, Geotek, 1986

24. Proposed Vaults South of $9720-5$, Geotek, 1985

25. Foundation Investigations for Y-12 Plant Expansion, U.S.A.C.O.E., 1967

26. Proposed Vault Addition to 9212 Building, Geotek, 1984

27. Vibro Seismic Survey (PRA9206), EDGE, 1989

28. Cross Borehole Shear Wave Study, vicinity of Building 9206, Robert Selfridge, (Draft Report), 1989

29. NRC Standard Review Plan, Nuclear Regulatory Commission 1989

30. Seismic Assessment Report, Watts Bar Nuclear Plant, 1989, Bechtel North American Power Corporation

31. Nuclear Fuel Recovery and Recycling Center, PSAR Exxon Nuclear Co., Inc., Jan. 1976

32. UCB/EERC-88/15 "Dynamic Moduli and Damping Ratios for Cohesive Soils" by J. I. Sun, R. Golesorkhi and H. B. Seed, Aug. 1988 
33. Liao, S. S. C., et al., "Overburden Correction Factors for SPT in Sand", ASCE Journal of Geotechnical Engineering, Vol. 112, No. 3, 1985

34. Natural Phenomena Hazards Modeling Project: Seismic Hazard Models for D.O.E. Sites, D. W. Coat \& R. C. Murray, Nov. 1984, Lawrence Livermore National Laboratory 
11.0 APPENDIX
A1 Spectral Acceleration Plots
A2 Amplification Ratio Plots
B Soil Liquefaction 'SLIQ' Computer Output
C Drawings 
-

-

- 
A1 Spectral Acceleration Plots 
-

-

○ 

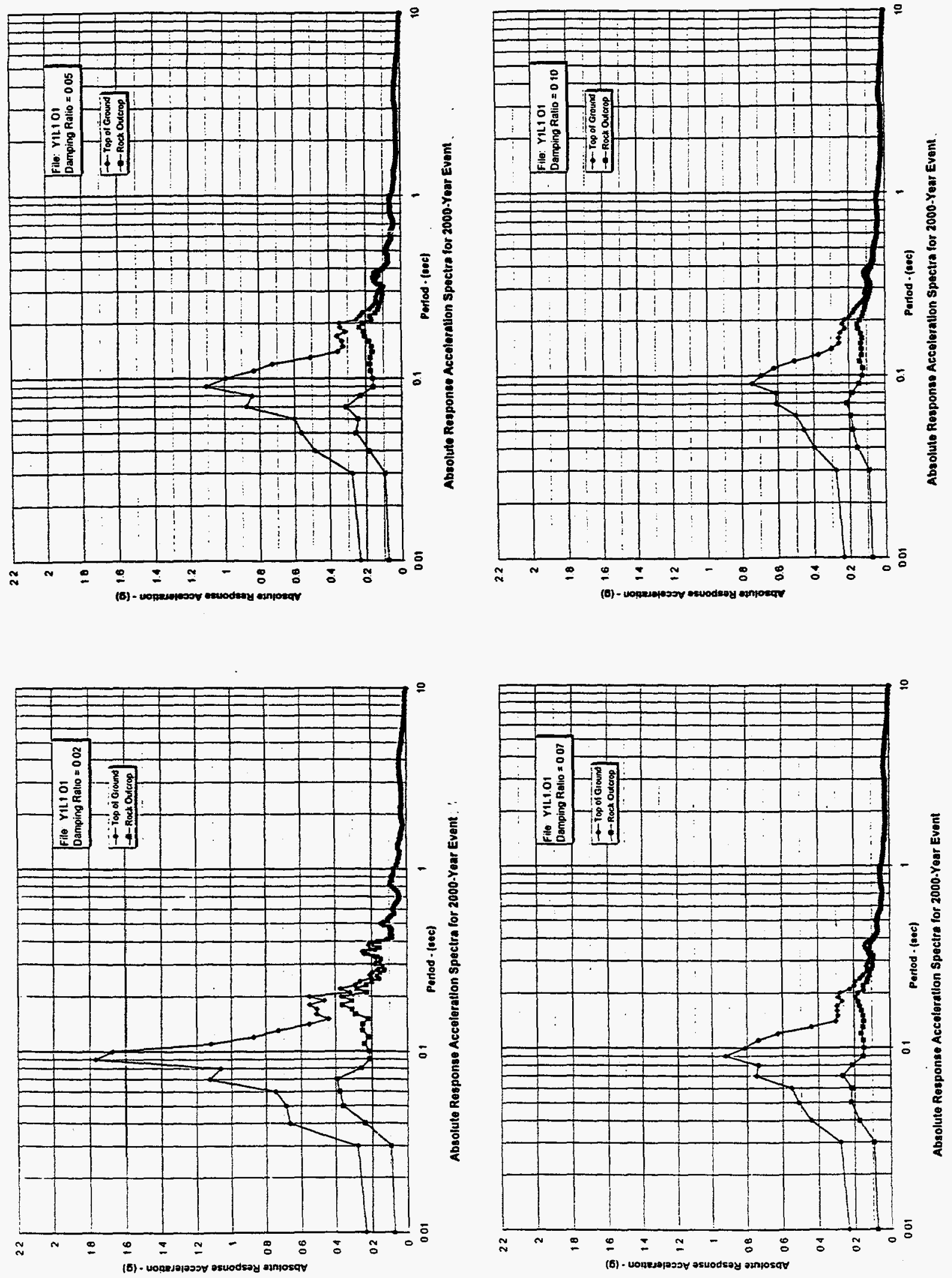

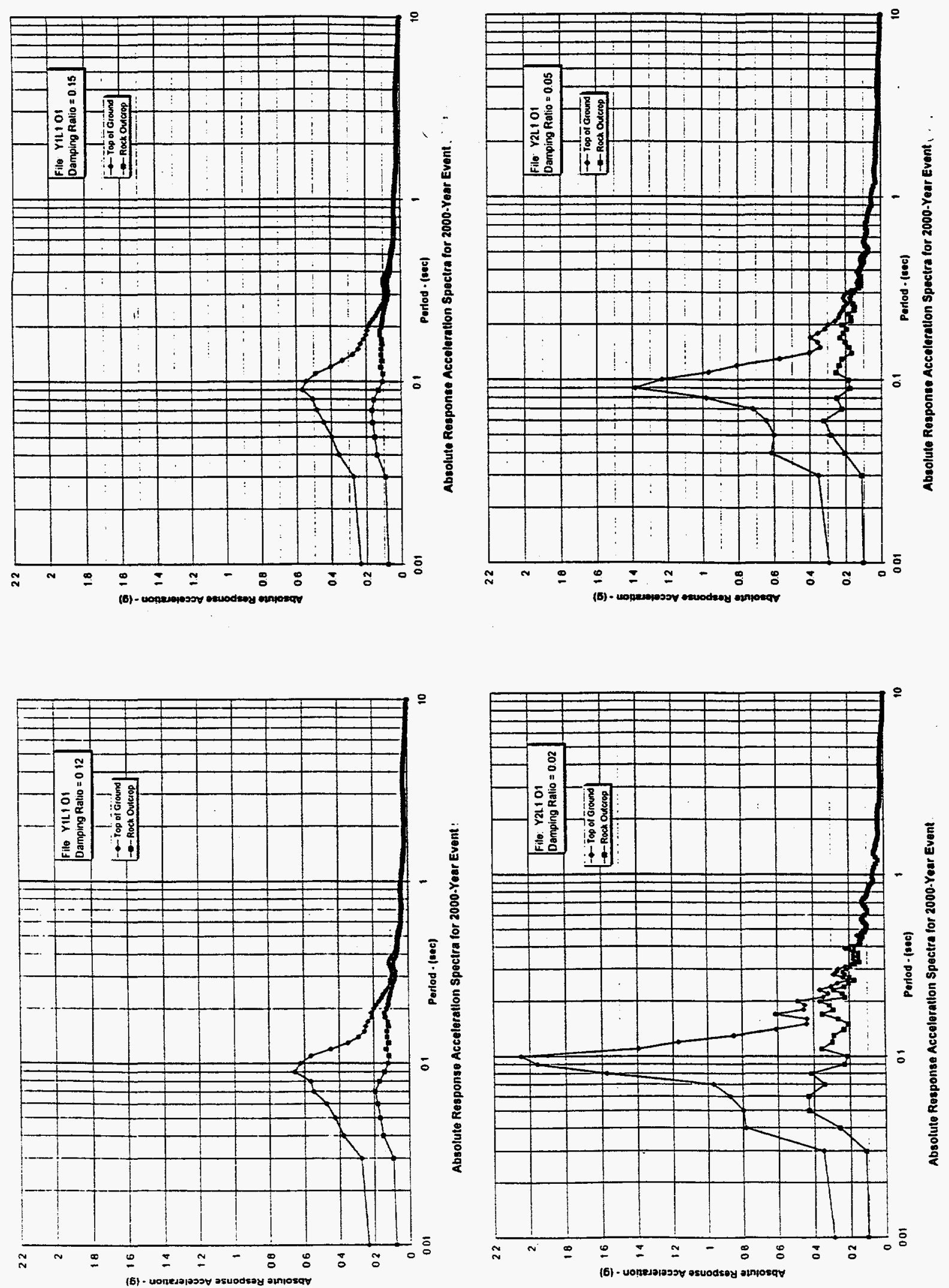

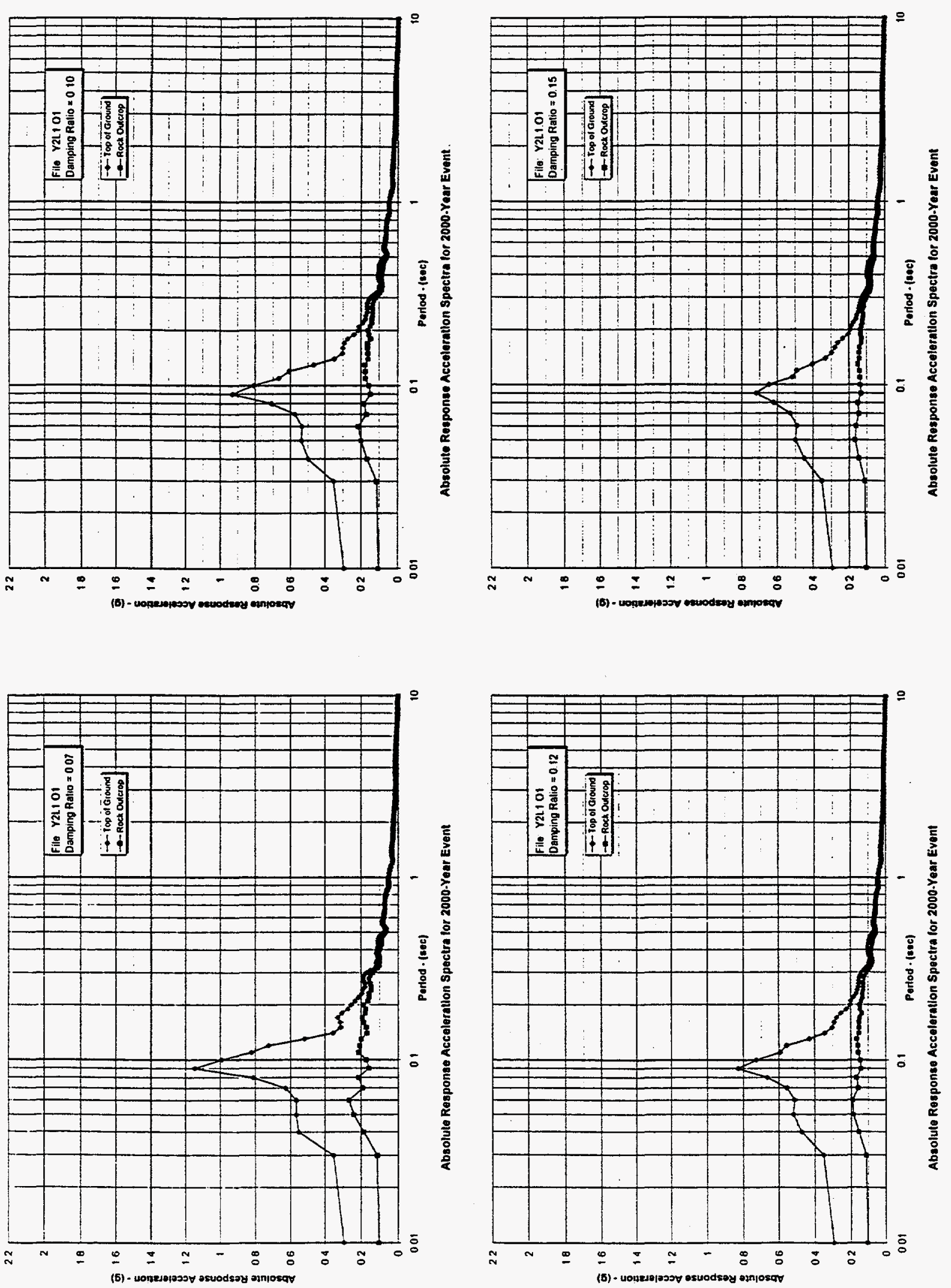

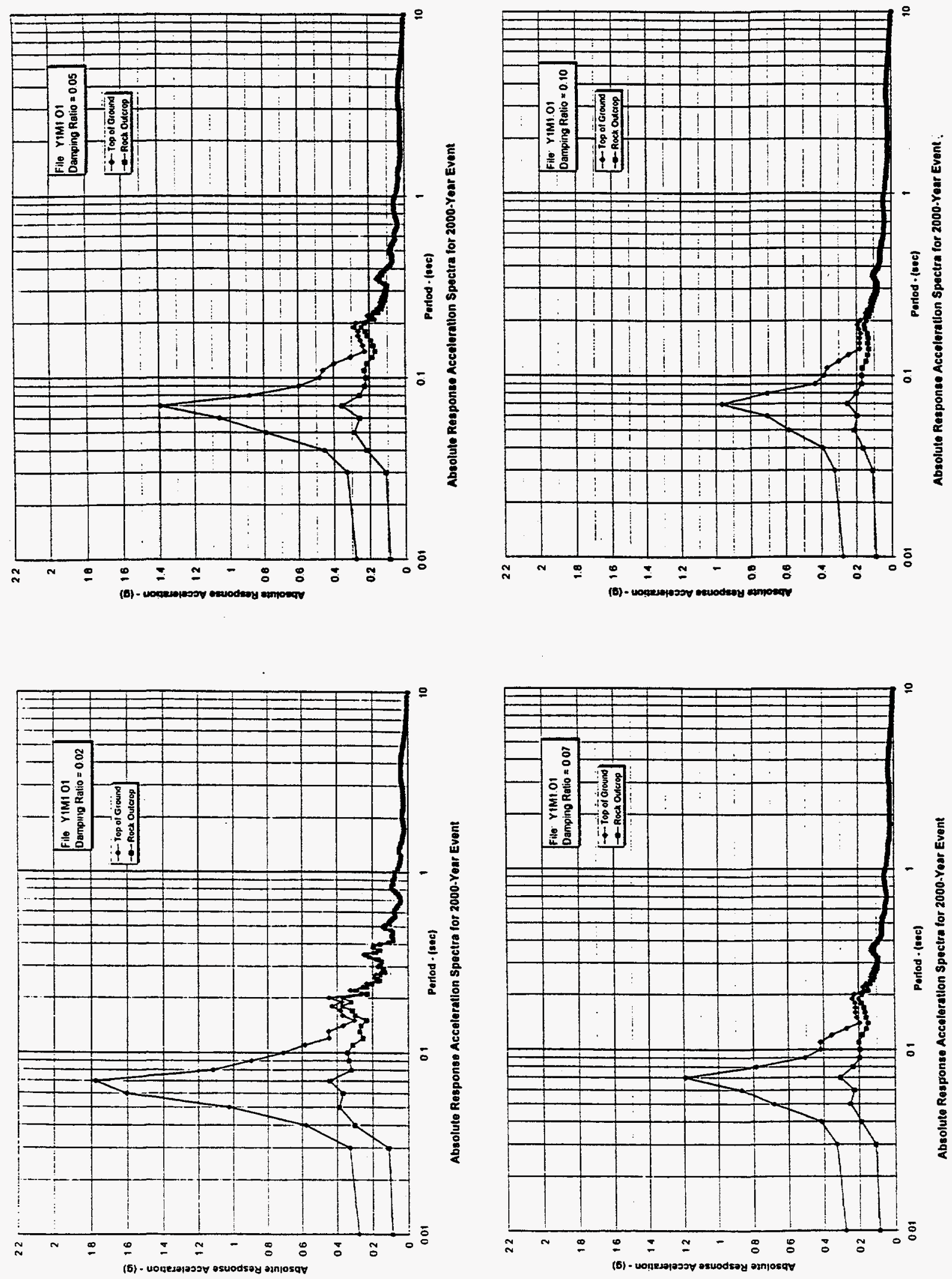

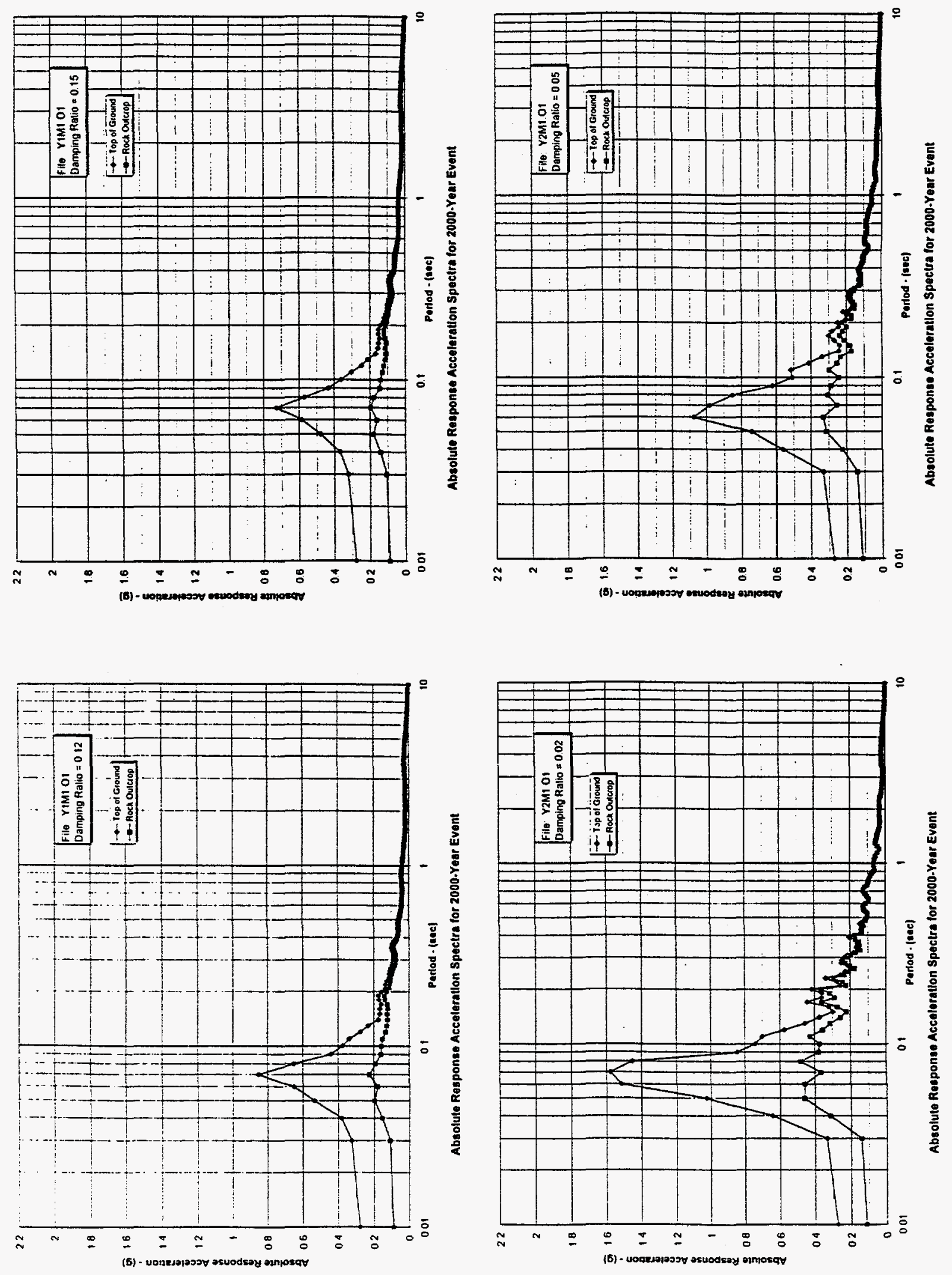

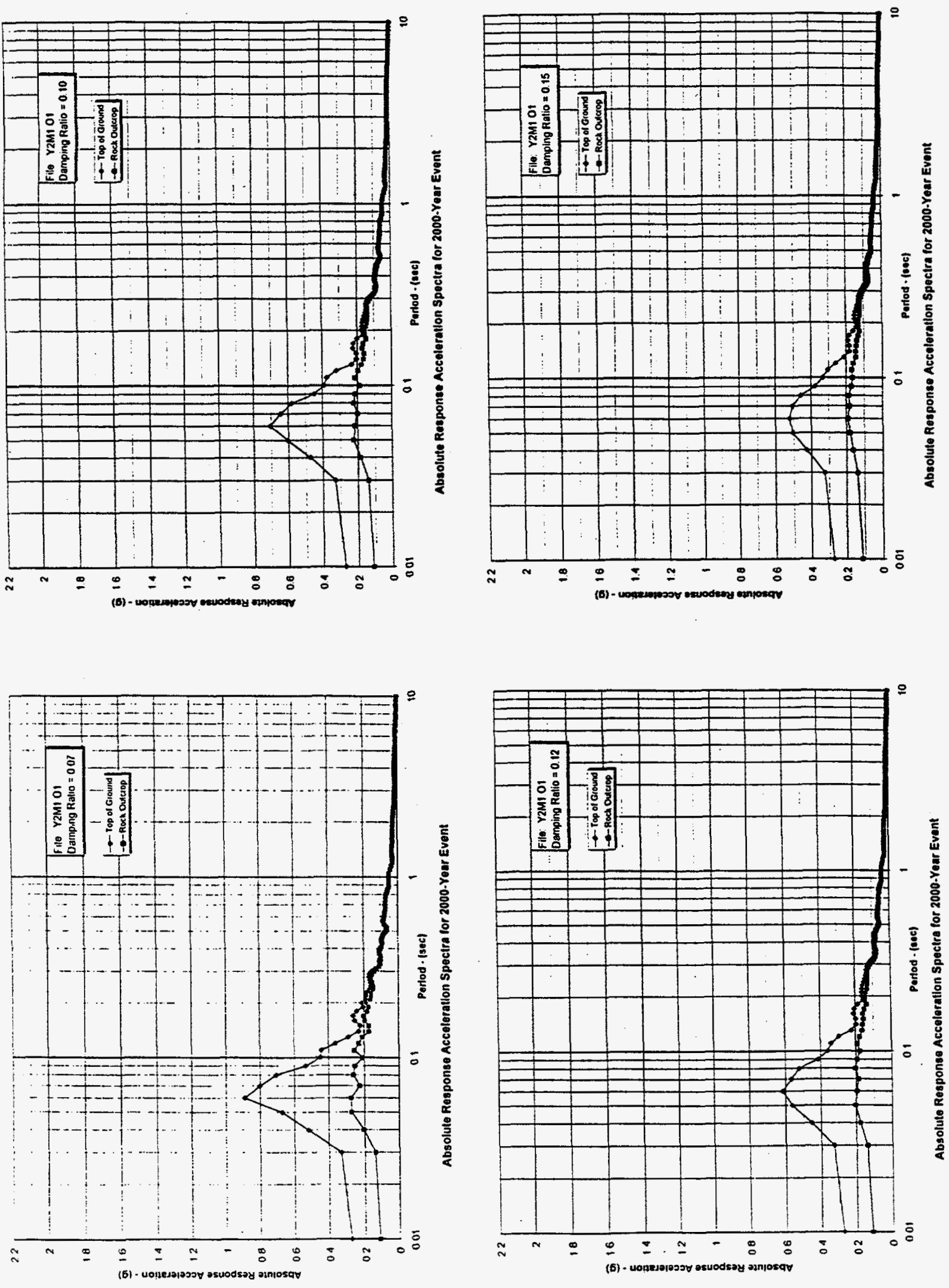

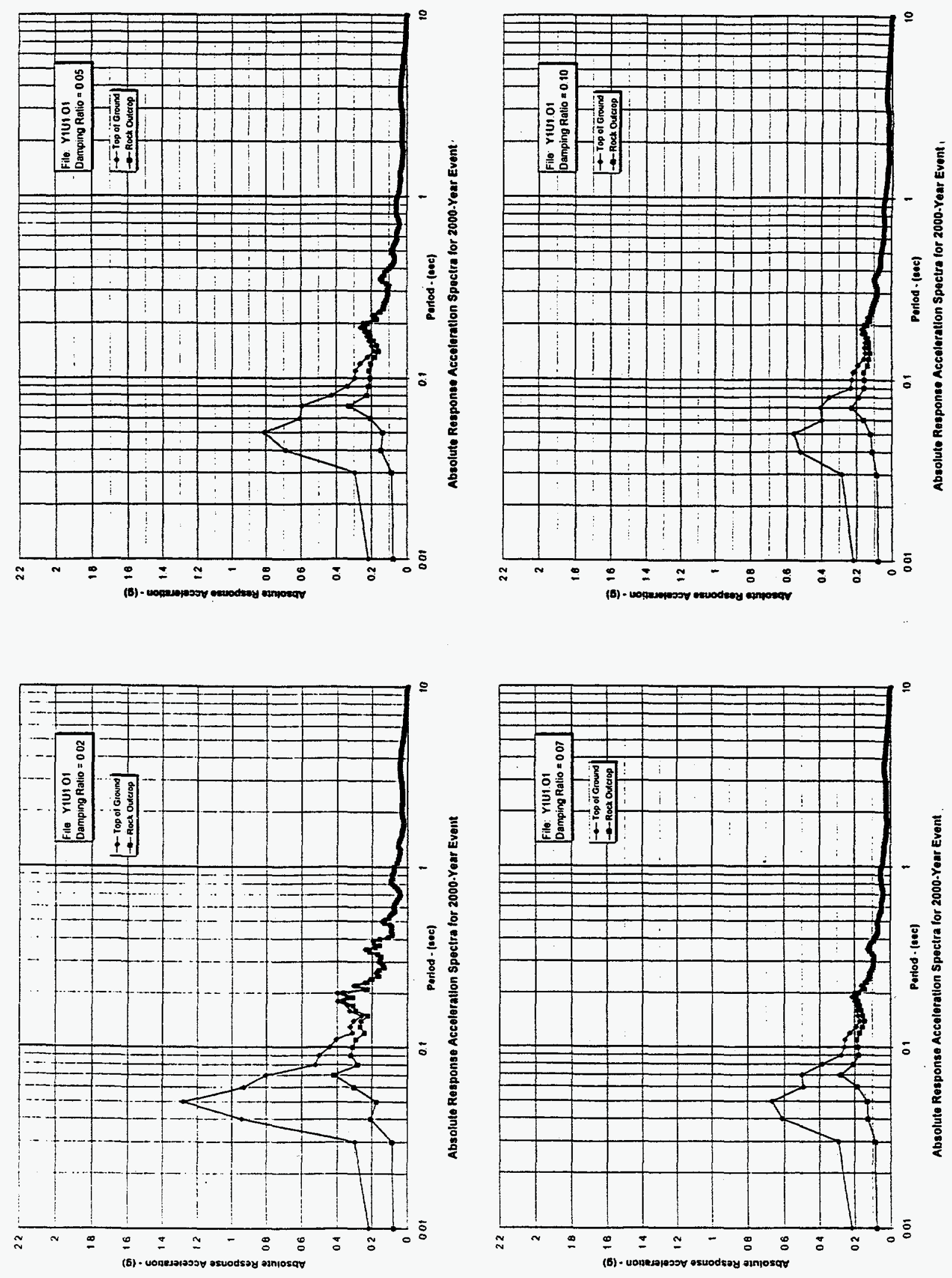

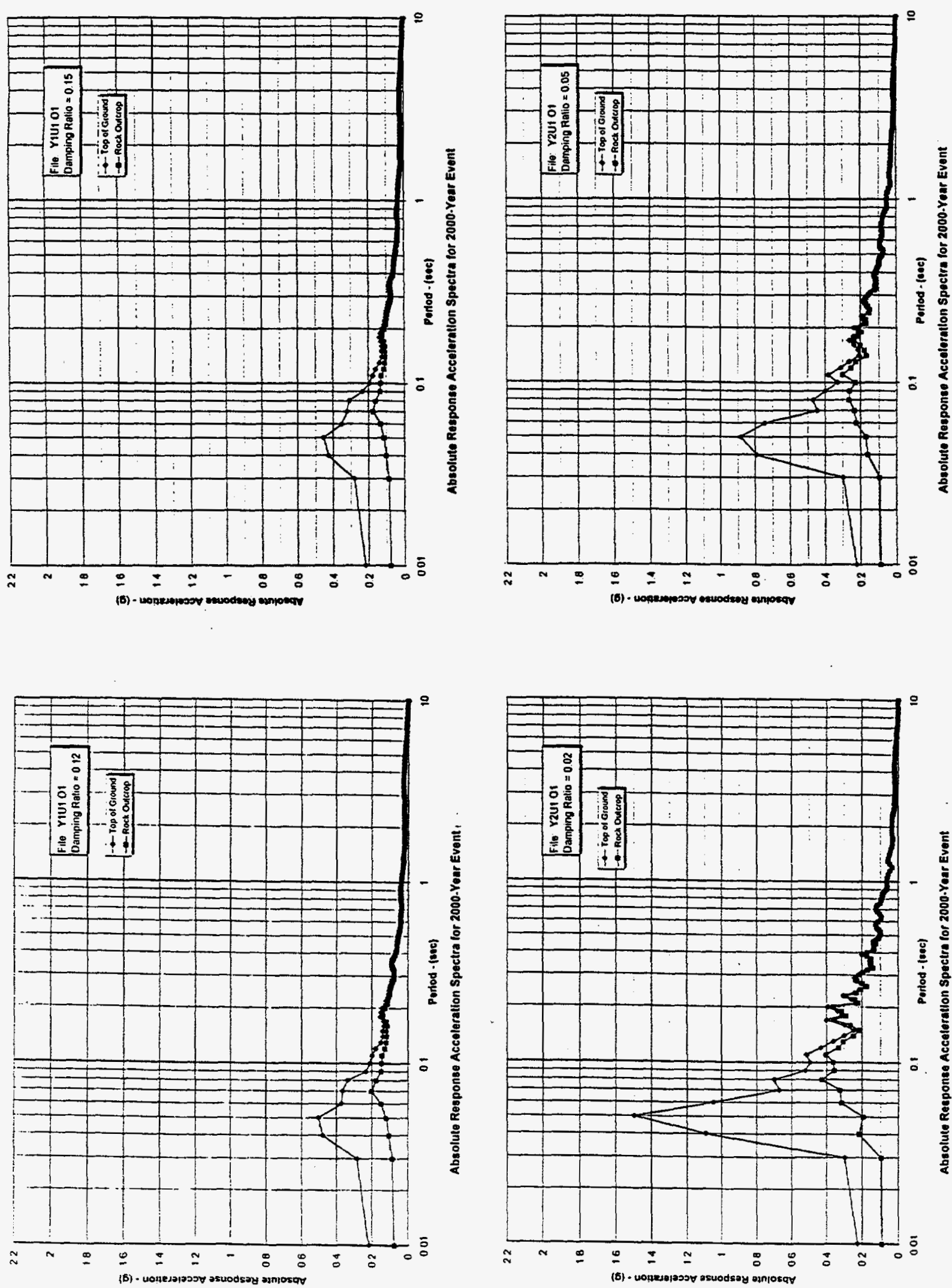

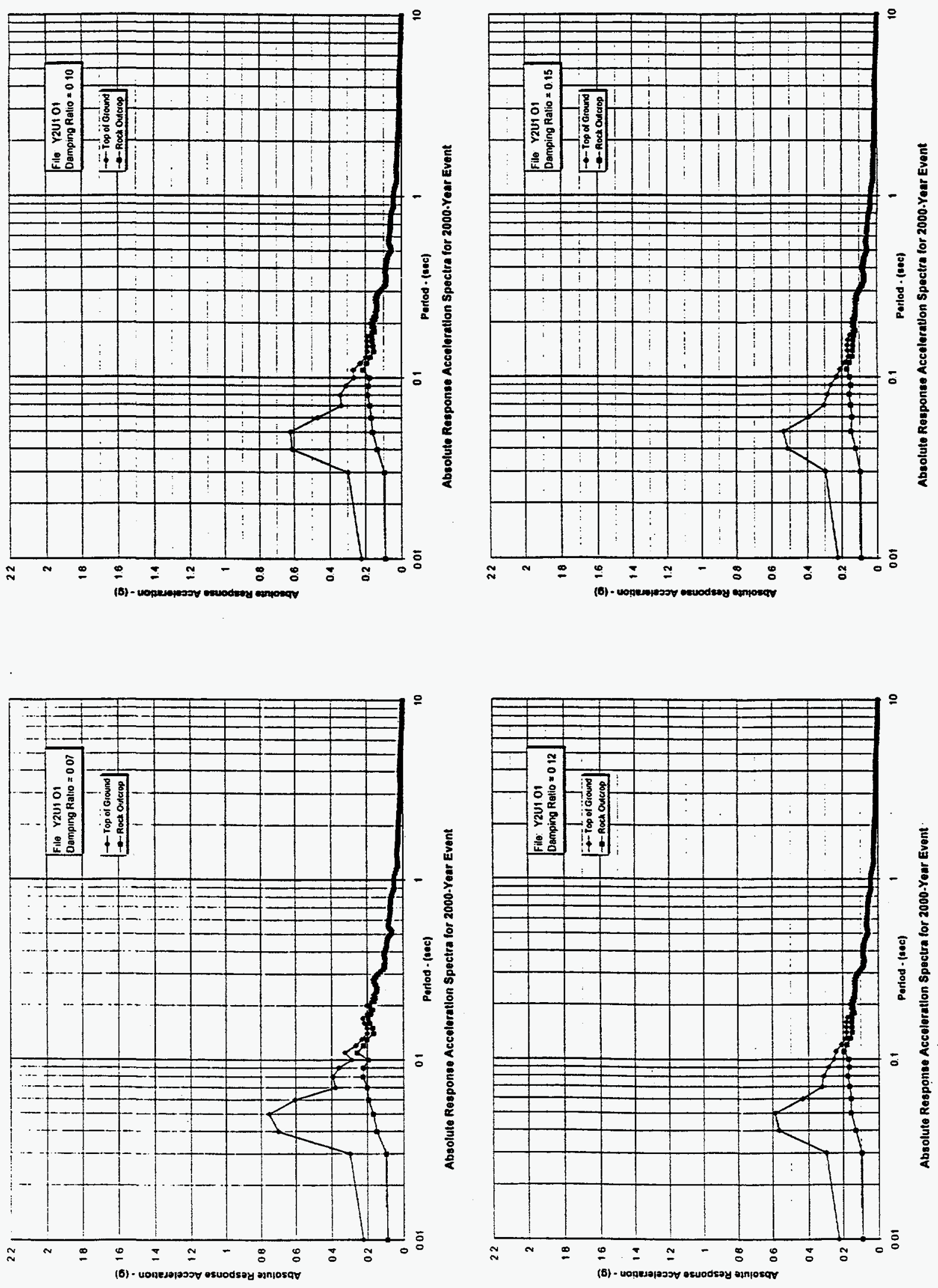

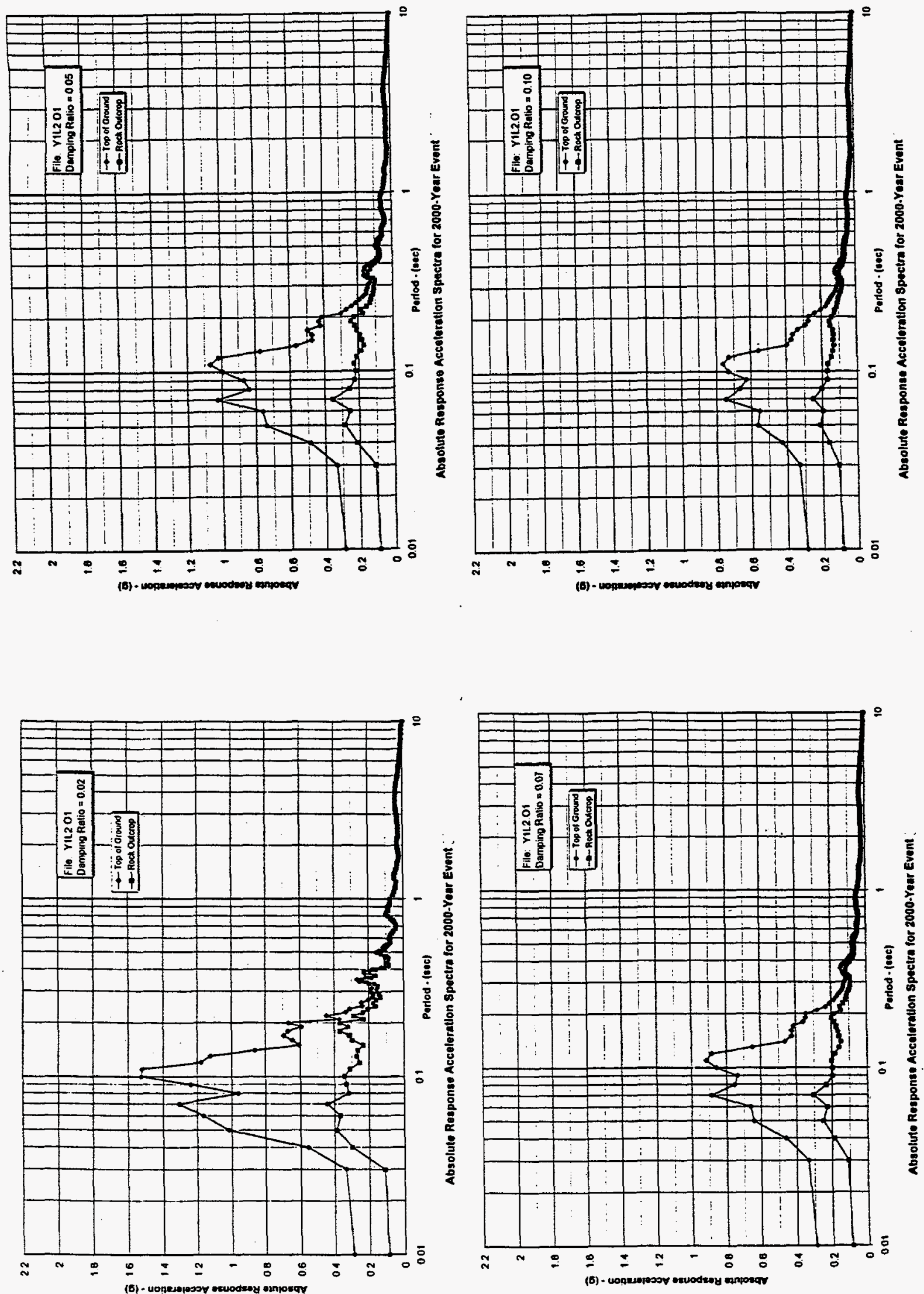

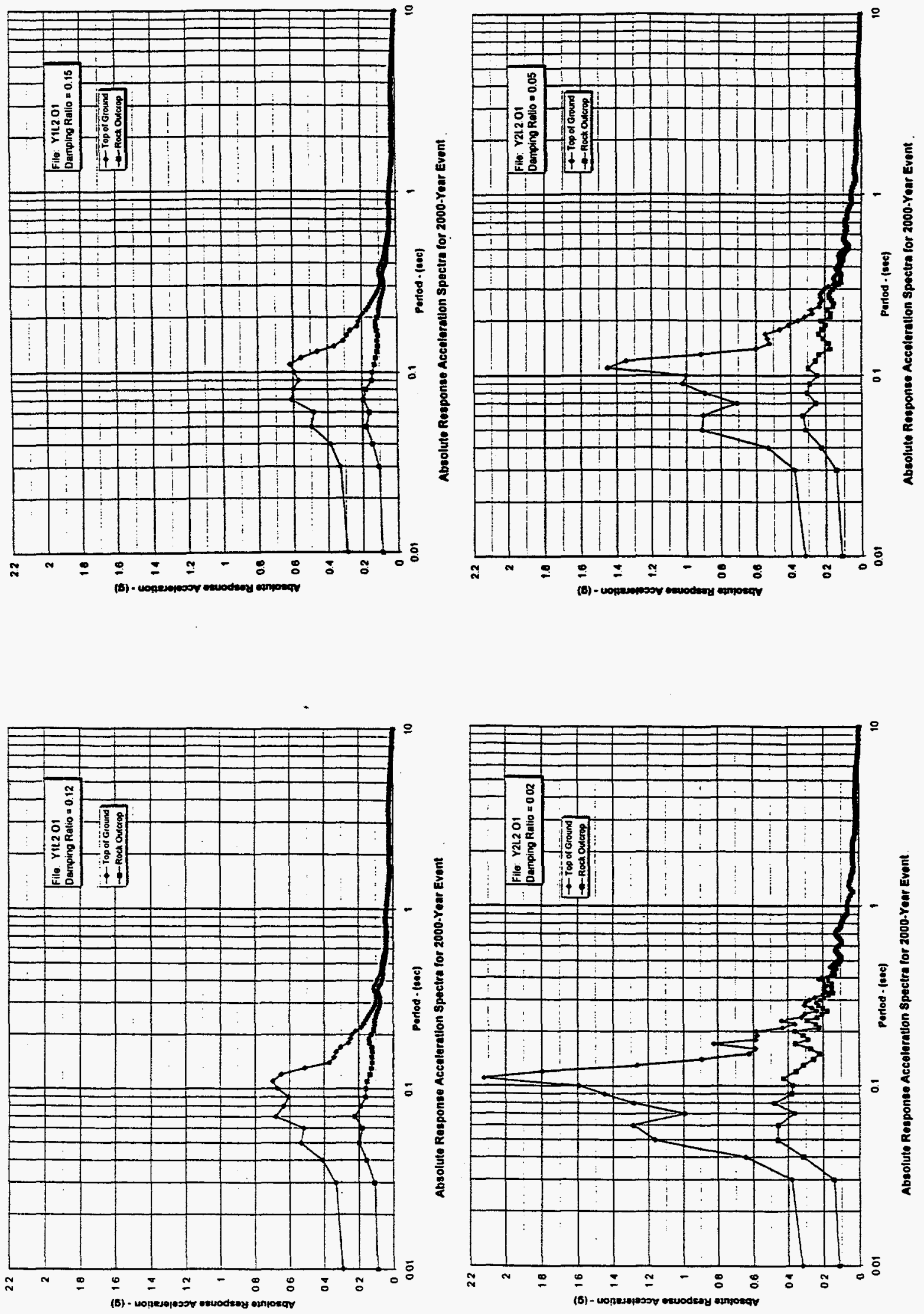

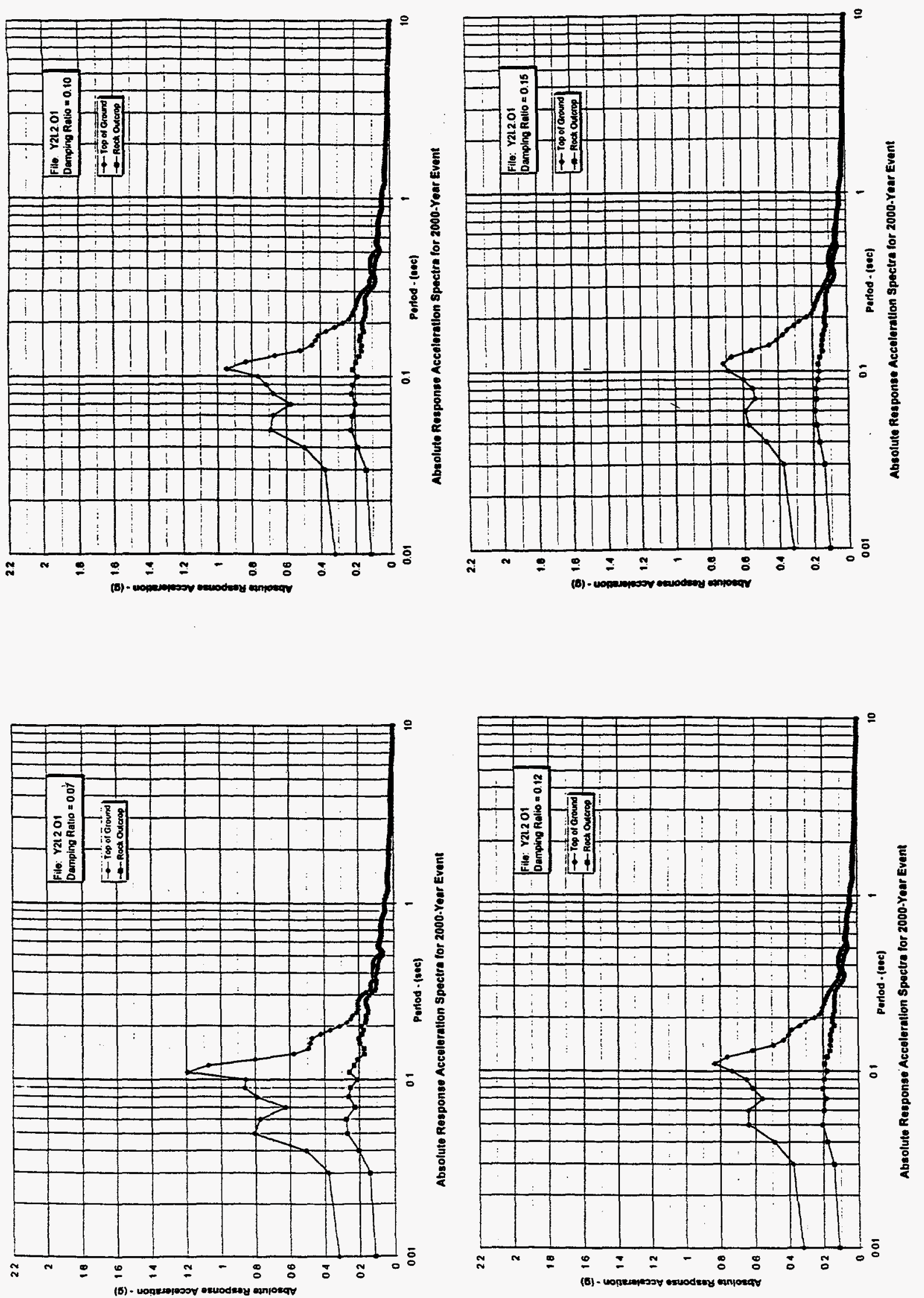

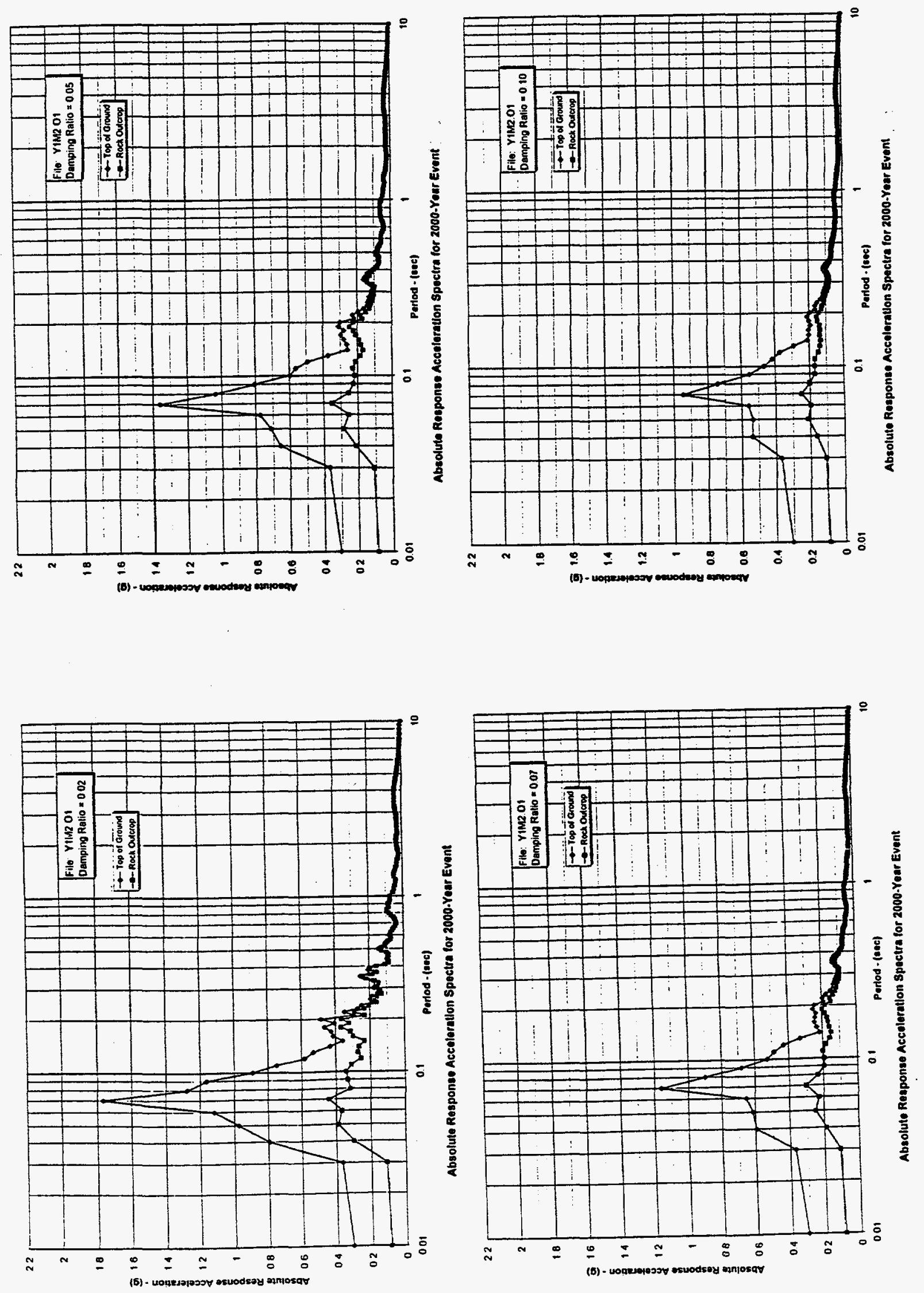

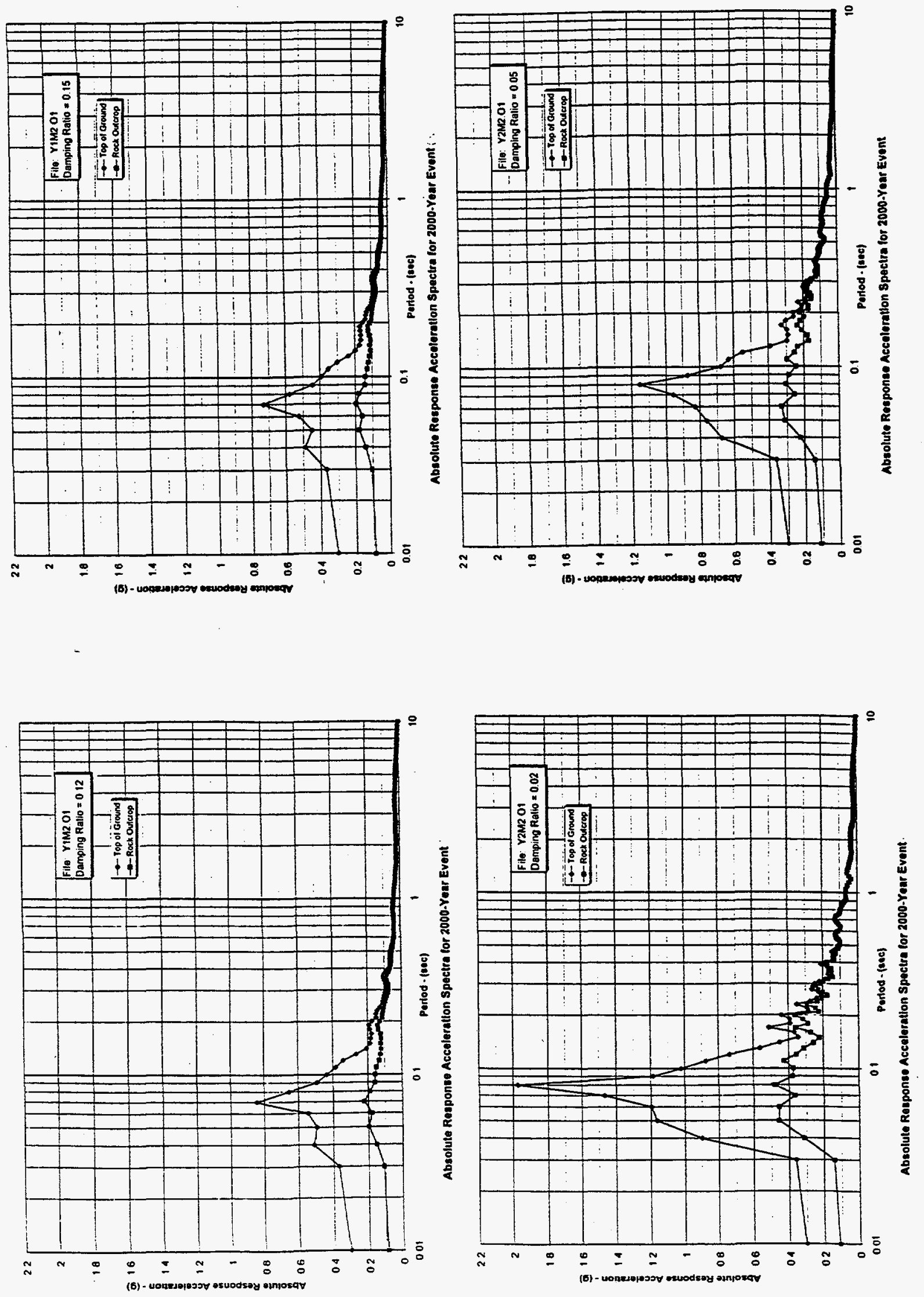

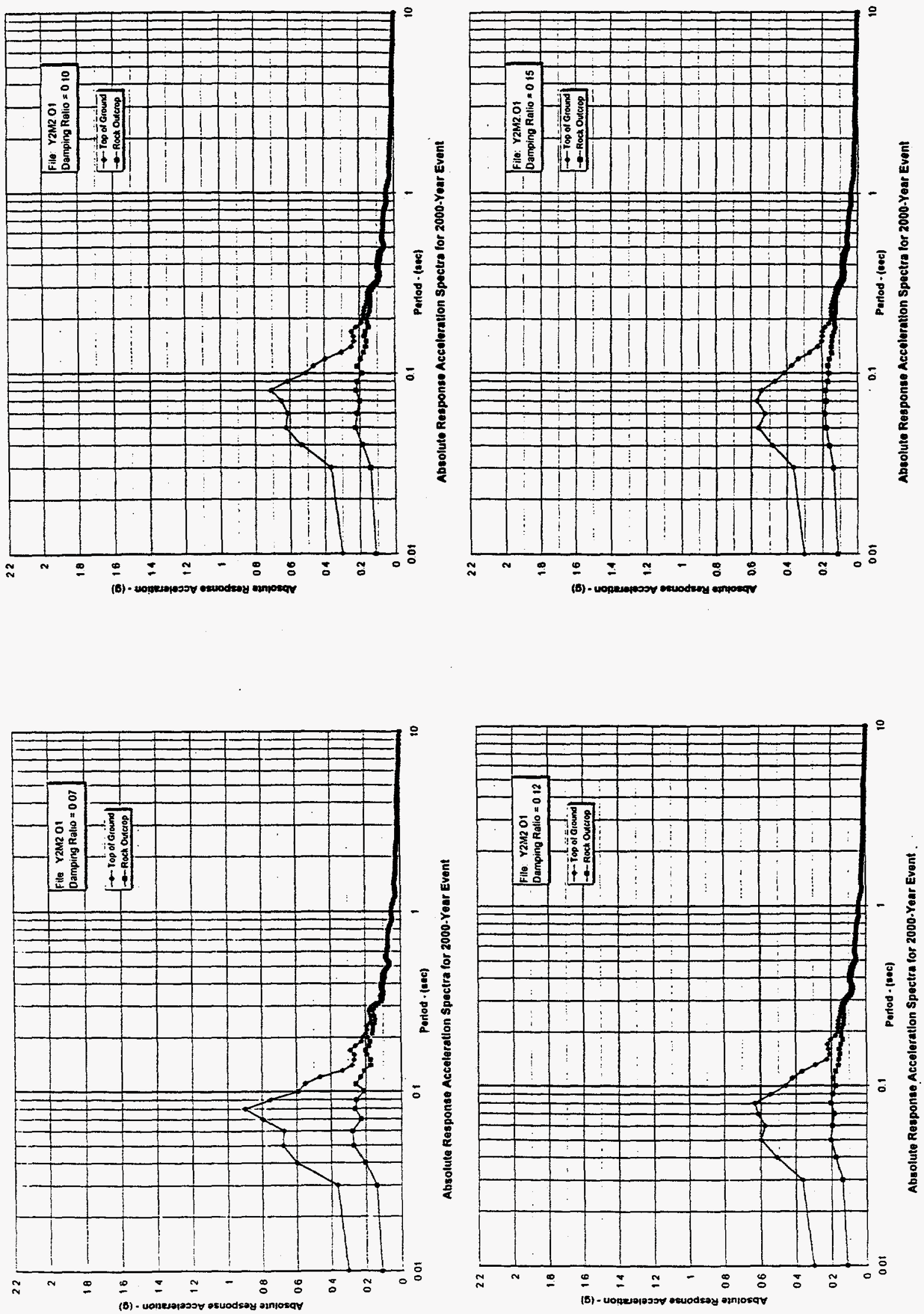

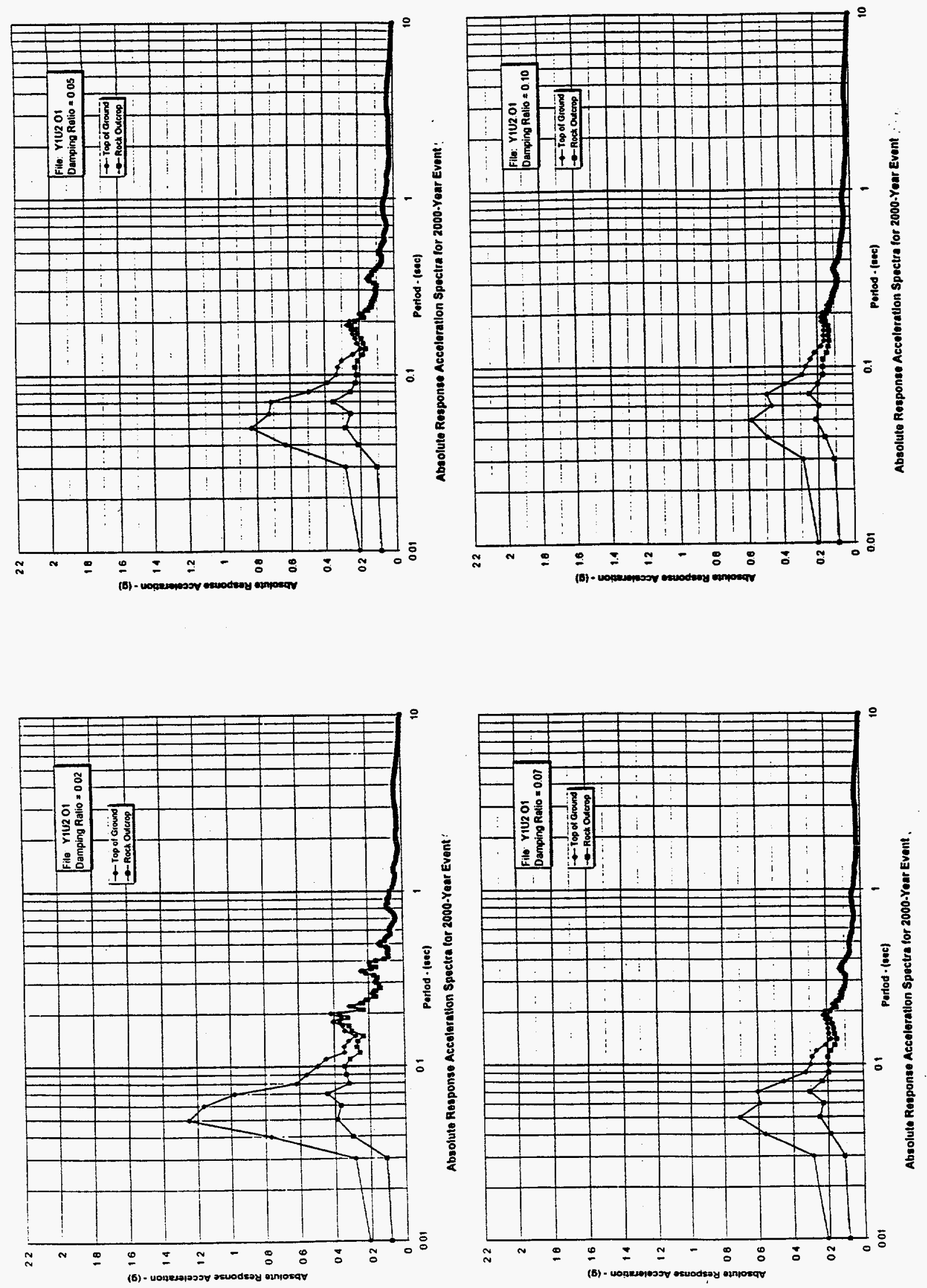

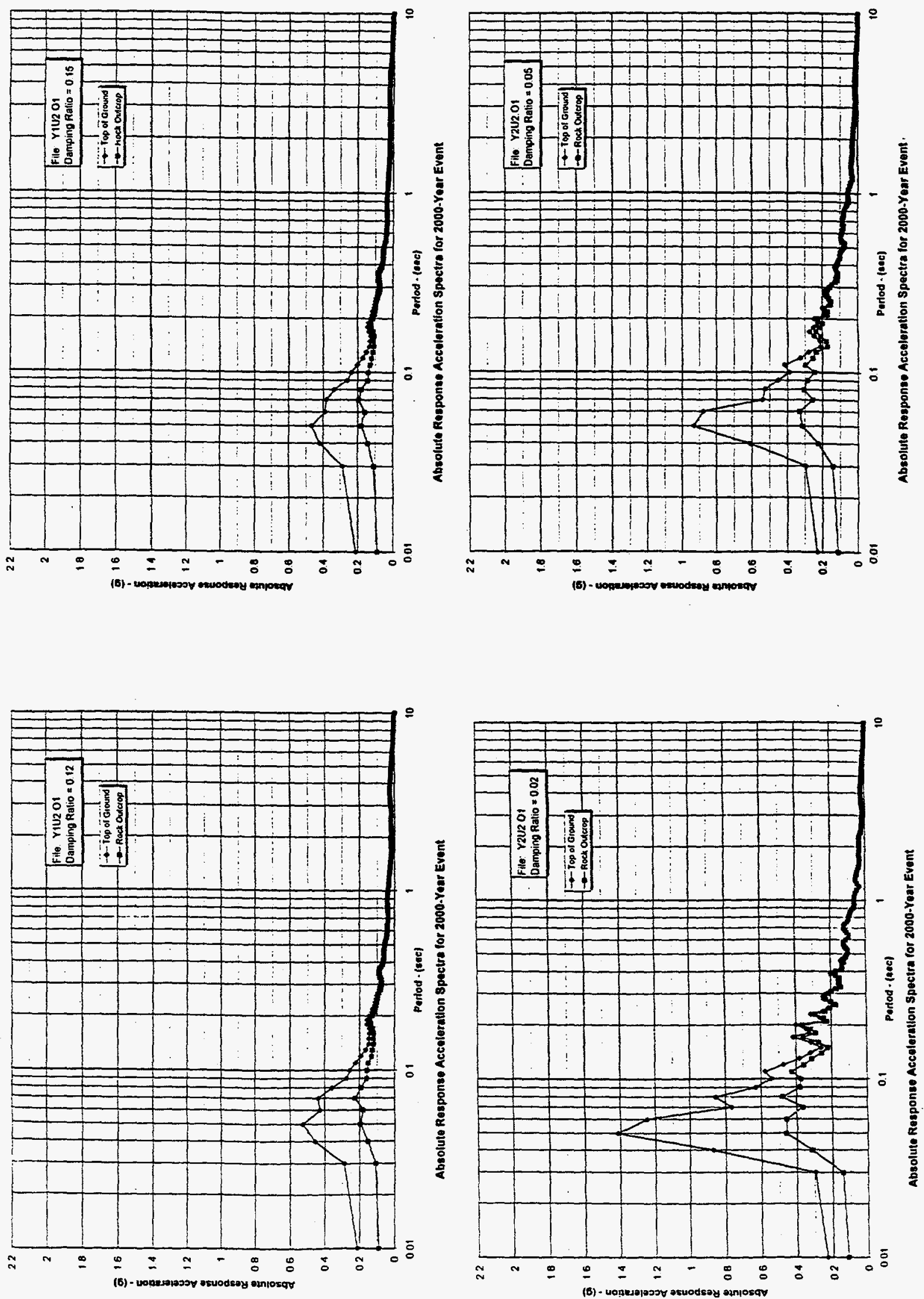

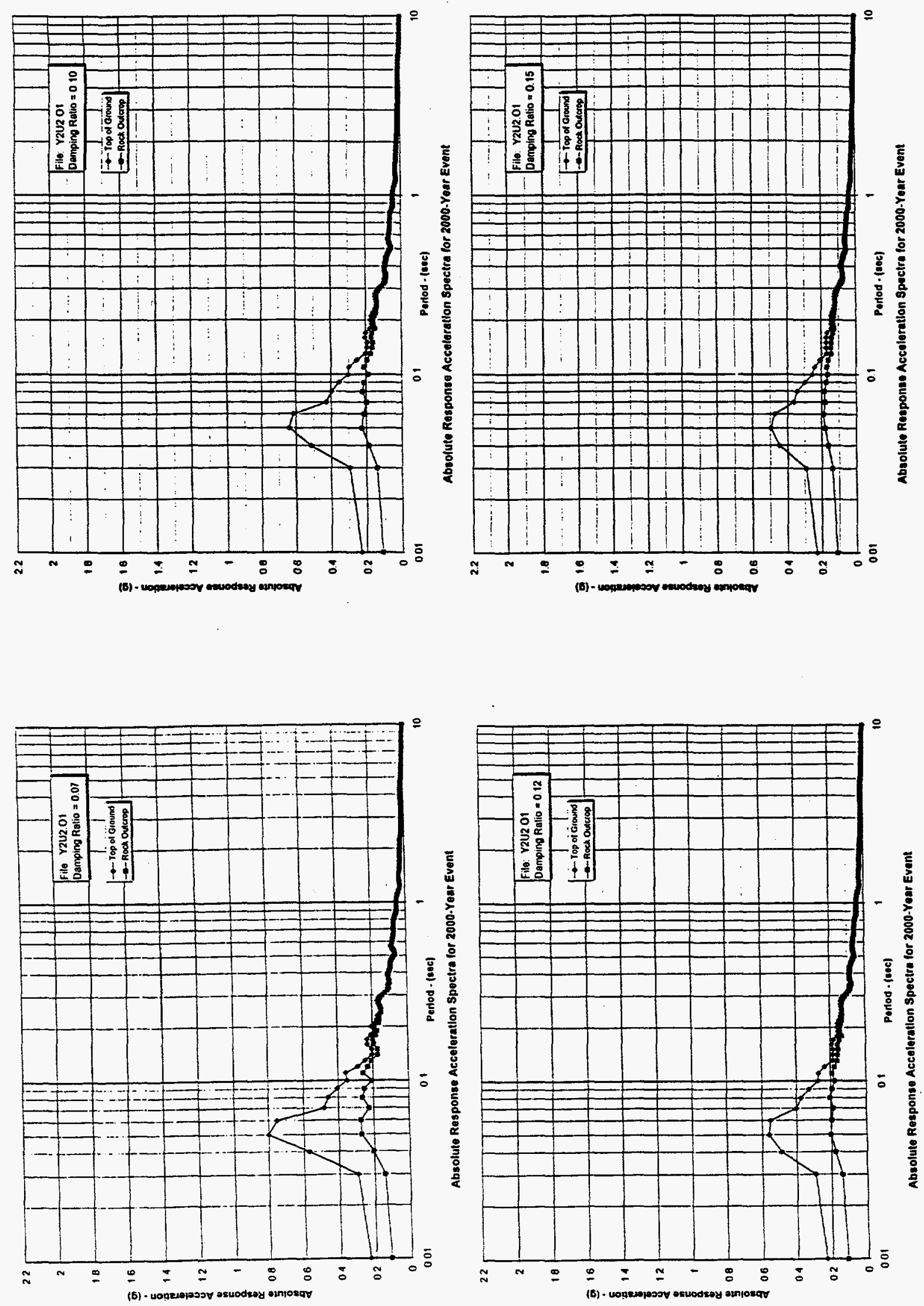

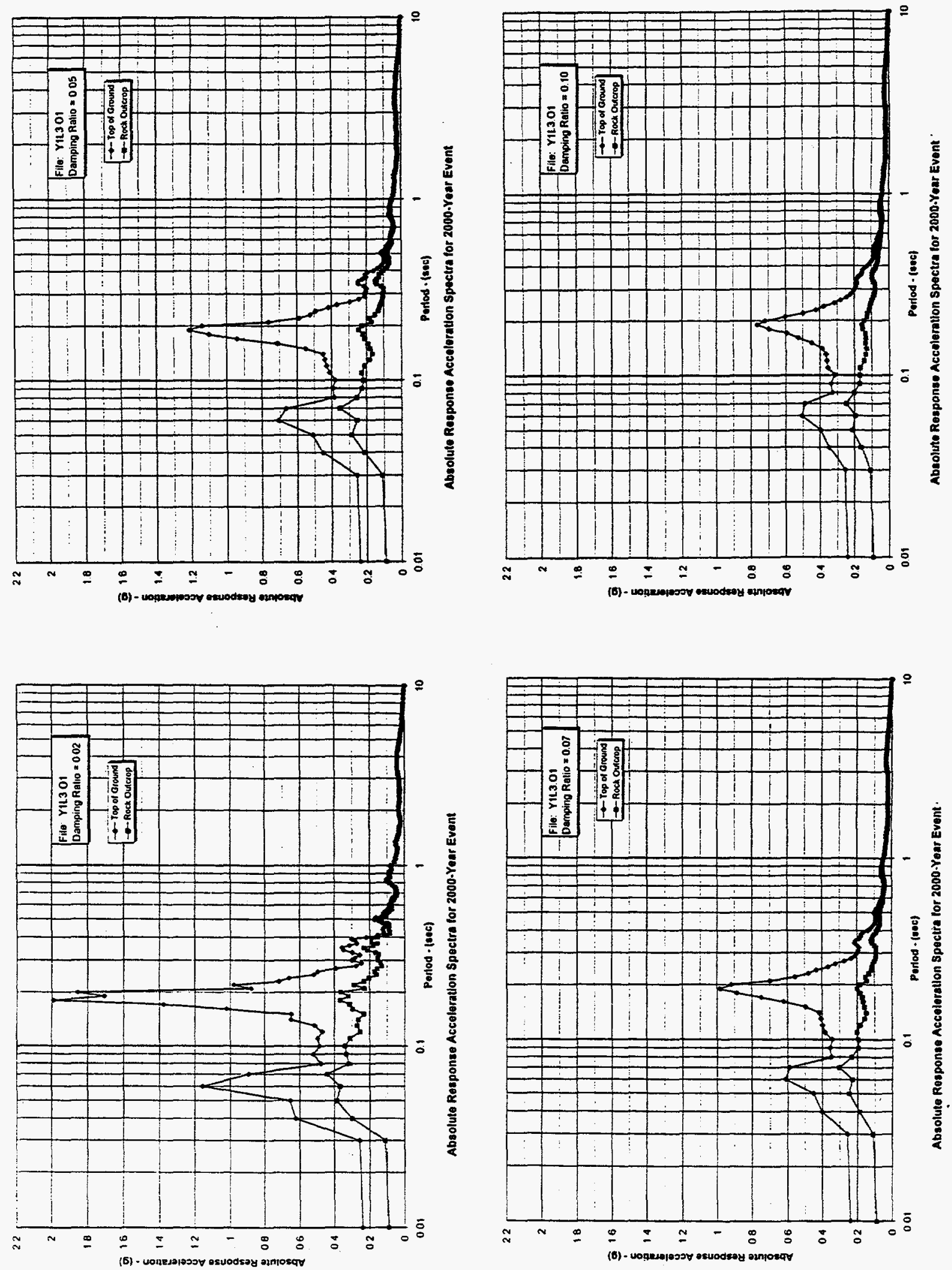

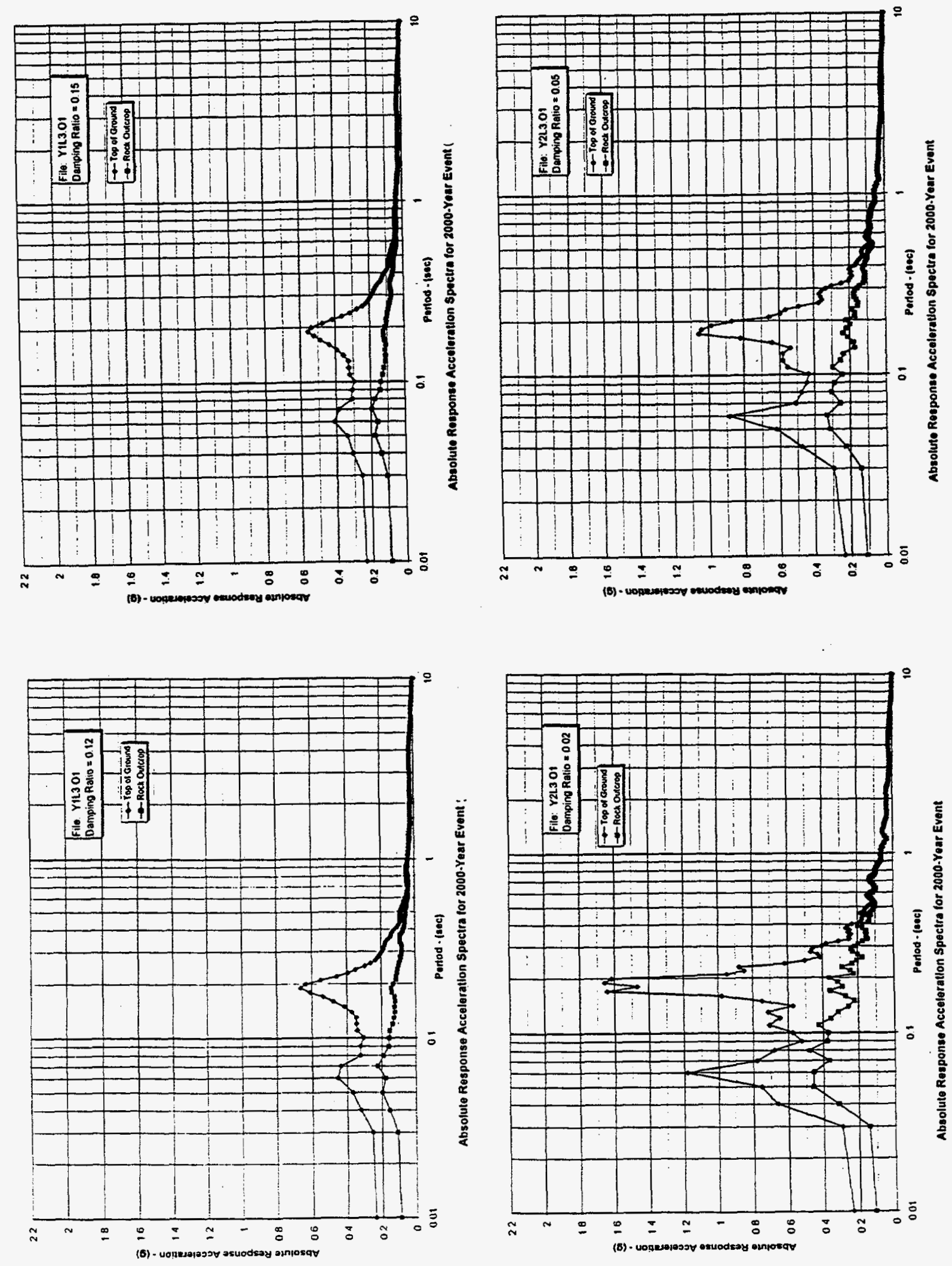

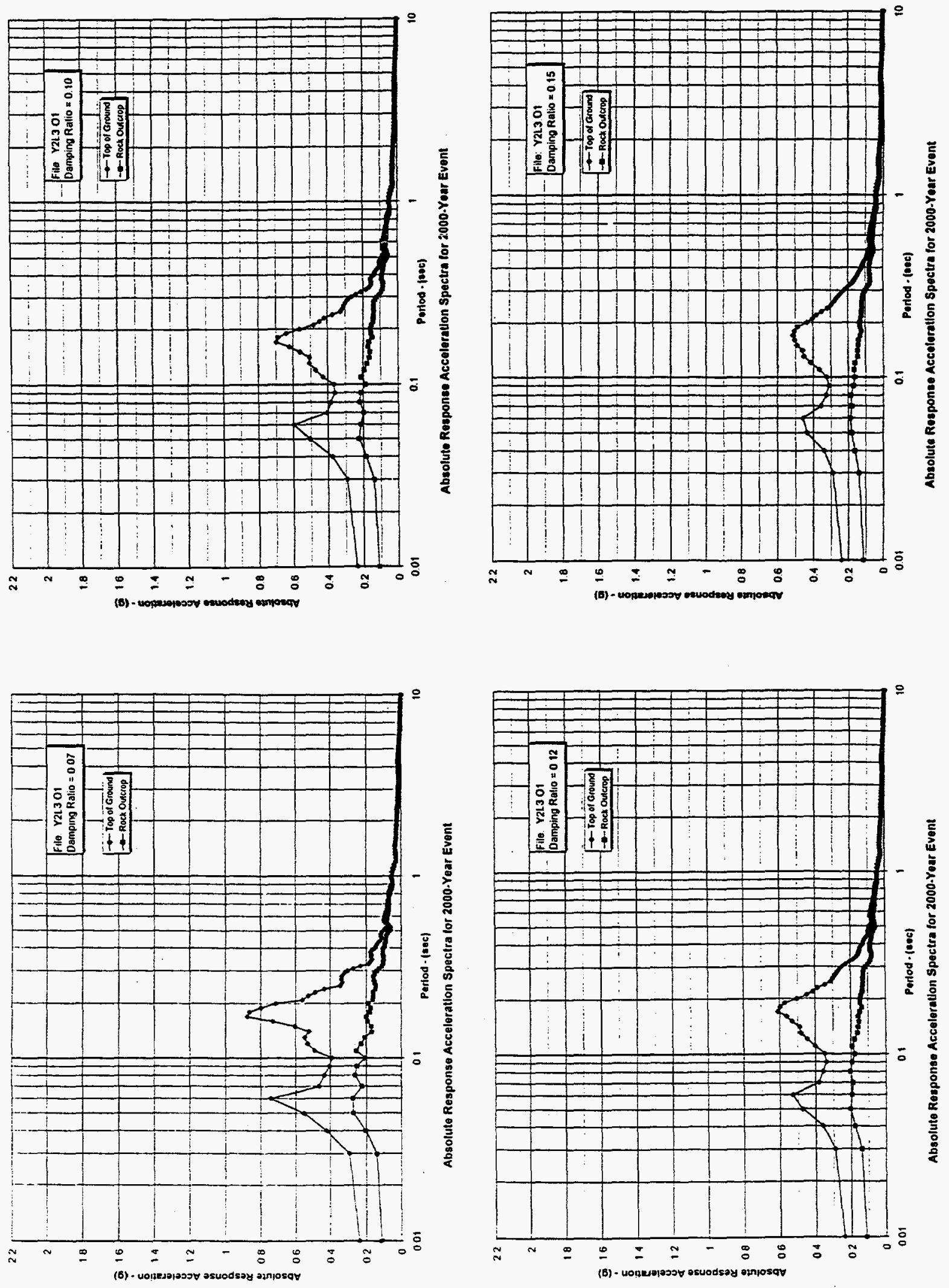

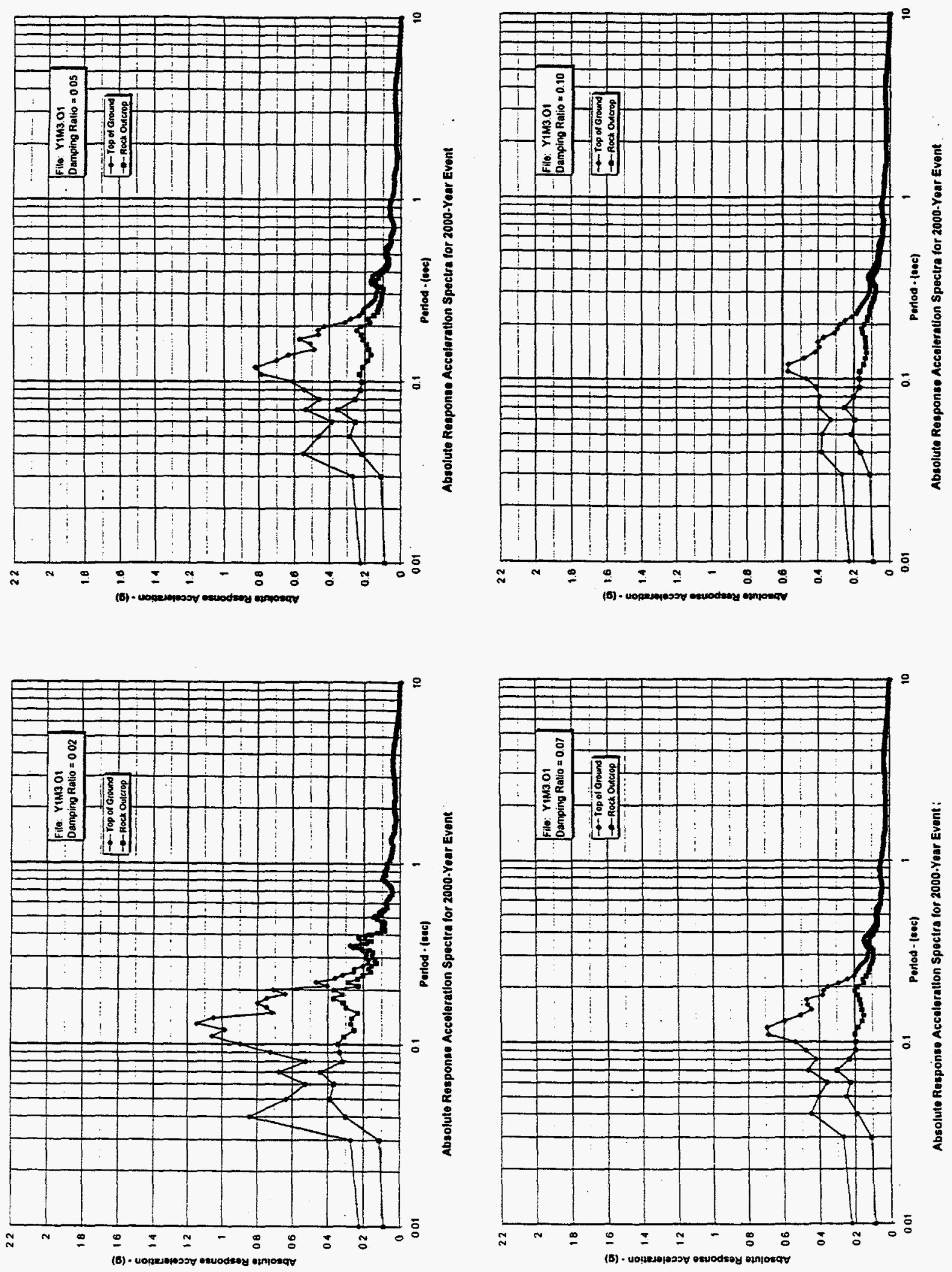

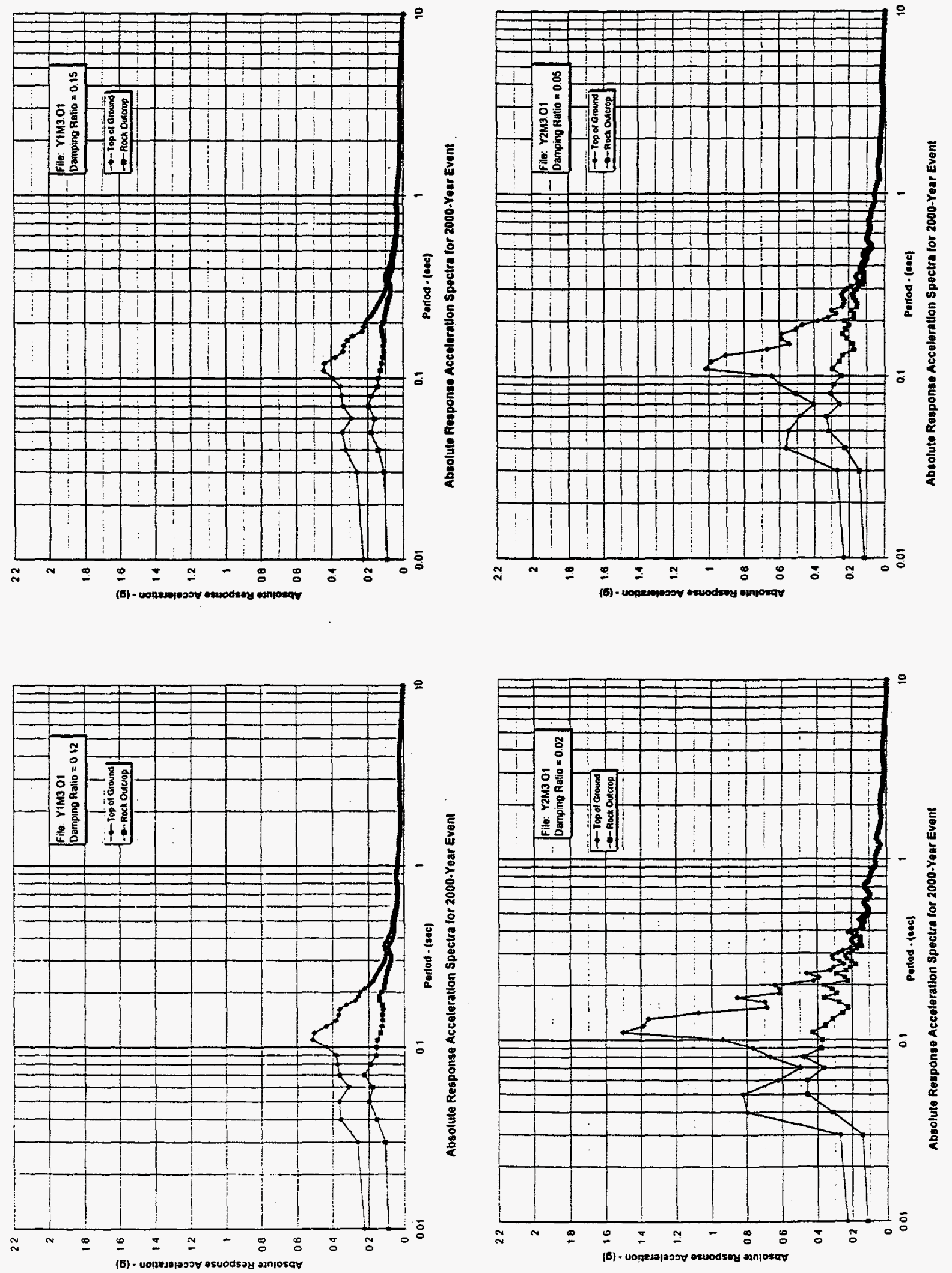

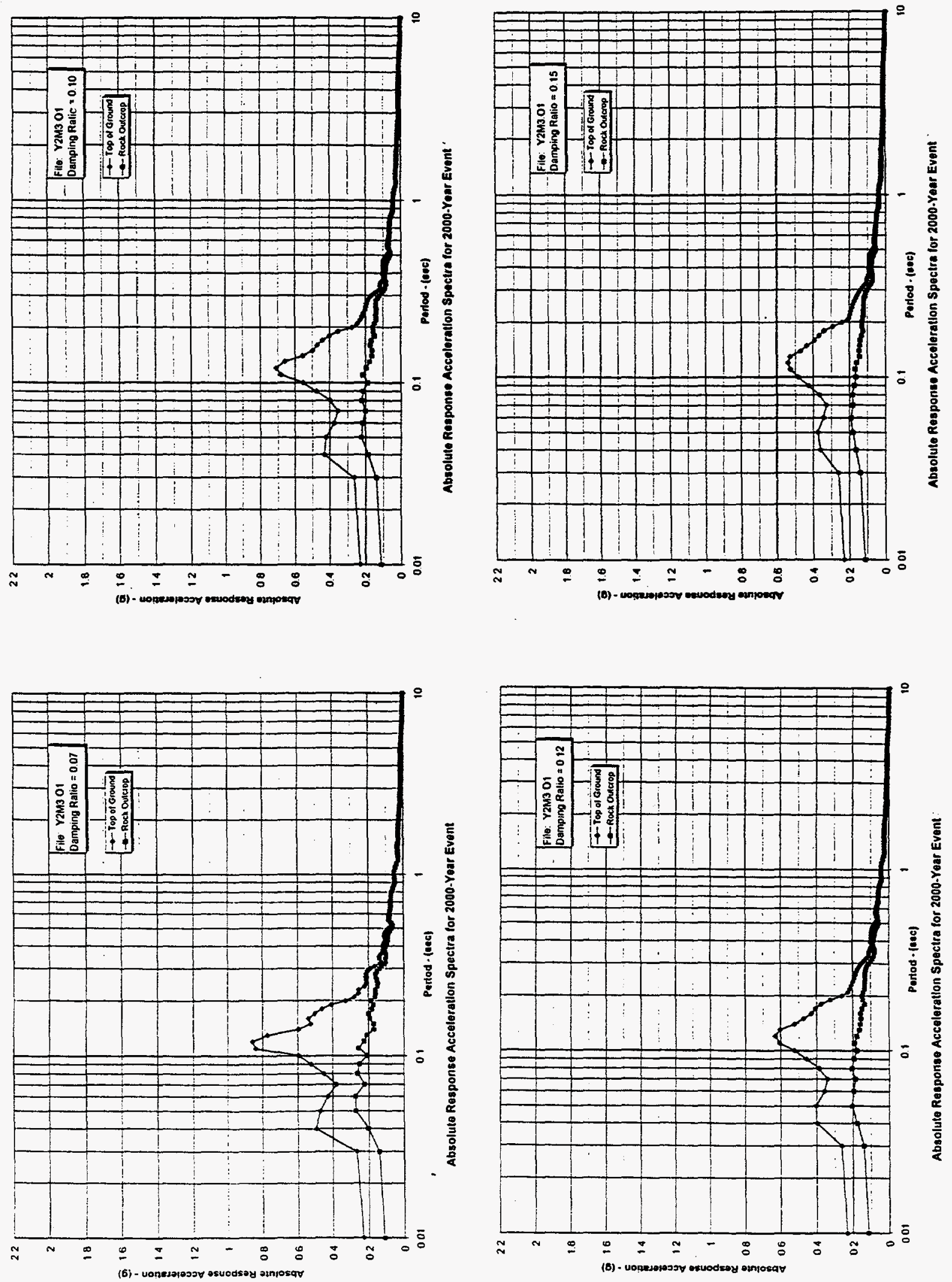

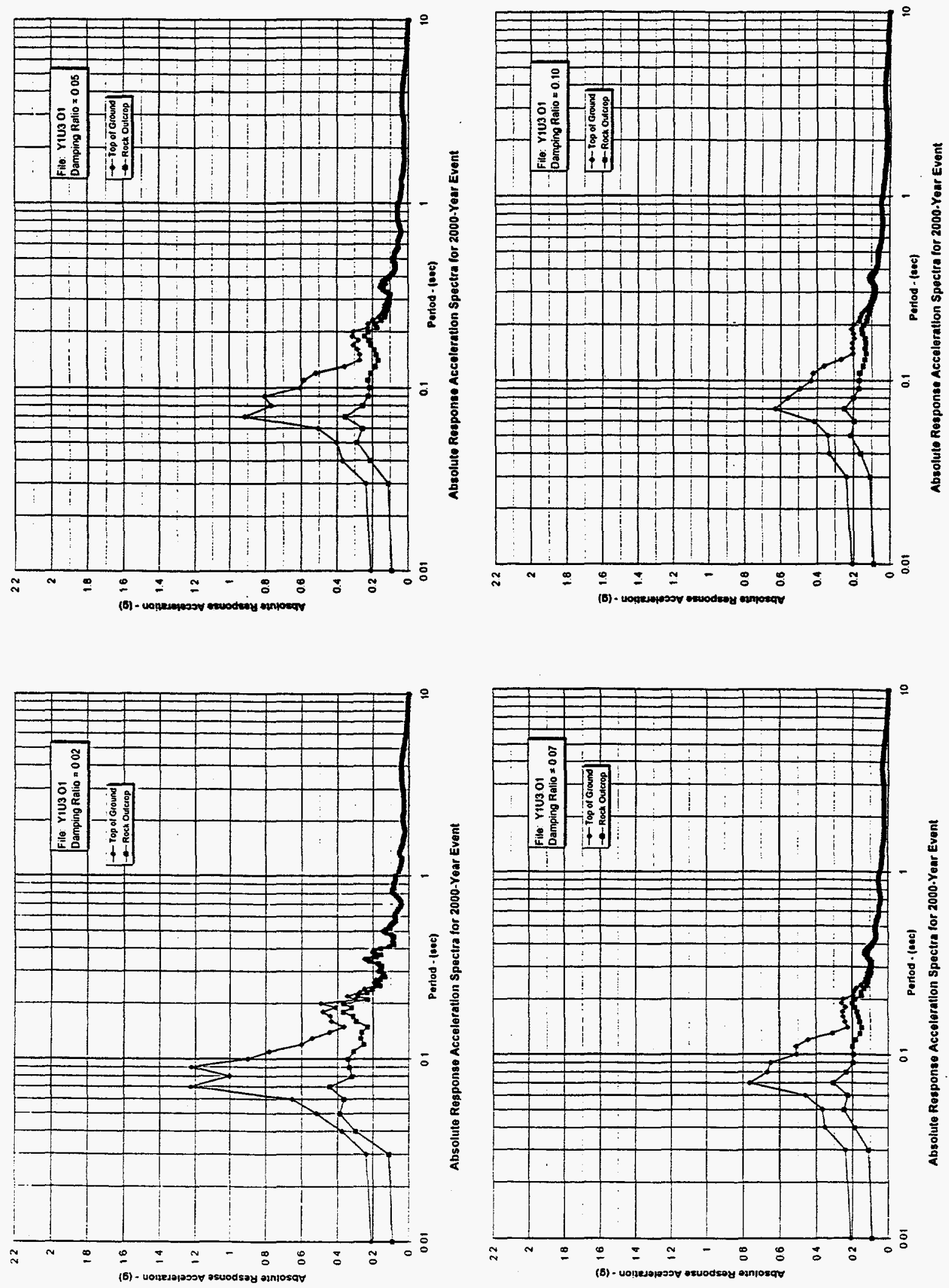

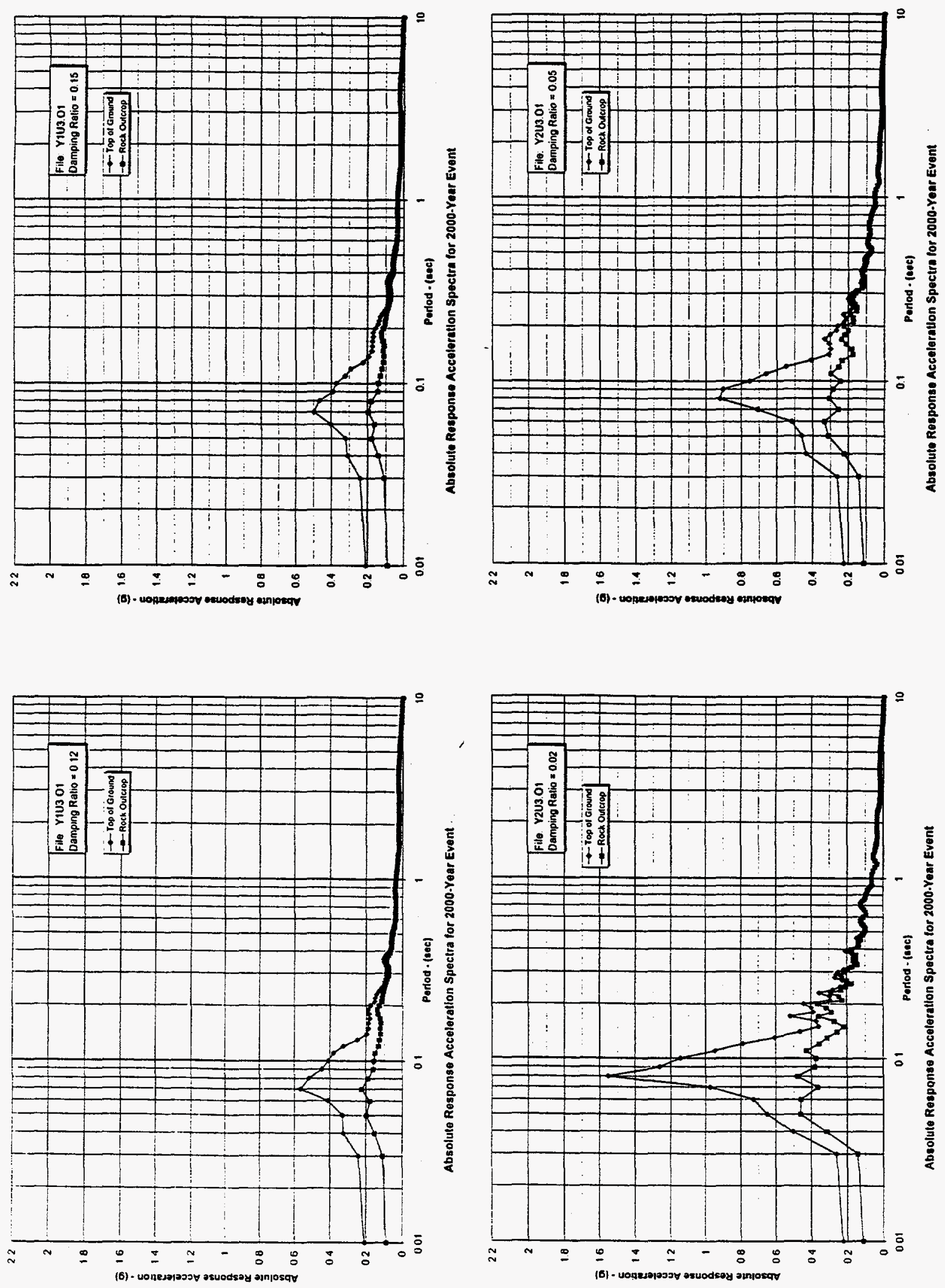

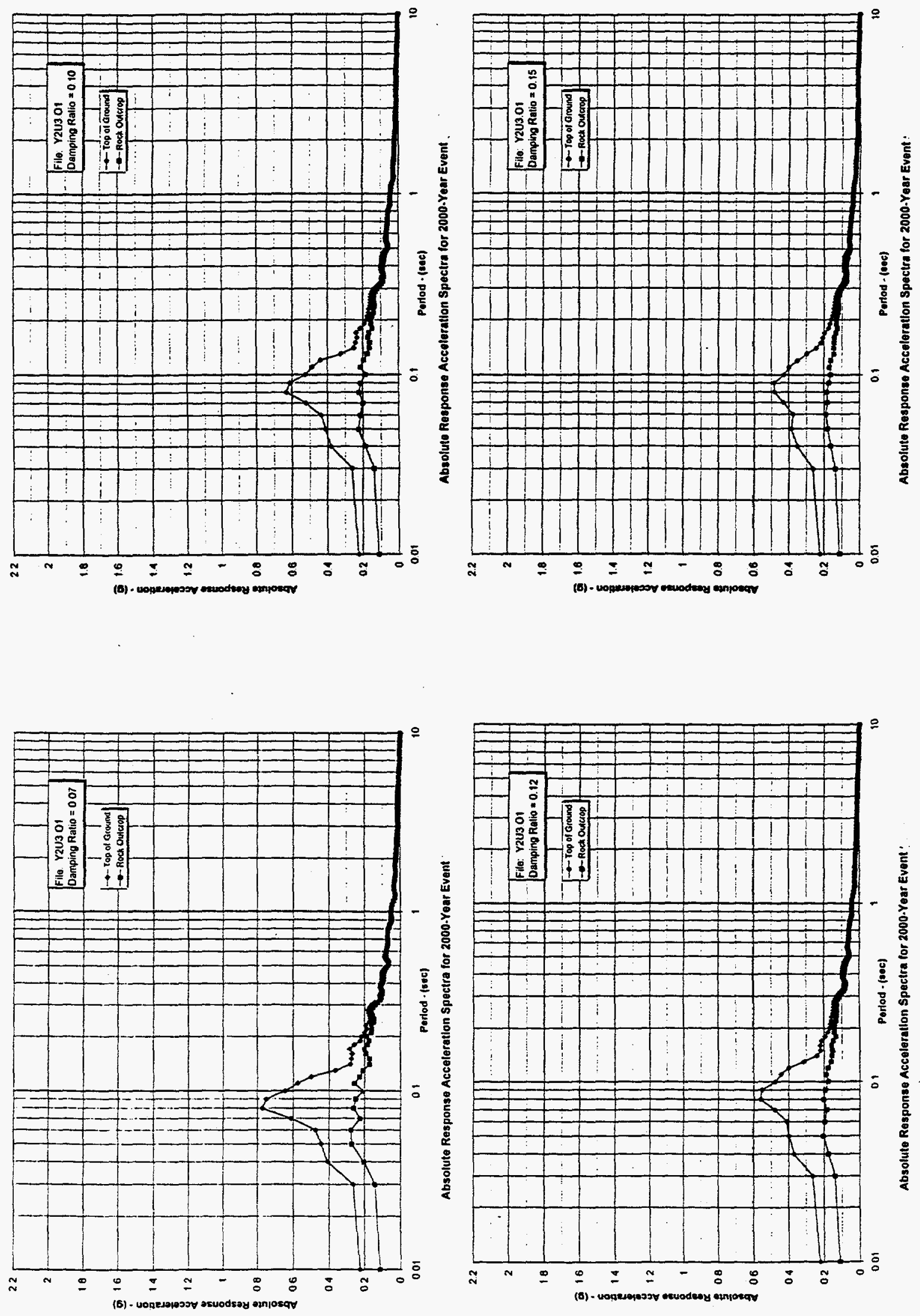

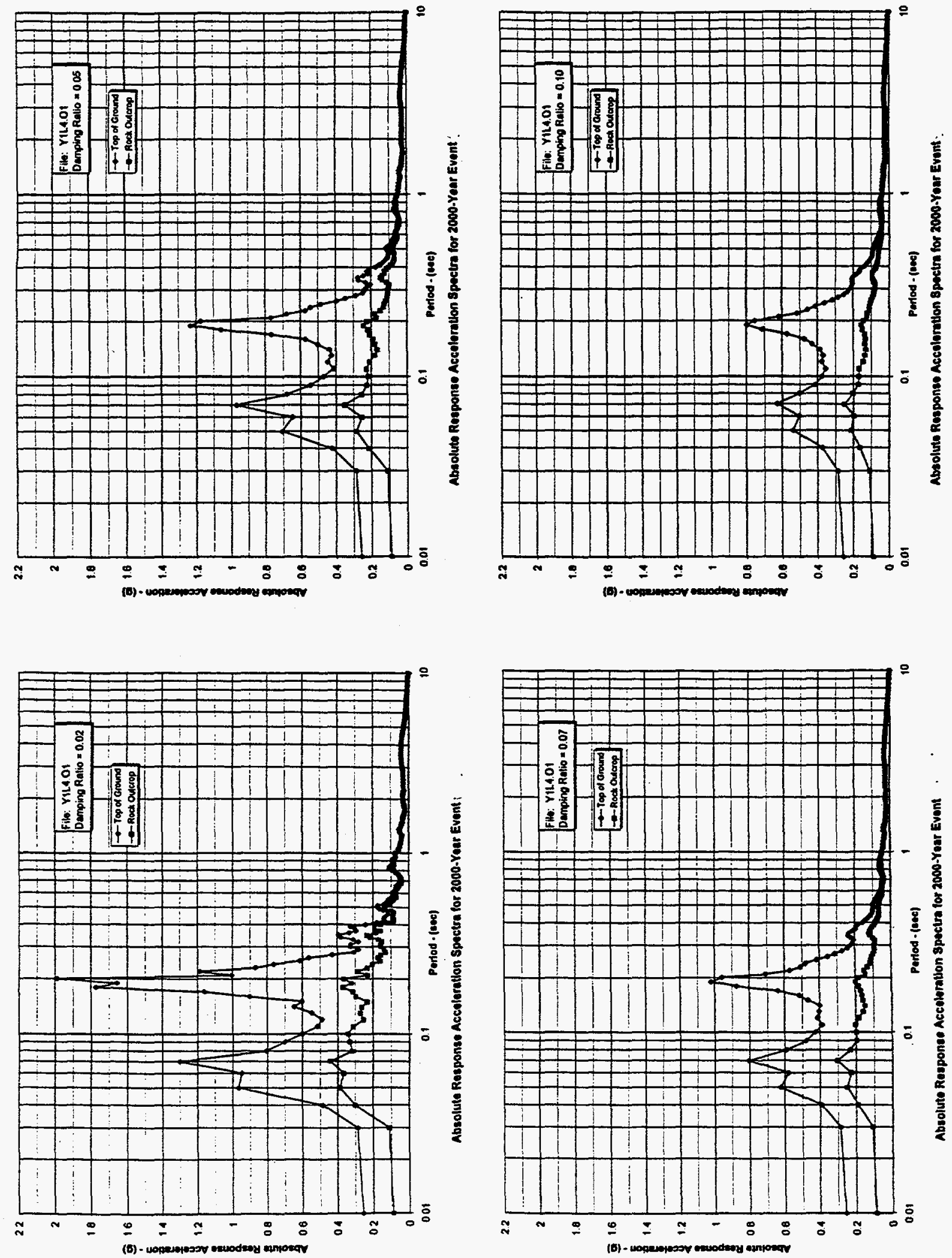

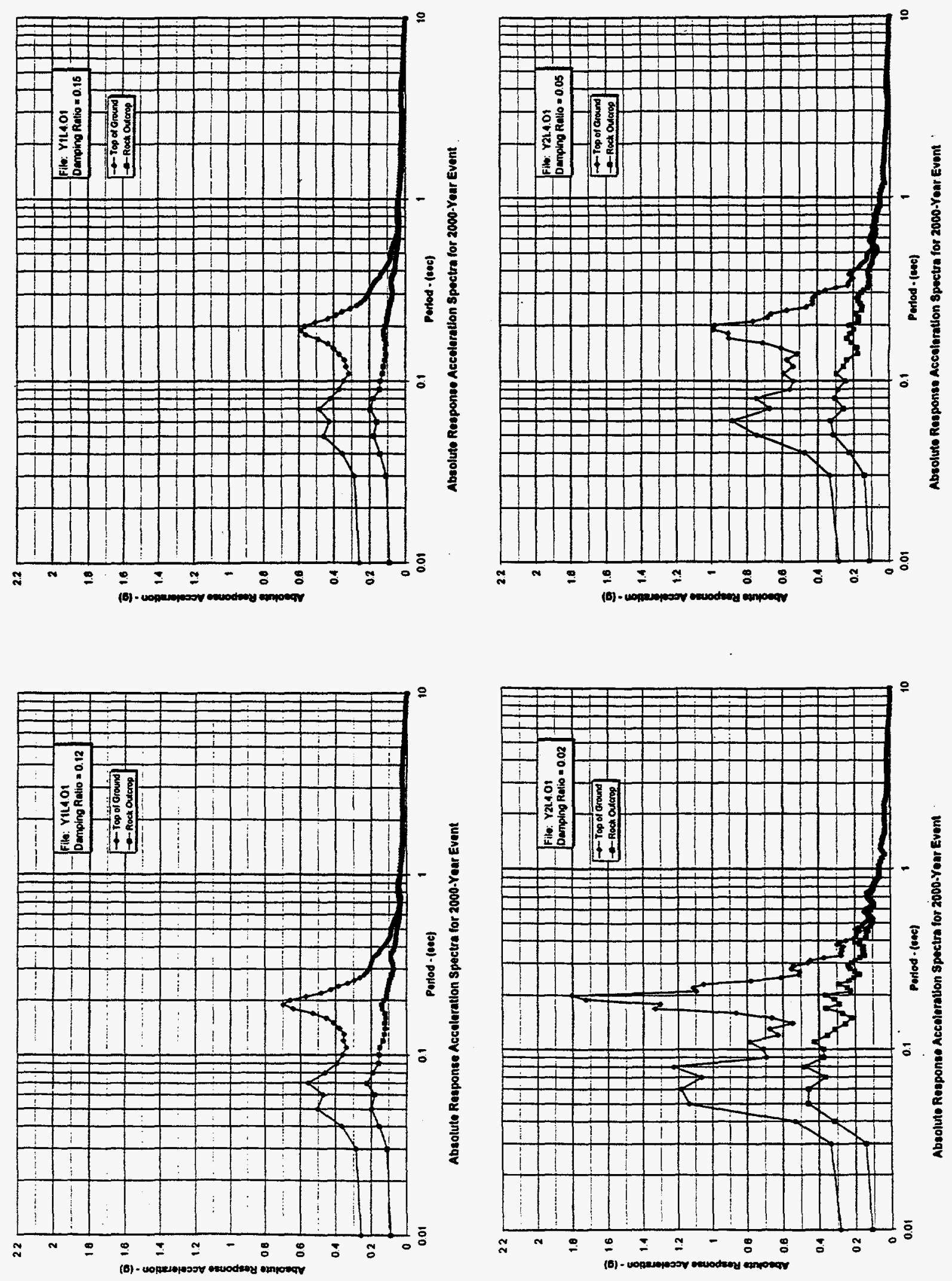

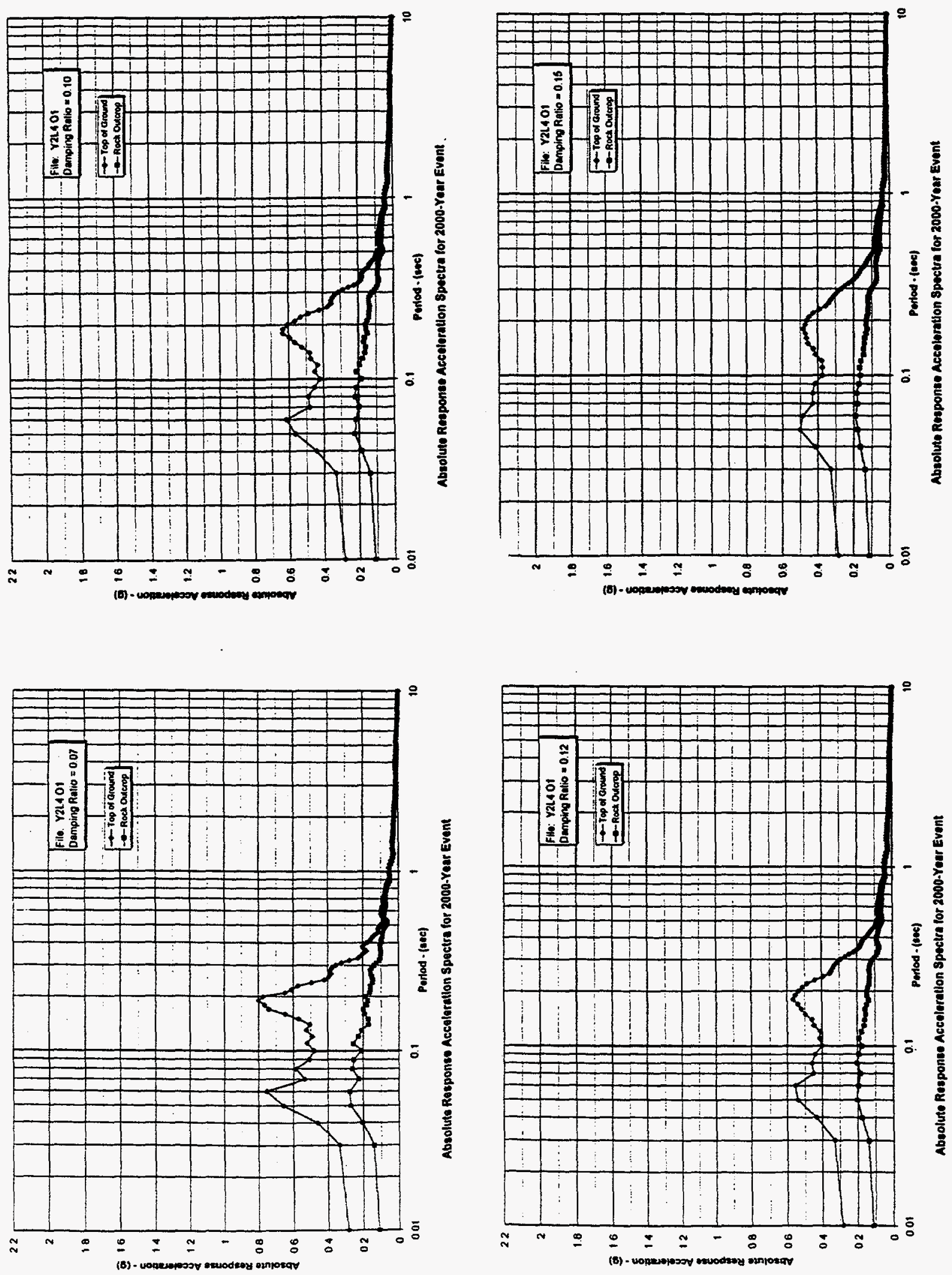

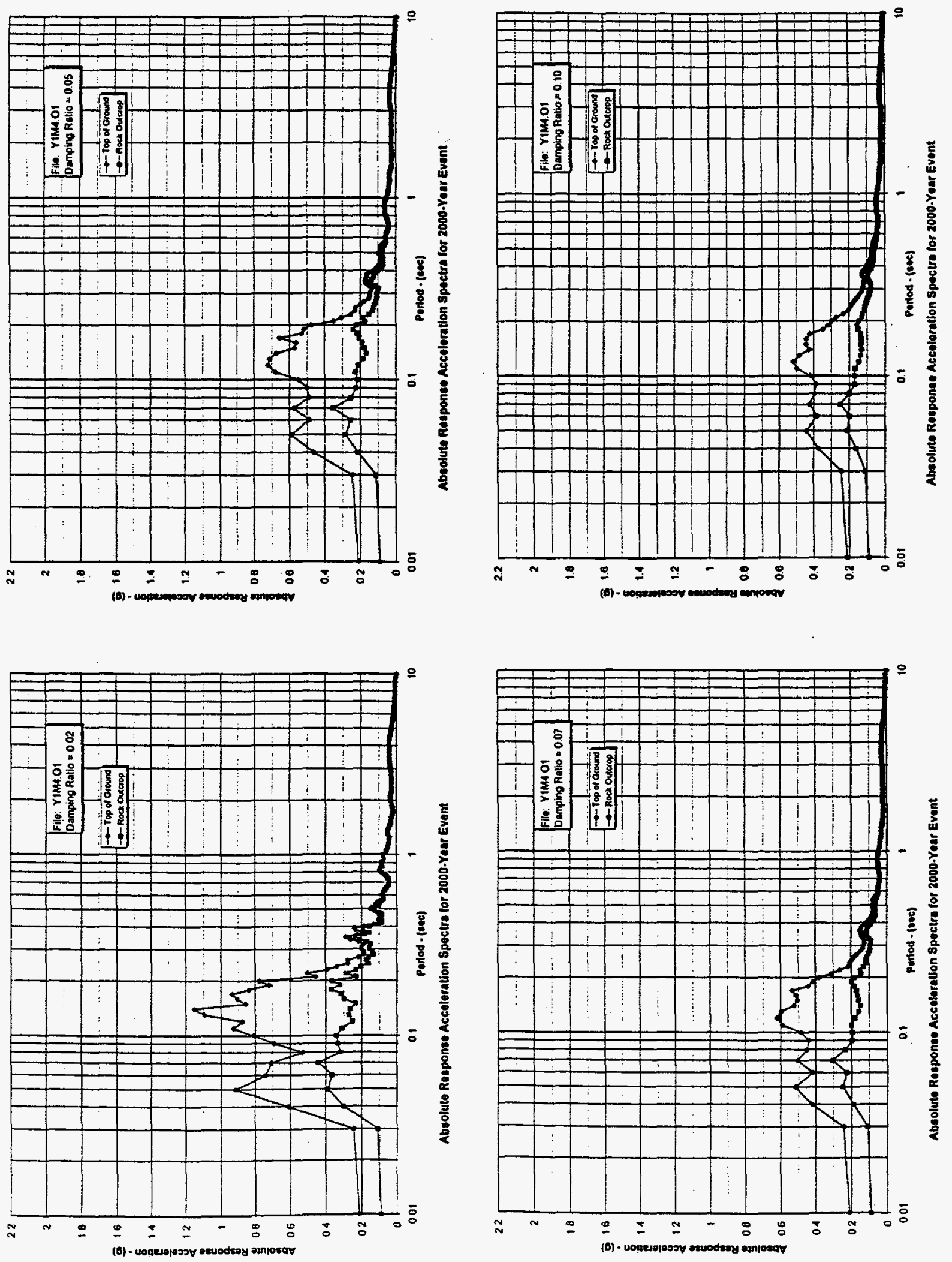

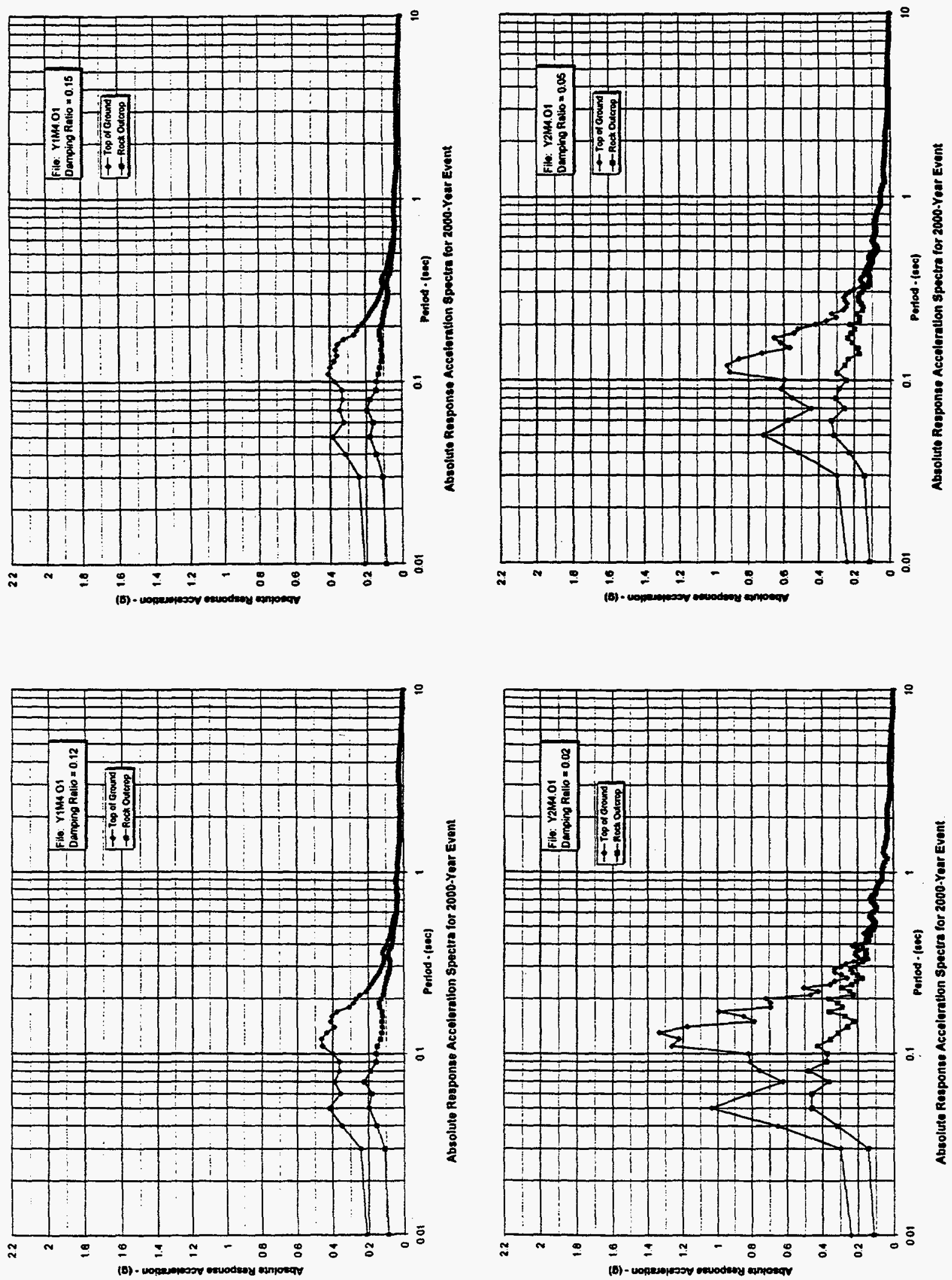

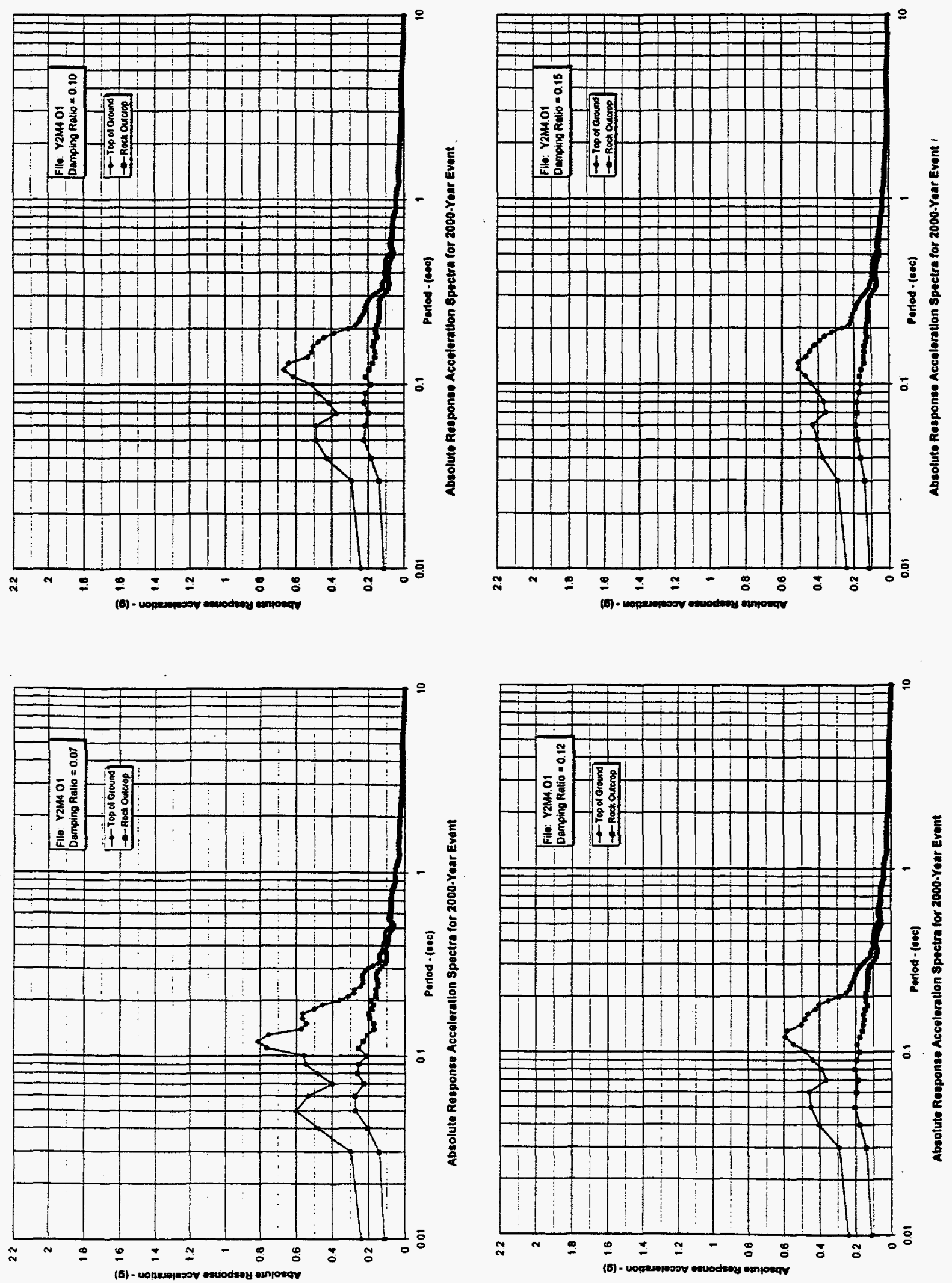

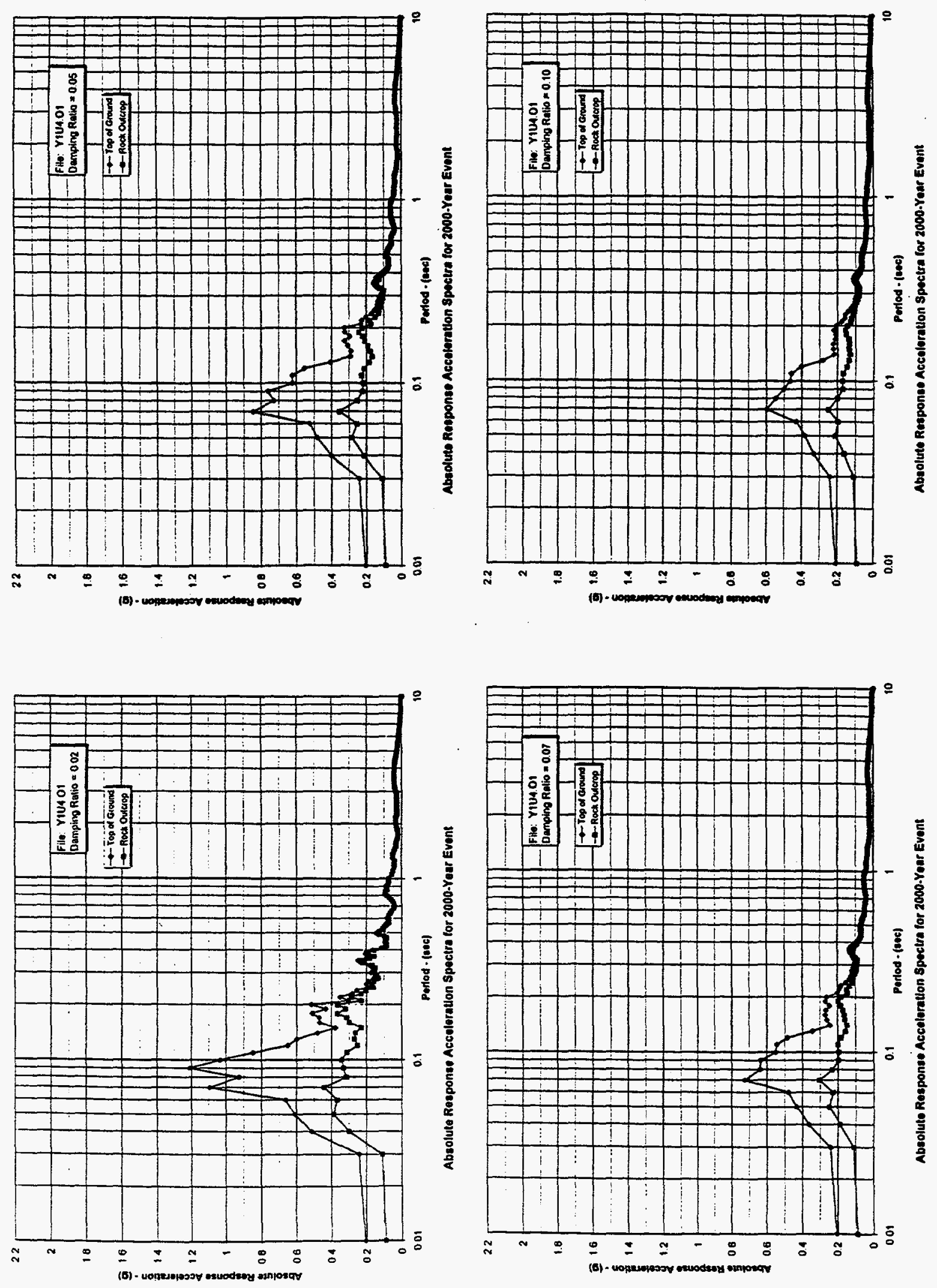

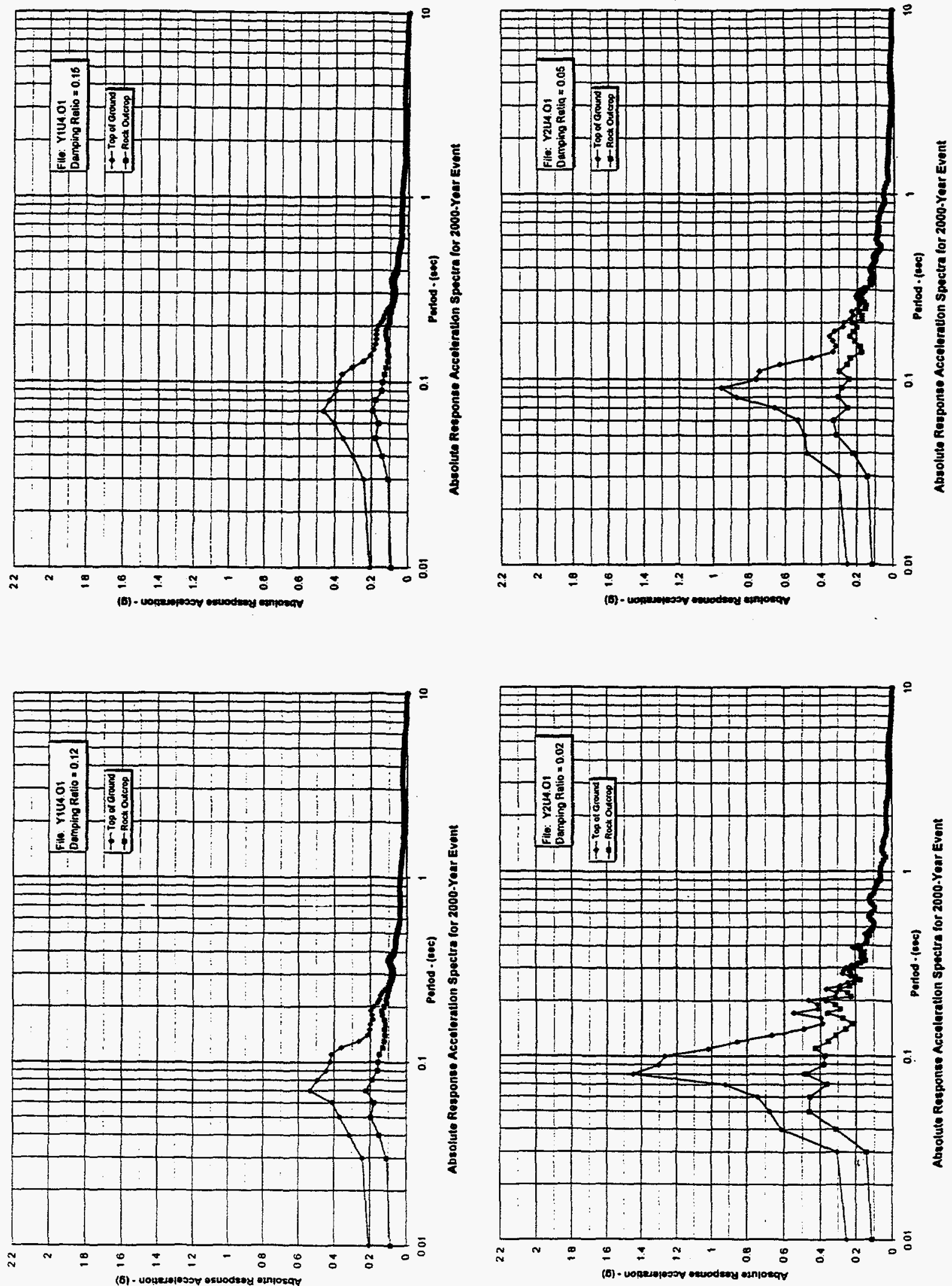

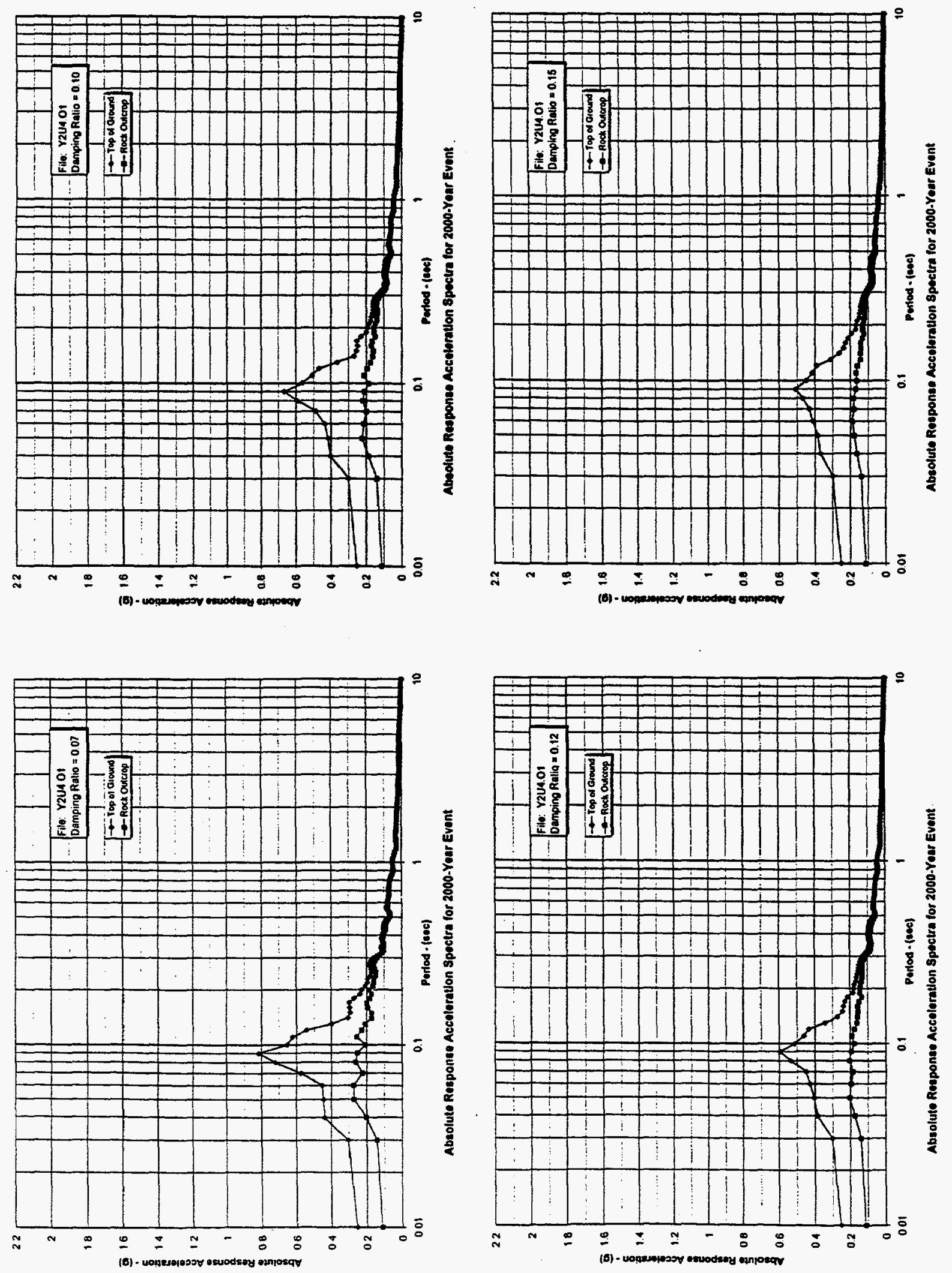

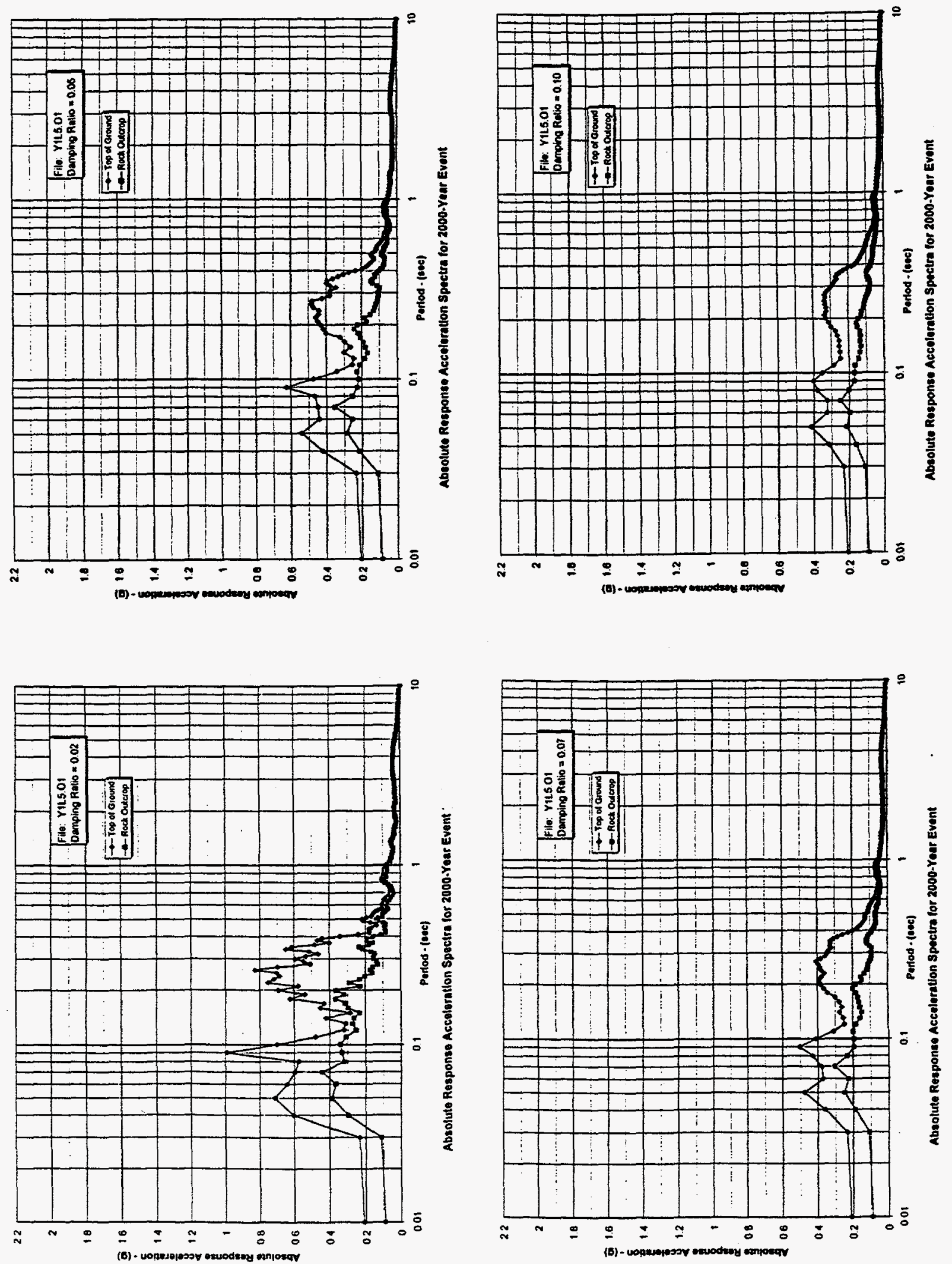

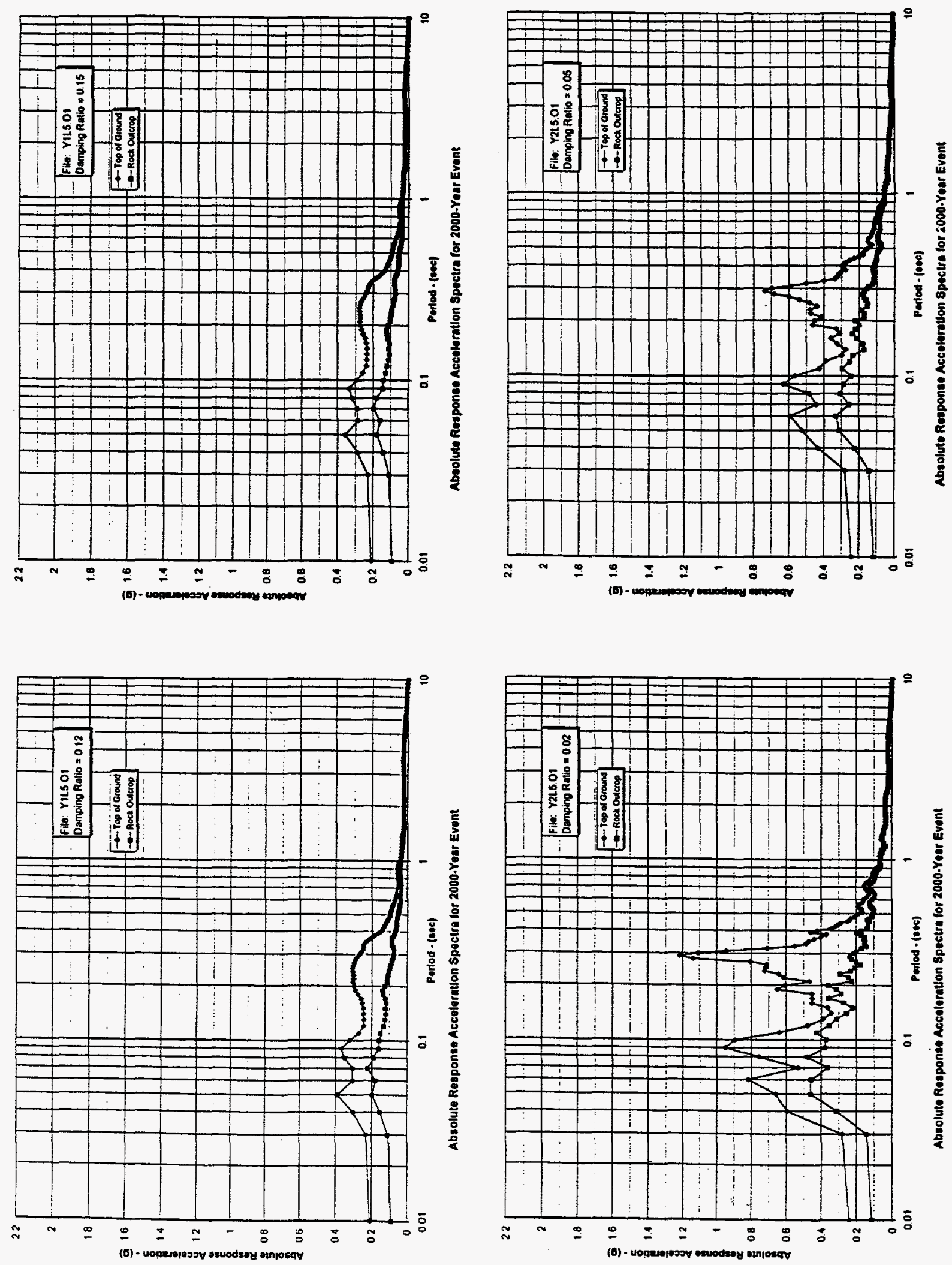

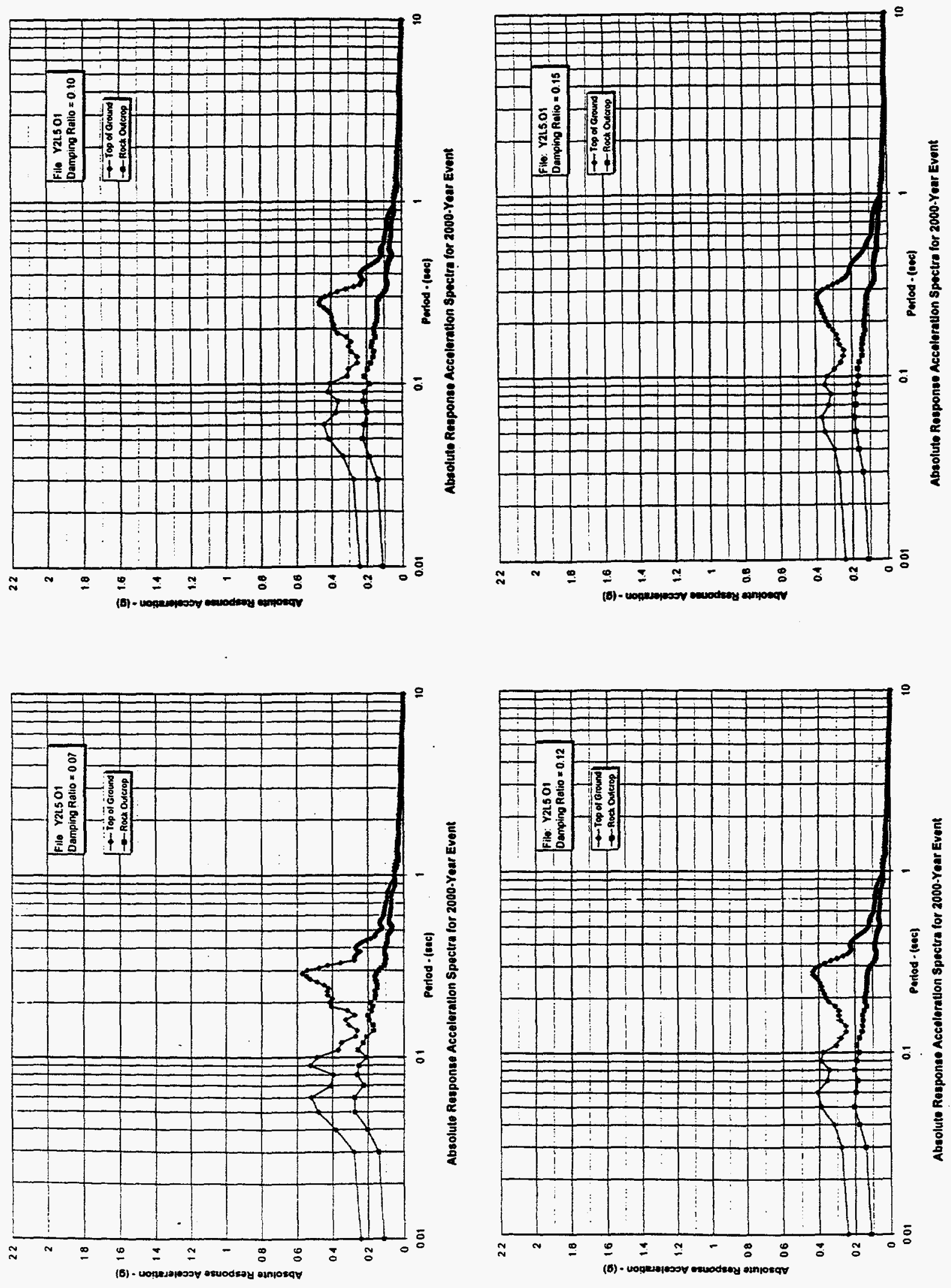

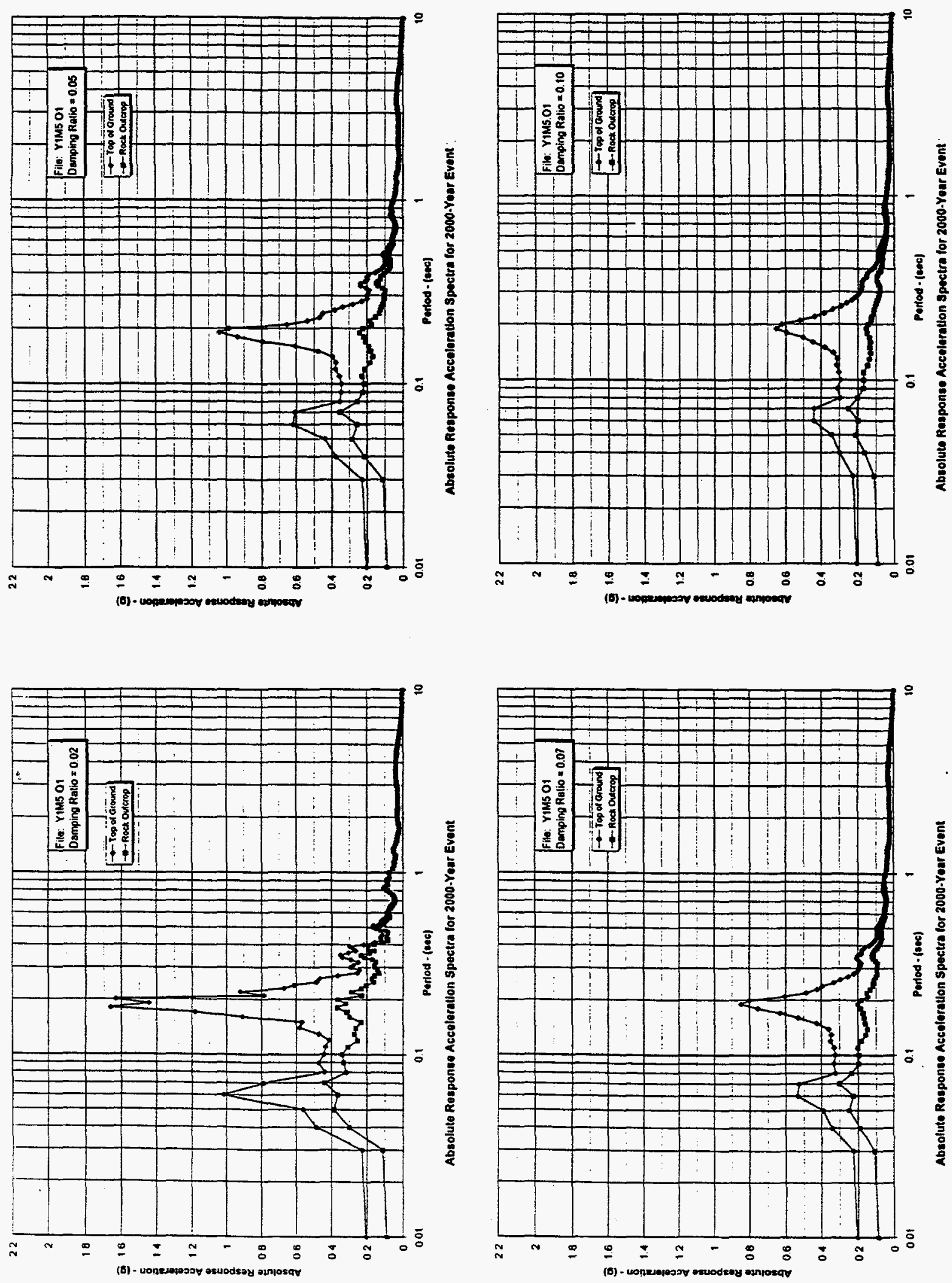

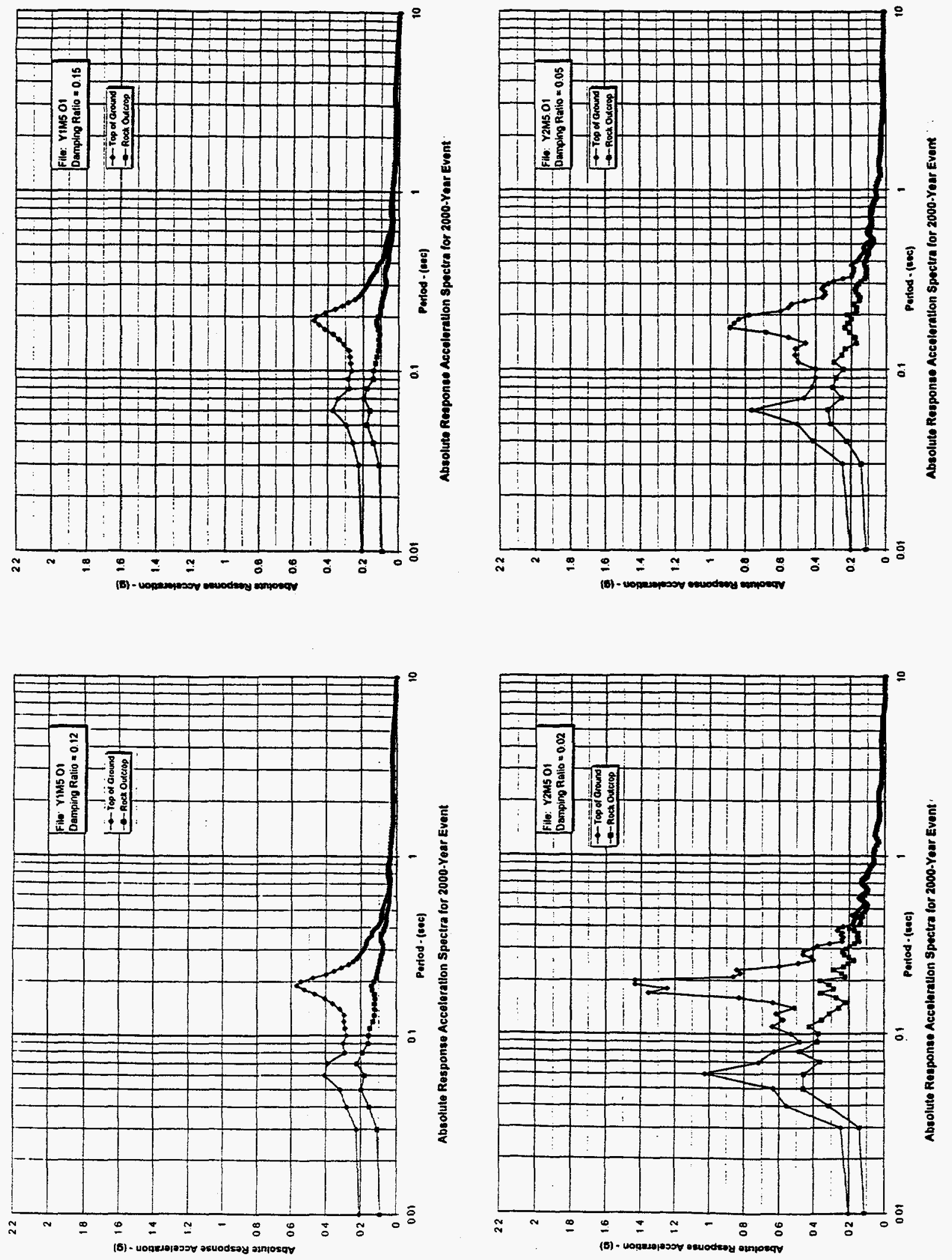

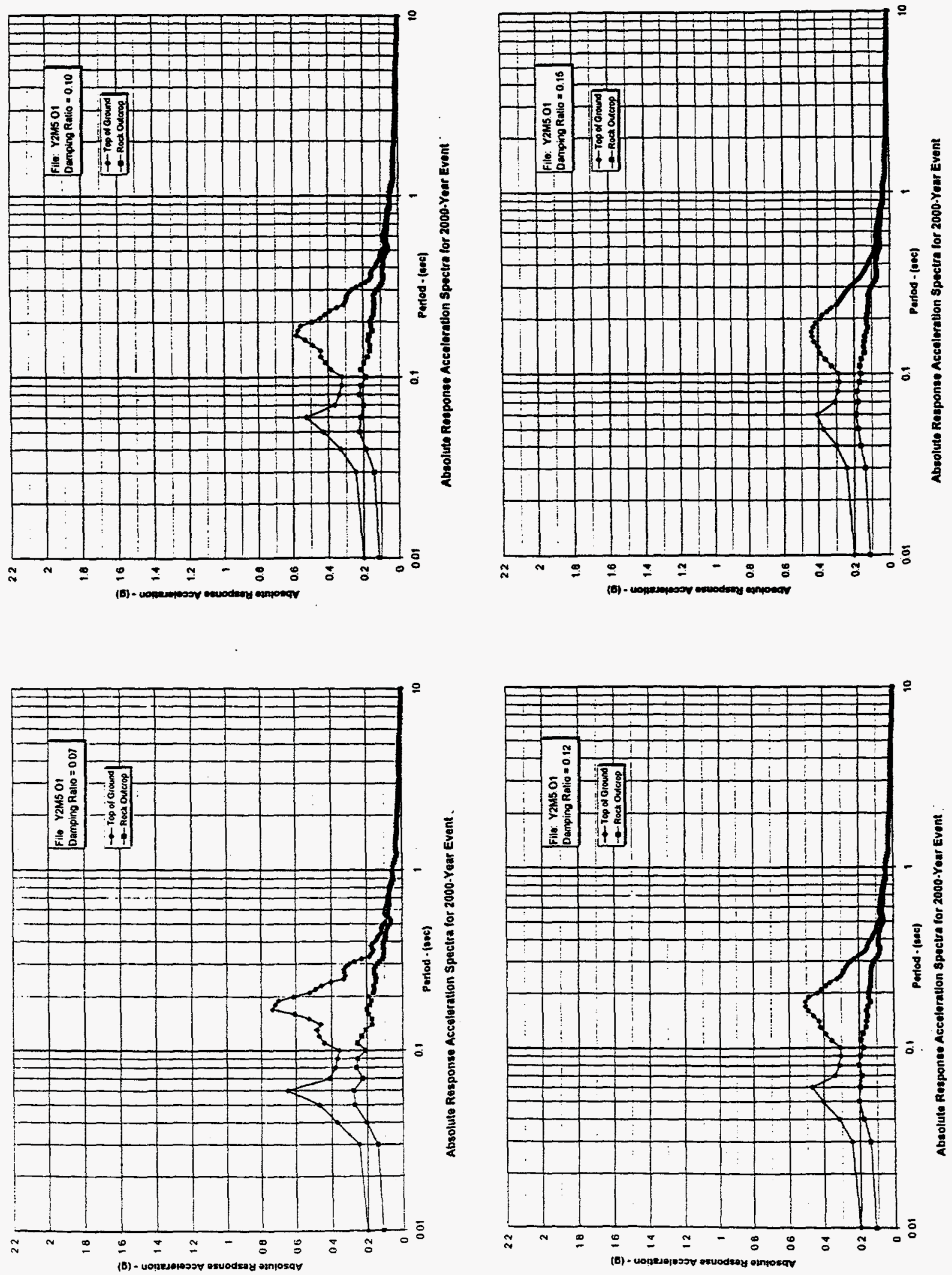

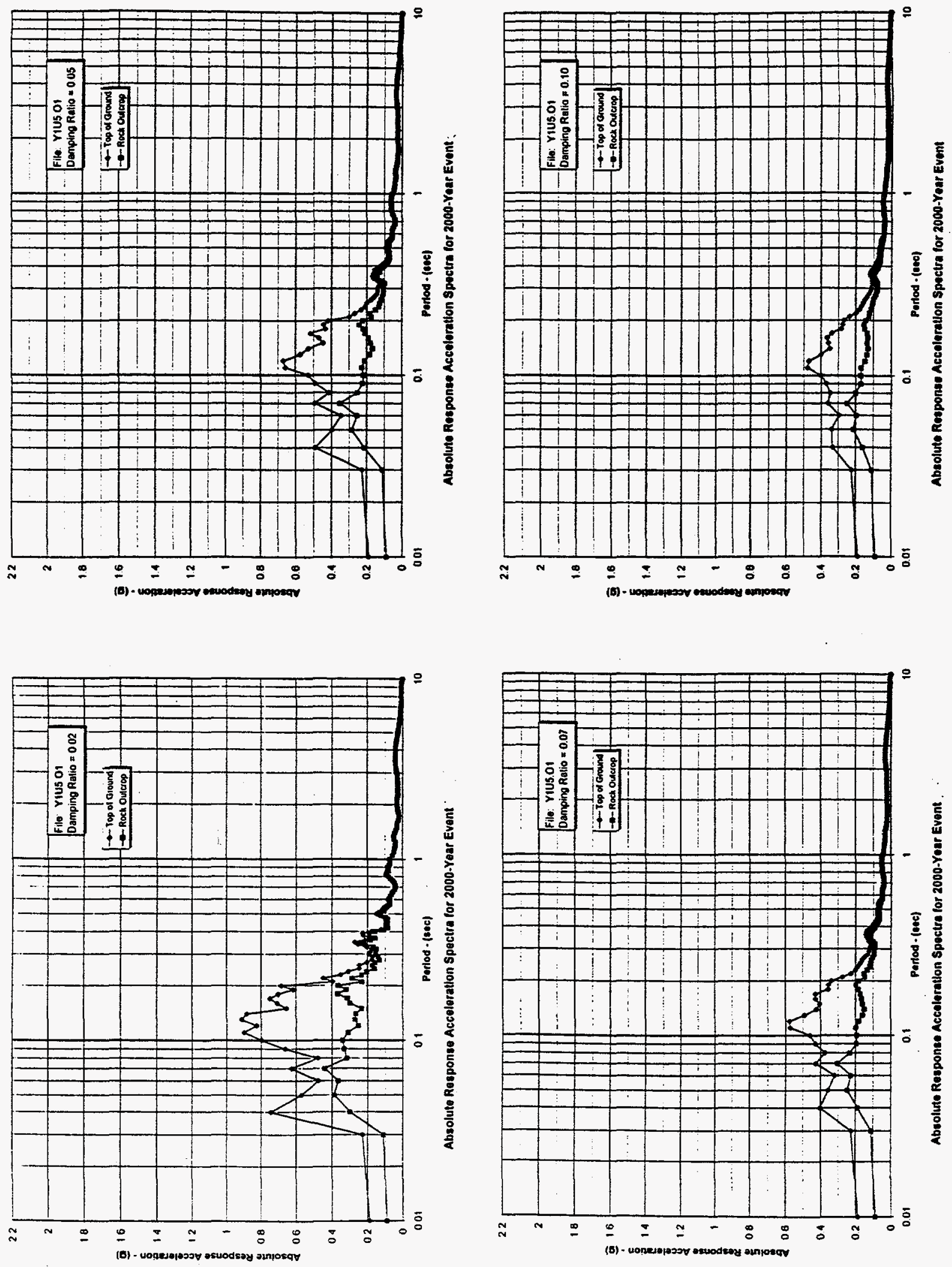

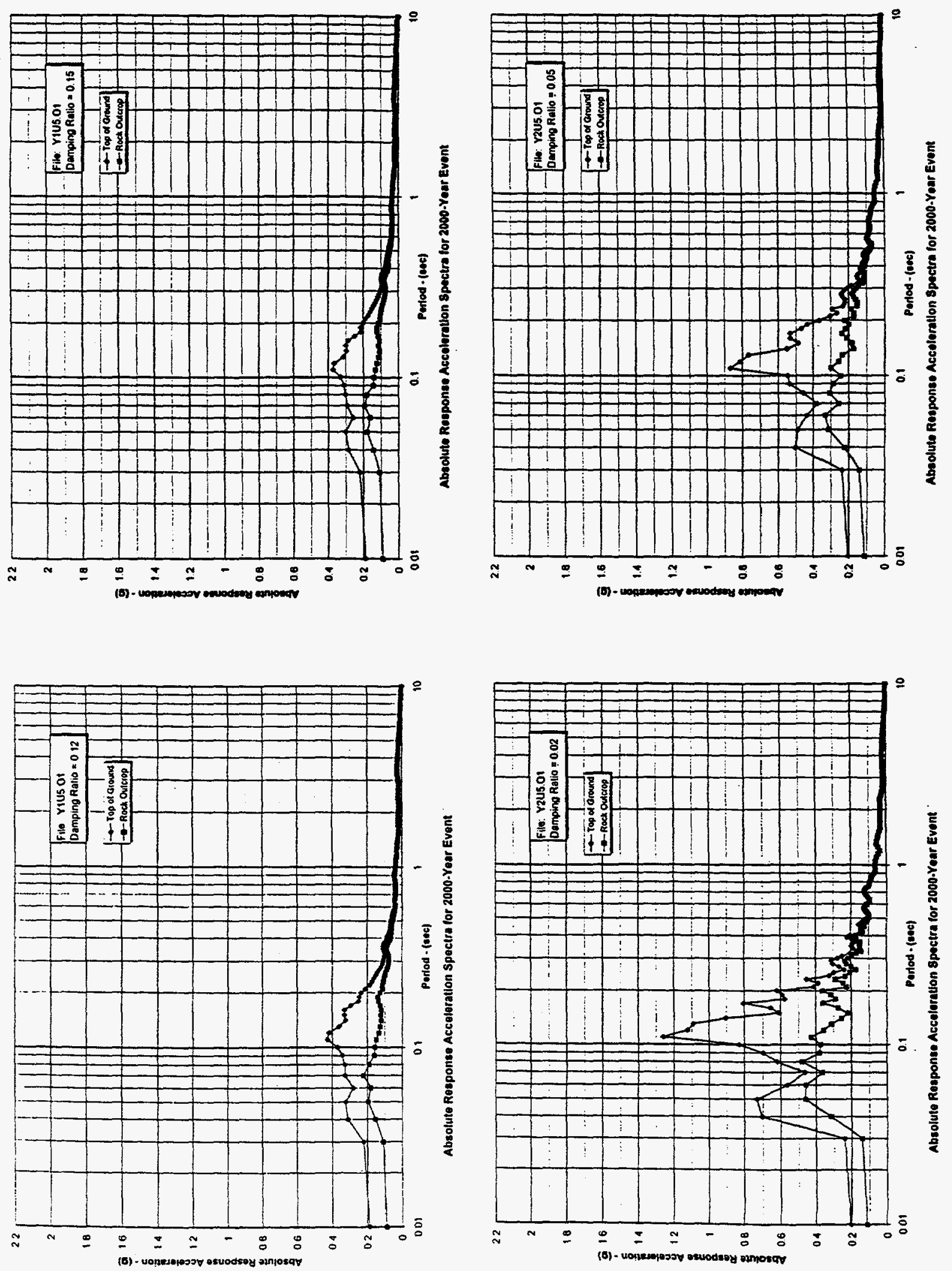

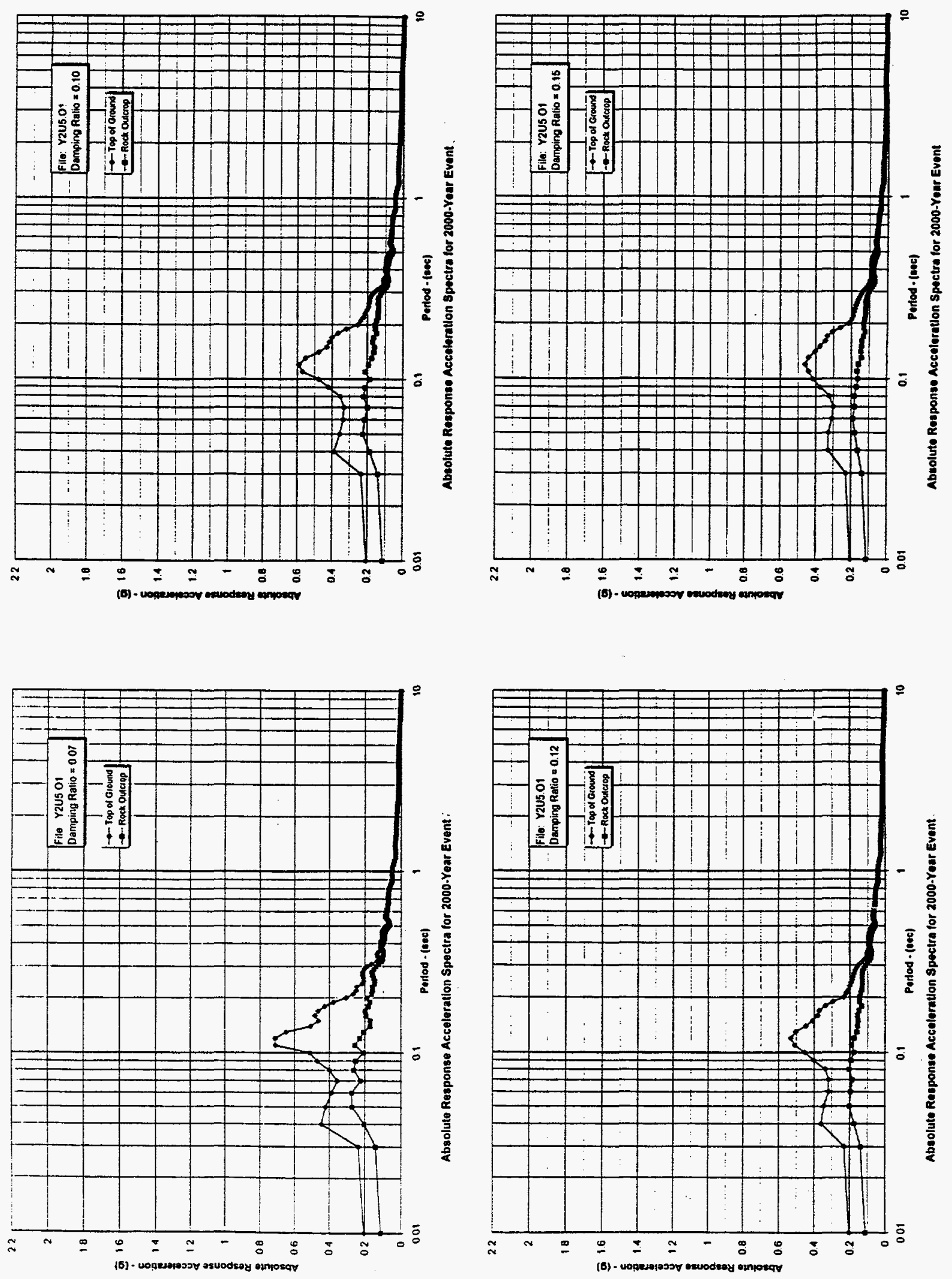

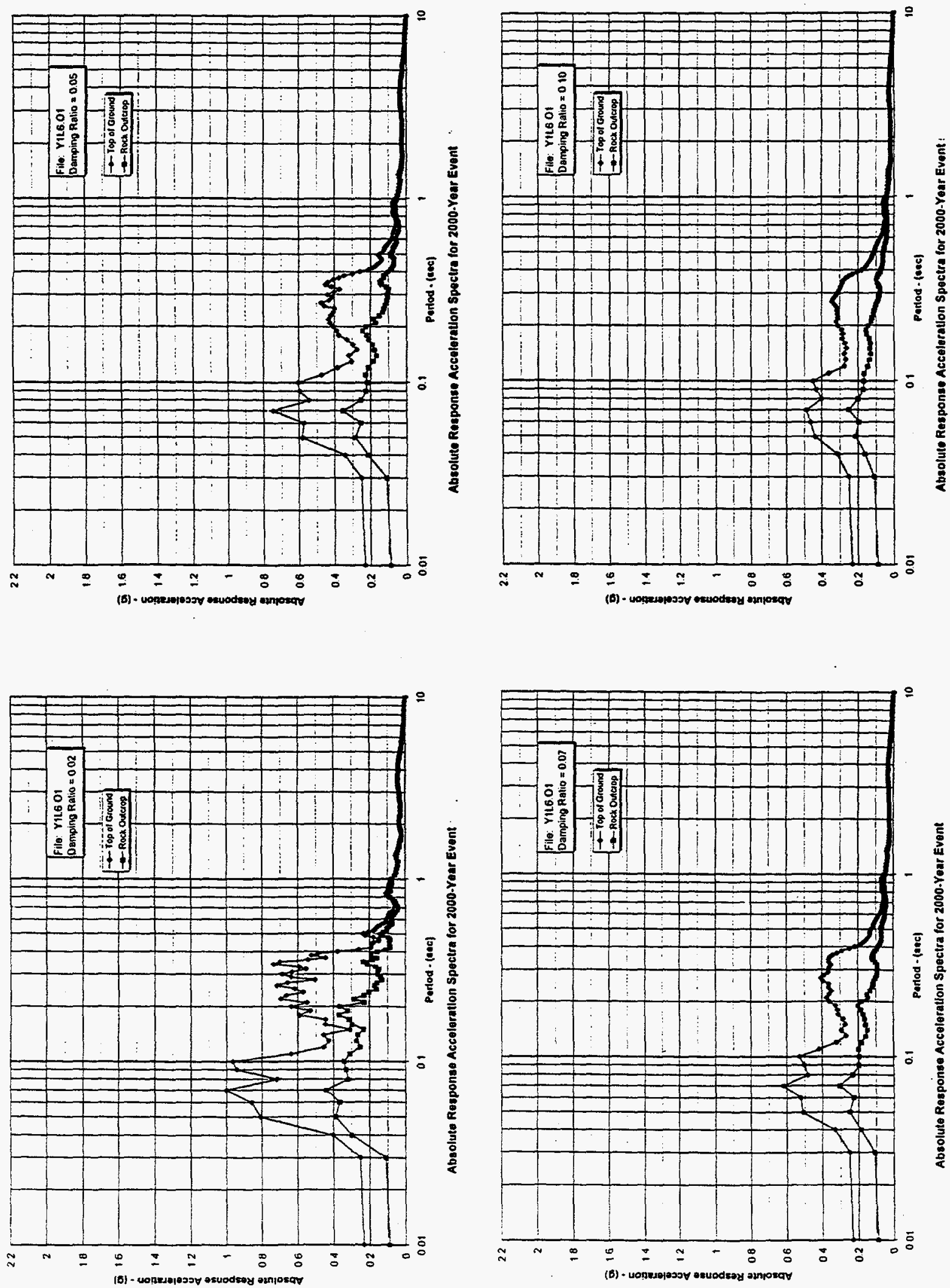

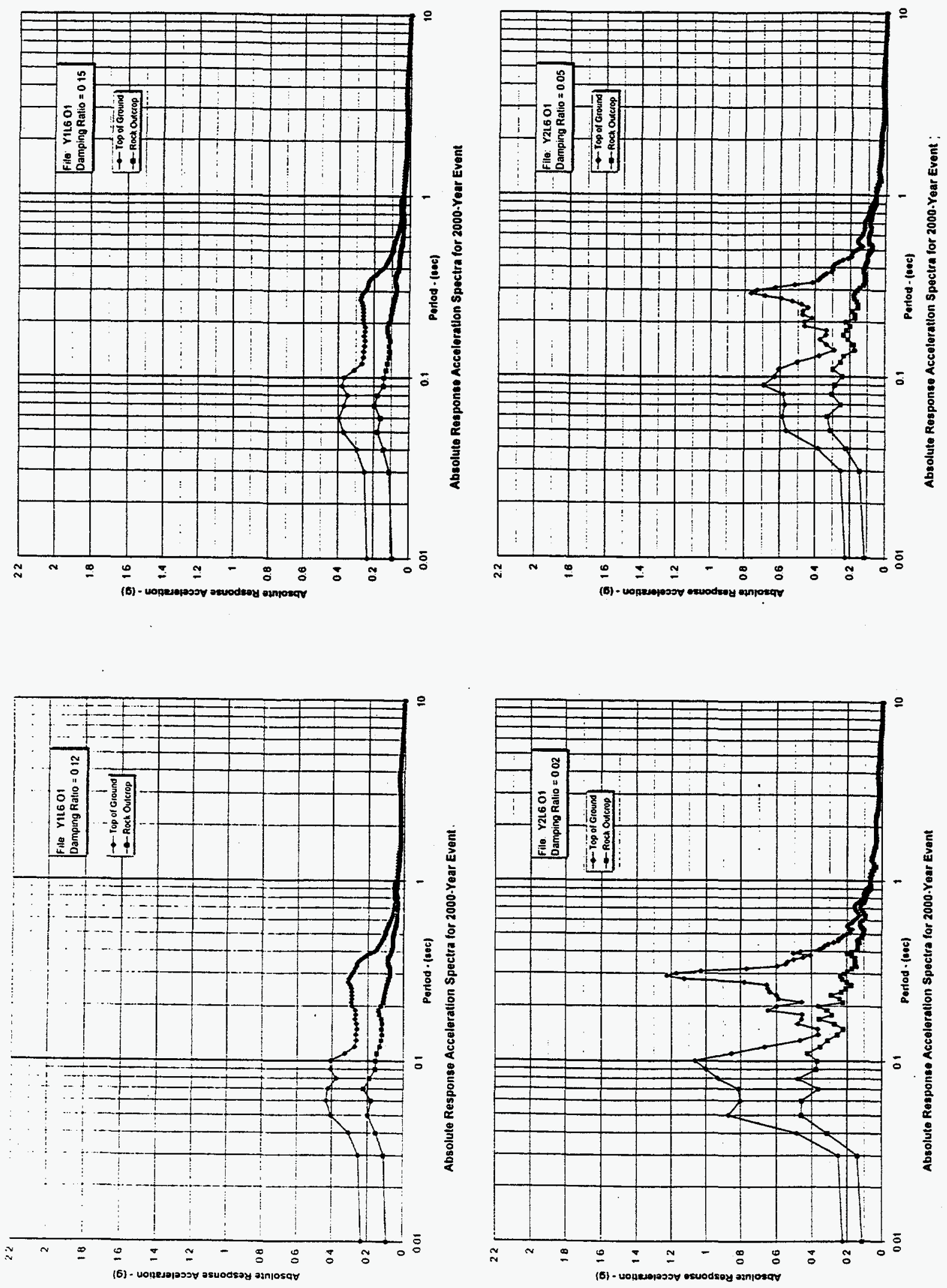

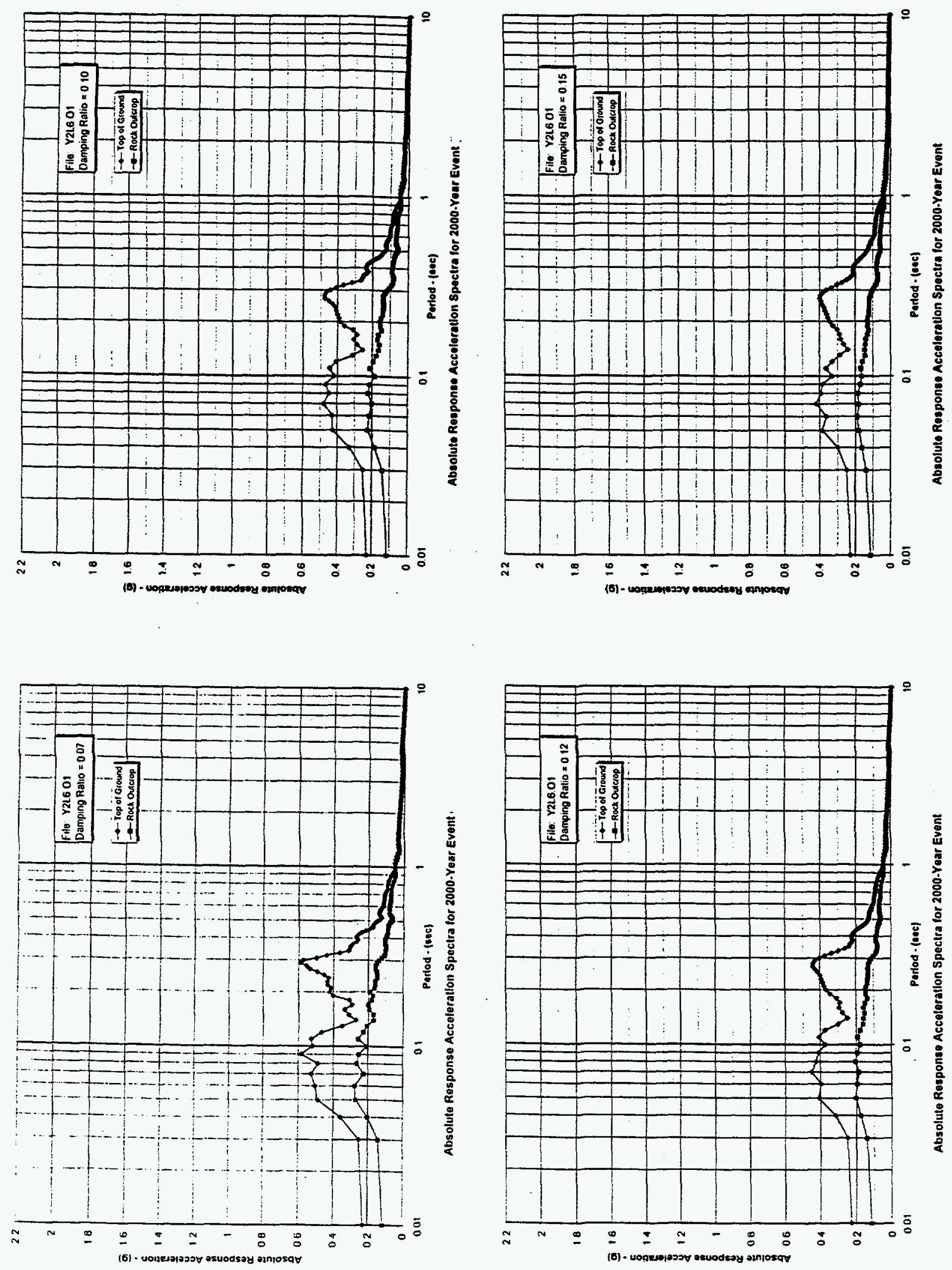

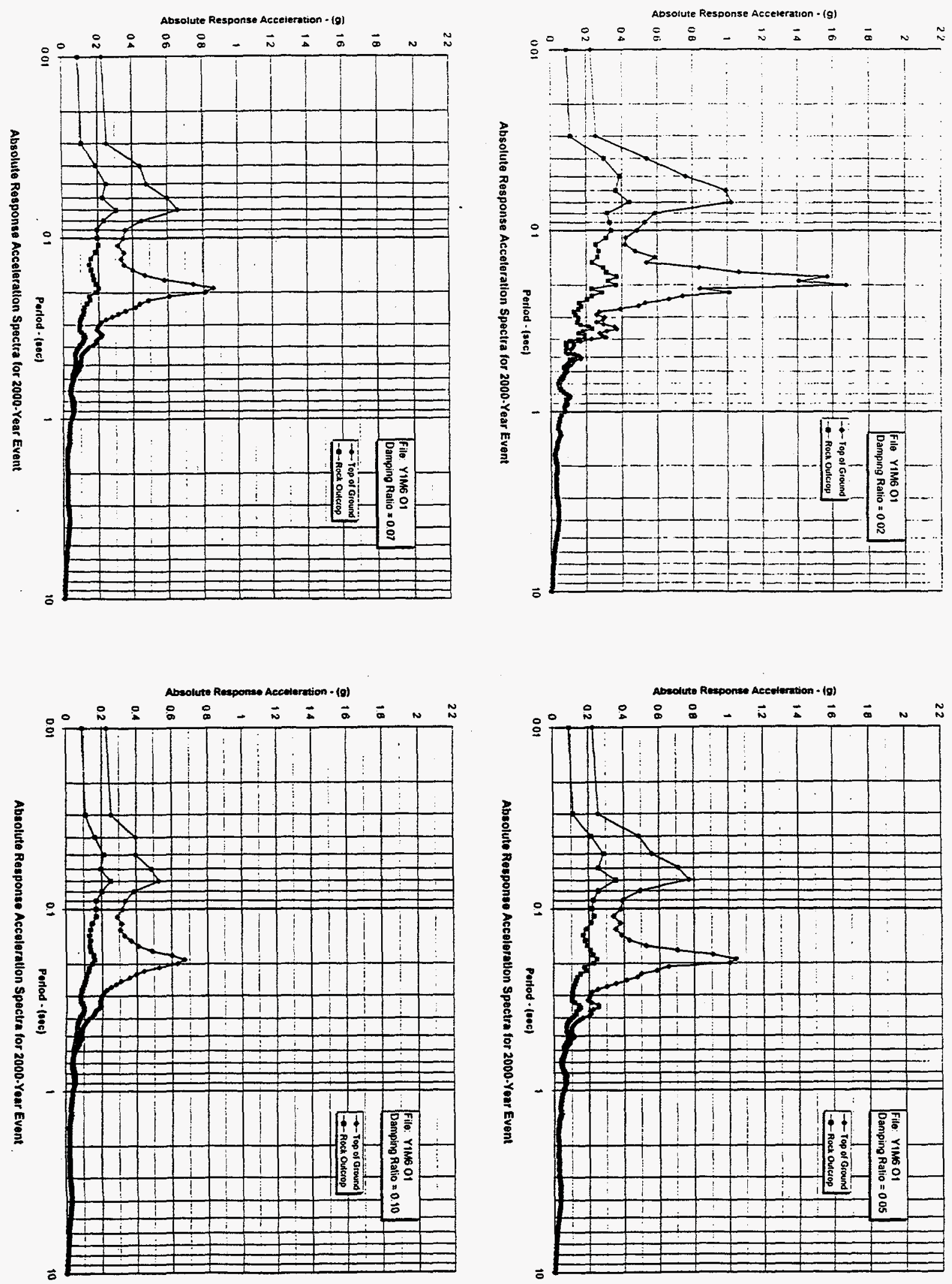

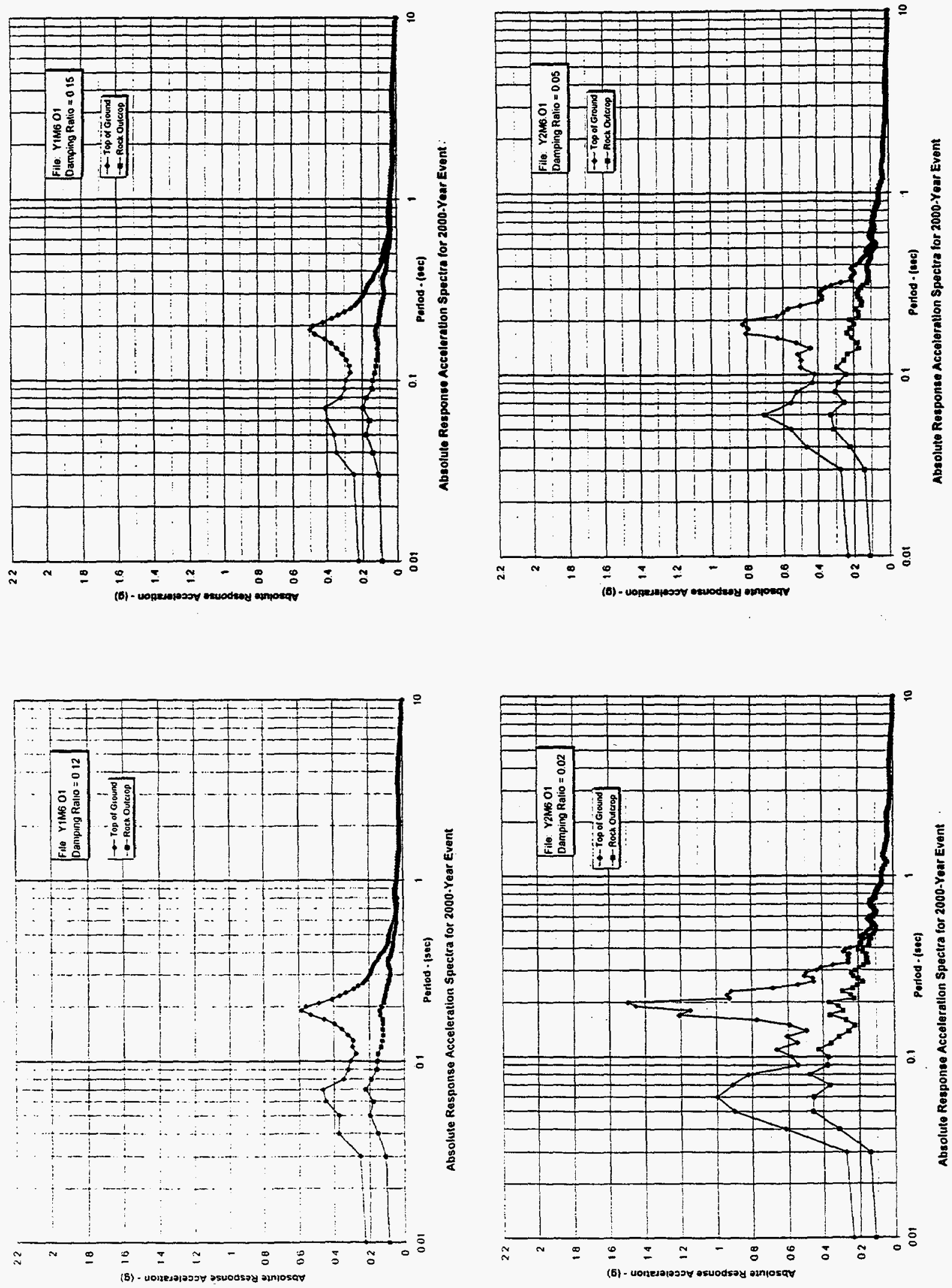

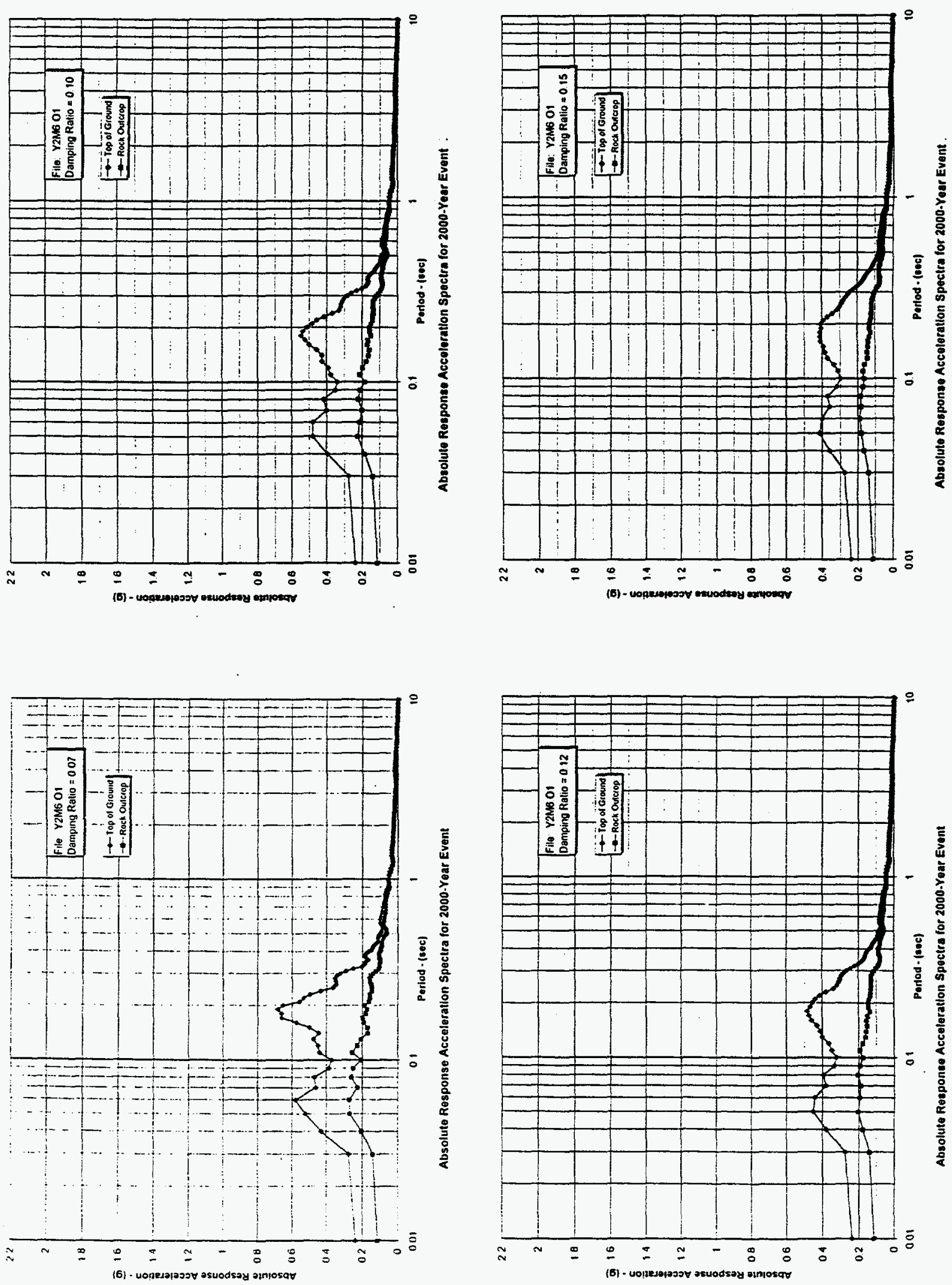

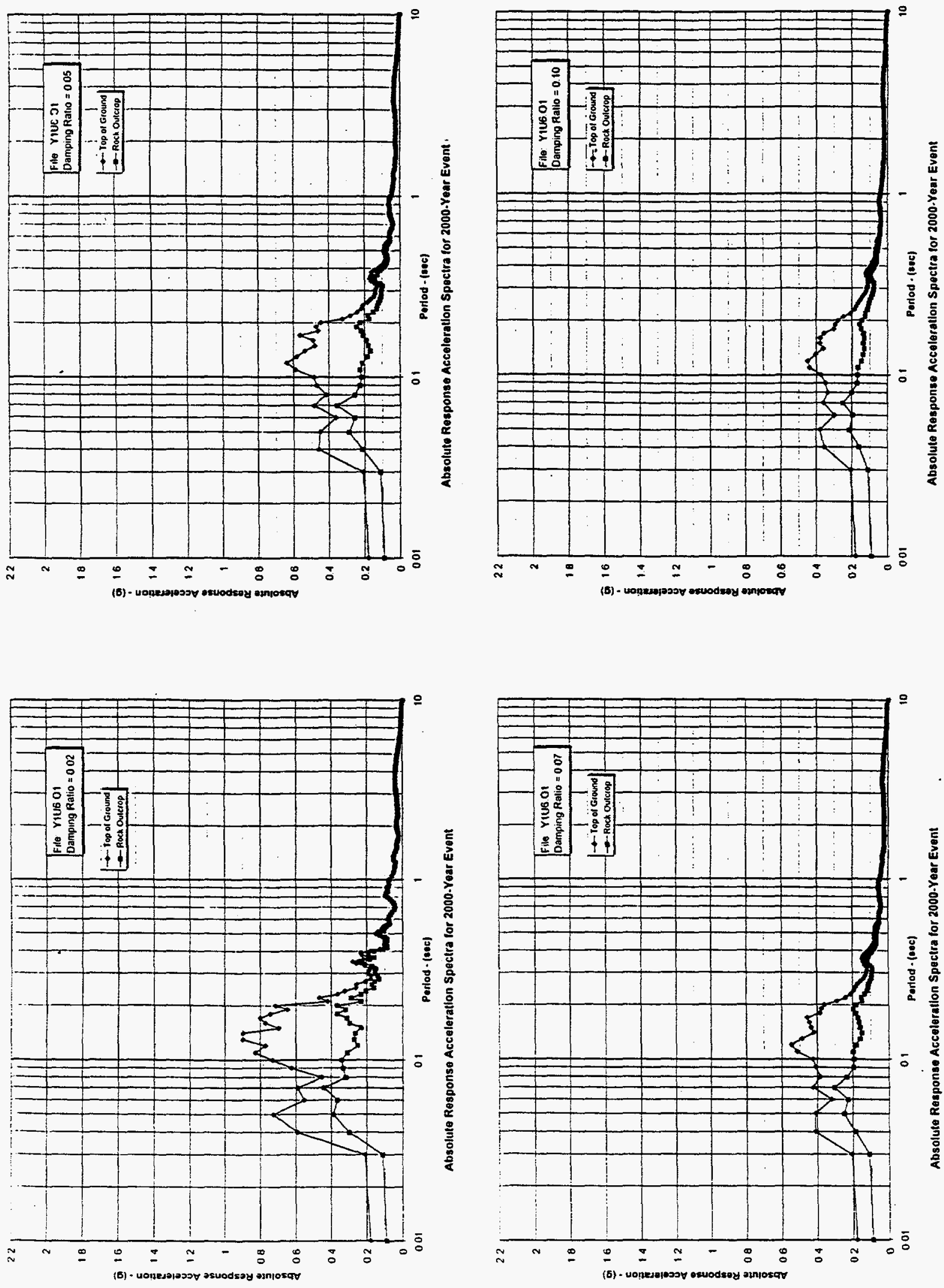

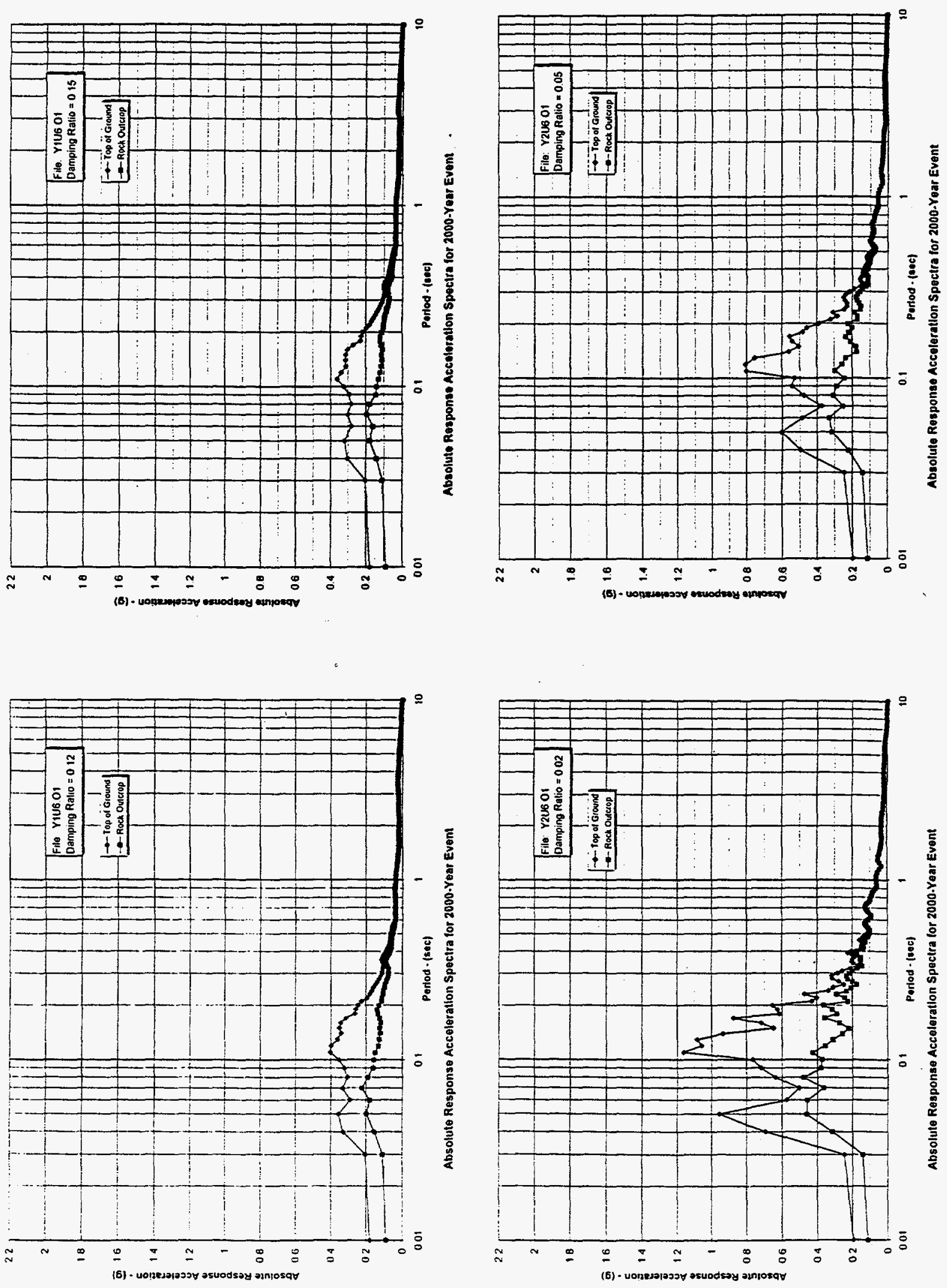

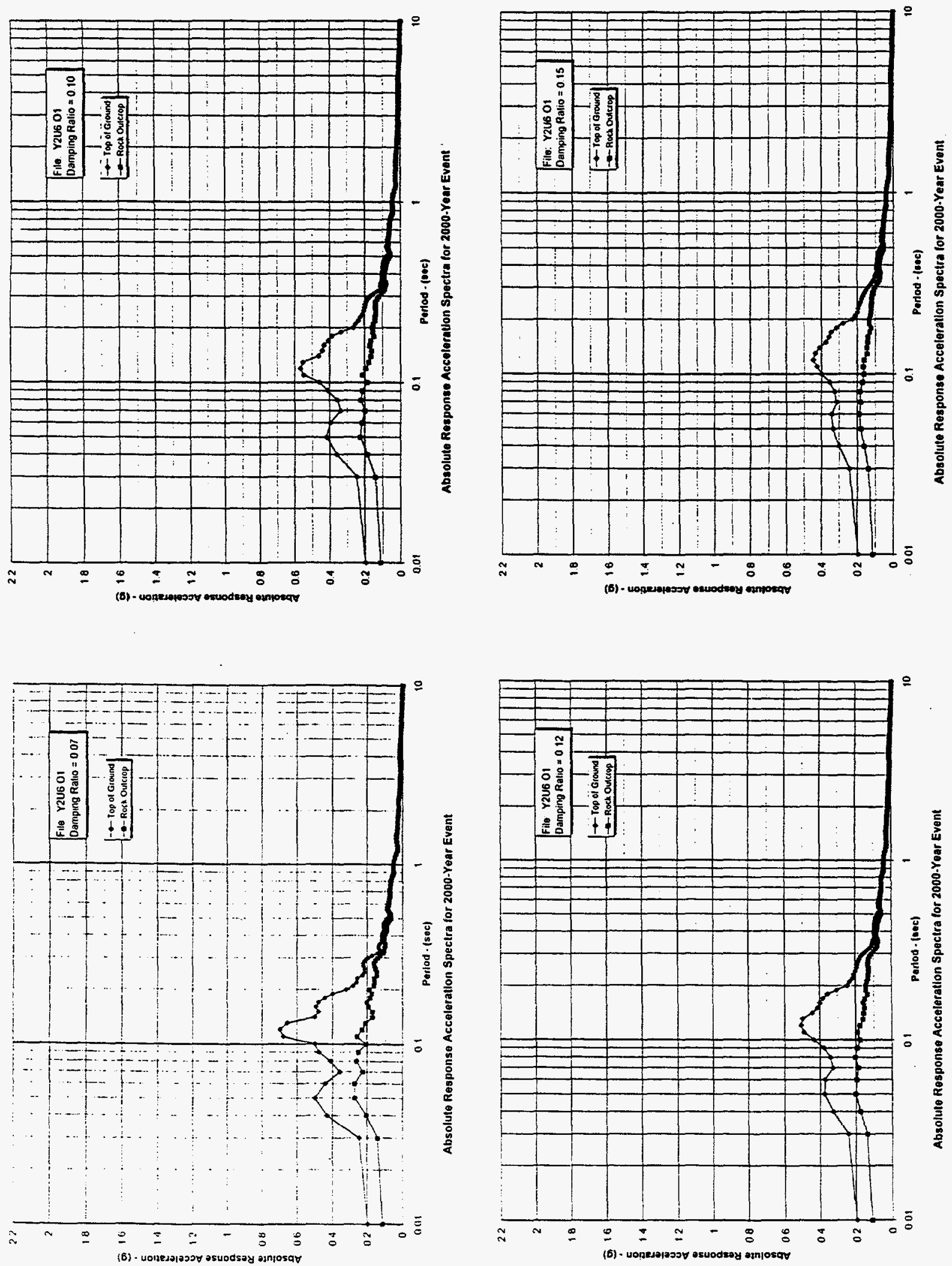

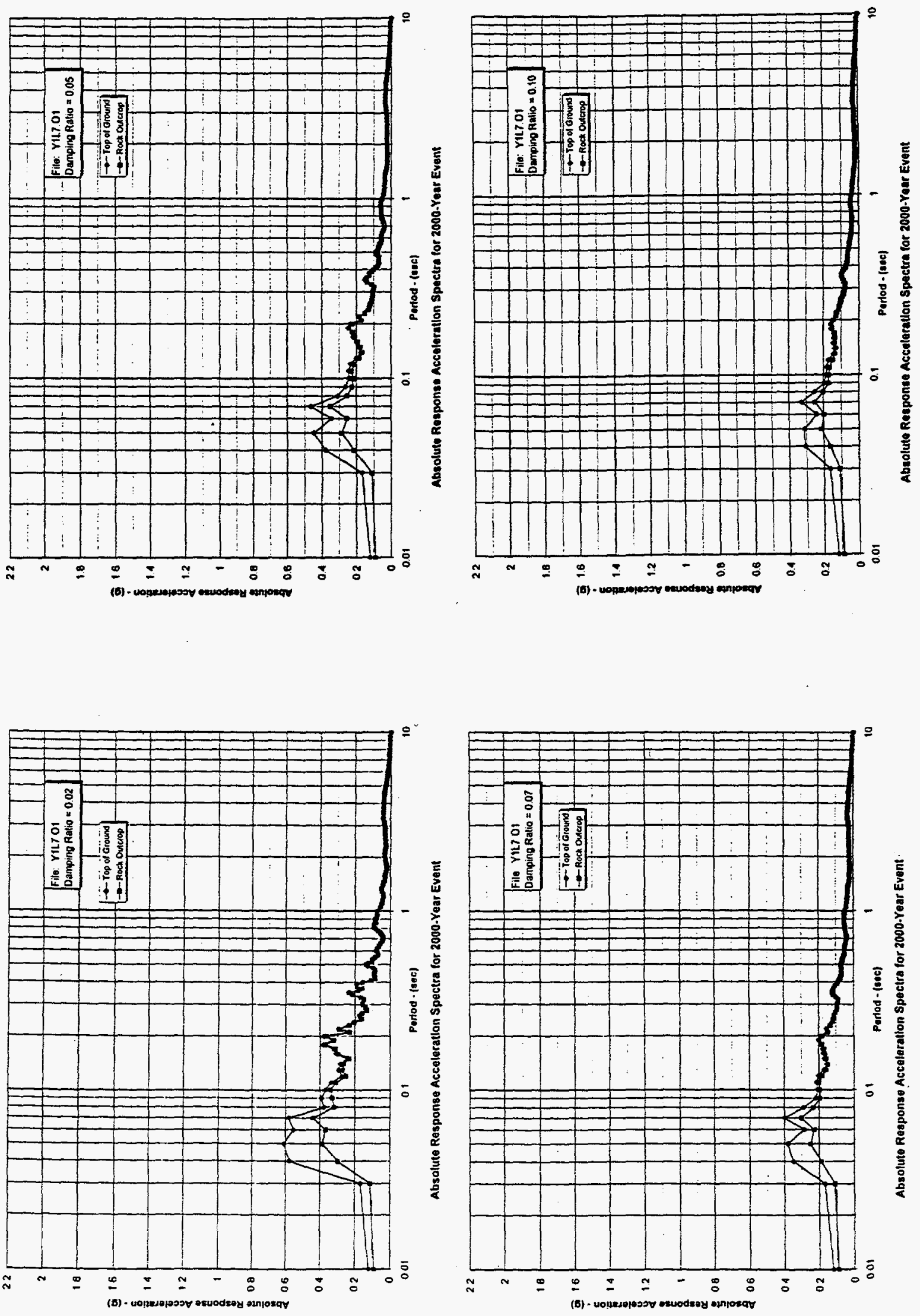

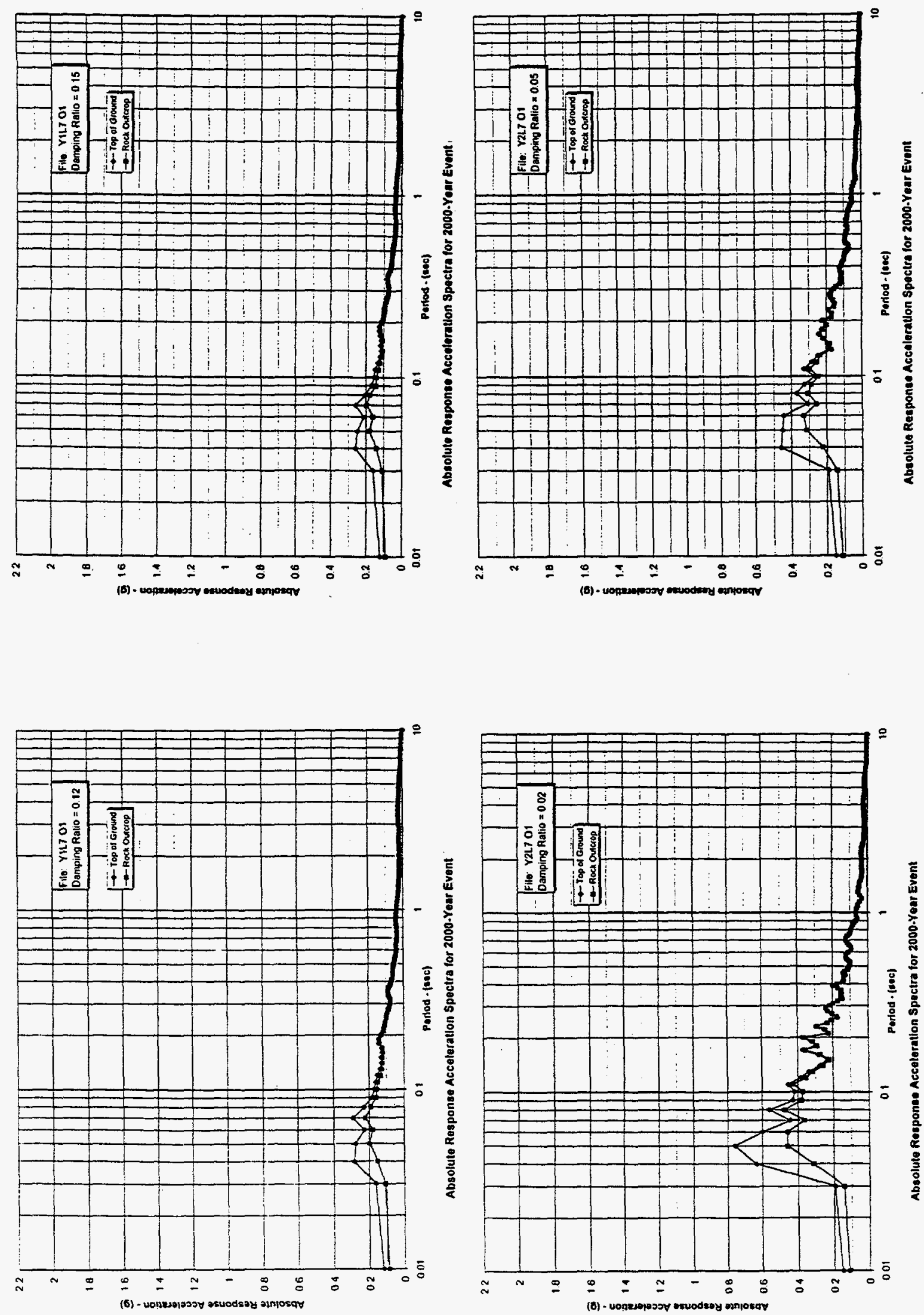

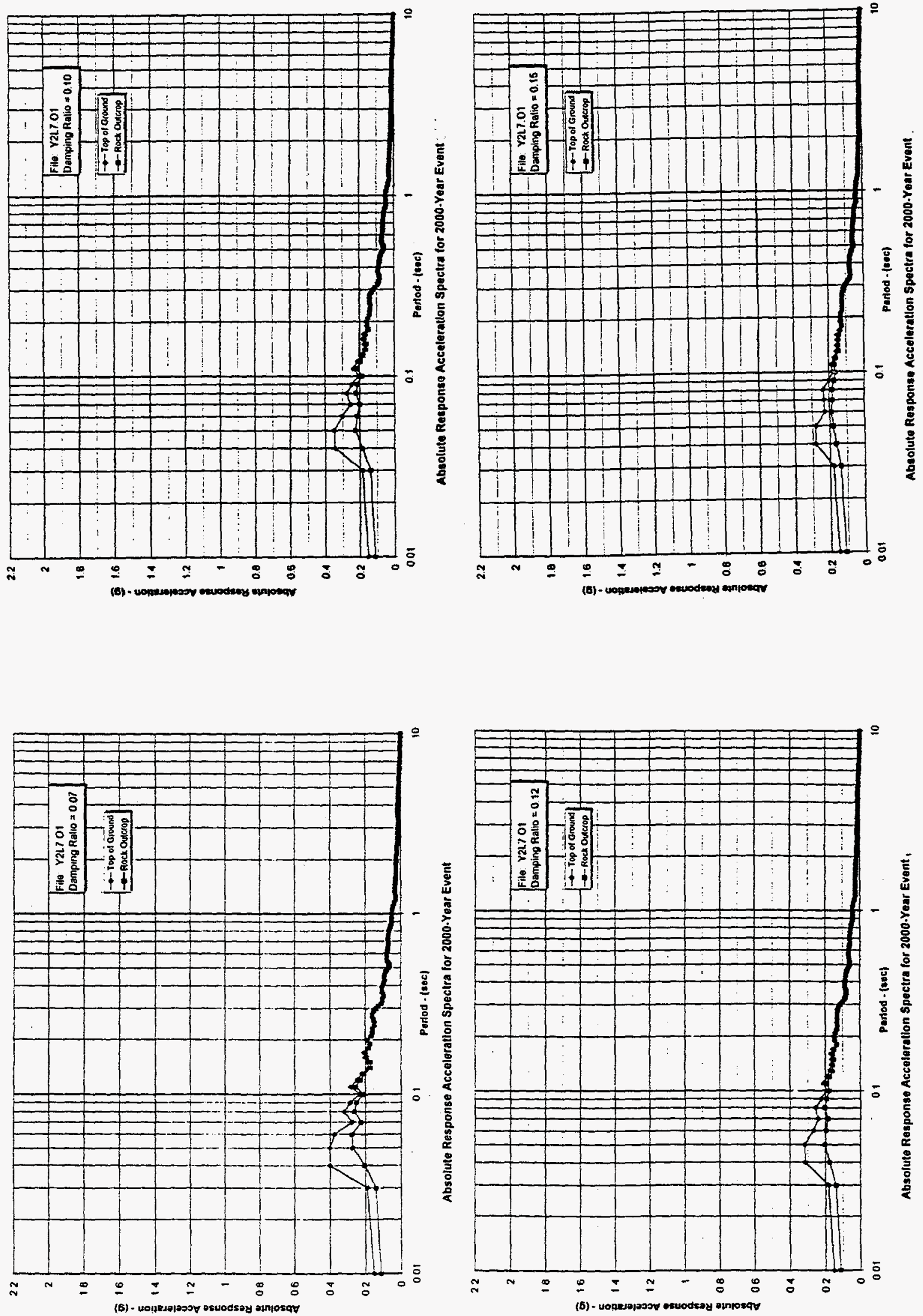

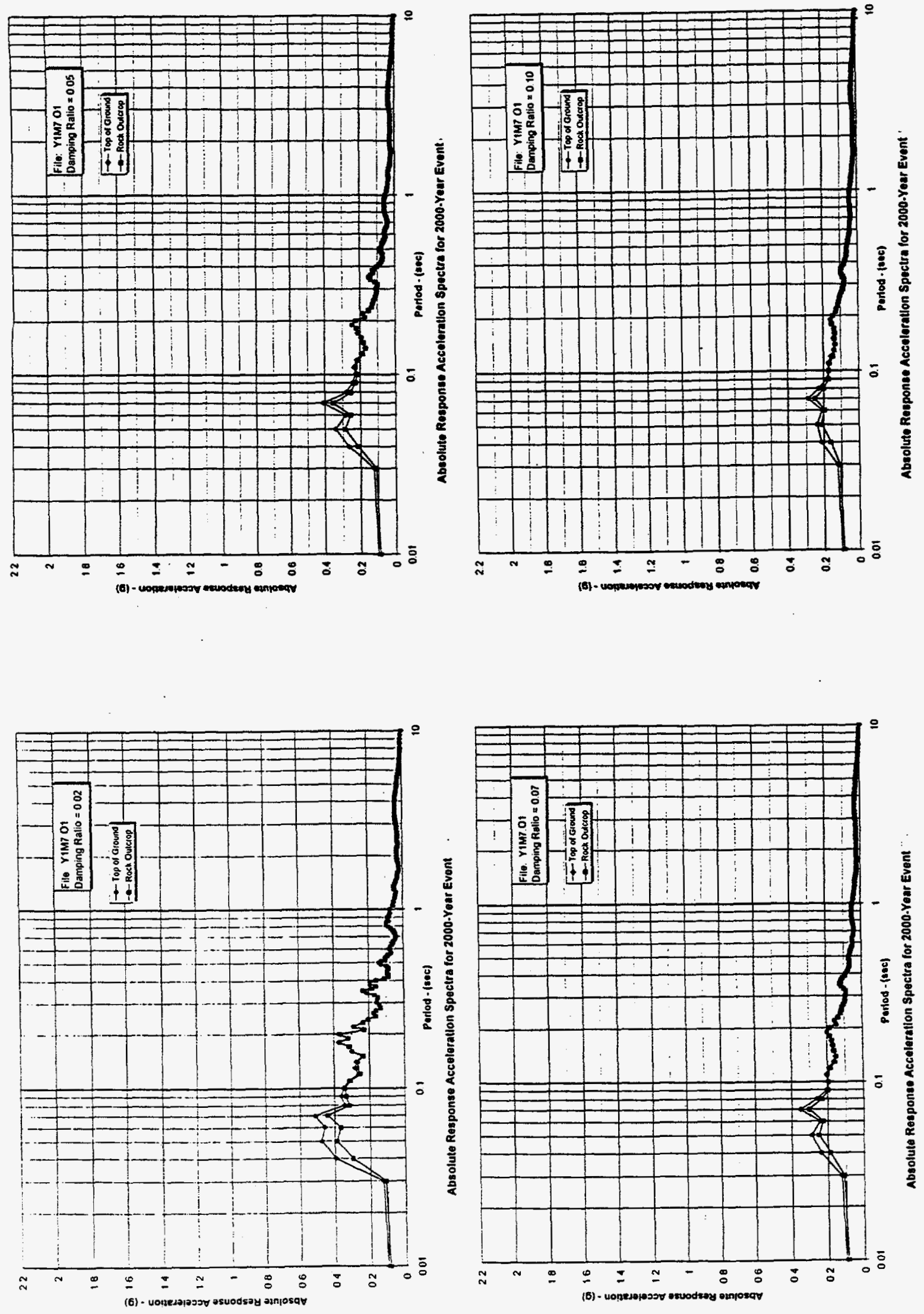

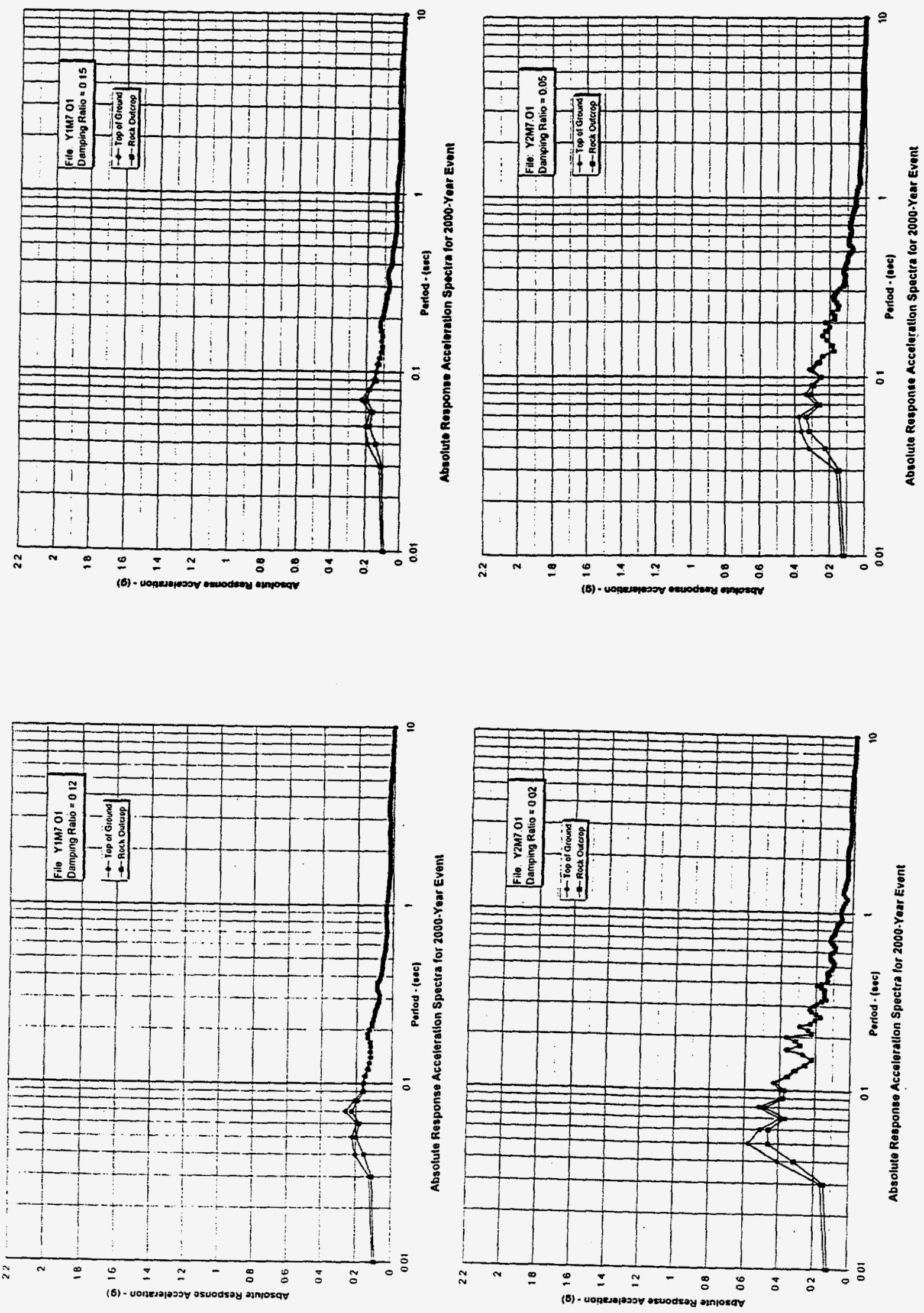

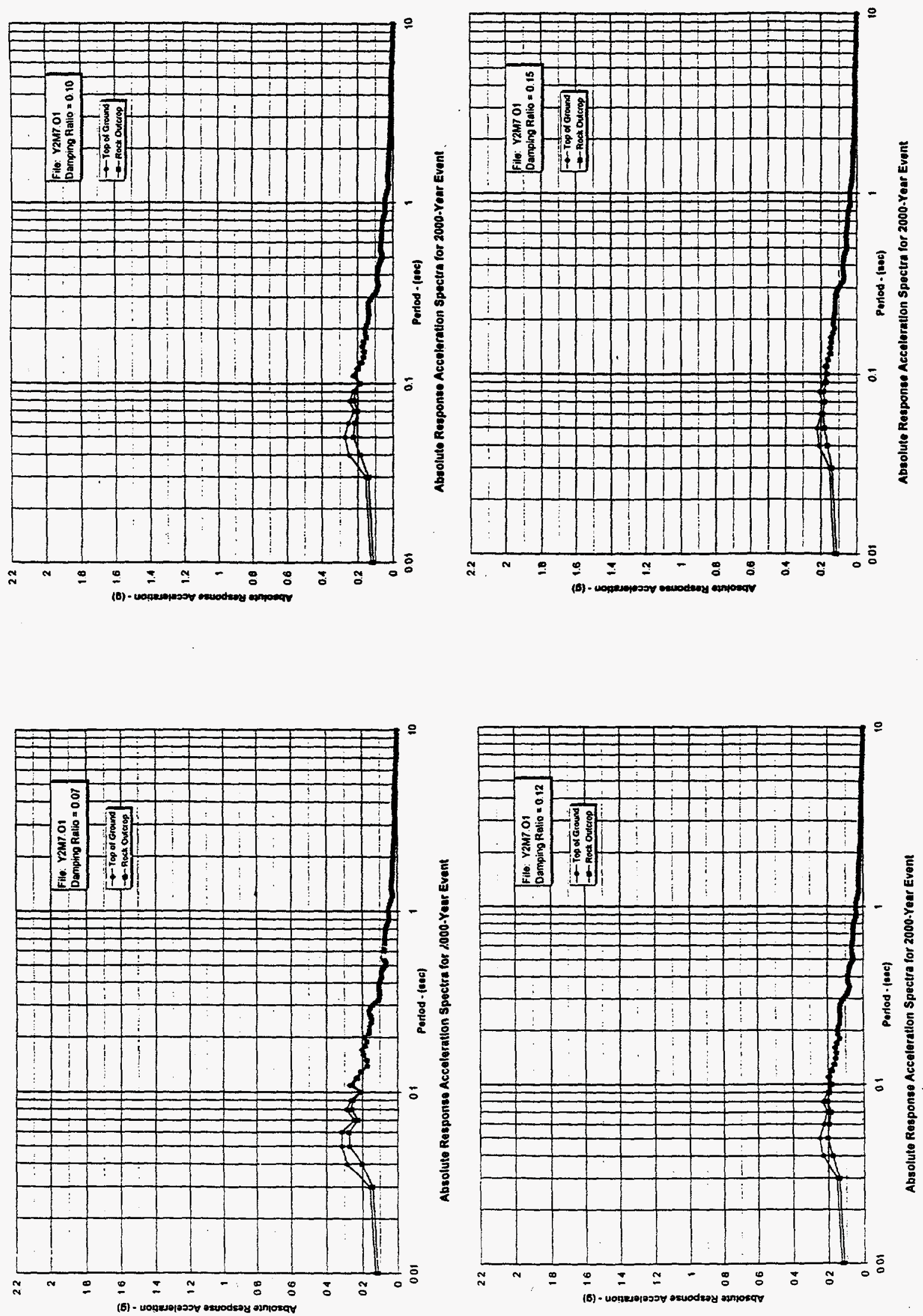

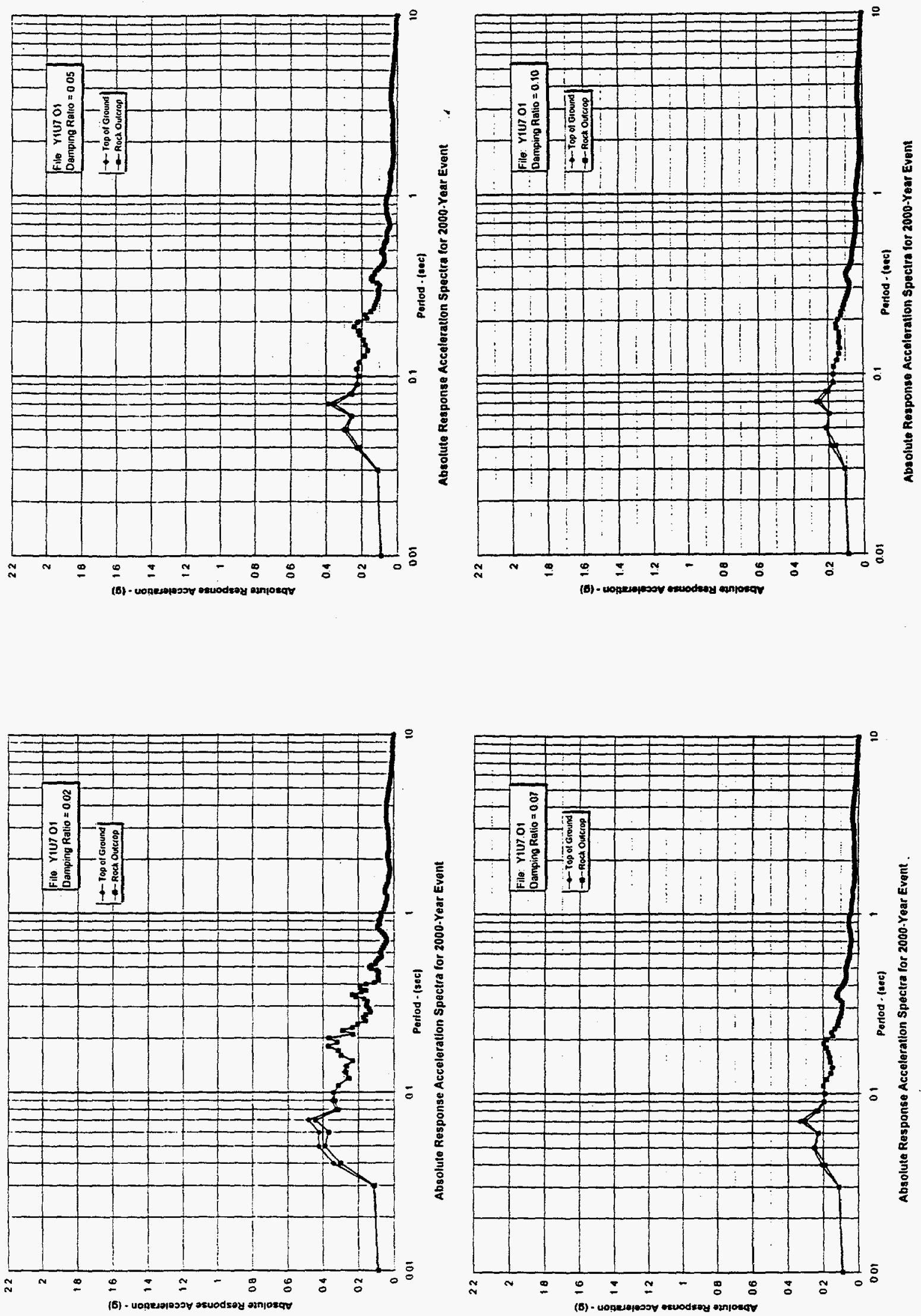

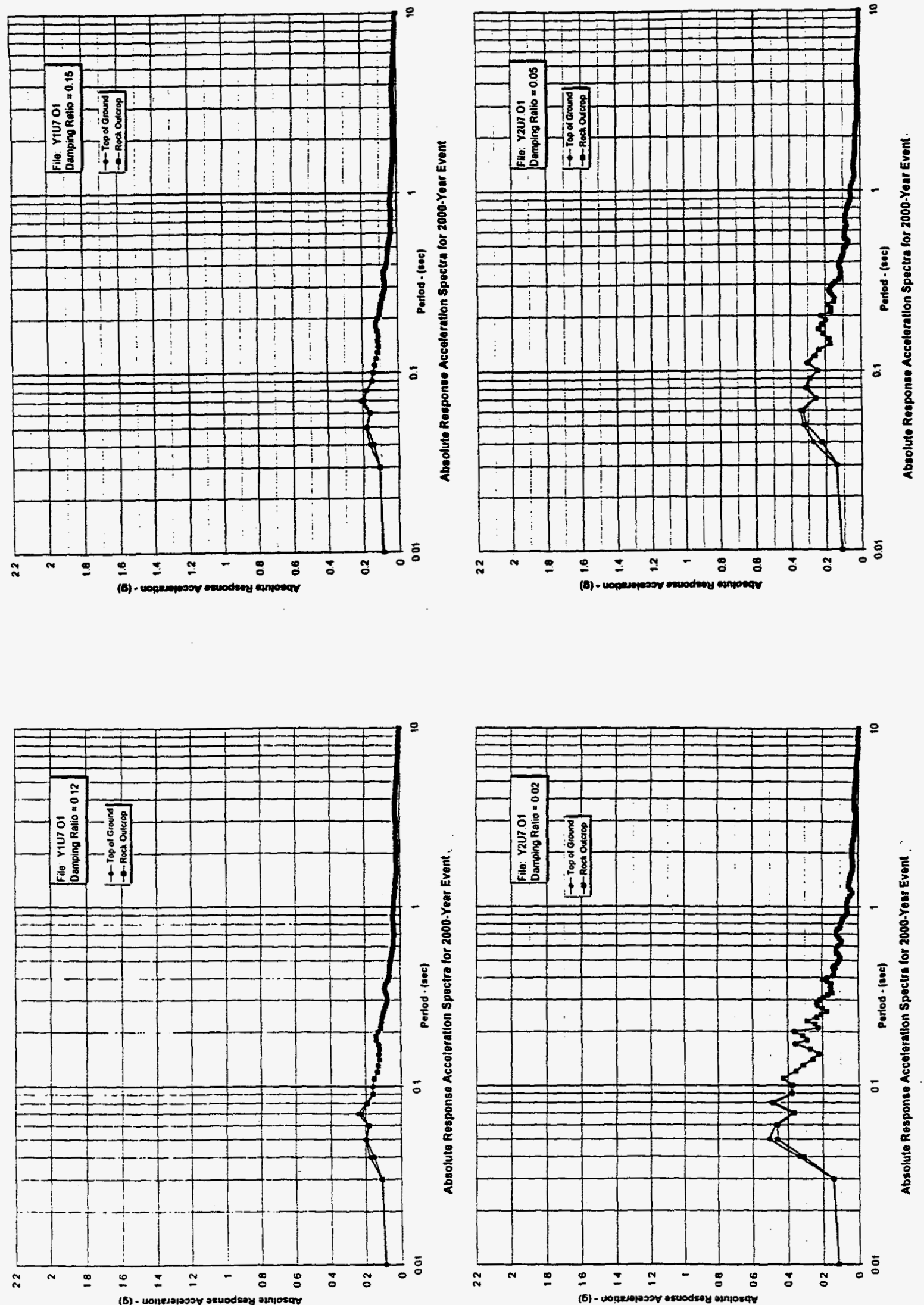

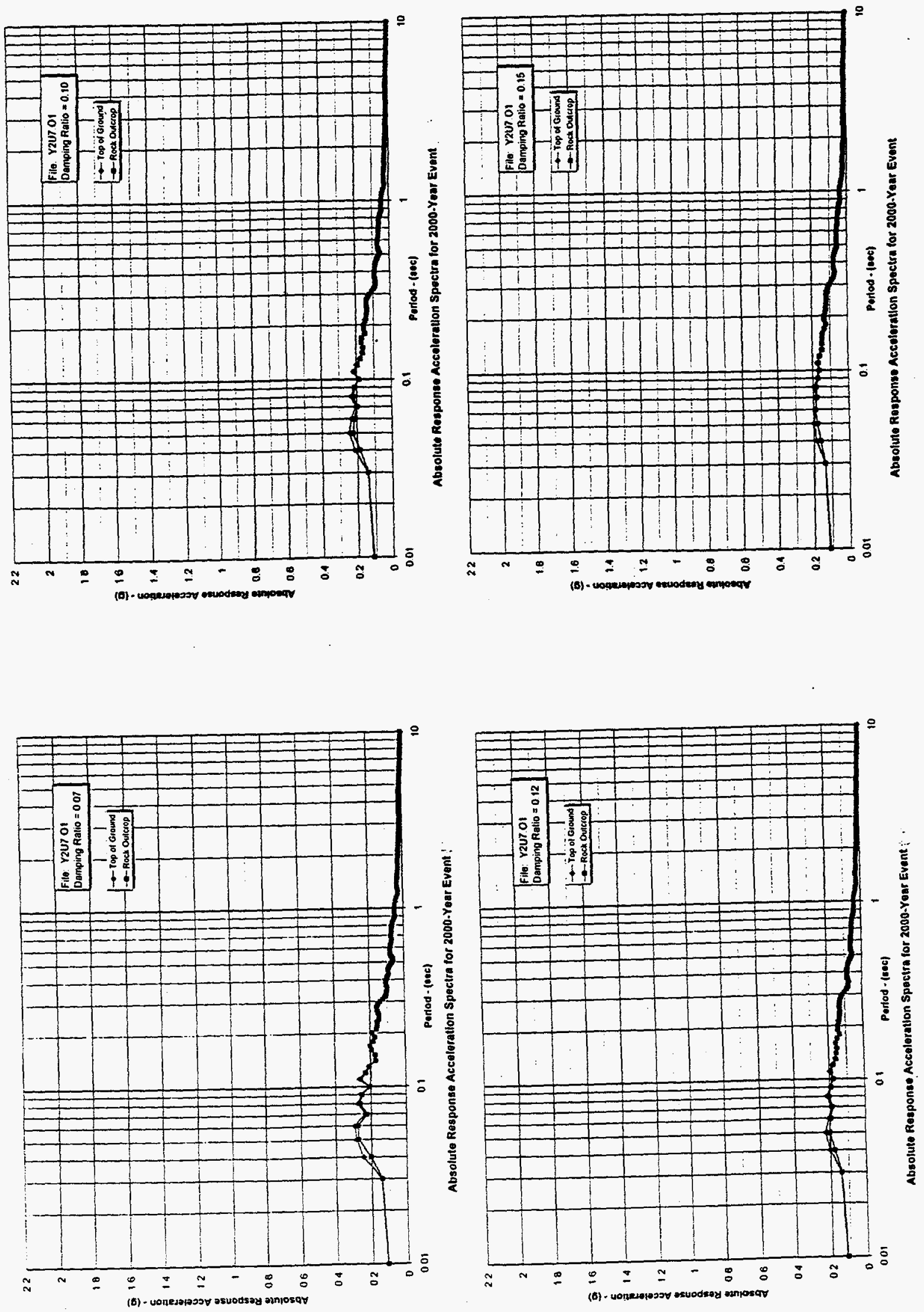
A2 Amplification Ratio Plots 

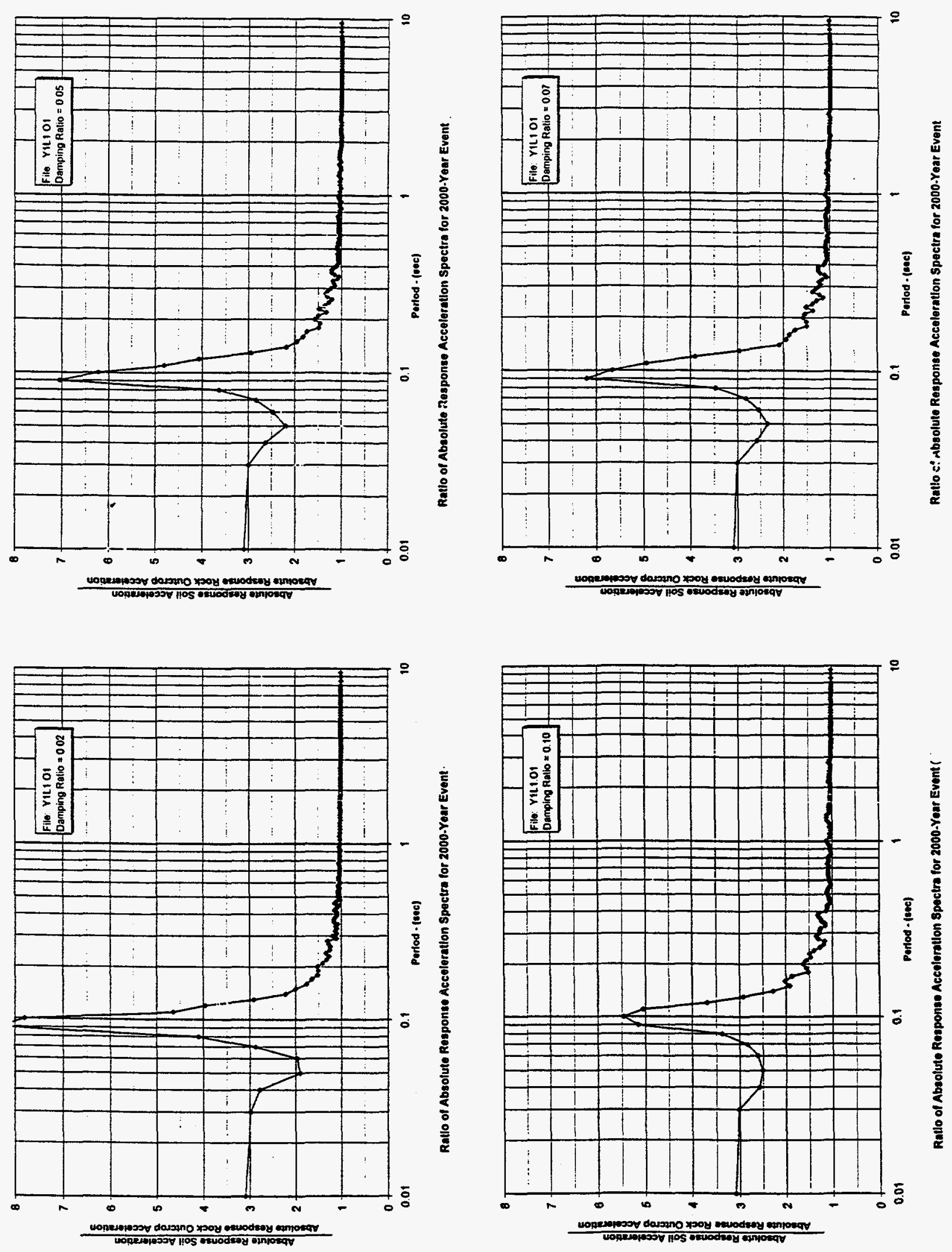

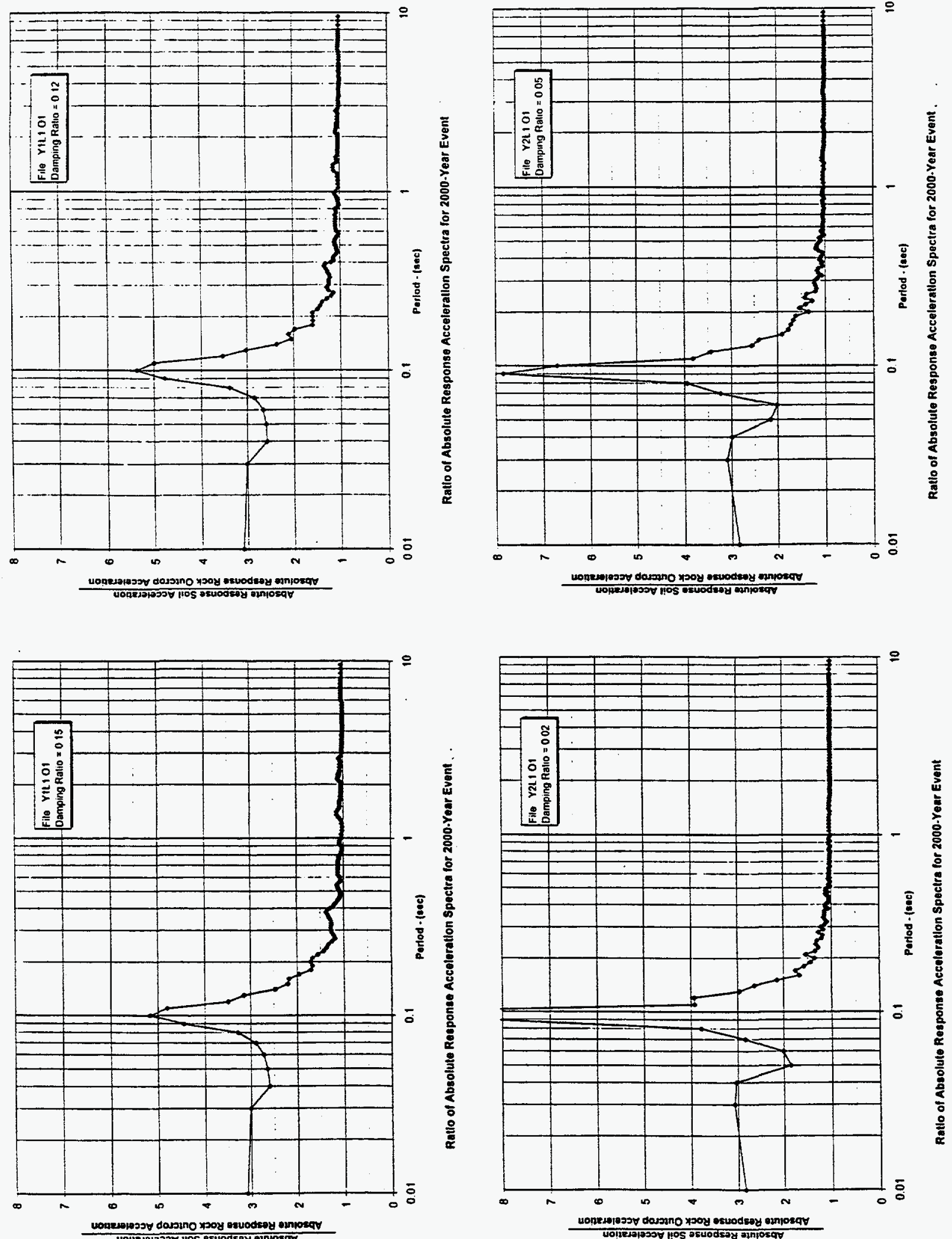

A2-2 


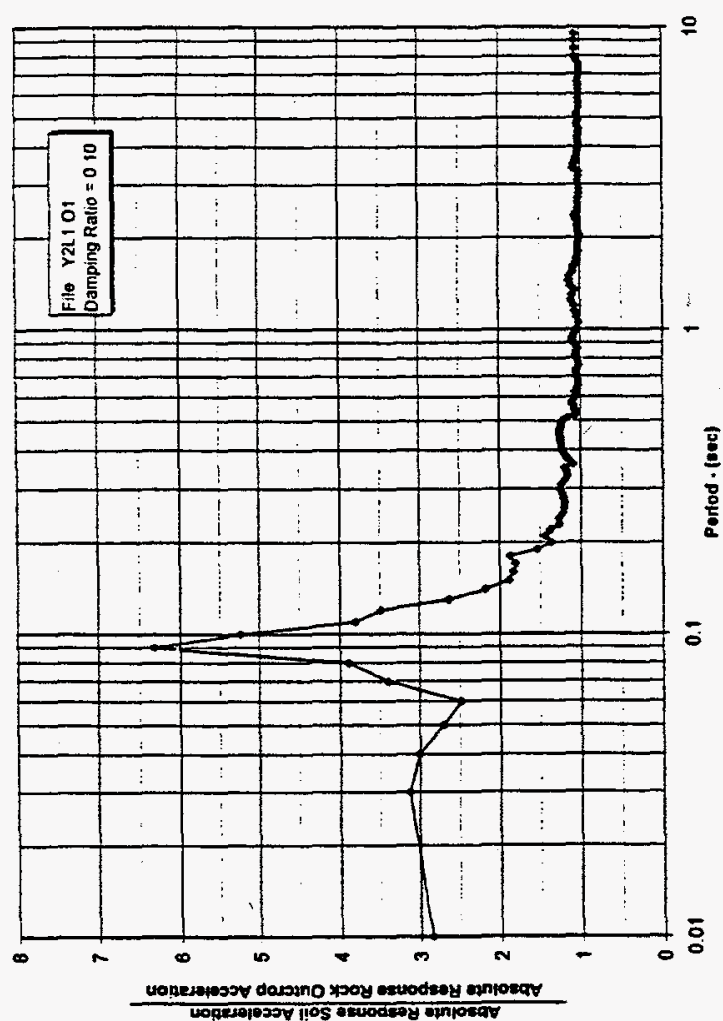

wopsoper
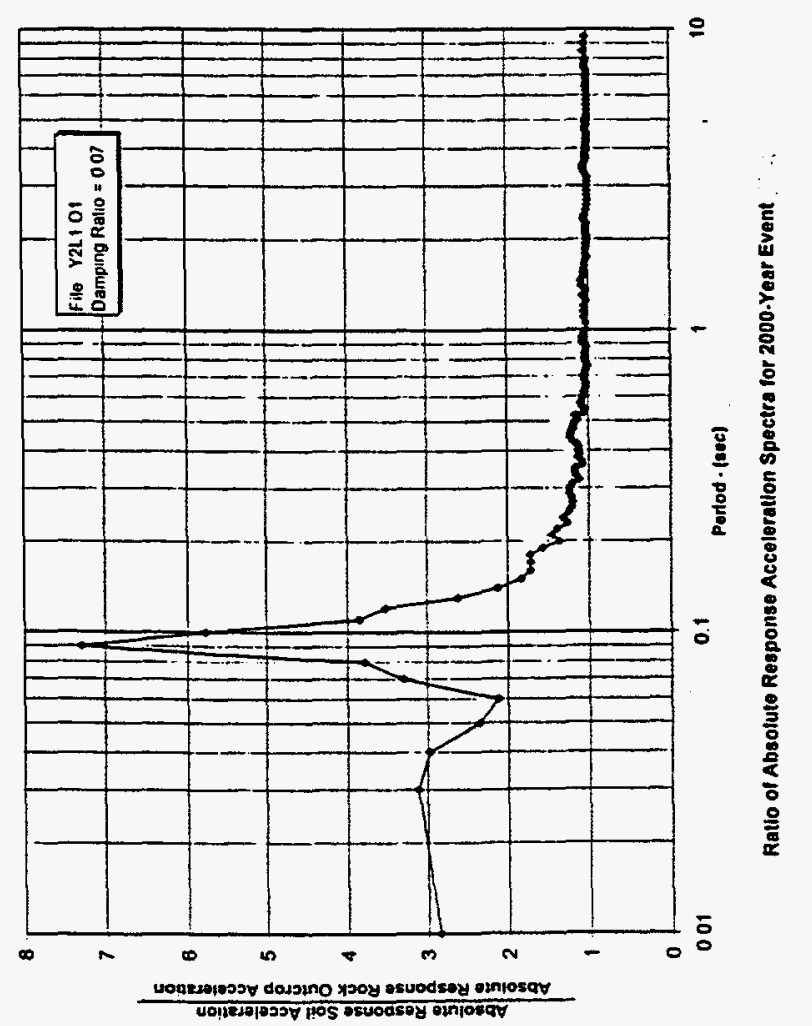

2

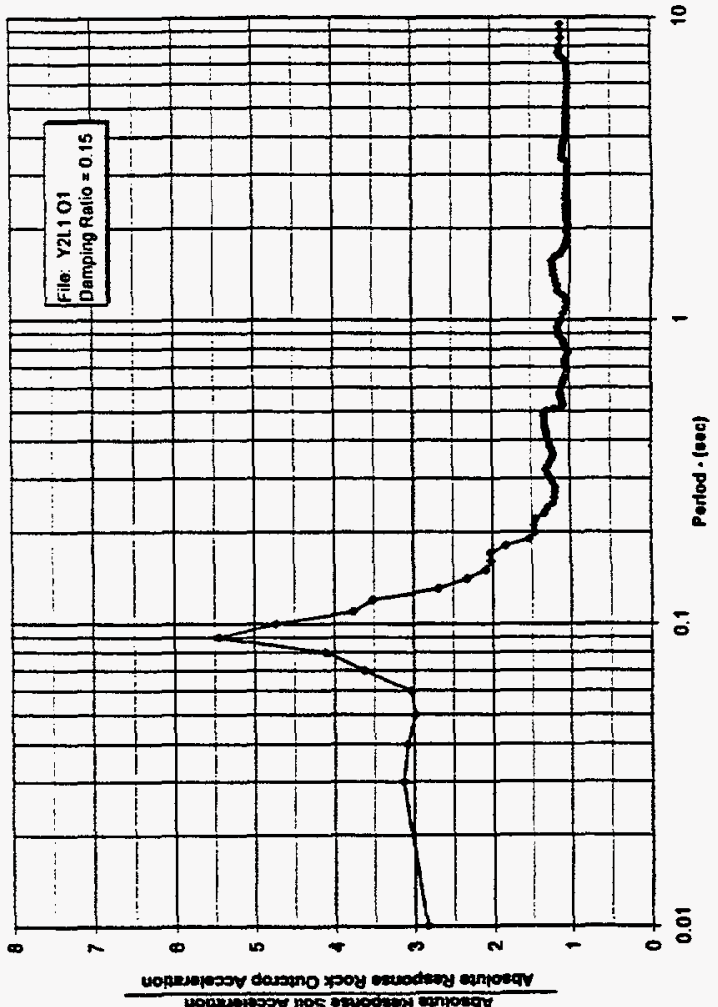

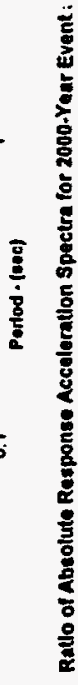
एop

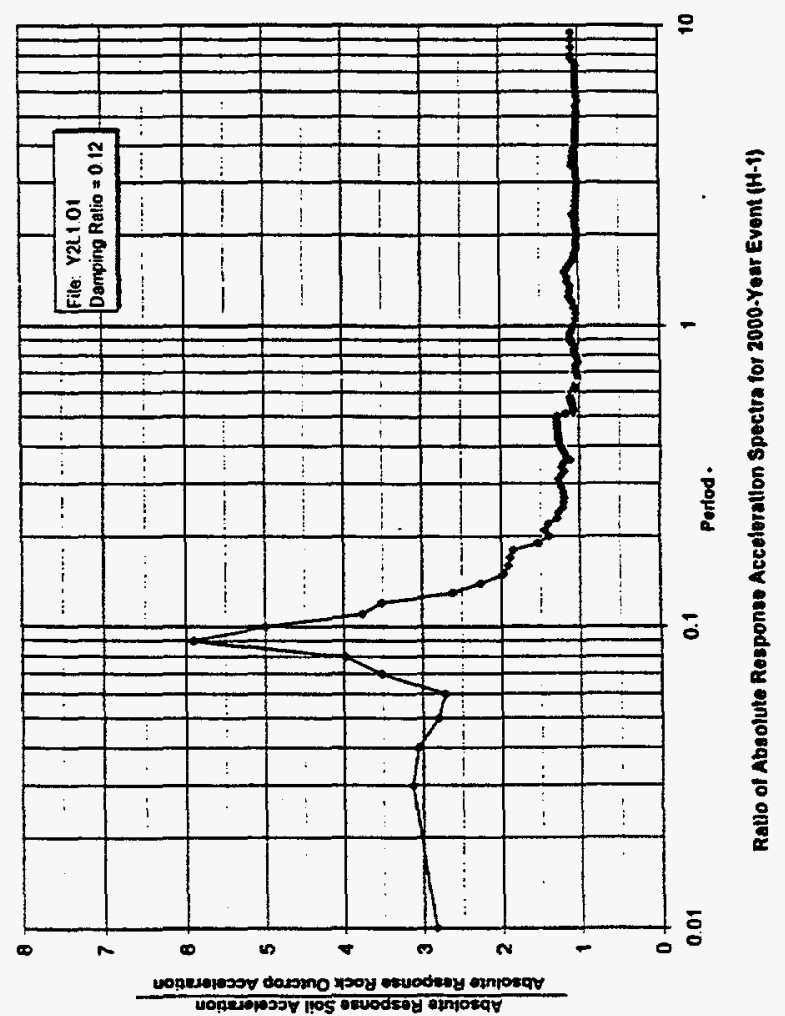

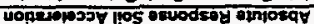




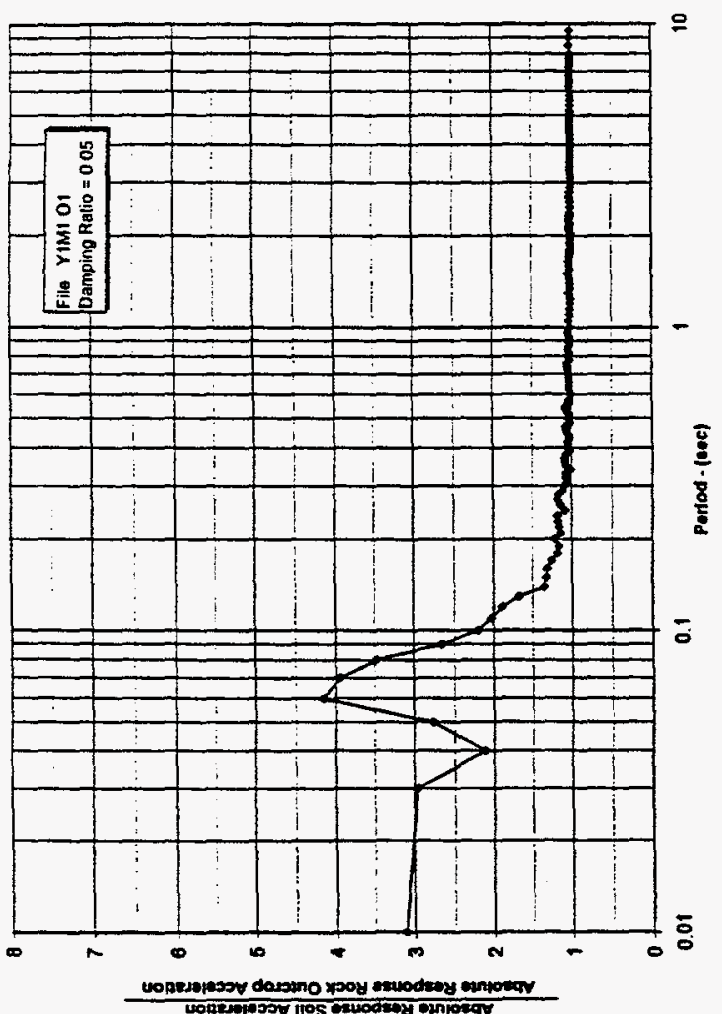

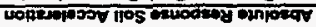
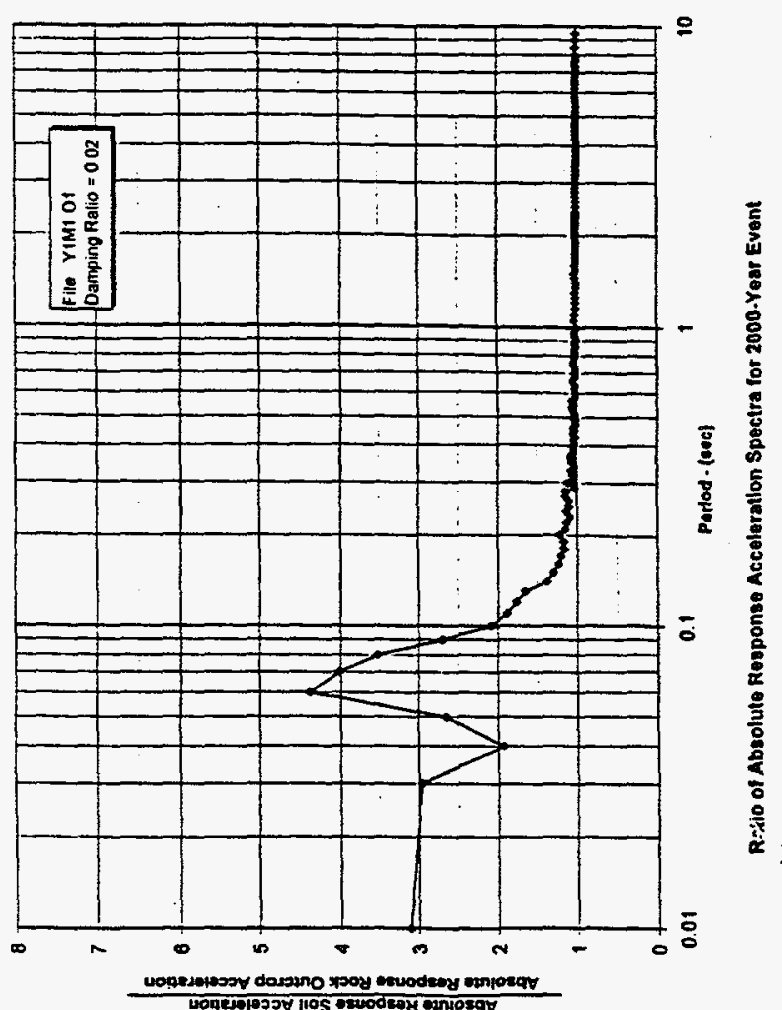
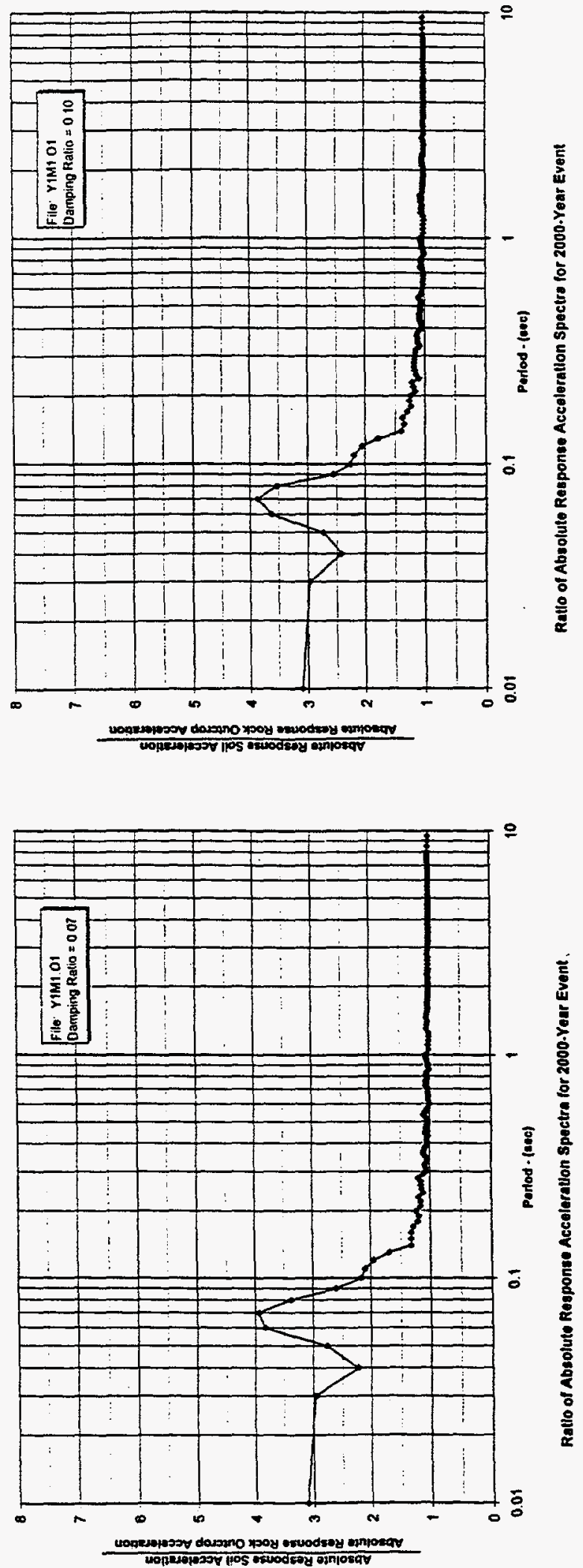

A2-4 

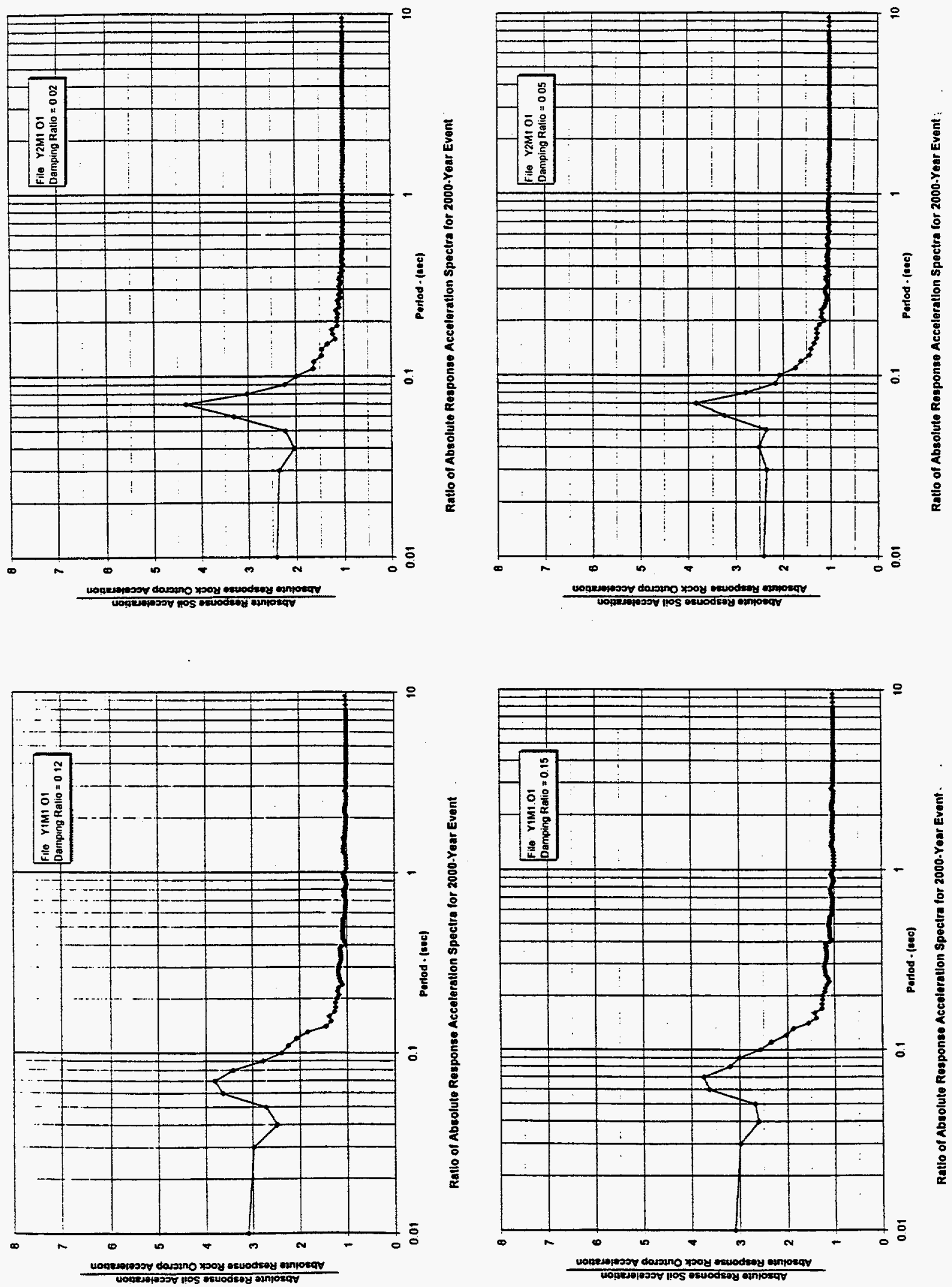

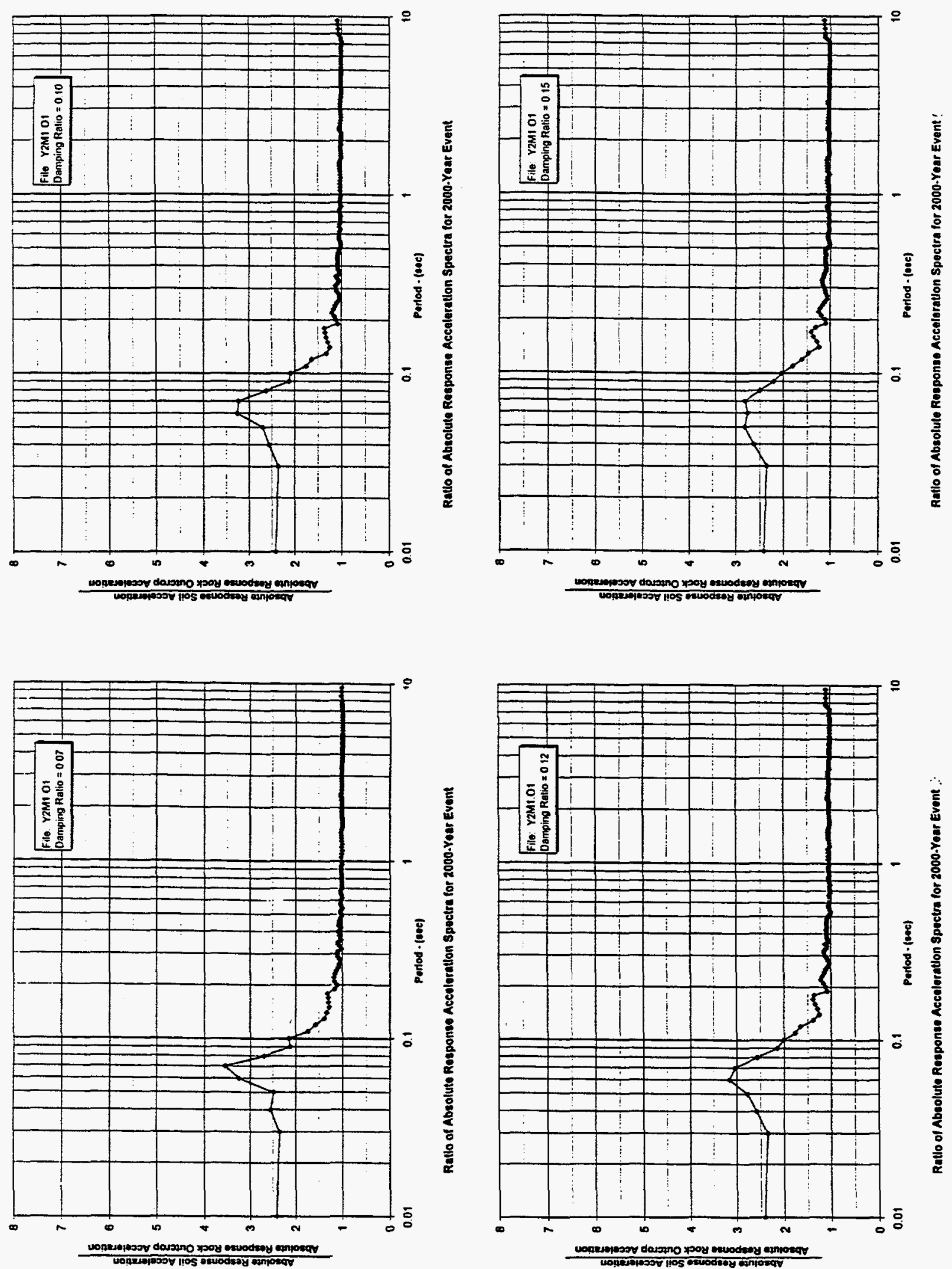

A2- 6 

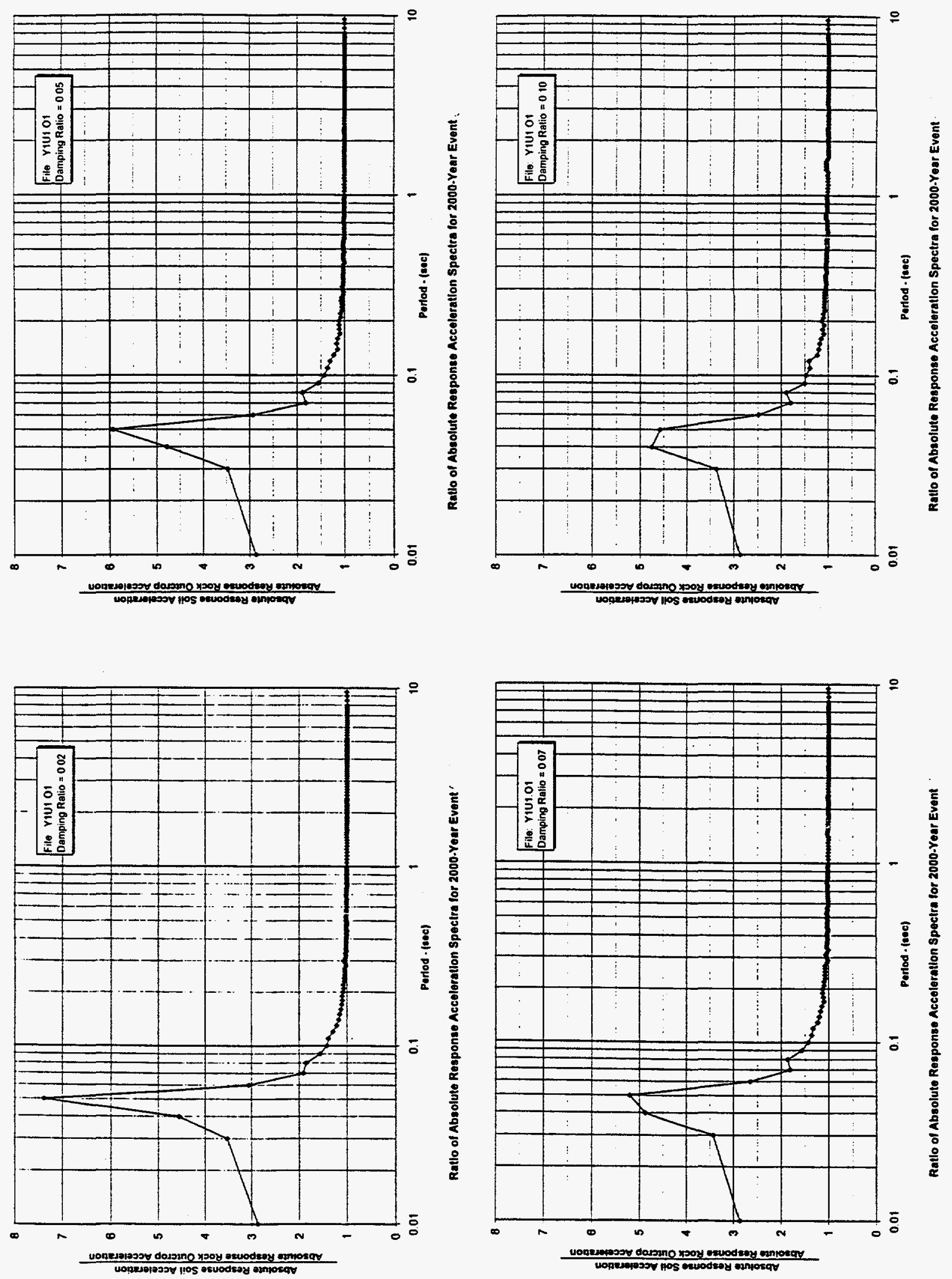

A2-7 

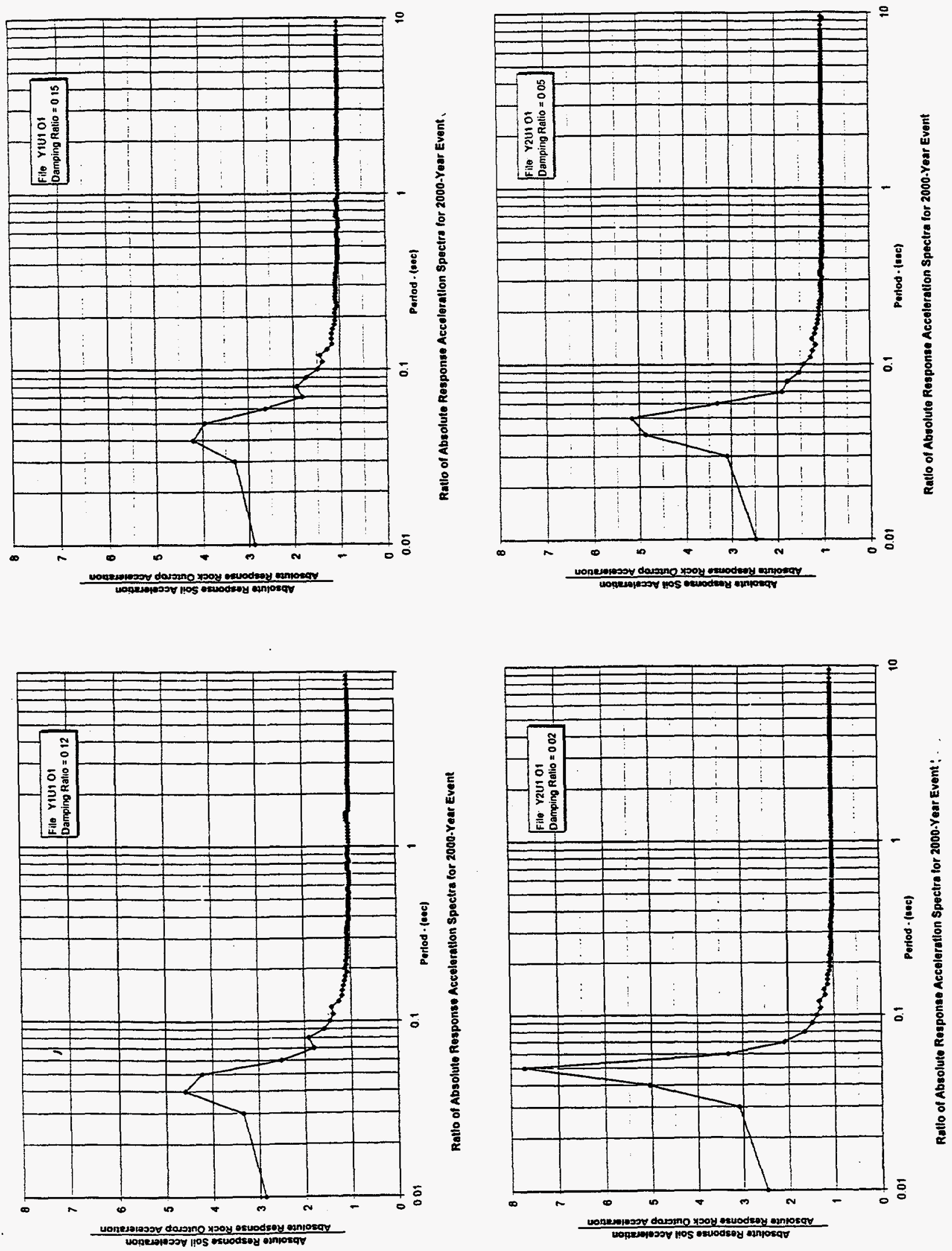

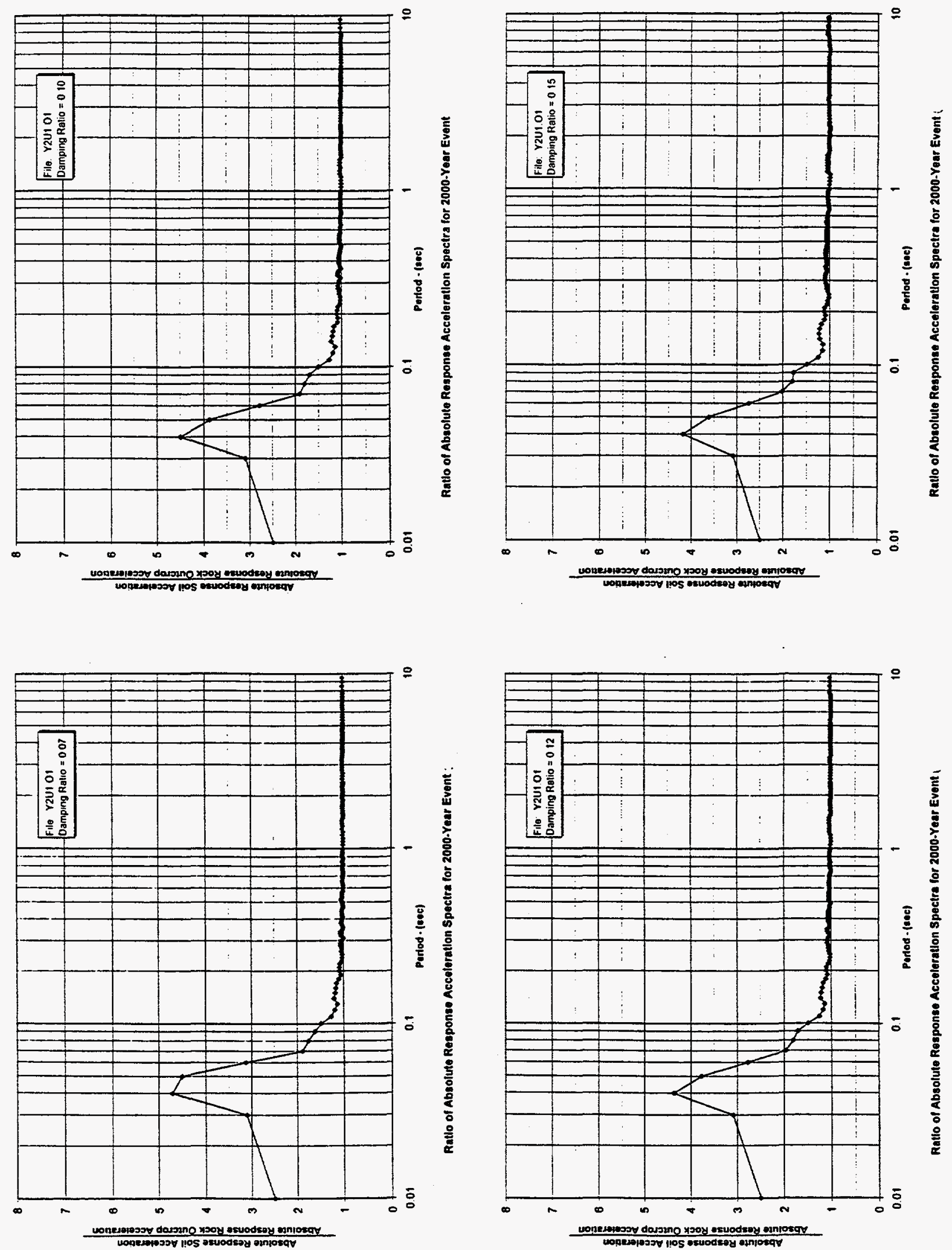

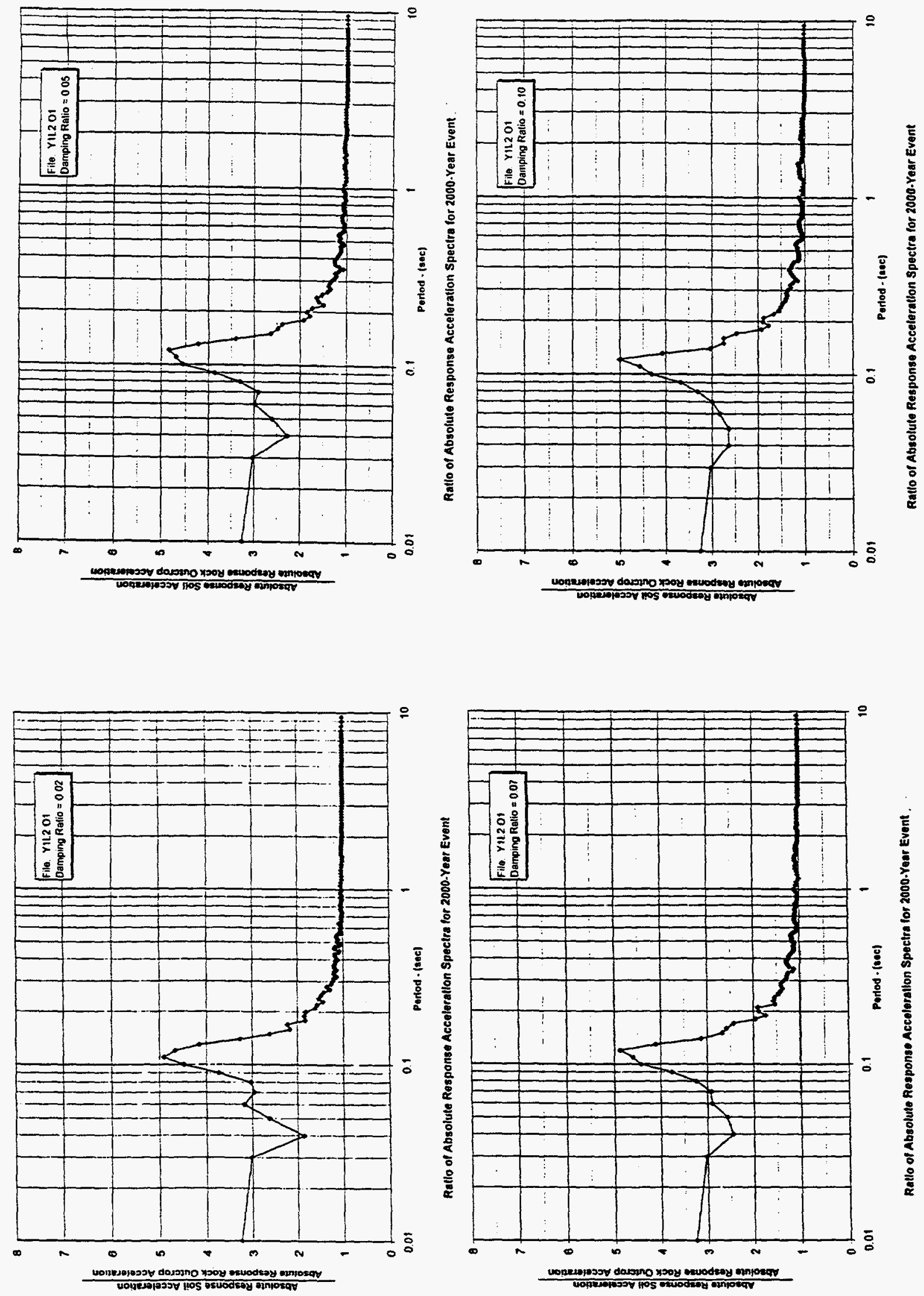

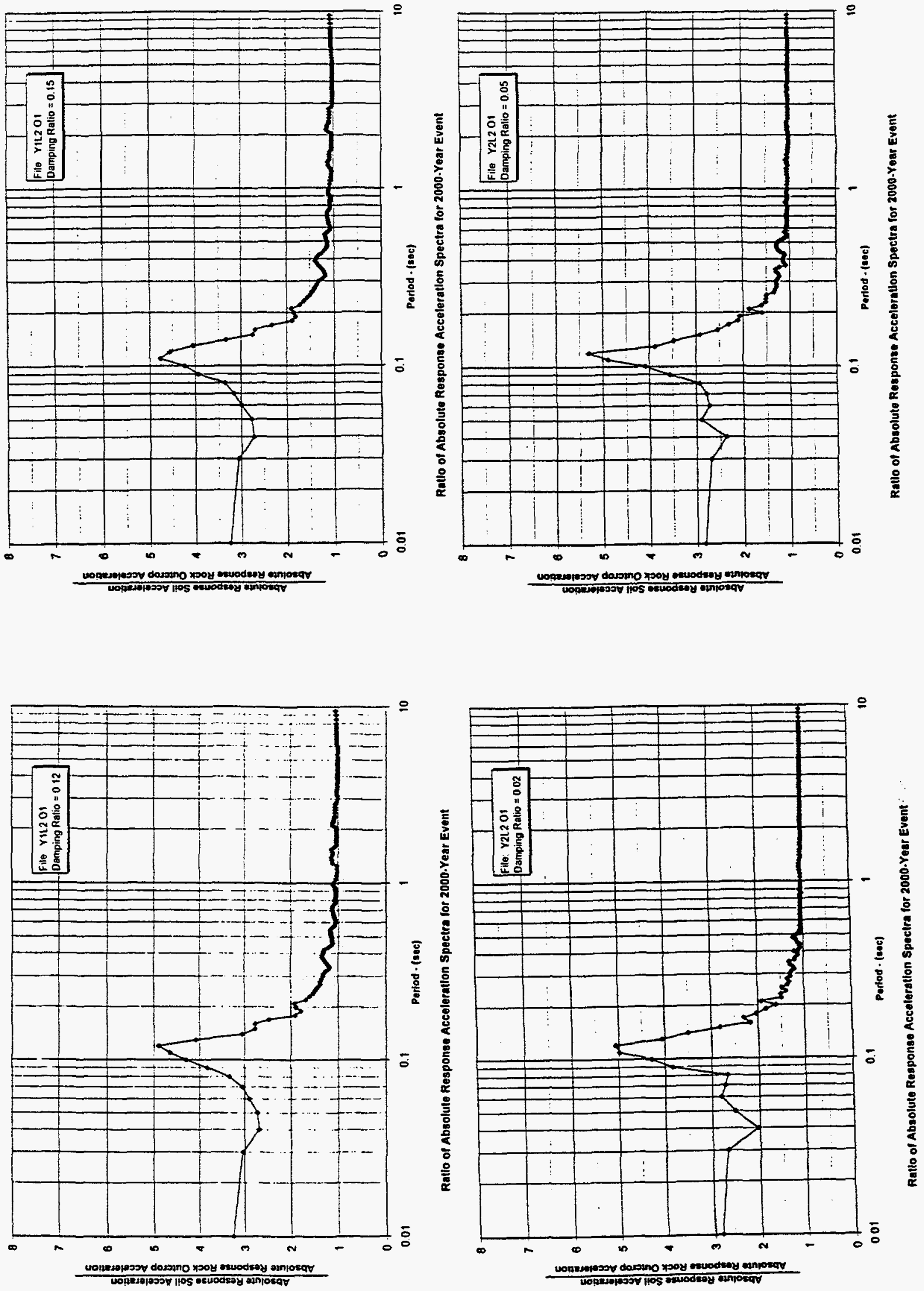

A2-11 

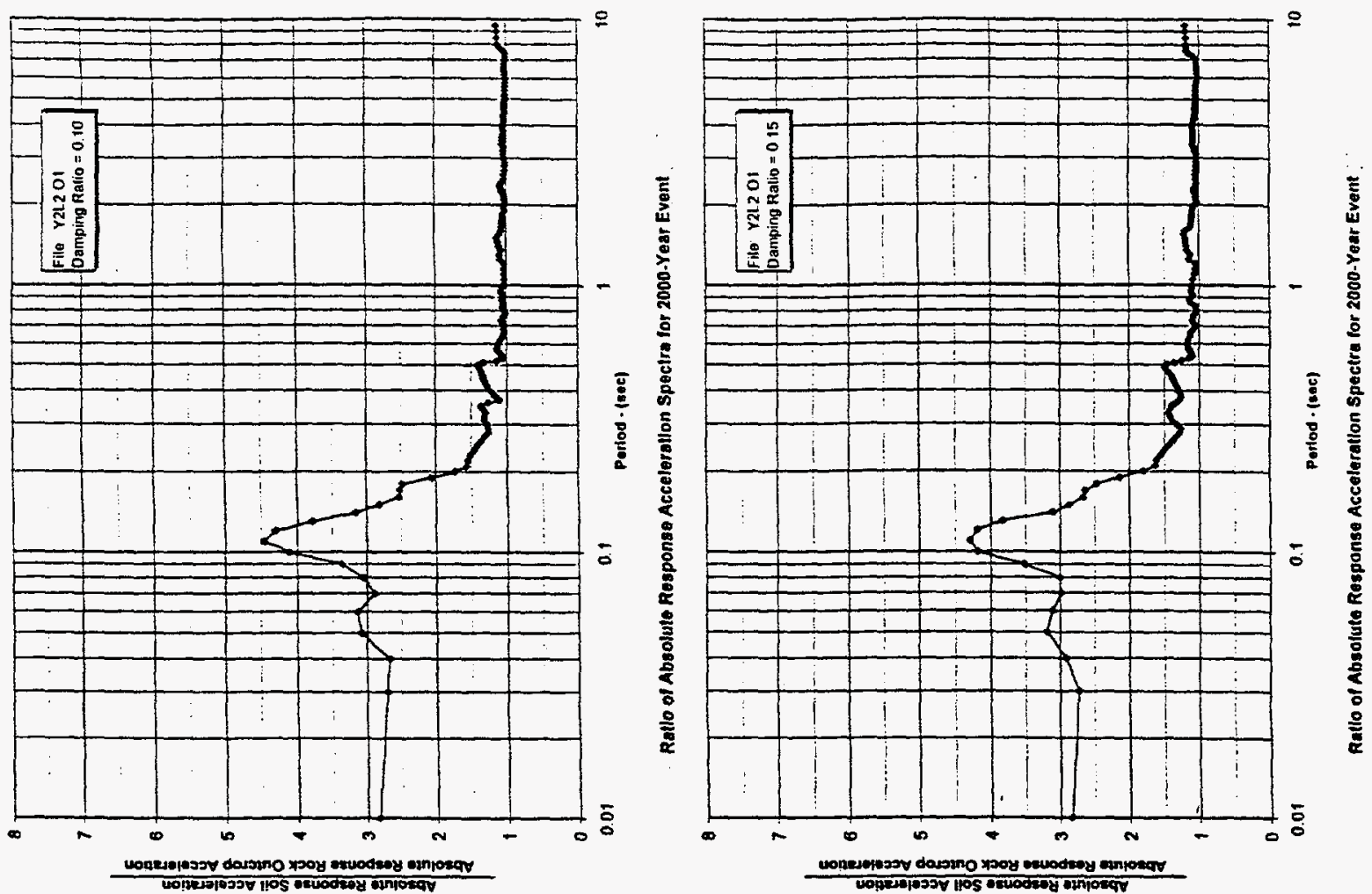

एक एका

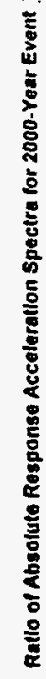
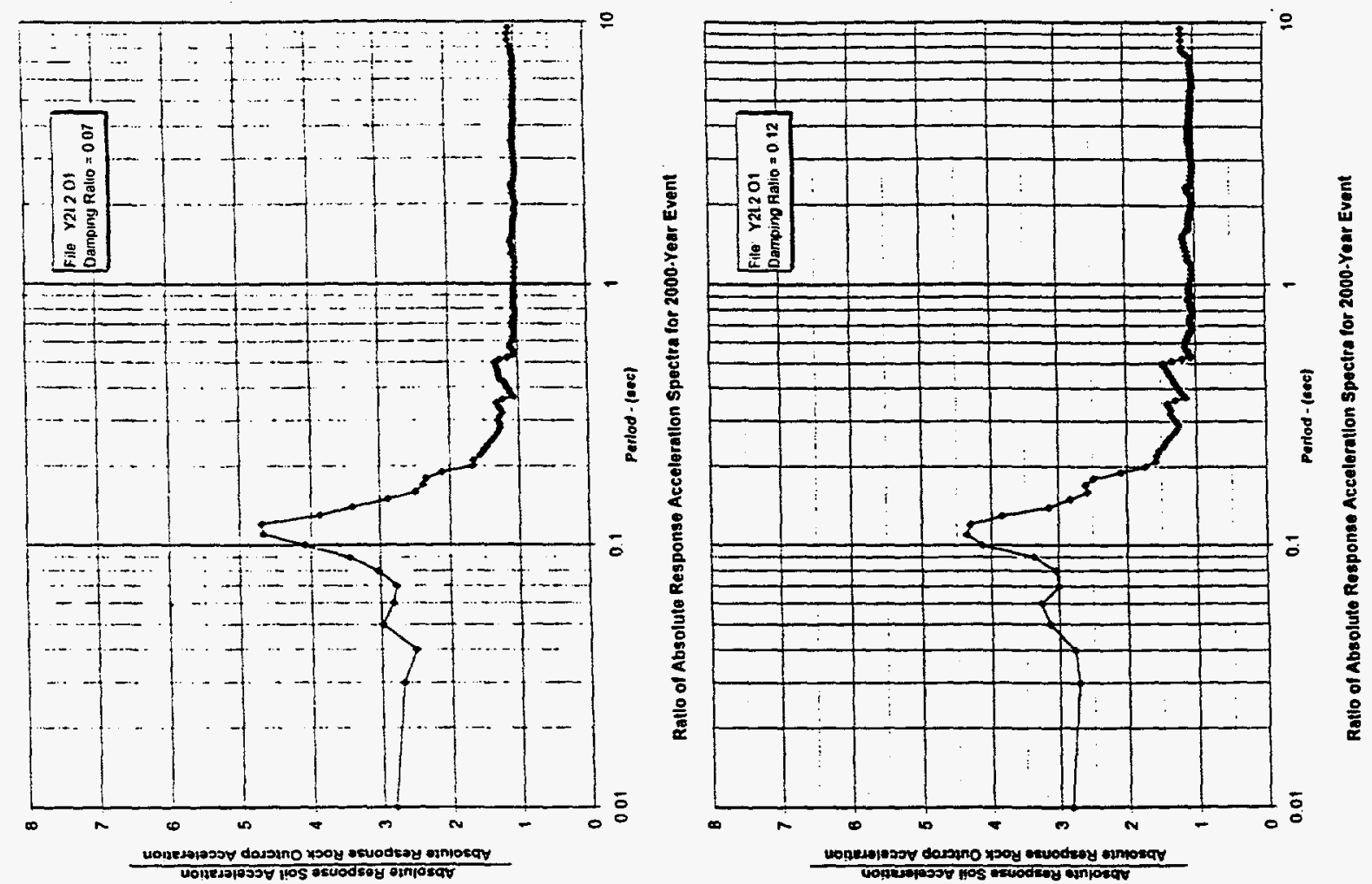

A2-12 

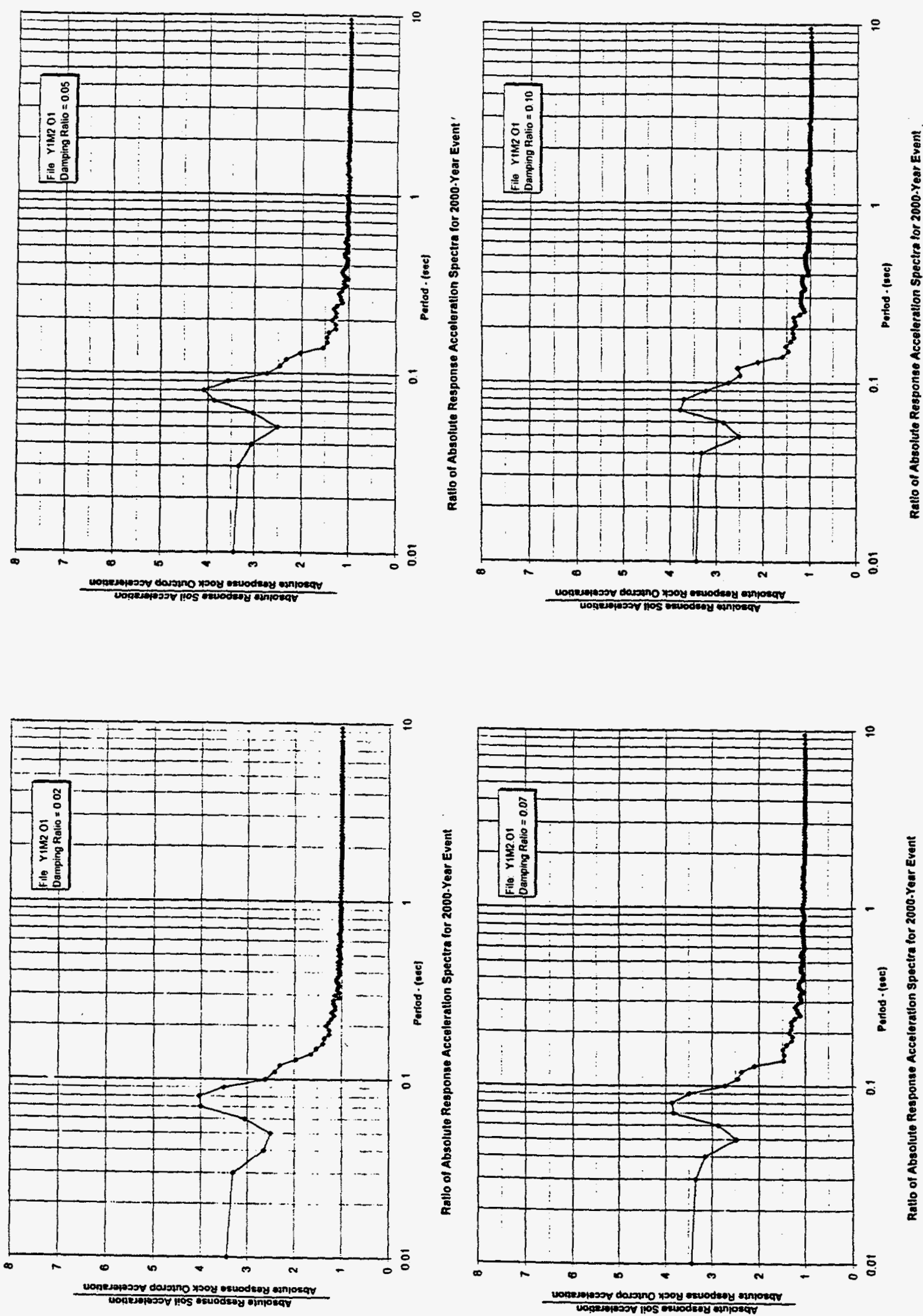

A2-13 

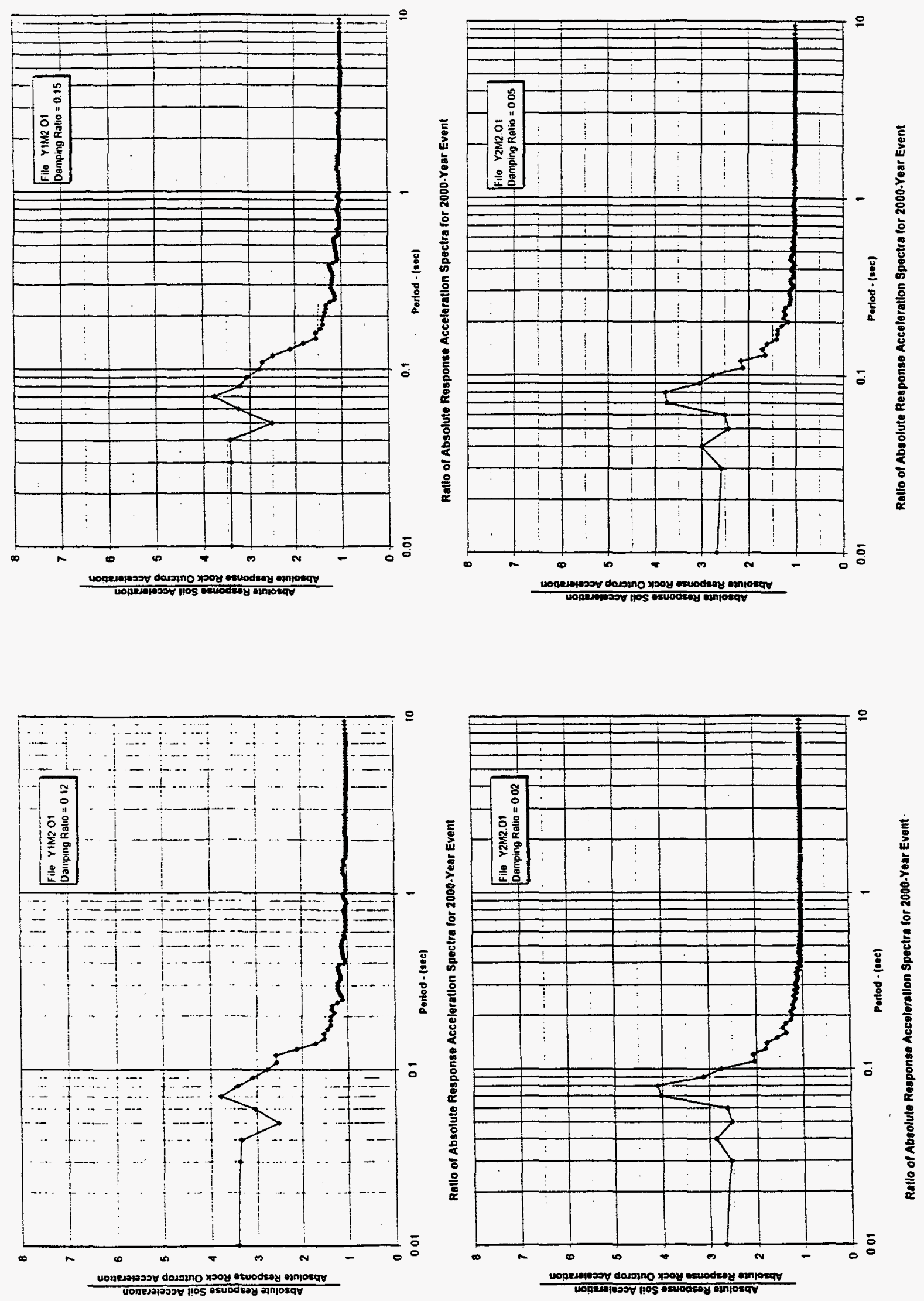

A2-14 

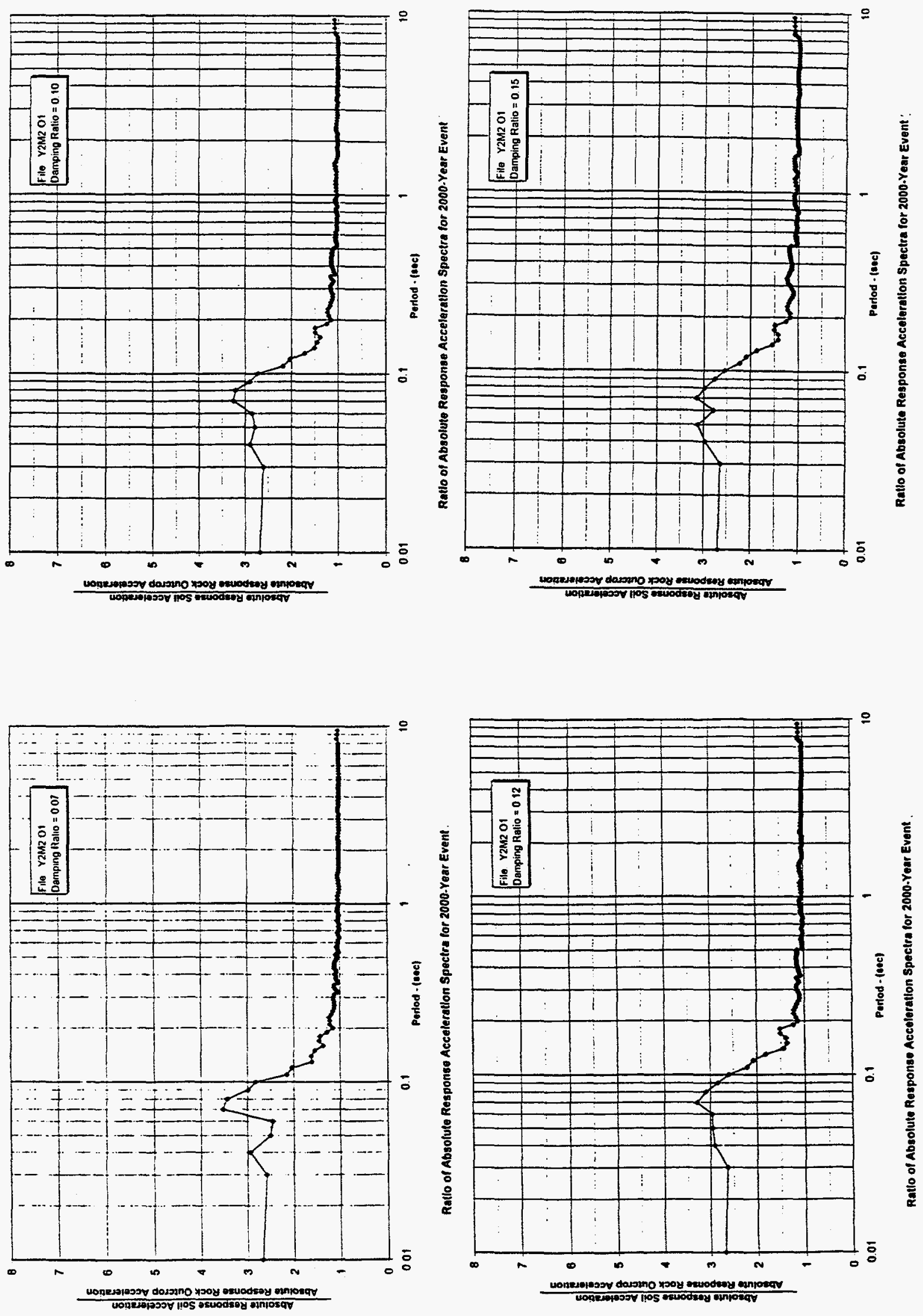

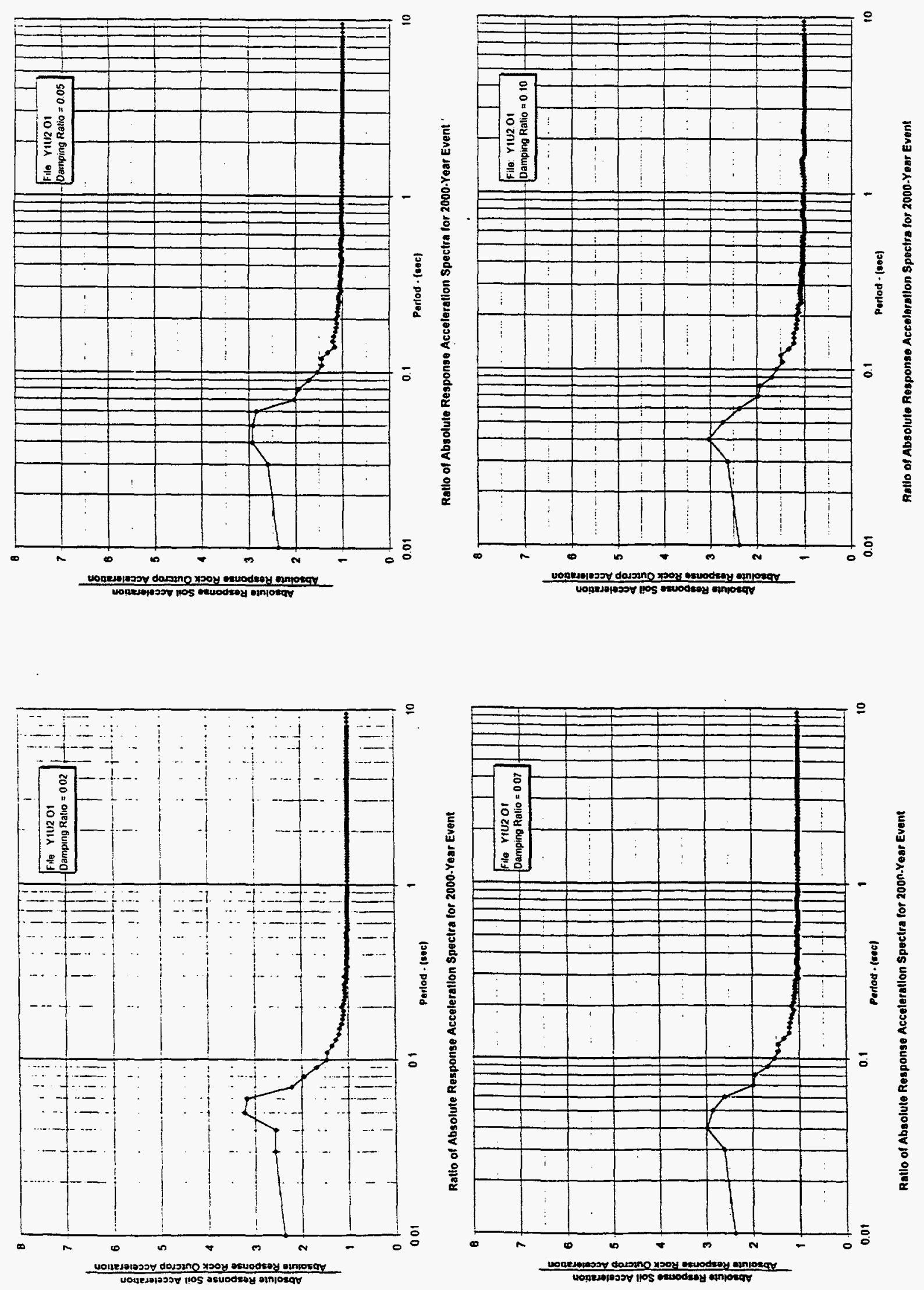

A2-16 

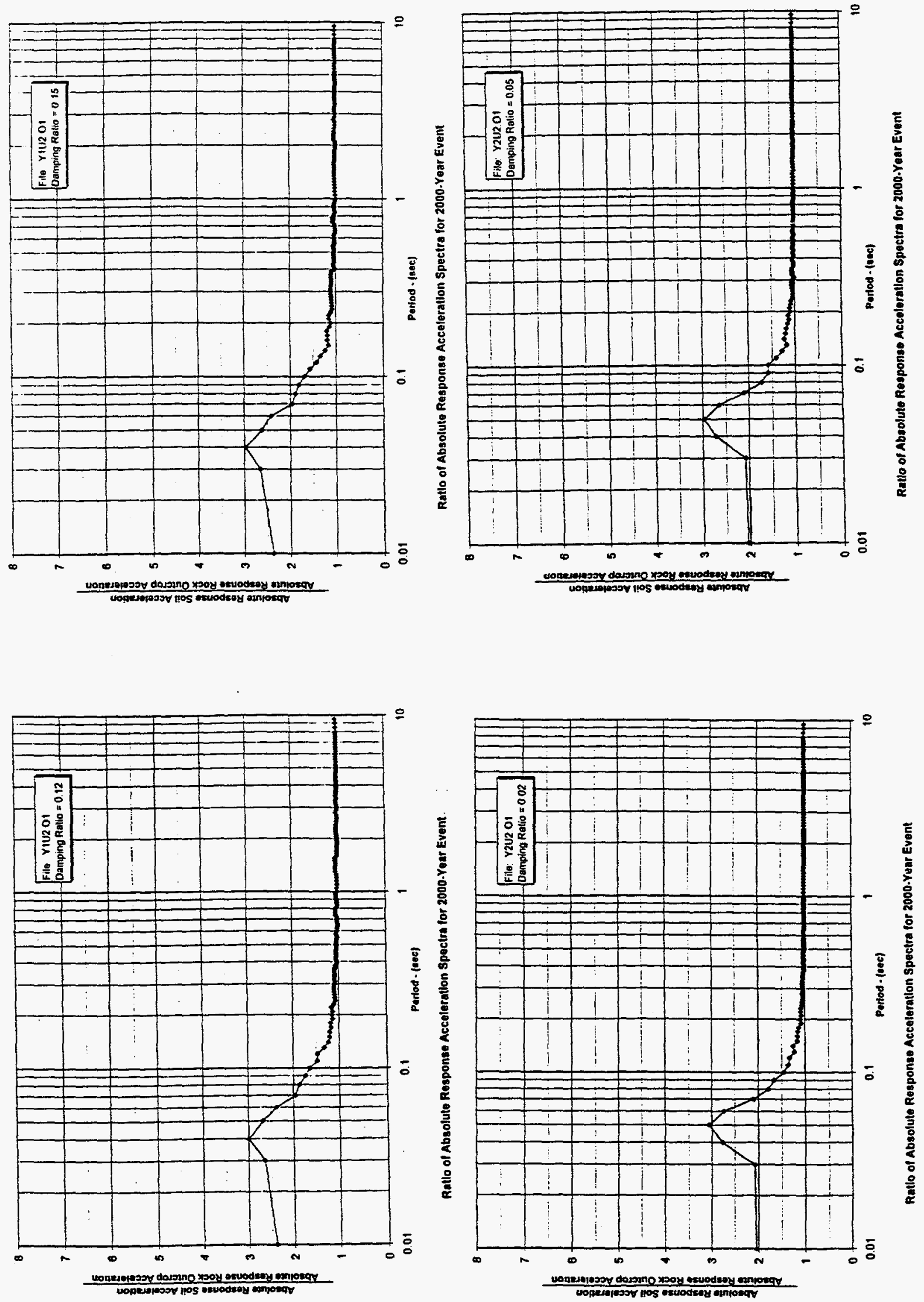

A2-17 

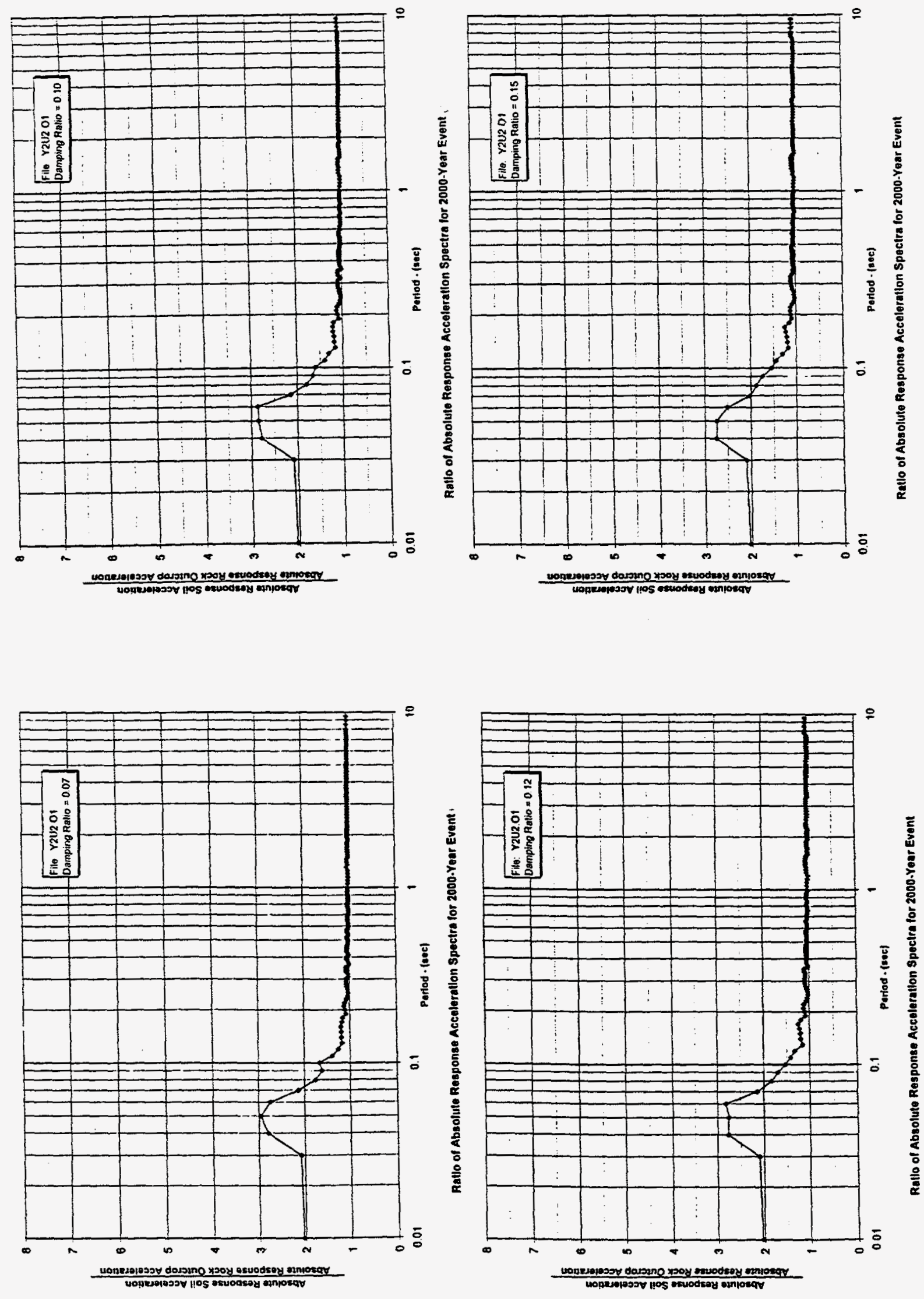

A2- -18 

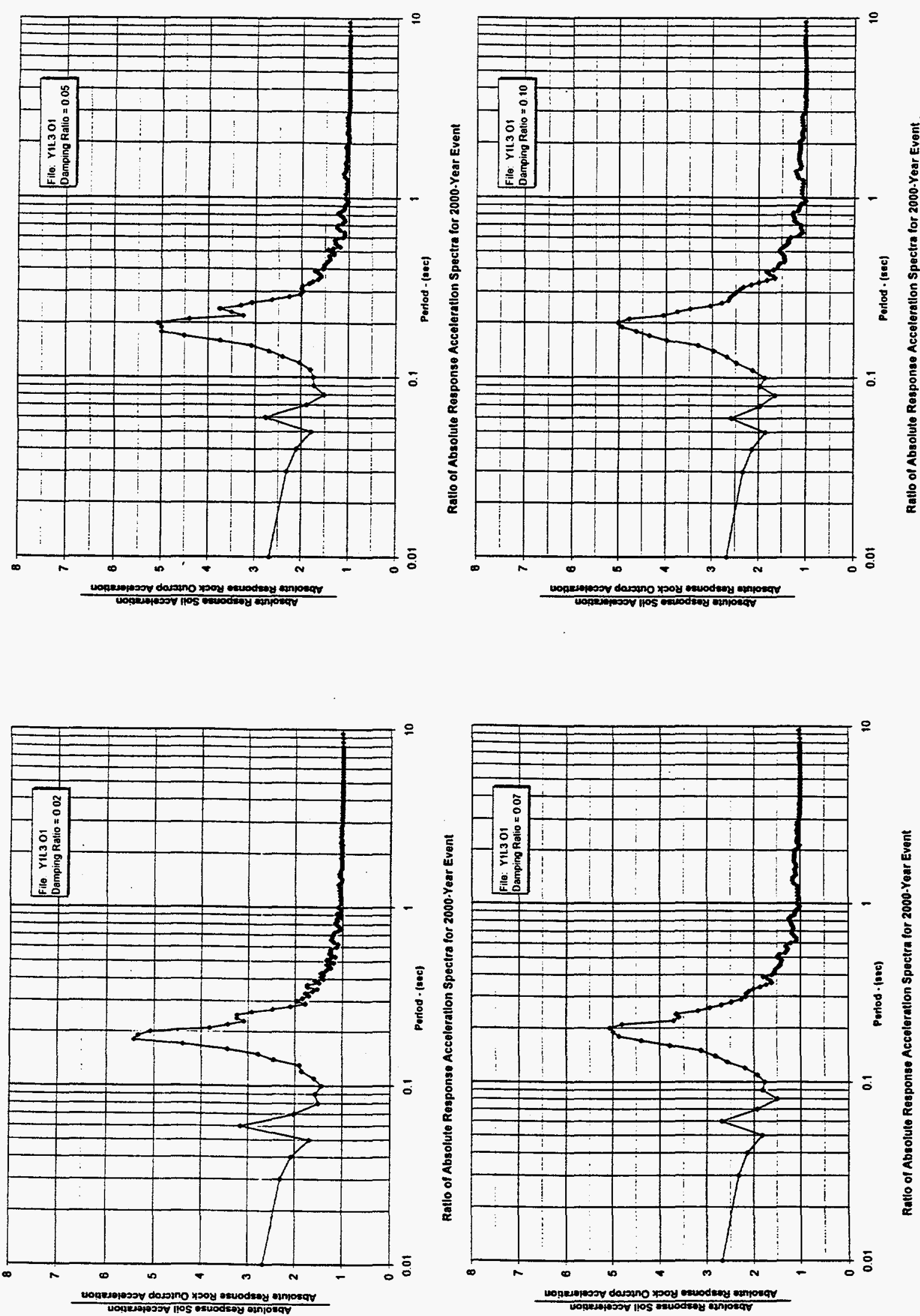

A2-19 

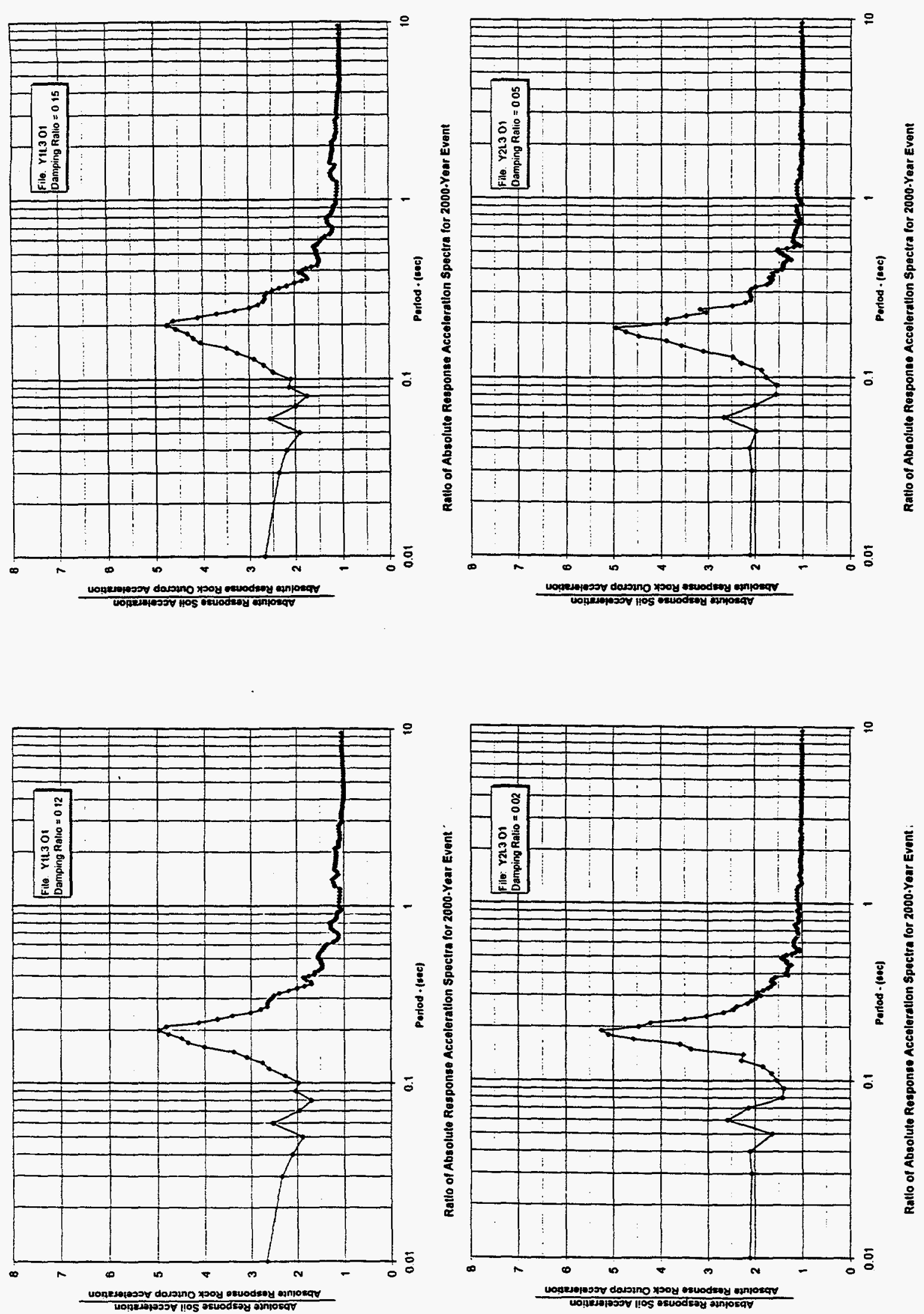

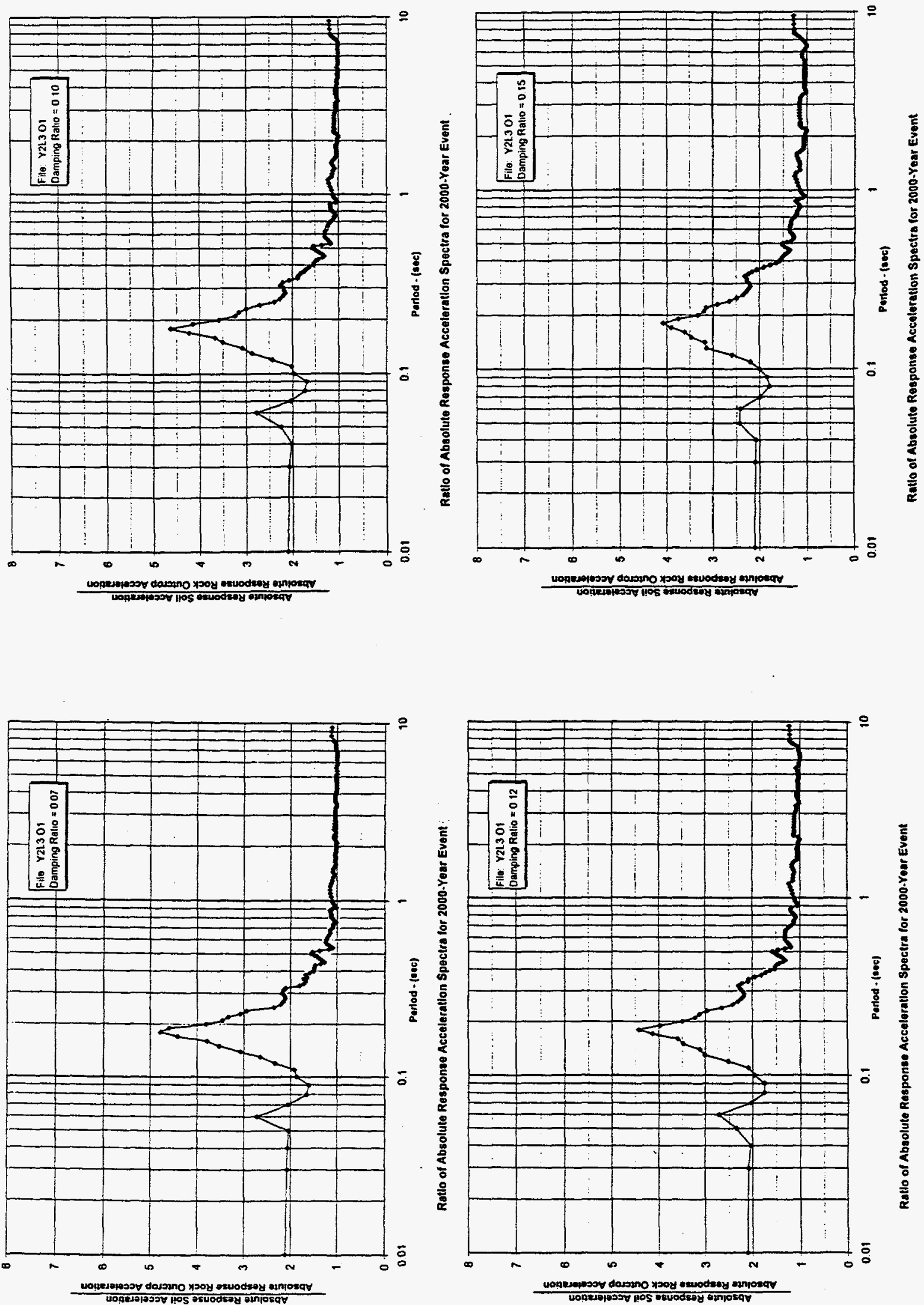

A2-21 


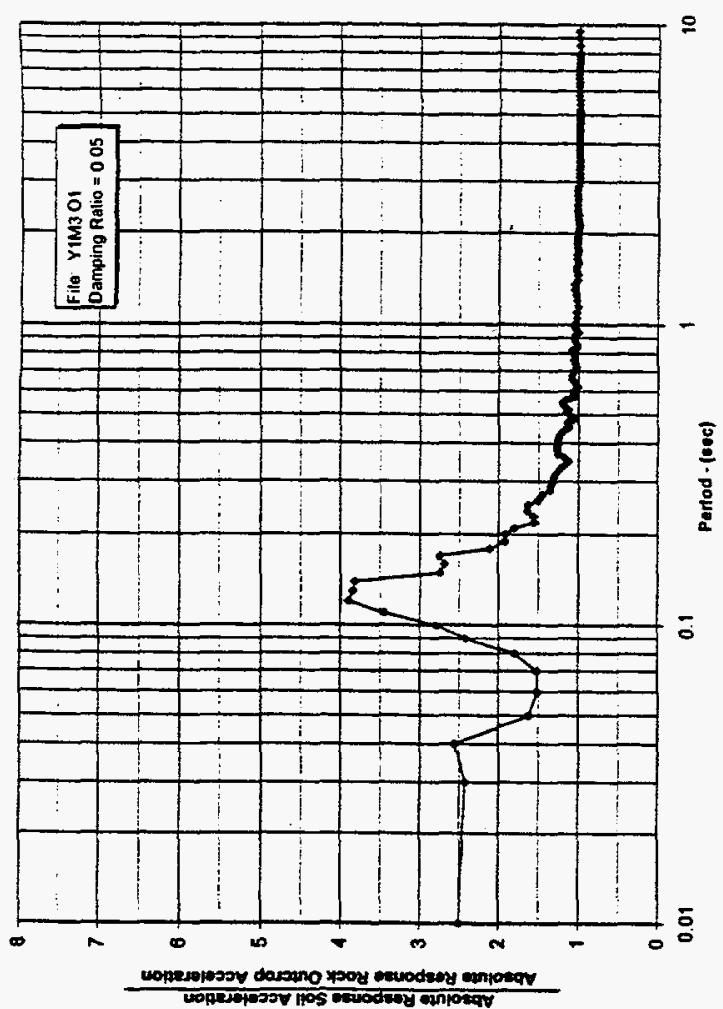

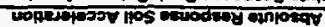
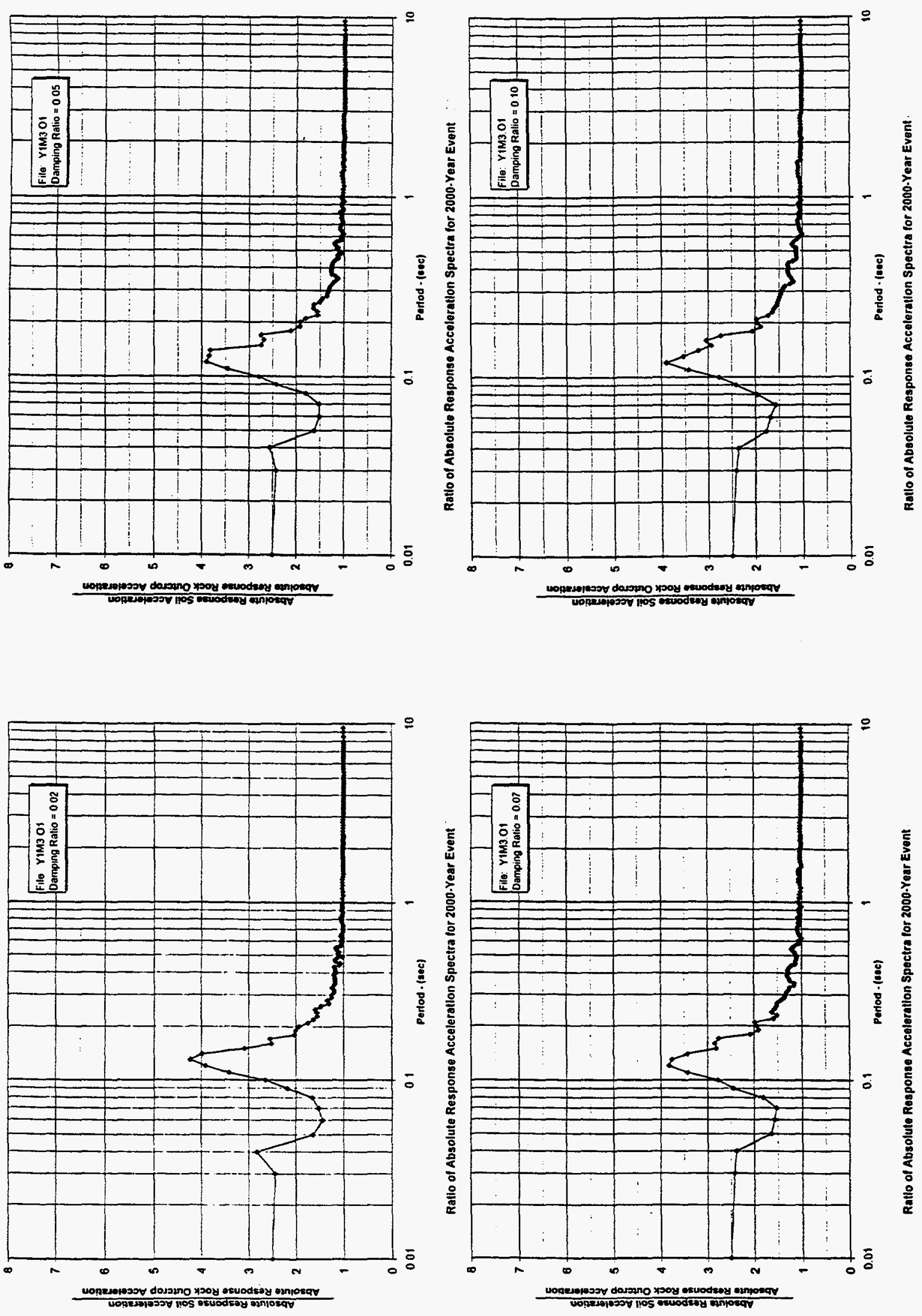


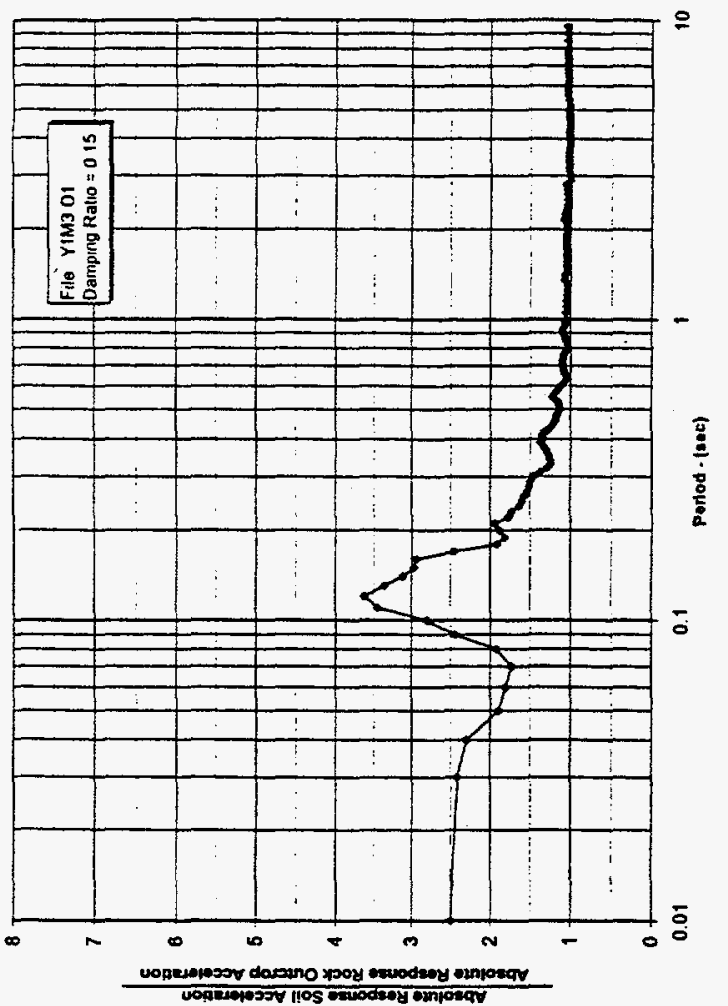

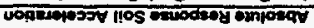
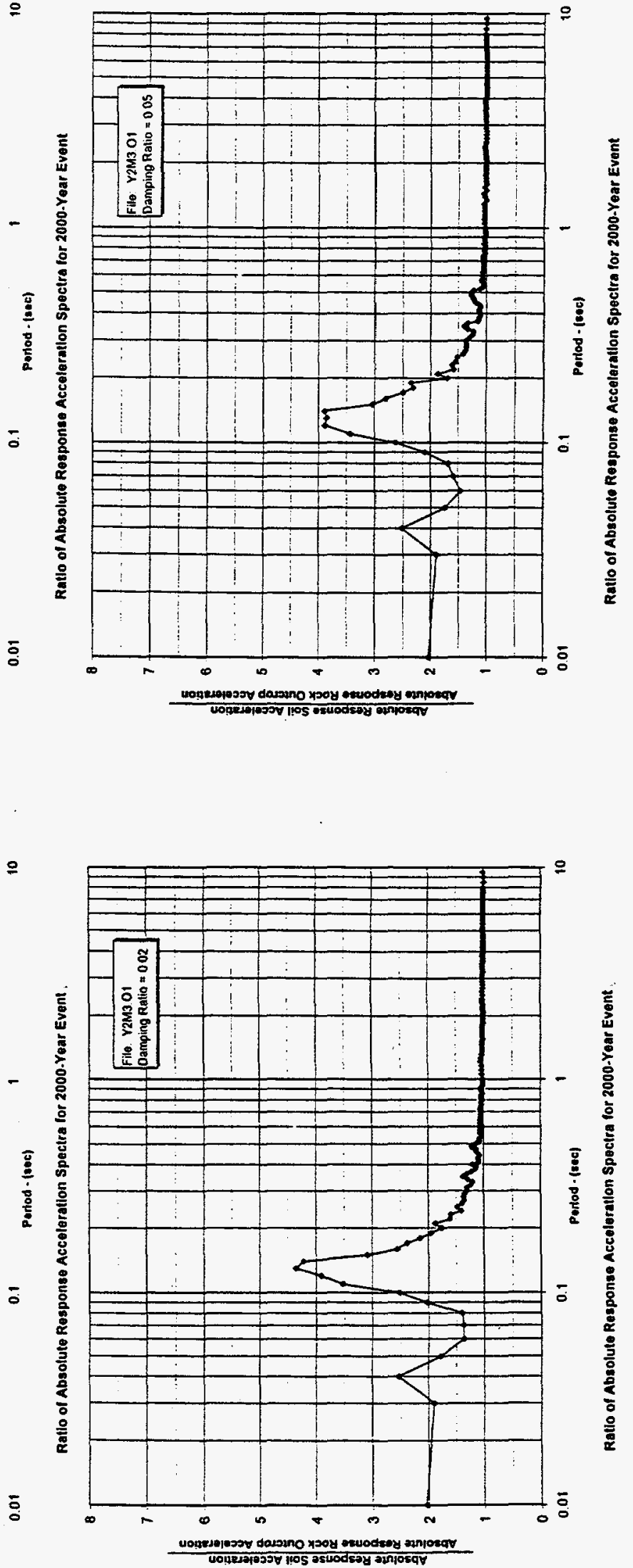

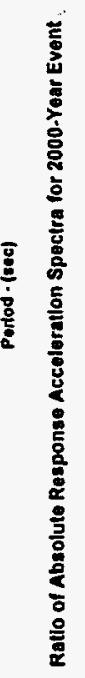



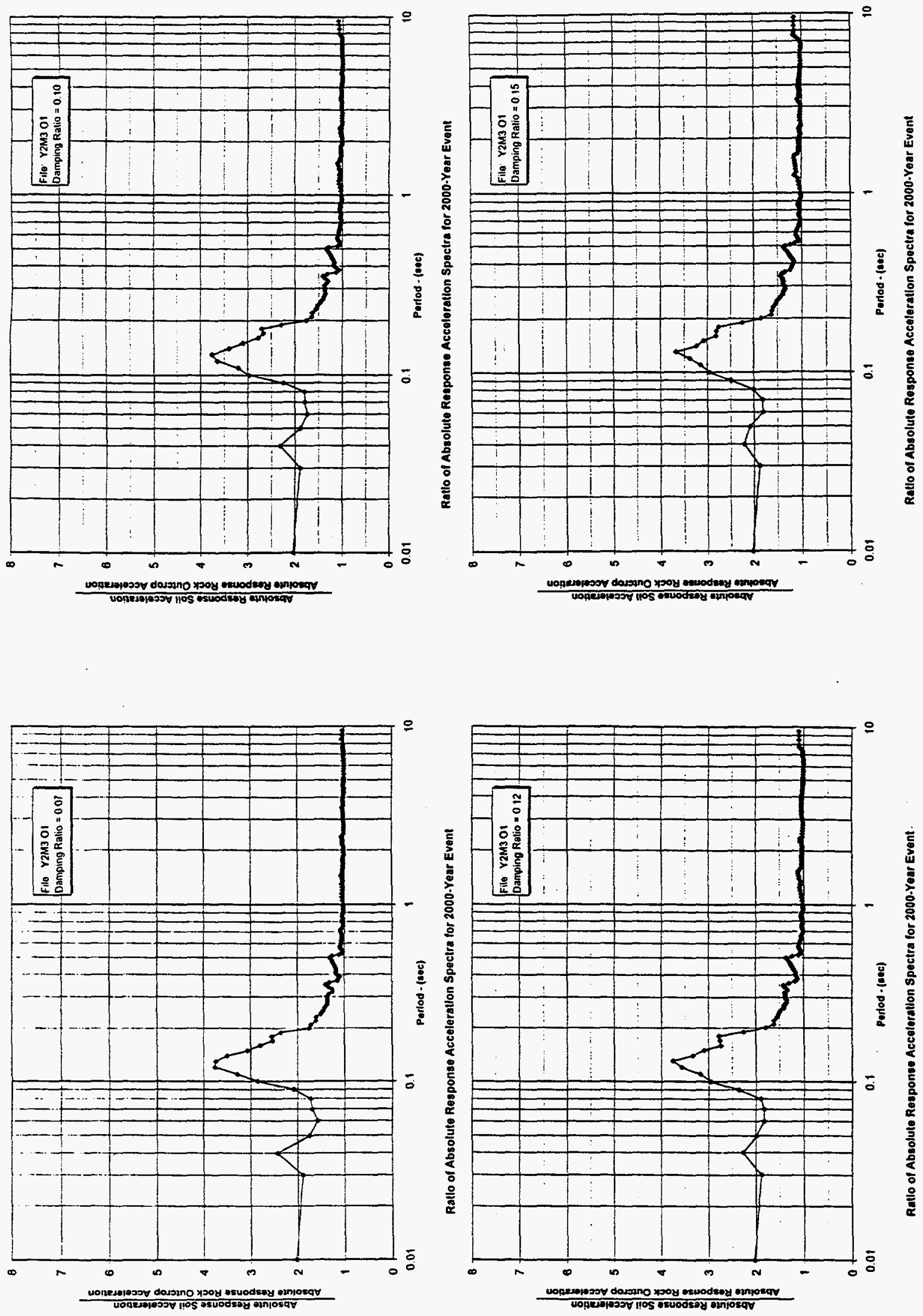

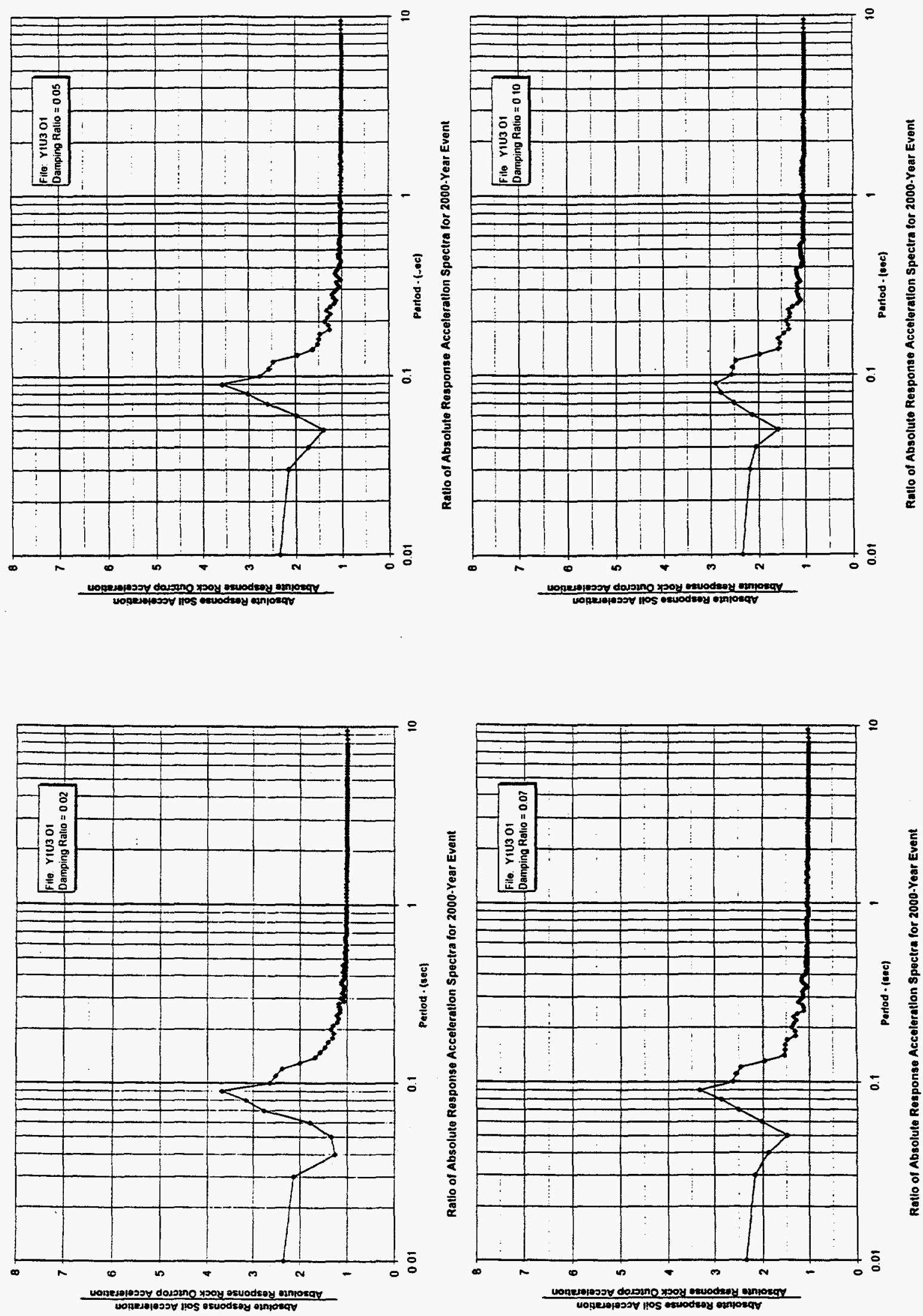

A2-25 

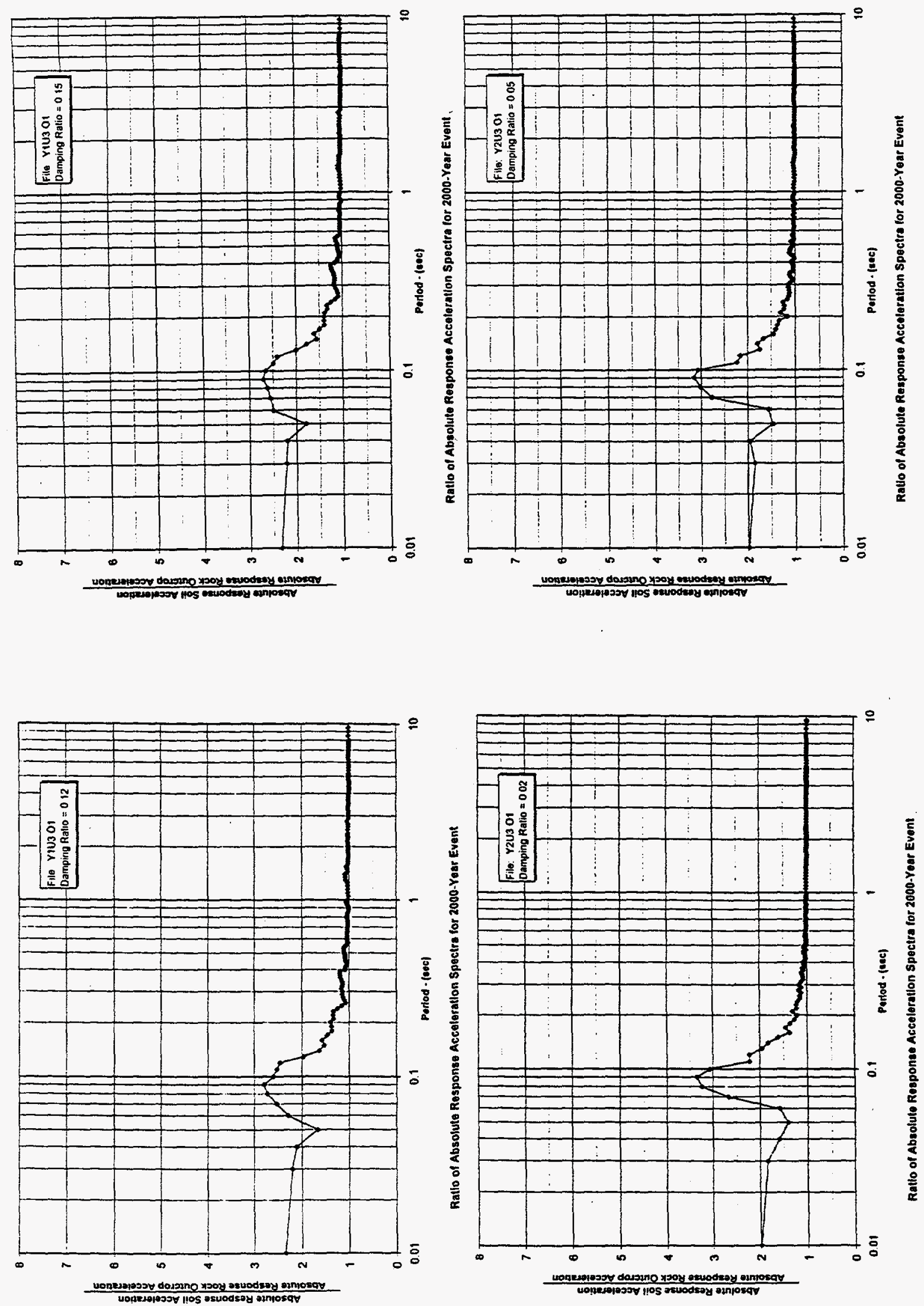

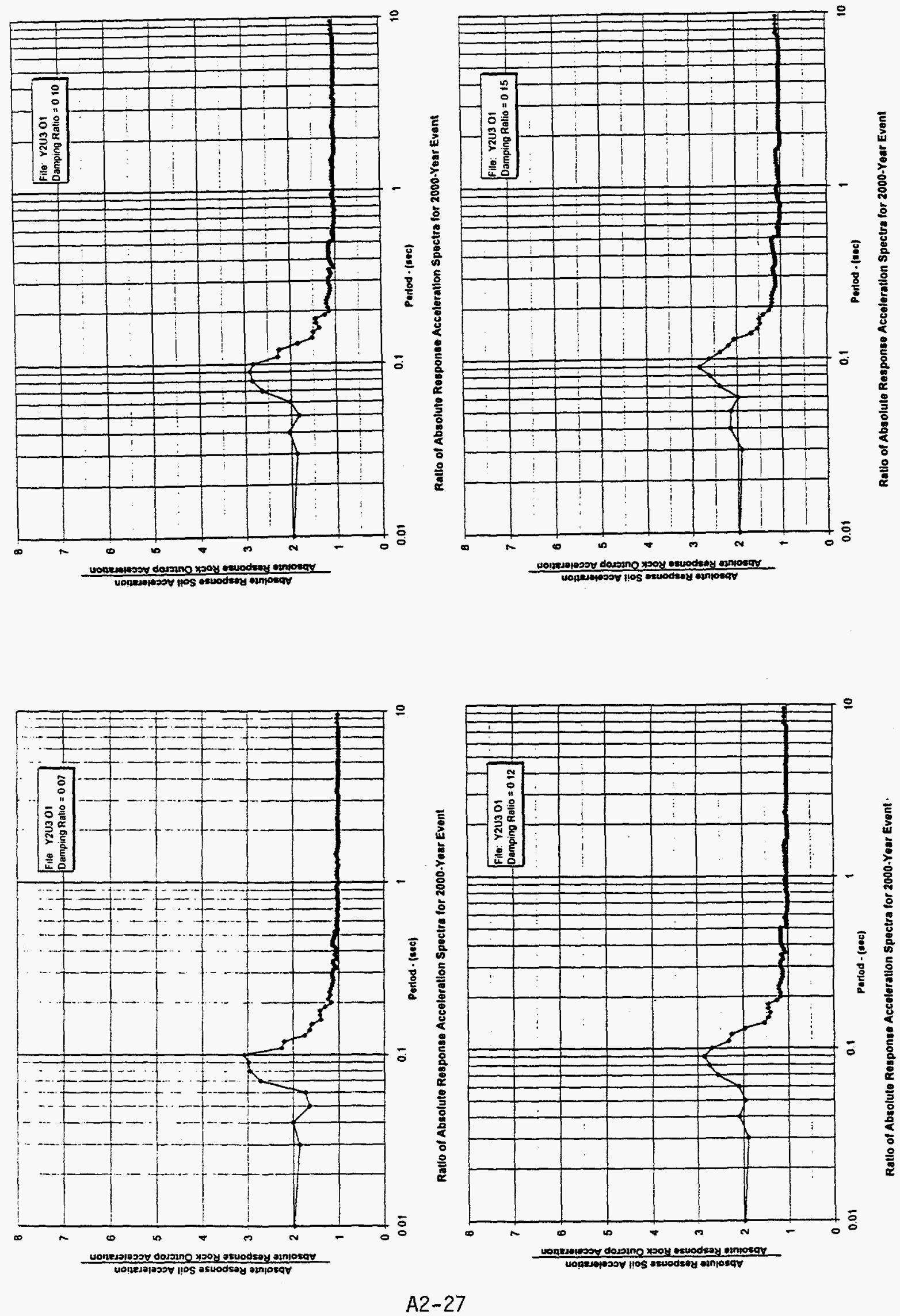

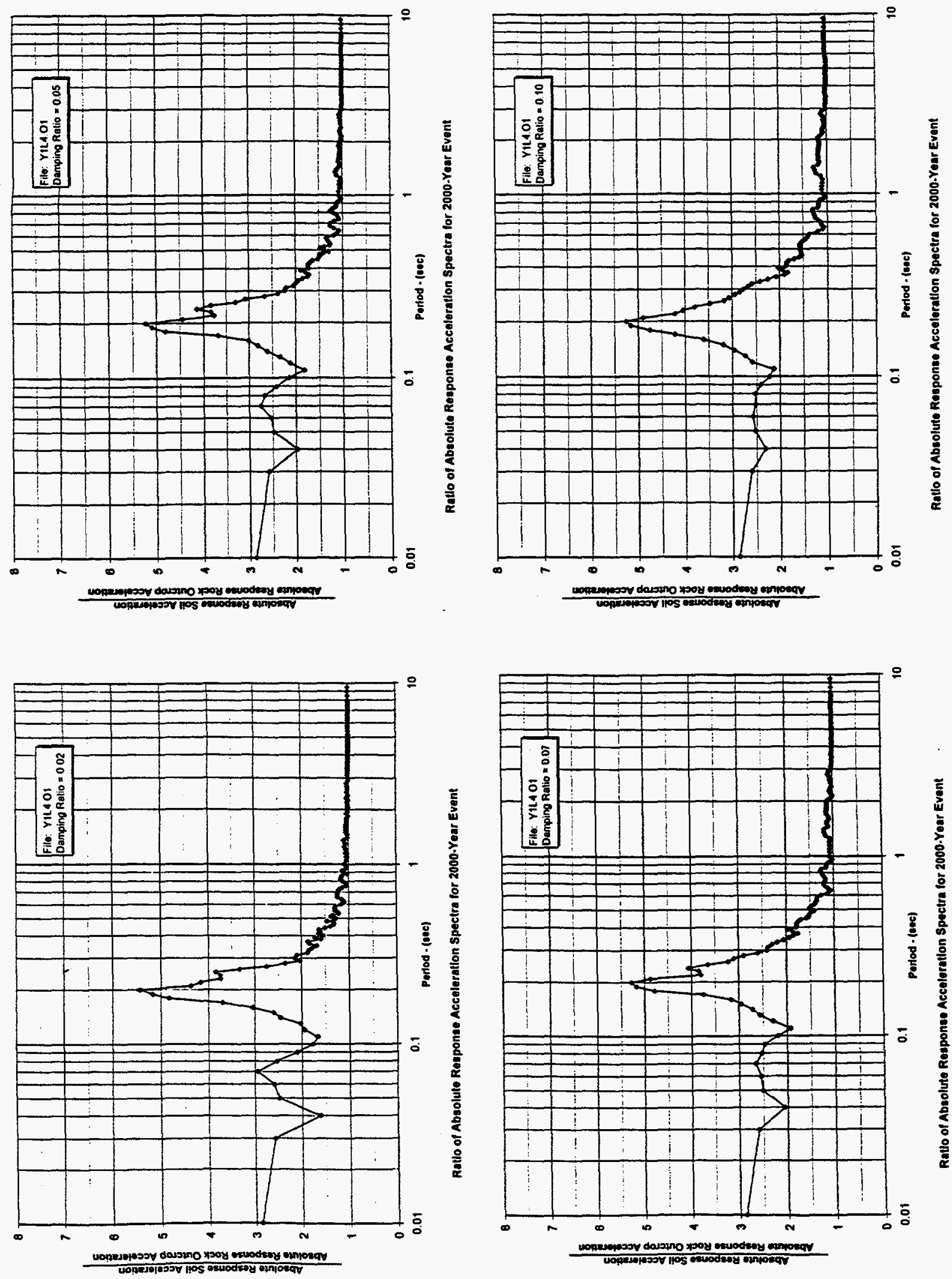

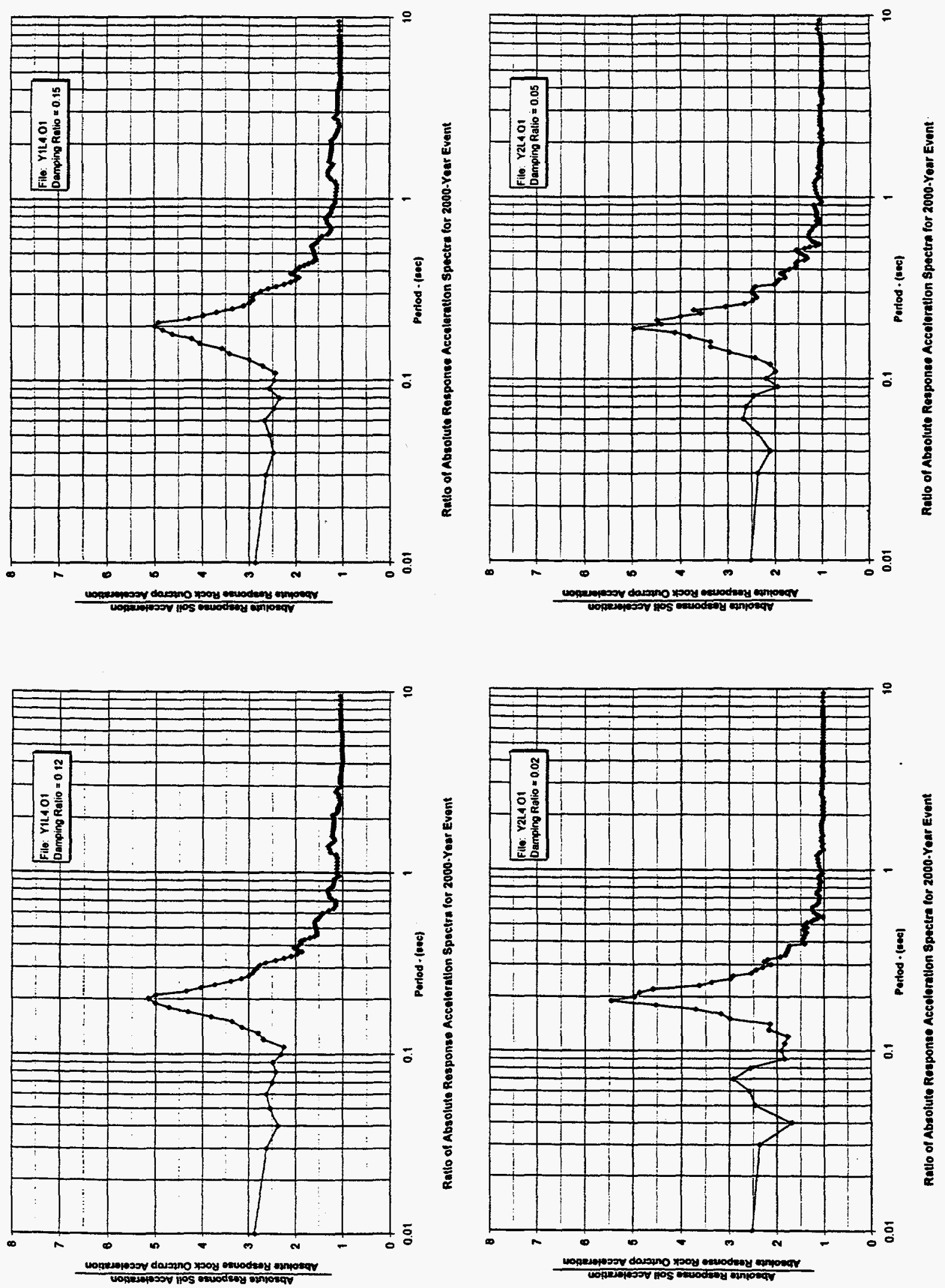

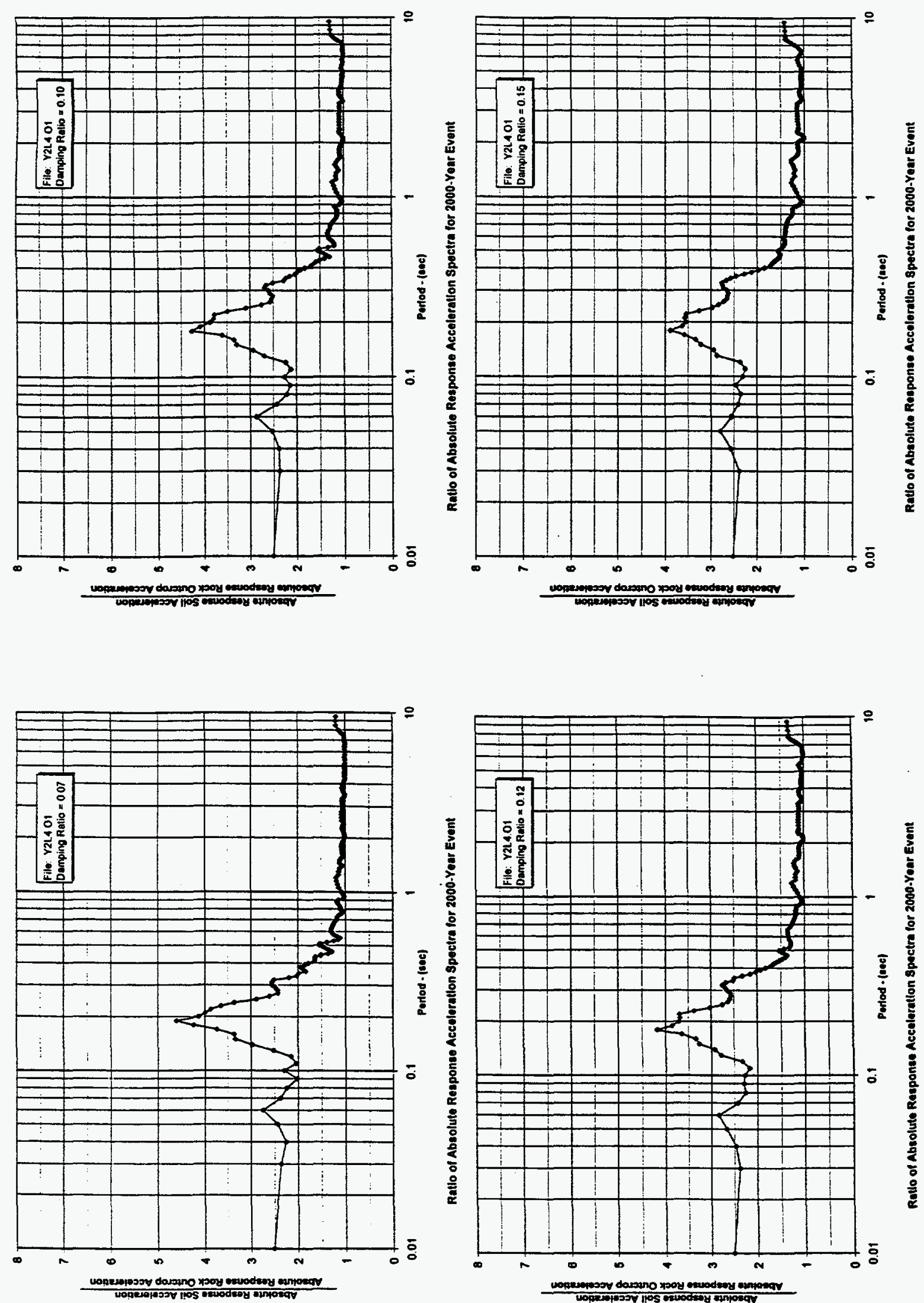

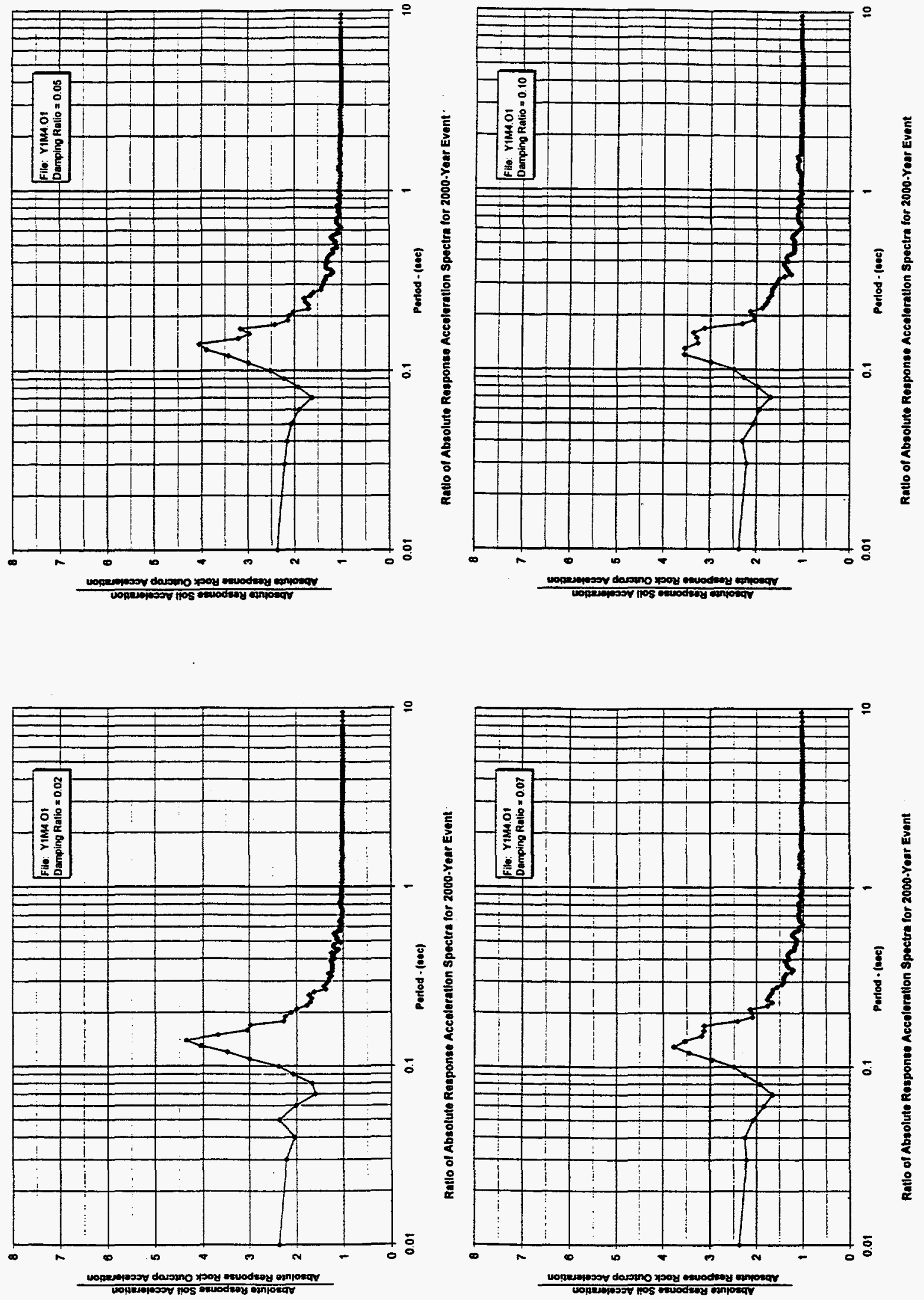

A2-31 

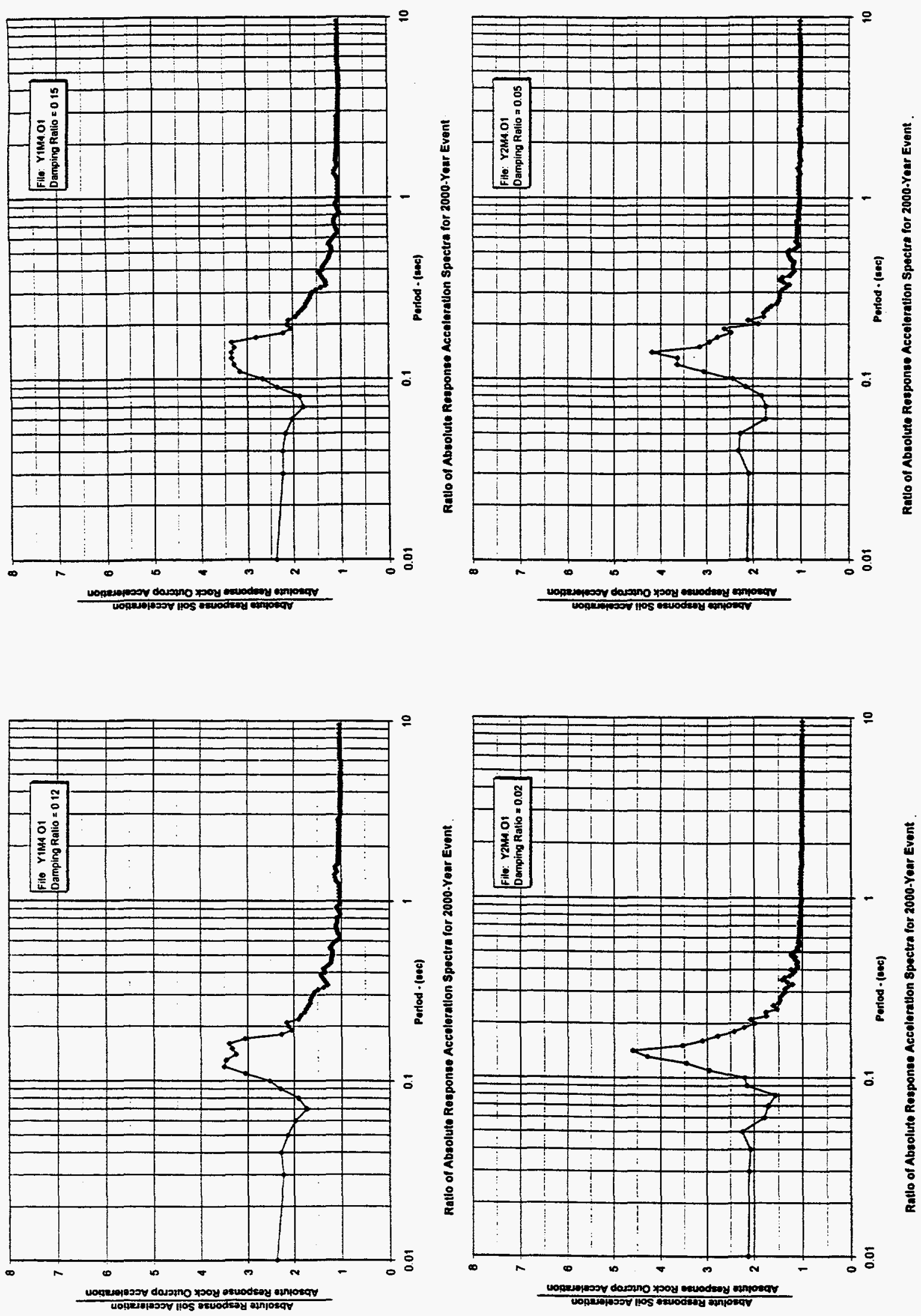

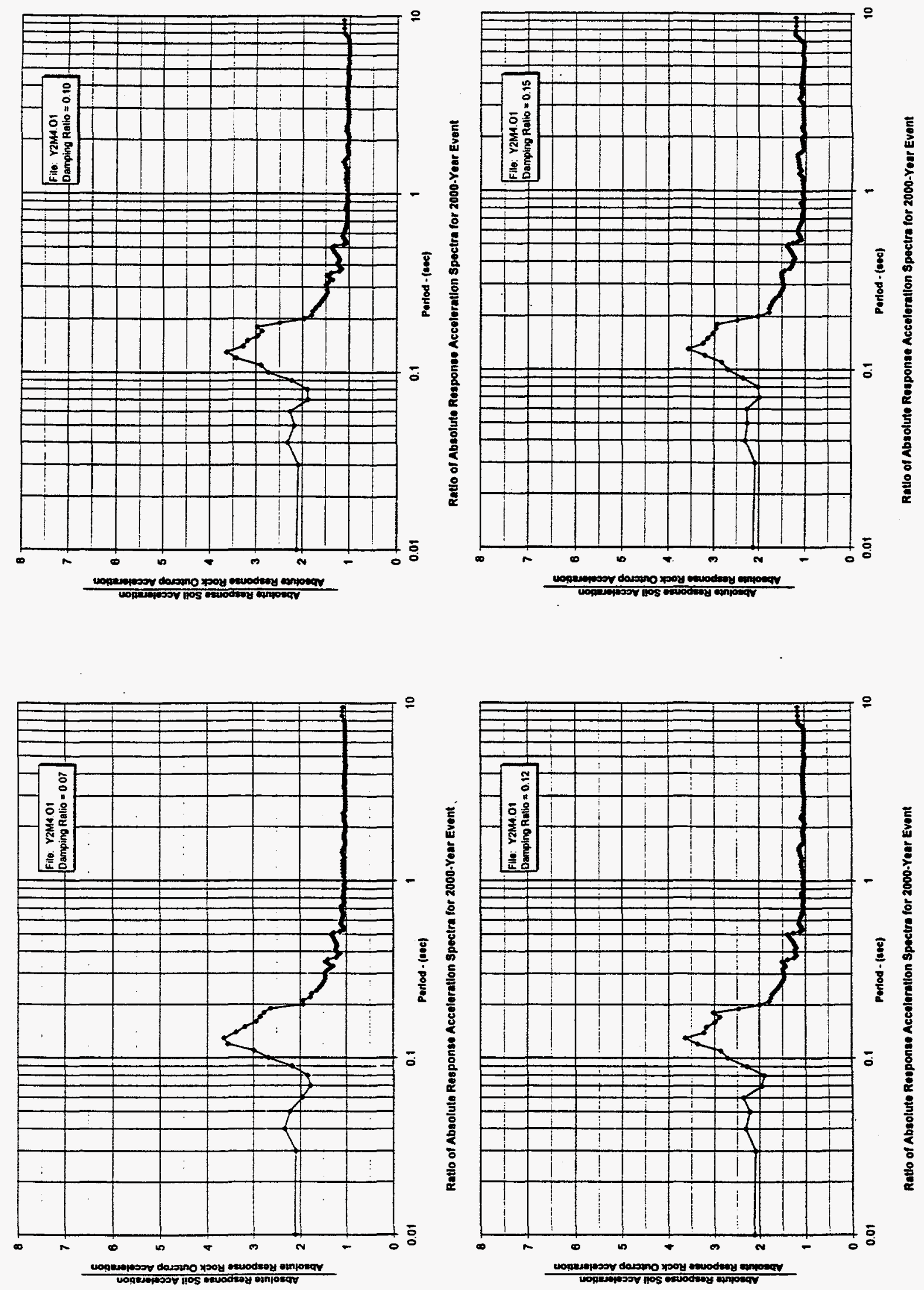

A2-33 

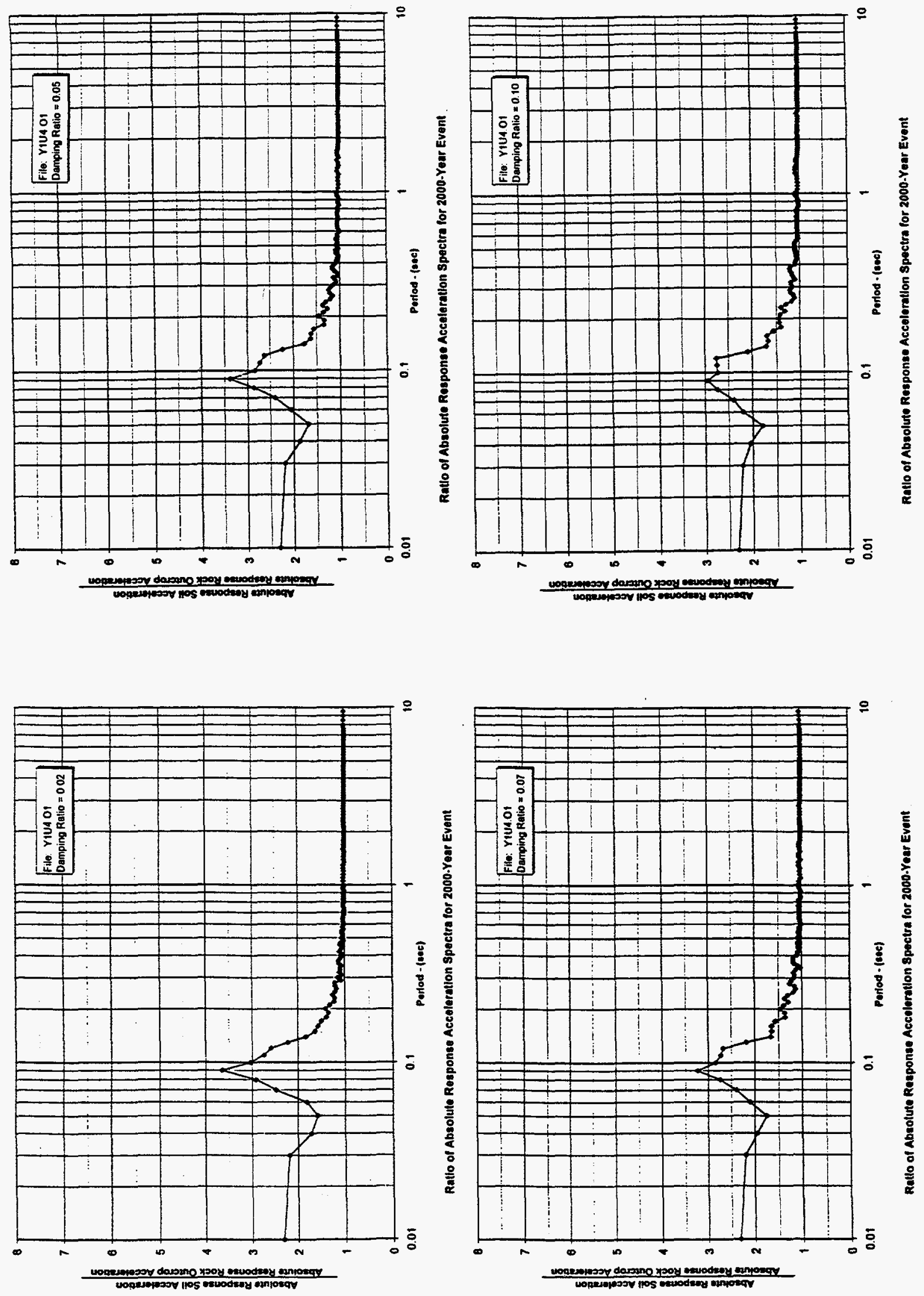

A2-34 

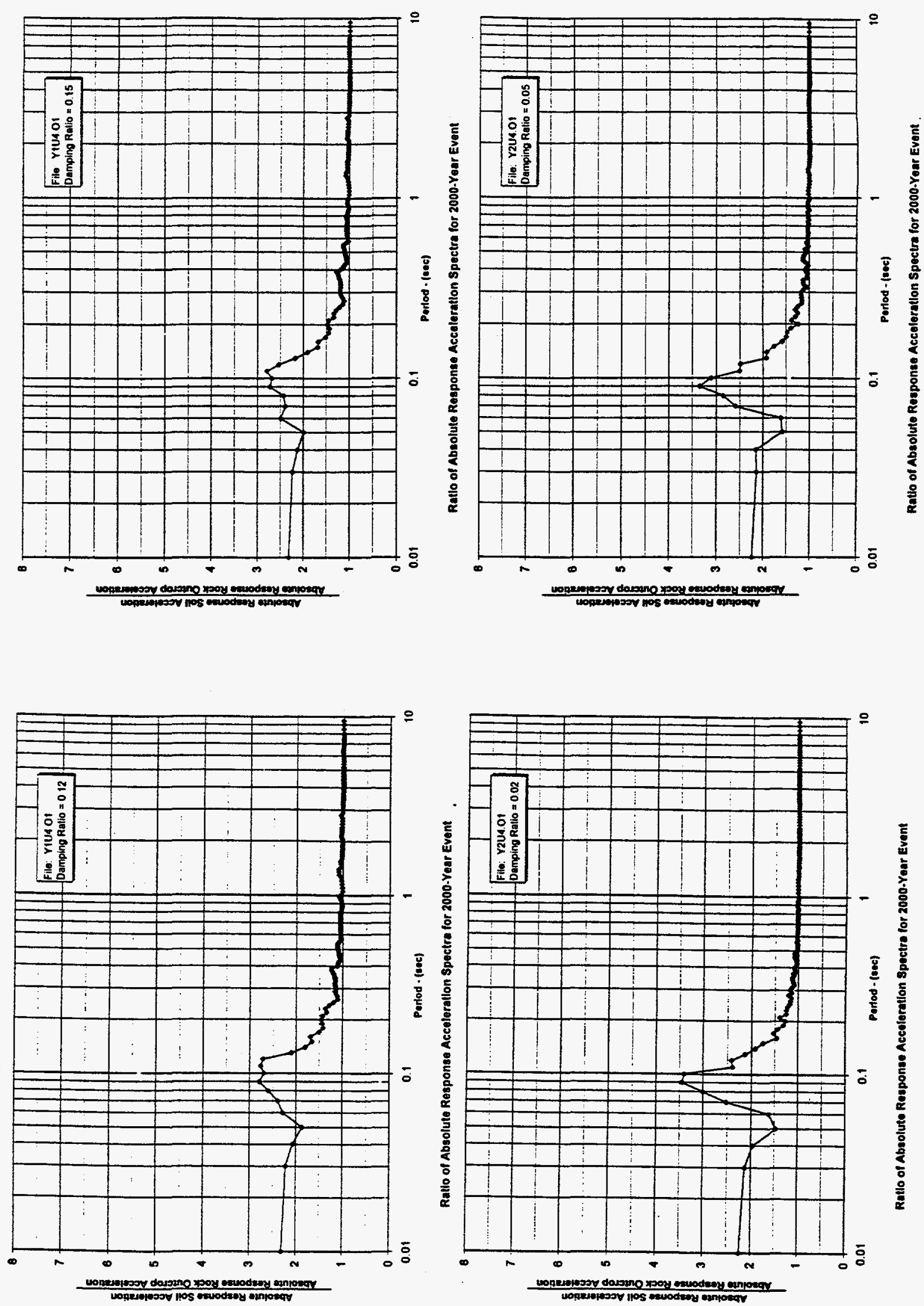

A2-35 

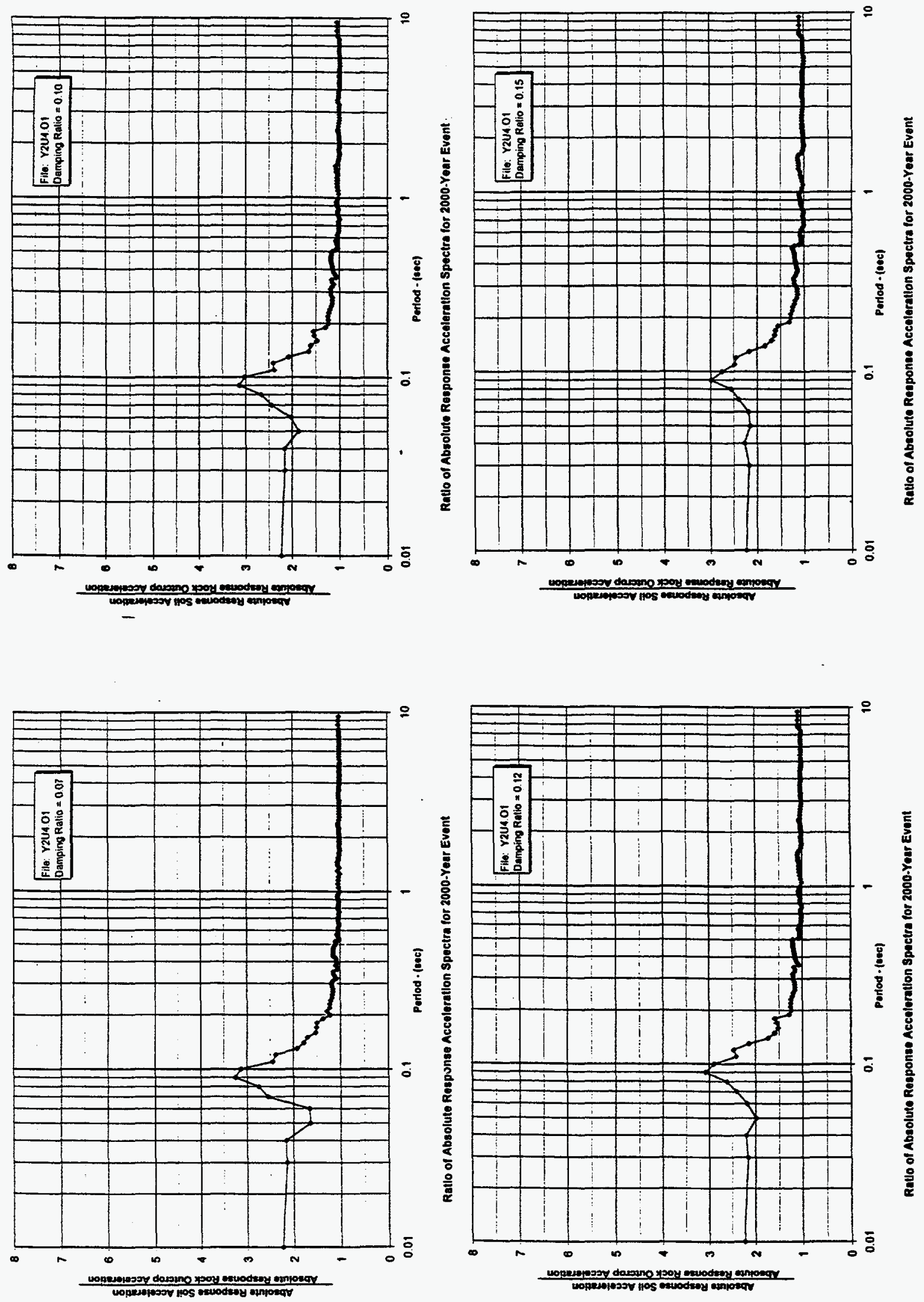

A2-36 

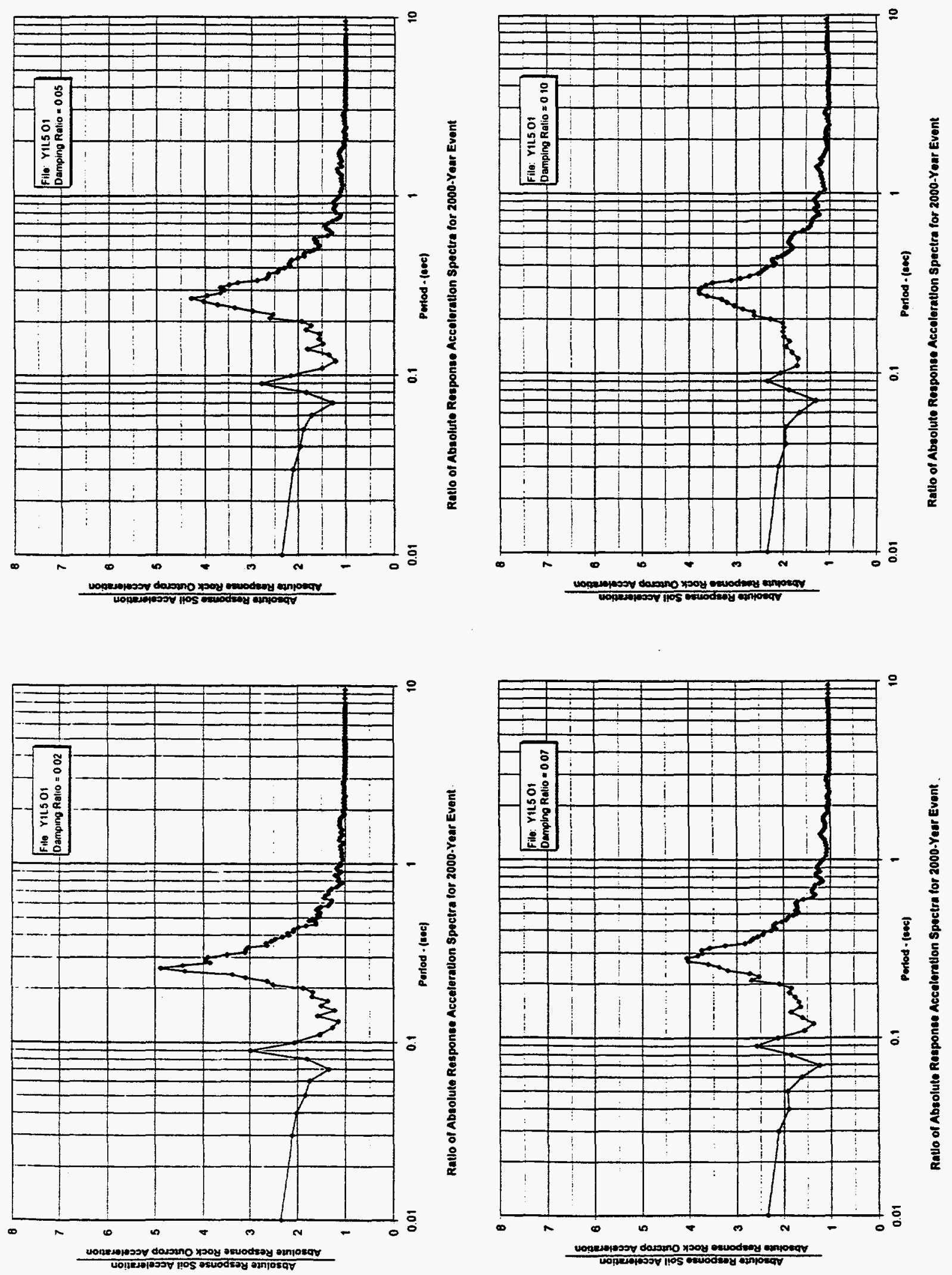

A2 -37 

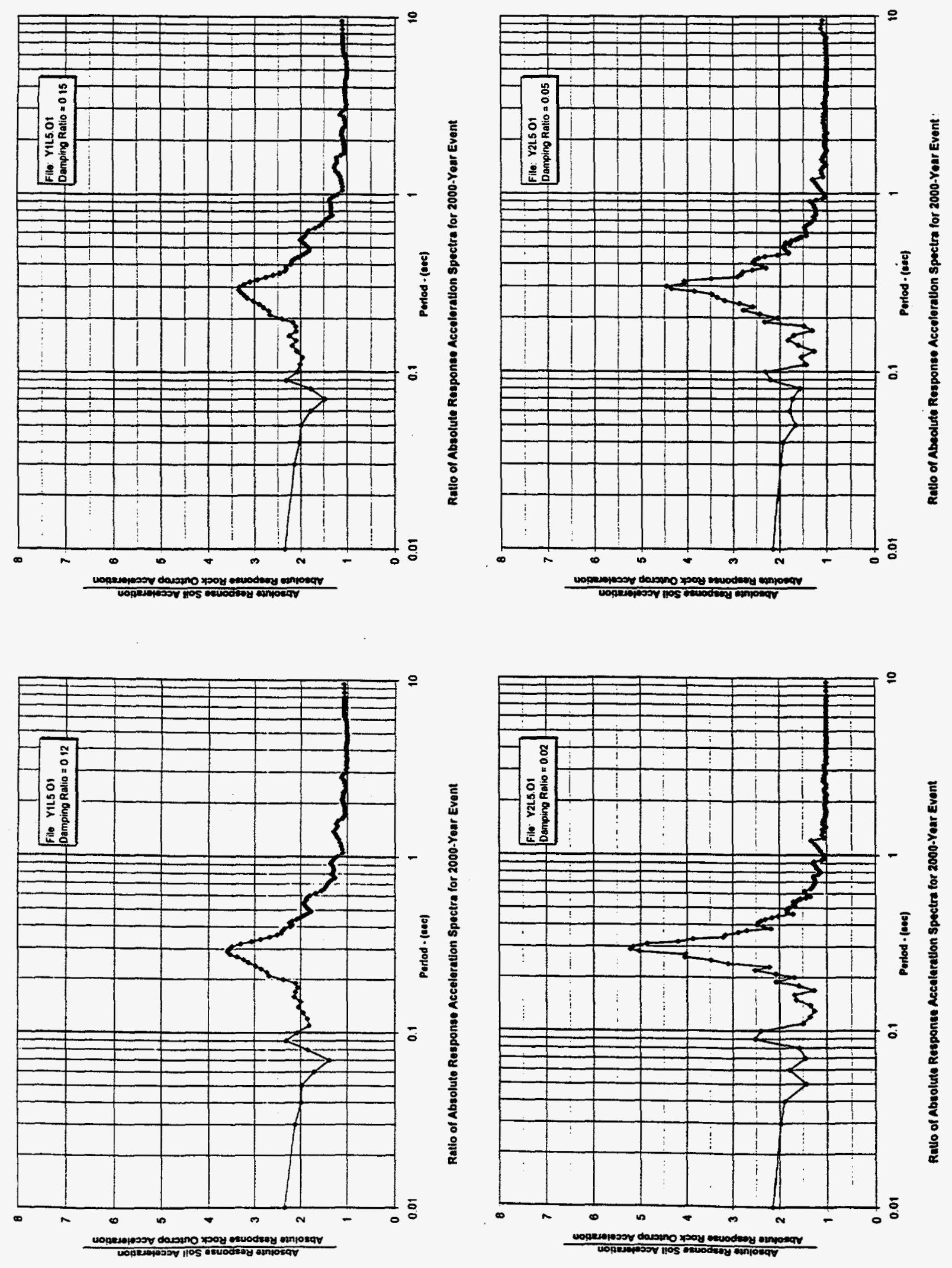

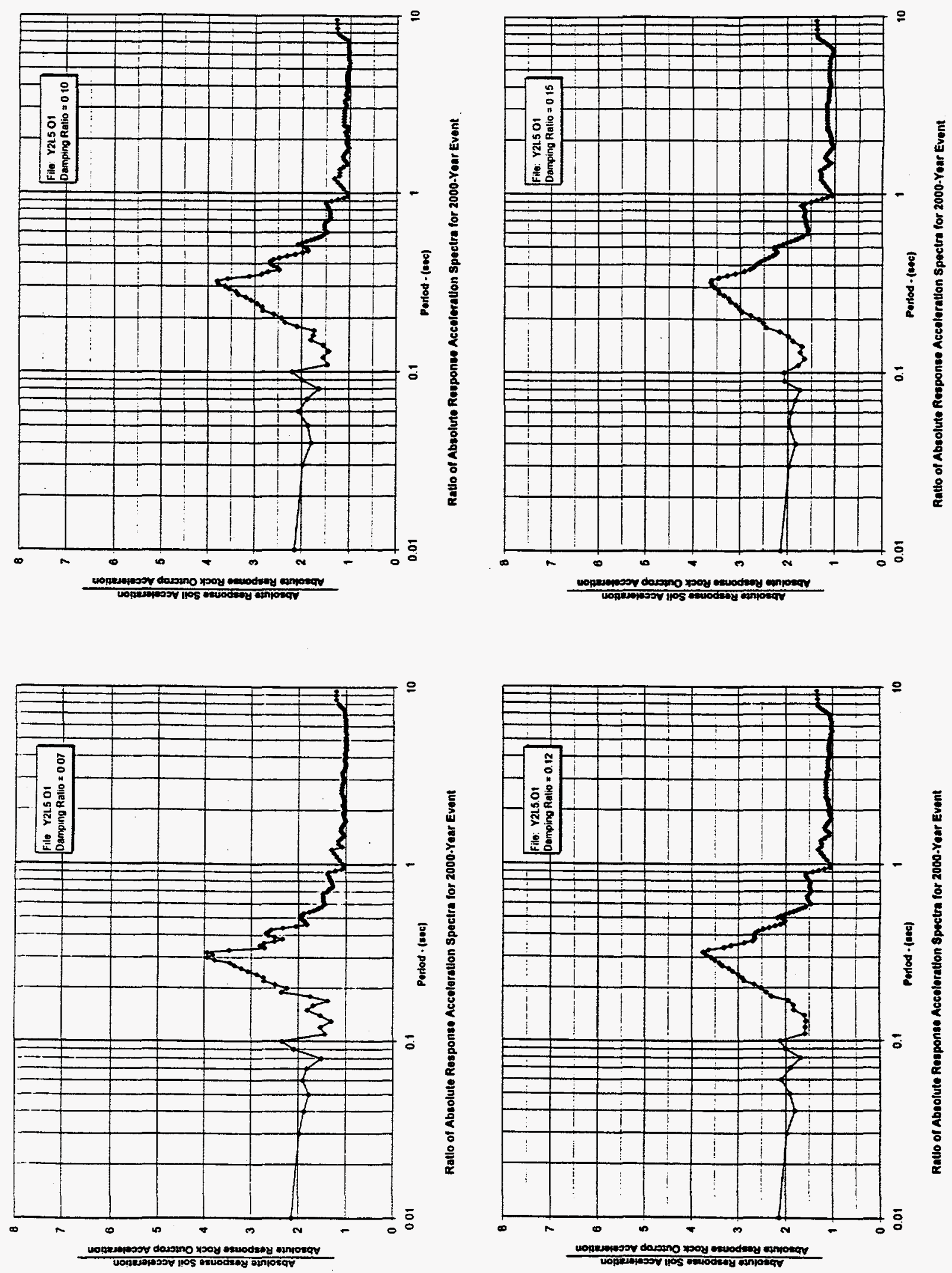

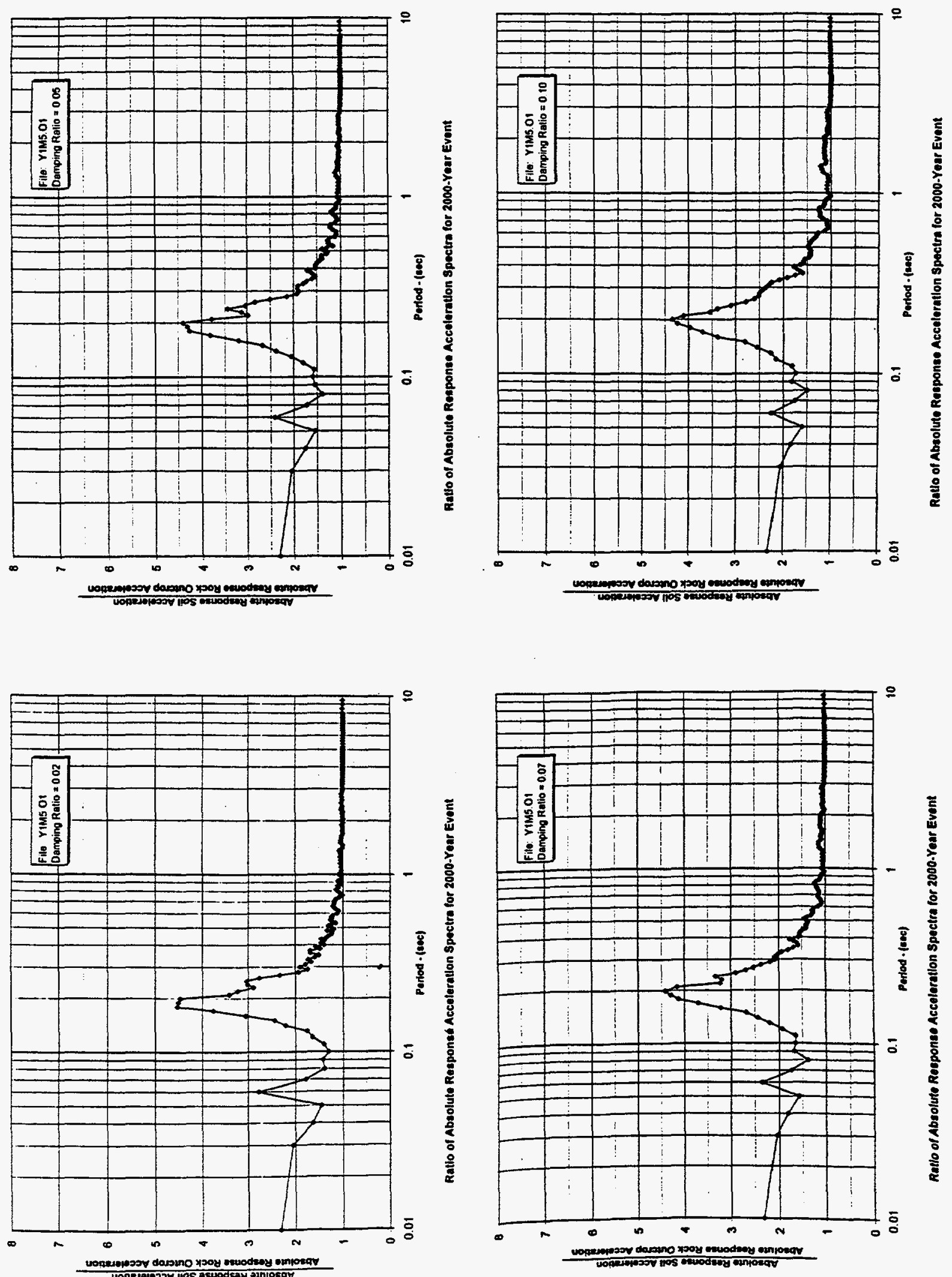

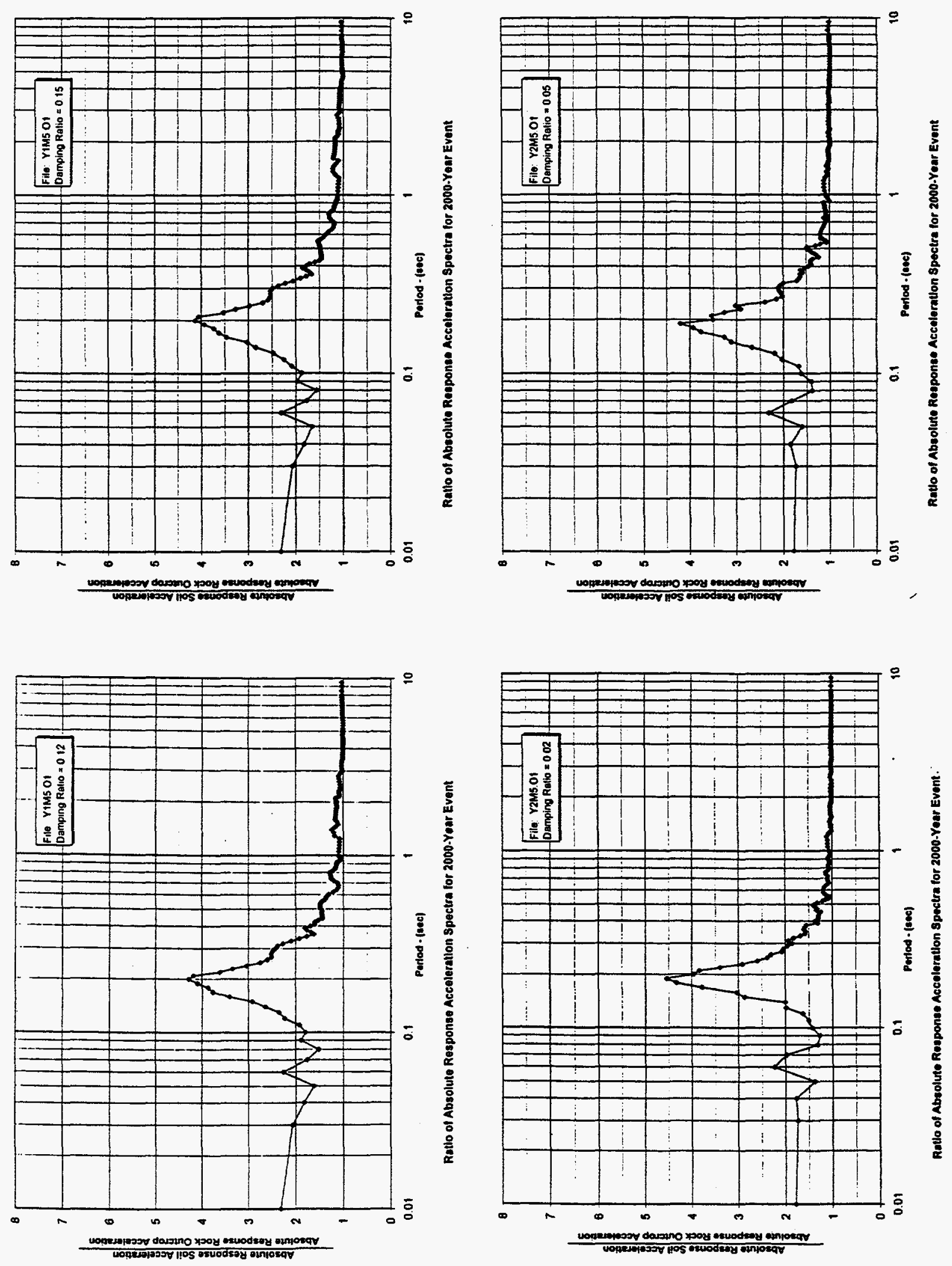

A2-41 

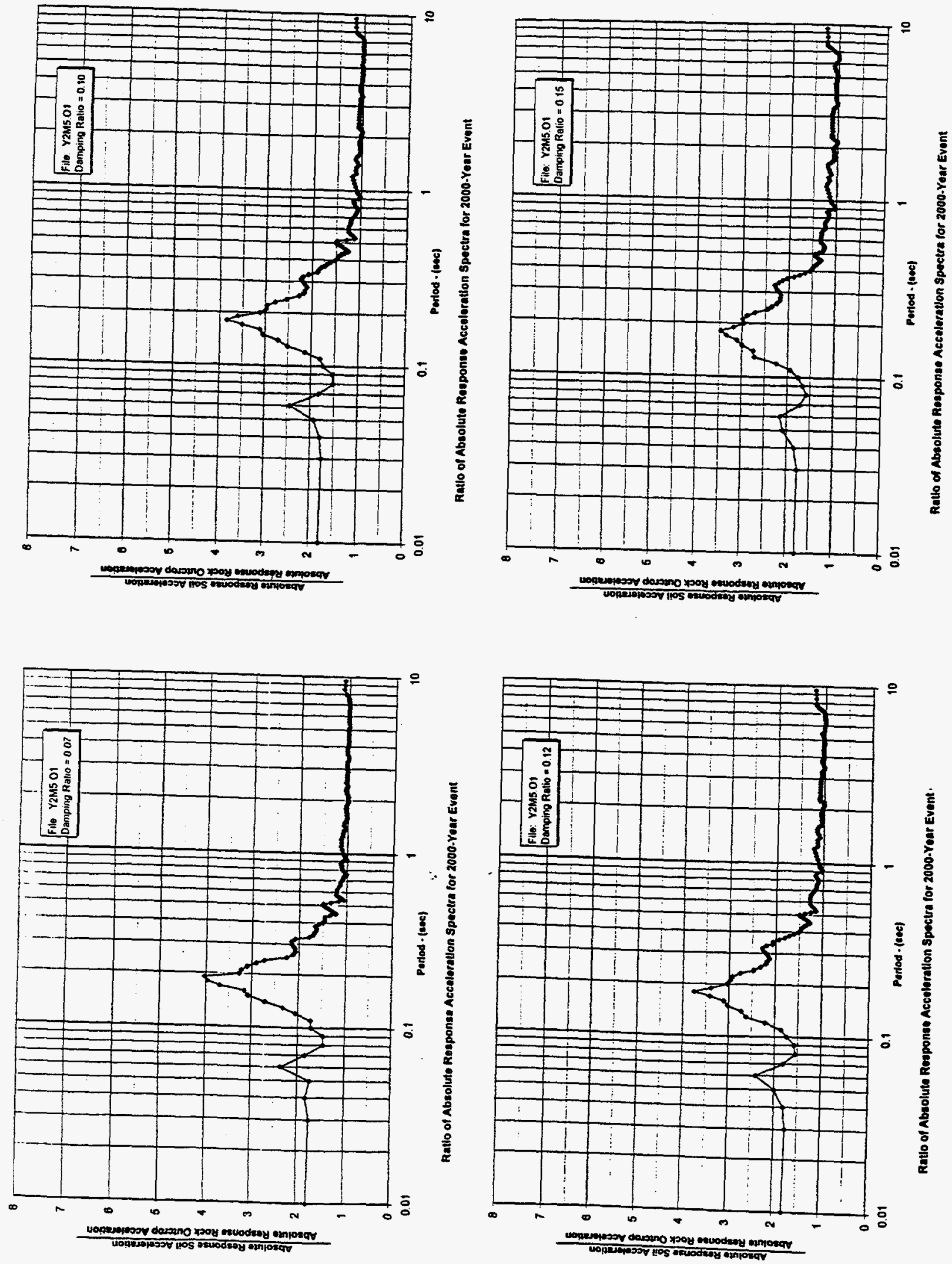

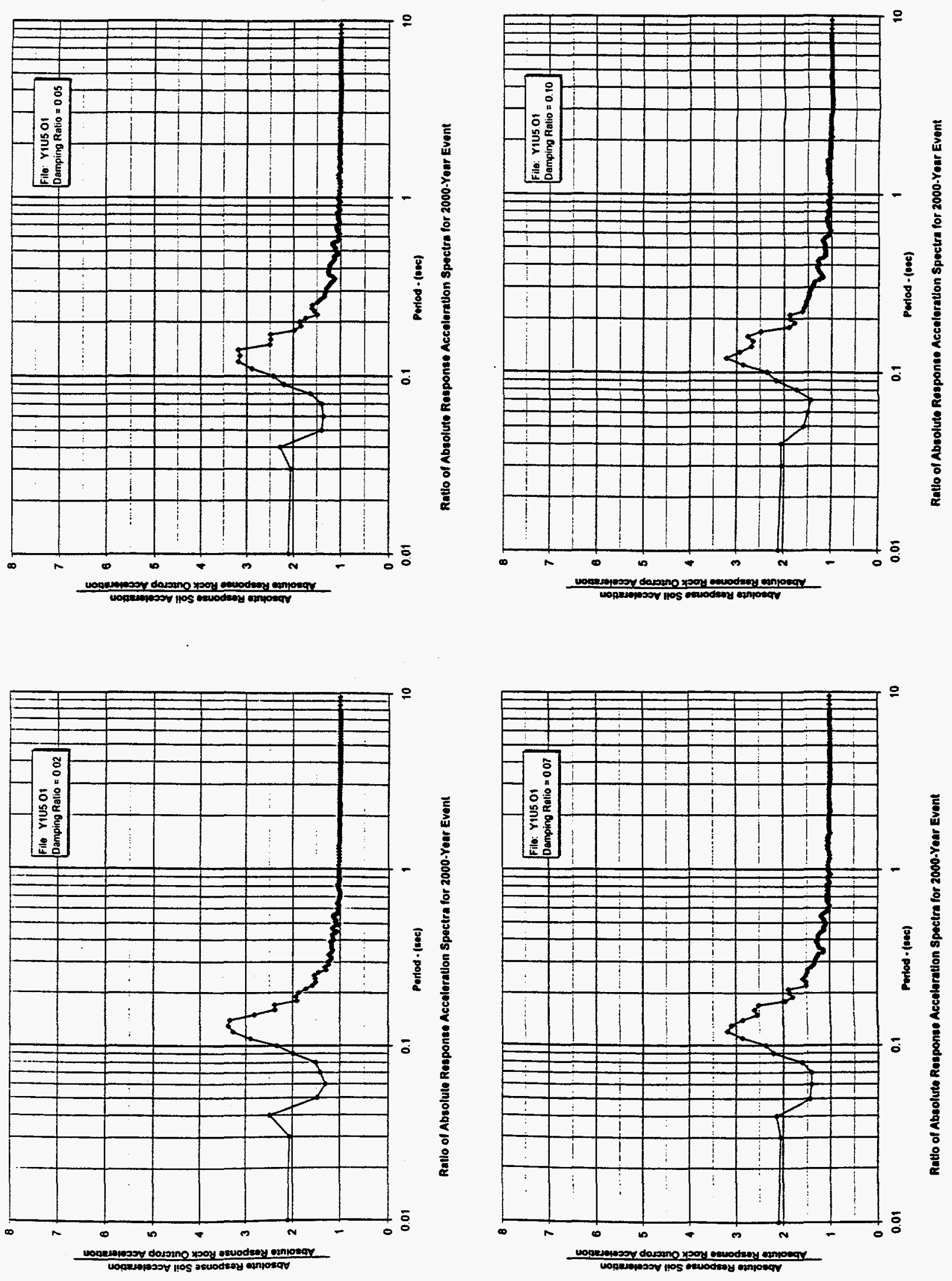

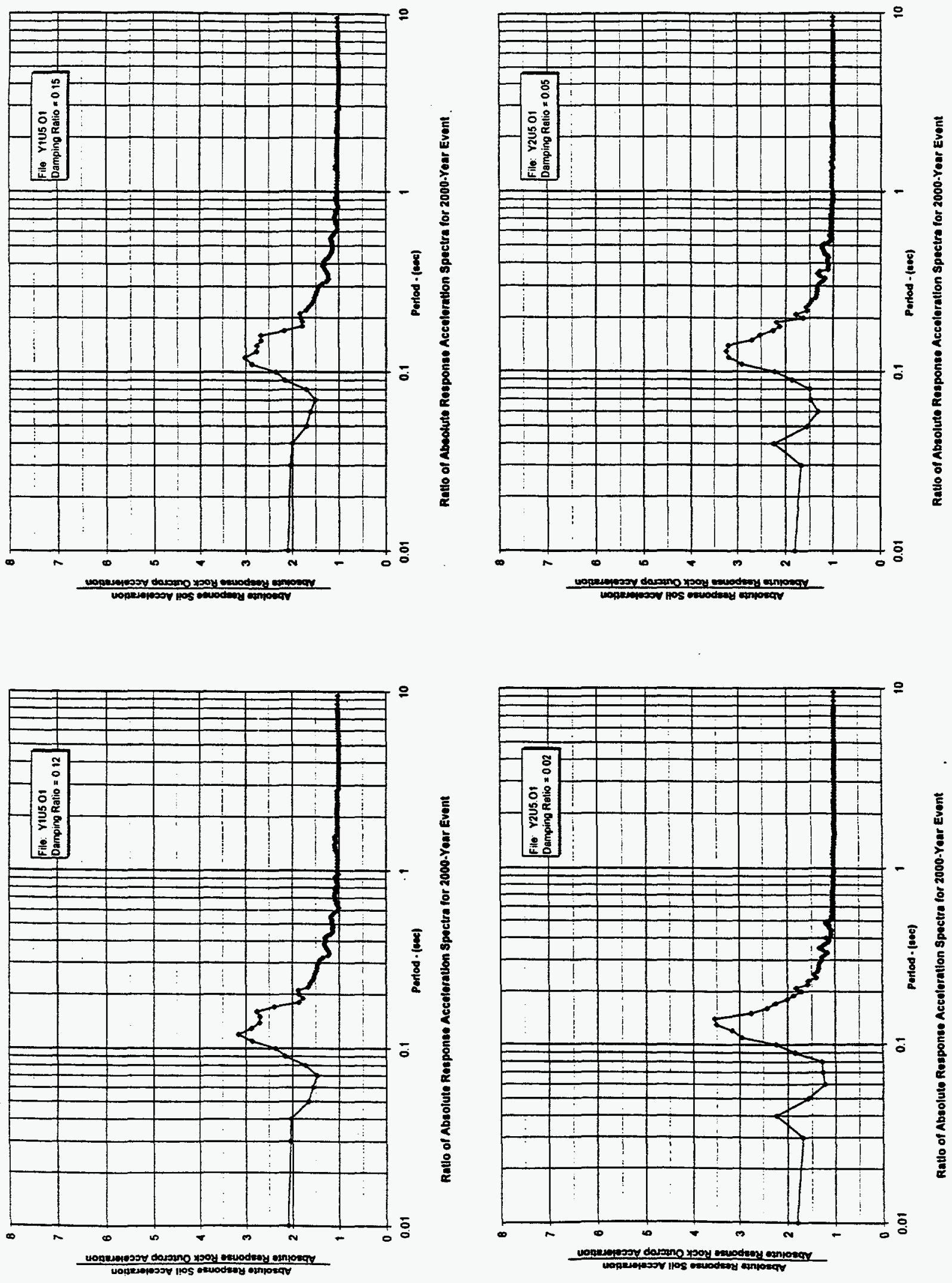

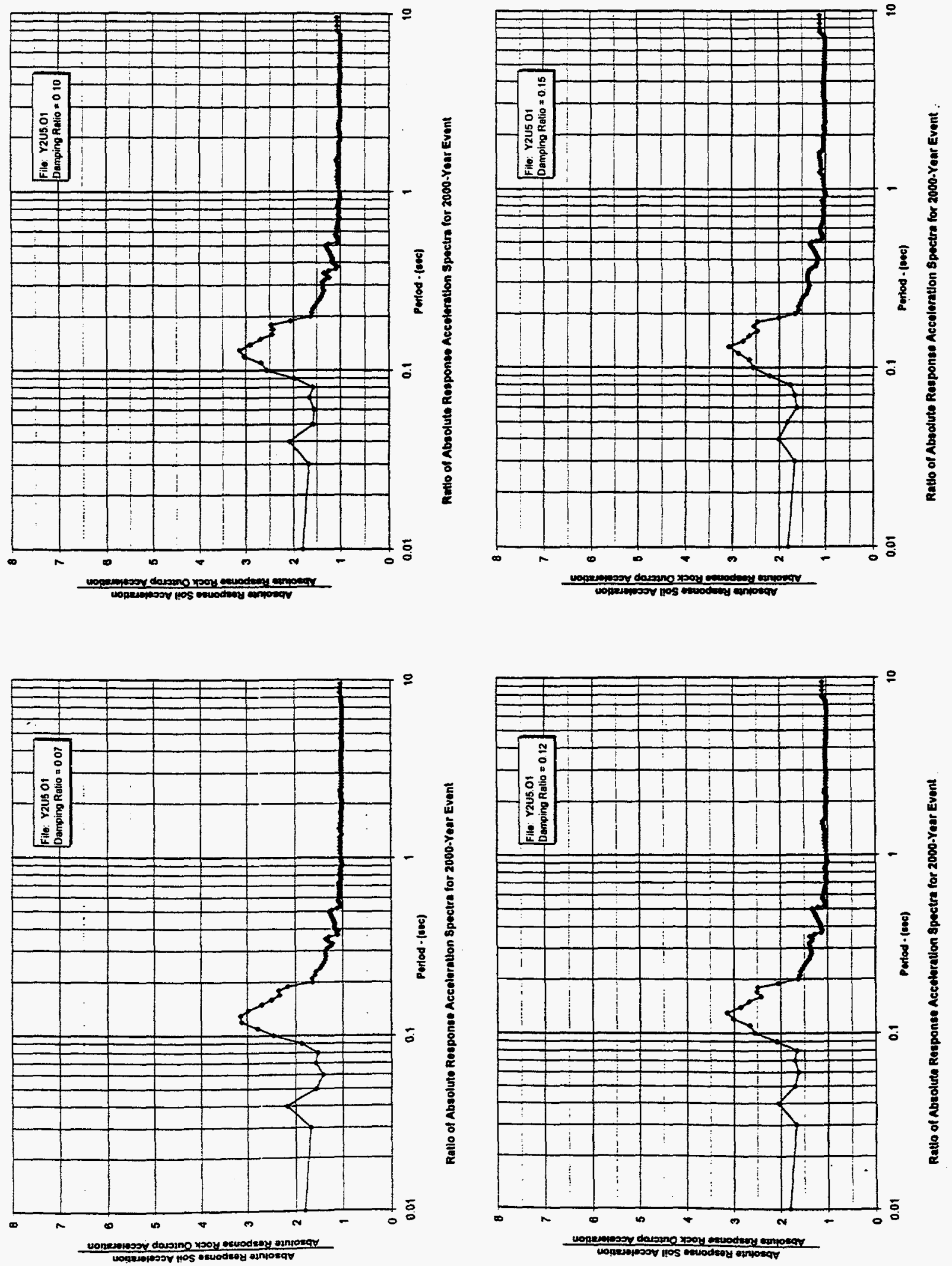

A2-45 

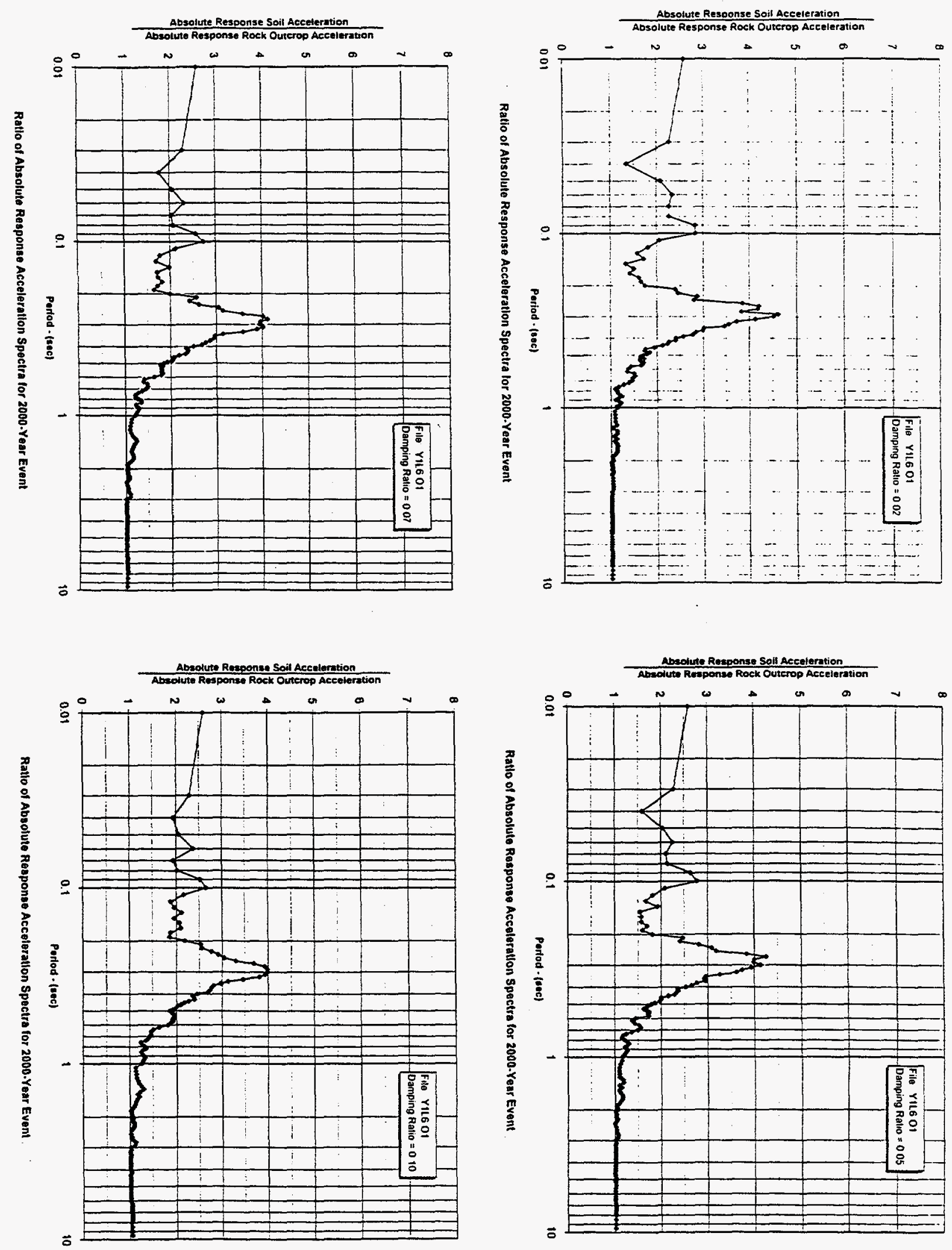

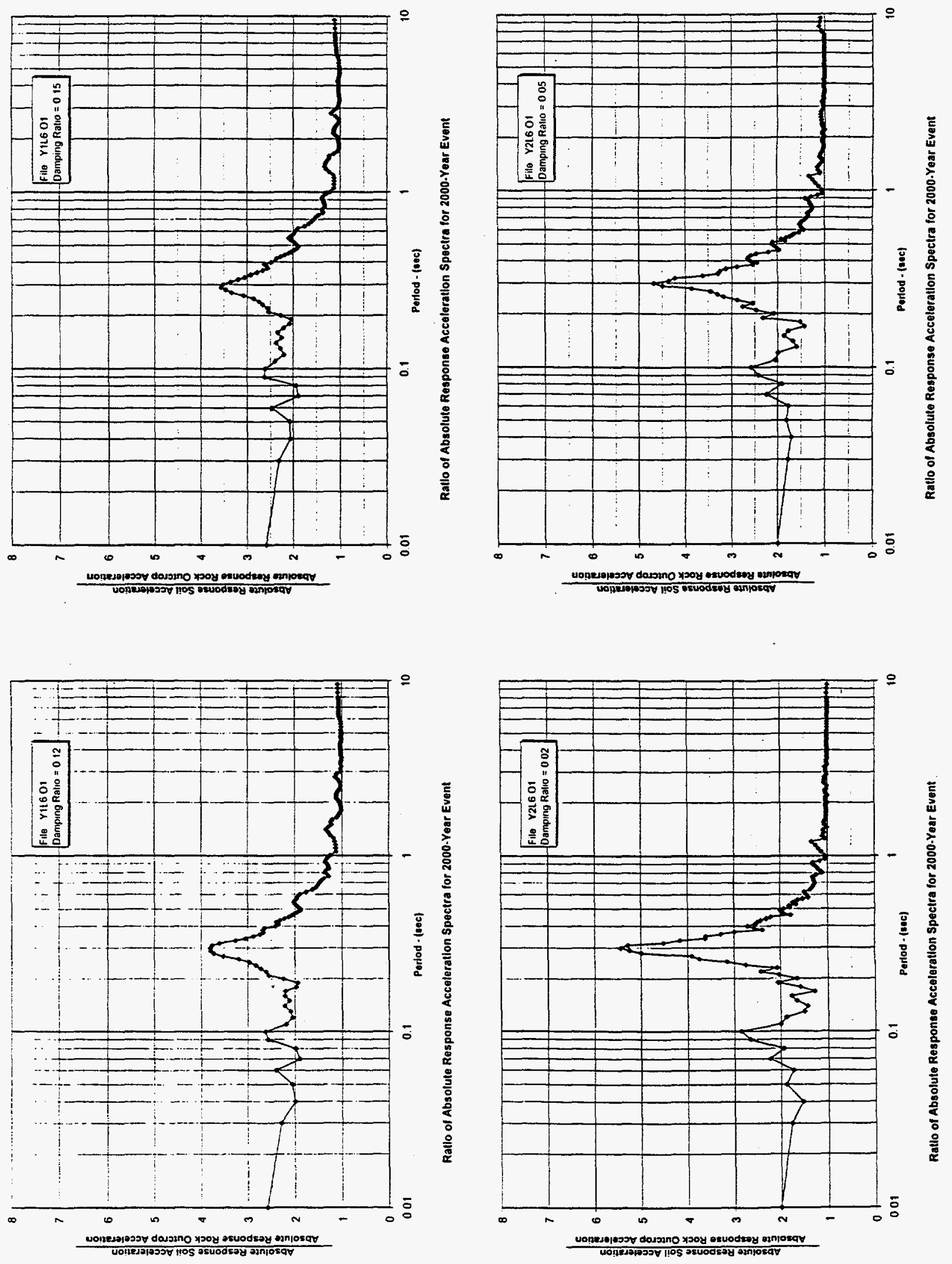

A2-47 
$87-2 \forall$
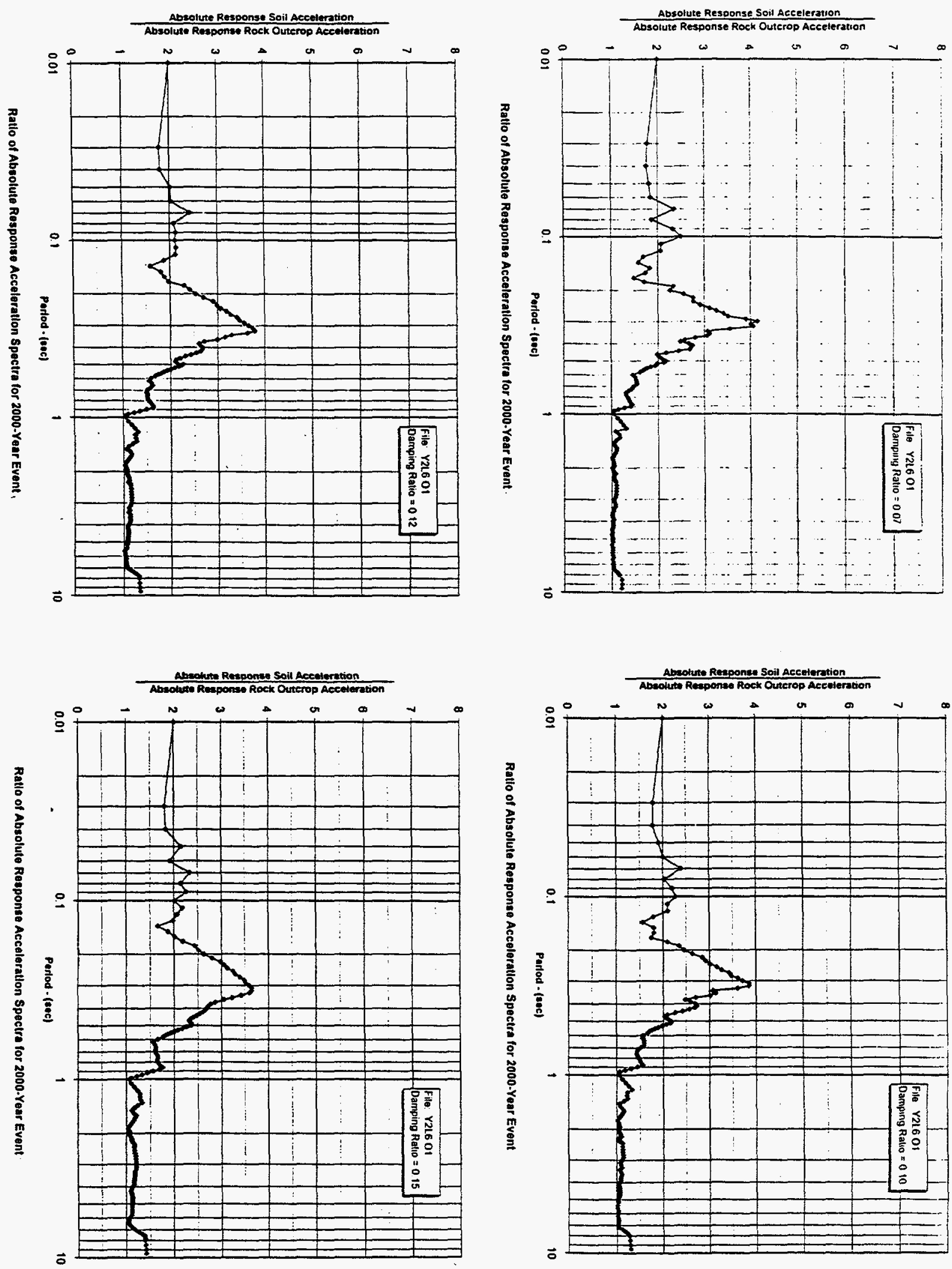

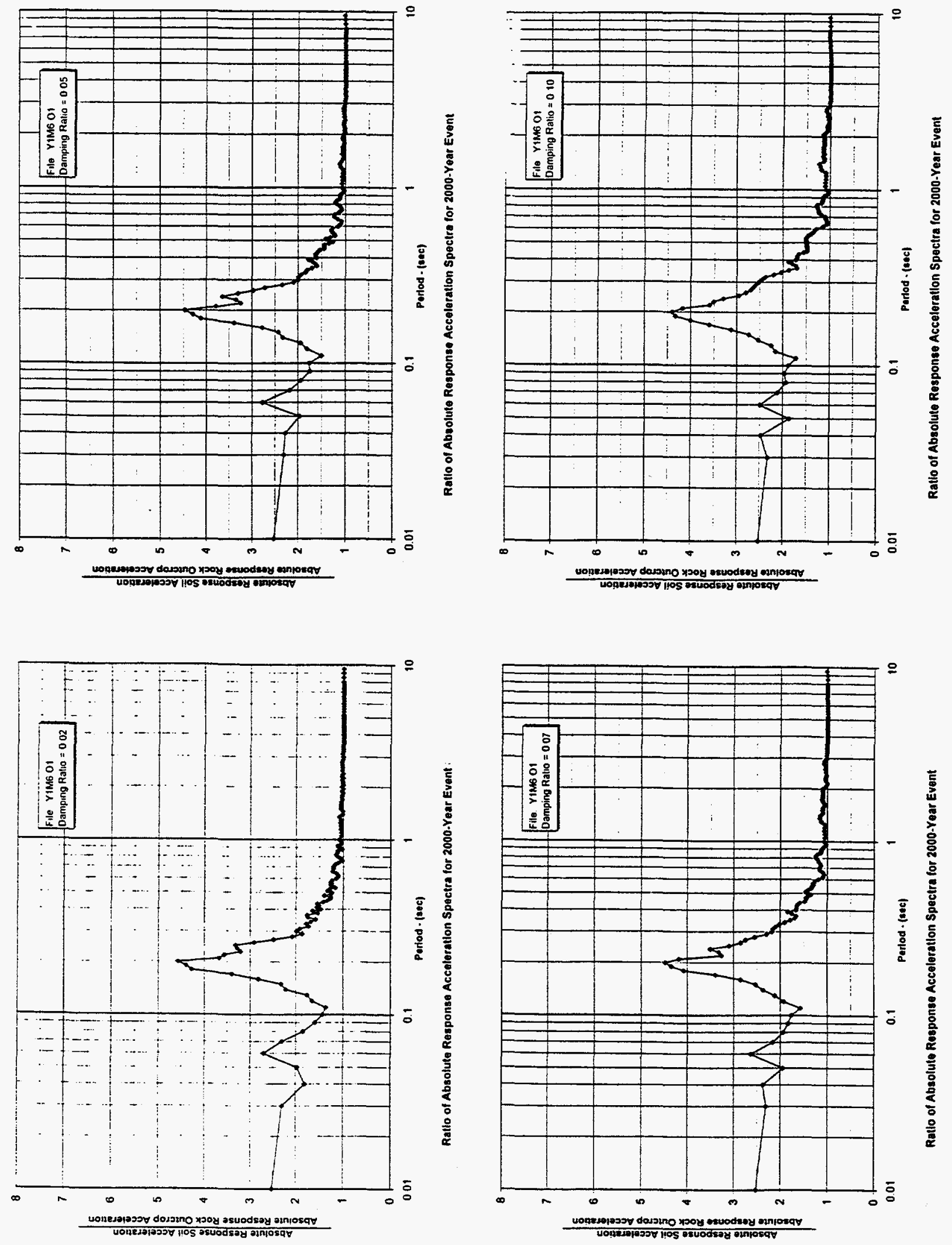

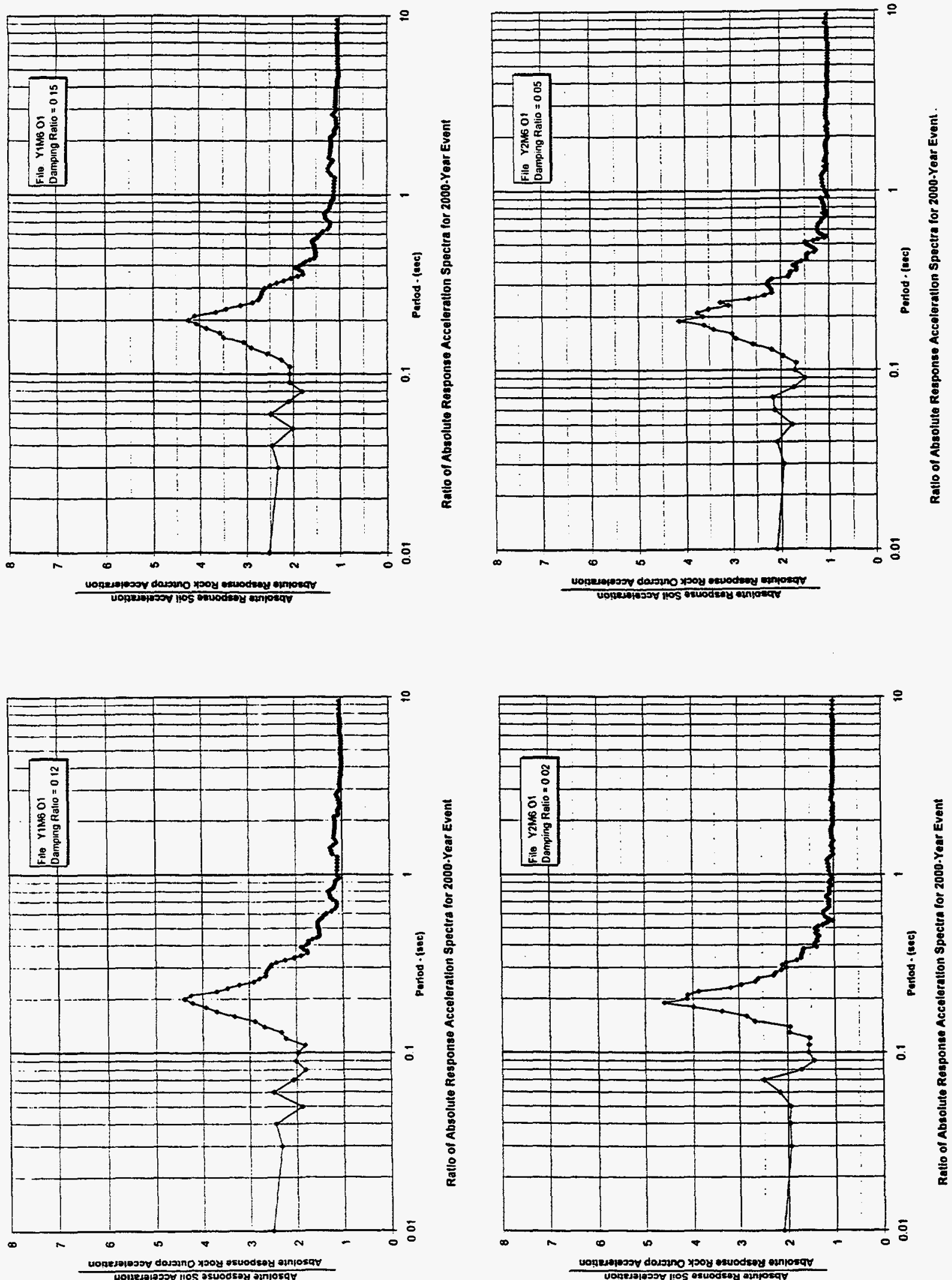

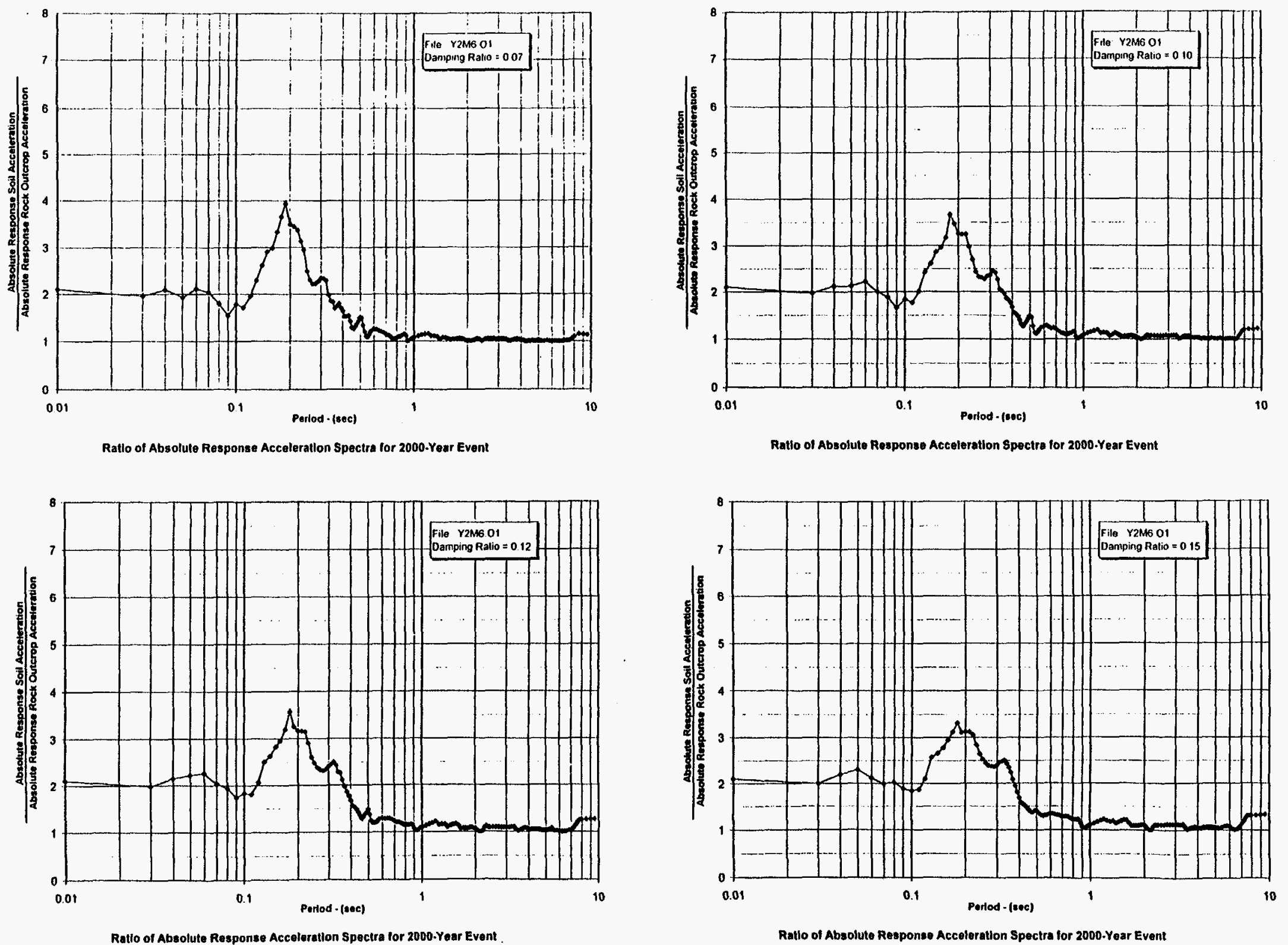

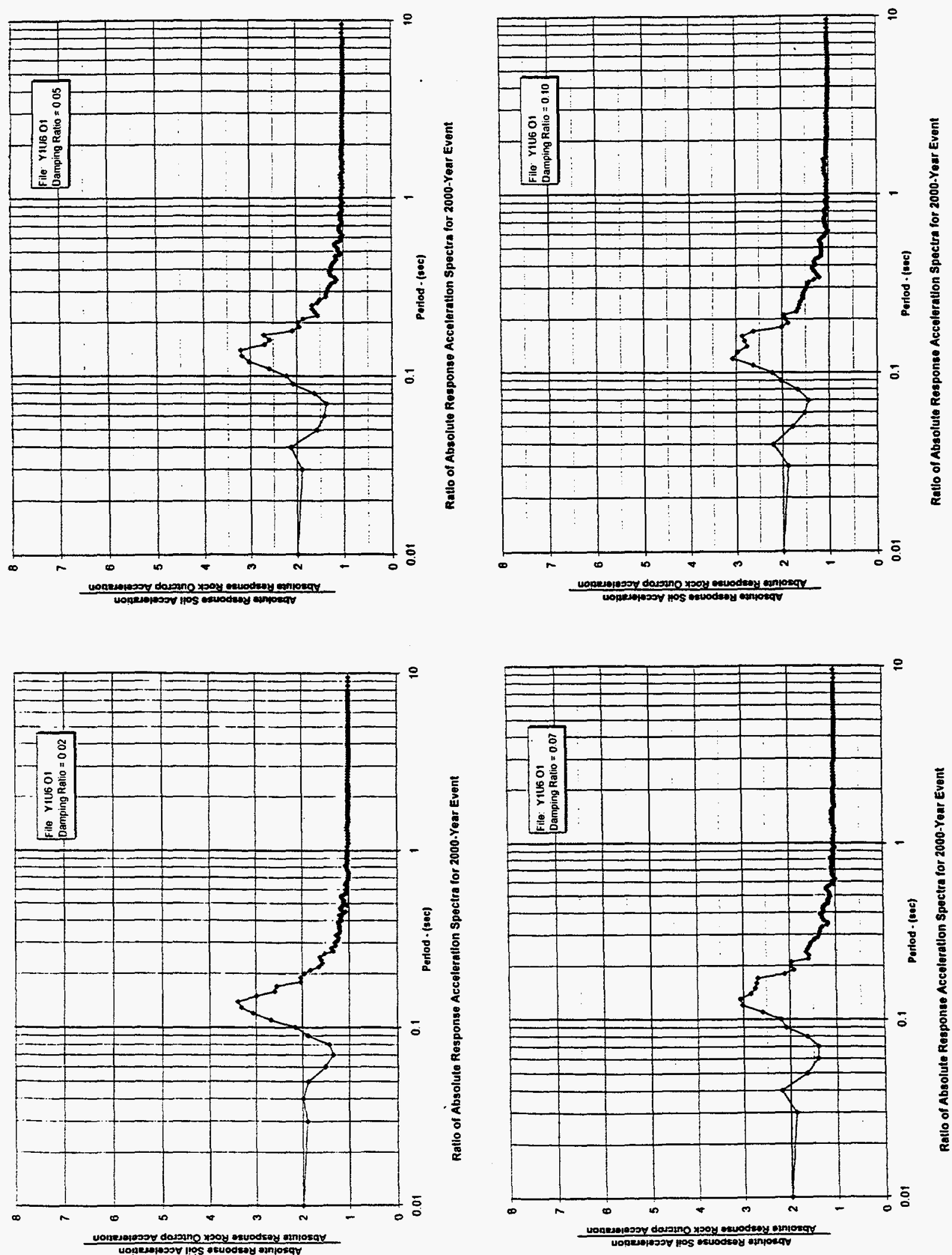

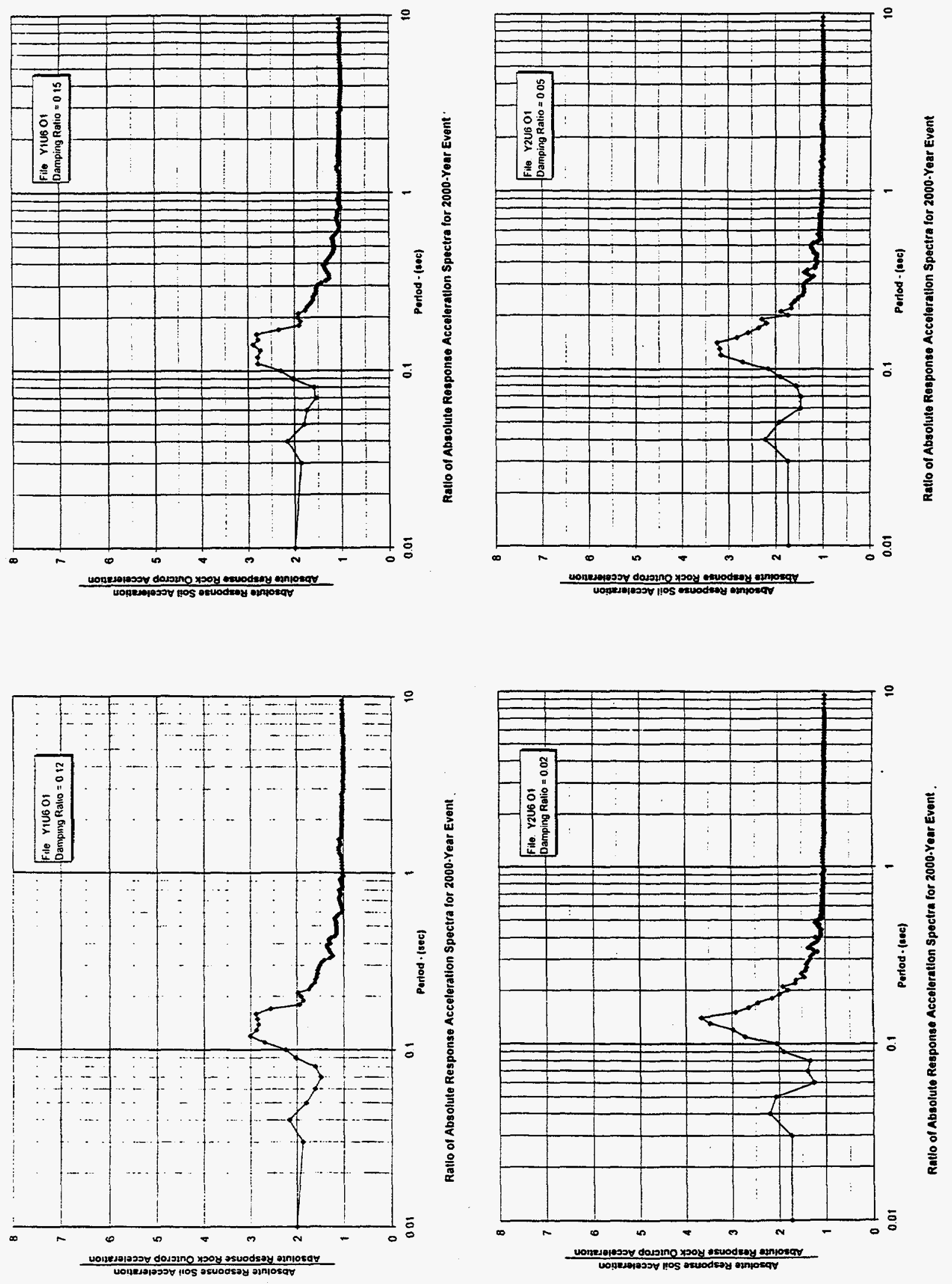

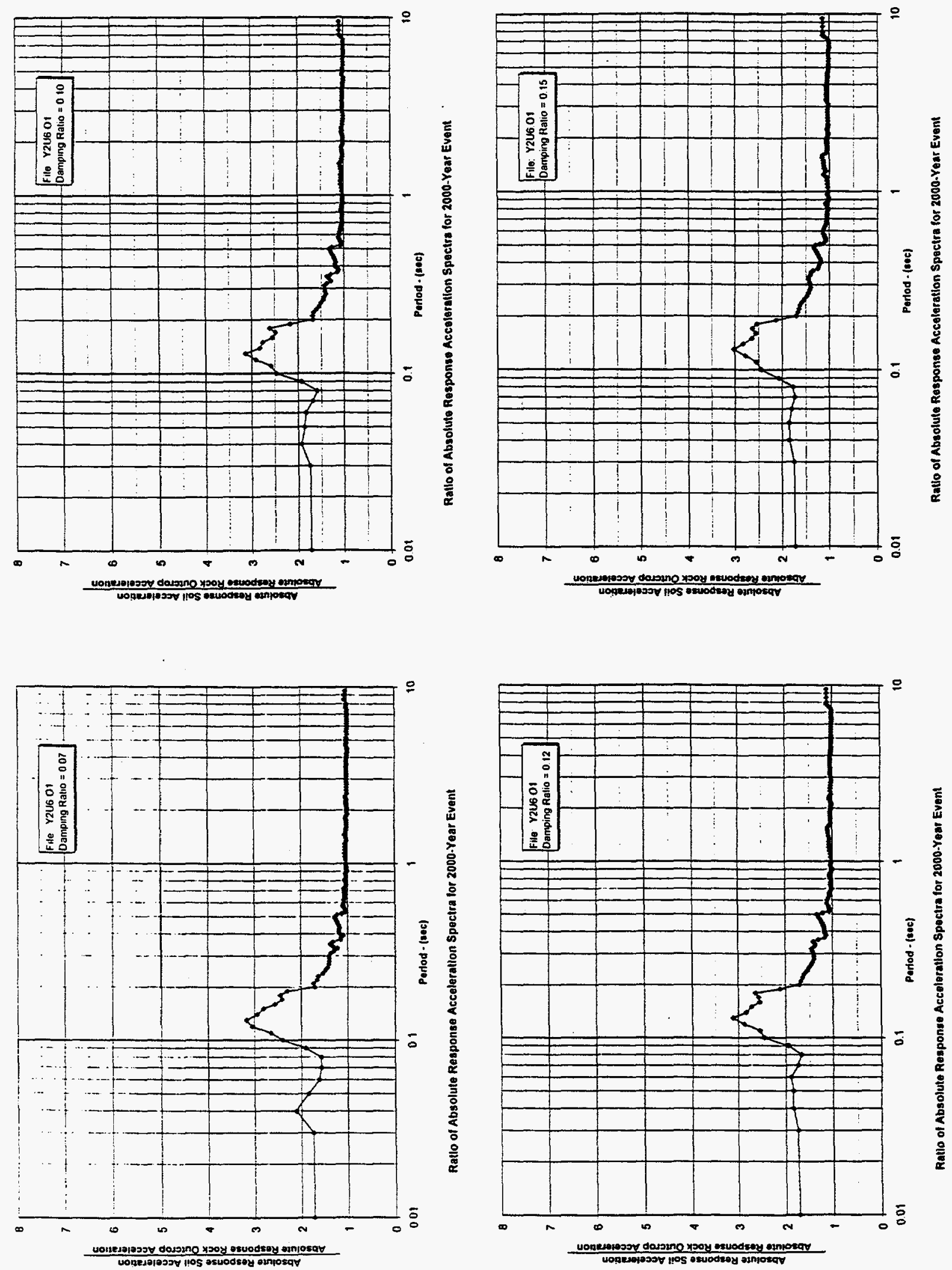

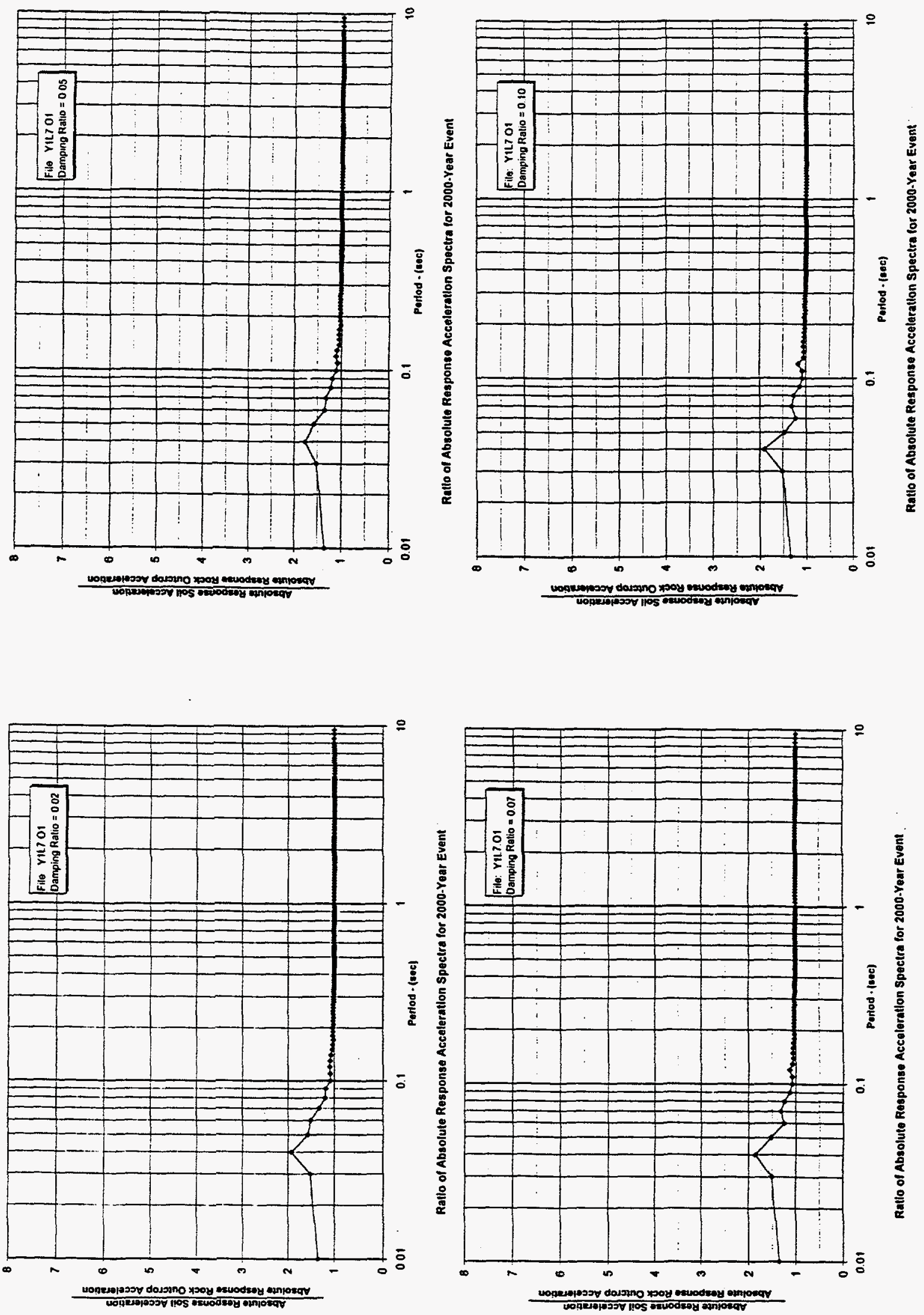

A2-55 

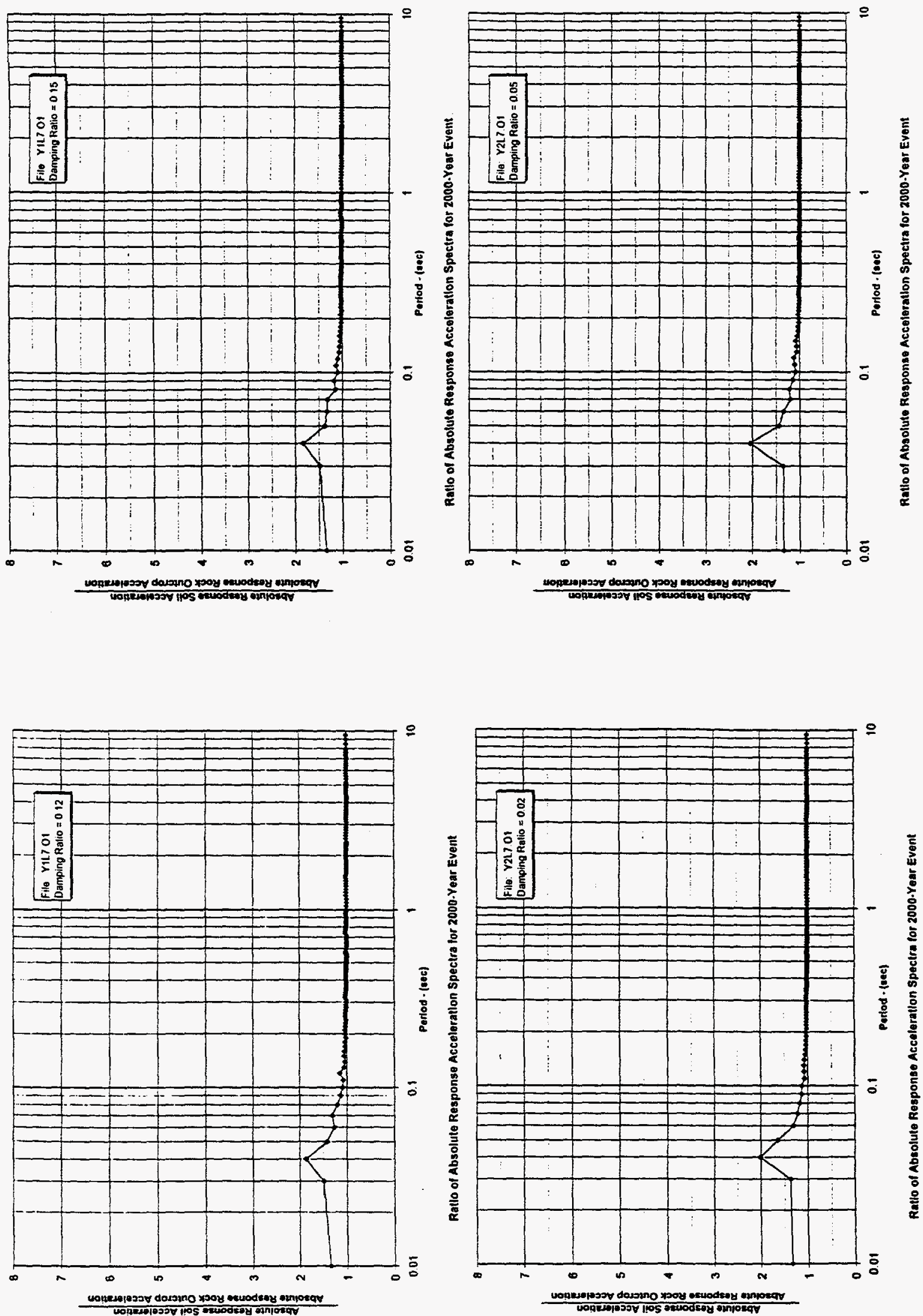

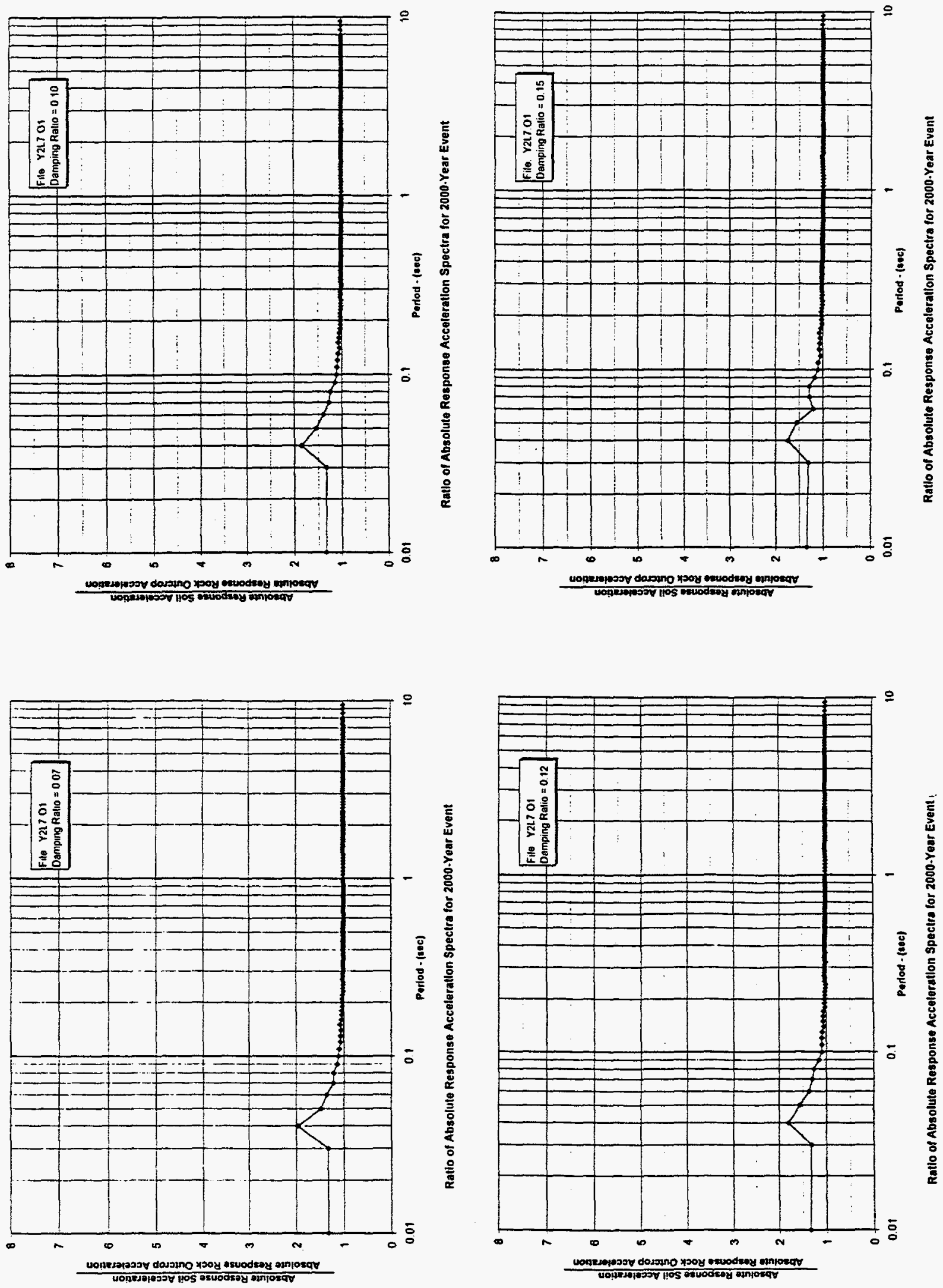

A2-57 

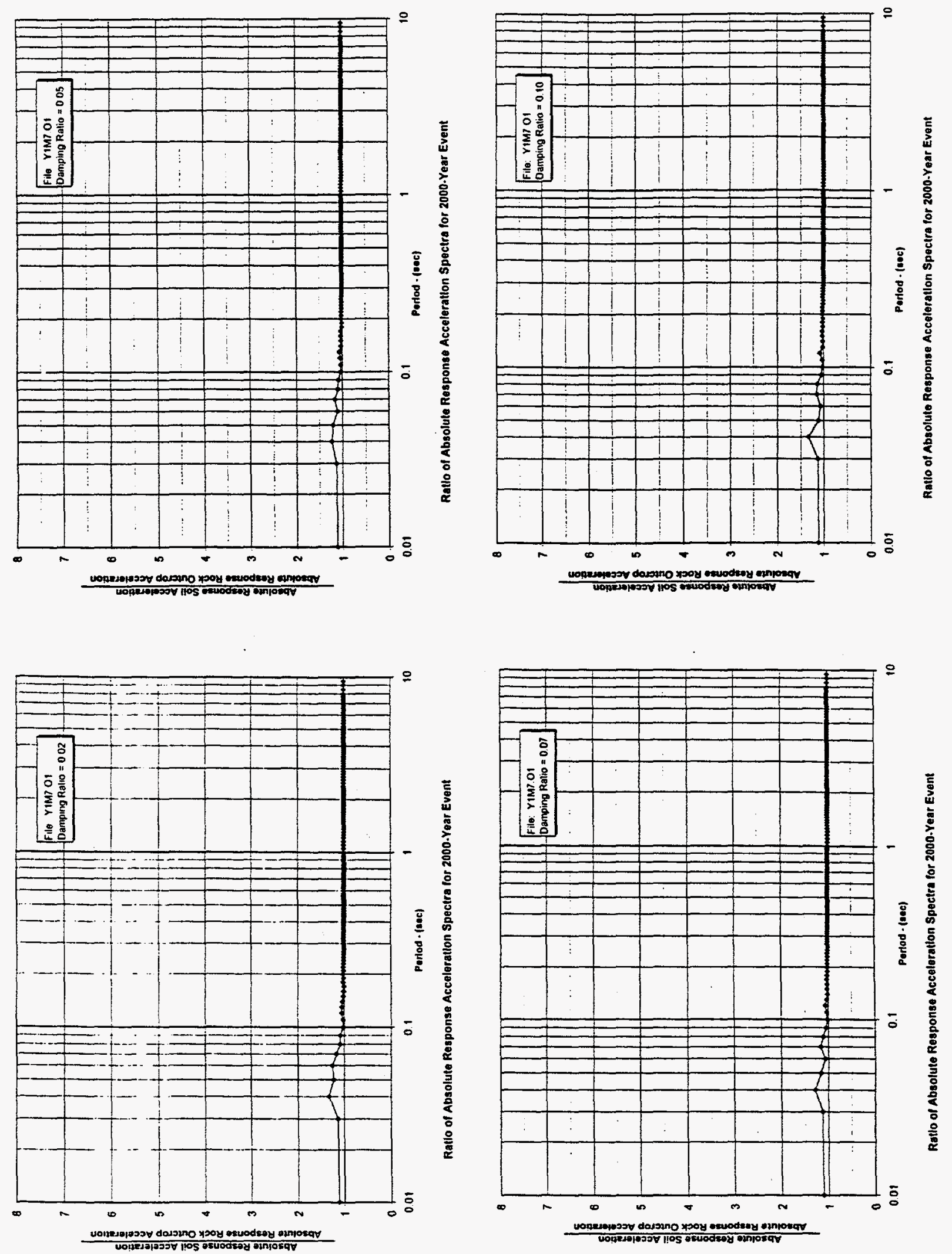

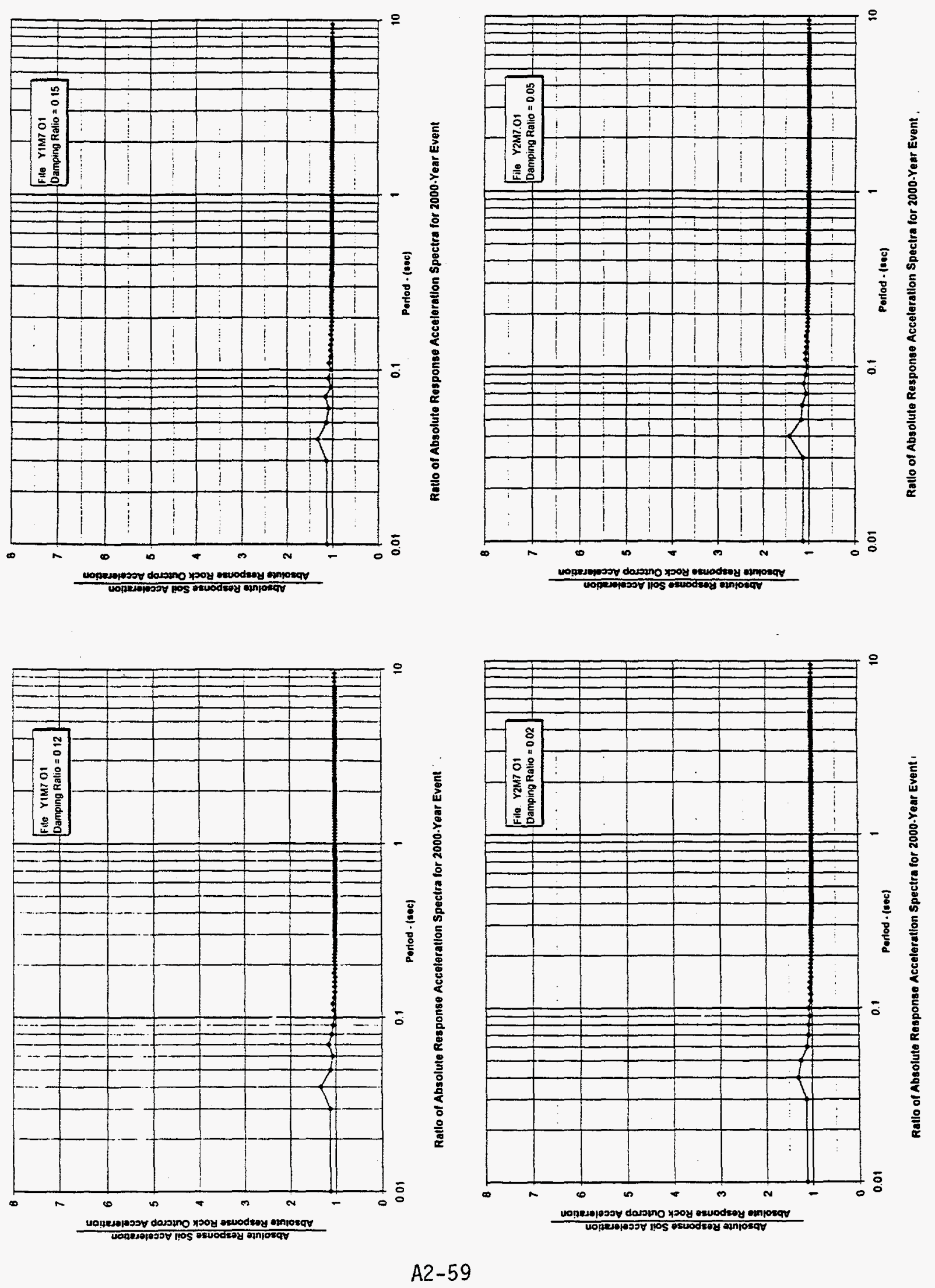

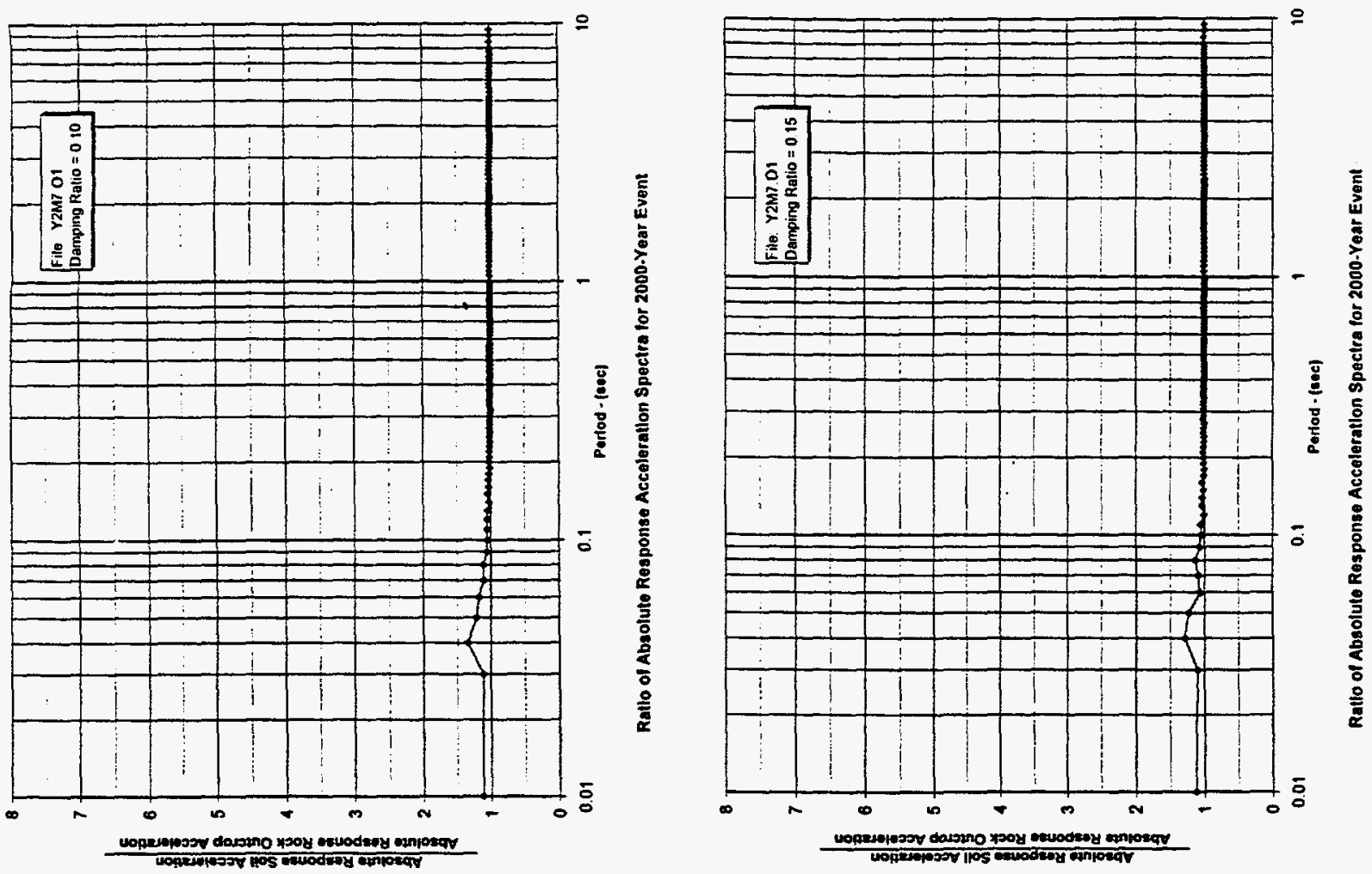

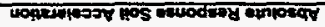
loin
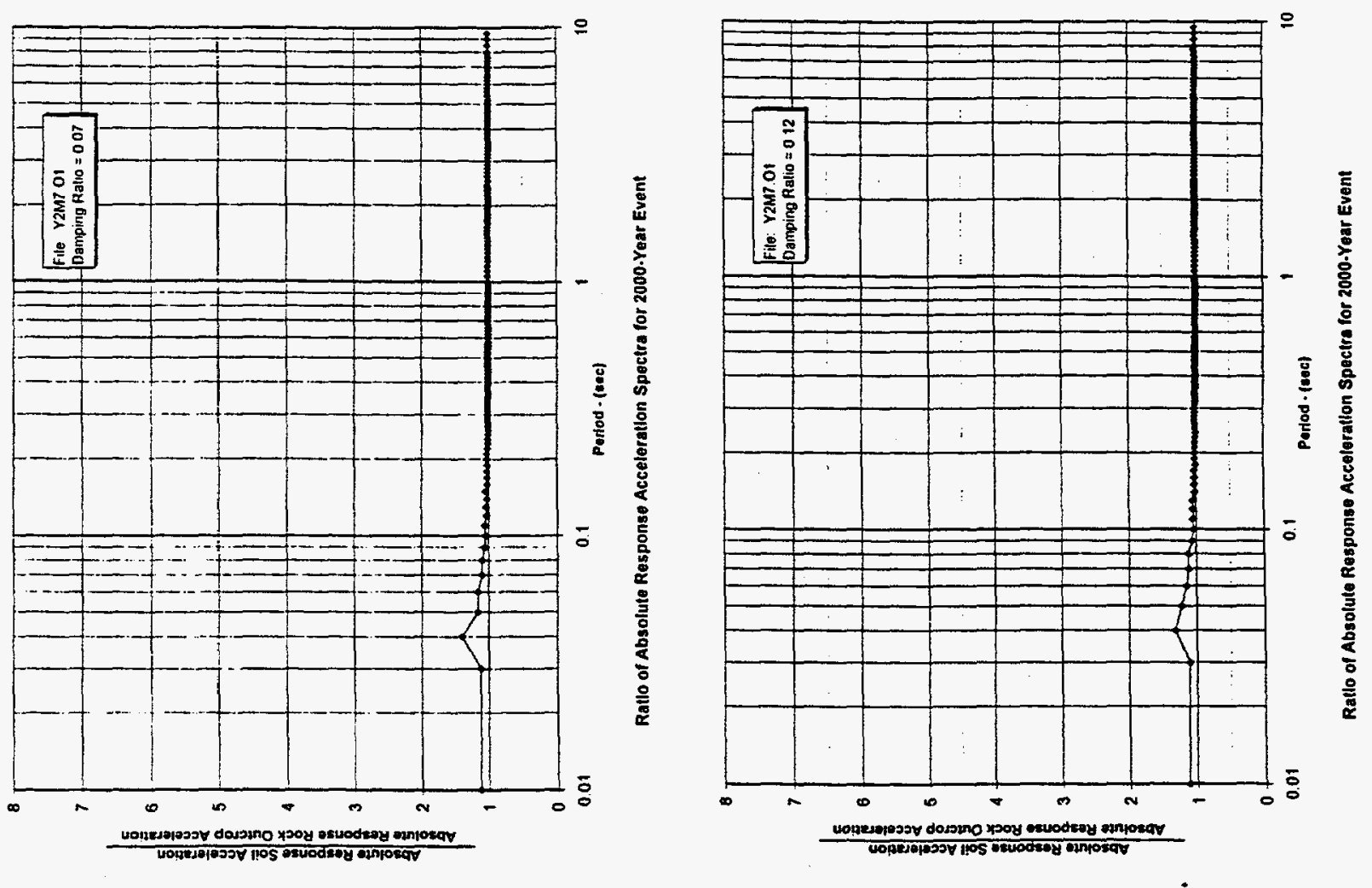

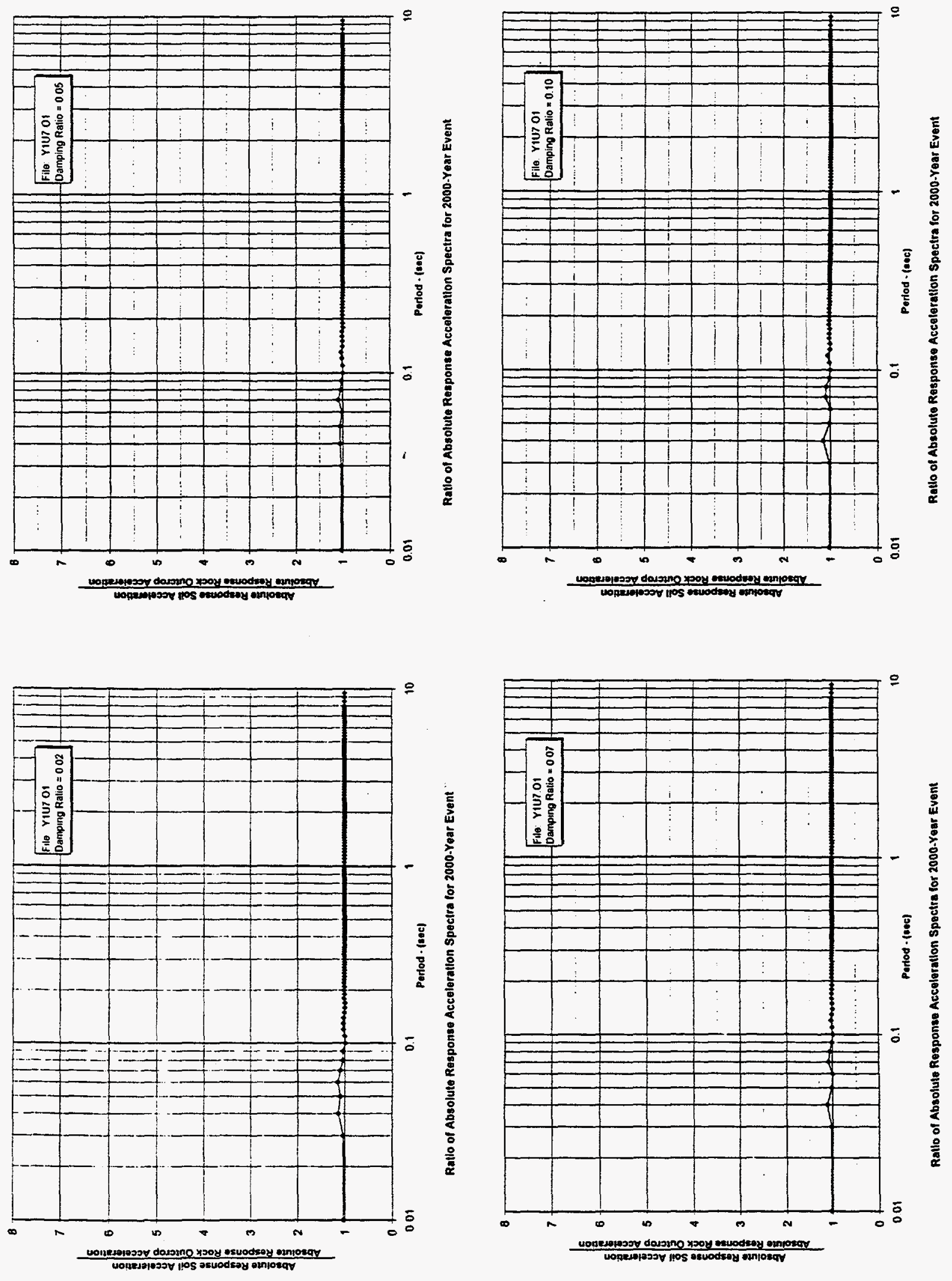

A2-61 

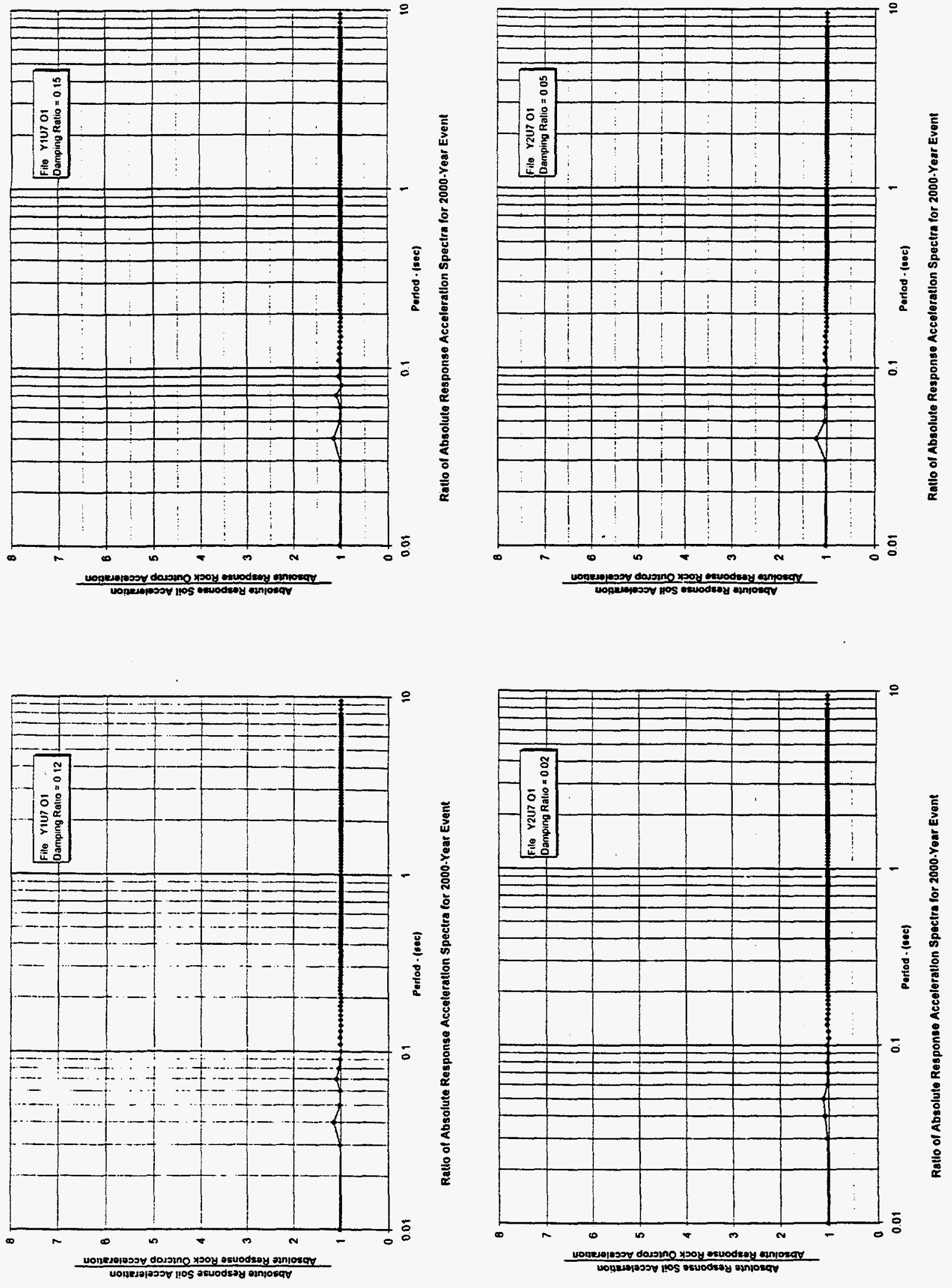


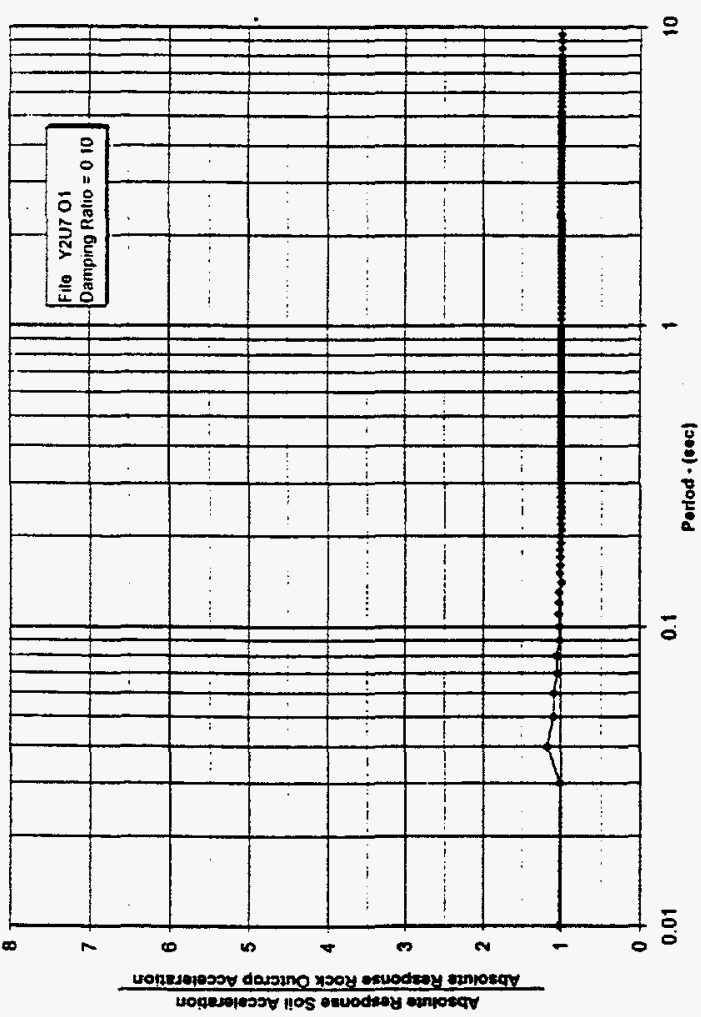

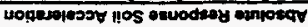
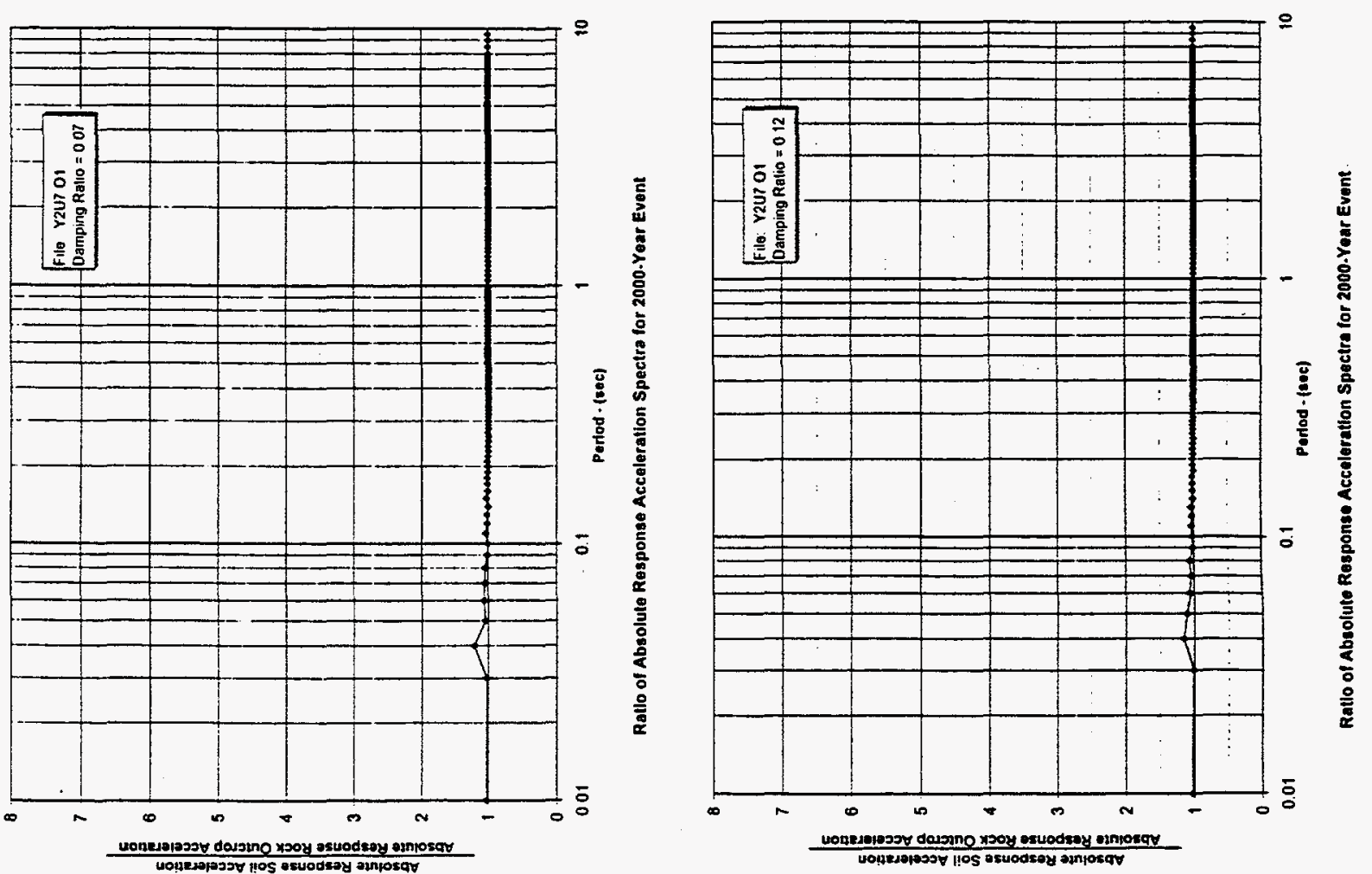

A2-63

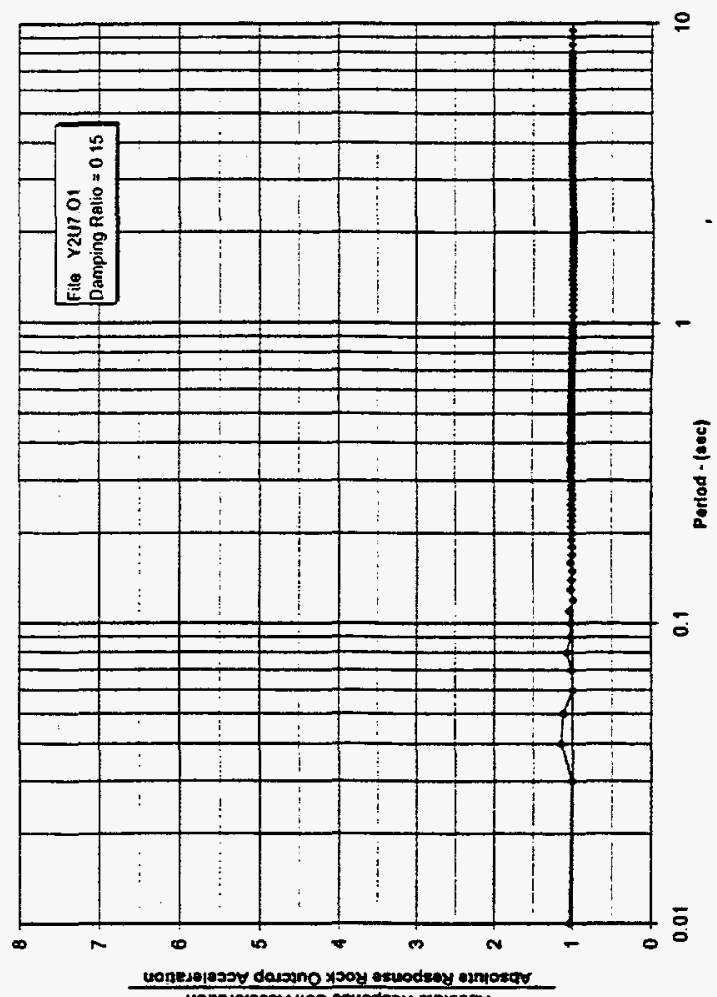

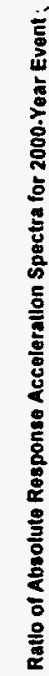

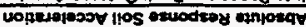


-

-

- 
B Soil Liquefaction 'SLIQ' Computer Output 
-

-

• 


\section{LIQUEFACTION POTENTIAL OF SILTY SAND}

TITLE: Y-12 SARUP, REF. 27, BORING V-1

TRIAL NO. 1

$\begin{array}{lll}\text { Magnitude of Earthquake }(\mathrm{M}) & =5.80 \\ \text { Ground Acceleration }(\mathrm{Ah}) \mathrm{max} & = & .12 \mathrm{~g} \\ \text { Percentage of silt particles } & =35.00 \\ \text { Unsaturated Unit Weight } & =120.00 \mathrm{pcf} \\ \text { Submerged Unit Weight } & =58.00 \mathrm{pcf} \\ \text { Depth of ground water table } & =5.00 \mathrm{ft} \\ \text { Correction factor for Rod Energy } & =1.00\end{array}$

Depth Total Pres rd EQ Shear Stress

(ft.) (psf) (psf)

$\begin{array}{cccc}2.50 & 300.00 & .996 & 23.29 \\ 5.00 & 600.00 & .991 & 46.38 \\ 7.50 & 901.00 & .987 & 69.33 \\ 10.00 & 1202.00 & .982 & 92.07 \\ 15.00 & 1804.00 & .973 & 136.91\end{array}$

$\begin{array}{llllll}\text { Depth Effective Cn N (N1)60 CSR Liq. Shear } & \text { N }\end{array}$ Pressure

(ft.) (psf) Stress

(psf)

$\begin{array}{rrrrrrr}2.50 & 300.00 & 2.58 & 12.0 & 30.98 & * * * * * * * & * * * * * * * \\ 5.00 & 600.00 & 1.83 & 36.0 & 65.73 & * * * * * * & * * * * * * * \\ 7.50 & 745.00 & 1.64 & 30.0 & 49.15 & * * * * * * & * * * * * * * \\ 10.00 & 890.00 & 1.50 & 62.0 & 92.94 & * * * * * * * & * * * * * * * \\ 15.00 & 1180.00 & 1.30 & 8.0 & 10.42 & .2609 & 307.86\end{array}$

\begin{tabular}{cccccc}
$\begin{array}{c}\text { Depth } \\
\text { (ft.) }\end{array}$ & $\begin{array}{c}\text { SDE } \\
\text { (psf) }\end{array}$ & (psf) & SLQ & \multicolumn{2}{c}{ FOS } \\
& & & & \\
2.5 & 23.3 & $* * * * * * *$ & $* * * * * * *$ & No liquefaction. \\
5.0 & 46.4 & $* * * * * * *$ & $* * * * * * *$ & No liquefaction. \\
7.5 & 69.3 & $* * * * * * *$ & $* * * * * * *$ & No liquefaction. \\
10.0 & 92.1 & $* * * * * * *$ & $* * * * * *$ & No liquefaction. \\
15.0 & 136.9 & 307.9 & 2.25 &
\end{tabular} 
TRIAL NO. 2

$\begin{array}{ll}\text { Magnitude of Earthquake }(\mathrm{M}) & =5.80 \\ \text { Ground Acceleration (Ah)max } & =.12 \mathrm{~g} \\ \text { Percentage of silt particles } & =10.00 \\ \text { Unsaturated Unit Weight } & =120.00 \mathrm{pcf} \\ \text { Submerged Unit Weight } & =58.00 \mathrm{pcf} \\ \text { Depth of ground water table } & =5.00 \mathrm{ft} \\ \text { Correction factor for Rod Energy } & =1.00\end{array}$

Depth Total Pres rd EQ Shear Stress

(ft.) (psf) (psf)

$\begin{array}{cccc}2.50 & 300.00 & .996 & 23.29 \\ 5.00 & 600.00 & .991 & 46.38 \\ 7.50 & 901.00 & .987 & 69.33 \\ 10.00 & 1202.00 & .982 & 92.07 \\ 15.00 & 1804.00 & .973 & 136.91\end{array}$

\begin{tabular}{|c|c|c|c|c|c|c|}
\hline $\begin{array}{l}\text { Depth } \\
\text { (ft.) }\end{array}$ & $\begin{array}{l}\text { Effective } \\
\text { Pressure } \\
\text { (psf) }\end{array}$ & $\mathrm{Cn}$ & $\mathrm{N}$ & (N1)6 & $\begin{array}{cc}60 & \text { CSR } \\
\text { Stress } \\
\text { (psf) }\end{array}$ & Liq. Shear \\
\hline 2.50 & 300.00 & 2.58 & 12.0 & 30.98 & $* * * * * * *$ & $* * * * * * *$ \\
\hline 5.00 & 600.00 & 1.83 & 36.0 & $65.73 *$ & $* * * * * * *$ & $* * * * * * *$ \\
\hline 7.50 & 745.00 & 1.64 & 30.0 & $49.15 *$ & $* * * * * * *$ & $* * * * * * *$ \\
\hline 10.00 & 890.00 & 1.50 & 62.0 & 92.94 & $* * * * * * *$ & $* * * * * * *$ \\
\hline 15.00 & 1180.00 & 1.30 & 8.0 & 10.42 & .1869 & 220.53 \\
\hline
\end{tabular}

Depth SDE SLQ FOS

(ft.) (psf) (psf) (SLQ/SDE)

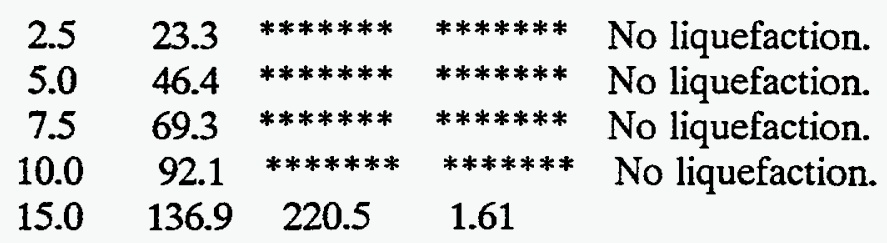

TRIAL NO. 3

Magnitude of Earthquake (M) $\quad=5.80$

Ground Acceleration (Ah) $\max =.12 \mathrm{~g}$

Percentage of silt particles $=5.00$

Unsaturated Unit Weight $\quad=120.00 \mathrm{pcf}$

Submerged Unit Weight $\quad=58.00 \mathrm{pcf}$ 
Depth of ground water table $=5.00 \mathrm{ft}$

Correction factor for Rod Energy $=1.00$

Depth Total Pres rd EQ Shear Stress

(ft.) (psf) (psf)

$\begin{array}{cccc}2.50 & 300.00 & .996 & 23.29 \\ 5.00 & 600.00 & .991 & 46.38 \\ 7.50 & 901.00 & .987 & 69.33 \\ 10.00 & 1202.00 & .982 & 92.07 \\ 15.00 & 1804.00 & .973 & 136.91\end{array}$

$\begin{array}{llllll}\text { Depth Effective Cn } & \text { N } & \text { (N1)60 CSR } & \text { Liq. Shear }\end{array}$ Pressure Stress

(ft.) (psf)

(psf)

$\begin{array}{rrrrrrr}2.50 & 300.00 & 2.58 & 12.0 & 30.98 & * * * * * * * & * * * * * * * \\ 5.00 & 600.00 & 1.83 & 36.0 & 65.73 & * * * * * * & * * * * * * * \\ 7.50 & 745.00 & 1.64 & 30.0 & 49.15 & * * * * * * & * * * * * * * \\ 10.00 & 890.00 & 1.50 & 62.0 & 92.94 & * * * * * * * & * * * * * * * \\ 15.00 & 1180.00 & 1.30 & 8.0 & 10.42 & .1557 & 183.77\end{array}$

Depth SDE SLQ FOS

(ft.) (psf) (psf) (SLQ/SDE)

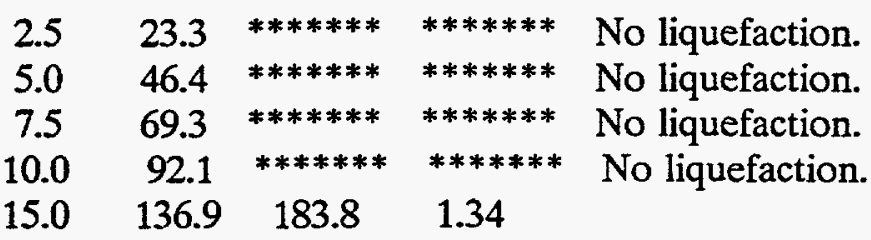

TRIAL NO. 4

Magnitude of Earthquake (M) $\quad=\quad 5.80$

Ground Acceleration (Ah)max $=.12 \mathrm{~g}$

Unsaturated Unit Weight $\quad=120.00 \mathrm{pcf}$

Submerged Unit Weight $\quad=58.00 \mathrm{pcf}$

Depth of ground water table $=5.00 \mathrm{ft}$

Correction factor for Rod Energy $=1.00$

Depth Total Pres rd EQ Shear Stress

(ft.) (psf) (psf)

$\begin{array}{llll}2.50 & 300.00 & .996 & 23.29\end{array}$

B -3 


$\begin{array}{cccc}5.00 & 600.00 & .991 & 46.38 \\ 7.50 & 901.00 & .987 & 69.33 \\ 10.00 & 1202.00 & .982 & 92.07 \\ 15.00 & 1804.00 & .973 & 136.91\end{array}$

$\begin{array}{llllll}\text { Depth Effective } & \text { Cn } & \text { N } & \text { (N1)60 CSR Liq. Shear }\end{array}$

(ft.) (psf) Stress

(psf)

$\begin{array}{rrrrrrr}2.50 & 300.00 & 2.58 & 12.0 & 30.98 & * * * * * * * & * * * * * * * \\ 5.00 & 600.00 & 1.83 & 36.0 & 65.73 & * * * * * * & * * * * * * * \\ 7.50 & 745.00 & 1.64 & 30.0 & 49.15 & * * * * * * & * * * * * * * \\ 10.00 & 890.00 & 1.50 & 62.0 & 92.94 & * * * * * * * & * * * * * * * \\ 15.00 & 1180.00 & 1.30 & 8.0 & 10.42 & .1557 & 183.77\end{array}$

Depth SDE SLQ FOS

(ft.) (psf) (psf) (SLQ/SDE)

$\begin{array}{ccccc}2.5 & 23.3 & * * * * * * * & * * * * * * * & \text { No liquefaction. } \\ 5.0 & 46.4 & * * * * * * * & * * * * * * * & \text { No liquefaction. } \\ 7.5 & 69.3 & * * * * * * & * * * * * * * & \text { No liquefaction. } \\ 10.0 & 92.1 & * * * * * * & * * * * * * * & \text { No liquefaction. } \\ 15.0 & 136.9 & 183.8 & 1.34 & \end{array}$




\section{LIQUEFACTION POTENTIAL OF SILTY SAND}

TITLE: Y-12 SARUP, REF.27, BORING V-1

TRIAL NO. 1

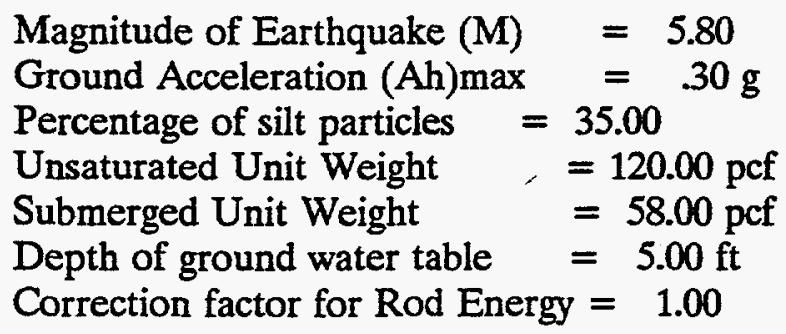

Depth Total Pres rd EQ Shear Stress

(ft.) (psf) (psf)

$\begin{array}{cccc}2.50 & 300.00 & .996 & 58.24 \\ 5.00 & 600.00 & .991 & 115.95 \\ 7.50 & 901.00 & .987 & 173.32 \\ 10.00 & 1202.00 & .982 & 230.17 \\ 15.00 & 1804.00 & .973 & 342.28\end{array}$

$\begin{array}{llllll}\text { Depth Effective } & \text { Cn } & \text { N } & \text { (N1)60 CSR } & \text { Liq. Shear }\end{array}$ Pressure

(ft.) (psf)

Stress

(psf)

$\begin{array}{rrrrrrr}2.50 & 300.00 & 2.58 & 12.0 & 30.98 & * * * * * * * & * * * * * * * \\ 5.00 & 600.00 & 1.83 & 36.0 & 65.73 & * * * * * * * & * * * * * * * \\ 7.50 & 745.00 & 1.64 & 30.0 & 49.15 & * * * * * * * & * * * * * * * \\ 10.00 & 890.00 & 1.50 & 62.0 & 92.94 & * * * * * * * & * * * * * * * \\ 15.00 & 1180.00 & 1.30 & 8.0 & 10.42 & .2609 & 307.86\end{array}$

Depth SDE SLQ FOS

(ft.) (psf) (psf) (SLQ/SDE)

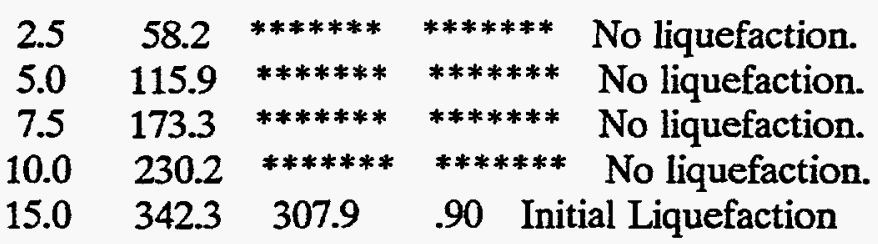

B -5 
TRIAL NO. 2

$$
\begin{array}{ll}
\text { Magnitude of Earthquake }(\mathrm{M}) & =5.80 \\
\text { Ground Acceleration (Ah)max } & =.30 \mathrm{~g} \\
\text { Percentage of silt particles } & =35.00 \\
\text { Unsaturated Unit Weight } & =120.00 \mathrm{pcf} \\
\text { Submerged Unit Weight } & =58.00 \mathrm{pcf} \\
\text { Depth of ground water table } & =10.00 \mathrm{ft} \\
\text { Correction factor for Rod Energy } & =1.00
\end{array}
$$

\begin{tabular}{|c|c|c|c|c|c|c|}
\hline $\begin{array}{l}\text { Depth } \\
\text { (ft.) }\end{array}$ & $\begin{array}{l}\text { Effective } \\
\text { Pressure } \\
\text { (psf) }\end{array}$ & $\mathrm{Cn}$ & $N$ & (N1)6C & $\begin{array}{cc}\text { SO } & \text { CSR } \\
\text { Stress } \\
\text { (psf) }\end{array}$ & Liq. Shear \\
\hline 2.50 & 300.00 & 2.58 & 12.0 & $30.98 *$ & $* * * * * * *$ & $* * * * * * *$ \\
\hline 5.00 & 600.00 & 1.83 & 36.0 & $65.73^{*}$ & $* * * * * * *$ & $* * * * * * *$ \\
\hline 7.50 & 900.00 & 1.49 & 30.0 & $44.72 *$ & $* * * * * *$ & $* * * * * * *$ \\
\hline 10.00 & 1200.00 & 1.29 & 62.0 & 80.04 & $* * * * * * *$ & $* * * * * * *$ \\
\hline 15.00 & 1490.00 & 1.16 & 8.0 & 9.27 & .2394 & 356.75 \\
\hline
\end{tabular}

\begin{tabular}{|c|c|c|c|c|}
\hline Dept & SDE & SLQ & FOS & \\
\hline & & & & \\
\hline 2.5 & 58.2 & $* * * * * * *$ & $* * * * * * *$ & No liquefaction. \\
\hline 5.0 & 115.9 & & $* * * * * * *$ & No liquefaction. \\
\hline 7.5 & 173.1 & $* * * * * * *$ & $* * * * * * *$ & No liquefaction. \\
\hline 10.0 & 229.8 & $* * * * * * *$ & $* * * * * * *$ & No liquefaction. \\
\hline 15.0 & 341.9 & 356.7 & 1.04 & \\
\hline
\end{tabular}

Depth Total Pres rd EQ Shear Stress

\begin{tabular}{cllc}
$(\mathrm{ft})$. & (psf) & \multicolumn{2}{c}{ (psf) } \\
& & & \\
2.50 & 300.00 & .996 & 58.24 \\
5.00 & 600.00 & .991 & 115.95 \\
7.50 & 900.00 & .987 & 173.13 \\
10.00 & 1200.00 & .982 & 229.79 \\
15.00 & 1802.00 & .973 & 341.90
\end{tabular}


TITLE: Y-12 SARUP, REF: 27, BORING V-3

TRIAL NO. 1

$$
\begin{array}{ll}
\text { Magnitude of Earthquake }(\mathrm{M}) & =5.80 \\
\text { Ground Acceleration (Ah)max } & =.12 \mathrm{~g} \\
\text { Percentage of silt particles } & =35.00 \\
\text { Unsaturated Unit Weight } & =120.00 \mathrm{pcf} \\
\text { Submerged Unit Weight } & =58.00 \mathrm{pcf} \\
\text { Depth of ground water table } & =5.00 \mathrm{ft} \\
\text { Correction factor for Rod Energy } & =1.00
\end{array}
$$

\begin{tabular}{|c|c|c|c|c|c|c|}
\hline $\begin{array}{l}\text { Depth } \\
\text { (ft.) }\end{array}$ & $\begin{array}{l}\text { Effective } \\
\text { Pressure } \\
\text { (psf) }\end{array}$ & $\mathrm{Cn}$ & $\mathrm{N}$ & (N1)60 & $50 \begin{array}{c}\text { CSR } \\
\text { Stress } \\
\text { (psf) }\end{array}$ & Liq. Shear \\
\hline 2.50 & 300.00 & 2.58 & 14.0 & $36.15^{*}$ & $* * * * * * *$ & $* * * * * * *$ \\
\hline 5.00 & 600.00 & 1.83 & 27.0 & $49.30^{*}$ & $* * * * * * *$ & $* * * * * * *$ \\
\hline 7.30 & 733.40 & 1.65 & 89.0 & $146.97 *$ & $* * * * * * *$ & $* * * * * * *$ \\
\hline 10.00 & 890.00 & 1.50 & 45.0 & $67.46 *$ & $* * * * * * *$ & $* * * * * * *$ \\
\hline 15.00 & 1180.00 & 1.30 & 36.0 & 46.87 & $* * * * * * *$ & $* * * * * * * *$ \\
\hline 20.00 & 1470.00 & 1.17 & 74.0 & 86.32 & $* * * * * * *$ & $* * * * * * *$ \\
\hline 25.00 & 1760.00 & 1.07 & 85.0 & $90.61=$ & $* * * * * * *$ & $* * * * * * *$ \\
\hline
\end{tabular}

Depth Total Pres rd EQ Shear Stress

(ft.) (psf) (psf)

$\begin{array}{cccc}2.50 & 300.00 & .996 & 23.29 \\ 5.00 & 600.00 & .991 & 46.38 \\ 7.30 & 876.92 & .987 & 67.50 \\ 10.00 & 1202.00 & .982 & 92.07 \\ 15.00 & 1804.00 & .973 & 136.91 \\ 20.00 & 2406.00 & .964 & 180.91 \\ 25.00 & 3008.00 & .939 & 220.32\end{array}$

Depth SDE SLQ FOS

(ft.) (psf) (psf) (SLQ/SDE)

$2.523 .3 * * * * * * * \quad * * * * * * * \quad$ No liquefaction.

$5.0 \quad 46.4 * * * * * * * \quad * * * * * * * \quad$ No liquefaction. 


$\begin{array}{ccccc}7.3 & 67.5 & * * * * * * * & * * * * * * * & \text { No liquefaction. } \\ 10.0 & 92.1 & * * * * * * * & * * * * * * & \text { No liquefaction. } \\ 15.0 & 136.9 & * * * * * * * & * * * * * * * & \text { No liquefaction. } \\ 20.0 & 180.9 & * * * * * * & * * * * * * & \text { No liquefaction. } \\ 25.0 & 220.3 & * * * * * * * & * * * * * * & \text { No liquefaction. }\end{array}$

TRIAL NO. 2

$\begin{array}{ll}\text { Magnitude of Earthquake }(\mathrm{M}) & =5.80 \\ \text { Ground Acceleration (Ah)max } & =.12 \mathrm{~g} \\ \text { Percentage of silt particles }= & 10.00 \\ \text { Unsaturated Unit Weight } & =120.00 \mathrm{pcf} \\ \text { Submerged Unit Weight } & =58.00 \mathrm{pcf} \\ \text { Depth of ground water table } & =5.00 \mathrm{ft} \\ \text { Correction factor for Rod Energy } & =1.00\end{array}$

$\begin{array}{lll}\begin{array}{l}\text { Depth } \\ \text { (ft.) }\end{array} & \begin{array}{l}\text { Total Pres } \\ \text { (psf) }\end{array} & \text { rd } \\ \text { (psf) }\end{array}$

$\begin{array}{cccc}2.50 & 300.00 & .996 & 23.29 \\ 5.00 & 600.00 & .991 & 46.38 \\ 7.30 & 876.92 & .987 & 67.50 \\ 10.00 & 1202.00 & .982 & 92.07 \\ 15.00 & 1804.00 & .973 & 136.91 \\ 20.00 & 2406.00 & .964 & 180.91 \\ 25.00 & 3008.00 & .939 & 220.32\end{array}$

$\begin{array}{llllll}\text { Depth Effective Cn N (N1)60 CSR Liq. Shear } & \text { C }\end{array}$ Pressure

(ft.) (psf) Stress

$\begin{array}{rrrrrrr}2.50 & 300.00 & 2.58 & 14.0 & 36.15 & * * * * * * * & * * * * * * * \\ 5.00 & 600.00 & 1.83 & 27.0 & 49.30 & * * * * * * * & * * * * * * * \\ 7.30 & 733.40 & 1.65 & 89.0 & 146.97 & * * * * * * * & * * * * * * * \\ 10.00 & 890.00 & 1.50 & 45.0 & 67.46 & * * * * * * * & * * * * * * * \\ 15.00 & 1180.00 & 1.30 & 36.0 & 46.87 & * * * * * * * & * * * * * * * \\ 20.00 & 1470.00 & 1.17 & 74.0 & 86.32 & * * * * * * * & * * * * * * * \\ 25.00 & 1760.00 & 1.07 & 85.0 & 90.61 & * * * * * * * & * * * * * * *\end{array}$

Depth SDE SLQ FOS

(ft.) (psf) (psf) (SLQ/SDE)

$\begin{array}{lllll}2.5 & 23.3 & * * * * * * * & * * * * * * * & \text { No liquefaction. } \\ 5.0 & 46.4 & * * * * * * * & * * * * * * * & \text { No liquefaction. } \\ 7.3 & 67.5 & * * * * * * * & * * * * * * * & \text { No liquefaction. }\end{array}$




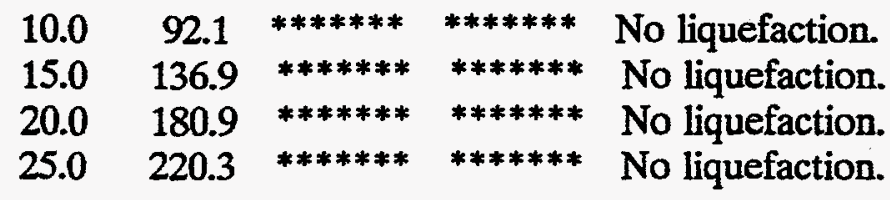

TRIAL NO. 3

$\begin{array}{ll}\text { Magnitude of Earthquake (M) } & =5.80 \\ \text { Ground Acceleration (Ah)max } & =.12 \mathrm{~g} \\ \text { Percentage of silt particles }= & 5.00 \\ \text { Unsaturated Unit Weight } & =120.00 \mathrm{pcf} \\ \text { Submerged Unit Weight } & =58.00 \mathrm{pcf} \\ \text { Depth of ground water table } & =5.00 \mathrm{ft} \\ \text { Correction factor for Rod Energy } & =1.00\end{array}$

Depth Total Pres rd EQ Shear Stress

(ft.) (psf) (psf)

$\begin{array}{cccc}2.50 & 300.00 & .996 & 23.29 \\ 5.00 & 600.00 & .991 & 46.38 \\ 7.30 & 876.92 & .987 & 67.50 \\ 10.00 & 1202.00 & .982 & 92.07 \\ 15.00 & 1804.00 & .973 & 136.91 \\ 20.00 & 2406.00 & .964 & 180.91 \\ 25.00 & 3008.00 & .939 & 220.32\end{array}$

$\begin{array}{llllll}\text { Depth Effective } & \mathrm{Cn} & \mathrm{N} & \text { (N1)60 CSR Liq. Shear }\end{array}$

(ft.) (psf)

(pst)

$\begin{array}{rrrrrrl}2.50 & 300.00 & 2.58 & 14.0 & 36.15 & * * * * * * * & * * * * * * * \\ 5.00 & 600.00 & 1.83 & 27.0 & 49.30 & * * * * * * * & * * * * * * * \\ 7.30 & 733.40 & 1.65 & 89.0 & 146.97 & * * * * * * * & * * * * * * * \\ 10.00 & 890.00 & 1.50 & 45.0 & 67.46 & * * * * * * * & * * * * * * * \\ 15.00 & 1180.00 & 1.30 & 36.0 & 46.87 & * * * * * * * & * * * * * * * \\ 20.00 & 1470.00 & 1.17 & 74.0 & 86.32 & * * * * * * * & * * * * * * * \\ 25.00 & 1760.00 & 1.07 & 85.0 & 90.61 & * * * * * * * & * * * * * * *\end{array}$

Depth SDE SLQ FOS

(ft.) (psf) (psf) (SLQ/SDE)

$\begin{array}{ccccc}2.5 & 23.3 & * * * * * * * & * * * * * * * & \text { No liquefaction. } \\ 5.0 & 46.4 & * * * * * * & * * * * * * * & \text { No liquefaction. } \\ 7.3 & 67.5 & * * * * * * & * * * * * * * & \text { No liquefaction. } \\ 10.0 & 92.1 & * * * * * * * & * * * * * * * & \text { No liquefaction. }\end{array}$




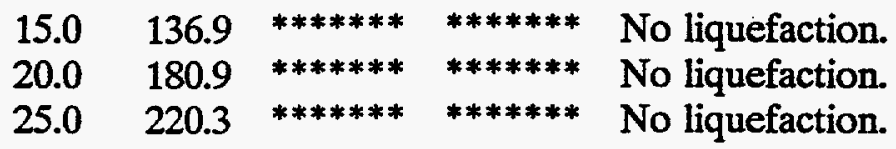

TRIAL NO. 4

$\begin{array}{lll}\text { Magnitude of Earthquake (M) } & =5.80 \\ \text { Ground Acceleration (Ah)max } & =\quad .12 \mathrm{~g} \\ \text { Unsaturated Unit Weight } & =120.00 \mathrm{pcf} \\ \text { Submerged Unit Weight } & =58.00 \mathrm{pcf} \\ \text { Depth of ground water table } & =5.00 \mathrm{ft} \\ \text { Correction factor for Rod Energy } & =1.00\end{array}$

Depth Total Pres rd EQ Shear Stress

(ft.) (psf) (psf)

$\begin{array}{cccc}2.50 & 300.00 & .996 & 23.29 \\ 5.00 & 600.00 & .991 & 46.38 \\ 7.30 & 876.92 & .987 & 67.50 \\ 10.00 & 1202.00 & .982 & 92.07 \\ 15.00 & 1804.00 & .973 & 136.91 \\ 20.00 & 2406.00 & .964 & 180.91 \\ 25.00 & 3008.00 & .939 & 220.32\end{array}$

\begin{tabular}{ccccccc}
$\begin{array}{c}\text { Depth } \\
\text { Pressure } \\
\text { (ft.) }\end{array}$ & $\begin{array}{c}\text { Effective } \\
\text { (psf) }\end{array}$ & & & \multicolumn{4}{c}{$\begin{array}{c}\text { Cn } \\
\text { (psf) }\end{array}$} & (N1)60 & $\begin{array}{c}\text { CSR } \\
\text { Liq. Shear }\end{array}$ \\
2.50 & 300.00 & 2.58 & 14.0 & 36.15 & $* * * * * * *$ & $* * * * * * *$ \\
5.00 & 600.00 & 1.83 & 27.0 & 49.30 & $* * * * * * *$ & $* * * * * * *$ \\
7.30 & 733.40 & 1.65 & 89.0 & 146.97 & $* * * * * * *$ & $* * * * * * *$ \\
10.00 & 890.00 & 1.50 & 45.0 & 67.46 & $* * * * * * *$ & $* * * * * * *$ \\
15.00 & 1180.00 & 1.30 & 36.0 & 46.87 & $* * * * * * *$ & $* * * * * * *$ \\
20.00 & 1470.00 & 1.17 & 74.0 & 86.32 & $* * * * * * *$ & $* * * * * * *$ \\
25.00 & 1760.00 & 1.07 & 85.0 & 90.61 & $* * * * * * *$ & $* * * * * * *$
\end{tabular}

\begin{tabular}{ccccc}
$\begin{array}{c}\text { Depth } \\
\text { (ft.) }\end{array}$ & \multicolumn{2}{c}{ SDE } & SLQ & FOS \\
& (ps) & (psf) & (SLQ/SDE) & \\
2.5 & 23.3 & $* * * * * * *$ & $* * * * * * *$ & No liquefaction. \\
5.0 & 46.4 & $* * * * * * *$ & $* * * * * * *$ & No liquefaction. \\
7.3 & 67.5 & $* * * * * * *$ & $* * * * * * *$ & No liquefaction. \\
10.0 & 92.1 & $* * * * * * *$ & $* * * * * * *$ & No liquefaction. \\
15.0 & 136.9 & $* * * * * *$ & $* * * * * *$ & No liquefaction. \\
20.0 & 180.9 & $* * * * * * *$ & $* * * * * * *$ & No liquefaction.
\end{tabular}


$25.0220 .3 * * * * * * * \quad * * * * * * * \quad$ No liquefaction. 


\section{LIQUEFACTION POTENTIAL OF SILTY SAND}

TITLE: Y-12 SARUP, REF. 27, BORING V-3

TRIAL NO. 1

$\begin{array}{lll}\text { Magnitude of Earthquake }(\mathrm{M}) & =5.80 \\ \text { Ground Acceleration (Ah)max } & =.30 \mathrm{~g} \\ \text { Percentage of silt particles }= & 35.00 \\ \text { Unsaturated Unit Weight } & =120.00 \mathrm{pcf} \\ \text { Submerged Unit Weight } & =58.00 \mathrm{pcf} \\ \text { Depth of ground water table } & =5.00 \mathrm{ft} \\ \text { Correction factor for Rod Energy } & =1.00\end{array}$

Depth Total Pres rd EQ Shear Stress

(ft.) (psf) (psf)

$\begin{array}{cccc}2.50 & 300.00 & .996 & 58.24 \\ 5.00 & 600.00 & .991 & 115.95 \\ 7.30 & 876.92 & .987 & 168.75 \\ 10.00 & 1202.00 & .982 & 230.17 \\ 15.00 & 1804.00 & .973 & 342.28 \\ 20.00 & 2406.00 & .964 & 452.28 \\ 25.00 & 3008.00 & .939 & 550.80\end{array}$

\begin{tabular}{|c|c|c|c|c|c|c|}
\hline $\begin{array}{l}\text { Depth } \\
\text { (ft.) }\end{array}$ & $\begin{array}{l}\text { Effective } \\
\text { Pressure } \\
\text { (psf) }\end{array}$ & $\mathrm{Cn}$ & $\mathbf{N}$ & (N1) 60 & $\begin{array}{cc}0 & \text { CSR } \\
\text { Stress } \\
(\mathrm{psf})\end{array}$ & Liq. Sh \\
\hline 2.50 & 300.00 & 2.58 & 14.0 & 36.15 & $* * * *$ & $* * *$ \\
\hline 5.00 & 600.0 & 1.83 & 27.0 & $49.30^{*}$ & $* * * * * * *$ & \\
\hline 7.30 & 733 & 1.65 & 89.0 & 146.97 & $* * * * * * *$ & $* * * * * * *$ \\
\hline 10.00 & 890.00 & 1.50 & 45.0 & $67.46 *$ & $* * * * * * *$ & $* * * * * * *$ \\
\hline 15.00 & 1180.00 & 1.30 & 36.0 & 46.87 & $* * * * * * *$ & $* * * * * * *$ \\
\hline 20.0 & 14 & 1.17 & 74.0 & 86.32 & $* * * * * * *$ & $* * * * * * *$ \\
\hline 25.00 & 00 & 07 & 85.0 & 90.61 & $* * * * *$ & $* * * * * * *$ \\
\hline
\end{tabular}

Depth SDE SLQ FOS

(ft.) (psf) (psf) (SLQ/SDE)

$\begin{array}{rrrrr}2.5 & 58.2 & * * * * * * * & * * * * * * * & \text { No liquefaction. } \\ 5.0 & 115.9 & * * * * * * * & * * * * * * * & \text { No liquefaction. }\end{array}$

$B-12$ 


$\begin{array}{rrrrr}7.3 & 168.8 & * * * * * * * & * * * * * * * & \text { No liquefaction. } \\ 10.0 & 230.2 & * * * * * * & * * * * * * & \text { No liquefaction. } \\ 15.0 & 342.3 & * * * * * * * & * * * * * * & \text { No liquefaction. } \\ 20.0 & 452.3 & * * * * * * & * * * * * * & \text { No liquefaction. } \\ 25.0 & 550.8 & * * * * * * & * * * * * * & \text { No liquefaction. }\end{array}$




\section{LIQUEFACTION POTENTIAL OF SILTY SAND}

TITLE: Y-12 SARUP, REF. 27, BORING V-6

TRIAL NO. 1

$\begin{array}{ll}\text { Magnitude of Earthquake }(\mathrm{M}) & =5.80 \\ \text { Ground Acceleration (Ah)max } & =.12 \mathrm{~g} \\ \text { Percentage of silt particles }= & 35.00 \\ \text { Saturated Unit Weight } & =120.40 \mathrm{pcf} \\ \text { Submerged Unit Weight } & =58.00 \mathrm{pcf} \\ \text { Depth of ground water table }= & .00 \mathrm{ft} \\ \text { Correction factor for Rod Energy } & =1.00\end{array}$

Depth Total Pres rd EQ Shear Stress

(ft.) (psf) (psf)

$\begin{array}{llll}2.50 & 301.00 & .996 & 23.37 \\ 5.00 & 602.00 & .991 & 46.53\end{array}$

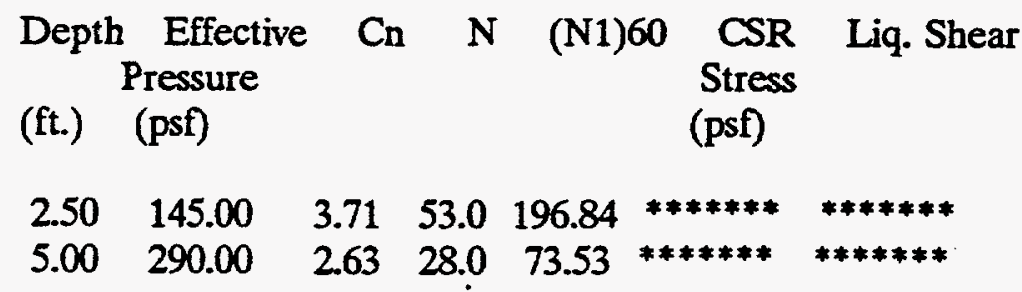

Depth SDE SLQ FOS

(ft.) (psf) (psf) (SLQ/SDE)

$\begin{array}{lllll}2.5 & 23.4 & * * * * * * * & * * * * * * * & \text { No liquefaction. } \\ 5.0 & 46.5 & * * * * * * * & * * * * * * * & \text { No liquefaction. }\end{array}$

TRIAL NO. 2

Magnitude of Earthquake (M) $\quad=\quad 5.80$

Ground Acceleration (Ah)max $=.12 \mathrm{~g}$

Percentage of silt particles $=10.00$

Saturated Unit Weight $\quad=120.40 \mathrm{pcf}$

Submerged Unit Weight $\quad=58.00 \mathrm{pcf}$

Depth of ground water table $=.00 \mathrm{ft}$

Correction factor for Rod Energy $=1.00$ 
Depth Total Pres rd EQ Shear Stress

(ft.) (psf) (psf)

$\begin{array}{llll}2.50 & 301.00 & .996 & 23.37 \\ 5.00 & 602.00 & .991 & 46.53\end{array}$

Depth Effective Cn N (N1)60 CSR Liq. Shear

(ft.) (psf)

Stress

$\begin{array}{llllcll}2.50 & 145.00 & 3.71 & 53.0 & 196.84 & * * * * * * * & * * * * * * * \\ 5.00 & 290.00 & 2.63 & 28.0 & 73.53 & * * * * * * * & * * * * * * *\end{array}$

Depth SDE SLQ FOS

(ft.) (psf) (psf) (SLQ/SDE)

$\begin{array}{lllll}2.5 & 23.4 & * * * * * * * & * * * * * * * & \text { No liquefaction. } \\ 5.0 & 46.5 & * * * * * * * & * * * * * * * & \text { No liquefaction. }\end{array}$

$5.046 .5 * * * * * * * \quad * * * * * * * \quad$ No liquefaction.

TRIAL NO. 3

Magnitude of Earthquake (M) $\quad=\quad 5.80$

Ground Acceleration (Ah)max $=.12 \mathrm{~g}$

Percentage of silt particles $=5.00$

Saturated Unit Weight $\quad=120.40 \mathrm{pcf}$

Submerged Unit Weight $\quad=58.00 \mathrm{pcf}$

Depth of ground water table $=.00 \mathrm{ft}$

Correction factor for Rod Energy $=1.00$

Depth Total Pres rd EQ Shear Stress

(ft.) (psf) (psf)

$\begin{array}{llll}2.50 & 301.00 & .996 & 23.37 \\ 5.00 & 602.00 & .991 & 46.53\end{array}$

Depth Effective $\mathrm{Cn} \quad \mathrm{N} \quad$ (N1)60 CSR Liq. Shear Pressure

(ft.) (psf)

(psf)

$\begin{array}{ccccccc}2.50 & 145.00 & 3.71 & 53.0 & 196.84 & * * * * * * * & * * * * * * * \\ 5.00 & 290.00 & 2.63 & 28.0 & 73.53 & * * * * * * * & * * * * * * *\end{array}$




\begin{tabular}{|c|c|c|c|c|}
\hline $\begin{array}{l}\text { Depth } \\
\text { (ft.) }\end{array}$ & $\begin{array}{l}\text { SD } \\
\text { (psf) }\end{array}$ & ${ }_{(\mathrm{psf})}$ SLC & $\begin{array}{r}\text { FOS } \\
\text { LQ/SDE) }\end{array}$ & \\
\hline 2.5 & 23.4 & $* * * * * * *$ & $* * * * * * *$ & No liquefactic \\
\hline 5.0 & 46.5 & $* * * * * * *$ & $* * * * * * *$ & No liquefactio \\
\hline
\end{tabular}

TRIAL NO. 4

$\begin{array}{lll}\text { Magnitude of Earthquake }(\mathrm{M}) & =5.80 \\ \text { Ground Acceleration (Ah)max } & =\quad .12 \mathrm{~g} \\ \text { Saturated Unit Weight } & =120.40 \mathrm{pcf} \\ \text { Submerged Unit Weight } & =58.00 \mathrm{pcf} \\ \text { Depth of ground water table } & =\quad .00 \mathrm{ft} \\ \text { Correction factor for Rod Energy } & =1.00\end{array}$

Depth Total Pres rd EQ Shear Stress

(ft.) (psf) (psf)

$\begin{array}{llll}2.50 & 301.00 & .996 & 23.37 \\ 5.00 & 602.00 & .991 & 46.53\end{array}$

Depth Effective Cn N (N1)60 CSR Liq. Shear

\begin{tabular}{|c|c|c|c|c|}
\hline (ft.) & $\begin{array}{l}\text { Pressure } \\
\text { (psf) }\end{array}$ & & & $\begin{array}{l}\text { Stress } \\
\text { (psf) }\end{array}$ \\
\hline & 145.00 & 3.71 & $\begin{array}{lll}53.0 & 196.84\end{array}$ & $* * * * * * *$ \\
\hline 5.00 & 290.00 & 2.63 & $28.0 \quad 73.53$ & $* * * * * * *$ \\
\hline
\end{tabular}

Depth SDE SLQ FOS

(ft.) (psf) (psf) (SLQ/SDE)

$2.523 .4 * * * * * * * \quad * * * * * * * \quad$ No liquefaction.

$5.0 \quad 46.5 \quad * * * * * * * \quad * * * * * * * \quad$ No liquefaction. 
TITLE: Y-12 SARUP, REF.27, BORING V-6

TRIAL NO. 1

$\begin{array}{ll}\text { Magnitude of Earthquake }(\mathrm{M}) & =5.80 \\ \text { Ground Acceleration (Ah)max } & =.30 \mathrm{~g} \\ \text { Percentage of silt particles } & =35.00 \\ \text { Unsaturated Unit Weight } & =120.00 \mathrm{pcf} \\ \text { Submerged Unit Weight } & =58.00 \mathrm{pcf} \\ \text { Depth of ground water table } & =2.00 \mathrm{ft} \\ \text { Correction factor for Rod Energy } & =1.00\end{array}$

Depth Total Pres rd EQ Shear Stress

(ft.) (psf) (psf)

$\begin{array}{rrrr}2.50 & 300.20 & .996 & 58.28 \\ 5.00 & 601.20 & .991 & 116.18\end{array}$

$\begin{array}{llll}5.00 & 601.20 & .991 & 116.18\end{array}$

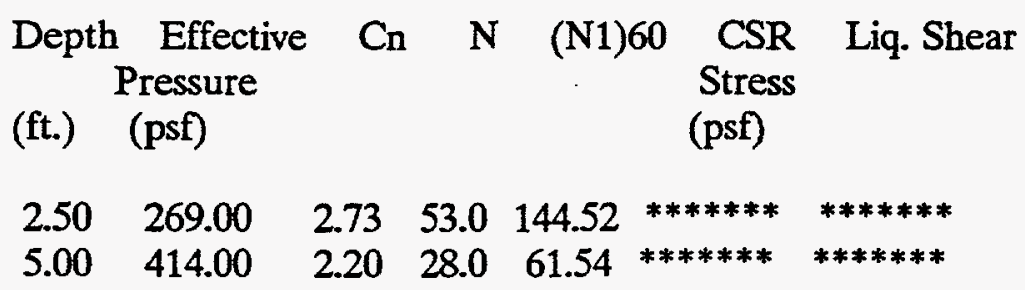

Depth SDE SLQ FOS

(ft.) (psf) (psf) (SLQ/SDE)

$\begin{array}{rrrrr}2.5 & 58.3 & * * * * * * * & * * * * * * * & \text { No liquefaction. } \\ 5.0 & 116.2 & * * * * * * * & * * * * * * * & \text { No liquefaction. }\end{array}$

TRIAL NO. 2

Magnitude of Earthquake (M) $=5.80$

Ground Acceleration (Ah) $\max =.30 \mathrm{~g}$

Percentage of silt particles $=35.00$

Saturated Unit Weight $\quad=120.00 \mathrm{pcf}$

Submerged Unit Weight $\quad=58.00 \mathrm{pcf}$

Depth of ground water table $=.00 \mathrm{ft}$

Correction factor for Rod Energy = 1.00 
$\begin{array}{llll}\begin{array}{l}\text { Depth } \\ \text { (ft.) }\end{array} & \begin{array}{l}\text { Total Pres } \\ \text { (psf) }\end{array} & \text { rd } & \text { EQ Shear Stress } \\ \text { (psf) }\end{array}$

$\begin{array}{rrrr}2.50 & 301.00 & .996 & 58.43 \\ 5.00 & 602.00 & .991 & 116.33\end{array}$

$\begin{array}{llllll}\text { Depth Effective Cn } & \text { N } & \text { (N1)60 CSR Liq. Shear }\end{array}$ Pressure

(ft.) (psf) Stress

$\begin{array}{lllll}2.50 & 145.00 & 3.71 & 53.0 & 196.84\end{array}$

$\begin{array}{lllll}5.00 & 290.00 & 2.63 & 28.0 & 73.53\end{array}$

(psf)

Depth SDE SLQ FOS

(ft.) (psf) (psf) (SLQ/SDE)

$2.5 \quad 58.4$

$5.0 \quad 116.3$

******* $\quad * * * * * * * \quad$ No liquefaction.

No liquefaction. 
C Drawings 
1012

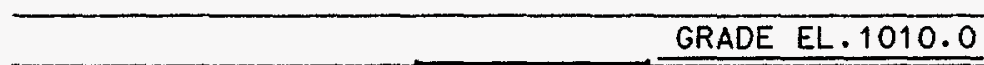

1008

1004

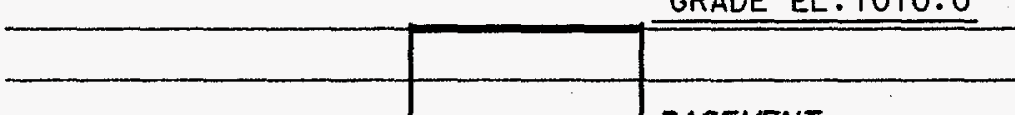

1000

BOTTOM OF

BLDG. EDN.

BASEMENT

SLAB EL.1004.0

GROUNO

WATER

TOP OF

WEATHEREQ

EL. 996.0

996

992

988

984

980

976

972

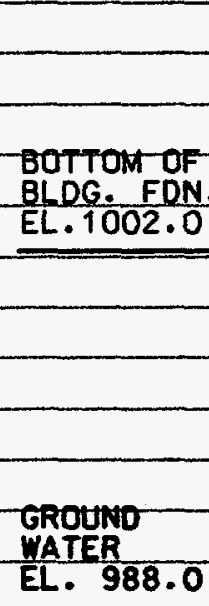

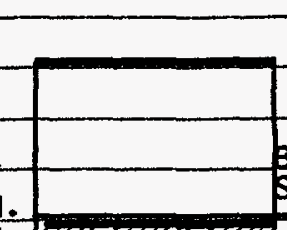

GRADE EL. $1 \mathrm{C}$

BASEMENT

SLAB EL. 1004
TOP OF

WEATHEREQ (11) $\pi$ II KJIUROCK EL.992 $\equiv \equiv \equiv \equiv$ OP OF-FRESt EL. $988.0 \equiv$ OROCK EL.988. 


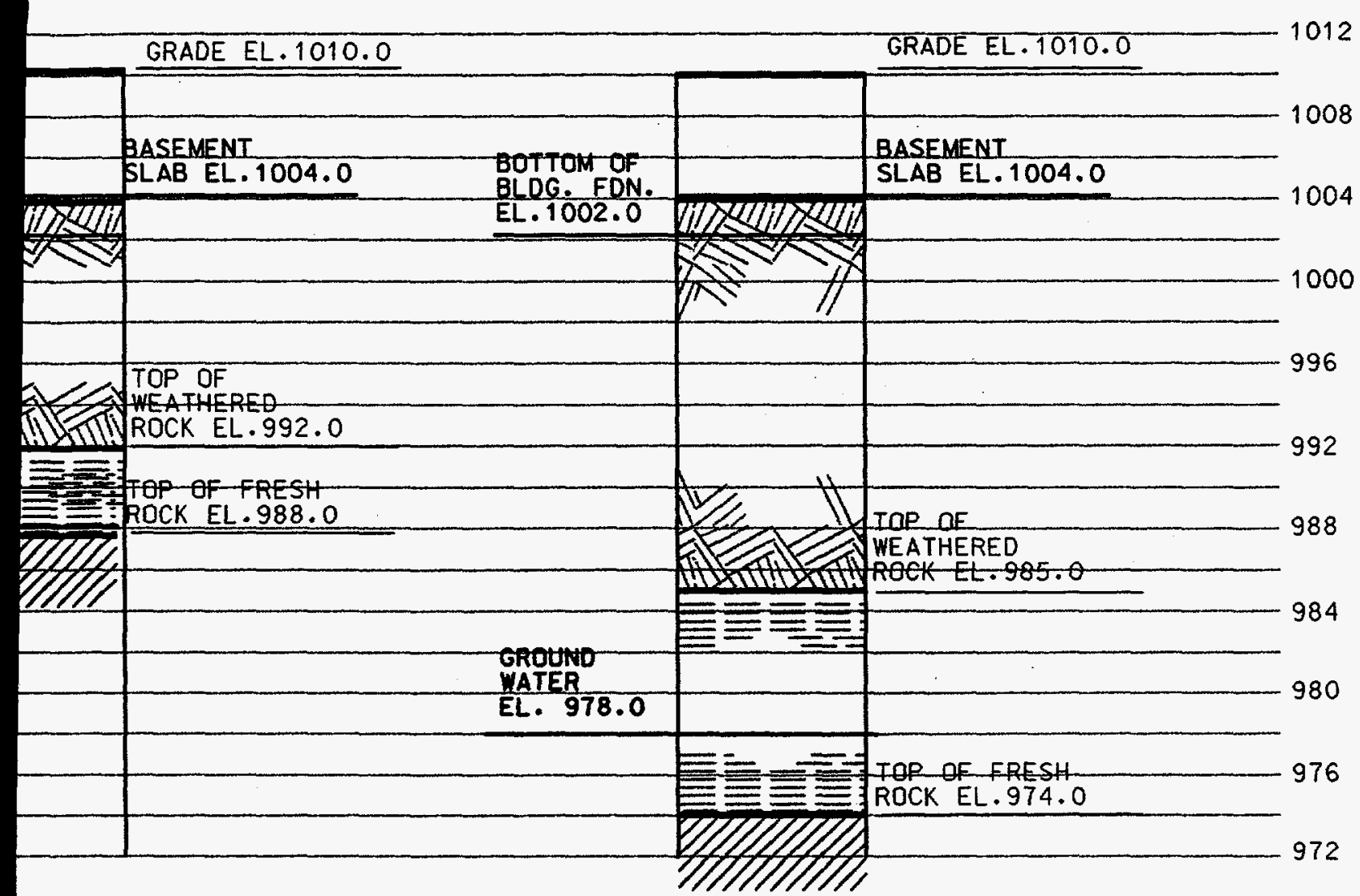




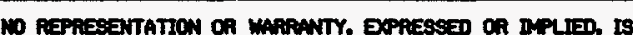

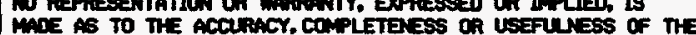

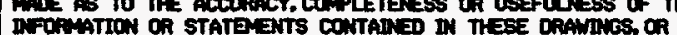

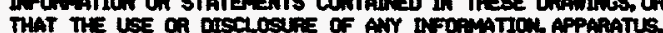

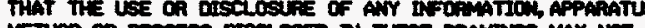

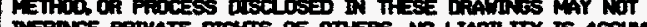

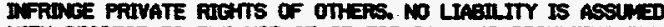

WTH RESPECT TO THE USE OF, OR FOR OAMAES RESULTDSG FROM THE LSE OF, AAY IMFOAMATJOM APPARATLS, METHCO OR PrOCESS

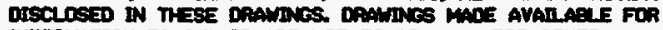
IIFOPACATZON TO BMOER AFE NOT TO BE USED FOR OTHEP PLPPOSES AHB AFE TO BE RETUPAED LPON REDVEST OF THE FCRWAFODNG CONTRACTOR
Document Rev. No.: EG-C-37 Review By:

Guideline Document: UCN-19586 12356

4-93)

Date:

Date:

\begin{tabular}{|c|c|c|c|c|}
\hline & & & & \\
\hline $\mathrm{XIN}$ & THIS DOCUMENT & & & \\
\hline 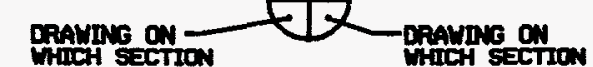 & CHANGE CONTROL & & & \\
\hline 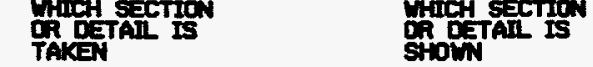 & SYSTEM. & & & \\
\hline SECTION AND & $\begin{array}{l}\text { ENGINEERING } \\
\text { PROCEDURE }\end{array}$ & REV & $\mathrm{cowh}_{\mathrm{m}}$ & $\mid \frac{9}{m}$ \\
\hline
\end{tabular}

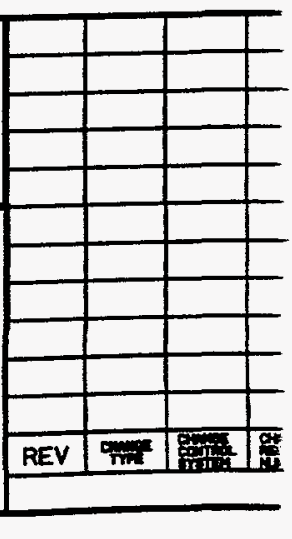




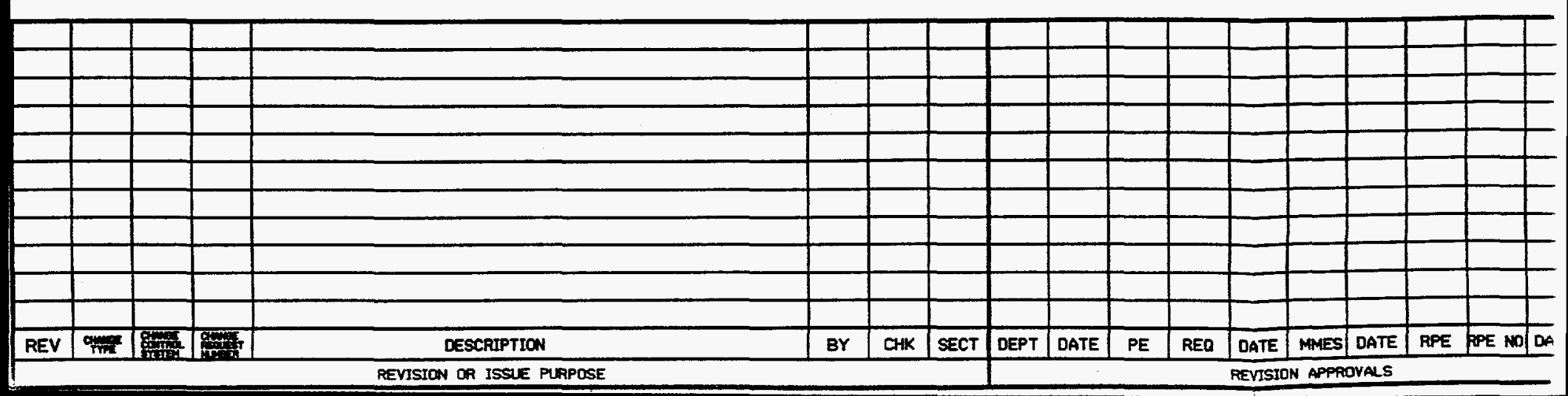




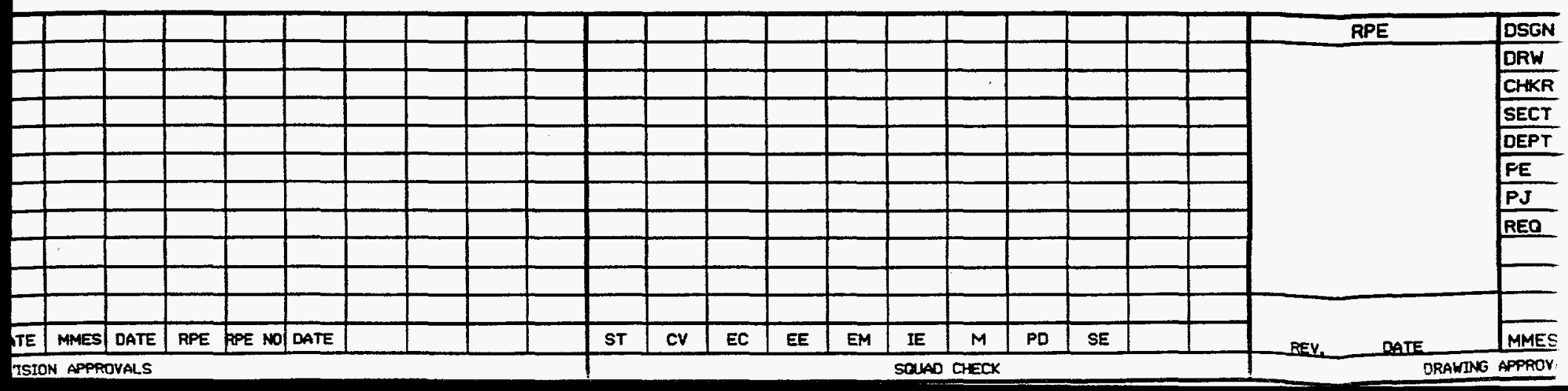




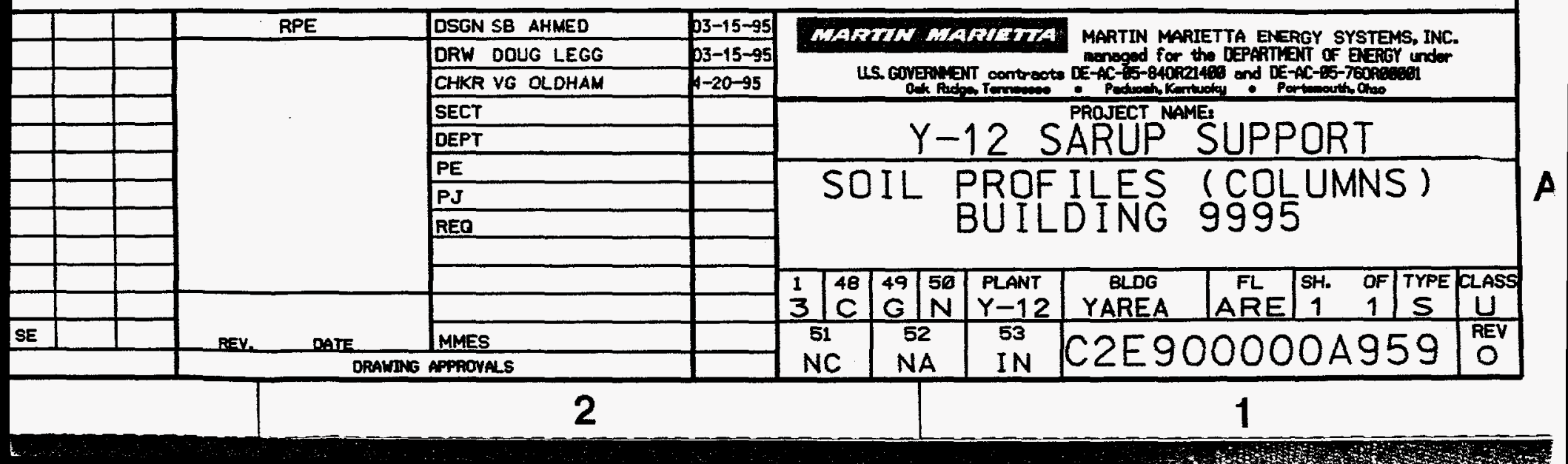




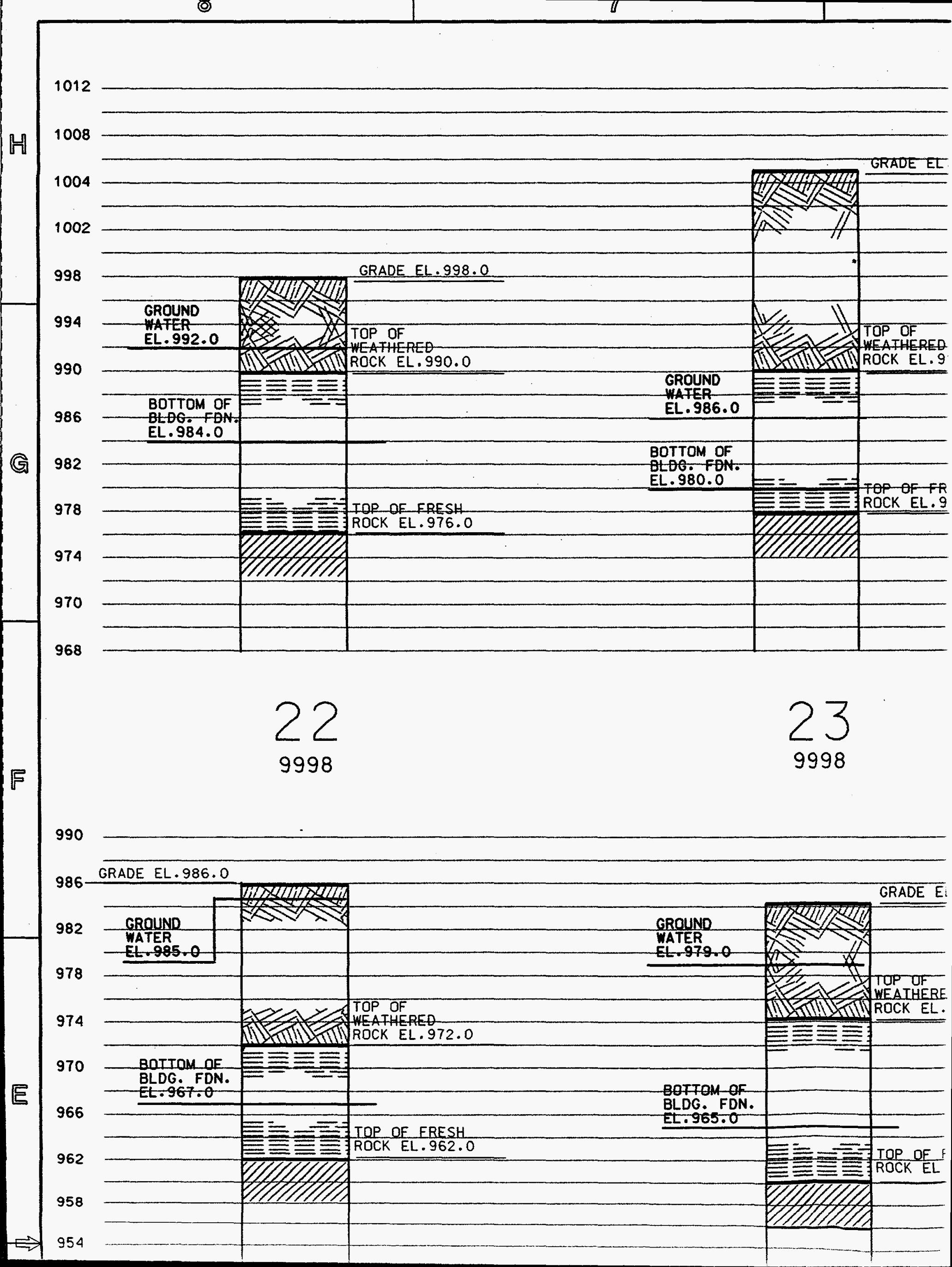


BOTTOM OF

11.0

to

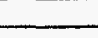

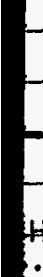

\section{BLDE. FDN. \\ EL. 1009.5}

0
GRADE Et.TOHT.O
TOP OF

WEATHERED

POCK-ET. 989.0

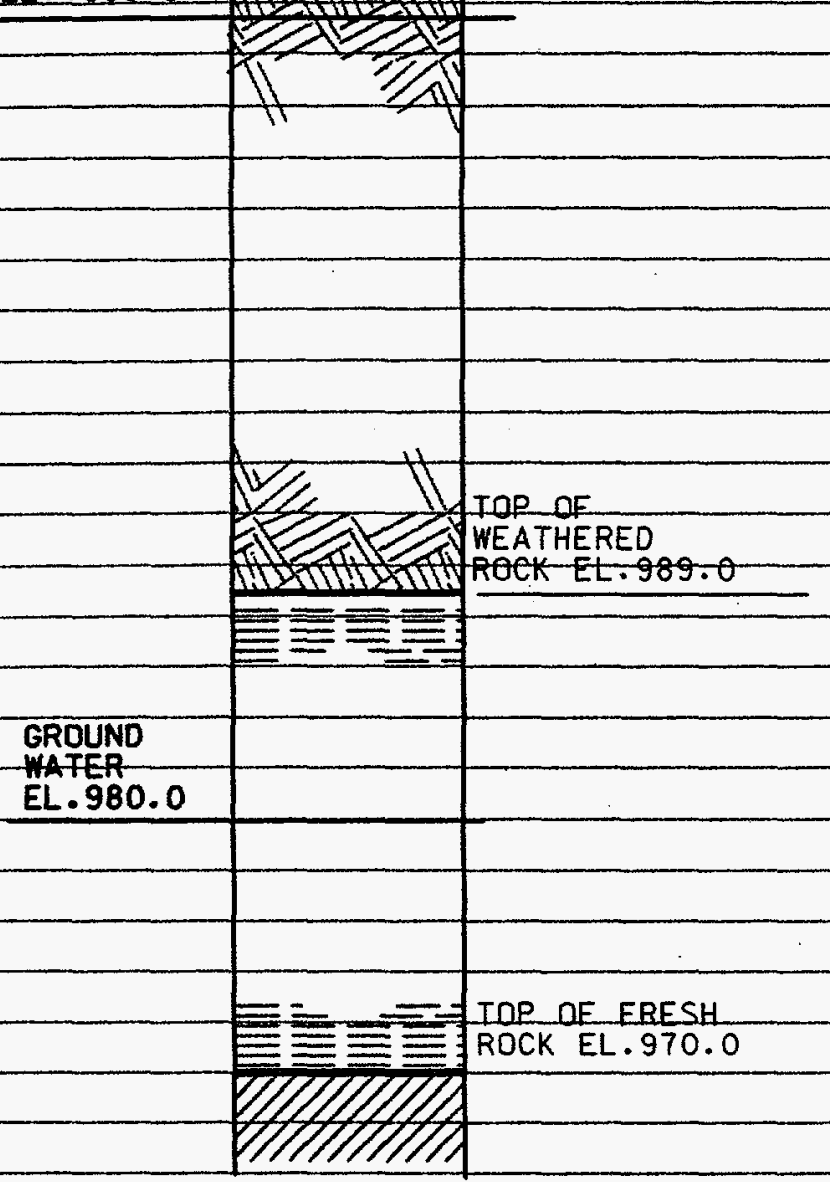

25

9996
BOTTOM OF

BLDG. FDN.

EL. 1009.5

-

GROUND

WATER

EL.980.0

26

9996
74.0

Grouno

WATER

EL.970.0

4.0 

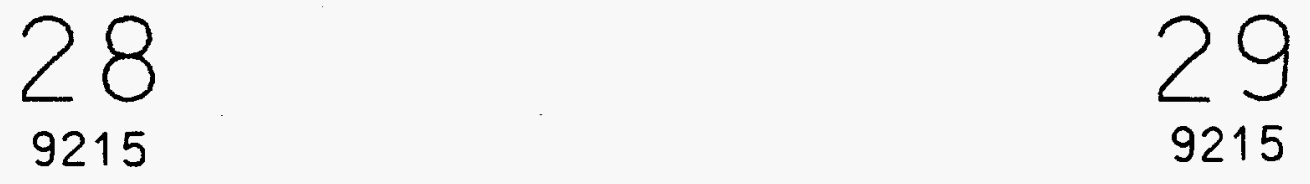

972

968

964

960

C

956

TOP OF WEATHERED

ROCK EL.956.0

952

948

944

940

936

932

字

10 REPRESENTATION OR WARAATT, EXPRESSED OR IMPLIED, IS MOE AS TO THE ACCURACY. COMPLETEEESS OR USERULESS OF THE

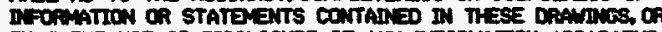

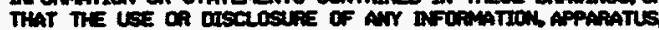

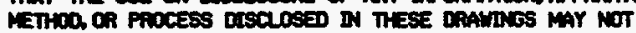

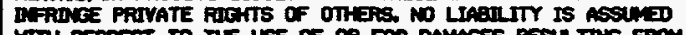
WTH RESPECT TO TRE USE $O F$. OR FOR DAWACES RESUL TING FROM

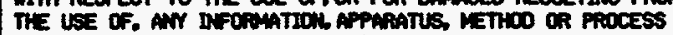

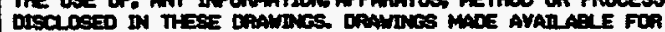

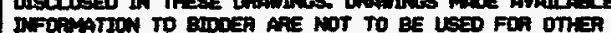

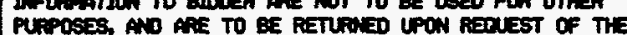

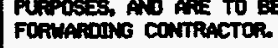

GRADE EL.958.0

GRADE EL. 958.0

GROUNO

WATER

EL.957.5
TOP OF

WEATHEREQ ROCK EL.95

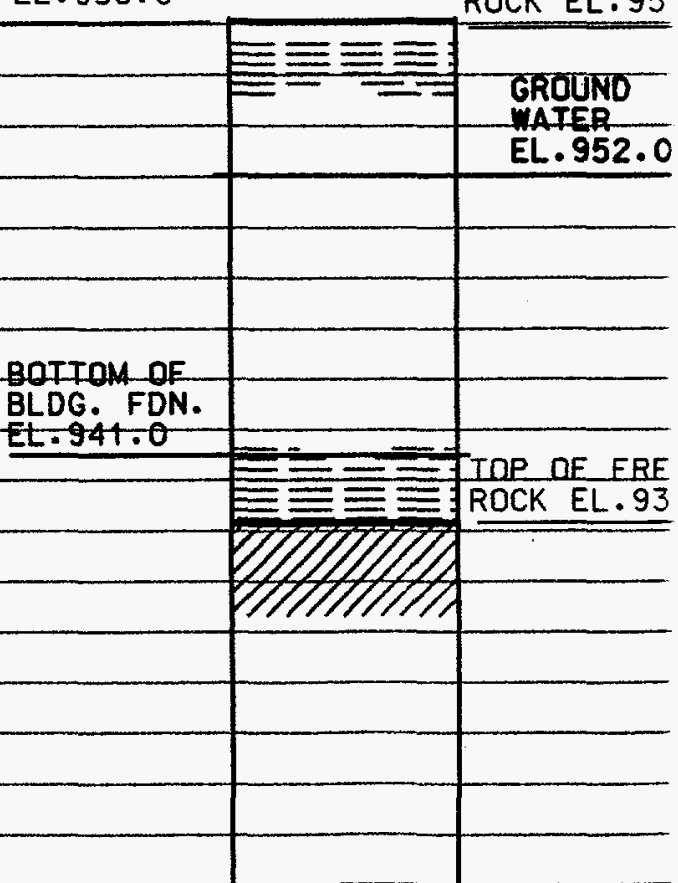

Document Rev. No.: EG-C-37 Reviow By: Guideline Document: UCN-19586 (12356 4-93)

\section{5 \\ 9204-2 \\ TUNNEL}

THIS DOCUMENT CONTROLLED BY

CHANGE CONTROL SYSTEM ENGINEERING PROCEDURE

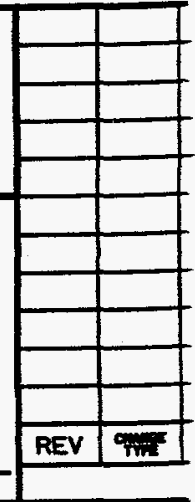




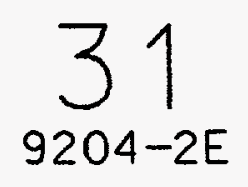

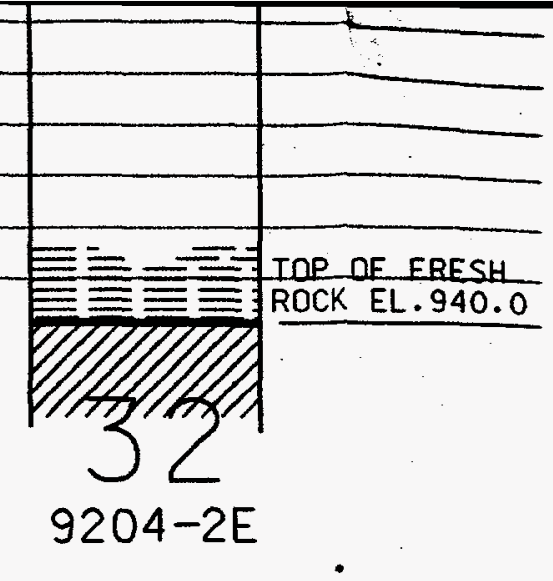
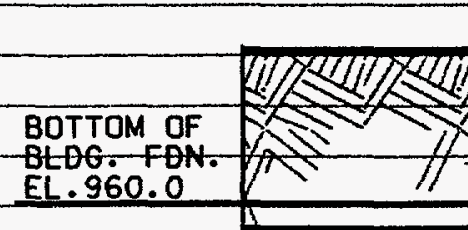

GRADE EL.966.0

TOP OF

WEATHERED

ROCK EL. 959.0

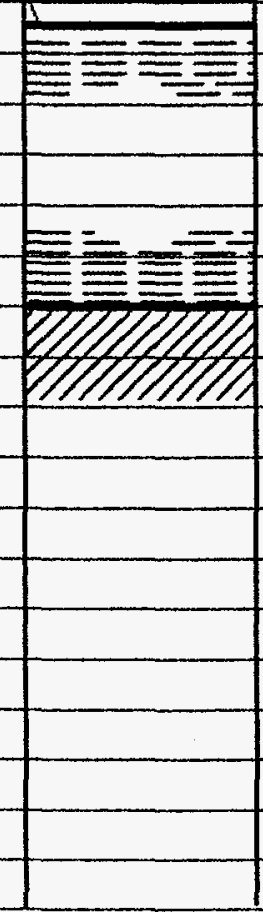

FOP OF FRESH

ROCK EL. 948.0

\section{7 \\ 9720-5}

38
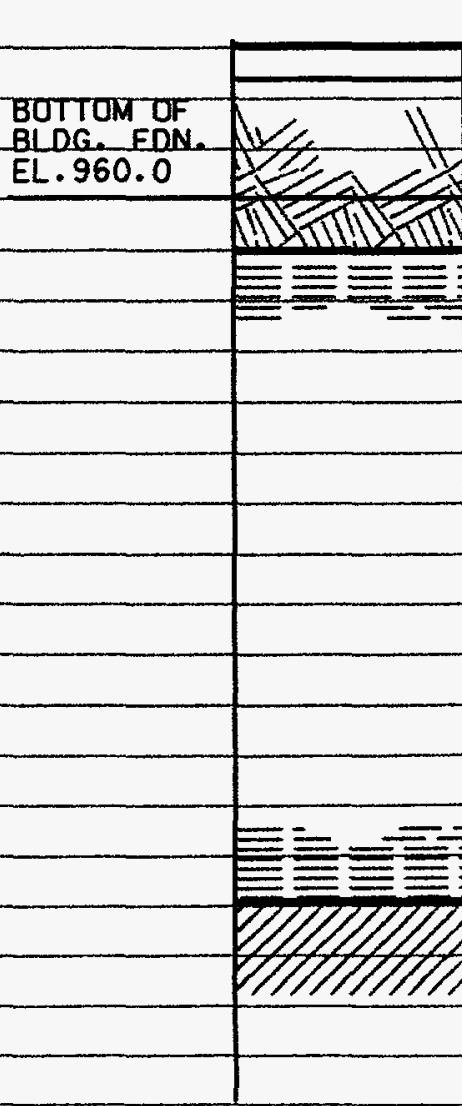

GRADE EL.966.0

TOP OF

WEATHERED

ROCK EL.958.0

TOP OE ERESH

ROCK EL. 944.0

9720-5

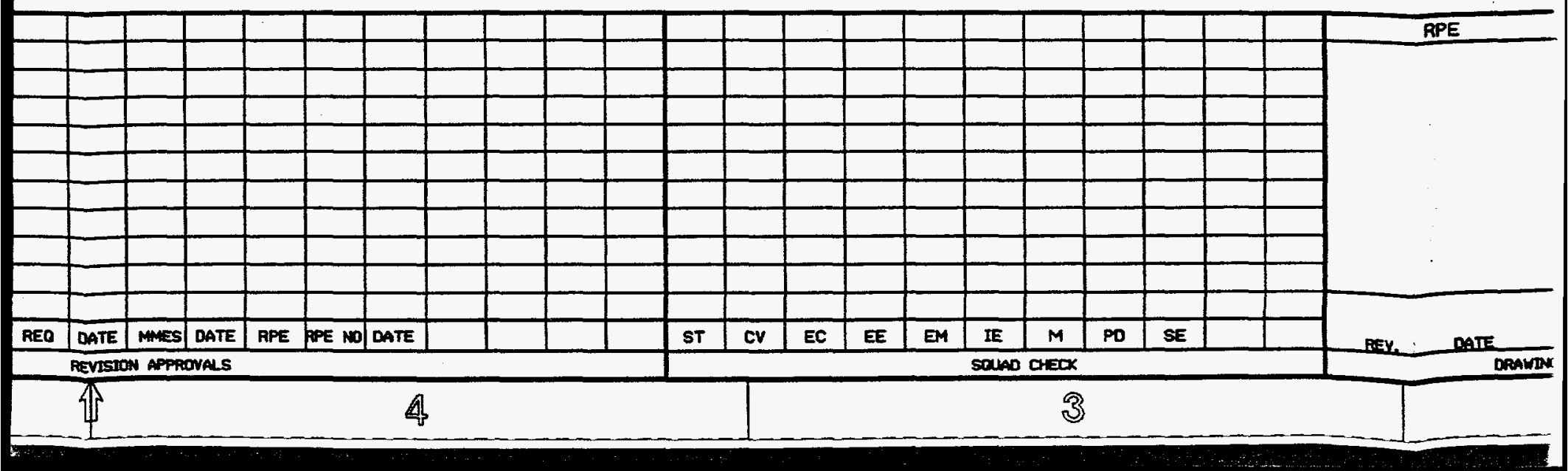




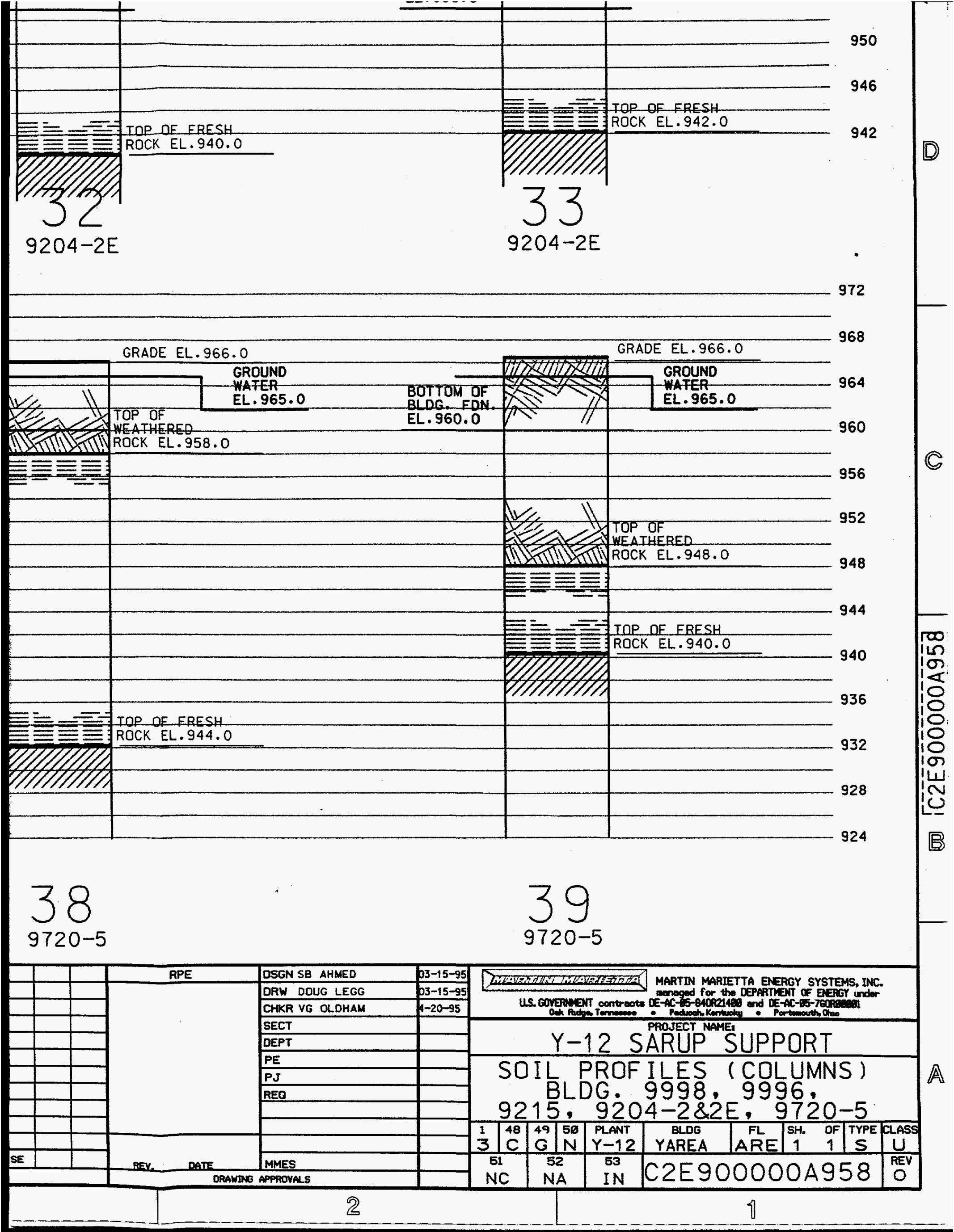


1016 BOTTOM OF BLDG. FDN.

1012

1008

1004

1000

996

992

988

984

980

976

972

1020

1016

1012 GRAOE EL.1011.0 $=$

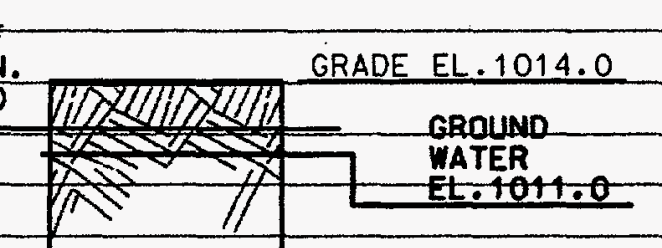

TOP OF

1if 1 III ROCK EL.998.0

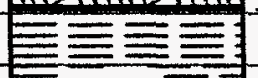

BOTTOM OF

BLDG. FDN. $E L .1012 .0$

\section{.}

GRADE EL.1014.0

GROUND

WATER

E. 101 
GRADE EL. 1014.0 卒
GROUND

WATER

EL .1011 .0

TUP UF

WFATHERED

ROCK EL.998.0

TOP OE FRESH

ROCK EL.974.0

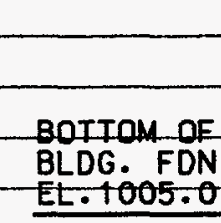

\section{BOTTOM OF BLDG. FDN. Et.997.0}
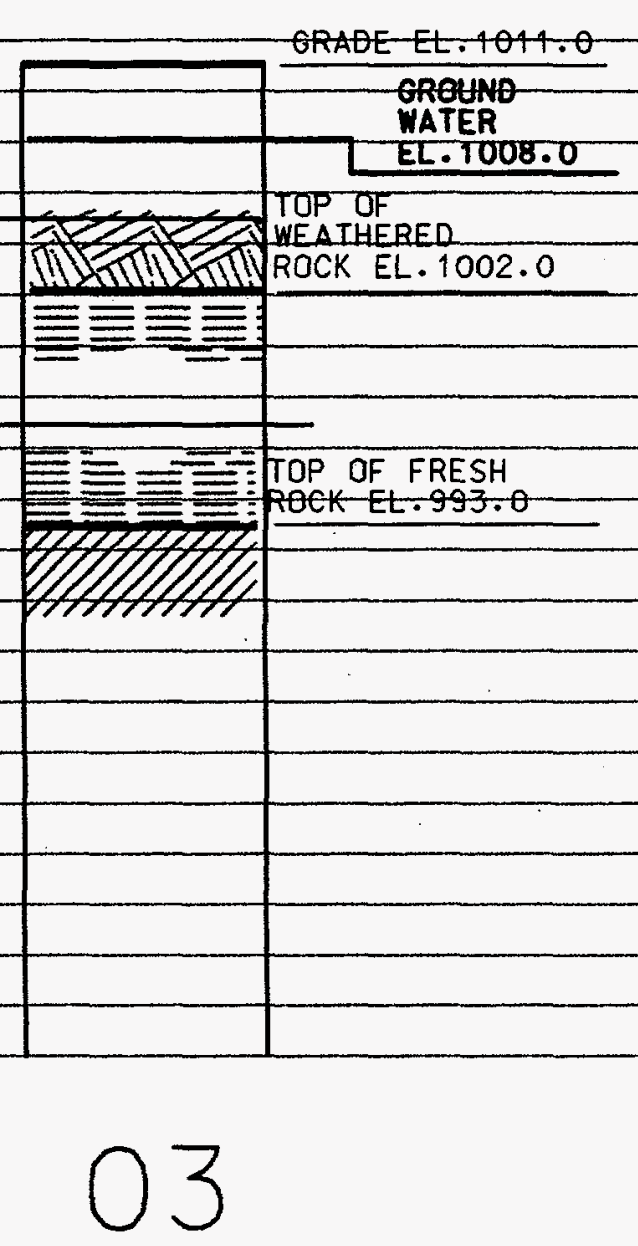

"E" WING
GRAOE-ET

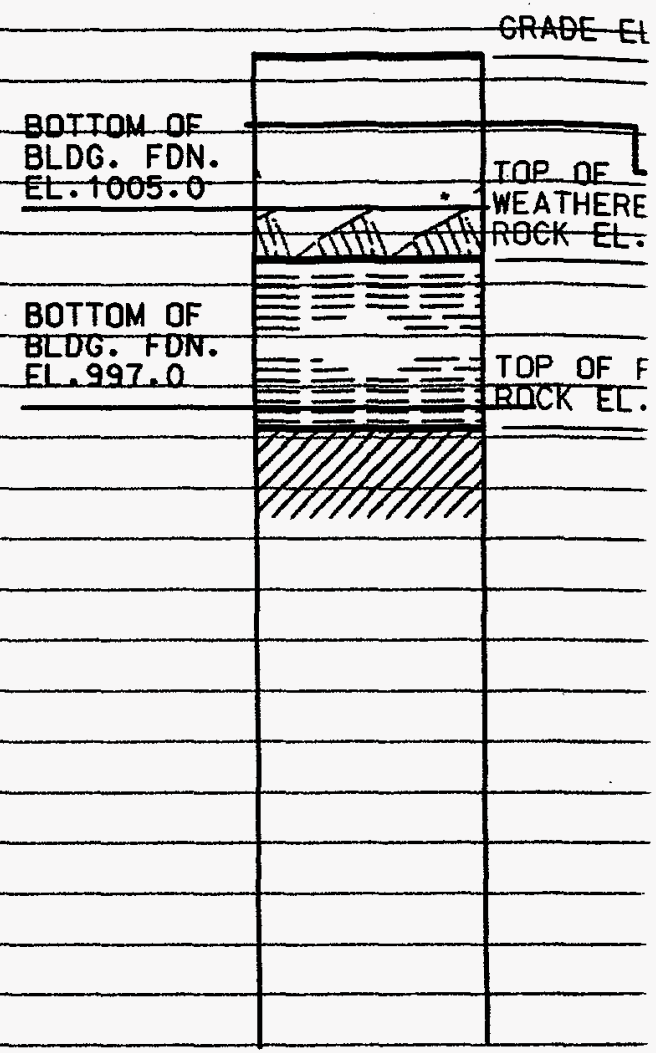

01

"E" WING
GRADE EL 1011.0

BOTIOM

BSMT. SLAB EL. 1007.0

TOP OF

WEATHERED

POCK EL.1001.0

TOP OF FRESH

ROCK EL 995.0

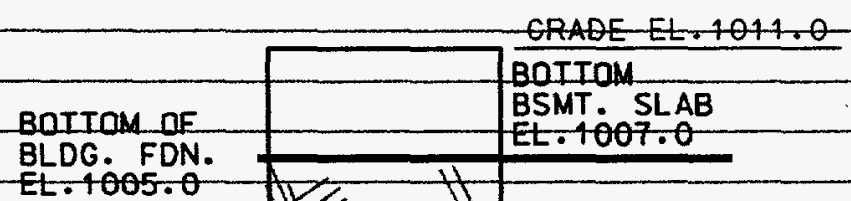

GRAQE EL.1011.0

BSMT. SLAB

inar af

WEATHERED

GROUND

WAFER
EL.998.0

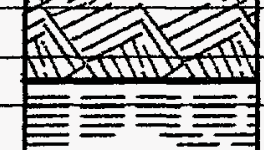

RECK EL. 4001.0

TOP OF FRESH

ROCK EL.992.0 
GRADE EL.1011.0

\begin{tabular}{l} 
GROUN \\
WATER \\
TOP OE LEL.100 \\
WEATHERED \\
ROCK EL.4003.0 \\
TOP OF FRESH \\
RECK EL.996.0 \\
\hline
\end{tabular}

14

W ING
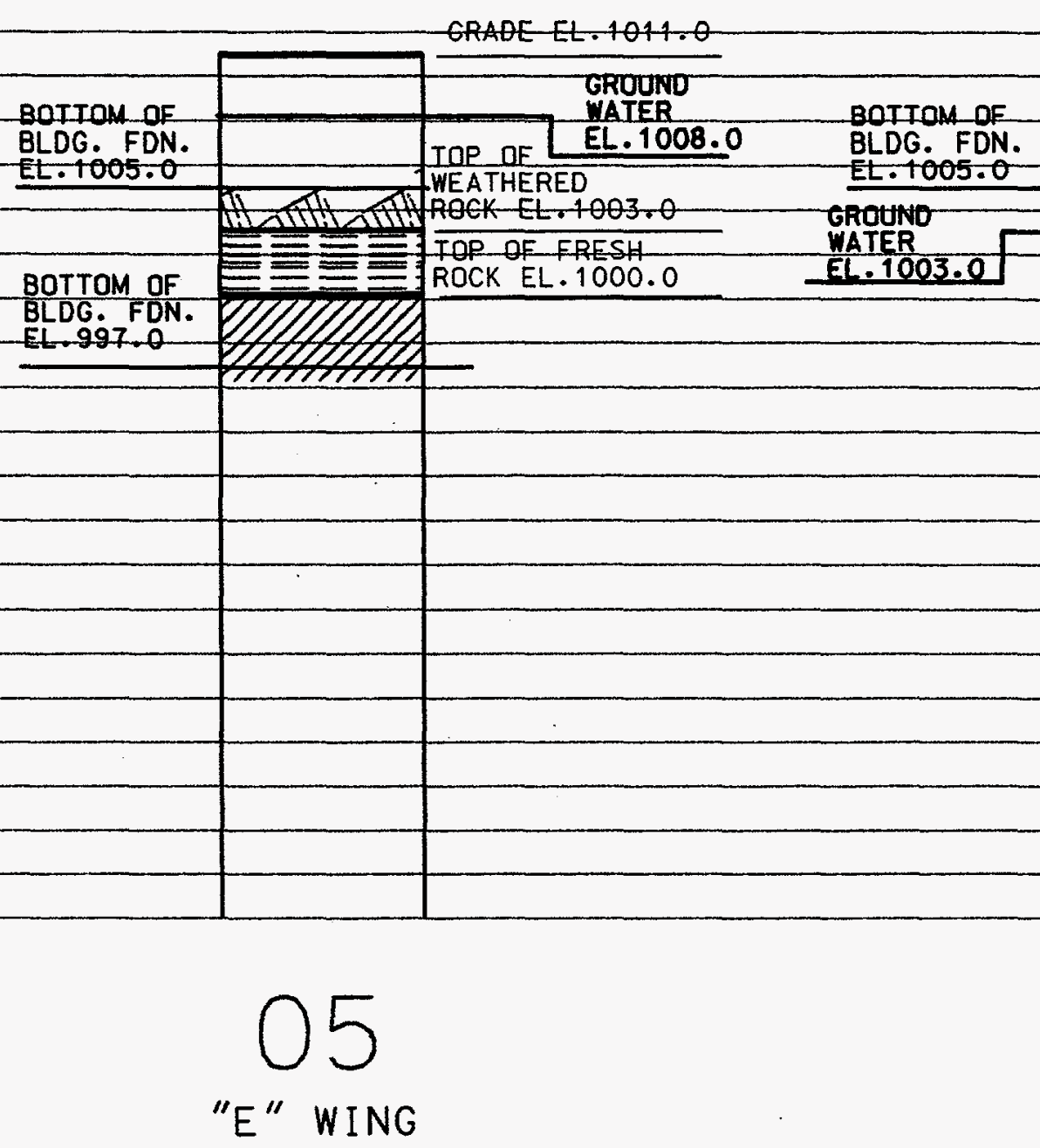

"D" WING HEADHOUSE

GRADE EL.1011.0

BOTTOM

BSMT. SLAB

EL.1007.0

TOP OF

WEATHERED

ROCK EL. 999.0

\section{GROUND \\ WATER \\ EL. 998.0}

TOP OF FRESH

ROCK EL.988.0
GPADE EL-1011.0

BOTTOM

BSMT. SLAB

BOTTOM OE

BLDG. FDN. EL. 1005.0

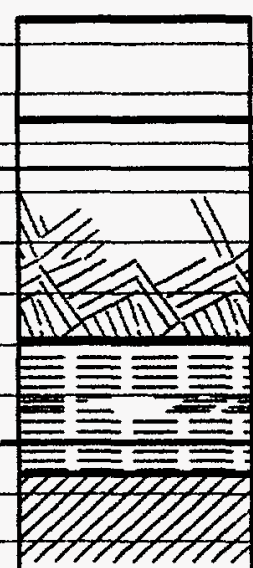

\section{EL.1007.0}

TOP OF

WE $\triangle$ THEBED

ROCK EL.998.0

GROUNO
WATER
EL. 994.0

FOP OF FRESH

ROCK EL.993.0
BOFTOM OF

BLDG. FDN.

EL. 1005.0 
GRADE EL-1011.0

BOTTOM

BOTTOM OF

BLDG. FDN.

EL. 1005.0

GROUNO

WATER
EL. 1003.0

TOP OF

ROCK EL. 1000.0 BSMT. SLAB

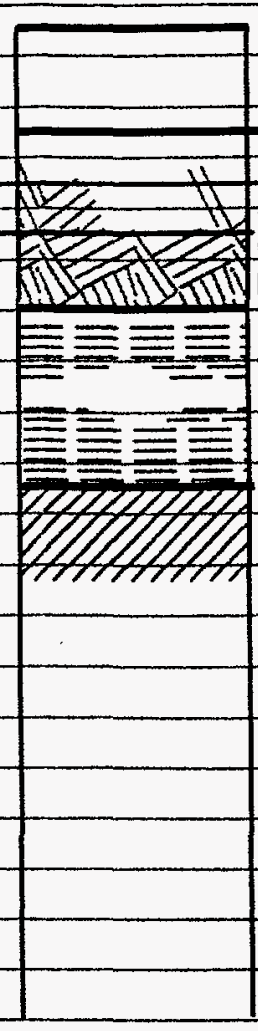

TOP OF FRESH

ROCK EL.993.0

WEATHERED

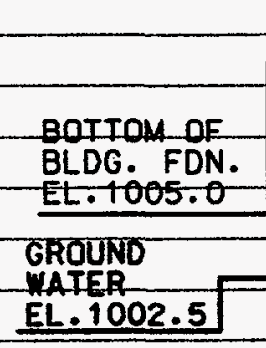

GRADEEL_. 1011 . 1012 BOTTOM

BSMT. SLAB

EL. 1007.0 1008

WATER

EL. 1002.5
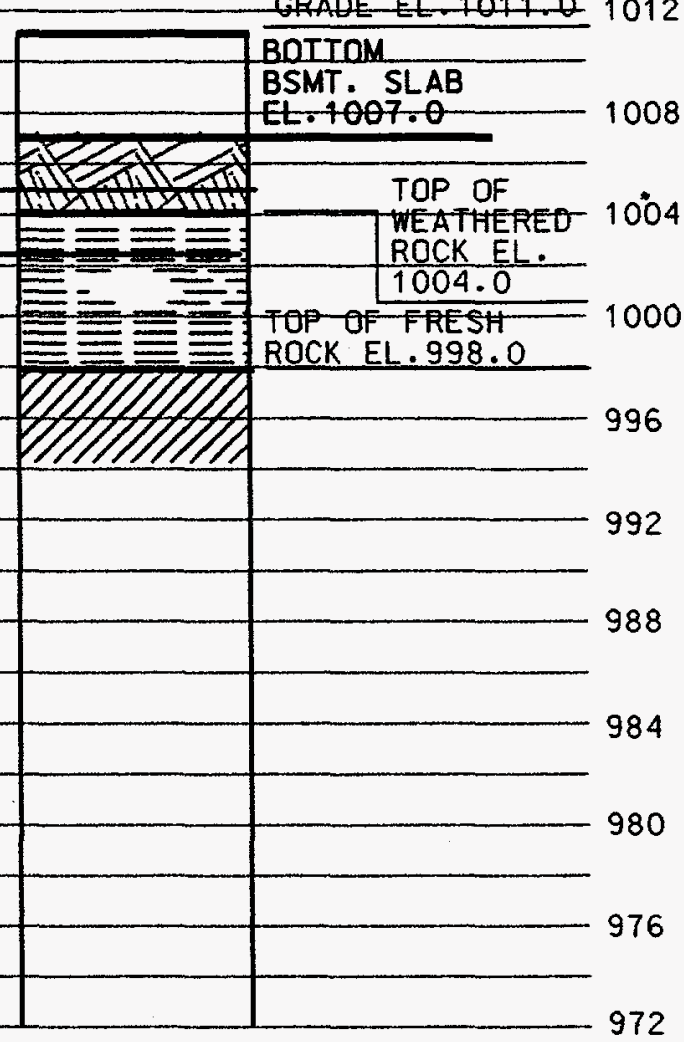

06

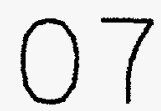

"D" WING

\section{"D" WING \\ HEADHOUSE}

GRAOE EL.TO11.0 BOTTOM BSMT. SLAB

BOTTOM OF BLDG. FDN. EL. 1005.0

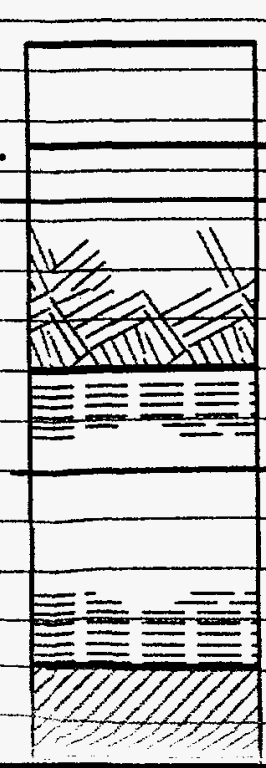

\section{EL. 1007.0}

TOP OF

WEATHERED

ROCK EL. 998.0

\begin{tabular}{l} 
GROUNO \\
WATER \\
EL. 994.0 \\
\hline
\end{tabular}




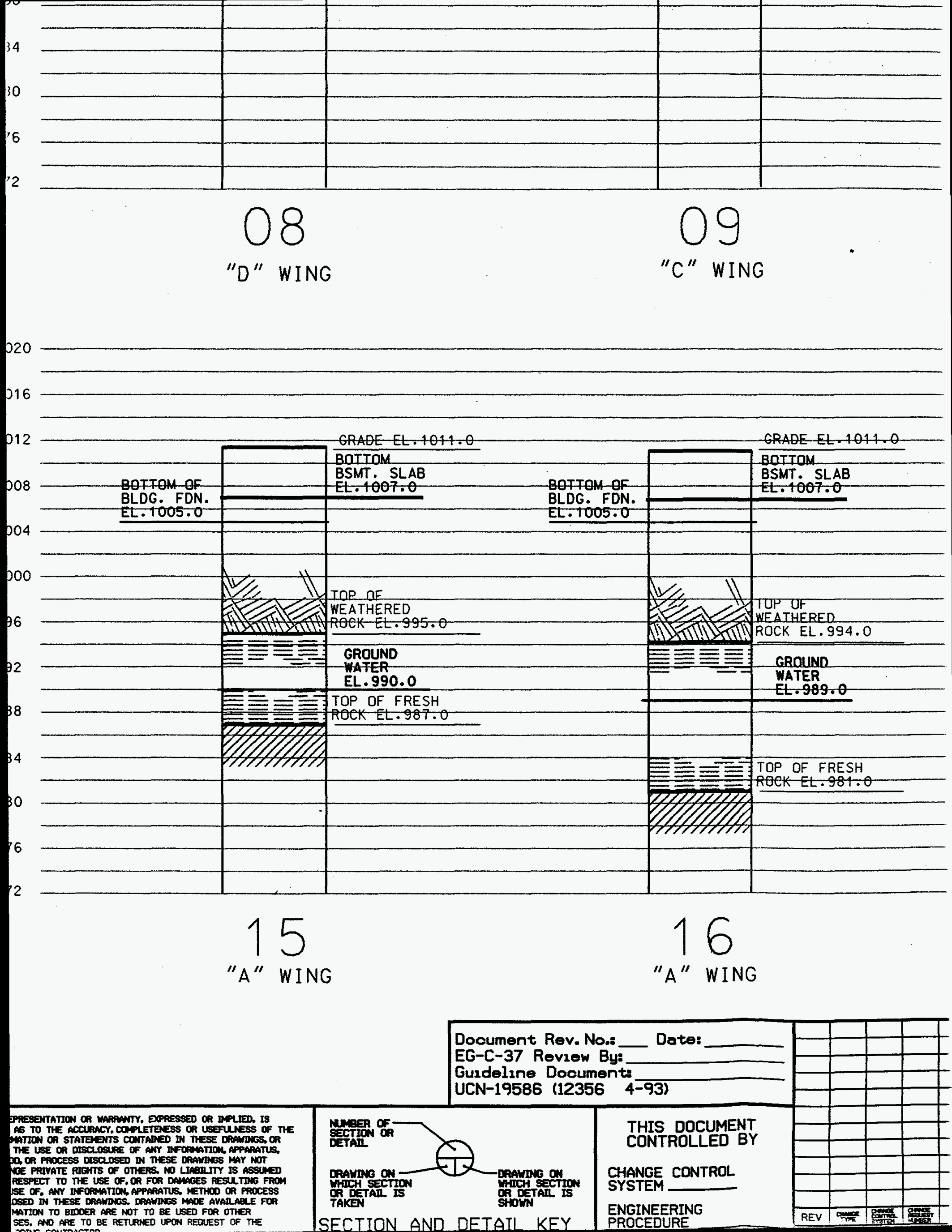




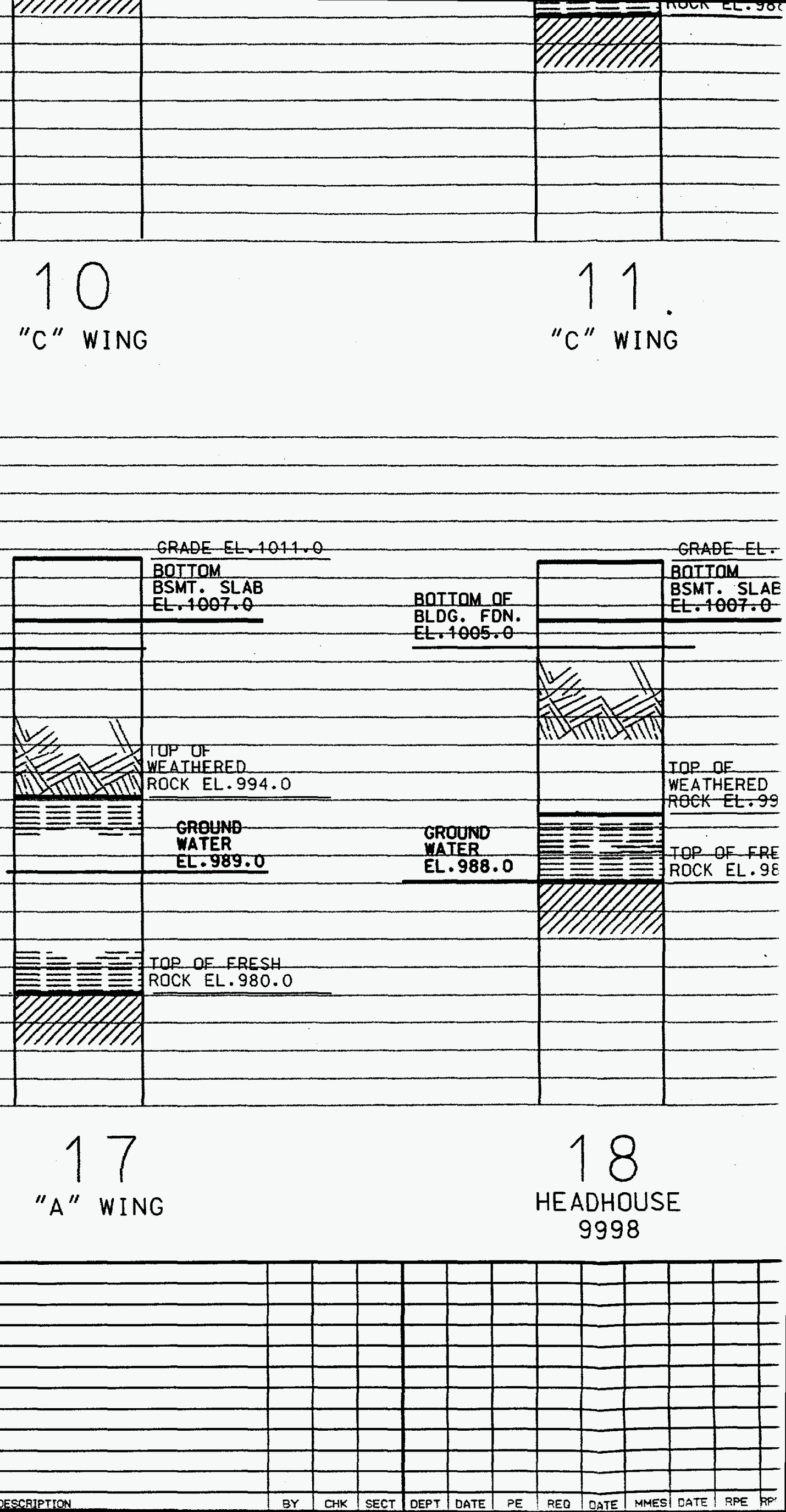



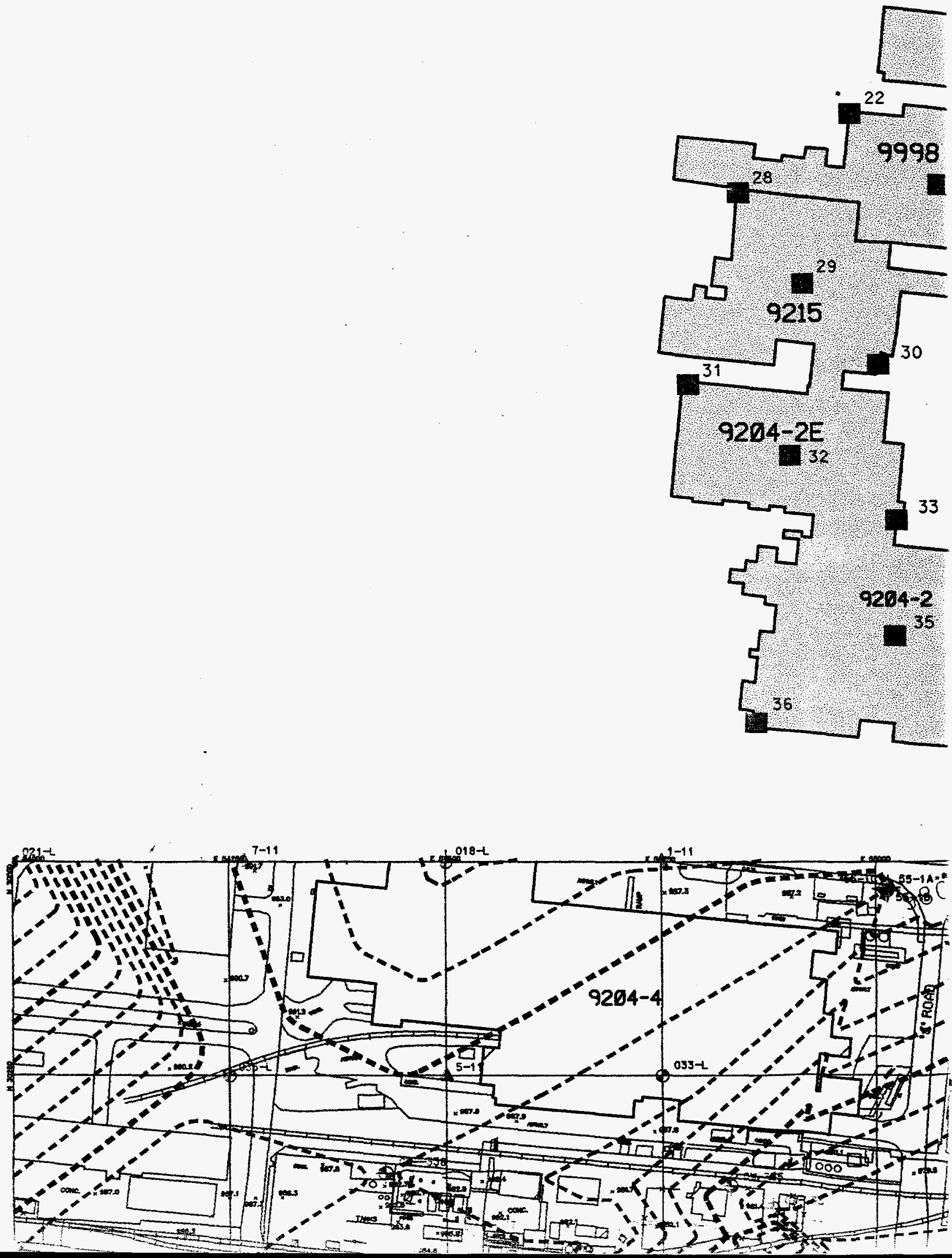


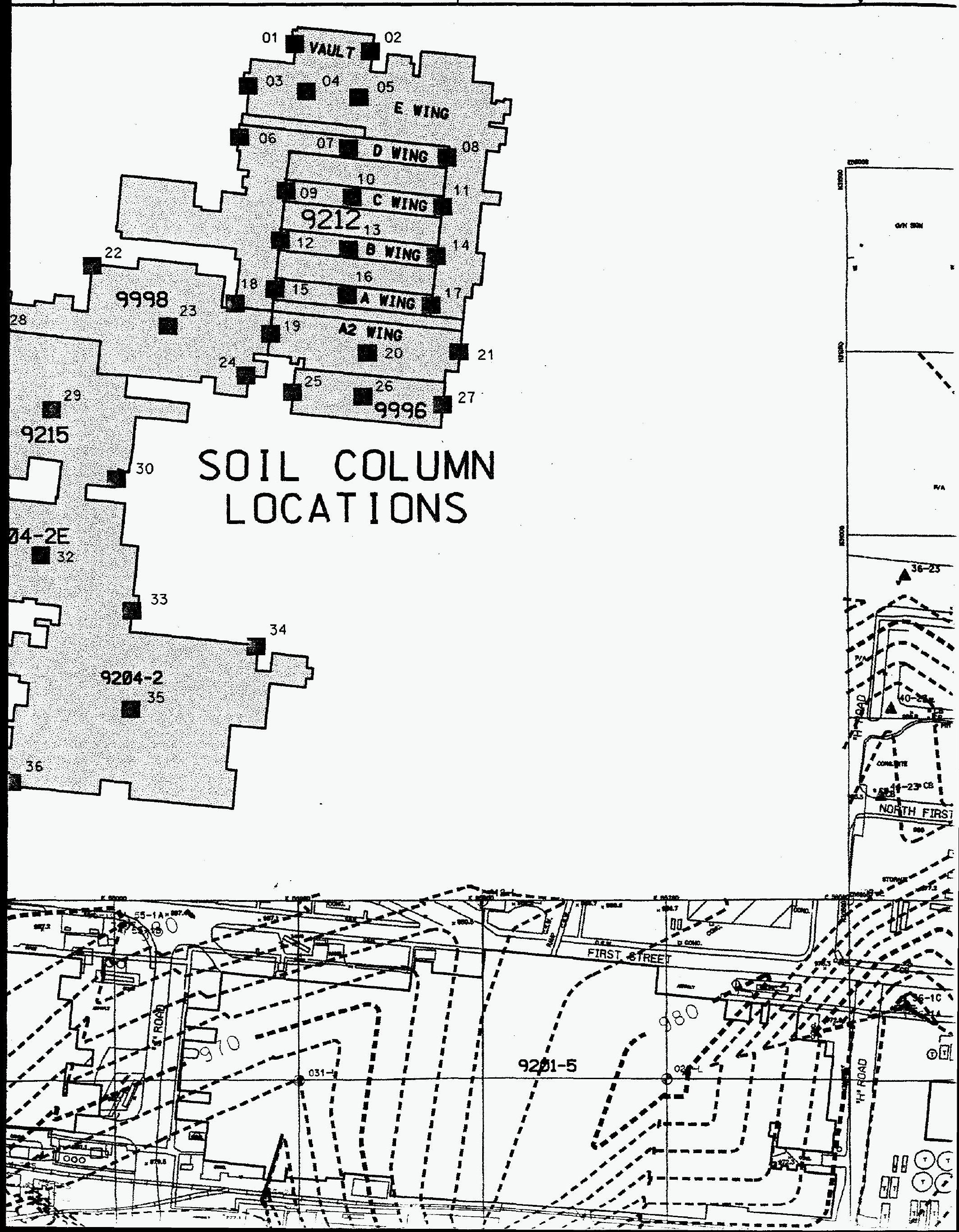




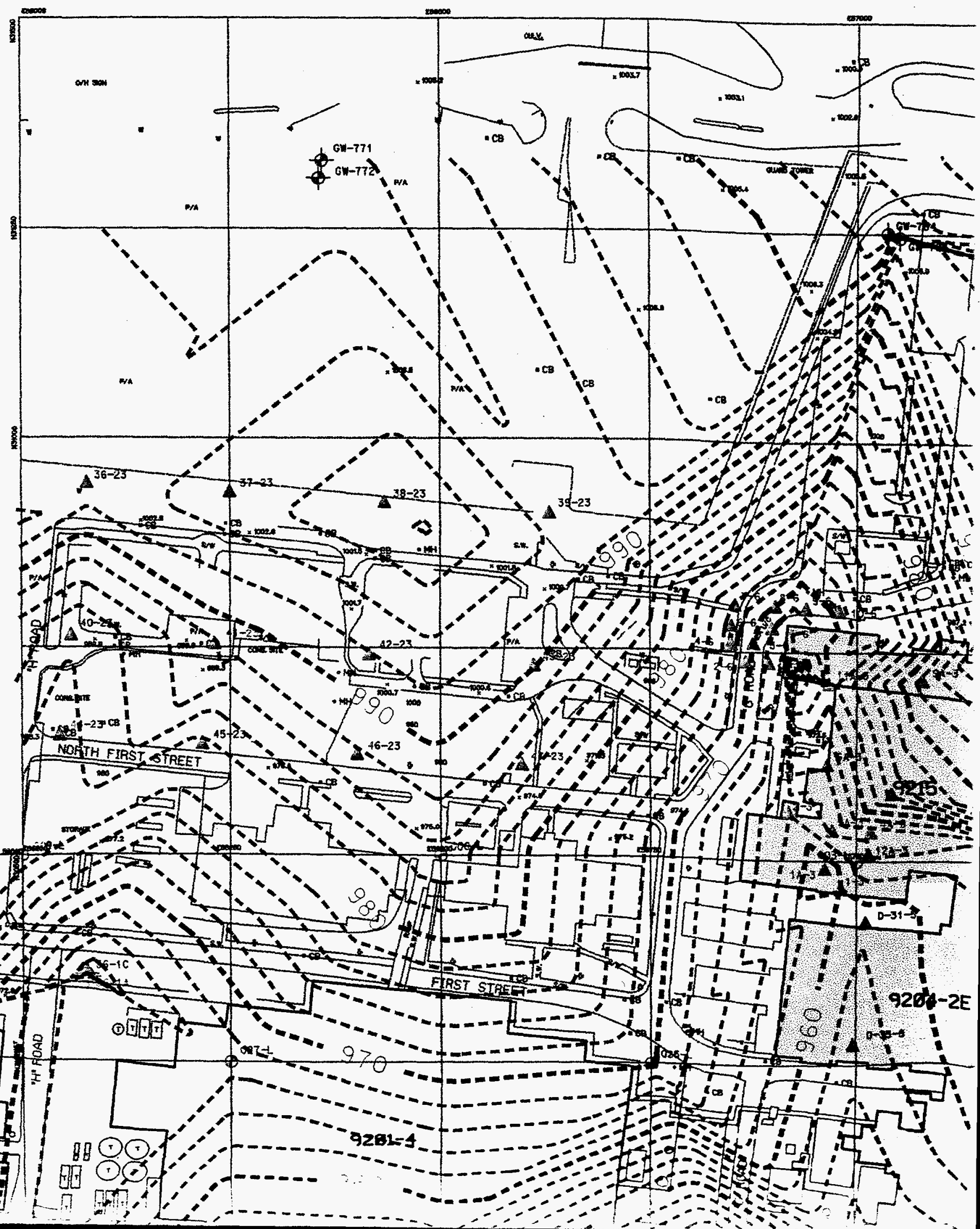




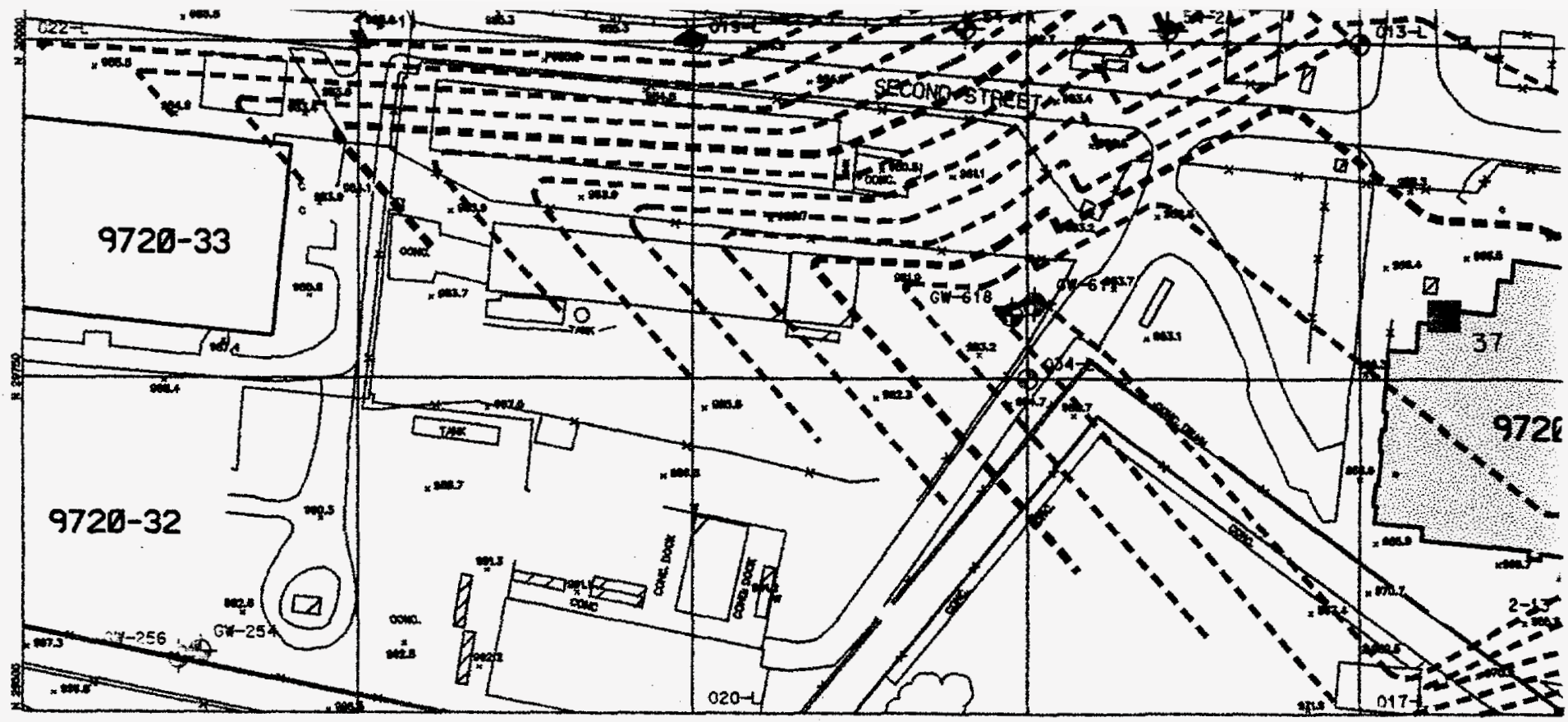

Document Rev. No.: EG-C-37 Roviow By: Guzdeline Document: UCN-19586 (12356

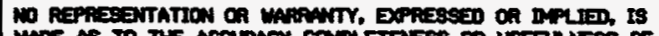

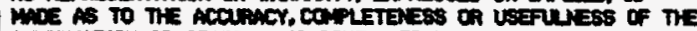

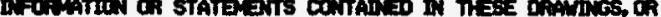

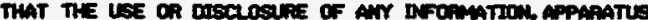

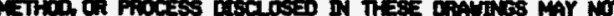

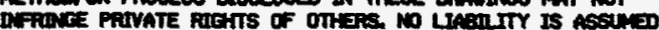

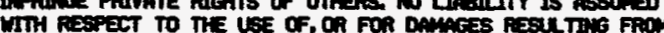

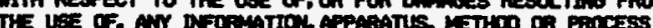
the Ue of

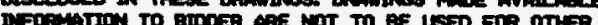

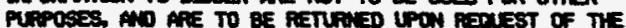
Foniminats contractor.

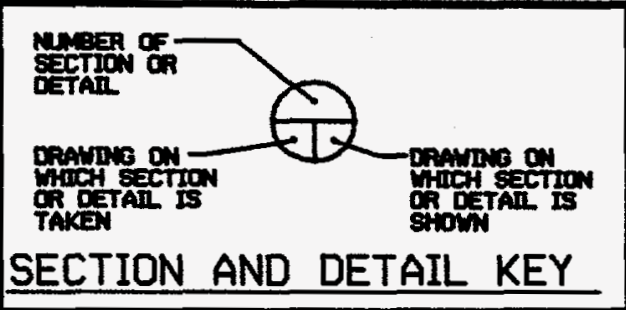

\section{THIS DOCUMENT} CONTROLLED BY

CHANGE CONTROL SYSTEM

ENGINEERING PROCEDURE
Dote:

$4-93$

4-93)

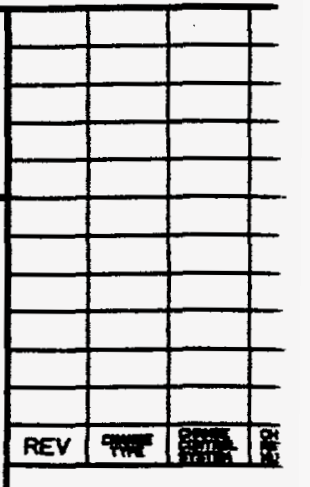



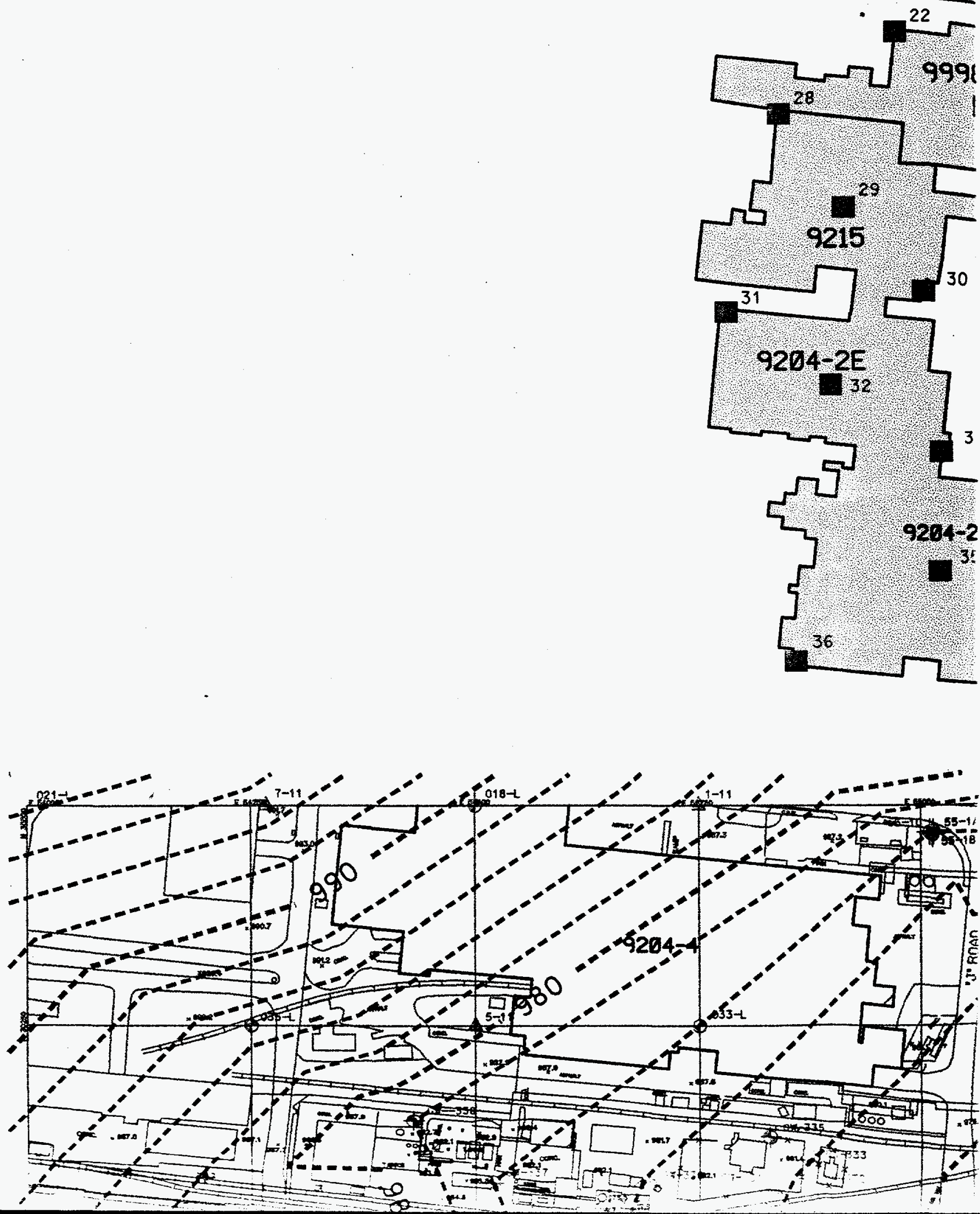


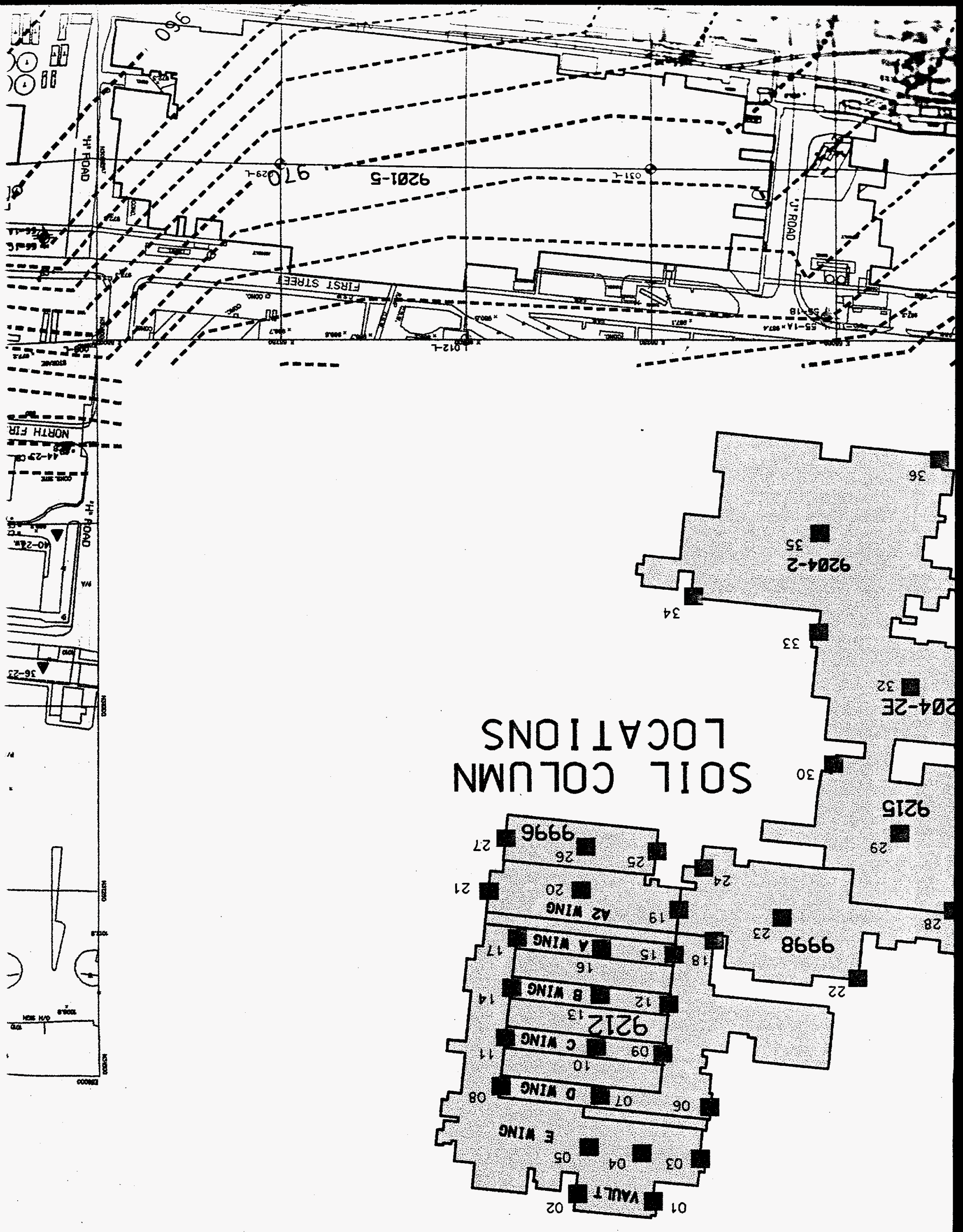




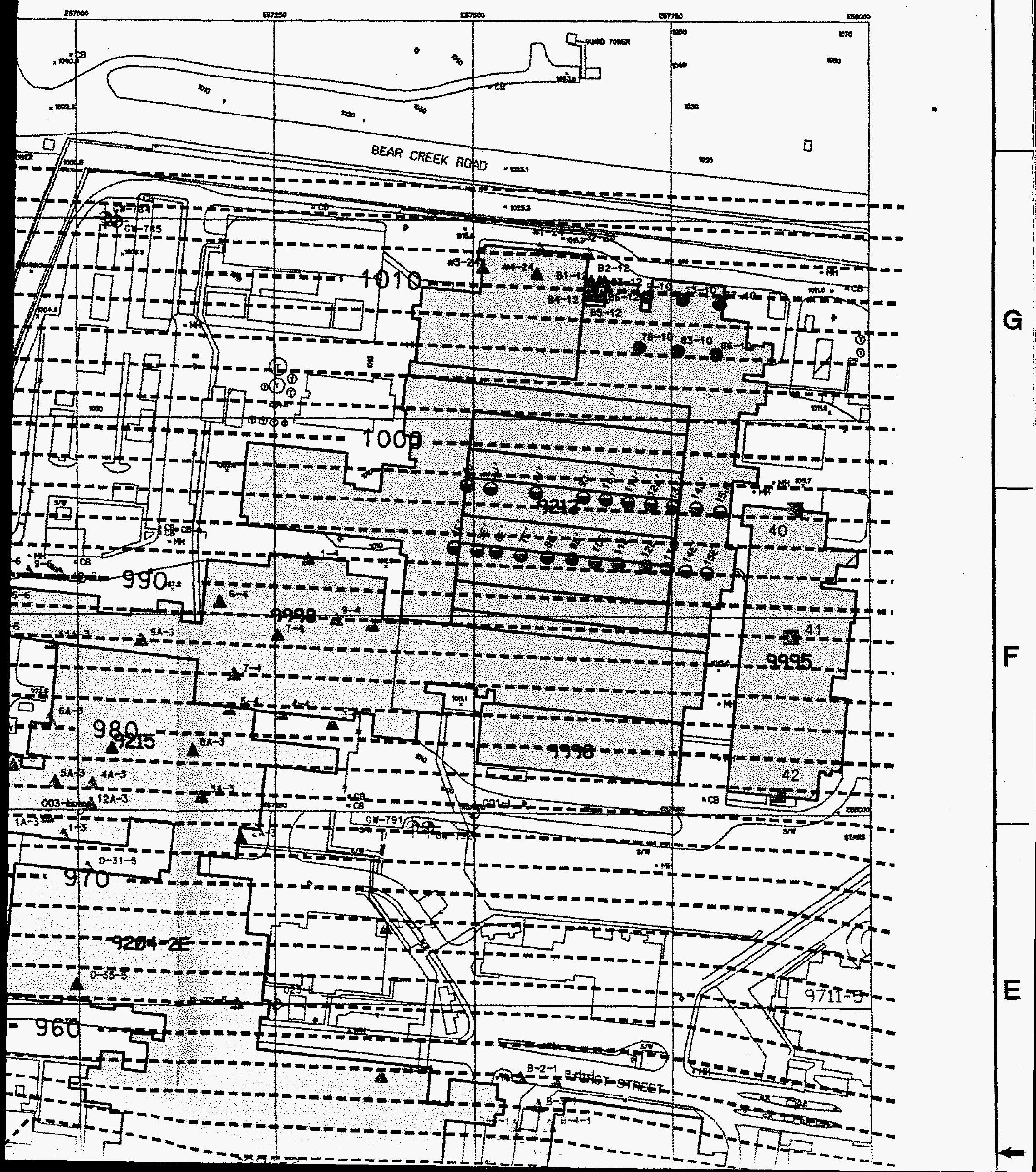




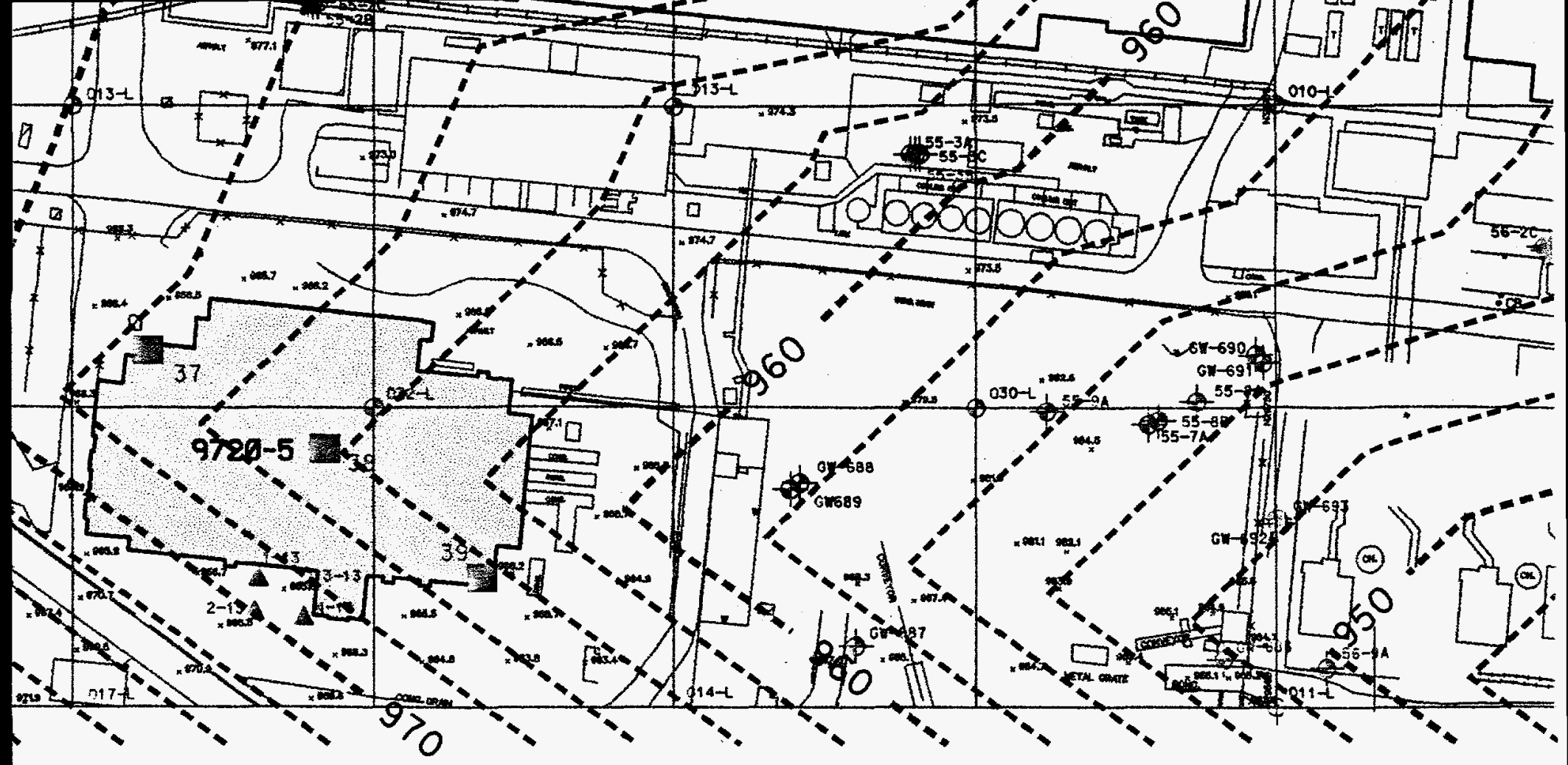

\begin{tabular}{|c|c|c|c|c|c|c|c|c|c|c|c|c|c|c|c|c|}
\hline & & & & & & & & & 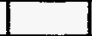 & 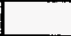 & & 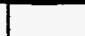 & . & 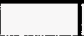 & - & \\
\hline & & & & & & & & & & & & & & & & \\
\hline & & & & & & & & & & & & 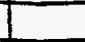 & & & & \\
\hline & & & & & & & & & & & & & & & $\square$ & \\
\hline & & & & & & & & & & & & & & & & \\
\hline & & & & & & & & & & & & & & & & \\
\hline & & & & & & & & & & & & & & & & \\
\hline & & & & & & & & & & & & & & & & \\
\hline & & & & & & & & & & & & & & & & \\
\hline & & & & & & & & & & & & & & & & \\
\hline & & & & & & & & & & & & & & & & \\
\hline REV & 整 & 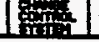 & 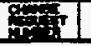 & DESCRIPTION & $B Y$ & CHK & SECT & DEPT & DATE & PE & RED & DATE & MAES & DATE & APE & BPE NO \\
\hline & & & & REVISTON OR ISSLE PLAPOSE & & & & & & & & REVISID & ON APPPC & DOVALS & & \\
\hline
\end{tabular}



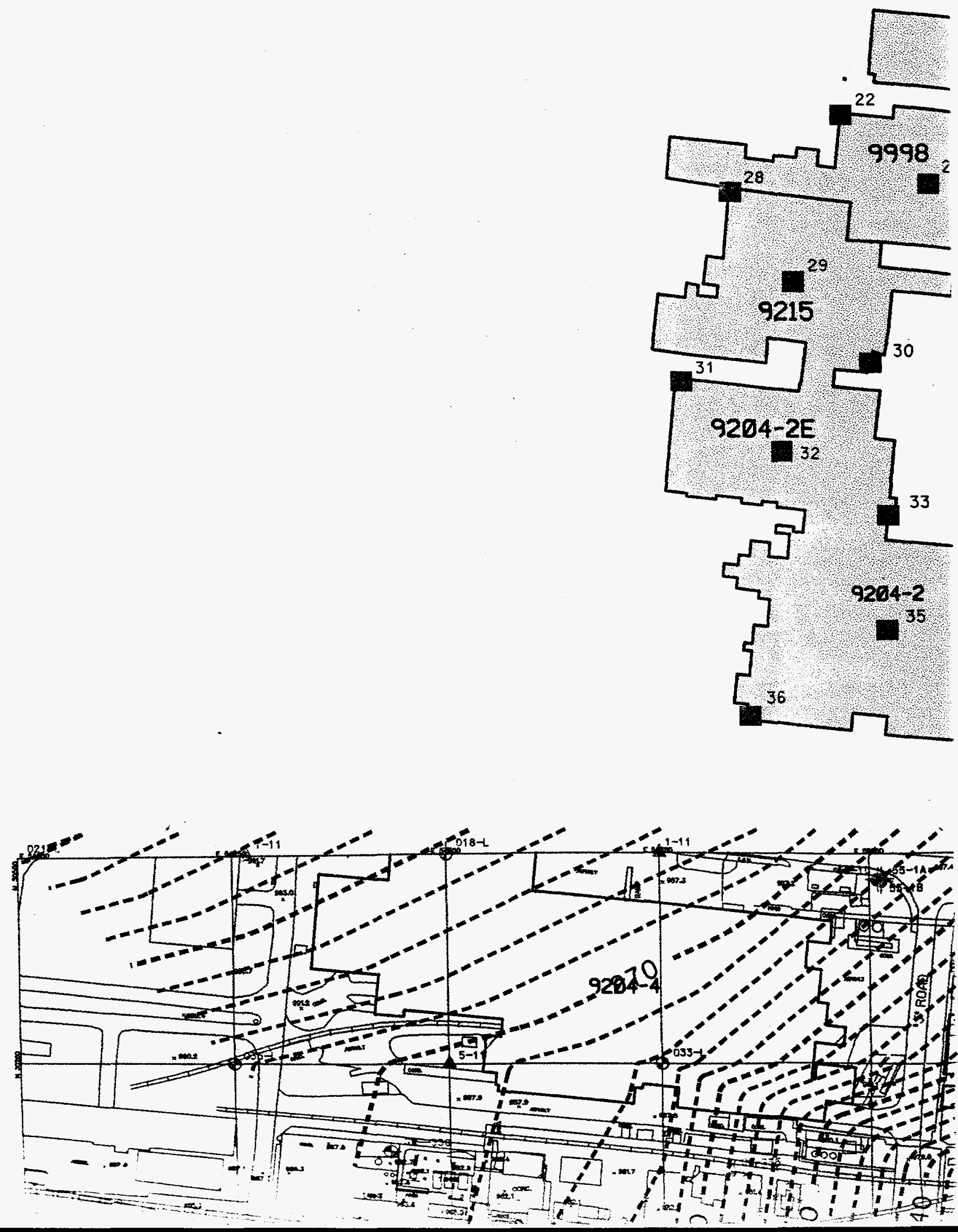


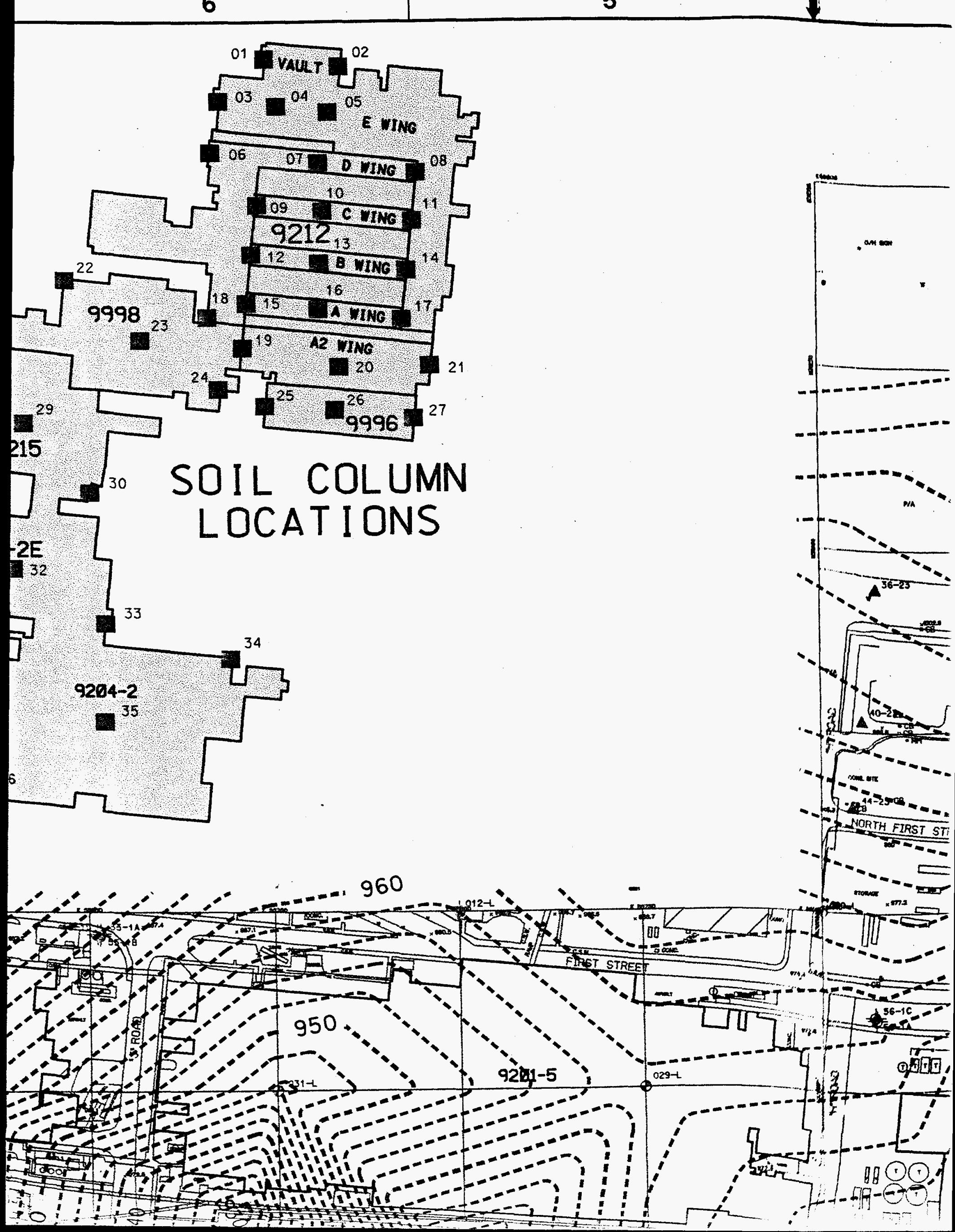




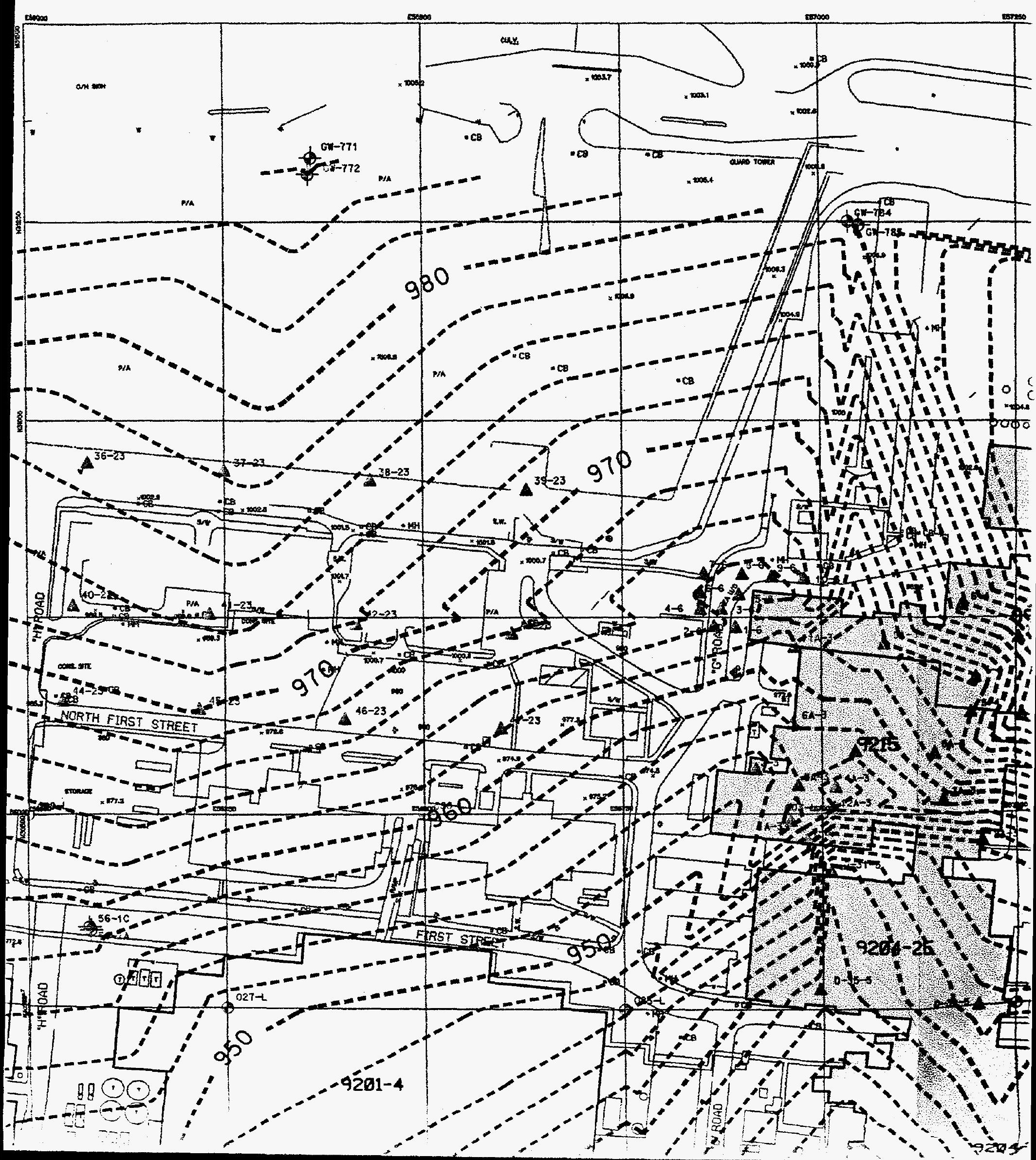




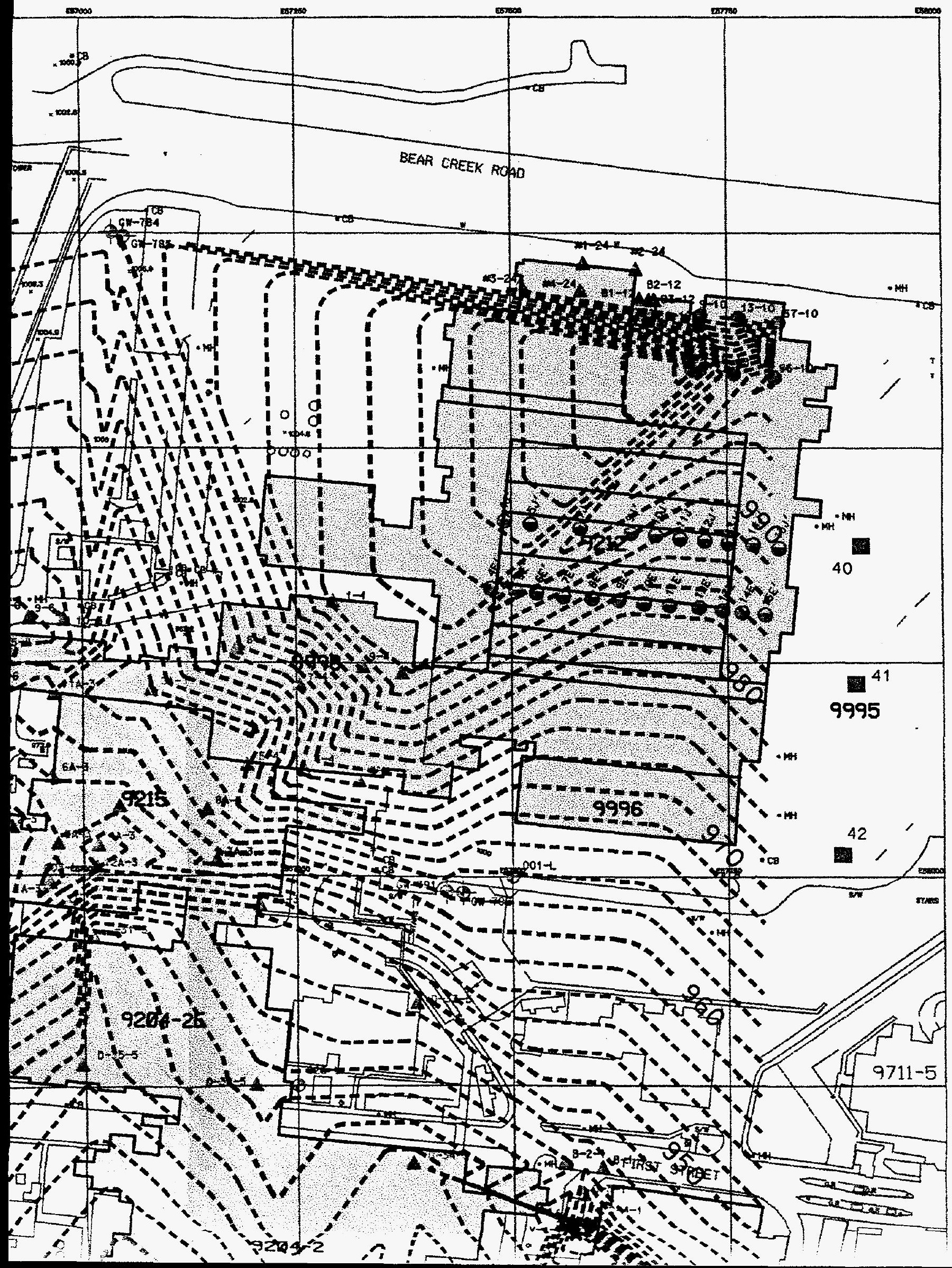




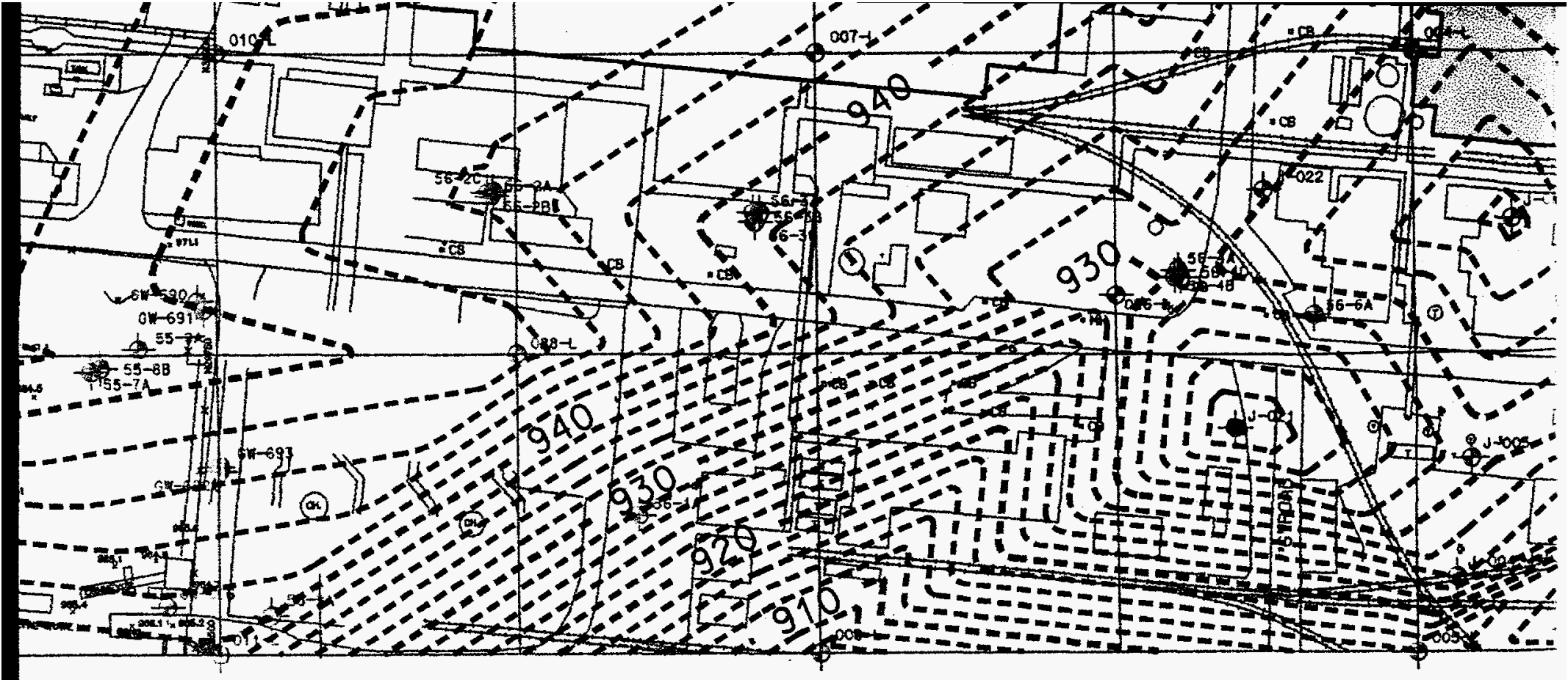

SOIL BORING

1 USR-III RAN 4781 FOMDATION ITVES FODLATION INVES
FOUDATIOA INVES

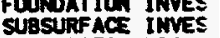
PILE DATA BLDG BUILOIMG 9212 FO C MICA INV OUDATIOA INVES EEOTECHMICIN IN CROSS BORE HOL VIEROSEISIMIC SL

EEOTECHAICAL IN

MONITORING

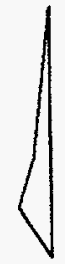

UPOATED SUBSURF/ CHESTMUT RIDEE OCTCBER 1993

CN ANDER YEAR IT IPPER EAST FORK PLANT FEBRUARY :

SOUNDING RE

\section{}

\begin{tabular}{l|l}
\hline & \\
\hline & \\
\hline & \\
\hline & \\
\hline & \\
\hline &
\end{tabular}

\begin{tabular}{|l|l|l|}
\hline & & \\
\hline & & \\
\hline & & \\
\hline & & \\
\hline & & \\
\hline & & \\
\hline & & \\
\hline & & \\
\hline & & \\
\hline & & \\
\hline
\end{tabular}

\begin{tabular}{|l|l|l|}
\hline & & \\
\hline & & \\
\hline & & \\
\hline & & \\
\hline & & \\
\hline & & \\
\hline & & \\
\hline & & \\
\hline & & \\
\hline ST & cr & \\
\hline
\end{tabular}

\begin{tabular}{l|l|l|l|l|l|l|l|l|l}
\hline & & & & & & & & & \\
\hline & & & & & & & & & \\
\hline & & & & & & & & & \\
\hline & & & & & & & & & \\
\hline & & & & & & & & \\
\hline & & & & & & & & & \\
\hline & & & & & & & & & \\
\hline & & & & & & & & & \multirow{2}{*}{ EC } \\
\hline
\end{tabular}



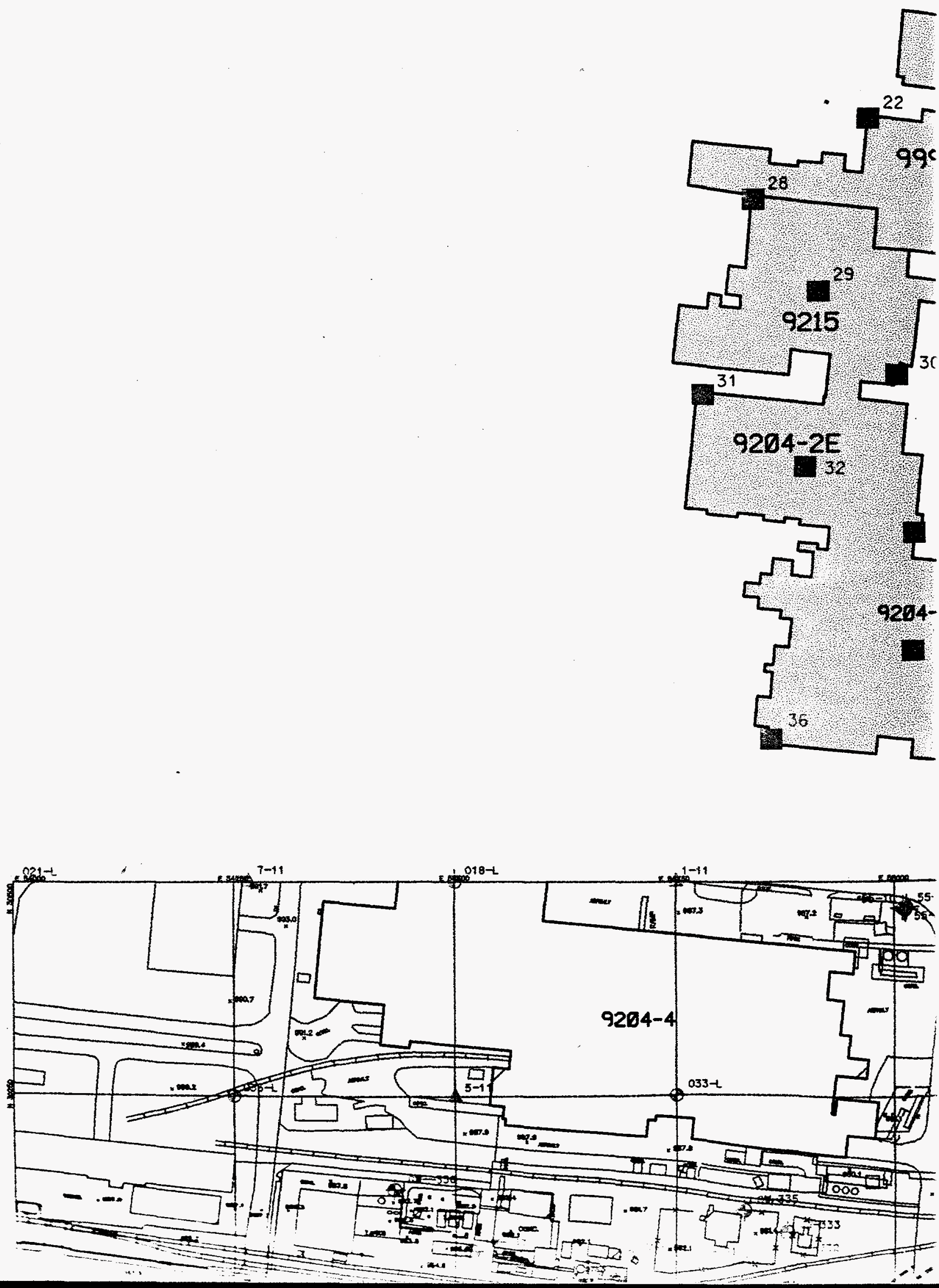


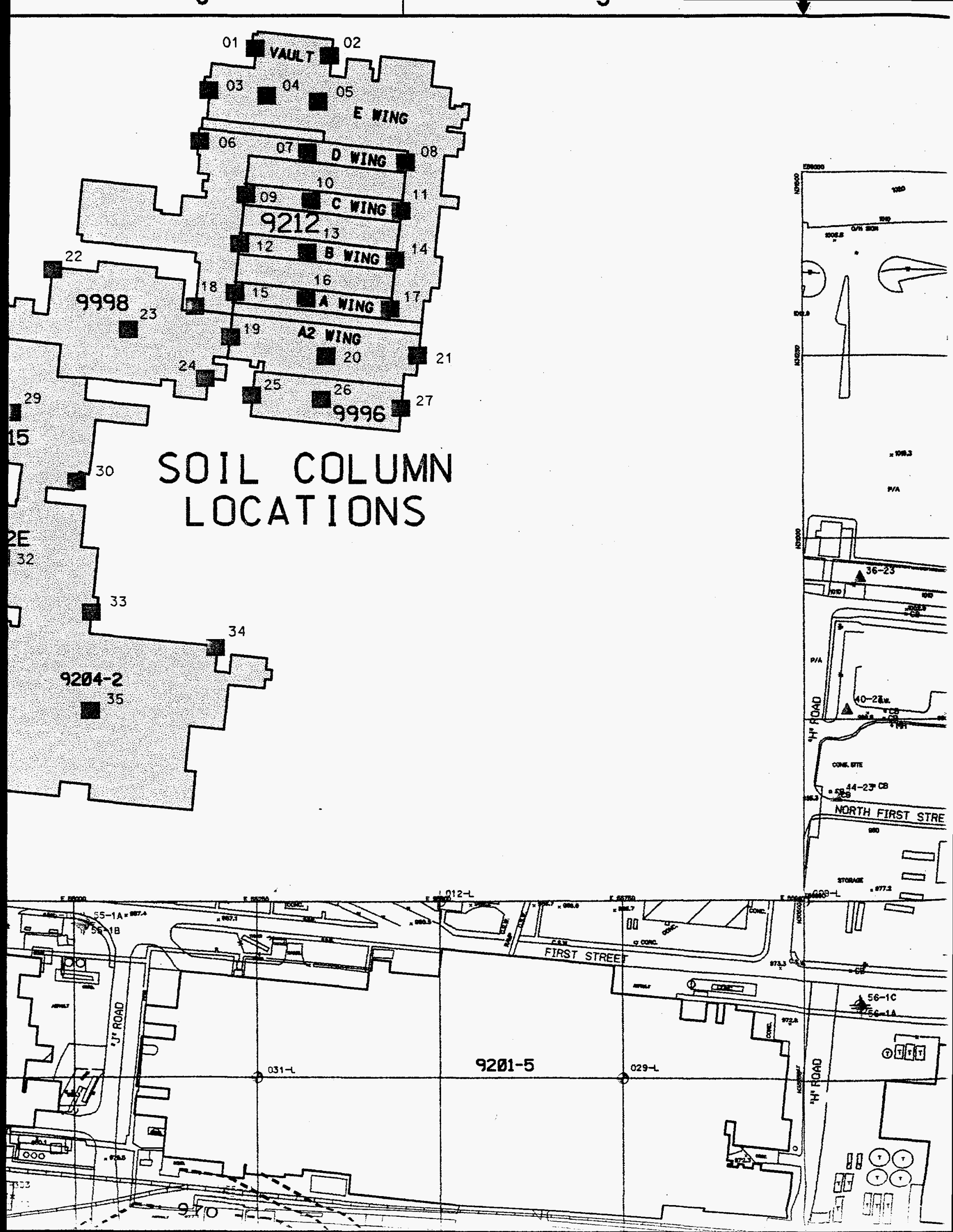




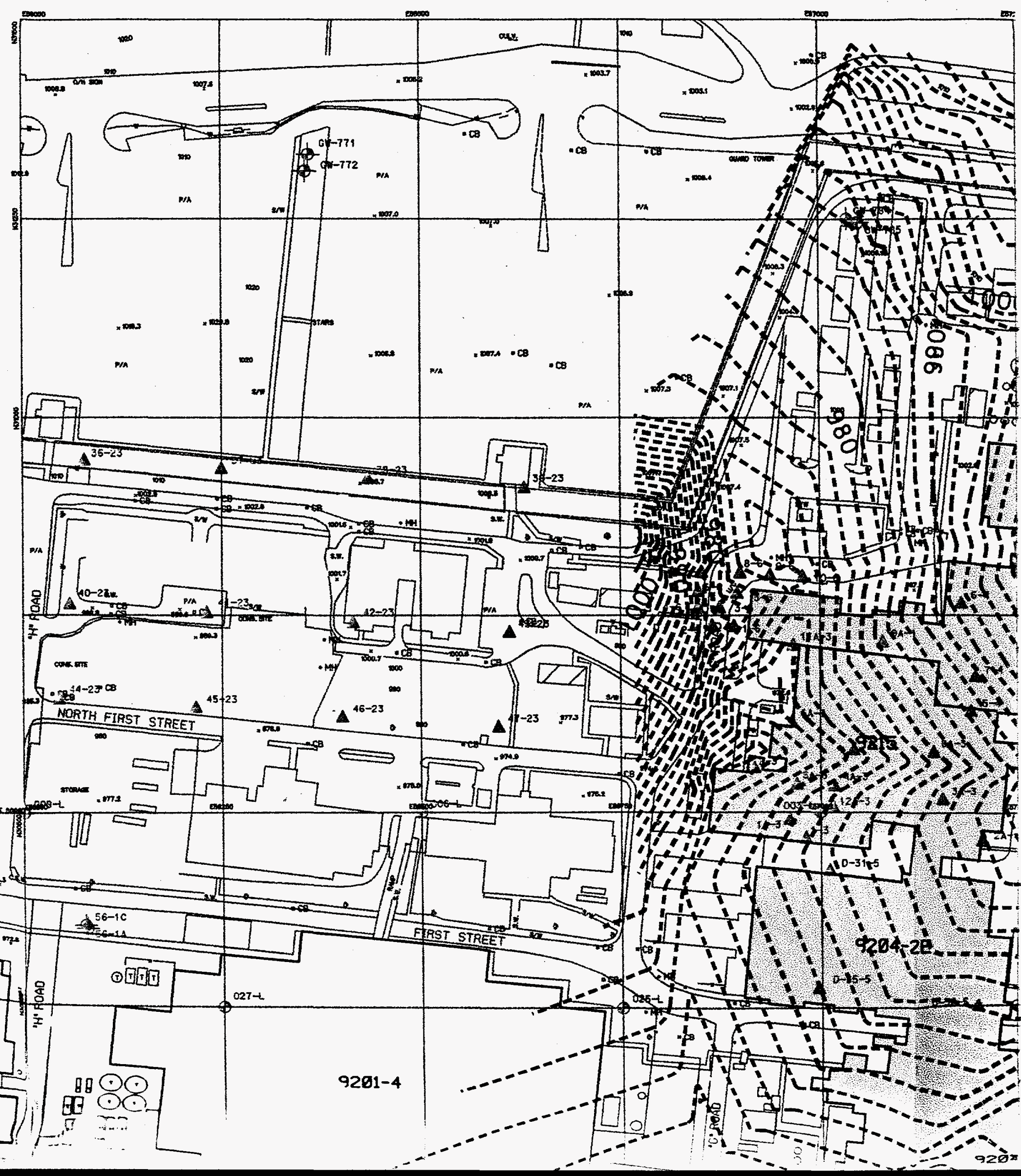




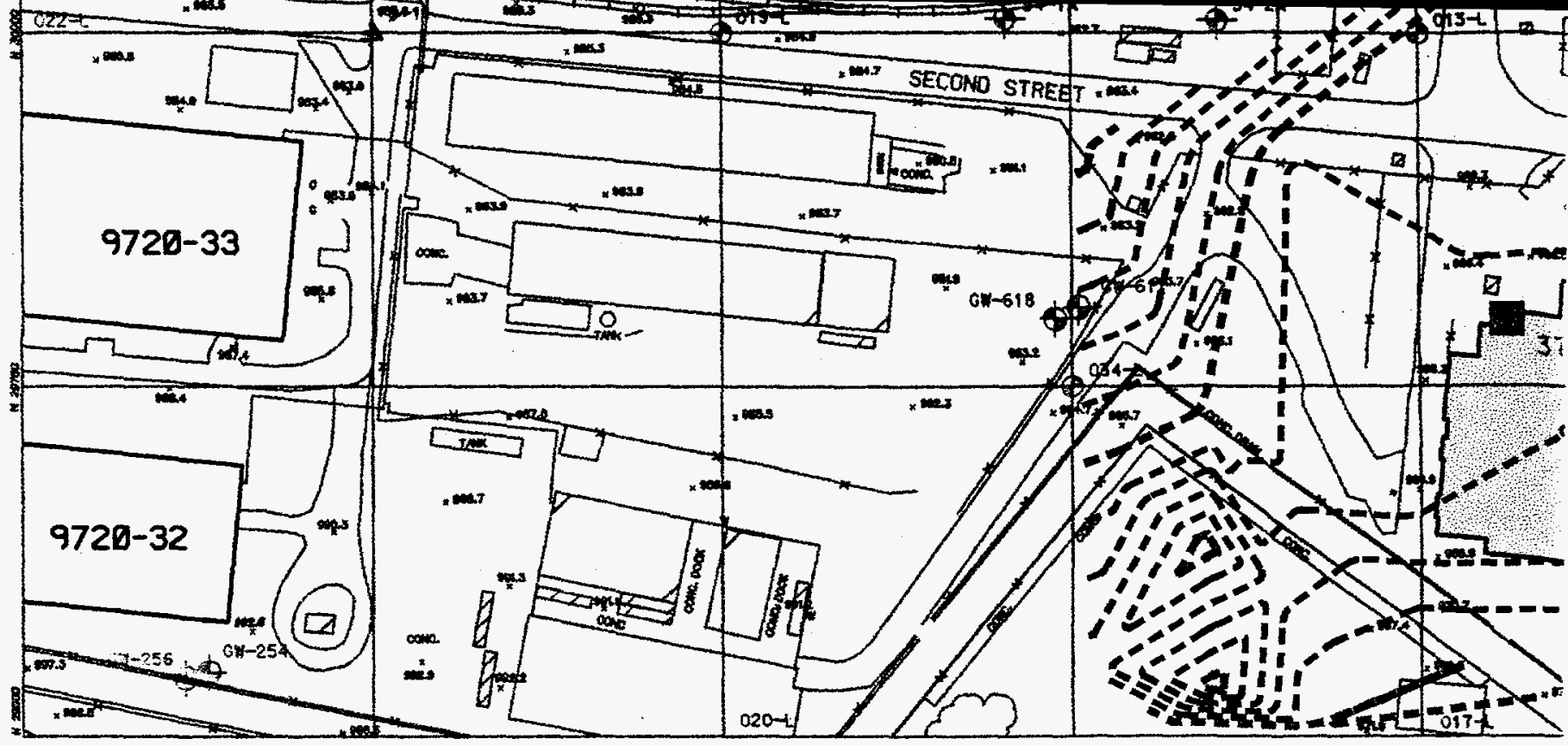

Document Rev. No.: EG-C-37 Review By: Guideline Document:

UCN-19586 (12356 4-93)

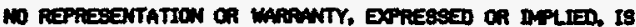

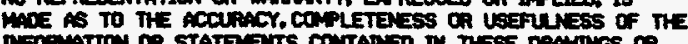

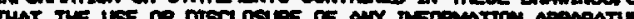

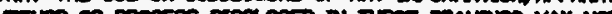

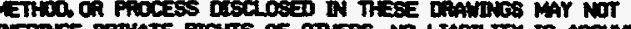

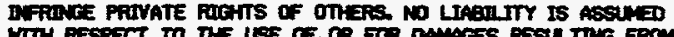

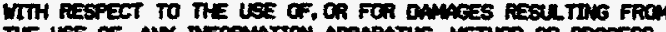

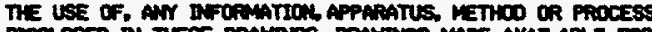

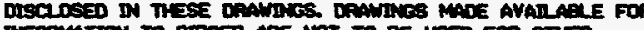

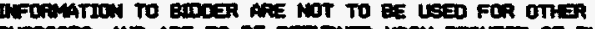

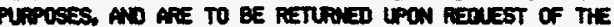
Fonifarions CONTRACTOR
Dato:

\section{THIS DOCUMENT CONTROLLED BY}

CHANGE CONTROL SYSTEM

ENGINEERING

PROCEDURE

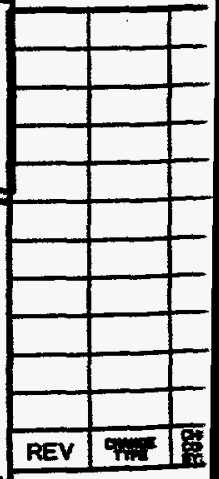




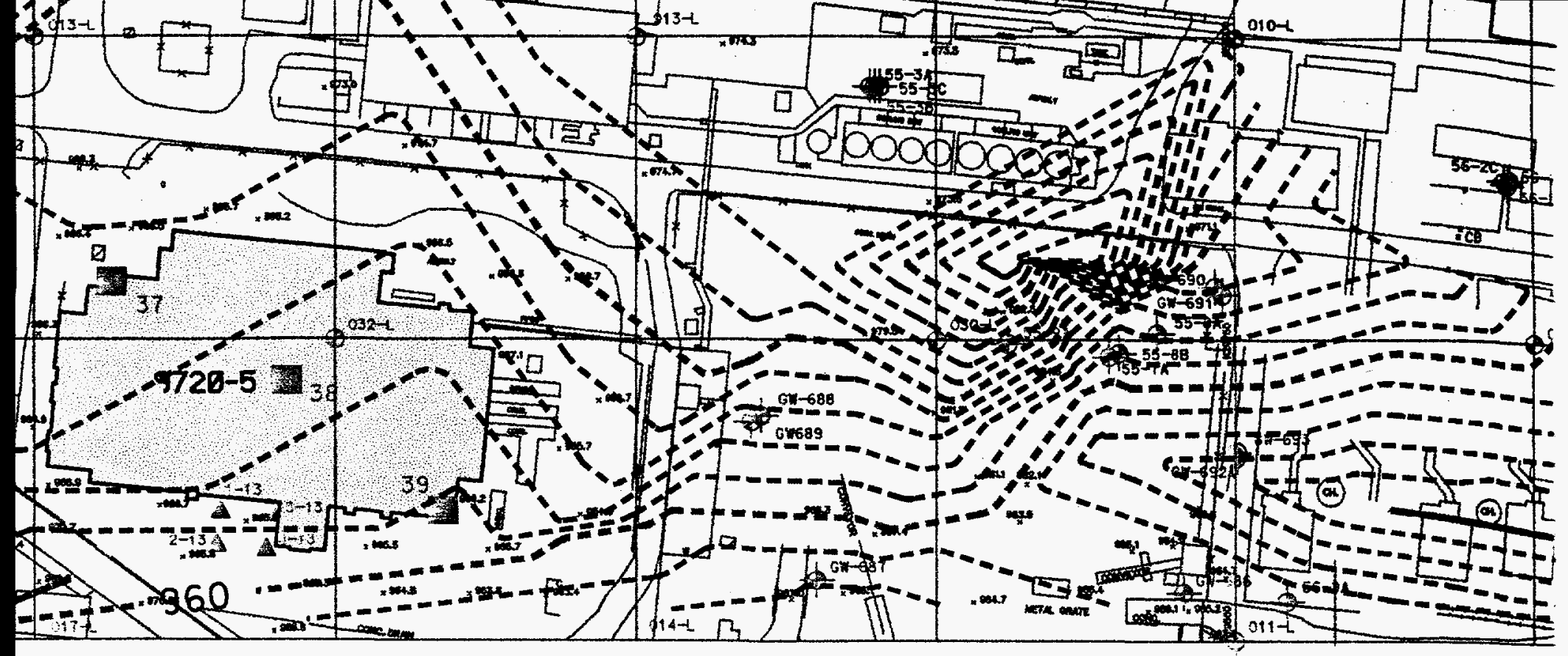

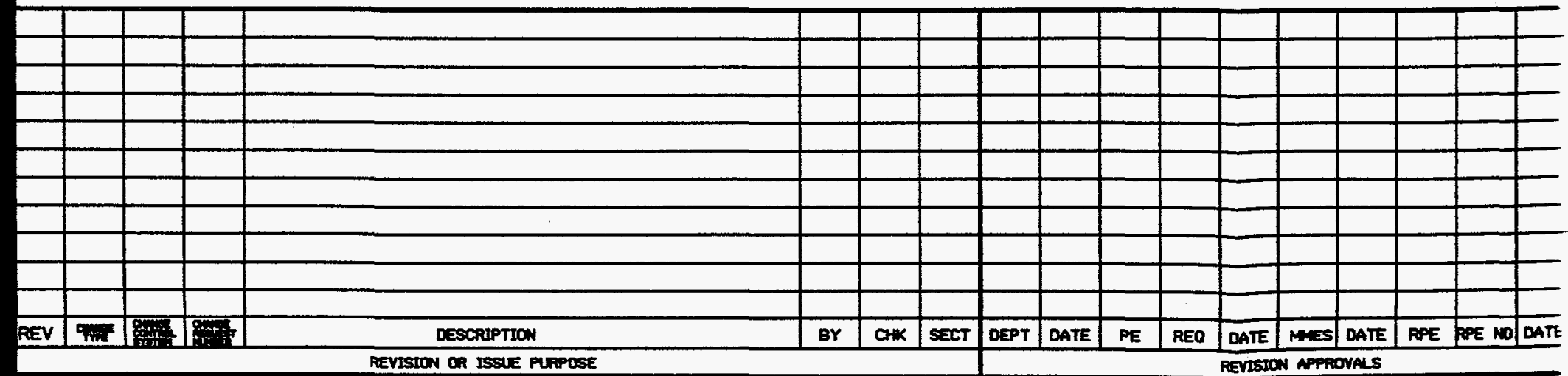




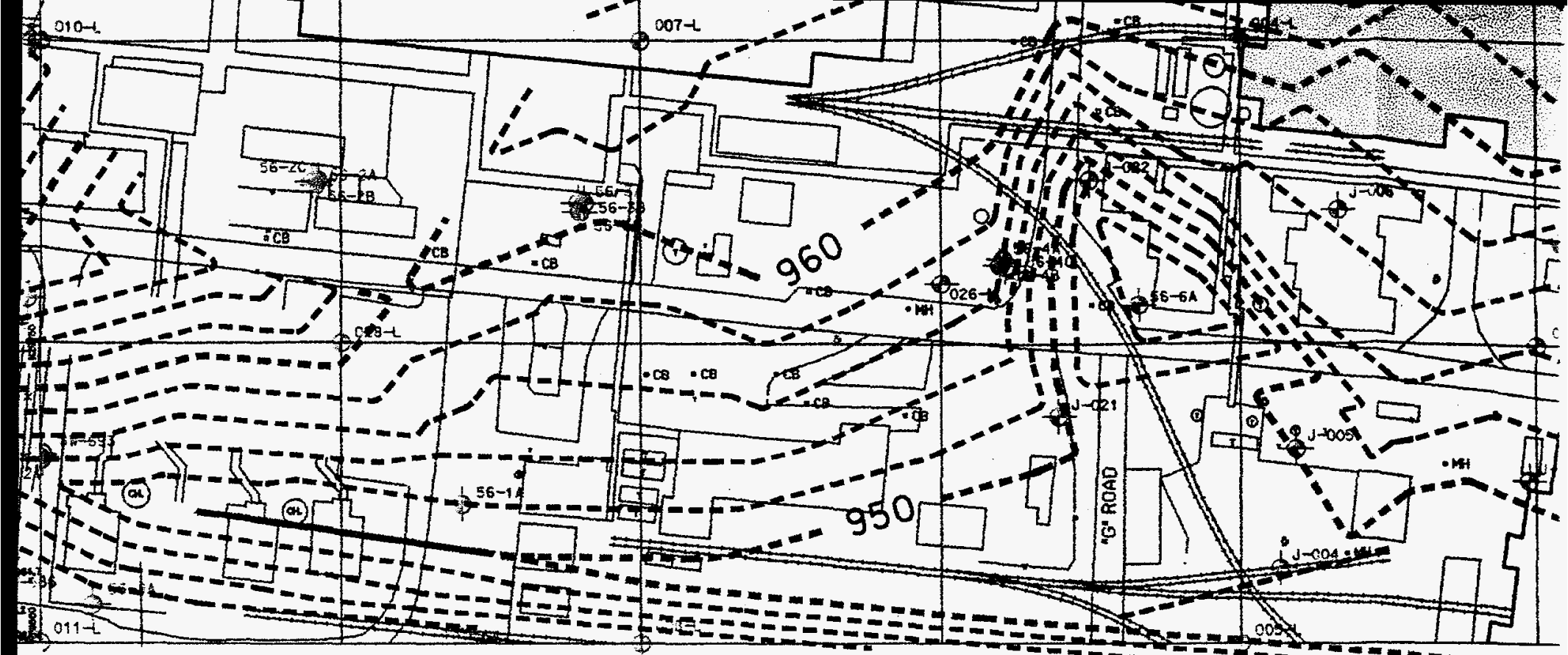



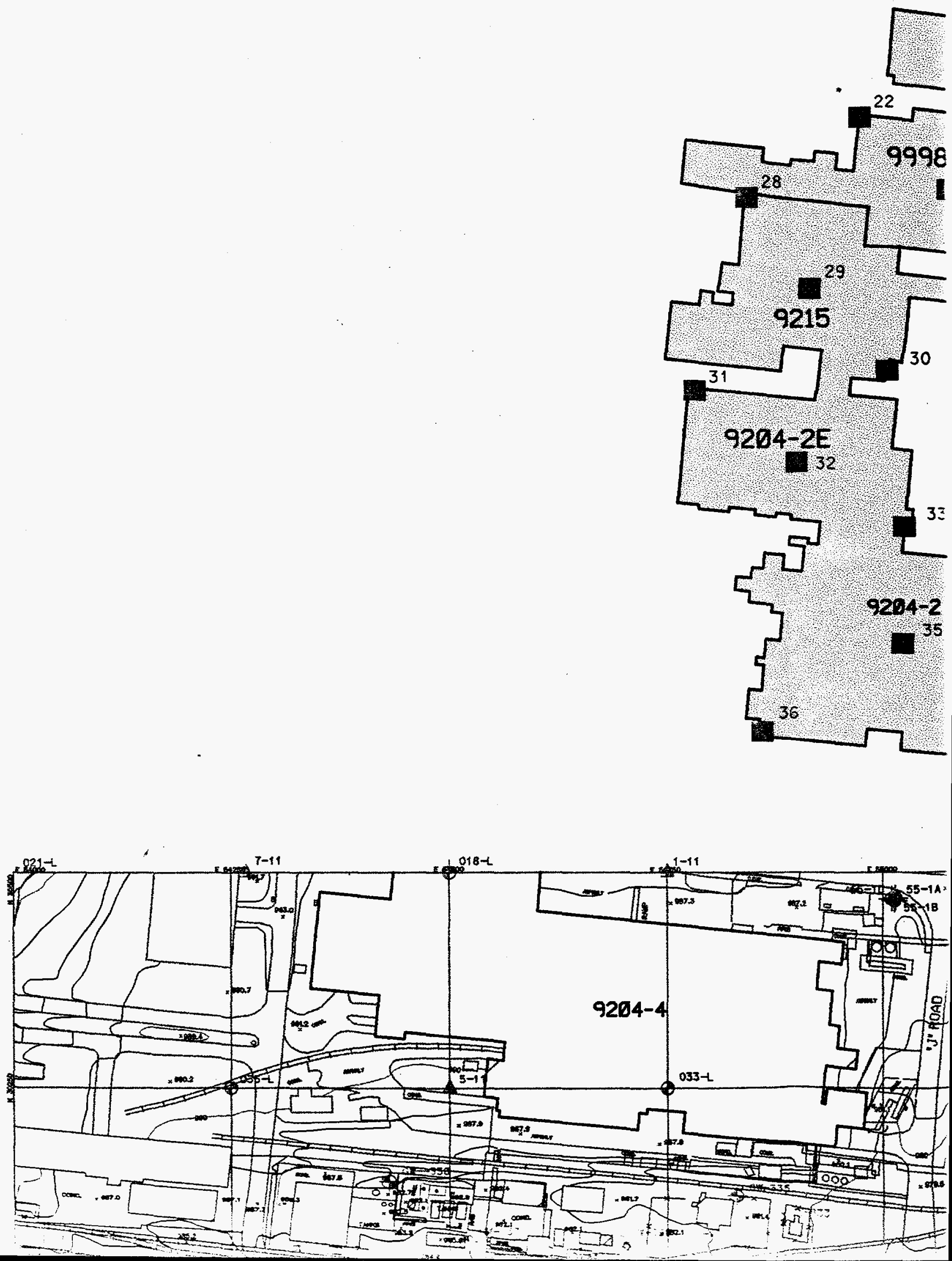


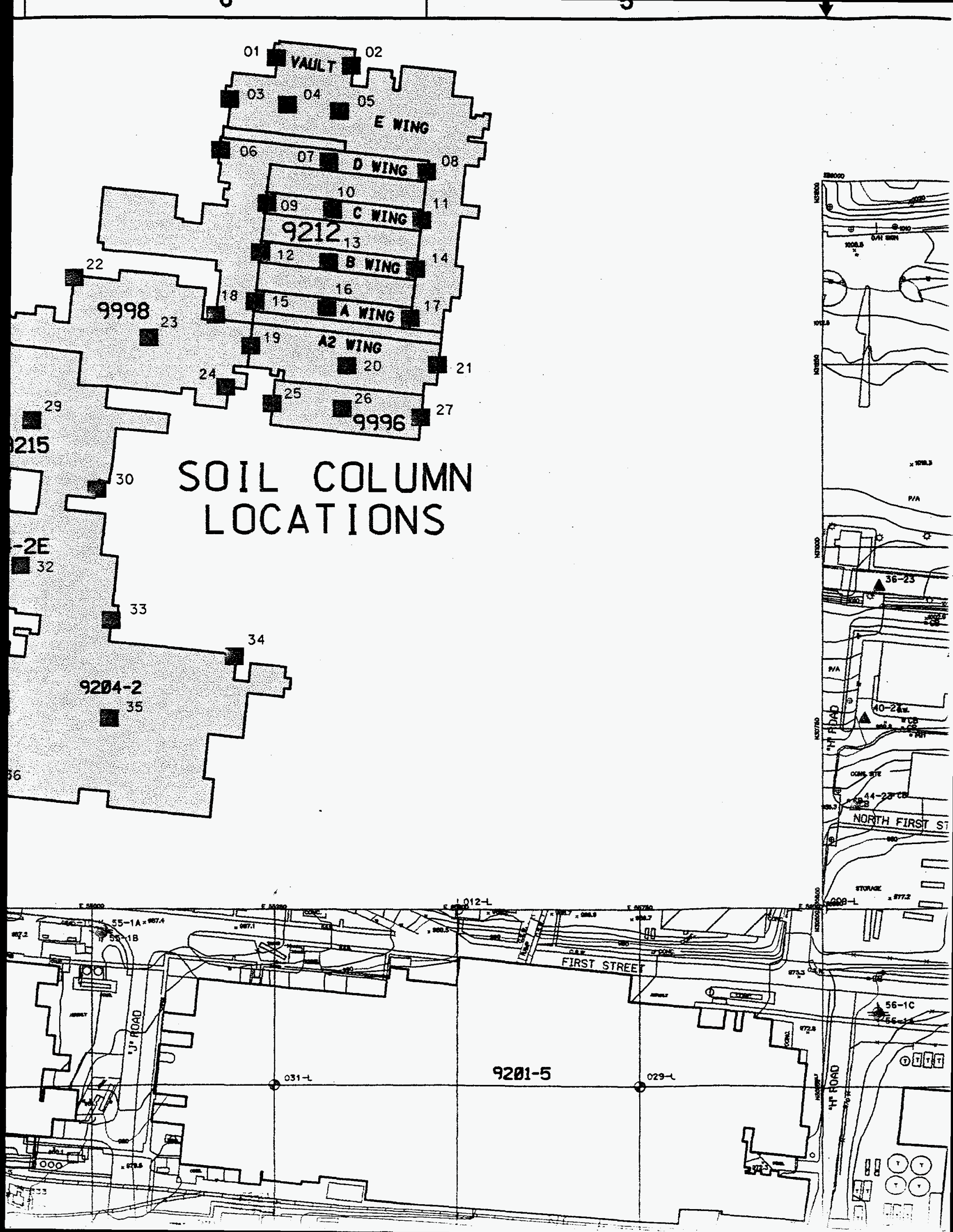




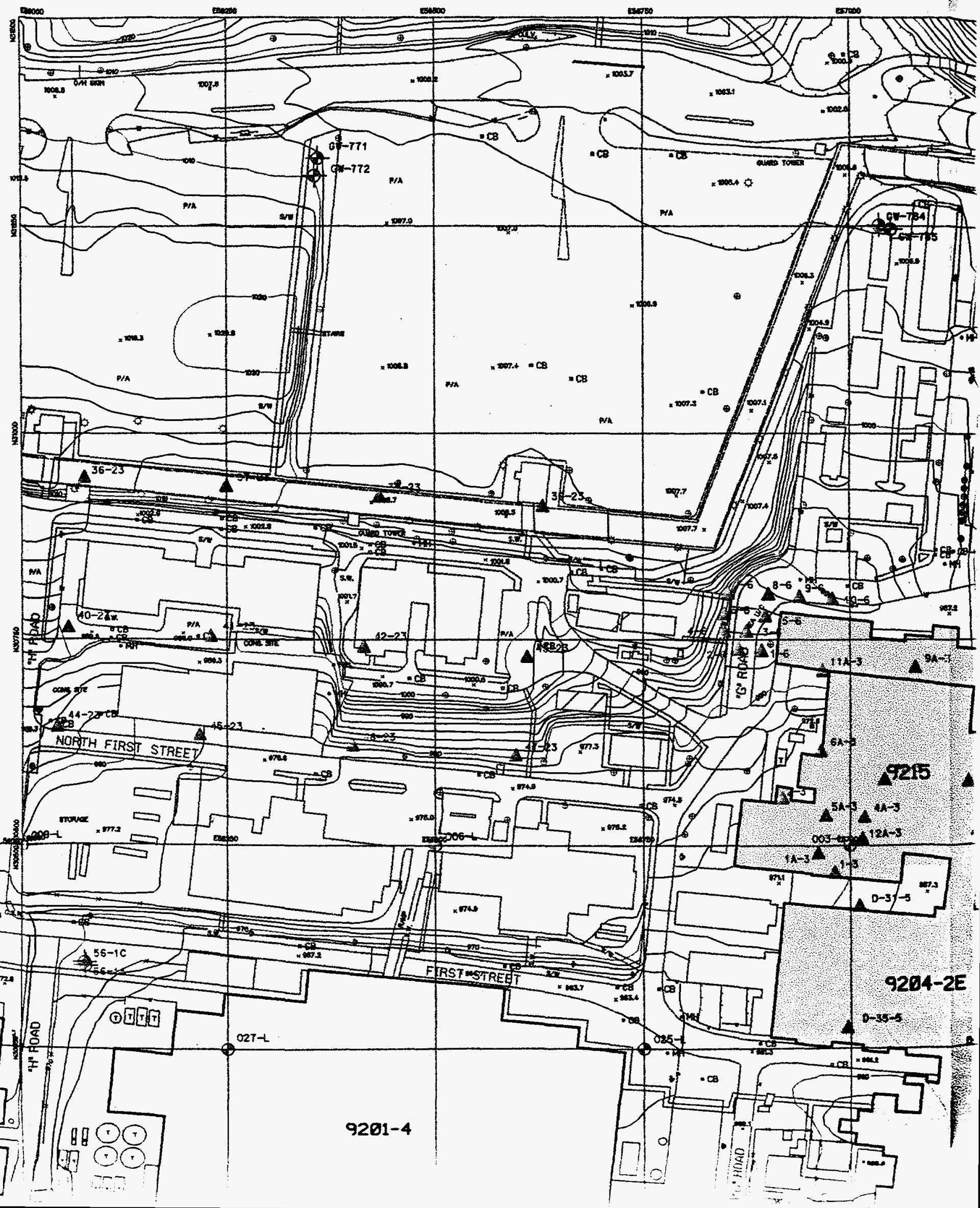




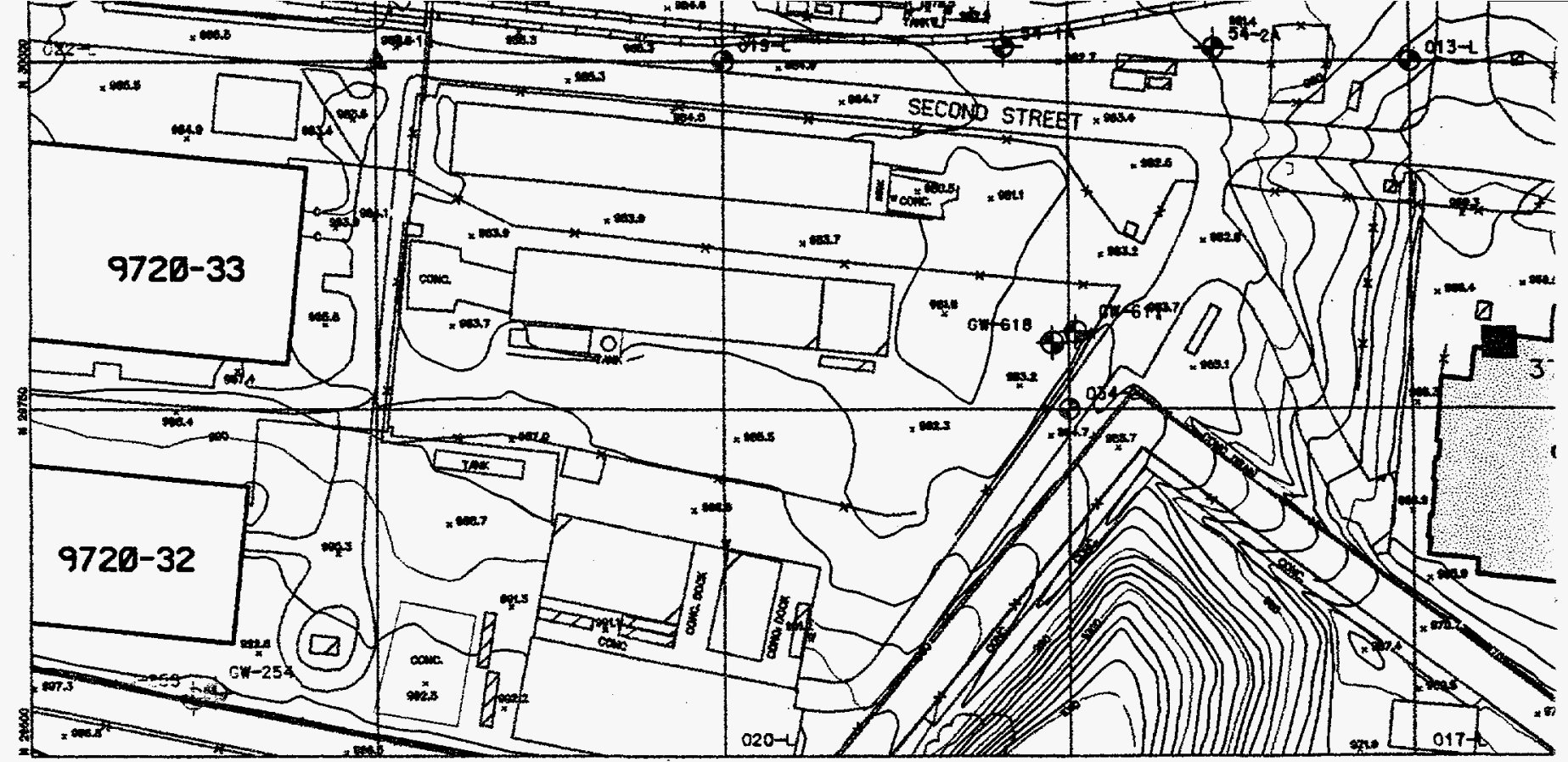

No RePRESENTATION OR MARRANTY, EXPRESSED OR MPLLED, IS MOE AS TO THE ACCARACY, COMPLETENESS OR USERUVESS OF THE DFCOMATION OR STATEMENTS CONTADYD IN THESE DRAWBNOS, OR THAT THE USE OR DISEL OSUFE OF ANY DFOOMATION APPARATLS EeTHOO, OR PROCESS DISCLOSED DN THESE DRAMNOS MaY hOT

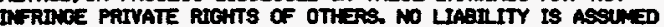
VITH FESPECT TO THE USE OF, OR FOR DAMAGES RESTR TING FROO ThE USE OF, ANY DIFORMATIOL, APPARATUS, METHOO OR PFDCCESS

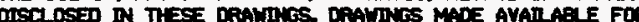

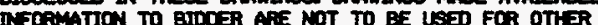

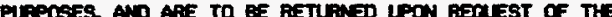
FOMAAOONG CONTRACTO
Document Rev. No.: EG-C-37 Review By: Gurdeline Document: UCN-19586 $\quad 12356$

SEMER of
SECTION AND DETAIL KEY
SETIII

Date:

4-93)
THIS DOCUMENT CONTROLLED BY

CHANGE CONTROL SYSTEM

ENGINEERING PROCEDURE

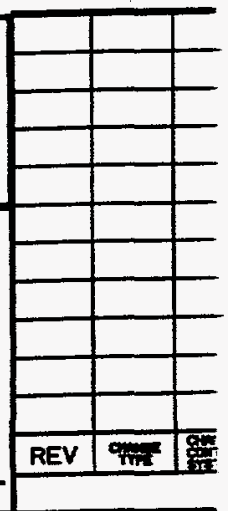




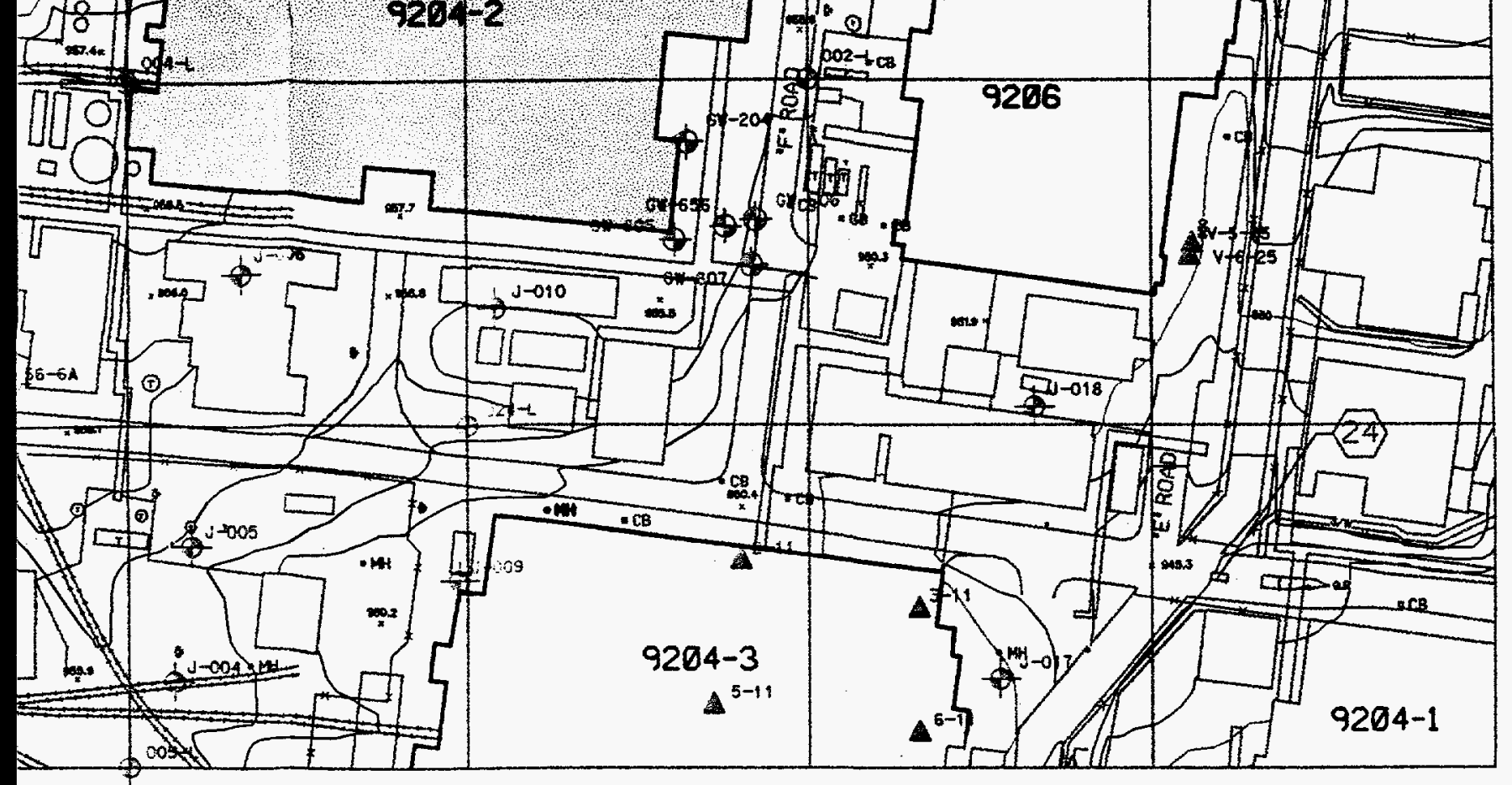

D

\section{SCIL BORING REFERENCES}

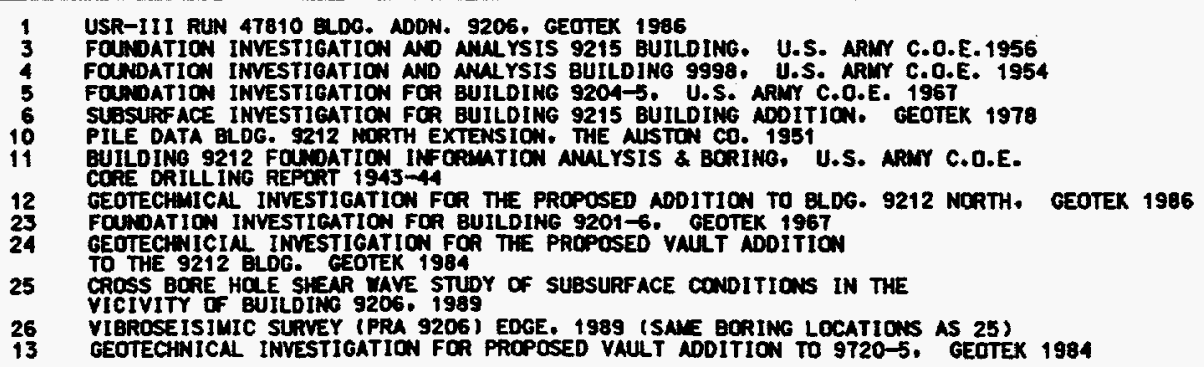

\section{MONITORING WELL REFERENCES}

UPDATEO SUBSURFACE DATA BASE FOA BEAR CREEX VALLEY. CHESTMUT RIDGE ALD PARTS OF BETHEL VALLEY REVISION 2. OCTOBER 1993

CALANOER YEAR 1993 GROUHDWATER OUALITY REPORT FOR THE UPPER EAST FORK POPLAR CREEK HYDROLOGIC REGINE. Y-12 PLANT FEBRUARY 1994

\section{SOUNDING REFERENCES}

ADOITION TO BUILDING 9212 SOMOING LOCATIONS A-1 AND 8-1 WINGS DRAWING E2F12902. 1950

\section{LEGEND}

SOIL COLUMN LOCATION
SOUNDING
SOIL BORING
SONITORING WELL
PILETED LORIVEN
EXISTING CONTOURS

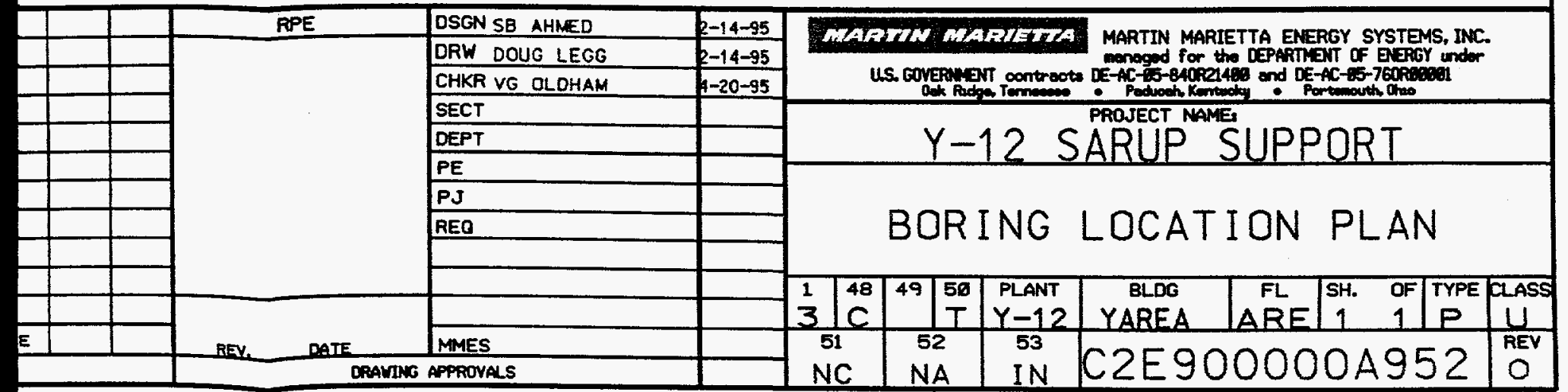

